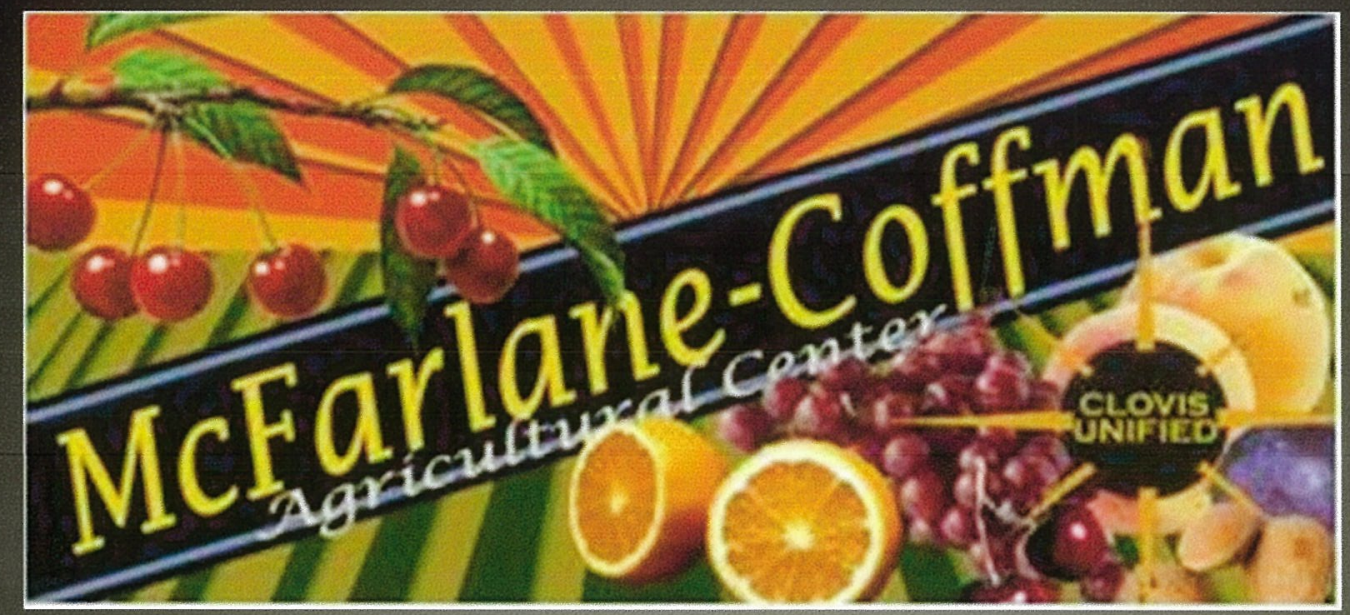

Ag Ed 539

Teacher Internship

Report

Jennifer A. Knight

Clovis East High School

Agriculture Department

Cal Poly San Luis obispo

Agriculture Education Masters

Program

Spring 2013

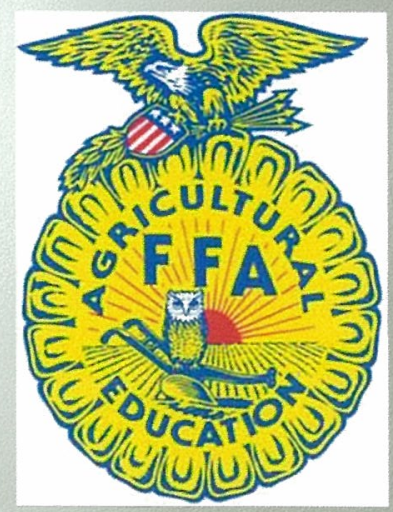




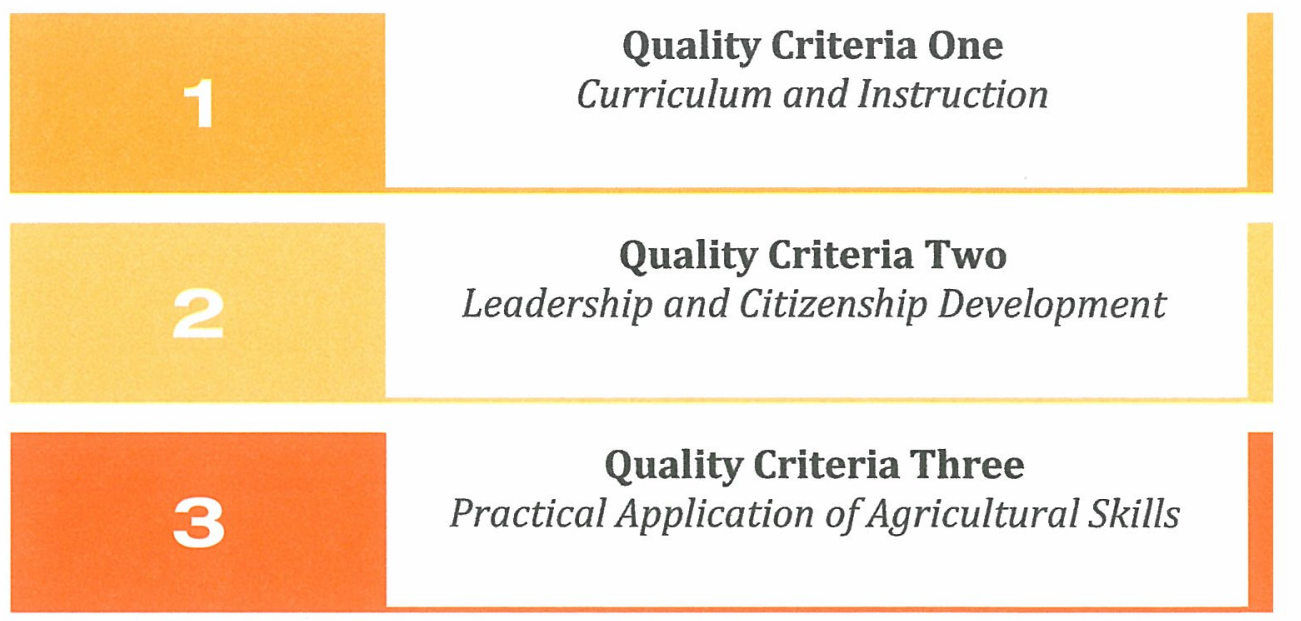

Quality Criteria One
Curriculum and Instruction

Quality Criteria Two

Leadership and Citizenship Development

$4 . \quad$ Qualified and Professional Personnel

5 Facilities, Equipment and Materials

Quality Criteria Five
ties, Equipment and Materials

Quality Criteria Six

Community, Business, and Industry Involvement

Quality Criteria Seven

Career Guidiance

7

Career Guidiance

Quality Criteria Eight

Program Promotion

8

Program Promotion

Quality Criteria Nine

9

Program Accountability and Planning

10

Quality Criteria Ten

Student to Teacher Ratio

11

Quality Criteria Eleven

Full Year Employment 


\section{Quality Criteria One}

\section{Curriculum and Instruction}

At the McFarlane Coffman Agriculture Center we offer a comprehensive agriculture experience for students from all Clovis Unified High Schools. Students are encouraged to select an agriculture career pathway that may relate to their career goal. The Pathways that we offer for our students are: Agrisceince, Agriculture Mechanics and Engineering, Animal Science, and Horticulture. Our schools master schedule is designed so students can take courses in a pathway sequence throughout their high school career. Students from other CUSD campuses can also set up their schedules so they can be over at Clovis East for one or more Ag Classes at a time. Our biggest weakness in this area would be that because we have five High Schools that we serve keeping all the counselors informed about our program and how the pathways operate.

Once a student is enrolled in an agriculture course they will be exposed to career opportunities in agriculture either by career instruction being integrated into the curriculum or by experiencing hands on learning at our Agriculture Center. As opportunities arise at the Ag Center we always take our students out to lean and see what other opportunities exist in the Agriculture field. For example we have the students watch the almonds being harvested in the fall with the shaker, watching the hoof trimmer come out and trim the cattle, or have them help install irrigation and grass in our new Ag Park. Our ROP classes also take students to the Career Skills Challenge event at Fresno City College. ROP courses also require students to create a professional portfolio and use it as a tool to seek job placement in the community. Guest speakers are also used in class time as a link to the community and as a way to spark interest in exciting agricultural careers while reinforcing the need for skills taught in school. I have in my classes assigned a career research paper, or interview assignment related to careers. I do see an need to improve career education in our department especially in our core science classes, but this may always remain a challenge with standards based assessments, benchmarks, and pacing guides. 
All students enrolled in Agriculture Education at the Ag Center will receive a grade for participation in FFA and SAE. This will be evidenced by the completion of the FFA Record Book. Participation in FFA and SAE will count as $10 \%$ of the student's grade in their Ag Classes. We are currently working on improving consistency, expectations, and scoring methods for this $10 \%$ of a student's grade. We would like our students to know with clarity what is expected as far as FFA and SAE participation and that it will be scored the same way no matter what Ag Class or Ag Teacher they have year to year. I am currently working with the staff on developing a standardized rubric for grading participation and the record books.

Technology is preferred as method for keeping FFA Record Books. Students are encouraged to complete record books on the e-record book. The biggest challenge we have is students seem to loøse previous years' books and when it comes time to fill out degree applications they are missing books. We are looking forward to the online FFA record book as a solution to this problem. As for now we will keep on file any paper books a student submits or save a copy on our computer of the kids books we have in our classes or SAE supervision areas. Twice during the year we also provide class time with laptops for students to work on their FFA record books. We also have record book workshops after school for students who need time to work on them or need our individual help.

We also aim to use technology throughout our agriculture program and facility. Students have access to laptops in each class, and can use the internet for projects and research. Our district also has a special Google sites login for staff and students where documents can be shared and lesson materials made available to students during non-class hours. At the farm facility technology is also used and applied; we have four technology driven greenhouses, climate controlled livestock barns, and environmental growth chambers.

We currently have four courses that receive A-G credit for the University Admission, Ag Biology, AP Environmental Science, Plant and Animal Physiology, and Botany. We also were successful in submitting two new courses for the upcoming school year; Floral Design is now approved and receives UC fine art credit, and Food Science which will receive 


\section{Ag Ed 539 Teacher Internship Report}

elective credit. We will continue to try and build up both of these courses and in the future if we add a food science lab on our facility we can try to submit for lab science credit for that course.

\section{Supporting Completion Materials}

Items A \& B- Agriculture Pathway and incoming upperclassmen course pathway map.

Items C,D,E, \& F- Various course outlines for the courses I taught this year. Applications for UC Certification of Agriculture Courses. I created and submitted the one for Food Science this year and will be teaching it next school year.

Item G- UC A-G requirements and list of Agriculture courses that meet these requirements.

Item H- Request for outside speaker form. I used this form to bring in several guest speakers this year. The speakers were one Animal Chiropractor and a Vet Tech to talk about career opportunities with my Vet Science students.

Item I- Targeted Occupational Clusters. Lists potential career fields students may be prepared for when they complete an agriculture course or pathway.

Item J- Pictures showing how the curriculum integrates career readiness and computer aided instruction. These are pictures showing the professional portfolios created by my students. They complete a Resume, Cover Letter, Job Application, go through a mock interview, and write a letter of introduction. They use my set of classroom laptop computers to complete this large final assignment. They also select a career and do research and write a paper about that career and what college/schooling is necessary for that type of career.

Item K- Screen shot of the Clovis FFA Webpage. We use technology as a way to reach members and parents anywhere, anytime. FFA results and applications are posted here.

Item L- FFA E Record Book Cover Page, included to show that E-Record books are utilized as part of the Ag Education Program. 


\section{Ag Ed 539 Teacher Internship Report}

Item M- Sheep SAE project completion and fair check sign off sheet. Included to show that Record Book completion and close out is required for students to receive a fair check.

Item N- Course Syllabi approved by the Agriculture Department Learning Director.

Discusses that Ag education is comprehensive and requires participation in FFA/SAE. Also mentions the creation of career documents such as Resume and Cover letter. 


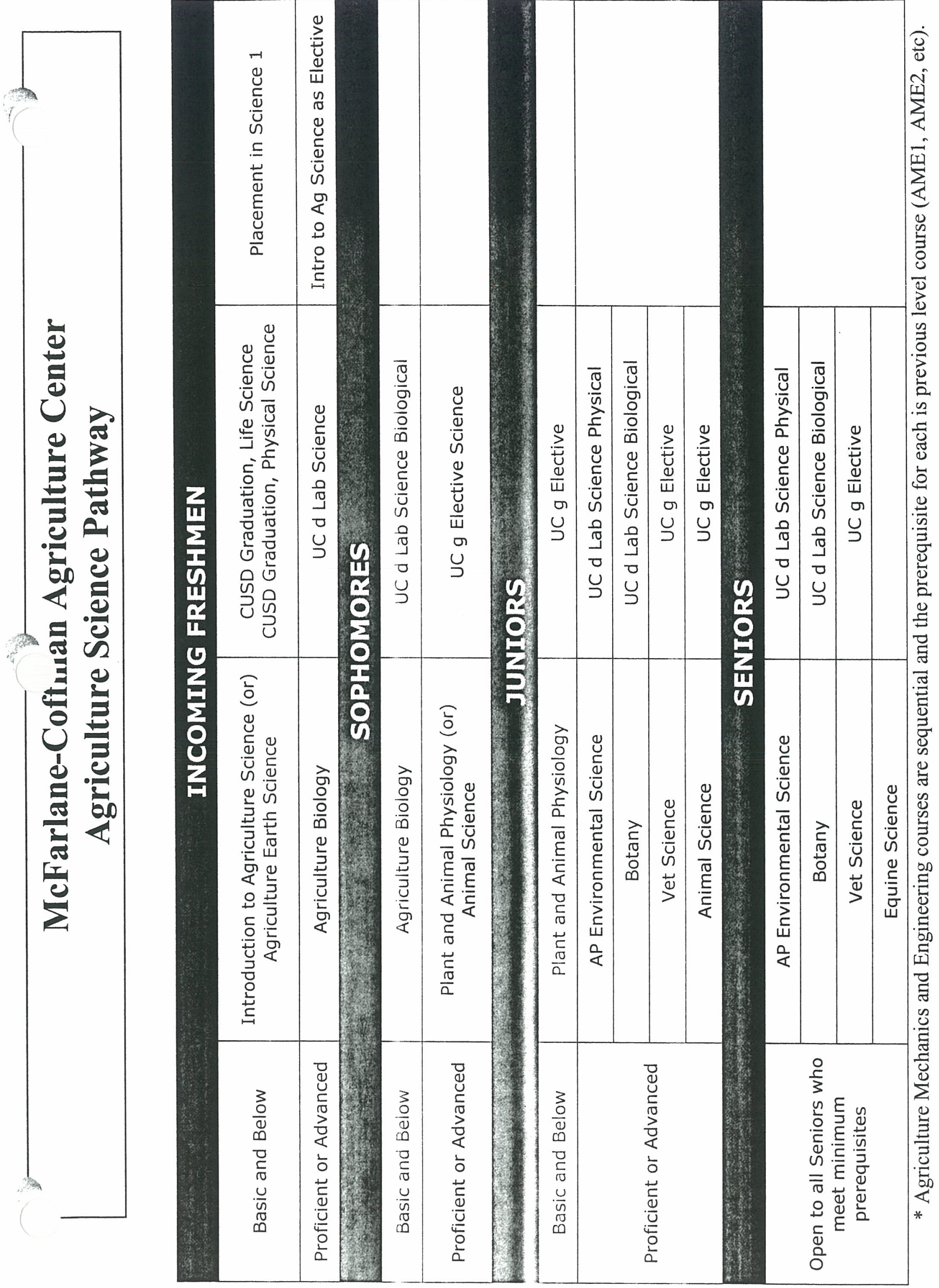




\section{Agriculture Pathways for Incoming Students 2010-11}

$7^{\text {th }}$ and $8^{\text {th }}$ Graders

Exploratory Agriculture

Incoming $9^{\text {th }}$ Graders

Agriculture Earth Science* (meets physical science for CUSD)

Introduction to Agriculture Science (meets life science for CUSD) AME 1

Ag Leadership

Incoming $10^{\text {th }}$ Graders

Agriculture Biology (meets U.C. Biology, lab D)

Plant and Animal Physiology (meets U.C. Elective, G)

Ornamental Horticulture

$\rightarrow$ AME 2 (meets U.C Elective, G)

Ag Leadership

Incoming $11^{\text {th }}$ and $12^{\text {th }}$ Graders

AP Environmental Science (meets U.C. Physical, lab D)

Botany (meets U.C. Biology, lab D)

AME 3

AME 4

Advance Animal Science

Vet Science ROP (meets U.C Elective, G)

Ag Engineering ROP

Ag Leadership

Agriculture Economics and Government (12 $2^{\text {th }}$ only)*

McFarlane-Coffman Agriculture Center

CLOVIS UNIFIED SCHOOL DISTRICT

*Upon approval 


\section{School Name: Clovis Unified Agriculture \\ Course Name: Botany \\ Course Instructor:Aireal Covey \\ Date: $2009-2010$

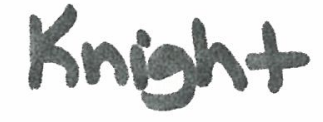

\begin{tabular}{|c|c|c|c|c|c|}
\hline 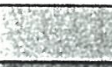 & 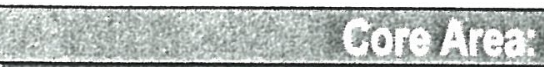 & Arigul & ne. & $2 x^{2} \times x^{2}$ & 36. \\
\hline 58 & 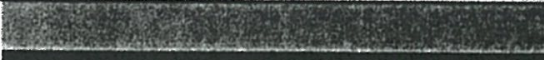 & Califo & mia Core Area & Ag \& Acad Stan & dards \\
\hline 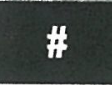 & Lesson Title & $\begin{array}{l}\mathrm{CA} \mathrm{Ag} \\
\text { Standard }\end{array}$ & CA Acad Topic & Sub Topic & Standard \\
\hline & Core Area UNIT 110: ECON & LIFORN & IA AGRICUL & TURE & \\
\hline & Core Area Topic & Local Proc & duction & & \\
\hline $\begin{array}{l}\text { Lesson } \\
\text { 111a: }\end{array}$ & Understanding State and Local Production & \begin{tabular}{|l} 
BC $1.1 / /$ \\
CMT 8.1 \\
// CMT \\
$8.2 / /$ \\
CMT 8.3
\end{tabular} & $\begin{array}{c}\text { LA- } \\
\text { ListenSpeak.9- } \\
\text { 12, LA- } \\
\text { Writing.9-12 }\end{array}$ & \begin{tabular}{|c} 
Speaking \\
Applications \\
(Genres and \\
Their \\
Characteristics) \\
// Writing \\
Strategies
\end{tabular} & $\begin{array}{c}\text { LS2.2// } \\
\text { W1.1 }\end{array}$ \\
\hline
\end{tabular}

Core Area Topic 112: California Agriculture and the National Economy

\begin{tabular}{|c|c|c|c|c|c|}
\hline & & & & & \\
\hline $\begin{array}{l}\text { Lesson } \\
\text { 112a: }\end{array}$ & $\begin{array}{l}\text { Understanding California Agriculture and the National } \\
\text { Economy }\end{array}$ & \begin{tabular}{|l} 
BC $1.1 / \|$ \\
CMT 8.1 \\
/ CMT \\
$8.2 / 1$ \\
CMT 8.3 \\
\end{tabular} & LA-Writing.9-12 & $\begin{array}{l}\text { Writing } \\
\text { Strategies }\end{array}$ & W1.1 \\
\hline & Core Area UNIT 120: AGRIC & JRE AN & D SOCIETY & & \\
\hline
\end{tabular}

Core Area Topic 121: Development of Ag and Our Society

\begin{tabular}{|c|l|c|c|c|c|}
\hline $\begin{array}{c}\text { Lesson } \\
121 \mathrm{~b}:\end{array}$ & Understanding the Imporance of Agriculture and Society & $\mathrm{BC} 1.1$ & $\begin{array}{c}\text { LA-Reading.9- } \\
12\end{array}$ & $\begin{array}{c}\text { Literary } \\
\text { Response and } \\
\text { Analysis }\end{array}$ & R3.3 \\
\hline $\begin{array}{c}\text { Lesson } \\
121 \mathrm{c}:\end{array}$ & Development of Agriculture and Society & $\mathrm{BC} 1.1$ & $\begin{array}{c}\text { LA-Reading.9- } \\
12\end{array}$ & $\begin{array}{c}\text { Reading } \\
\text { Comprehension } \\
\text { (Focus on } \\
\text { Informational } \\
\text { Materials) }\end{array}$ & R2.2 \\
\hline
\end{tabular}

Core Area Topic 122: Agricultural Trends

\begin{tabular}{|c|l|c|c|c|c|}
\hline $\begin{array}{c}\text { Lesson } \\
\text { 122a: }\end{array}$ & Determining Trends in Agriculture & BC 1.1 & $\begin{array}{c}\text { Sci-Biology.9- } \\
12\end{array}$ & Genetics & GEN.5 \\
\hline
\end{tabular}

Core Area Topic 123: Agencies for Agriculture

\begin{tabular}{|c|l|l|l|c|c|}
\hline $\begin{array}{c}\text { Lesson } \\
\text { 123a: }\end{array}$ & Agencies for Agriculture & AB 4.7 & $\begin{array}{c}\text { LA-Reading.9- } \\
12\end{array}$ & $\begin{array}{c}\text { Reading } \\
\text { omprehension } \\
\text { (Focus on } \\
\text { Informational } \\
\text { Materials) }\end{array}$ & R2.2 \\
\hline \multicolumn{2}{|c|}{ Core Area UNIT 130: AGRICULTURE AND CALIFORNIA RESOURCES } \\
\hline
\end{tabular}

Core Area Topic 131: Ag Resources

\begin{tabular}{|c|l|c|c|c|c|}
\hline $\begin{array}{c}\text { Lesson } \\
\text { 131a: }\end{array}$ & $\begin{array}{l}\text { Determining the Kinds and Importance of Natural } \\
\text { Resources }\end{array}$ & BC 1.2 & $\begin{array}{c}\text { Sci- } \\
\text { EarthScience.9 } \\
12\end{array}$ & $\begin{array}{c}\text { Biogeochemical } \\
\text { Cycles }\end{array}$ & BC.7 \\
\hline $\begin{array}{c}\text { Lesson } \\
\text { 131b: }\end{array}$ & $\begin{array}{l}\text { Understanding California's Agriculture and Natural } \\
\text { Resources }\end{array}$ & $\begin{array}{c}\text { NR 7.3/1/ } \\
\text { NR 7.5 // } \\
\text { NR 7.8 }\end{array}$ & $\begin{array}{c}\text { Eci- } \\
\text { Science.9- } \\
12\end{array}$ & $\begin{array}{c}\text { Biogeochemical } \\
\text { Cycles }\end{array}$ & BC.7 \\
\hline
\end{tabular}


Core Area Topic 132: Agriculture and the Environment

\begin{tabular}{|c|c|c|c|c|c|}
\hline $\begin{array}{c}\text { Lesson } \\
\text { 132a: }\end{array}$ & Understanding Agriculture and the Environment & $\begin{array}{l}\mathrm{BC} 1.2 / / \\
\mathrm{BC} 1.19 \\
/ / \mathrm{NR} 7.2\end{array}$ & \begin{tabular}{|c} 
Sci- \\
EarthScience.9. \\
12
\end{tabular} & \begin{tabular}{|c|} 
Structure and \\
Composition of \\
the Atmosphere
\end{tabular} & SCA.8 \\
\hline
\end{tabular}

\begin{tabular}{|c|l|c|c|c|c|}
\hline \multicolumn{5}{|c|}{ Core Area Topic 133: Energy and the Environment } \\
\hline $\begin{array}{c}\text { Lesson } \\
\text { 133a: }\end{array}$ & $\begin{array}{l}\text { Determining the Kinds and Importance fo Natural } \\
\text { Resources }\end{array}$ & BC 1.2 & $\begin{array}{c}\text { Sci- } \\
\text { EarthScience.9- } \\
12\end{array}$ & $\begin{array}{c}\text { Biogeochemical } \\
\text { Cycles }\end{array}$ & BC.7 \\
\hline $\begin{array}{c}\text { Lesson } \\
\text { 133b: }\end{array}$ & Determining Energy Use in Agriculture & NR 7.2 & LA-Writing.9-12 & $\begin{array}{c}\text { Writing } \\
\text { Strategies }\end{array}$ & W1.1 \\
\hline
\end{tabular}

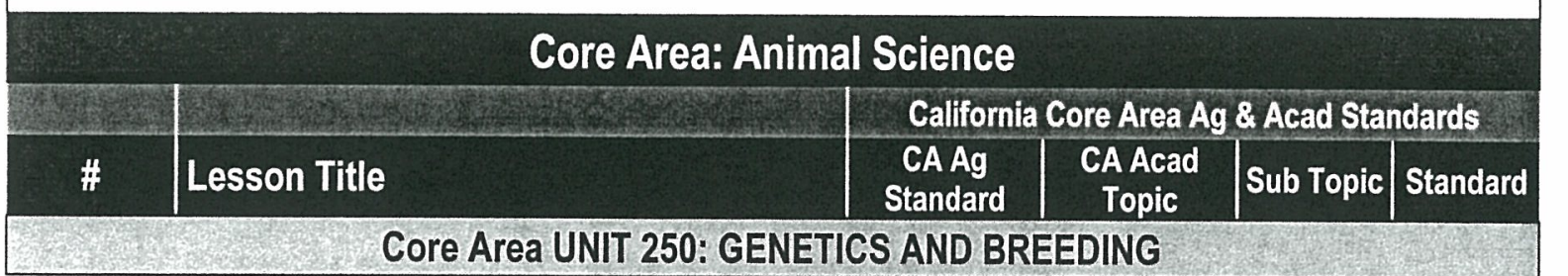

Core Area Topic 251: Fertilization

\begin{tabular}{|c|l|c|c|c|c|}
\hline $\begin{array}{c}\text { Lesson } \\
251 \mathrm{~d}:\end{array}$ & Understanding Genetics & $\begin{array}{c}\text { AS 2.5// BC } \\
1.13\end{array}$ & $\begin{array}{c}\text { Sci-Biology.9- } \\
12\end{array}$ & $\begin{array}{c}\text { Evolution, } \\
\text { Genetics }\end{array}$ & $\begin{array}{c}\text { EVOL.8, } \\
\text { GEN.2, } \\
\text { GEN.4, } \\
\text { GEN.5 }\end{array}$ \\
\hline $\begin{array}{c}\text { Lesson } \\
251 \mathrm{e}:\end{array}$ & Understanding Molecular Biotechnology & AS 2.5 & $\begin{array}{c}\text { Sci-Biology.9- } \\
12\end{array}$ & $\begin{array}{c}\text { Evolution, } \\
\text { Genetics }\end{array}$ & $\begin{array}{c}\text { EVOL.8, } \\
\text { GEN.5 }\end{array}$ \\
\hline Lesson 251f: & Understanding Organismic Biotechnology & $\begin{array}{c}\text { AS 2.4 // BC } \\
1.15 / / \text { BC 1.15 }\end{array}$ & $\begin{array}{c}\text { Sci-Biology.9- } \\
12\end{array}$ & Genetics & GEN.5 \\
\hline
\end{tabular}

\begin{tabular}{|c|c|c|c|c|c|}
\hline \multicolumn{6}{|c|}{ Core Area Topic 252: Inheritance } \\
\hline $\begin{array}{l}\text { Lesson } \\
\text { 252d: }\end{array}$ & Understanding Genetics & $\begin{array}{c}\text { AS } 2.5 / / B C \\
1.13\end{array}$ & $\begin{array}{c}\text { Sci-Biology.9- } \\
12\end{array}$ & $\begin{array}{l}\text { Evolution, } \\
\text { Genetics }\end{array}$ & $\begin{array}{l}\text { EVOL.8, } \\
\text { GEN.2, } \\
\text { GEN.4, } \\
\text { GEN.5 }\end{array}$ \\
\hline $\begin{array}{l}\text { Lesson } \\
\text { 252e: }\end{array}$ & Understanding Molecular Biotechnology & AS 2.5 & \begin{tabular}{|c|} 
Sci-Biology.9- \\
12 \\
\end{tabular} & $\begin{array}{l}\text { Evolution, } \\
\text { Genetics }\end{array}$ & $\begin{array}{c}\text { EVOL.8, } \\
\text { GEN.5 }\end{array}$ \\
\hline Lesson $252 f$ & Understanding Organismic Biotechnology & $\begin{array}{c}\text { AS } 2.4 / / \mathrm{BC} \\
1.15\end{array}$ & \begin{tabular}{|c} 
Sci-Biology.9- \\
12
\end{tabular} & Genetics & GEN.5 \\
\hline
\end{tabular}

\begin{tabular}{|c|l|c|c|c|c|}
\hline \multicolumn{2}{|c}{ Core Area Topic 253: Geno- and Phenotype } \\
\hline $\begin{array}{c}\text { Lesson } \\
\text { 253d: }\end{array}$ & Understanding Genetics & $\begin{array}{c}\text { AS 2.5// BC } \\
1.13\end{array}$ & $\begin{array}{c}\text { Sci-Biology.9- } \\
12\end{array}$ & $\begin{array}{c}\text { Evolution, } \\
\text { Genetics }\end{array}$ & $\begin{array}{c}\text { GVOL.8, } \\
\text { GEN.2, } \\
\text { GEN.4, } \\
\text { GEN.5 }\end{array}$ \\
\hline $\begin{array}{c}\text { Lesson } \\
\text { 253e: }\end{array}$ & Understanding Molecular Biotechnology & AS 2.5 & $\begin{array}{c}\text { Sci-Biology.9- } \\
12\end{array}$ & $\begin{array}{c}\text { Evolution, } \\
\text { Genetics }\end{array}$ & $\begin{array}{c}\text { EVOL.8, } \\
\text { GEN.5 }\end{array}$ \\
\hline Lesson 253f: Understanding Organismic Biotechnology & $\begin{array}{c}\text { AS 2.4// BC } \\
1.15\end{array}$ & $\begin{array}{c}\text { Sci-Biology.9- } \\
12\end{array}$ & Genetics & GEN.5 \\
\hline
\end{tabular}

\begin{tabular}{|c|l|c|c|c|c|}
\hline \multicolumn{9}{|c|}{ Core Area Topic 254: Dominant and Recessive Genes } \\
\hline $\begin{array}{c}\text { Lesson } \\
254 \mathrm{~d}:\end{array}$ & Understanding Genetics & $\begin{array}{c}\text { AS 2.5// BC } \\
1.13\end{array}$ & $\begin{array}{c}\text { Sci-Biology.9- } \\
12\end{array}$ & $\begin{array}{c}\text { Evolution, } \\
\text { Genetics }\end{array}$ & $\begin{array}{c}\text { EVOL.8, } \\
\text { GEN.2, } \\
\text { GEN.4, } \\
\text { GEN.5 }\end{array}$ \\
\hline $\begin{array}{c}\text { Lesson } \\
254 \mathrm{e}:\end{array}$ & Understanding Molecular Biotechnology & AS 2.5 & $\begin{array}{c}\text { Sci-Biology.9- } \\
12\end{array}$ & $\begin{array}{c}\text { Evolution, } \\
\text { Genetics }\end{array}$ & $\begin{array}{c}\text { EVOL.8, } \\
\text { GEN.5 }\end{array}$ \\
\hline Lesson 254f: Understanding Organismic Biotechnology & $\begin{array}{c}\text { AS 2.4// BC } \\
1.15\end{array}$ & $\begin{array}{c}\text { Sci-Biology.9- } \\
12\end{array}$ & Genetics & GEN.5 \\
\hline
\end{tabular}


Core Area Topic 255: Mutation

\begin{tabular}{|c|l|c|c|c|c|}
\hline $\begin{array}{c}\text { Lesson } \\
\text { 255b: }\end{array}$ & Understanding Natural Reproduction Technology & $\begin{array}{c}\text { AS } 2.4 / / \mathrm{BC} \\
1.13\end{array}$ & $\begin{array}{c}\text { Sci-Biology.9- } \\
12\end{array}$ & $\begin{array}{c}\text { Evolution, } \\
\text { Genetics }\end{array}$ & $\begin{array}{c}\text { EVOL.7, } \\
\text { GEN.2, } \\
\text { GEN.3 }\end{array}$ \\
\hline $\begin{array}{c}\text { Lesson } \\
\text { 255d: }\end{array}$ & Understanding Genetics & $\begin{array}{c}\text { AS } 2.5 / / \mathrm{BC} \\
1.13\end{array}$ & $\begin{array}{c}\text { Sci-Biology.9- } \\
12\end{array}$ & $\begin{array}{c}\text { Evolution, } \\
\text { Genetics }\end{array}$ & $\begin{array}{c}\text { EVOL.8, } \\
\text { GEN.2, } \\
\text { GEN.4, } \\
\text { GEN.5 }\end{array}$ \\
\hline $\begin{array}{c}\text { Lesson } \\
\text { 255e: }\end{array}$ & Understanding Molecular Biotechnology & AS 2.5 & $\begin{array}{c}\text { Sci-Biology.9- } \\
12\end{array}$ & $\begin{array}{c}\text { Evolution, } \\
\text { Genetics }\end{array}$ & $\begin{array}{c}\text { EVOL.8, } \\
\text { GEN.5 }\end{array}$ \\
\hline Lesson 255f: Understanding Organismic Biotechnology & AS 2.4// BC \\
1.15 & $\begin{array}{c}\text { Sci-Biology.9- } \\
12\end{array}$ & Genetics & GEN.5 \\
\hline
\end{tabular}

\begin{tabular}{|c|l|c|c|c|c|}
\hline \multicolumn{2}{|c|}{ Core Area Topic 256: Genetics Glossary } \\
\hline $\begin{array}{c}\text { Lesson } \\
\text { 256d: }\end{array}$ & Understanding Genetics & $\begin{array}{c}\text { AS 2.5 // BC } \\
1.13\end{array}$ & $\begin{array}{c}\text { Sci-Biology.9-9- } \\
12\end{array}$ & $\begin{array}{c}\text { Evolution, } \\
\text { Genetics }\end{array}$ & $\begin{array}{c}\text { EVOL.8, } \\
\text { GEN.2, } \\
\text { GEN.4, } \\
\text { GEN.5 }\end{array}$ \\
\hline $\begin{array}{c}\text { Lesson } \\
\text { 256e: }\end{array}$ & Understanding Molecular Biotechnology & AS 2.5 & $\begin{array}{c}\text { Sci-Biology.9- } \\
12\end{array}$ & $\begin{array}{c}\text { Evolution, } \\
\text { Genetics }\end{array}$ & $\begin{array}{c}\text { EVOL.8, } \\
\text { GEN.5 }\end{array}$ \\
\hline Lesson 256f: Understanding Organismic Biotechnology & $\begin{array}{c}\text { AS 2.4// BC } \\
1.15\end{array}$ & $\begin{array}{c}\text { Sci-Biology.9- } \\
12\end{array}$ & Genetics & GEN.5 \\
\hline \multicolumn{7}{|c|}{ Core Area UNIT 260: NUTRITION AND FEEDS } \\
\hline
\end{tabular}

Core Area Topic 265: Protein Needs

\begin{tabular}{|c|c|c|c|c|c|c|}
\hline $\begin{array}{l}\text { Lesson } \\
265 a \text { : }\end{array}$ & Understanding Nutrients and Their Importance & \multicolumn{2}{|c|}{$\begin{array}{c}\mathrm{AS} 2.2 / / \mathrm{BC} \\
1.14\end{array}$} & \multicolumn{2}{|c|}{\begin{tabular}{|c|c|}
$\begin{array}{c}\text { Sci-Biology.9- } \\
12\end{array}$ & Cell Biology \\
\end{tabular}} & CB.1 \\
\hline \multicolumn{7}{|c|}{ Core Area: Plant Science } \\
\hline & & \multicolumn{5}{|c|}{ California Core Area Ag \& Acad Standards } \\
\hline$\#$ & Lesson Title & $\begin{array}{c}\mathrm{CAAg} \\
\text { Standard }\end{array}$ & \multicolumn{2}{|c|}{ CA Acad Topic } & Sub Topic & Standard \\
\hline \multicolumn{7}{|c|}{ Core Area UNIT 310: BASIC BOTANY } \\
\hline \multicolumn{7}{|c|}{ Core Area Topic 311: Plant Parts } \\
\hline $\begin{array}{l}\text { Lesson } \\
\text { 311a: }\end{array}$ & Examining Plant Structures and Functions & $\begin{array}{c}\mathrm{BC} 1.17 / / \mathrm{PS} \\
3.2\end{array}$ & Sci-Bi & iology.9-12 & Cell Biology & CB.1 \\
\hline
\end{tabular}

\begin{tabular}{|c|c|c|c|c|c|}
\hline \multicolumn{6}{|c|}{ Core Area Topic 312: Function of Plant Parts } \\
\hline $\begin{array}{l}\text { Lesson } \\
\text { 312a: }\end{array}$ & Examining Plant Structures and Functions & $\begin{array}{c}\text { BC 1.17 // PS } \\
3.2\end{array}$ & Sci-Biology.9-12 & $\begin{array}{c}\text { Cell Biology, } \\
\text { Ecology, } \\
\text { Evolution }\end{array}$ & $\begin{array}{l}\text { CB.1, } \\
\text { ECO.6, } \\
\text { EVOL.7, } \\
\text { EVOL.8 }\end{array}$ \\
\hline \multicolumn{6}{|c|}{ Core Area Topic 314: Classification of Plants by Life Cycle } \\
\hline $\begin{array}{c}\text { Lesson } \\
\text { 314a: }\end{array}$ & Understanding Plant Life Cycles & $\begin{array}{c}\mathrm{BC} 1.17 / / \mathrm{PS} \\
3.3\end{array}$ & Sci-Biology.9-12 & Evolution & EVOL.8 \\
\hline \multicolumn{6}{|c|}{ Core Area Topic 315: Plant Propagation by Seed } \\
\hline $\begin{array}{c}\text { Lesson } \\
315 a:\end{array}$ & Propgating Plants Sexually & PS 3.4 & Sci-Biology.9-12 & Genetics & $\begin{array}{l}\text { GEN.2, } \\
\text { GEN.3 }\end{array}$ \\
\hline
\end{tabular}

\begin{tabular}{|c|l|c|c|c|c|}
\hline \multicolumn{5}{|c}{ Core Area Topic 316: Plant Propogation by Vegative } \\
\hline 316a: & Propagating Plants Asexually & PS 3.4 & Sci-Biology.9-12 & Genetics & GEN.5 \\
\hline
\end{tabular}

\begin{tabular}{|c|c|c|c|c|c|}
\hline \multicolumn{6}{|c|}{ Core Area Topic 318: Photosynthesis } \\
\hline $\begin{array}{l}\text { Lesson } \\
\text { 318a: }\end{array}$ & Examining Photosynthesis & $\begin{array}{c}\text { BC 1.17 // PS } \\
3.3\end{array}$ & $\begin{array}{l}\text { Sci-Biology.9-12, } \\
\text { Sci- }\end{array}$ & $\begin{array}{l}\text { Cell Biology, } \\
\text { Ecology // }\end{array}$ & $\begin{array}{c}\text { CB.1, } \\
\text { ECO.6// }\end{array}$ \\
\hline
\end{tabular}




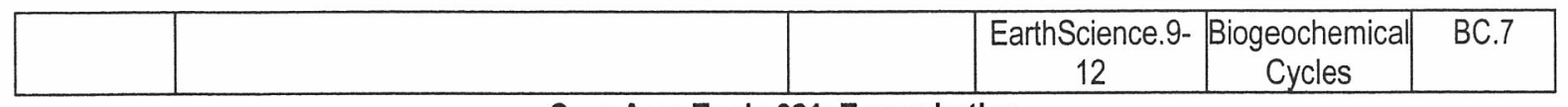

\section{Core Area Topic 321: Transpiration}

\begin{tabular}{|c|c|c|c|c|c|}
\hline $\begin{array}{l}\text { Lesson } \\
\text { 321a: }\end{array}$ & Understanding Plant Physiology & \begin{tabular}{|l|}
$\mathrm{BC} 1.17 / / \mathrm{OH}$ \\
$5.2 / / \mathrm{PS} 3.17$
\end{tabular} & Sci-Biology.9-12 & Cell Biology & CB.1 \\
\hline \multicolumn{6}{|c|}{ Core Area Topic 323: Plant Growth Requirements } \\
\hline $\begin{array}{l}\text { Lesson } \\
\text { 323a: }\end{array}$ & $\begin{array}{l}\text { Understanding Light, Temperature, Air, and } \\
\text { Water Effects on Plant Growth }\end{array}$ & \begin{tabular}{|c|}
$\mathrm{BC} 1.17 / / \mathrm{PS}$ \\
3.3
\end{tabular} & $\begin{array}{c}\text { Sci-Biology.9-12, } \\
\text { Sci- } \\
\text { EarthScience.9- } \\
12\end{array}$ & $\begin{array}{l}\text { Cell Biology } / 1 \\
\text { Energy in the } \\
\text { Earth System }\end{array}$ & $\begin{array}{l}\text { CB.1// } \\
\text { ENGY.4 }\end{array}$ \\
\hline $\begin{array}{l}\text { Lesson } \\
\text { 323b: }\end{array}$ & Determining Nutrient Functions and Utilization & $\begin{array}{c}\mathrm{OH} 5.6 / / \mathrm{PS} \\
3.10\end{array}$ & $\begin{array}{l}\text { Sci-Chemistry.9- } \\
12\end{array}$ & $\begin{array}{l}\text { Atomic and } \\
\text { Molecular } \\
\text { Structure, } \\
\text { Chemical } \\
\text { Bonds, } \\
\text { Reaction } \\
\text { Rates, } \\
\text { Solutions } \\
\end{array}$ & $\begin{array}{l}\text { AMS.1, } \\
\text { CB.2, } \\
\text { RR.8, } \\
\text { SOL.6 }\end{array}$ \\
\hline \multicolumn{6}{|c|}{ Core Area UNIT 330: SOILS } \\
\hline \multicolumn{6}{|c|}{ Core Area Topic 331: Soil and Plant Culture } \\
\hline $\begin{array}{l}\text { Lesson } \\
\text { 331a: }\end{array}$ & Determining the Nature of Soil & \begin{tabular}{|c|}
$\mathrm{BC} 1.16 / / \mathrm{PS}$ \\
3.6 \\
\end{tabular} & Sci-Physics.9-12 & $\begin{array}{l}\text { Motion and } \\
\text { Forces }\end{array}$ & MF.1 \\
\hline
\end{tabular}

Core Area Topic 332: Soil Texture and Structure

\begin{tabular}{|c|l|c|c|c|c|}
\hline $\begin{array}{c}\text { Lesson } \\
\text { 332a: }\end{array}$ & Understanding Soil Texture and Structure & $\begin{array}{c}\text { BC 1.16 / PS } \\
3.6\end{array}$ & Sci-Physics.9-12 & $\begin{array}{c}\text { Motion and } \\
\text { Forces }\end{array}$ & MF.1 \\
\hline
\end{tabular}

\begin{tabular}{|c|c|c|c|c|c|}
\hline \multicolumn{6}{|c|}{ Core Area Topic 333: Influence of Texture on Soil } \\
\hline $\begin{array}{l}\text { Lesson } \\
\text { 333a: }\end{array}$ & Understanding Soil Texture and Structure & \begin{tabular}{|c|}
$\mathrm{BC} 1.16 / / \mathrm{PS}$ \\
3.6
\end{tabular} & Sci-Physics.9-12 & $\begin{array}{l}\text { Motion and } \\
\text { Forces }\end{array}$ & MF.1 \\
\hline \multicolumn{6}{|c|}{ Core Area Topic 334: Soil Erosion and Conservation } \\
\hline $\begin{array}{l}\text { Lesson } \\
\text { 334a: }\end{array}$ & $\begin{array}{l}\text { Understanding Soil Erosion and Management } \\
\text { Practices }\end{array}$ & \begin{tabular}{|c|}
$\mathrm{BC} 1.19 / / \mathrm{NR}$ \\
$7.4 / / \mathrm{OH} 5.5 / /$ \\
$\mathrm{PS} 3.8$
\end{tabular} & $\begin{array}{c}\text { Sci- } \\
\text { EarthScience.9- } \\
12 \\
\end{array}$ & $\begin{array}{l}\text { California } \\
\text { Geology }\end{array}$ & CG.9 \\
\hline \multicolumn{6}{|c|}{ Core Area UNIT 340: PLANT NUTRITION } \\
\hline \multicolumn{6}{|c|}{ Core Area Topic 341: Nutrients Essential to Plant Growth } \\
\hline $\begin{array}{l}\text { Lesson } \\
\text { 341a: }\end{array}$ & Determining Plant Nutrients and Fertility & $\begin{array}{c}\mathrm{BC} 1.16 / / \mathrm{PS} \\
3.3\end{array}$ & $\begin{array}{c}\text { Sci-Chemistry.9- } \\
12\end{array}$ & $\begin{array}{c}\text { Acids and } \\
\text { Bases, } \\
\text { Reaction Rates }\end{array}$ & $\begin{array}{l}\text { AB. } 5 \\
\text { RR.8 }\end{array}$ \\
\hline
\end{tabular}

\begin{tabular}{|c|l|c|c|c|c|}
\hline \multicolumn{6}{|c|}{ Core Area Topic 342: Sources of Primary Plant Nutrients } \\
\hline $\begin{array}{c}\text { Lesson } \\
\text { 342a: }\end{array}$ & Understanding Soil Chemistry & $\begin{array}{c}\text { BC 1.16 // OH } \\
5.6 / / \text { PS 3.6 }\end{array}$ & $\begin{array}{c}\text { Sci-Chemistry.9- } \\
12\end{array}$ & $\begin{array}{c}\text { Acids and } \\
\text { Bases, } \\
\text { Chemical } \\
\text { Bonds }\end{array}$ & AB.5, CB.2 \\
\hline $\begin{array}{c}\text { Lesson } \\
\text { 342b: }\end{array}$ & Determining Fertilizer Formulations & $\begin{array}{c}\text { OH 5.6 // PS } \\
3.10\end{array}$ & $\begin{array}{c}\text { Math-Algebra1.8- } \\
12\end{array}$ & $\begin{array}{c}\text { Reasonbolic } \\
\text { Calculations and } \\
\text { with Symbols }\end{array}$ & A1.15 \\
\hline \multicolumn{7}{|c|}{ Core Area UNIT 360: PEST MANAGEMENT } \\
\hline
\end{tabular}

\section{Core Area UNIT 360: PEST MANAGEMENT}

Core Area Topic 361: Intro to Plant Pests

\begin{tabular}{|c|l|c|l|l|l|}
\hline $\begin{array}{c}\text { Lesson } \\
\text { 361a: }\end{array}$ & Managing Plant Pests & $\begin{array}{c}\text { BC 1.18// PS } \\
3.5\end{array}$ & Sci-Biology.9-12 & Physiology & PHY.10 \\
\hline $\begin{array}{c}\text { Lesson } \\
\text { 361b: }\end{array}$ & Introduction to Plant Pests & PS 3.5 & Sci-Biology.9-12 & Physiology & PHY.10 \\
\hline
\end{tabular}




\begin{tabular}{|c|c|c|c|c|c|}
\hline $\begin{array}{l}\text { Lesson } \\
\text { 362a: }\end{array}$ & Managing Weeds & \begin{tabular}{|c|}
$\mathrm{BC} 1.8 / / \mathrm{PS}$ \\
3.1 \\
\end{tabular} & Sci-Biology.9-12 & Cell Biology & CB.1 \\
\hline \multicolumn{6}{|c|}{ Core Area Topic 366: Disease Control: Cutural } \\
\hline $\begin{array}{l}\text { Lesson } \\
\text { 366a: }\end{array}$ & Managing Crop Diseases & \begin{tabular}{|c|}
$\mathrm{BC} 1.18 / / \mathrm{PS}$ \\
3.5
\end{tabular} & Sci-Biology.9-12 & Physiology & PHY.10 \\
\hline \multicolumn{6}{|c|}{ Core Area Topic 367: Disease Control: Chemical } \\
\hline $\begin{array}{l}\text { Lesson } \\
\text { 367a: }\end{array}$ & Determining the Kinds of Pesticides & PS 3.5 & Sci-Biology.9-12 & Cell Biology & CB.1 \\
\hline \multicolumn{6}{|c|}{ Core Area Topic 368: Biology of Insects } \\
\hline $\begin{array}{l}\text { Lesson } \\
\text { 368a: }\end{array}$ & Managing Insect Pests & \begin{tabular}{|c|} 
BC 1.18 //PS \\
3.5
\end{tabular} & Sci-Biology.9-12 & Cell Biology & CB.1 \\
\hline \multicolumn{6}{|c|}{ Core Area Topic 369: Insect Control } \\
\hline $\begin{array}{l}\text { Lesson } \\
\text { 369a: }\end{array}$ & Managing Insect Pests & \begin{tabular}{|c|c|} 
BC 1.18 //PS & \\
3.5 & \\
\end{tabular} & Sci-Biology.9-12 & Cell Biology & CB.1 \\
\hline \multicolumn{6}{|c|}{ Core Area: Agricultural Business Management } \\
\hline & & \multicolumn{4}{|c|}{ California Core Area Ag \& Acad Standards } \\
\hline$\#$ & Lesson Title & $\begin{array}{c}\mathrm{CA} \mathrm{Ag} \\
\text { Standard }\end{array}$ & $\begin{array}{c}\text { CA Acad } \\
\text { Topic }\end{array}$ & Sub Topic & Standard \\
\hline \multicolumn{6}{|c|}{ Core Area UNIT 410: RECORD KEEPING } \\
\hline \multicolumn{6}{|c|}{ Core Area Topic 411: Intro to Record Keeping } \\
\hline $\begin{array}{l}\text { Lesson } \\
\text { 411a: }\end{array}$ & Introduction to Record Keeping & \begin{tabular}{|c}
$\mathrm{AB} 4.3 / / \mathrm{BC}$ \\
1.4 \\
\end{tabular} & LA-Writing.9-12 & $\begin{array}{c}\text { Writing } \\
\text { Strategies }\end{array}$ & W1.1 \\
\hline \multicolumn{6}{|c|}{ Core Area Topic 412: Record Book: Topic Cover, Front Page, and Planning Calendar } \\
\hline $\begin{array}{l}\text { Lesson } \\
\text { 412a: }\end{array}$ & Introduction to Record Keeping & \begin{tabular}{|c}
$\mathrm{AB} 4.3 / / \mathrm{BC}$ \\
1.4 \\
\end{tabular} & LA-Writing.9-12 & $\begin{array}{c}\text { Writing } \\
\text { Strategies }\end{array}$ & W1.1 \\
\hline \multicolumn{6}{|c|}{ Core Area Topic 413: Record Book: Calendar } \\
\hline $\begin{array}{l}\text { Lesson } \\
\text { 413a: }\end{array}$ & Introduction to Record Keeping & \begin{tabular}{|c|}
$\mathrm{AB} 4.3 / / \mathrm{BC}$ \\
1.4 \\
\end{tabular} & LA-Writing.9-12 & $\begin{array}{c}\text { Writing } \\
\text { Strategies }\end{array}$ & W1.1 \\
\hline \multicolumn{6}{|c|}{ Core Area Topic 414: Rocrod Book: Business Agreements/Budget } \\
\hline $\begin{array}{l}\text { Lesson } \\
\text { 414a: }\end{array}$ & Making Business Agreements and Budgets & BC 1.4 & LA-Writing.9-12 & \begin{tabular}{c|} 
Writing \\
Applications \\
(Genres and \\
Their \\
Characteristics)
\end{tabular} & W2.2 \\
\hline \multicolumn{6}{|c|}{ Core Area Topic 415: Record Book: Journal } \\
\hline $\begin{array}{l}\text { Lesson } \\
\text { 415a: }\end{array}$ & Completing Record Book Journals & $\begin{array}{c}\mathrm{AB} 4.3 / / \mathrm{BC} \\
1.4\end{array}$ & \begin{tabular}{|c|}
$\begin{array}{c}\text { Math- } \\
\text { Algebra1.8-12 }\end{array}$ \\
\end{tabular} & \begin{tabular}{c|} 
Symbolic \\
Reasoning and \\
Calculations \\
with Symbols \\
\end{tabular} & A1.5 \\
\hline \multicolumn{6}{|c|}{ Core Area UNIT 430: AGRICULTURAL MARKETING } \\
\hline \multicolumn{6}{|c|}{ Core Area Topic 441: Computers and You } \\
\hline $\begin{array}{l}\text { Lesson } \\
\text { 441a: }\end{array}$ & Computer Terminology and Equipment & BC 1.5 & \begin{tabular}{|c|} 
\\
LA-Reading.9- \\
12 \\
\end{tabular} & $\begin{array}{l}\text { Word Analysis, } \\
\text { Fluency, and } \\
\text { Systematic } \\
\text { Vocabulary } \\
\text { Development, } \\
\text { Reading } \\
\text { Comprehension } \\
\text { (Focus on } \\
\text { Informational } \\
\end{array}$ & R1.1, R2.2 \\
\hline
\end{tabular}




\begin{tabular}{|c|c|c|c|c|c|c|}
\hline & & & & & Materials) & \\
\hline $\begin{array}{l}\text { Lesson } \\
441 \mathrm{~b}:\end{array}$ & Introduction to Computers in Agriculture & \multicolumn{2}{|c|}{$\begin{array}{l}\mathrm{BC} 1.3 / / \mathrm{BC} \\
1.4 / / \mathrm{BC} 1.5\end{array}$} & Math-Trig.8-12 & \begin{tabular}{|c|}
$\begin{array}{c}\text { Trigonometric } \\
\text { Functions }\end{array}$ \\
\end{tabular} & TRIG.12 \\
\hline $\begin{array}{l}\text { Lesson } \\
441 \mathrm{c} \text { : }\end{array}$ & Using the Internet & \multicolumn{2}{|l|}{$\mathrm{BC} 1.5$} & $\begin{array}{c}\text { LA-Reading.9- } \\
12\end{array}$ & \begin{tabular}{|c|} 
Reading \\
Comprehension \\
(Focus on \\
Informational \\
Materials) \\
\end{tabular} & $\mathrm{R} 2.2$ \\
\hline \multicolumn{7}{|c|}{ Core Area Topic 442: Introduction to Personal Computers } \\
\hline $\begin{array}{l}\text { Lesson } \\
\text { 442a: }\end{array}$ & Computer Terminology and Equipment & \multicolumn{2}{|l|}{ BC 1.5} & \begin{tabular}{|c|} 
\\
LA-Reading.9- \\
12 \\
\end{tabular} & \begin{tabular}{|c|} 
Word Analysis, \\
Fluency, and \\
Systematic \\
Vocabulary \\
Development, \\
Reading \\
Comprehension \\
(Focus on \\
Informational \\
Materials) \\
\end{tabular} & R1.1, R2.2 \\
\hline \multicolumn{7}{|c|}{ Core Area: FFA Leadership Development } \\
\hline & 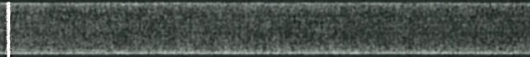 & \multicolumn{5}{|c|}{ Callifornia Core Area Ag \& Acad Standards } \\
\hline \# & Lesson Title & $\begin{array}{c}\mathrm{CAAg} \\
\text { Standard }\end{array}$ & & A Acad Topic & Sub Topic & Standard \\
\hline \multicolumn{7}{|c|}{ Core Area UNIT 540: CRITICAL THINKING } \\
\hline \multicolumn{7}{|c|}{ Core Area Topic 541: The Critical Thinker } \\
\hline $\begin{array}{l}\text { Lesson } \\
\text { 541a: }\end{array}$ & Understanding Problem Solving & $\begin{array}{c}A B 4.1 / / A B \\
4.8\end{array}$ & & $\begin{array}{l}\text { Sci- } \\
\text { perimentation.9- } \\
12\end{array}$ & $\begin{array}{c}\text { Scientific } \\
\text { Progress } \\
\text { through } \\
\text { Investigations }\end{array}$ & SPI.1 \\
\hline $\begin{array}{l}\text { Lesson } \\
541 \mathrm{~b} \text { : }\end{array}$ & The Critical Thinker & BC 1.6 & & A-Reading.9-12 & \begin{tabular}{|c|} 
Reading \\
Comprehension \\
(Focus on \\
Informational \\
Materials) \\
\end{tabular} & $\mathrm{R} 2.2$ \\
\hline \multicolumn{7}{|c|}{ Core Area Topic 542: Problem Solving } \\
\hline $\begin{array}{l}\text { Lesson } \\
\text { 542a: }\end{array}$ & Understanding Problem Solving & $\begin{array}{c}A B 4.1 / / A B \\
4.8\end{array}$ & & $\begin{array}{c}\text { Sci- } \\
\text { perimentation.9- } \\
12\end{array}$ & $\begin{array}{c}\text { Scientific } \\
\text { Progress } \\
\text { through } \\
\text { Investigations }\end{array}$ & SPI.1 \\
\hline \multicolumn{7}{|c|}{ Core Area Topic 543: Critical Processes } \\
\hline $\begin{array}{l}\text { Lesson } \\
\text { 543a: }\end{array}$ & Understanding Problem Solving & $\begin{array}{c}A B 4.1 / / A B \\
4.8\end{array}$ & Expe & $\begin{array}{c}\text { Sci- } \\
\text { Derimentation.9- } \\
12 \\
\end{array}$ & \begin{tabular}{|c} 
Scientific \\
Progress \\
through \\
Investigations
\end{tabular} & SPI.1 \\
\hline \multicolumn{7}{|c|}{ Core Area: Agricultural Mechanics } \\
\hline 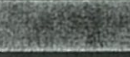 & 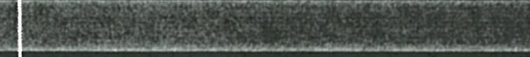 & \multicolumn{5}{|c|}{ California Core Area Ag \& Acad Standards } \\
\hline$\#$ & Lesson Title & \multicolumn{2}{|c|}{$\begin{array}{c}\mathrm{CAAg} \\
\text { Standard }\end{array}$} & CA Acad To & opic Sub Topic & Standard \\
\hline \multicolumn{7}{|c|}{ Core Area UNIT 910: MEASURING AND LAYOUT } \\
\hline
\end{tabular}


Core Area Topic 911: Measuring

\begin{tabular}{|c|c|c|c|c|c|}
\hline $\begin{array}{l}\text { Lesson } \\
\text { 911a: }\end{array}$ & Using English and Metric Measurements & $\begin{array}{c}\mathrm{AM} 6.14 / / \mathrm{BC} \\
1.9\end{array}$ & $\begin{array}{c}\text { Math- } \\
\text { Geometry.8-12 }\end{array}$ & $\begin{array}{c}\text { Basic } \\
\text { Geometry } \\
\text { Skills and } \\
\text { Concepts }\end{array}$ & GEO.8 \\
\hline
\end{tabular}

Core Area Topic 912: Calculating Area and Volume

\begin{tabular}{|c|c|c|c|c|c|}
\hline $\begin{array}{l}\text { Lesson } \\
\text { 912a: }\end{array}$ & Determining Area and Volume & $\begin{array}{c}\text { AM } 6.14 / / \mathrm{BC} \\
1.9\end{array}$ & $\begin{array}{c}\text { Math- } \\
\text { Geometry.8-12 }\end{array}$ & $\begin{array}{c}\text { Basic } \\
\text { Geometry } \\
\text { Skills and } \\
\text { Concepts }\end{array}$ & GEO.10 \\
\hline
\end{tabular}




\section{COURSE DATA}

Title: $\quad$ Exploratory Agricultural Science

Grade Level: 7-8 Semester/Year Course: Semester

Prerequisite(s): $\quad$ None

Please indicate if requesting the following: (High School Only)

a Repeatable Course

a Meets C.U.S.D. Graduation Requirements

a Meets UC Honors Admittance Requirements

a Meets UC Regular Admittance Requirements

a Meets CSU Fine Arts Requirements but not UC

Course Description: Exploratory Agricultural Science is a semester long class designed to introduce the intermediate level student to the top industry in California. Using a hands-on approach to instruction students will explore concepts in Agricultural Engineering, Animal Science, Plant Science, Natural Resources, and Global Agricultural Business. In addition, each student will be given the opportunity utilize the Central Valley Applied Agriculture and Technology Center located at the Ronald Reagan Educational Center.

\section{Special Notes:}

\section{Course Offered At:}

a Buchanan High School

- Clovis High School

- Clovis East High School

a Clovis West High School

a Enterprise High School

a Gateway High School

- Alta Sierra Intermediate School

$\checkmark$ Clark Intermediate School

- Kastner Intermediate School

$\checkmark$ Reyburn Intermediate School 
Textbook (Primary textbook if more than one is used):

Title: _Exploring Agriscience

Edition:

Author:__ Ray V. Herren

Publisher: _ Delmar Publishers

Copyright Year: 1996 Adoption Year:

\section{COURSE PROFILE}

\section{Course Goals:}

1. Provide students with a strong foundation of Agriculture and it's allied industries.

2. Promote active learning of science concepts using agriculture-based hands on acti $y_{\text {ities. }}$

3. Give students a better understanding of themselves and their role in our ecosystem.

4. Expose students to the opportunities available to them within the district agriculture program

\section{Course Objectives:}

1. Discuss why agriculture is important in our everyday lives.

2. Discuss why agriculture was necessary to the development of civilizations.

3. Explain why agriculture and all of life depends on soil.

4. Describe the functions and uses of the various plant parts.

5. Explain how agriculture is working to protect the environment.

6. Discuss the importance of the livestock industry in the United States.

7. Discuss some of the ways companion animals are used to assist humans.

8. Explain the importance of Agricultural Engineering in sustainable agriculture.

9. Discuss careers available in Agriculture and its Allied Industries.

\section{Instructional Methods:}

1. Farm laboratory investigation

2. Lectures and class discussion

3. Video, internet, and other multimedia

4. Textbooks 
5. Periodicals

6. Guest Speakers

7. Enrichment activities (class projects, field trips, etc.)

\section{Evaluation Procedures:}

1. Skills Proficiency

2. Written and Oral Testing

3. Projects and Labs

4. Overall Participation 


\section{Clovis Unified School District}

David E. Cash, Ed.D., District Superintendent

\section{Proposal}

\section{New Course of Study}

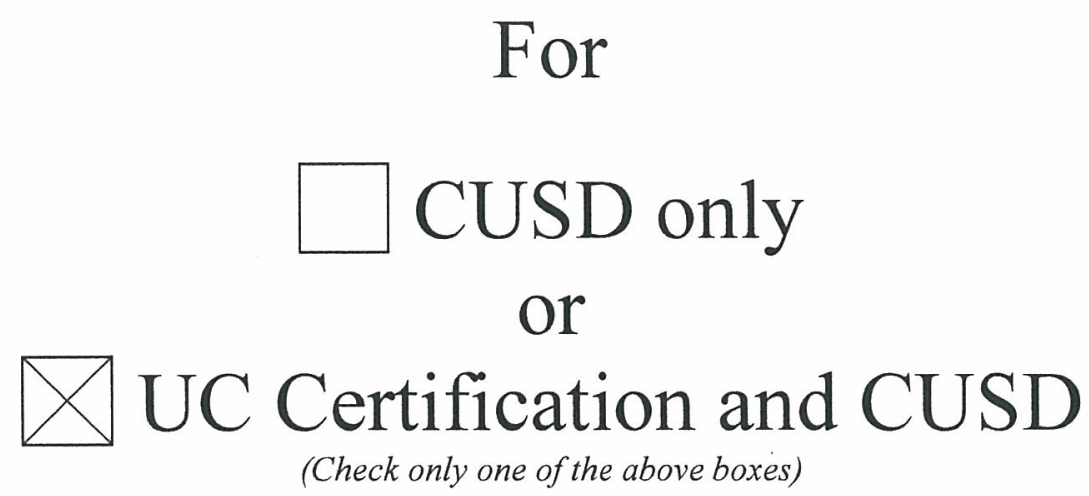

IS THIS AN ELECTIVE? $\square$ YES $\square$ NO

$>$ IS THIS AN NCLB CORE SUBJECT? $\square$ YES $\square$ NO

$\checkmark$ IF YES, WHICH CORE SUBJECT(S)

\section{English}

Reading/Language Arts

Mathematics

Science

History $\square$ Geography $\square$ Civics/Government $\square$ Economics Arts (Visual) $\square$ Arts (Performing)

Foreign Language

Self-Contained Elementary Multiple Subjects

$>$ IS THIS COURSE REPEATABLE? $\square$ YES $\square$ NO

$>$ IS THIS COURSE PASS/FAIL? $\square$ YES $\square$ NO

Course Title: Animal Science

\begin{tabular}{ll|ll}
\hline School(s): Clovis East High School & Department: Agriculture
\end{tabular}

\begin{tabular}{|l|l|}
\hline Title & Name \\
\hline Department Chairperson & Kevin Woodard \\
\hline Learning Director & Greg Sansom \\
\hline Principal & Darin Tocky \\
\hline rea Superintendent & Rosalie Baker \\
\hline hief Curriculum Officer & Michelle Steagall, Ed.D. \\
\hline Deputy Superintendent & Dan Kaiser, Ed.D. \\
\hline
\end{tabular}




\section{Course Codes}

istructions: Complete the CUSD Course and CBEDS Assignment Codes using the pull down menus under ine word "Choose."

\section{CUSD Course Code:}

(For help see below)

Choose Choose Choose

\section{CBEDS Assignment Code:}

(For help see bottom of this page)

$339 \mathrm{TT}$
4

4020
0

2

2

0

To determine the CUSD Course Code use the following table to identify the first 3 characters of the unique course code to be assigned to this course if approved.

\section{District Course Numbering Guide ( $\underline{\mathrm{D}} \underline{\mathrm{C}} \mathrm{T}$ T)}

"D"

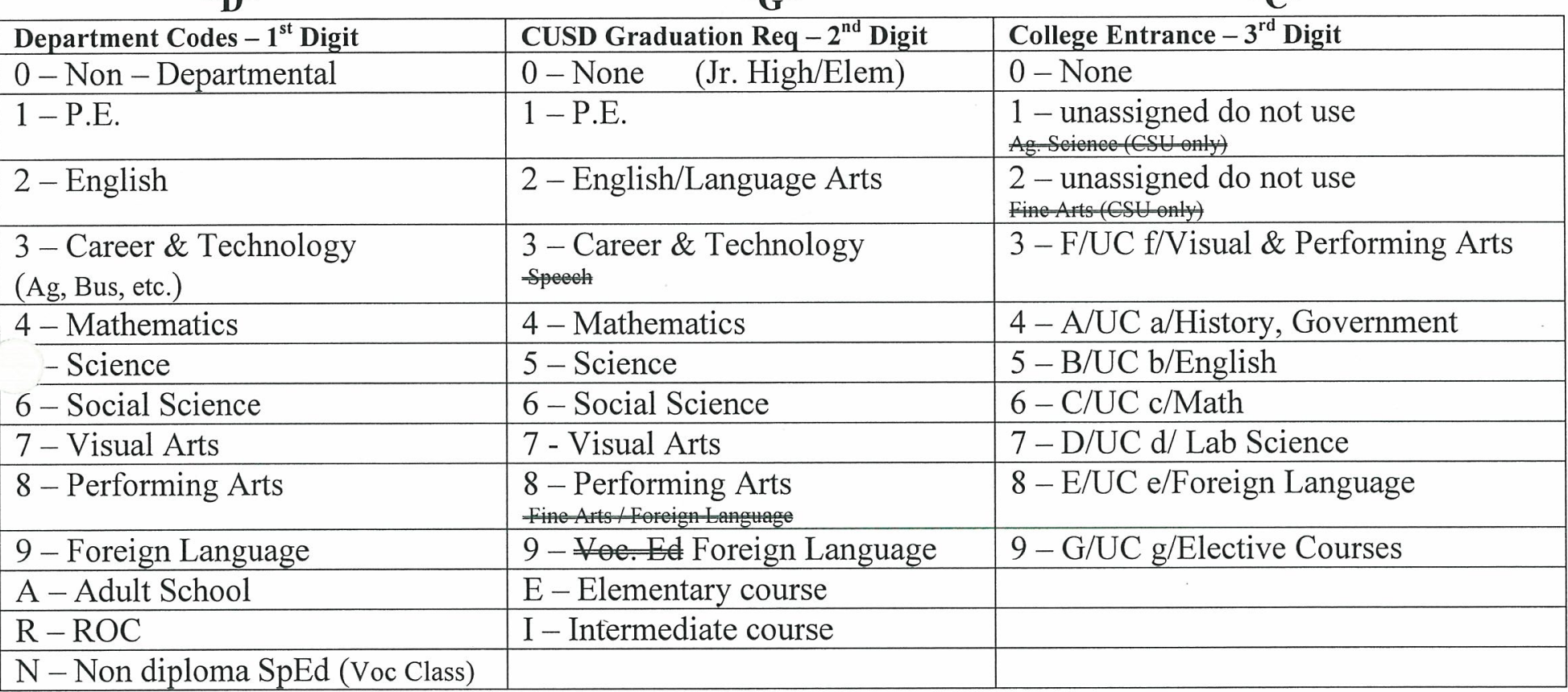

\section{CBEDS Assignment Code}

To determine the CBEDS Assignment Code refer to the CBEDS course definition binder located at your school in the Deputy Principal's office or log on to the following web site.

\section{http://www.cde.ca.gov/ds/sd/cb/subjects.asp}

Whether you use the web site or the definition binder follow the steps listed below.

Locate the content area your course to which your proposed course would be assigned.

Review all the course titles and descriptions listed in that content area.

Choose the course description that best matches the content that will be covered in the proposed course.

Enter the CBEDS number attached to that course description in the CBEDS Assignment Code box above. 


\section{Instructions:}

Complete pages 3 through 4 if this proposal is for CUSD only. If this proposal is for UC Certification and CUSD, complete pages 5 through 7.

\section{Course Description - CUSD Only}

Course Title:

Animal Science

Transcript Abbreviation:

Limited to 17 characters including spaces.

Grade Levels That Can Enroll: Lowest $11 \quad$ Course Length: 1 year

Highest 12

Course Offered At (check all that apply):

$\square$ Alta Sierra Intermediate School

$\square$ Clark Intermediate School

$\square$ Granite Ridge Intermediate School

Kastner Intermediate School

Reyburn Intermediate School

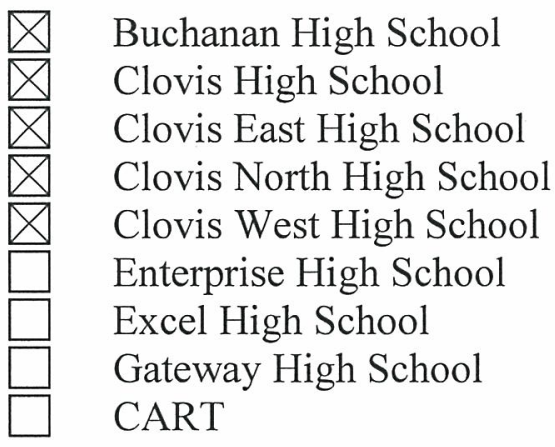

\section{High Schools Only}

Please check the appropriate box(s) you are requesting for which this course should be approved.

Course is Repeatable

Course is for elective credit only

Course meets CUSD Content Requirement for Graduation

Course Description (to be used in the CUSD Course Description Handbook):

This course will provide the student with principles in Animal Science focusing on the areas livestock (mammalian) production, anatomy, physiology, reproduction, nutrition, respiration, and genetics. This course is intended to successfully prepare those students who plan on majoring in Agricultural Sciences or Veterinary Science at a college or university.

Prerequisite(s):

Algebra and Agriculture Biology or Biology

Special Notes:

This class is recommended to take prior to enrolling in the Veterinary Science Course. 


\section{Course Profile}

Course Goals:

To give students fu

Course Objectives:

Students will be able to:

1. Assemble and use laboratory apparatus, tools and material in a skillful manner, giving attention to accident prevention and safety.

2. Gather the qualitative and quantitative information needed for developing and testing inferences and hypotheses by making purposeful, objective observations of things and events.

3. Understand the make up of the body and its functions.

4. Understand how to apply the knowledge of heredity and genetics to mammalian production.

5. Understand evolution and natural selection and how it relates to production agriculture.

6. Record observations accurately and organize data and ideas in ways that enhance their usefulness.

7. communicate with others (oral and written) in a manner that is consistent with the knowledge of scientific conventions, and facilities the learning of the listeners or readers.

8. Use the metric system effectively in measuring and quantifying substances.

\section{Instructional Methods:}
A. Laboratory Activities and Experiments
B. Lecture and discussion
C. Reading research assignments
D. Written and oral reports
E. Homework assignments
F. Audio-Visual presentations
G. Projects

\section{Evaluation procedures:}
A. Tests and quizzes
B. Homework assignments/term paper
C. Notebook/Portfolio
D. Laboratory Activities
E. SAE Project and Record Book
F. Leadership development activities

\section{Course Outline: \\ A. Economic Impact \\ 1. Content and methodology \\ 2. Demographics \\ 3. Social economic balance \\ 4. Plant and animal balance \\ 5. Human health and nutrition}




\section{Scientific classification system}

B. Plants, Animals, and their Management

1. History and principles

2. Habitat

3. New scientific principals

4. Behavioral modification and manipulation

C. Animal Anatomy and Physiology

1. Analysis of body systems

2. Physiological function of hormones and auxins

3. Reproductive physiology

4. Process of digestion

D. Animal Breeding and Genetics

1. Process of mitosis and meiosis

2. Cell theory of inheritance

3. Heritability percentage of traits

4. Artificial insemination

5. Embryo transplants

E. Animal Phenotypic Selection and Evaluation

1. External anatomy

2. Skeletal identification and position

3. Muscle volume

4. Fat deposition

5. Productivity and performance

F. Animal Health Care

1. Diseases and parasites

2. Predisposing factors and conditions

3. Biological preparation, antibiotics

4. Sanitation requirements and procedures

5. Laws involving human consumption, food product retention

G. Animal Nutrition and Feeds

1. Classes of nutrients and requirements

2. Animal nutrient requirements

3. Analysis of macro and micro animals

4. Vitamin roles

5. Nutrient deficiencies

6. Balancing rations and feed practices

7. Photosynthesis

H. Common Integument and its Derivation

1. Epithelium, mesothelium and endothelium

2. Skin and it's function

3. Mammary glands

4. Physiology of lactation

I. The Nervous System 


\begin{tabular}{|c|c|c|}
\hline & $\begin{array}{l}1 . \\
2 . \\
3 . \\
4 .\end{array}$ & $\begin{array}{l}\text { The brain and its function } \\
\text { The spinal cord } \\
\text { The peripheral nervous system } \\
\text { The autonomic system }\end{array}$ \\
\hline J. & $\begin{array}{l}\text { Res } \\
1 . \\
2 . \\
3 . \\
4 .\end{array}$ & $\begin{array}{l}\text { atory System and Respiration } \\
\text { Structure of mammalian respiratory system } \\
\text { Physiology of respiration } \\
\text { Mechanics of breathing } \\
\text { Plant respiration }\end{array}$ \\
\hline $\mathrm{K}$. & $\begin{array}{l}\text { An } \\
1 . \\
2 . \\
3 .\end{array}$ & $\begin{array}{l}1 \text { Research Presentation } \\
\text { Current animal research and investigation } \\
\text { Data presentation } \\
\text { Summarization and conclusion }\end{array}$ \\
\hline L. & & $\begin{array}{l}\text { sional Opportunities in Animal Science } \\
\text { Animal research fields } \\
\text { Other related animal science fields }\end{array}$ \\
\hline M. & $\begin{array}{l}\mathrm{Ag} \\
1 . \\
2 . \\
3 . \\
4 .\end{array}$ & $\begin{array}{l}\text { Itural Inter-Personal \& Leadership Development } \\
\text { Completion of a Supervised Agricultural Experience Program and data collection } \\
\text { Development of listening, speaking, writing \& reading skill activities } \\
\text { Critical thinking \& group team building activities } \\
\text { Agriculture presentations }\end{array}$ \\
\hline
\end{tabular}




\section{Course Description - UC Certification}

For new courses being submitted to UC for "a-g" certification and CUSD

\section{A. COVER PAGE}

Please refer to instructions if you need help (see page 8)

\begin{tabular}{|c|c|}
\hline $\begin{array}{l}\text { Date of Submission (Please include Month, Day and Year) } \\
\text { Adopted } 2003\end{array}$ & \\
\hline $\begin{array}{l}\text { 1. Course Title } \\
\text { Animal Science }\end{array}$ & $\begin{array}{l}\text { 9. Subject Area } \\
\qquad a \text { History/Social Science }\end{array}$ \\
\hline 2. Transcript Title(s)/Abbreviation(s) - 17 character limit & $\begin{array}{l}\text { b } \square \text { English } \\
\text { c } \square \text { Mathematics }\end{array}$ \\
\hline 3. Transcript Course Code(s)/Number(s) & d $\square$ Laboratory Science \\
\hline $\begin{array}{l}\text { 4. School } \\
\text { Clovis East High School }\end{array}$ & $\begin{array}{l}\text { e } \square \text { Language other than English } \\
\text { f } \square \text { Visual \& Performing Arts }\end{array}$ \\
\hline $\begin{array}{l}\text { 5. District } \\
\text { Clovis Unified School District }\end{array}$ & $\begin{array}{c}\square \text { Intro } \square \text { Advanced } \\
\mathrm{g} \otimes \text { College Prep Elective }\end{array}$ \\
\hline $\begin{array}{l}\text { 6. City } \\
\text { Clovis }\end{array}$ & 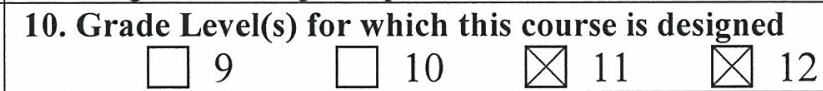 \\
\hline 7. School / District Web Site & $\begin{array}{c}\text { 11. Seeking "Honors" Distinction? } \\
\square \quad \text { Yes } \quad \square \text { No }\end{array}$ \\
\hline $\begin{array}{l}\text { 8. School Course List Contact } \\
\text { Name: } \\
\text { Title/Position: } \\
\text { Phone: } \\
\text { E-mail: }\end{array}$ & 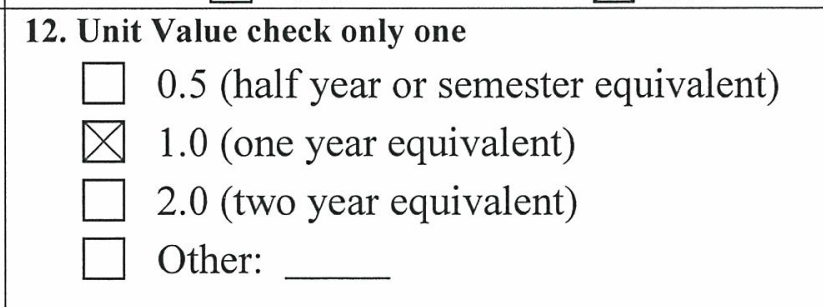 \\
\hline $\begin{array}{llll}\text { 13. Is this an Internet-based course? } & \square \text { Yes } \\
\text { If "Yes", who is the provider? } & \square \text { UCCP }\end{array}$ & $\begin{array}{l}\text { No } \\
\text { PASS/Cyber High }\end{array}$ \\
\hline
\end{tabular}

14. Complete outlines are not needed for courses that were previously approved by UC. If course was previously approved, indicate in which category it falls.

A course reinstated after removal within 3 years. Year removed from list?

Same course title? $\square$ Yes $\square$ No

If no, previous course title?

An identical course approved at another school in same district. Which school?

Same course title? $\square$ Yes $\square$ No

If no, course title at other school?

Alternative course title for course with identical content at this school

Title of previously-approved identical course:

Approved Advanced Placement (AP) or International Baccalaureate (IB) course

Approved UC College Prep (UCCP) Initiative course

Year-long VPA course replacing two approved successive semester-long courses in the same discipline

Approved P.A.S.S. course

Approved ROP/C course. Name of ROP/C?

Other. Explain: 


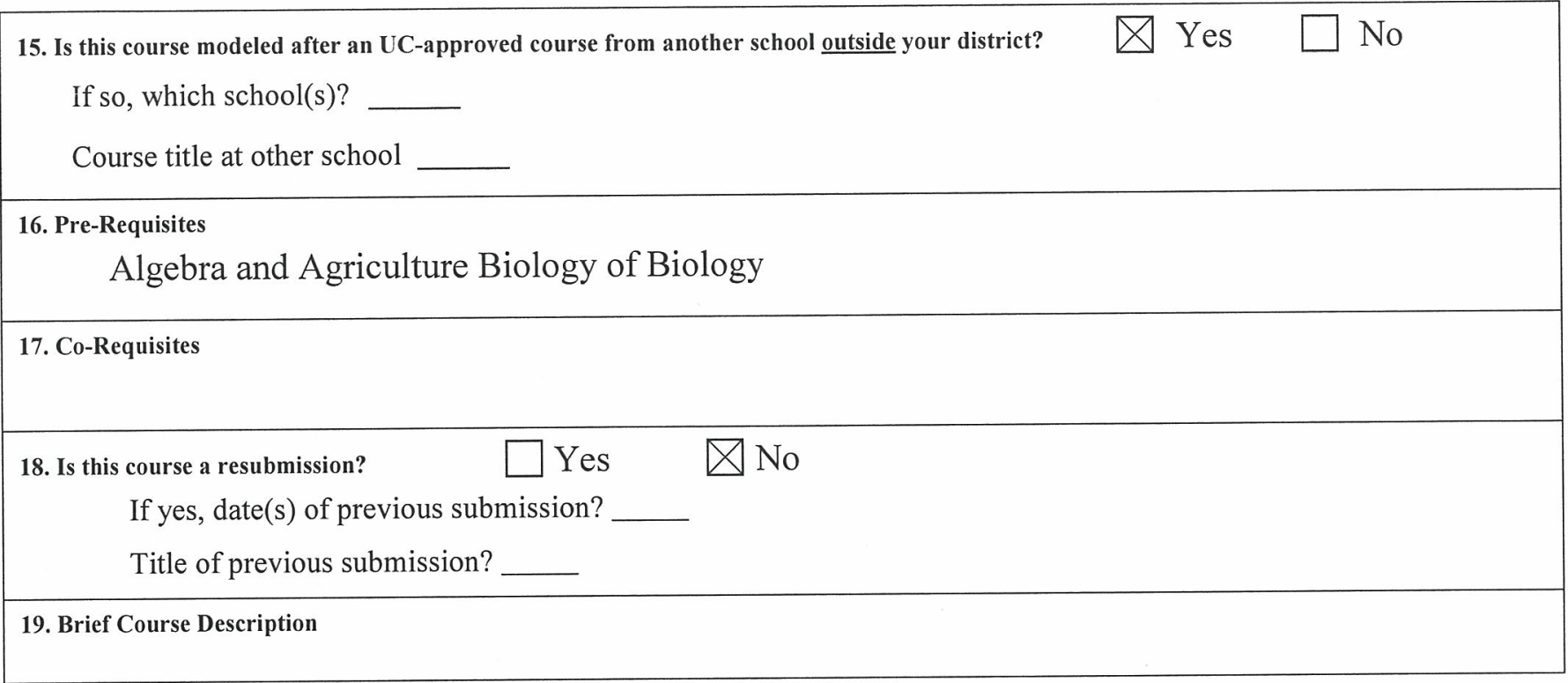

\section{B. COURSE CONTENT}

Please refer to instructions (see page 8)

\section{Course Goals and/or Major Student Outcomes}

This course will provide the student with principles in Animal Science focusing on the areas livestock (mammalian) production, anatomy, physiology, reproduction, nutrition, respiration, and genetics. This course is intended to successfully prepare those students who plan on majoring in Agricultural Sciences or Veterinary Science at a college or university.

\section{Course Objectives}

1. Assemble and use laboratory apparatus, tools, and materials in a skillful manner, giving attention to accident prevention and safety.

2. Gather the qualitative and quantitative information needed for developing and testing inferences and hypotheses by making purposeful, objective observations of things and events.

3. Understand the make up of the body and its functions.

4. Understand how to apply the knowledge of heredity and genetics to mammalian production.

5. Understand evolution and natural selection and how it relates to production agriculture.

6. Record observations accurately and organize data and ideas in ways that enhance their usefulness.

7. Communicate with others (oral and written) in a manner that is consistent with the knowledge of scientific conventions, and facilities the learning of the listeners or readers.

\section{Use the metric system effectively in measuring and quantifying substances.}

\section{Course Outline}

A. Economic Impact

1. Content and methodology

2. Demographics

3. Social economic balance

4. Plant and animal balance

5. Human health and nutrition 
6. Scientific classification system

B. Plants, Animals, and their Management

1. History and principles

2. Habitat

3. New scientific principals

4. Behavioral modification and manipulation

C. Animal Anatomy and Physiology

1. Analysis of body systems

2. Physiological function of hormones and auxins

3. Reproductive physiology

4. Process of digestion

D. Animal Breeding and Genetics

1. Process of mitosis and meiosis

2. Cell theory of inheritance

3. Heritability percentage of traits

4. Artificial insemination

5. Embryo transplants

E. Animal Phenotypic Selection and Evaluation

1. External anatomy

2. Skeletal identification and position

3. Muscle volume

4. Fat deposition

5. Productivity and performance

F. Animal Health Care

1. Diseases and parasites

2. Predisposing factors and conditions

3. Biological preparation, antibiotics

4. Sanitation requirements and procedures

5. Laws involving human consumption, food product retention

G. Animal Nutrition and Feeds

1. Classes of nutrients and requirements

2. Animal nutrient requirements

3. Analysis of macro and micro animals

4. Vitamin roles

5. Nutrient deficiencies

6. Balancing rations and feed practices

7. Photosynthesis

H. Common Integument and its Derivation

1. Epithelium, mesothelium and endothelium

2. Skin and it's function

3. Mammary glands

4. Physiology of lactation

I. The Nervous System 
1. The brain and its function

2. The spinal cord

3. The peripheral nervous system

4. The autonomic system

J. Respiratory System and Respiration

1. Structure of mammalian respiratory system

2. Physiology of respiration

3. Mechanics of breathing

4. Plant respiration

K. Animal Research Presentation

1. Current animal research and investigation

2. Data presentation

3. Summarization and conclusion

M. Professional Opportunities in Animal Science

1. Animal research fields

2. Other related animal science fields

M. Agricultural Inter-Personal \& Leadership Development

1. Completion of a Supervised Agricultural Experience Program and data collection

2. Development of listening, speaking, writing \& reading skill activities

3. Critical thinking \& group team building activities

4. Agriculture presentations

\section{Texts \& Supplemental Instructional Materials}

A. Laboratory Activities and Experiments

B. Lecture and discussion

C. Reading research assignments

D. Written and oral reports

E. Homework assignments

F. Audio-Visual presentations

G. Projects

\section{Key Assignments}

A. Weekly academic textbook assignments.

B. Weekly laboratory activities and report

C. Animal Science Term Paper

D. Supervised Agricultural Experience Project and Record Book

E. Student Seminar Presentation related to Animal Science Topic

F. Portfolio of Laboratory Exercises

\section{Instructional Methods and/or Strategies}

A. Laboratory Activities and Experiments

B. Lecture and Discussion

C. Reading research Assignments

D. Written and Oral Reports

E. Homework Assignments

F. Audio-Visual Presentations

G. Projects 
26. Assessment Methods and/or Tools

A. Tests and Quizzes $\quad 40 \%$

B. Homework Assignments/Term Paper $15 \%$

C. Notebook/Portfolio $05 \%$

D. Laboratory Activities $20 \%$

E. SAE Project \& Record Book $\quad 10 \%$

F. Leadership Development Activities $\quad 10 \%$ 


\section{Clovis Unified School District}

Janet L. Young, Ed.D., District Superintendent

\section{Proposal}

\section{New Course of Study}

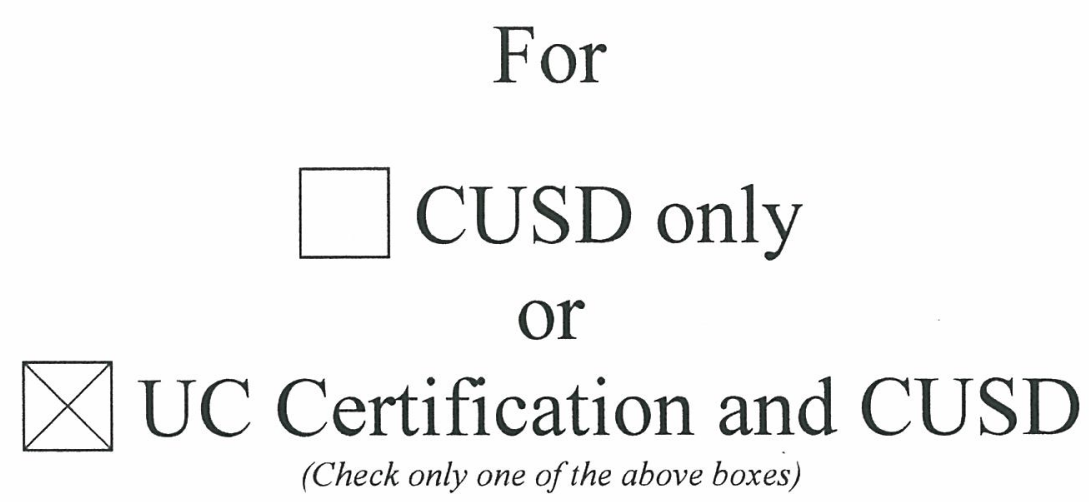

IS THIS AN ELECTIVE? $\square$ YES $\square$ NO

$>$ IS THIS AN NCLB CORE SUBJECT? $\square$ YES $\square$ NO

$\checkmark$ IF YES, WHICH CORE SUBJECT(S)

\section{English}

Reading/Language Arts

Mathematics

Science

History $\square$ Geography $\square$ Civics/Government $\square$ Economics Arts (Visual) $\square$ Arts (Performing)

Foreign Language

Self-Contained Elementary Multiple Subjects

$>$ IS THIS COURSE REPEATABLE? $\square$ YES $\square$ NO

$>$ IS THIS COURSE PASS/FAIL? $\square$ YES $\square$ NO

Course Title: Food Science

School(s): Clovis East High School 1 Department: Agriculture

\begin{tabular}{|l|l|}
\hline Title & Name \\
\hline Department Chairperson & Kevin Woodard \\
\hline Learning Director & Stephanie Hanks \\
\hline Principal & Darin Tockey \\
\hline rea Superintendent & Rosalie Baker \\
\hline Associate Superintendent & Carlo Prandini, Ph.D. \\
\hline Administrator, Curriculum \& Instruction & Robyn Castillo, Ed.D. \\
\hline
\end{tabular}




\section{Course Codes}

istructions: Complete the CUSD Course and CBEDS Assignment Codes using the pull down menus under ule word "Choose."

\section{CUSD Course Code:}

(For help see below)

\section{CBEDS Assignment Code:}

(For help see bottom of this page)

$3 \quad 39 / \mathrm{G} / \mathrm{UC}$ g/Elective $\quad \mathrm{T} \quad \mathrm{T}$
4
0
9

8

To determine the CUSD Course Code use the following table to identify the first 3 characters of the unique course code to be assigned to this course if approved.

\section{District Course Numbering Guide ( $\underline{\mathbb{D}} \underline{\mathrm{G}} \underline{\mathrm{C}} \mathrm{T}$ T)}

\begin{tabular}{|c|c|c|}
\hline "D" & “G" & "C C" \\
\hline Department Codes $-1^{\text {st }}$ Digit & CUSD Graduation Req $-2^{\text {nd }}$ Digit & College Entrance $-3^{\text {rd }}$ Digit \\
\hline 0 - Non - Departmental & $0-$ None $\quad$ (Jr. High/Elem) & $0-$ None \\
\hline $1-$ P.E. & $1-$ P.E. & 1 - unassigned do not use \\
\hline 2 - English & 2 - English/Language Arts & 2 - unassigned do not use \\
\hline $\begin{array}{l}3 \text { - Career \& Technology } \\
\text { (Ag, Bus, etc.) }\end{array}$ & $\begin{array}{l}3 \text { - Career \& Technology } \\
\text {. }\end{array}$ & $3-\mathrm{F} / \mathrm{UC}$ f/Visual \& Performing Arts \\
\hline 4-Mathematics & 4 - Mathematics & $4-\mathrm{A} / \mathrm{UC}$ a/History, Government \\
\hline - Science & $5-$ Science & $5-\mathrm{B} / \mathrm{UC} \mathrm{b} /$ English \\
\hline $6-$ Social Science & $6-$ Social Science & $6-\mathrm{C} / \mathrm{UC} \mathrm{c} / \mathrm{Math}$ \\
\hline 7 -Visual Arts & 7 - Visual Arts & $7-\mathrm{D} / \mathrm{UC} \mathrm{d} / \mathrm{Lab}$ Science \\
\hline $8-$ Performing Arts & 8- Performing Arts & $8-\mathrm{E} / \mathrm{UC}$ e/Foreign Language \\
\hline 9 - Foreign Language & 9 - Ed Foreign Language & 9- G/UC g/Elective Courses \\
\hline A - Adult School & E - Elementary course & \\
\hline $\mathrm{R}-\mathrm{ROC}$ & I - Intermediate course & \\
\hline N - Non diploma SpEd (Voc Class) & & \\
\hline
\end{tabular}

\section{CBEDS Assignment Code}

To determine the CBEDS Assignment Code refer to the CBEDS course definition binder located at your school in the Deputy Principal's office or log on to the following web site.

\section{http://www.cde.ca.gov/ds/sd/cb/subjects.asp}

Whether you use the web site or the definition binder follow the steps listed below.

Locate the content area your course to which your proposed course would be assigned.

Review all the course titles and descriptions listed in that content area.

Choose the course description that best matches the content that will be covered in the proposed course.

Enter the CBEDS number attached to that course description in the CBEDS Assignment Code box above. 


\section{Instructions:}

Complete pages 3 through 4 if this proposal is for CUSD only. If this proposal is for UC Certification and CUSD, complete pages 5 through 7.

\section{Course Description - CUSD Only}

Course Title: Food Science

Transcript Abbreviation: Food Science

Limited to 17 characters including spaces.

Grade Levels That Can Enroll: Lowest $10 \quad$ Course Length: $\quad$ Year Long (1 year)

Highest 12

Course Offered At (check all that apply):

$\square$ Alta Sierra Intermediate School

$\square$ Clark Intermediate School

Granite Ridge Intermediate School

Buchanan High School

Clovis High School

Clovis East High School

Clovis North High School

Clovis West High School

Enterprise High School

Excel High School

Gateway High School

CART

\section{High Schools Only}

Please check the appropriate box(s) you are requesting for which this course should be approved.

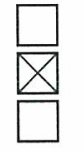

Course is Repeatable

Course is for elective credit only

Course meets CUSD Content Requirement for Graduation

Course Description (to be used in the CUSD Course Description Handbook):

FOOD SCIENCE applies fundamental scientific principles to the research, development, manufacturing, packaging, storage, and marketing of food products. This applied, laboratory-based course, which focuses on food processing, is designed to educate students about functional components of foods, food safety, nutrition, sensory evaluation, quality assurance, new product development, food chemistry, food microbiology, food processing and engineering

\section{Prerequisite(s):}

Algebra and Agiculture Biology or Biology

\section{Special Notes:}

Agriculture students are expected to participate in the comprehensive agriculture program model which includes FFA and Supervised Agriculture Experience. 


\begin{tabular}{|ll|}
\hline- & Homework assignments \\
- & Class and lab participation \\
- & Labobook/portfolio \\
- & Individual or group project based learning assignments \\
- & Safety awareness/evaluation in the classroom or lab \\
\hline
\end{tabular}

\section{Course Outline:}

A. Overview of Food Science
a. Parts/segments of the Food Industry
b. Division by major product lines
c. International Trade

B. Food Industry Trends
a. U.S. Food expenditures compared to other countries
b. World food needs
c. Food consumption trends
d. Trends in meal purchases
e.Identification of allied industries

C.Safety and Laboratory Procedures
a. Tools and Layout
b. Safety Measures
c. Weights and Measures
d. Lab Organization

D.Nutrition and Digestion
a. Nutritional Needs RDA or DRI
b. Functions of Energy- Carbohydrates, fats, and proteins in the body.
c. Calorie Content
d. Essential Amino Acids
e. Water and Fat soluble vitamins
f. Minerals
g. Digestion process
h. Organs of digestion
i. Diet and Human health

E. Chemistry of Foods
a. Chemical properties of elements
b. Elemental Bonding
c. Oxidation-reduction reactions
d. Divisions of metabolism
e. Carbohydrates
f. Proteins
g. Lipids
h. Minerals
i. Water Essentials
j. Miscellaneous chemicals
a. Sanitation
b. Food-Borne Illness
c. Regulations
G.Operations in Food Processing
a. Material Handling

F.Food Safety 
b. Miscellaneous Operations

i.Particle size reduction

ii. Separating foods

iii. Mixing

iv. Heat Transfer

v. Drying and dehydration

vi. Formed foods

vii. Overlapping operations

H. Packaging

a.Packaging concepts

i.Types of food packaging

ii.Tests of packaging properties

iii. Special features

iv. Environmental concerns

v.Innovations

I.Dairy Processing
a. Milk
b. Butter
c. Ice Cream
d. Cheese
e.USDA Grades

J.Meat, Poultry, and Eggs
a. Production
b. Conversion of muscle to meat
c. Meat products
d. Factors affecting tenderness
e. Meat Cookery
f. Meat Grading
g. Value added and specialty meat products
h. Egg production
i. Egg Quality
j. Egg Grading

K.Cereal Grains, Legumes, and Oilseeds

a. Grains

i. Structure and composition

ii. Types

b. Starches

i. Properties

ii. Factors to control when cooking with starch

c. Flour

i. Milling

ii. Classes and Grades

d. Corn

i. Refining

ii. Corn by products

e. Oilseeds

i. Composition and uses

f. Legumes

i. Composition and uses

g. Soy Beans

i. Products from extraction 


\begin{tabular}{|l|}
\hline h. Baking Process \\
i. Leavening \\
L. Fruits and Vegetables \\
a. Structure and Physiology \\
b. Grading \\
c. Storage \\
d. Processing \\
M. Fats and Oils \\
a. Composition and Processes \\
$\quad$ i. Saturated, Unsatureated, and Trans fats \\
$\quad$ ii. Fatty Acids \\
$\quad$ iii. Sources of fats and oils \\
$\quad$ iv. Function in Foods \\
N. Investigation and Experimentation \\
a. Lab Research Trials \\
O. Regulation and Labeling \\
a. Agencies and Regulations \\
b. Labeling \\
c. Nutritional Identification \\
P. Career Opportunities in Food Science \\
Q. Supervised Agriculture Experience \\
R. FFA Organization and Leadership \\
\hline
\end{tabular}




\section{Course Description - UC Certification}

For new courses being submitted to UC for "a-g" certification and CUSD

\section{A. COVER PAGE}

Please refer to instructions if you need help (see page 8)

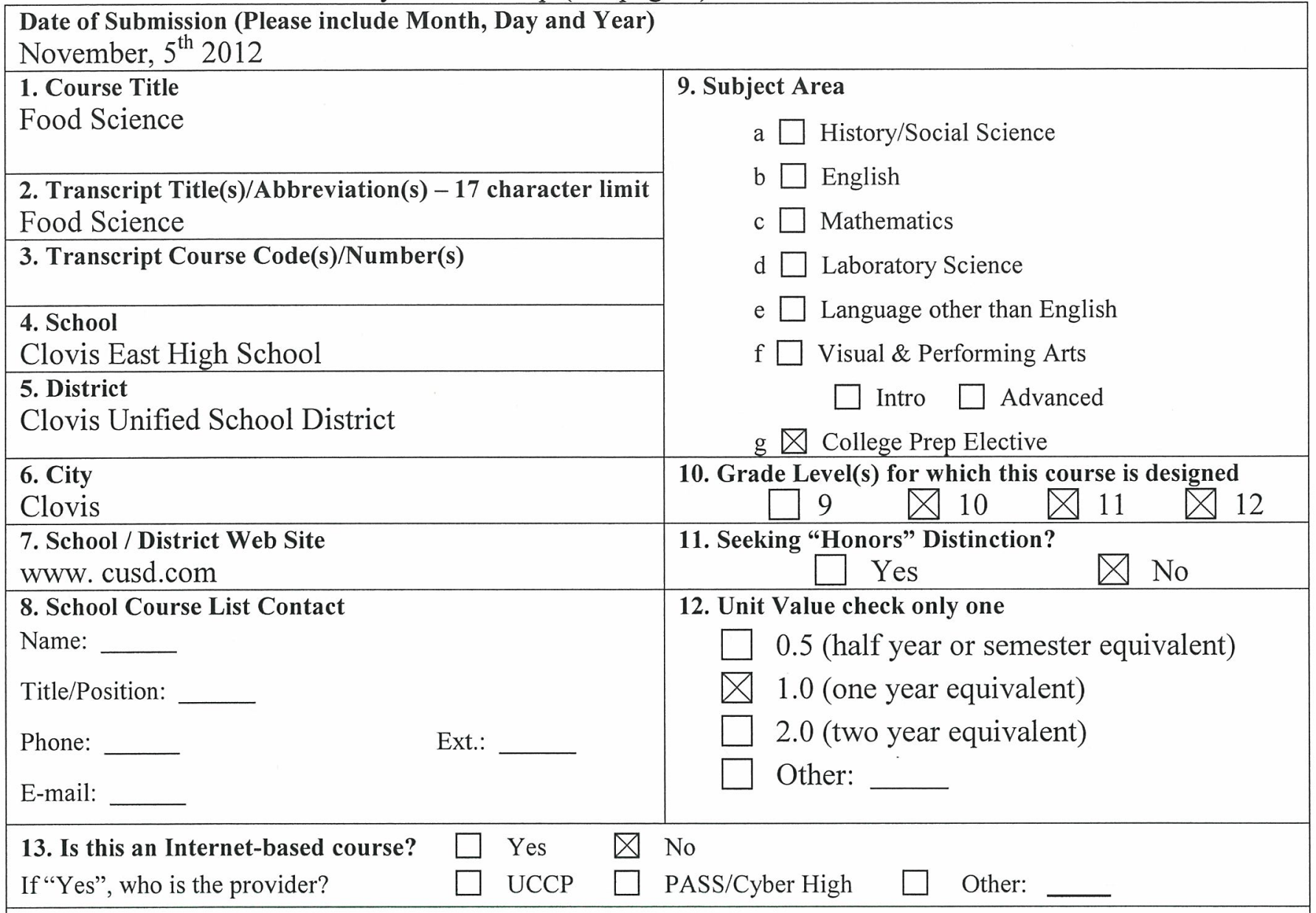

14. Complete outlines are not needed for courses that were previously approved by UC. If course was previously approved, indicate in which category it falls.

A course reinstated after removal within 3 years. Year removed from list?

$$
\text { Same course title? } \square \text { Yes } \square \text { No }
$$

If no, previous course title?

An identical course approved at another school in same district. Which school?

Same course title? $\square$ Yes $\square$ No

If no, course title at other school?

Alternative course title for course with identical content at this school

Title of previously-approved identical course:

Approved Advanced Placement (AP) or International Baccalaureate (IB) course

Approved UC College Prep (UCCP) Initiative course

Year-long VPA course replacing two approved successive semester-long courses in the same discipline

Approved P.A.S.S. course

Approved ROP/C course. Name of ROP/C?

Other. Explain: 
15. Is this course modeled after an UC-approved course from another school outside your district?

If so, which school(s)? Central West High School, and Gustine High School

Course title at other school Food Science

16. Pre-Requisites

Algebra, Agriculture Biology or Biology

17. Co-Requisites

18. Is this course a resubmission?

Yes

$\bigotimes$ No

If yes, date(s) of previous submission?

Title of previous submission?

\section{Brief Course Description}

FOOD SCIENCE applies fundamental scientific principles to the research, development, manufacturing, packaging, storage, and marketing of food products. This applied, laboratory-based course, which focuses on food processing, is designed to educate students about functional components of foods, food safety, nutrition, sensory evaluation, quality assurance, new product development, food chemistry, food microbiology, food processing and engineering

\section{B. COURSE CONTENT}

Please refer to instructions (see page 8)

\section{Course Goals and/or Major Student Outcomes}

To give students a basic background in food science in order to prepare them for college level courses in food science. Expose students to career opportunities in the food science and processing industries. Create educated consumers who know how to properly and safely handle and prepare food.

\section{Course Objectives}

Understand the parts of the food industry.

- Identify the chemistry of foods.

- Understand the nutrition and digestion of foods.

- Describe quality factors of foods.

- Have an understanding of unit operations in food processing.

- Discuss the reasons for varied methods of food preservation including canning, dehydration, cooking, and chilling.

- Identify and describe types and uses of food packaging.

- Describe and understand the process of milk production and processing.

- Describe the production and processing of meat from cattle, sheep, swine, and poultry.

- Describe the general composition of grains, legumes, and oilseeds.

- Identify the parts of a plant considered a vegetable or fruit.

- Understand Food Safety and HACCP

- Name and describe microorganisms that cause foodborne illness.

- Identify the agencies and laws that regulate food safety and food labeling.

- Use laboratory equipment and scientific thinking to conduct food research trials. 
- Analyze data and create lab reports.

- Understand the economic importance of value added food products.

- Discuss fermentation and its relationship to food processing.

- Discuss the purpose of food additives.

- Discuss the process of caramelization .

- Identify qualities that make the sensory characteristics of food.

- Discuss the principles of food microbiology.

- $\quad$ Explain solutions, colloids, sols, gels, foams, and emulsions.

\section{Course Outline}

Overview of Food Science

a. Parts/segments of the Food Industry

b. Division by major product lines

c. International Trade

B. Food Industry Trends

a. U.S. Food expenditures compared to other countries

b. World food needs

c. Food consumption trends

d. Trends in meal purchases

e.Identification of allied industries

C.Safety and Laboratory Procedures
a. Tools and Layout
b. Safety Measures
c. Weights and Measures
d. Lab Organization

D.Nutrition and Digestion
a. Nutritional Needs RDA or DRI
b. Functions of Energy-Carbohydrates, fats, and proteins in the body.
c. Calorie Content
d. Essential Amino Acids
e. Water and Fat soluble vitamins
f. Minerals
g. Digestion process
h. Organs of digestion
i. Diet and Human health

E. Chemistry of Foods
a. Chemical properties of elements
b. Elemental Bonding
c. Oxidation-reduction reactions
d. Divisions of metabolism
e. Carbohydrates
f. Proteins
g. Lipids
h. Minerals
i. Water Essentials
j. Miscellaneous chemicals
a. Sanitation
b. Food-Borne Illness

F.Food Safety 
c. Regulations

G.Operations in Food Processing

a. Material Handling

b. Miscellaneous Operations

i.Particle size reduction

ii. Separating foods

iii. Mixing

iv. Heat Transfer

v. Drying and dehydration

vi. Formed foods

vii. Overlapping operations

H. Packaging

a.Packaging concepts

i.Types of food packaging

ii.Tests of packaging properties

iii. Special features

iv. Environmental concerns

v.Innovations

I.Dairy Processing
a. Milk
b. Butter
c. Ice Cream
d. Cheese
e.USDA Grades

J.Meat, Poultry, and Eggs
a. Production
b. Conversion of muscle to meat
c. Meat products
d. Factors affecting tenderness
e. Meat Cookery
f. Meat Grading
g. Value added and specialty meat products
h. Egg production
i. Egg Quality
j. Egg Grading

K.Cereal Grains, Legumes, and Oilseeds

a. Grains

i. Structure and composition

ii. Types

b. Starches

i. Properties

ii. Factors to control when cooking with starch

c. Flour

i. Milling

ii. Classes and Grades

d. Corn

i. Refining

ii. Corn by products

e. Oilseeds

i. Composition and uses

f. Legumes 
i. Composition and uses

g. Soy Beans

i. Products from extraction

h. Baking Process

i. Leavening

L. Fruits and Vegetables

a. Structure and Physiology

b. Grading

c. Storage

d. Processing

M. Fats and Oils

a. Composition and Processes

i. Saturated, Unsatureated, and Trans fats

ii. Fatty Acids

iii. Sources of fats and oils

iv. Function in Foods

N. Investigation and Experimentation

a. Lab Research Trials

O. Regulation and Labeling

a. Agencies and Regulations

b. Labeling

c. Nutritional Identification

P. Career Opportunities in Food Science

Q. Supervised Agriculture Experience

R. FFA Organization and Leadership

\section{Texts \& Supplemental Instructional Materials}

Introduction to Food Science by Rick Parker, Delmar Learning 2003

(Supplemental) Food Processing Principles and Applications by J. Scott Smith, Blackwell Publishing

(Supplemental) Principlas of Meat Science by John C. Forrest, Freeman Publishing

\section{Key Assignments}

Weekly academic textbook assignments

Weekly laboratory activities and reports

Food Science Term Paper

Value Added product development

Food Research Trials

Student Demonstrations

Notebook/Portfolio Crteation

\section{Instructional Methods and/or Strategies}

Direct instruction and discussion

- Teacher developed materials

- Operation of equipment

- Group and Independent project based learning assignment(s)

- Cooperative learning

- Audio-Visual presentations

- Web quests

- $\quad$ Reading a writing assignments

- Investigation and research assignments

- Business and or community partnerships and guest speakers

- $\quad$ Student exhibitions and presentations 
- School and community survey's

- Lab Activities

\section{Assessment Methods and/or Tools}

- Tests and quizzes

- Homework assignments

- $\quad$ Class and lab participation

- Notebook/portfolio

- Laboratory activities

- Individual or group project based learning assignments

- $\quad$ Safety awareness/evaluation in the classroom or lab

- $\quad$ Presentations and exhibitions 


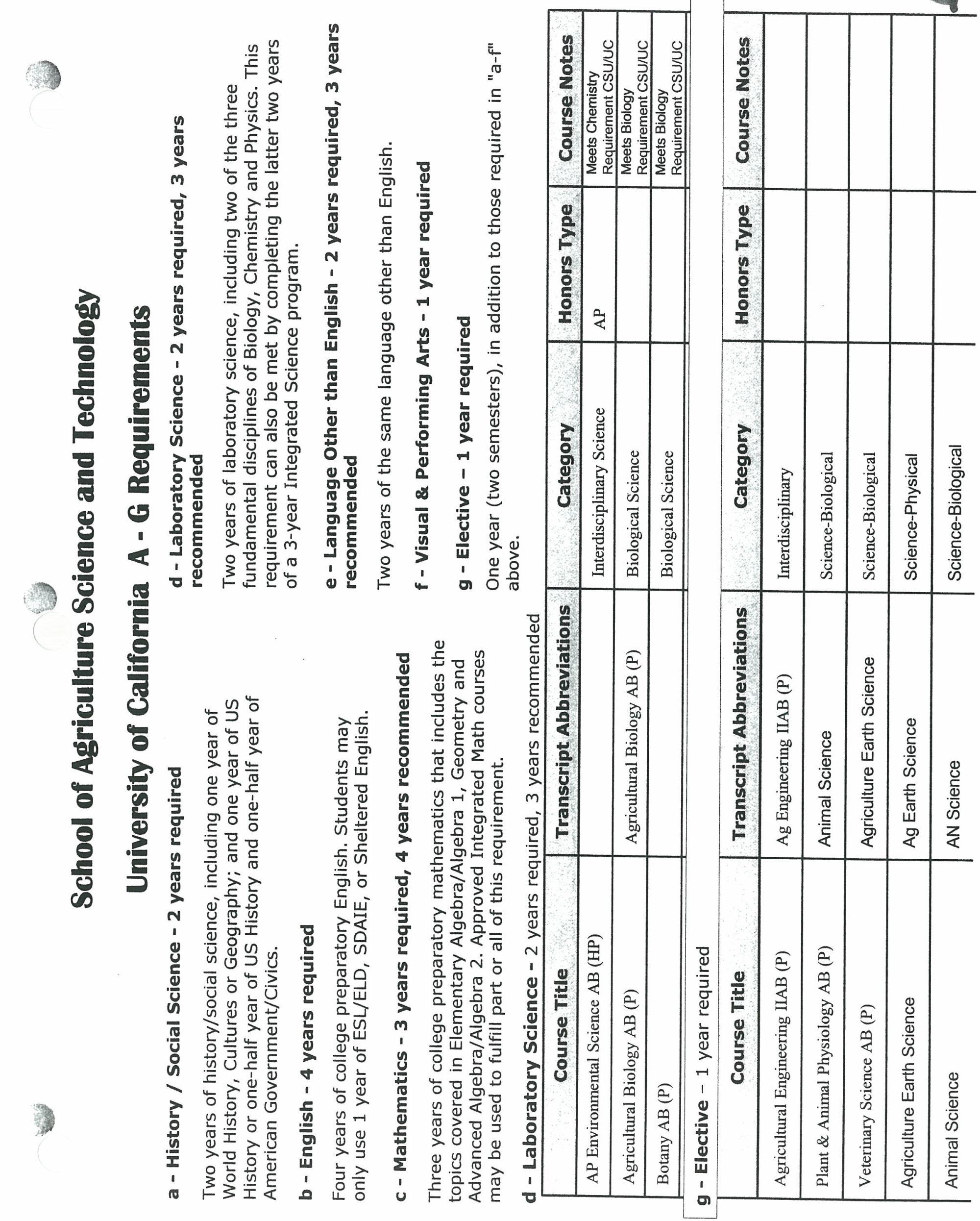


Clovis Unified School District

Career Education Department

Request for Outside Speaker

Speaker:

Date of Presentation:

Affiliation:

Topic:

Time/Periods:

Location:

Location:

Requesting Staff Member

Learning Director's Signature

Speaker:

Date of Presentation:

Affiliation:

Topic:

Time/Periods:

Location:

Clovis Unified School District

Career Education Department

Request for Outside Speaker

Deputy Principal's Signature

Date

\section{Request for Outside Speaker}




\section{TARGETED OCCUPATIONAL CLUSTERS}

Agriculture Production

Grower, Manager

Farmhand

Irrigator

Hydroponics

Farm Foreman

Farm Mechanic

Dairy Farmer

Dairy Farm Manager

Milker

Artificial Inseminator

Veterinarian

Veterinarian Assistant

Agriculture Instructor

Agriculture Supplies and Service
Agriculture Products and Processing

Machine Operator

Lift Operator

Truck Driver

Food Inspector

Laboratory Technician

Plant Manager

Plant Electrician/Mechanic

Line Foreman

Produce Department Manager

Inspector

Fieldsman, Grower Relations

Produce Buyer

Warehouseman

Creamery Manager

Produce Buyer

Processor Fieldsman

Agriculture Consultant

Laboratory Technician

Field Technician

Custom Equipment Operator

Warehouse Manager

Stock clerk

Retail Sales and Marketing

Quality Control/Inspector

Chemical Applicator

Agriculture Labor Consultant

Pest Control Advisor

Agriculture Journalism

Water Management

Conservation

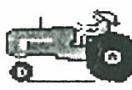




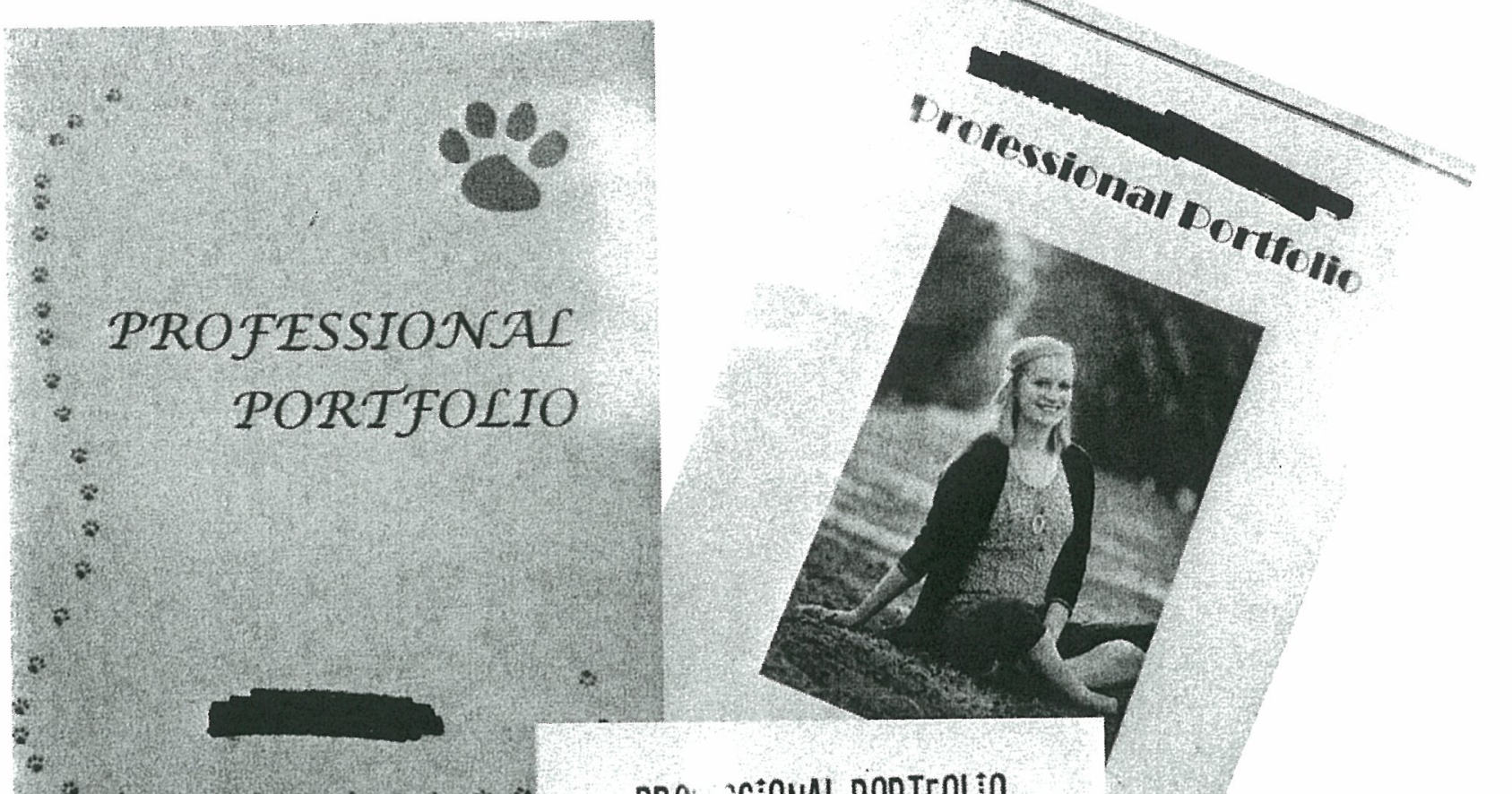

\section{PROA SSIONAL PORTFOLIO}
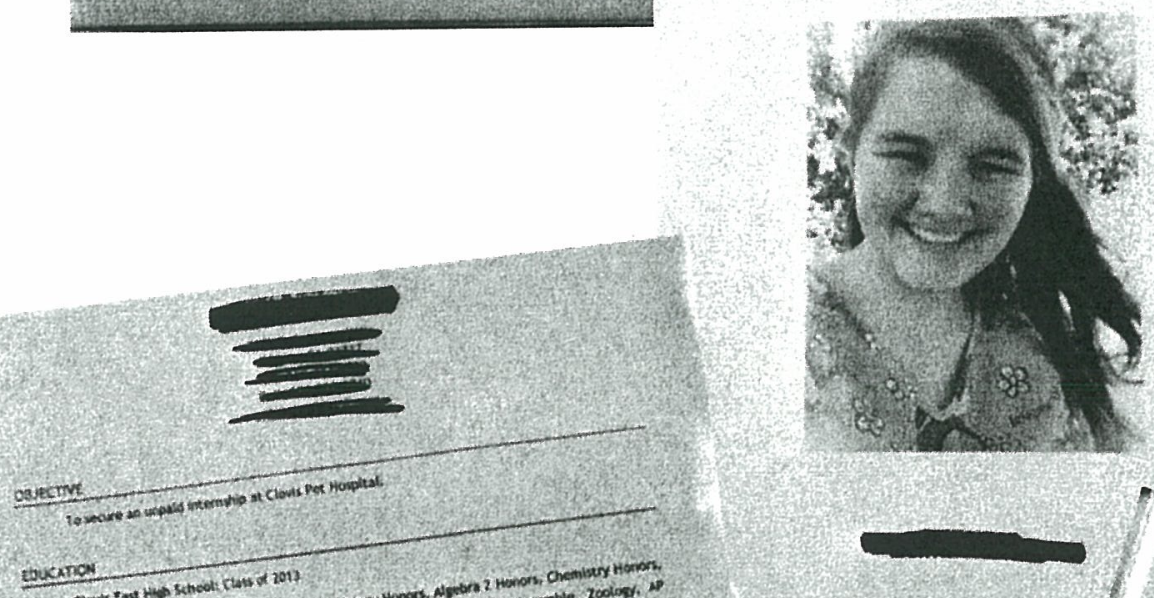

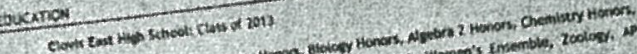

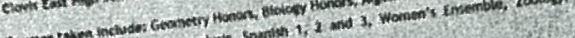

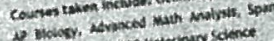

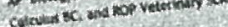

on:s"

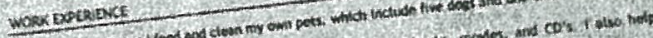

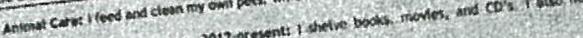

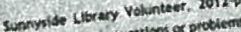

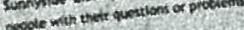

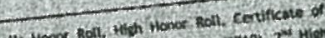
actidver. pertect the

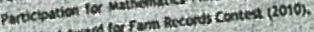

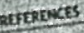

andor Ray izodosv resciet

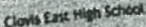

550. 237.0000

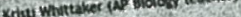
Cloms enut Hivis stood 359327.4000

syrati omatz ifomily friend Resbum interpidate school 359.377 .4500

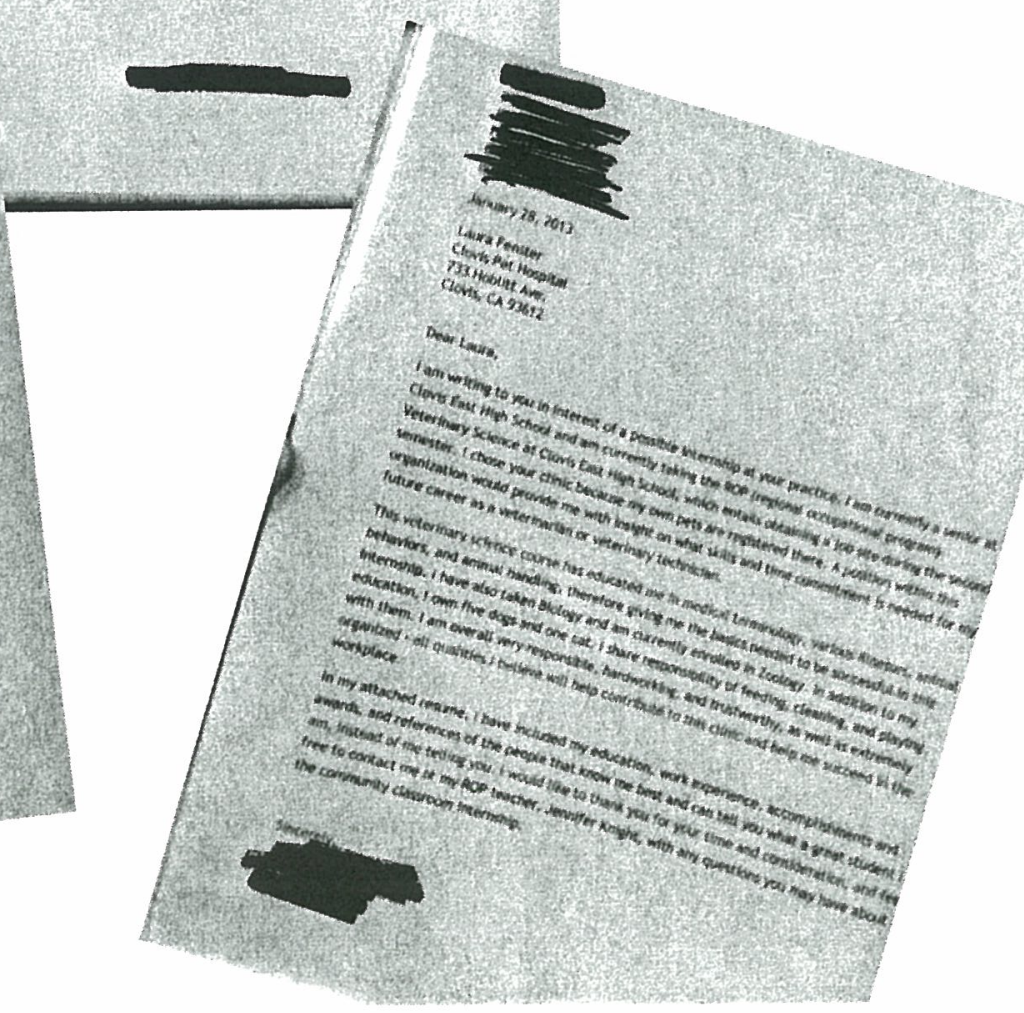




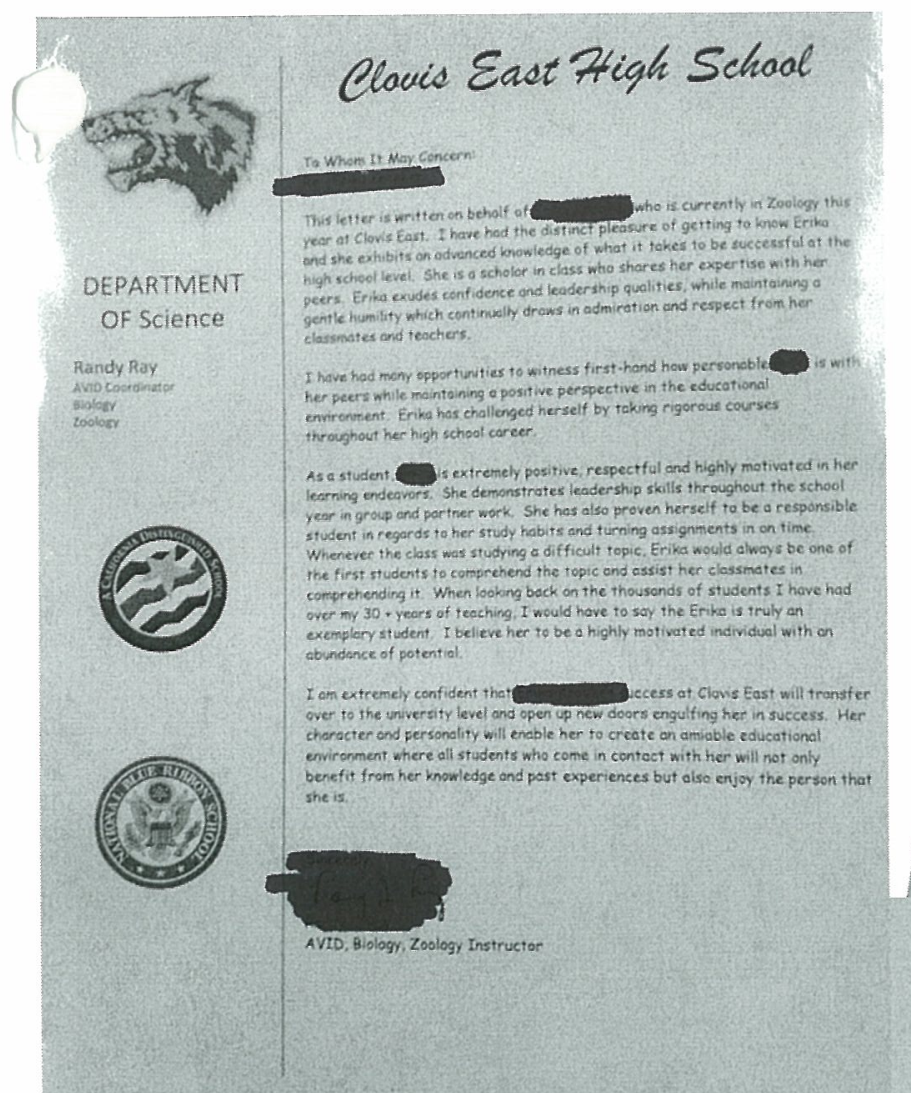

2940 Lechard Averue - Covis, Cafifornia 93619 , Phone (559) $327-4000$ - Fax (559) $327-4190$

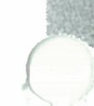

ROP Veterinary Science

30 August 2012

Clovis East High

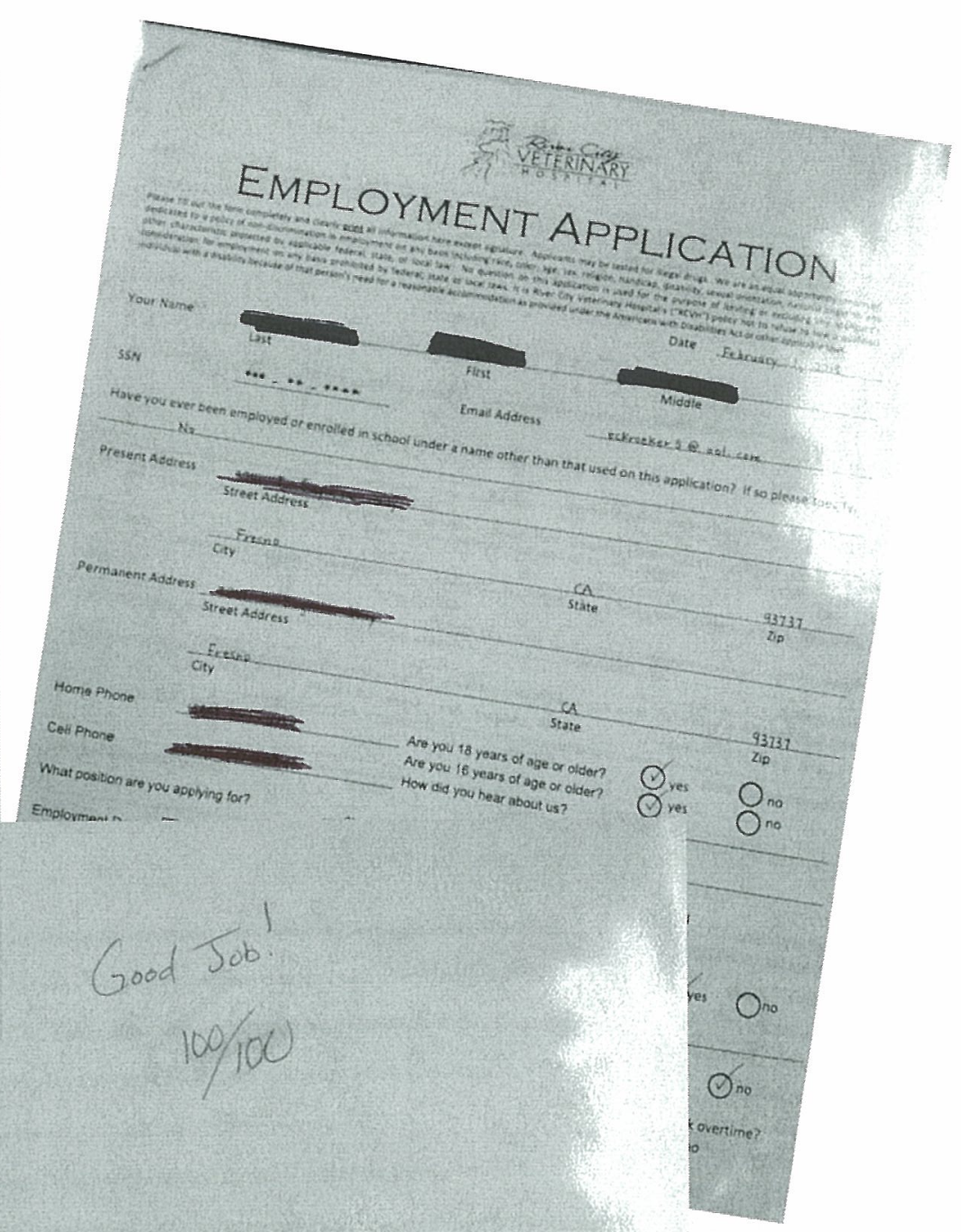

Vet Career/College Paper

Small animal veterinarians, also known as companion animat veterinarians, generally care for, diagnose and treat a variety of animals kept as pets, including dogs, cats, rabbits, birds. and reptiles. Typically, small animal veterinarians perform health exams, give vaccinations, prescribe and administer medications, draw blood, perform surgeries, treat wounds, and put terminally ill animals to sleep. Other duties may include cleaning teeth, performing post-surgical follow up exams, monitoring the reproductive health of breeding animals, assisting with birth problems, using ultrasound machines, and taking $x$-rays. In addition, small animal veterinarians communicate with pet owners about general care, behavior, nutrition, or breeding. Small animal veterinarians not only need compassion and a love for animals, but also need to be prepared to work long and irregular hours and deal with agitated or injured animals.

Future small animal veterinarians attend college for an average six to eight years. Those interested in this field can prepare by studying sciences like chemistry and biology in high school, and by volunteering in animal shelters to gain experience with small animals. First, you must complete a pre-veterinary program, which is usually three to four years, and maintain a high GPA. While veterinary schools differ in their undergraduate requirements, students should take 

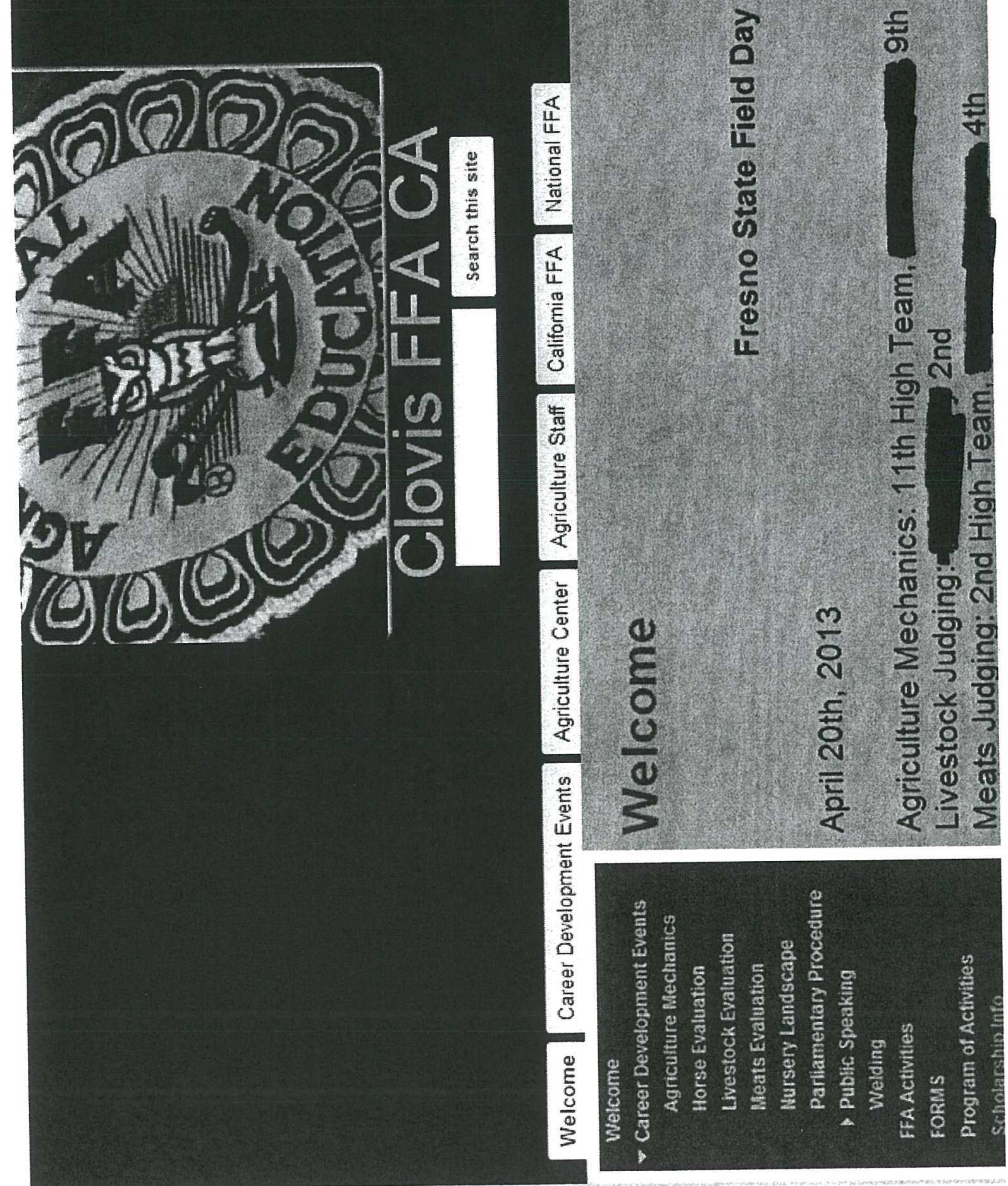


\section{CALIFORNIA AGRICULTURAL EDUCATION RECORD BOOK}

Name:

School: $\quad$ Clovis East High School

Chapter: $\quad$ Clovis

Year in Agriculture: 3

Beginning Date: $\quad$ 01/01/12 Months: 12

Ending Date: $\quad 12 / 31 / 12$

Instructor(s): $\quad$ Mrs. Covey, Mr. Woodard, Mr. Dias

Mrs. Knight, Mr. Gambril, Mr. Valdı

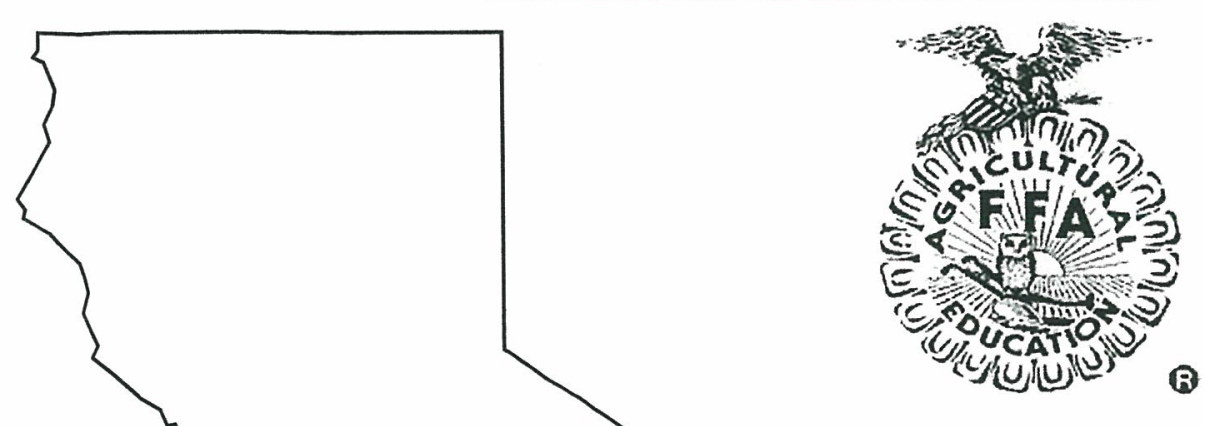




\section{$M$}

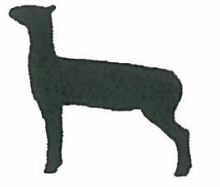

\section{Sheep Exhibitor Project Completion Sign Off Sheet}

NAME OF EXHIBITOR:

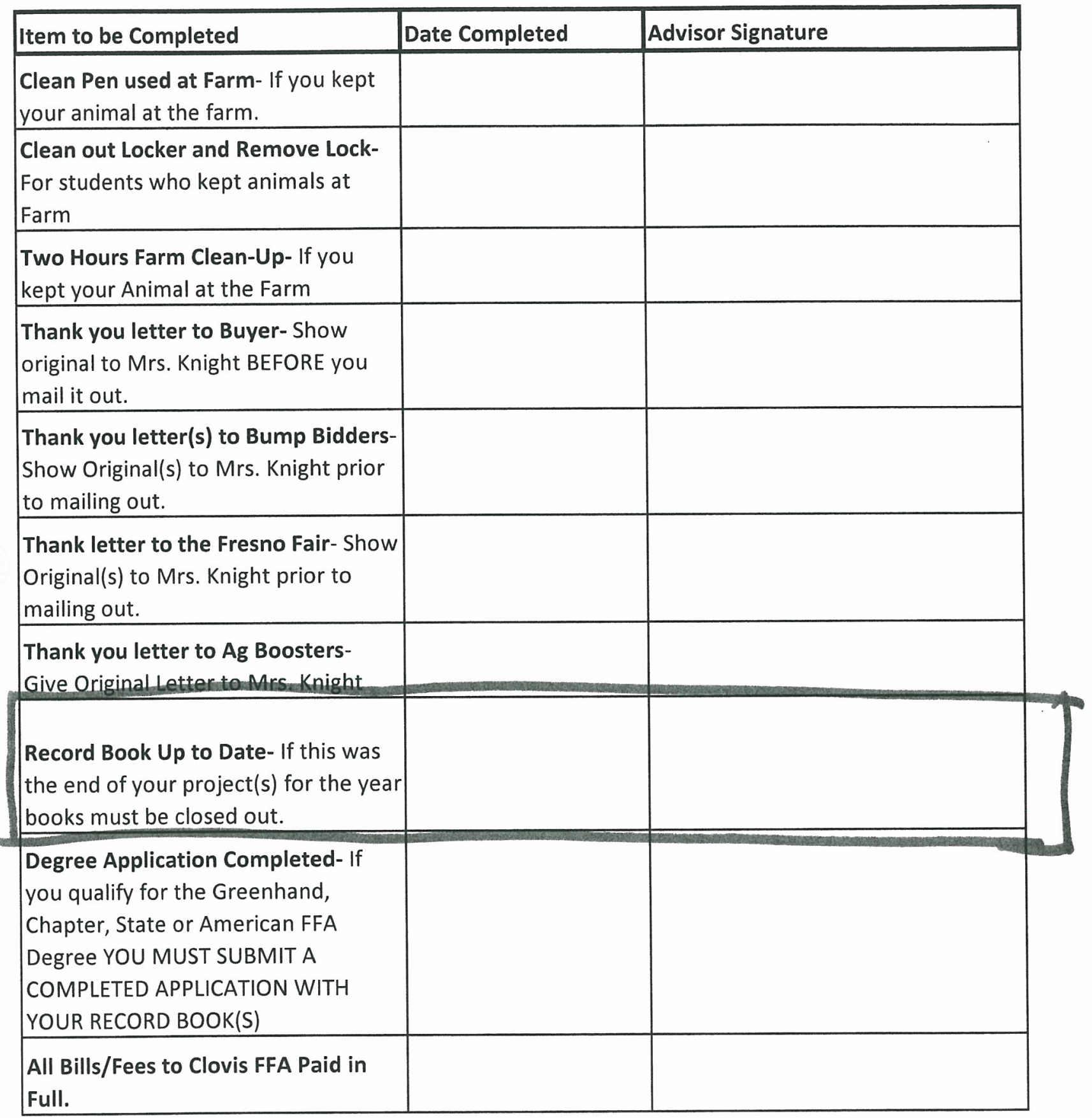

Check Received/Sign Off Completed 
Regan Educational Center

McFarlane Coffman Ag Center Room 903

Mrs. Knight

jenniferknight@cusd.com

$559-327-4343$ or $559-327-4327$

\section{Course Information:}

Course work provides for the study of classification systems, anatomy, physiology, nutrition, reproduction, mitosis, meiosis, respiration, genetics, and health care of domesticated animals. Curriculum includes lab and fieldwork utilizing scientific methodology while reinforcing writing and presentation skills. Students will seek placement at a Community Classroom site (Vet Clinic or Animal Facility) during the second semester of instruction and will be expected to complete their class hours as an unpaid assistant or job shadow participant. This class is designed to meet EVERY day $6^{\text {th }}$ and $7^{\text {th }}$ period(s) to allow for increased instructional hours in the Fall and time for job site participation in the Spring semester.

\section{Suggested Materials:}

- 3 ring binder $1.5^{\prime \prime}$ to 2 " (To use for Vet Science only)

- 8 tab dividers for vet science 3 ring binder

- Writing instrument (Pen or Pencil)

- Lined paper

- Composition or spiral notebook for work summaries (Spring Semester)

- Clear sheet protectors for portfolio materials (Spring Semester)

***If a student is unable to acquire the recommended materials the student should contact the teacher to obtain materials.

\section{Classwork and Participation:}

- Students are expected to participate actively in class by completing daily warm ups, taking notes, keeping resource materials organized, completing activities and labs, and doing homework.

- Agriculture Education is a comprehensive program and requires students to participate in a Supervised Agriculture Experience Project component as well as FFA leadership activities and events.

\section{Homework and Projects:}

- Homework may be assigned 3-4 times a week.

- Students will complete several research projects.

- Students will complete a Resume and Cover Letter that they will use to find a community classroom location placement.

A portfolio will be due as the Spring semester final project.

- Torn, crumpled, decorated, or sloppy work will not be accepted.

- Student work should have the students first and last name, course title, period, date, and assignment number in the top right corner.

Late or make up work:

- Late work will be accepted during the CURRENT unit of study for half credit. 
- Make up work is available for EXCUSED absences. It is the student's responsibility to collect make up work upon returning to class. Make-up school days are equal to number of days school days missed. This policy may change, depending on the particular situation.

\section{Testing Policy:}

- Missed tests must be made up within one week of student returning to school, an appointment should be made at lunch, break, before or after school.

- Students may re take a test once if they scored below 75\%. The max score that can be earned on a re take is $75 \%$

\section{Grade Breakdown FALL SEMESTER:}

- Tests \& Quizzes

$30 \%$

- Class \& Homework

$25 \%$

- Labs \& Projects

$30 \%$

- Binders

$10 \%$

FFA and SAE

$5 \%$

- SAE Credit by completion of FFA record book

- FFA Points awarded by attending 1 activity for each 6 week grading period.

\section{Grade Breakdown SPRING SEMESTER:}

- Special Presentations $10 \%$

- Job Site Journal 10\%

Final Portfolio $\quad 40 \%$

- Time Cards and Hours Worked 30\%

- Points can be deducted for:

- Not calling in absences or tardies

- Turning in time cards late

- Not meeting hour requirements

- Quality of work on Job Site

$10 \%$

$\circ$ (Instructor and Supervisor Evaluations)

\section{Behavior and Discipline:}

- Please refer to the student handbook. ALL CEHS and CUSD rules will be strictly enforced. * Character Counts pillars and principals will also be a part of our classroom environment.

\section{Extra Credit and Incentives:}

- Incentive tickets will be awarded in class for exemplary participation, as a reward, or for displays of Character Counts.

- Tickets may be used for the following: (The 3 P's)

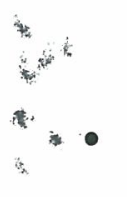

- (Points) Extra Credit Points at the end of a grading period

$\circ$ (Potty) Exchange 1 ticket for a restroom trip

- (Prizes) Exchange for various prizes

Other extra credit may be assigned as the instructor sees fit but is not frequently available, and will not replace the need to complete regular assignments.

\footnotetext{
The Clovis Unified School District does not discriminate on the basis of race, color, disability, or national origin in admission or access to and treatment of employment in its programs and activities as required by Title VI, Title IX and Section 504. Complaints: Contact Dr. Cheryl Rogers, Human Resources, (559) 327-9308.

Favor de llamar a la oficina para assistencia en espanol.

Thov tiv tauj peb lub hoob kas yog xav tau kev pab nrog lus Hmoob.
} 


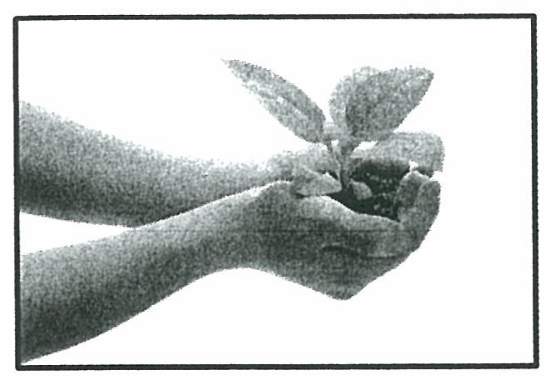

\section{Botany}

Clovis East High School

McFarlane Coffman Ag Center Room 903

Mrs. Knight

jenniferknight@cusd.com

559-327-4343 or 559-327-4327

Course Information:

Botany is the Biology of plants, and will include concepts like plant classification, plant anatomy and physiology, growth principals, reproduction, soil science, biotechnology, and weed science and entomology.

Suggested Materials:

- 3 ring binder 1" to 1.5" (To use for Botany and leave in the classroom)

- 5 tab dividers for Botany 3 ring binder

- Writing instrument (Pen or Pencil)

- Lined paper to write notes or journal entries

***If a student is unable to purchase the recommended materials, the student should contact the teacher and materials will be provided.

\section{Classwork and Participation:}

- Students are expected to participate actively in class by completing daily warm ups, taking notes, keeping resource materials organized, completing activities and labs, and doing homework.

- Agriculture Education is a comprehensive program and requires students to participate in a Supervised Agriculture Experience Project component as well as FFA leadership activities and events.

Homework and Projects:

- Homework may be assigned 2-3 times a week.

Students will complete an agriscience research project.

- Torn, crumpled, decorated, or sloppy work will not be accepted.

- Student work should have the students first and last name, course title, period, date, and assignment number in the top right corner.

Late or make up work:

- Late work will be accepted during the CURRENT unit of study for half credit. 
- Make-up work is available for EXCUSED absences. It is the student's responsibility to collect make up work upon returning to class. Make-up school days are equal to number of days school days missed. This policy may change, depending on the particular situation.

\section{Testing Policy:}

- Missed tests must be made up within one week of student returning to school, an appointment should be made at lunch, break, before or after school.

- Students may re-take a test once if they scored below $75 \%$. The max score that can be earned on a re take is $75 \%$

\section{Grade Breakdown:}

- Tests \& Quizzes

- Class \& Homework

- Labs \& Projects

- Binders FFA and SAE

$25 \%$
$25 \%$
$35 \%$
$10 \%$
$5 \%$

- SAE Credit by completion of FFA record book

- FFA Points awarded by attending 1 activity for each 6 week grading period.

\section{Behavior and Discipline:}

- Please refer to the student handbook. ALL CEHS rules will be strictly enforced.

- Character Counts pillars and principals will also be a part of our classroom environment.

\section{Extra Credit and Incentives:}

- Incentive tickets will be awarded in class for exemplary participation, as a reward, or for displays of Character Counts.

- Tickets may be used for the following: (The 3 P's)

- (Points) Extra Credit Points at the end of a grading period

- (Potty) Exchange 1 ticket for a restroom trip

- (Prizes) Exchange for various prizes

- Other extra credit may be assigned as the instructor sees fit but is not frequently available, and will not replace the need to complete regular assignments.

\footnotetext{
The Clovis Unified School District does not discriminate on the basis of race, color, disability, or national origin in admission or access to and treatment of employment in its programs and activities as required by Title VI, Title IX and Section 504. Complaints: Contact Dr. Cheryl Rogers, Human Resources, (559) 327-9308.

Favor de llamar a la oficina para assistencia en espanol.

Thov tiv tauj peb lub hoob kas yog xav tau kev pab nrog lus Hmoob.
} 


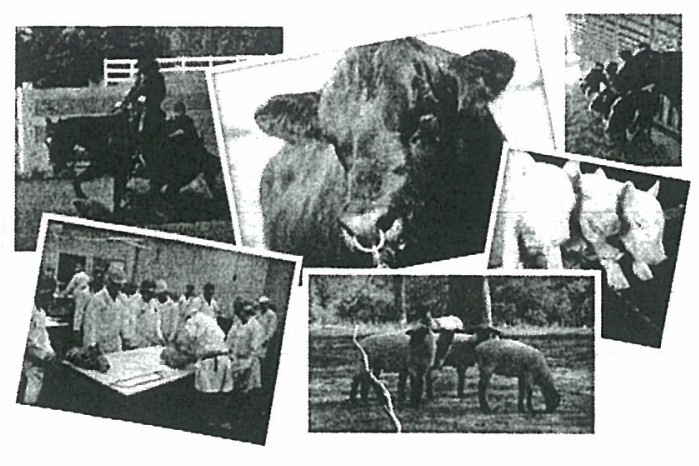

\section{Animal Science}

Clovis East High School

McFarlane Coffman Ag Center Room 903

Mrs. Knight

jenniferknight@cusd.com

559-327-4343 or 559-327-4327

\section{Course Information:}

This course will provide the student with principals in Animal Science focusing on the areas of livestock (Mammalian) production, anatomy, physiology, reproduction, nutrition, respiration, and genetics. This course is intended to prepare those students who plan on majoring in Agriculture or Animal Sciences at a college or University.

\section{Suggested Materials:}

- 3 ring binder 1" to 1.5" (To use for Animal Science and leave in the classroom)

- 5 tab dividers for Animal science 3 ring binder

- Writing instrument (Pen or Pencil)

- Lined paper to write notes or journal entries

***If a student is unable to purchase the recommended materials, the student should contact the teacher and materials will be provided.

\section{Classwork and Participation:}

- Students are expected to participate actively in class by completing daily warm ups, taking notes, keeping resource materials organized, completing activities and labs, and doing homework.

- Agriculture Education is a comprehensive program and requires students to participate in a Supervised Agriculture Experience Project component as well as FFA leadership activities and events.

\section{Homework and Projects:}

- Homework may be assigned 2-3 times a week.

- Students will complete an agriscience research project.

- Torn, crumpled, decorated, or sloppy work will not be accepted.

- Student work should have the students first and last name, course title, period, date, and assignment number in the top right corner.

\section{Late or make up work:}

- Late work will be accepted during the CURRENT unit of study for half credit.

- Make-up work is available for EXCUSED absences. It is the student's responsibility to collect make up work upon returning to class. Make-up school days are equal to number of days school days missed. This policy may change, depending on the particular situation. 


\section{Testing Policy:}

- Missed tests must be made up within one week of student returning to school, an appointment should be made at lunch, break, before or after school.

- Students may re-take a test once if they scored below $75 \%$. The max score that can be earned on a re-take is $75 \%$

\section{Grade Breakdown:}

- Tests \& Quizzes 25\%

- Class \& Homework 25\%

- Labs \& Projects $\quad 35 \%$

- Binders 10\%

- FFA and SAE $5 \%$

- SAE Credit by completion of FFA record book

- FFA Points awarded by attending 1 activity for each 6 week grading period.

\section{Behavior and Discipline:}

- Please refer to the student handbook. ALL CEHS rules will be strictly enforced.

- Character Counts pillars and principals will also be a part of our classroom environment.

\section{Extra Credit and Incentives:}

- Incentive tickets will be awarded in class for exemplary participation, as a reward, or for displays of Character Counts.

- Tickets may be used for the following: (The 3 P's)

- (Points) Extra Credit Points at the end of a grading period

- (Potty) Exchange 1 ticket for a restroom trip

- (Prizes) Exchange for various prizes

- Other extra credit may be assigned as the instructor sees fit but is not frequently available, and will not replace the need to complete regular assignments.

\footnotetext{
The Clovis Unified School District does not discriminate on the basis of race, color, disability, or national origin in admission or access to and treatment of employment in its programs and activities as required by Title VI, Title IX and Section 504. Complaints: Contact Dr. Cheryl Rogers,
Human Resources, (559) 327-9308.

Favor de llamar a la oficina para assistencia en espanol.

Thov tiv tauj peb lub hoob kas yog xav tau kev pab nrog lus Hmoob.
} 


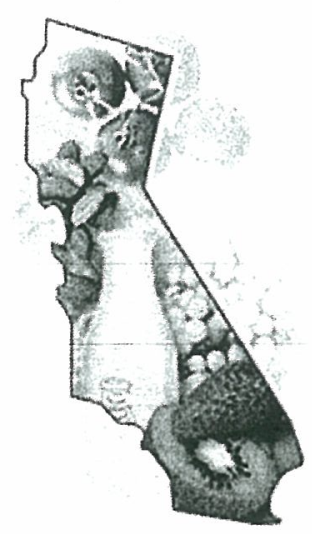

\title{
Exploring Agriculture
}

\author{
Regan Educational Center \\ McFarlane Coffman Ag Center Room 903 \\ Mrs. Knight \\ jenniferknight@cusd.com \\ $559-327-4343$ or $559-327-4327$
}

\section{Course Information:}

This one semester course will provide Junior High School students with an introduction and overview of Agriculture. Topics may include California agriculture, Animal Sciences, Crop Science, and Natural Resources. It will also serve as an introduction to the Agriculture Program available at the High School level.

\section{Suggested Materials:}

- 70 page spiral bound notebook (To use for this class only)

- Writing instrument (Pen or Pencil)

- Lined paper to write notes or complete homework

***If a student is unable to acquire the recommended materials the student should contact the teacher to obtain materials.

\section{Classwork and Participation:}

- Students are expected to participate actively in class by completing daily warm ups, taking notes, keeping resource materials organized, completing activities and labs, and doing homework.

- Students will have the opportunity to work outdoors at our state of the art Ag Center, and they may get dirty. Participation and work ethic will also be evaluated as part of the student's grade.

- Agriculture Education is a comprehensive program and students may have the opportunity to participate in additional activities that are part of the FFA organization.

\section{Homework and Projects:}

- Homework may be assigned, but students should have time to work on assignments during class.

- Torn, crumpled, decorated, or sloppy work will not be accepted.

- Student work should have the students first and last name, course title, period, date, and assignment number in the top right corner.

\section{Late or make up work:}

- Late work will be accepted during the CURRENT unit of study for full credit.

- Make up work is available for EXCUSED absences. It is the student's responsibility to collect make up work upon returning to class. Make-up school days are equal to number of days school days missed. This policy may change, depending on the particular situation. 


\section{Testing Policy:}

- Missed tests must be made up within one week of student returning to school, an appointment should be made at lunch, break, before or after school.

- Students may re take a test once if they scored below $75 \%$. The max score that can be earned on a re-take is $85 \%$

\section{Grade Breakdown:}

- Tests \& Quizzes $\quad 15 \%$

- Class \& Homework 25\%

- Projects and Presentations $\quad 30 \%$

- Notebooks 20\%

- Character Counts \& Work Ethic $\quad 10 \%$

\section{Behavior and Discipline:}

- Please refer to the student handbook. ALL Regan Educational Center rules will be strictly enforced.

- Character Counts pillars and principals will also be a part of our classroom environment.

\section{Extra Credit and Incentives:}

- Incentive tickets will be awarded in class for exemplary participation, as a reward, or for displays of Character Counts.

- Tickets may be used for the following: (The 3 P's)

- (Points) Extra Credit Points at the end of a grading period

- (Potty) Exchange 1 ticket for a restroom trip

- (Prizes) Exchange for various prizes

- Extra credit may be available for attendance at certain FFA events.

The Clovis Unified School District does not discriminate on the basis of race, color, disability, or national origin in admission or access to and treatment of employment in its programs and activities as required by Title VI, Title IX, and Section 504. Complaints: Contact Dr. Cheryl Rogers, Human Resources, (559) 327-9308.

Favor de llamar a la oficina para asistencia en español.

Thov tiv tauj peb lub hoob kas yog xav tau kev pab nrog lus Hmoob. 


\section{Quality Criteria Two}

\section{Leadership and Citizenship Development}

The Clovis FFA Chapter was Chartered in 1932 (Chapter Number 0042) and has been a well noted and competitive chapter ever since. We have had 44 State Champion CDE Teams, 24 National Champion Teams, and 7 State and 1 National FFA Officer. I am proud to say that I am a past Clovis FFA member, and was part of the above mentioned successes. Our first State Farmer was John Coffman in 1941 and our Ag Center is now named after him, he still to this day contributes advice and support for our program as part of our Advisory Board. I am also very proud of the fact that many past Clovis FFA members are still involved in helping our program be successful today. Many serve in our Ag Boosters group and help serve judges for our local contests. Clovis FFA is still a powerhouse of leadership today, as we participate in more than the 12 activities required of an active Chapter, and when we do participate we do it with the intention of winning!

Our Chapter's Program of Work is revised annually by our Ag Staff and Chapter FFA Officers and submitted to our Regional Supervisor Mr. Parker. Our program of work is comprehensive and covers the three circles of Ag Education; it gives specific information to students and parents about the opportunities that exist for our agriculture students. In all agriculture classes it states on our course syllabi that students will be graded on their FFA and SAE participation for $5 \%$ of their grade. It is expected that students participate in three FFA activities at any level per semester. This will be verified by sign in sheets or attendance rosters kept by the Ag Staff. We are currently working as a staff to standardize this process and make the way students are scored for FFA participation consistent in all Ag Classes. This will be accomplished by the scoring rubric we develop for the FFA Record Book. We also will be increasing the percentage from 5 to $10 \%$ as a student should not be able to receive an " $\mathrm{A}$ " grade without FFA/SAE participation. I was advised to leave the percentages at $5 \%$ for my first year this year, even though I wished it was more.

In order to ensure all students are affiliated we complete the student data sheets with our classes during the first month of school. This also helps serve as a means to 
discuss and promote courses we offer in our department and for the students to make plans on which activities they would like to attend for the year in order to earn their $5 \%$ toward their grade. To assist our students in FFA planning and filling out their record books we create an FFA Activities Chart for the entire year, this is a one page document that lists all activities by month for the whole school year.

In order to motivate our students to be involved in the Chapter Activities and beyond we have a point awards system that recognizes our top 20 FFA members each year at our Spring Awards Banquet. Our top 20 will receive a special trip for their outstanding accomplishment, last year they went white water rafting. Looking into the future we would like to improve how we use this point award system. What we have in mind is to collect point sheets or data monthly and have the chapter Vice President and Historian update it each month. Then the tally sheet can be posted so members know where they stand and can set goals for reaching a certain level or points or making the top 20. We would also like to incorporate an FFA Bucks Program, where students can earn FFA Bucks for participation in certain fundraising events. The Bucks would act as cash that could be used for payment toward FFA sponsored events like State Conference or buying an FFA Jacket just to name a few options. Also by posting the point awards this could give us a more accurate idea of what percentage of our members are actively involved. If we see only a small percentage involved then we could investigate ways to increase involvement within the Chapter.

\section{Supporting Completion Materials}

Item A- Clovis FFA Program of Activities. Classroom copies printed, then posted on the Clovis FFA Web Page.

Item B- Picture of the Clovis FFA Charter and Picture of the FFA Wall on the front of our Ag Department Building.

Item C- Copies of Course Syllabi showing FFA as a graded component of each Ag Class. 
Item D- Copy of the FFA activities Chart provided for all members and Ag Staff at the beginning of each school year.

Item E- FFA Top Twenty points application.

Item F- Pictures from the 2012-2013 FFA Scrapbook

Item G \& H- Travel Requests. Copy of an overnight trip request form I submitted for the FFA Parli-Pro Contest at UC Davis. Copy of a FFA Field Trip Permission slip that I made for the MFE/ALA Trip this year.

Item I \& J- Open PO in my name for reimbursement for FFA associated costs. Copy of Remittance form that is to be completed and submitted with receipts for reimbursement.

\section{** Ag Ed 539 Special Project}

-Part of my special project was to create new policies for FFA officers and Chapter FFA Officer Elections. Please see these items in the 539 Special Projects Section. 

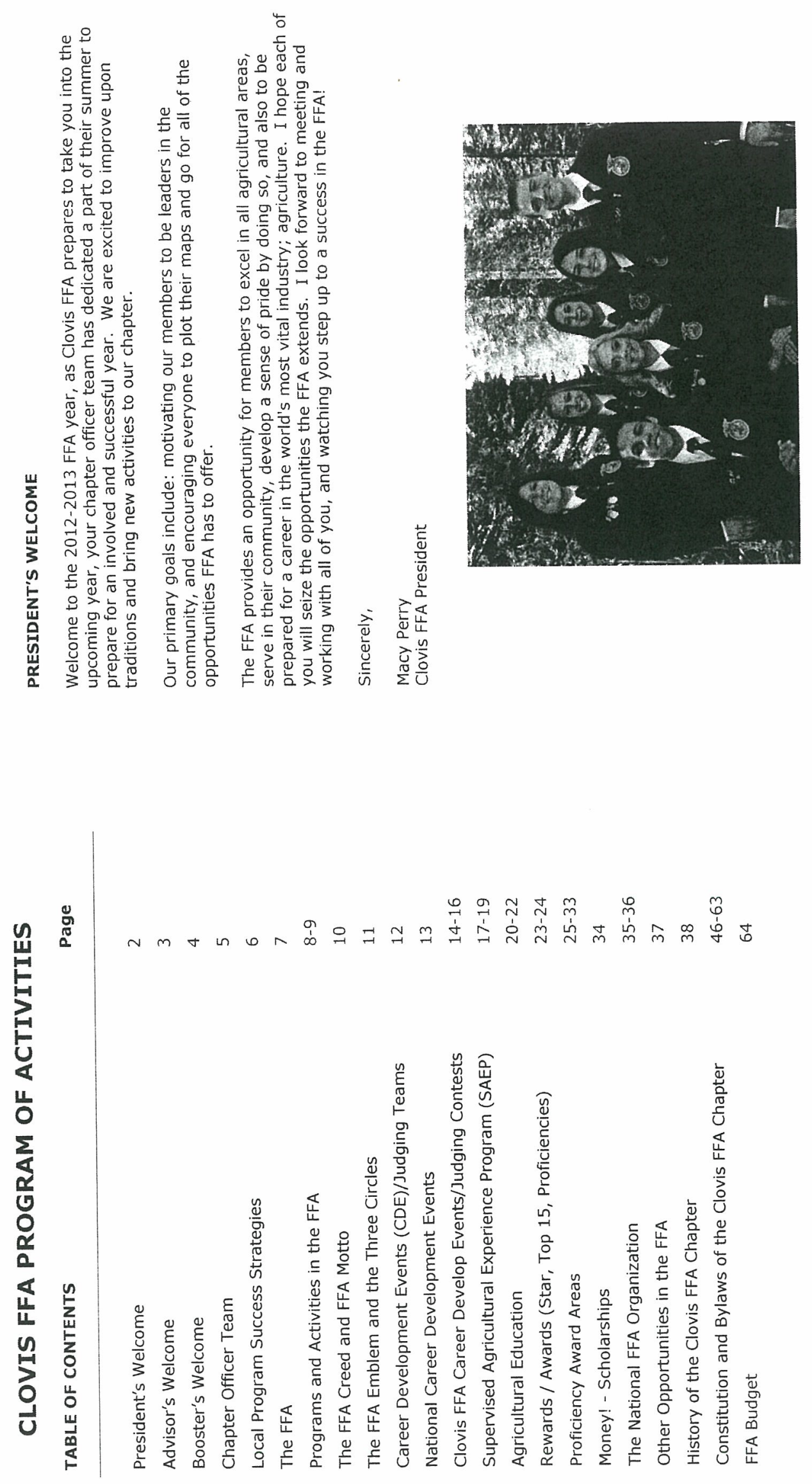

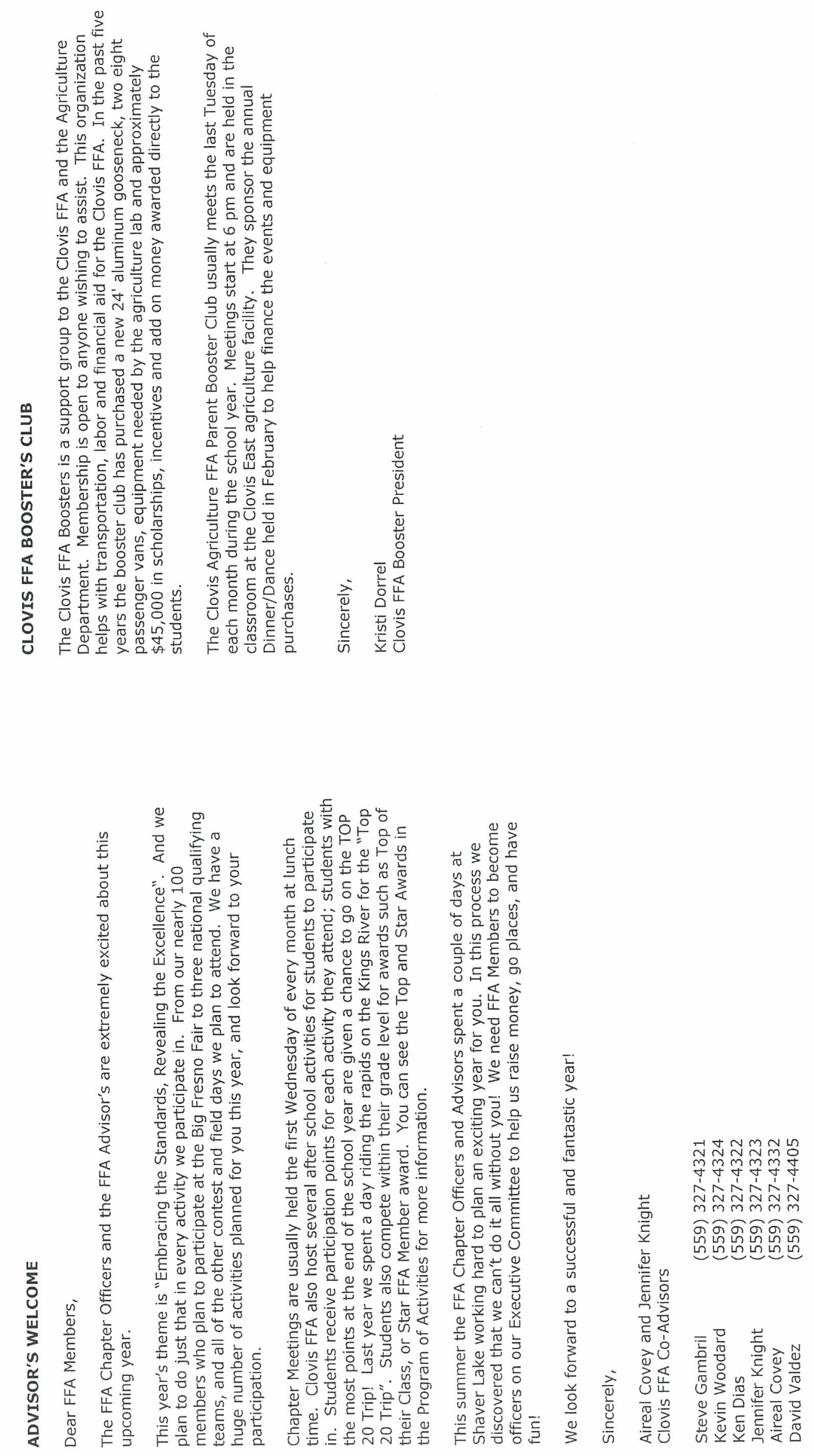

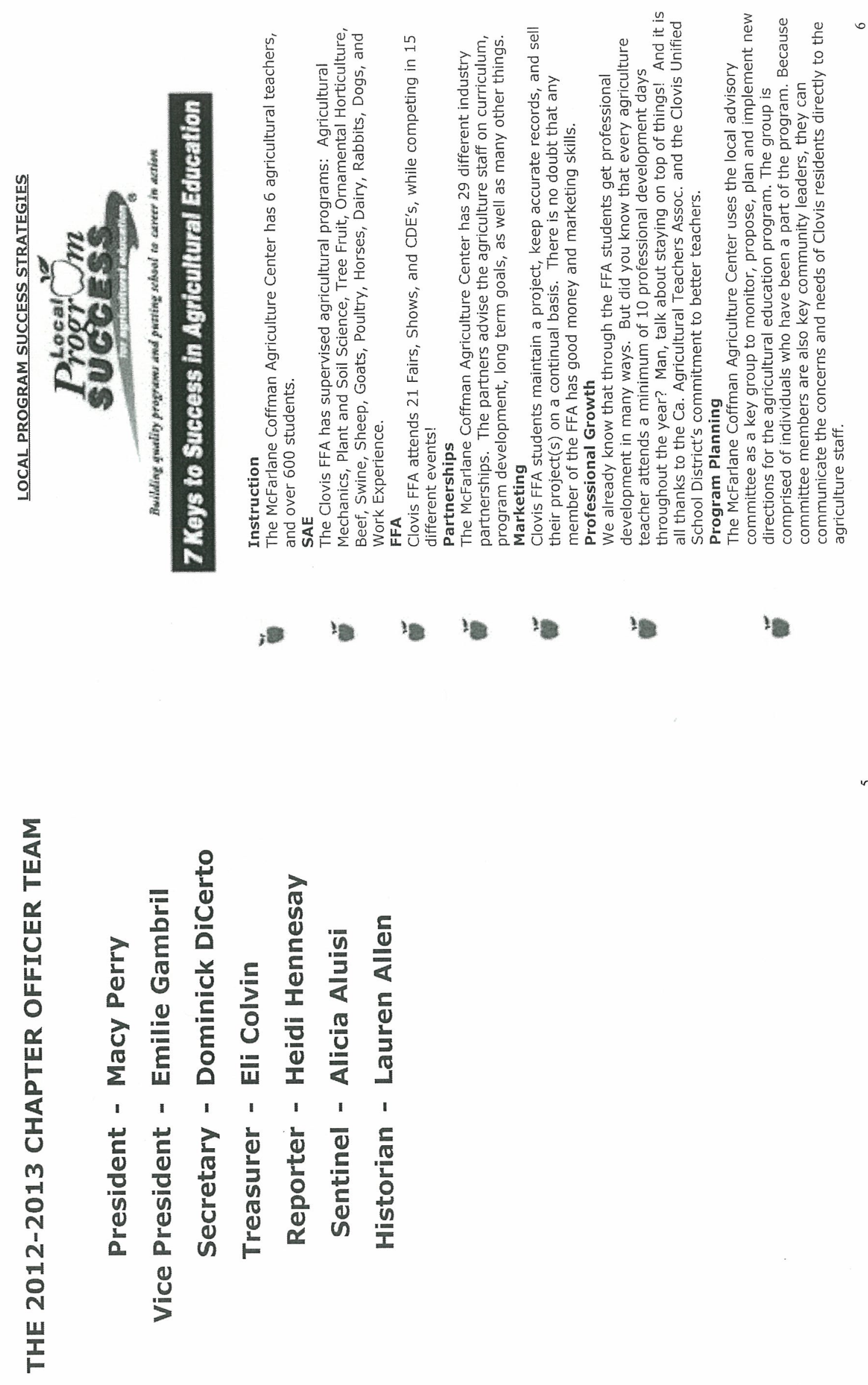

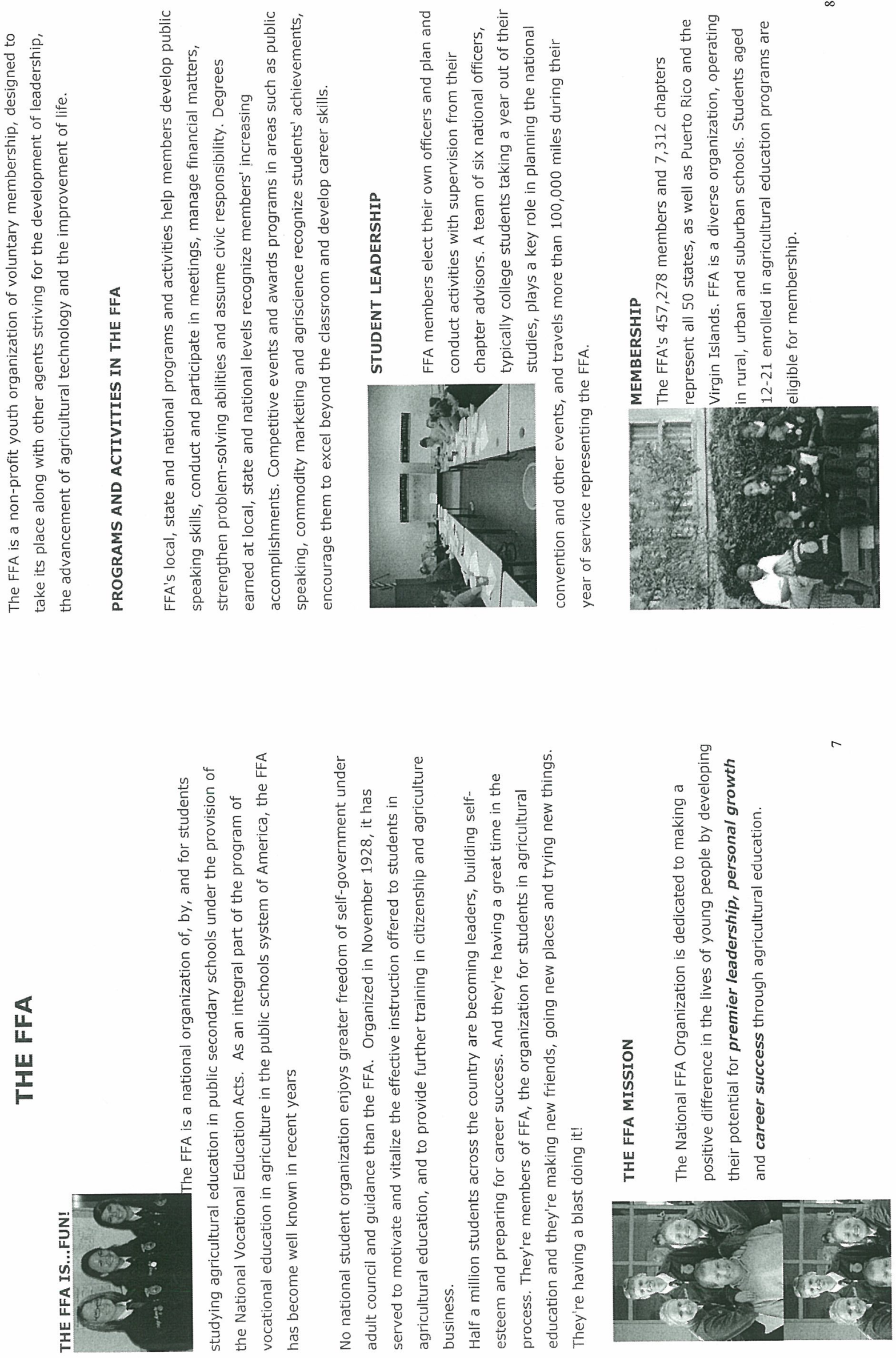

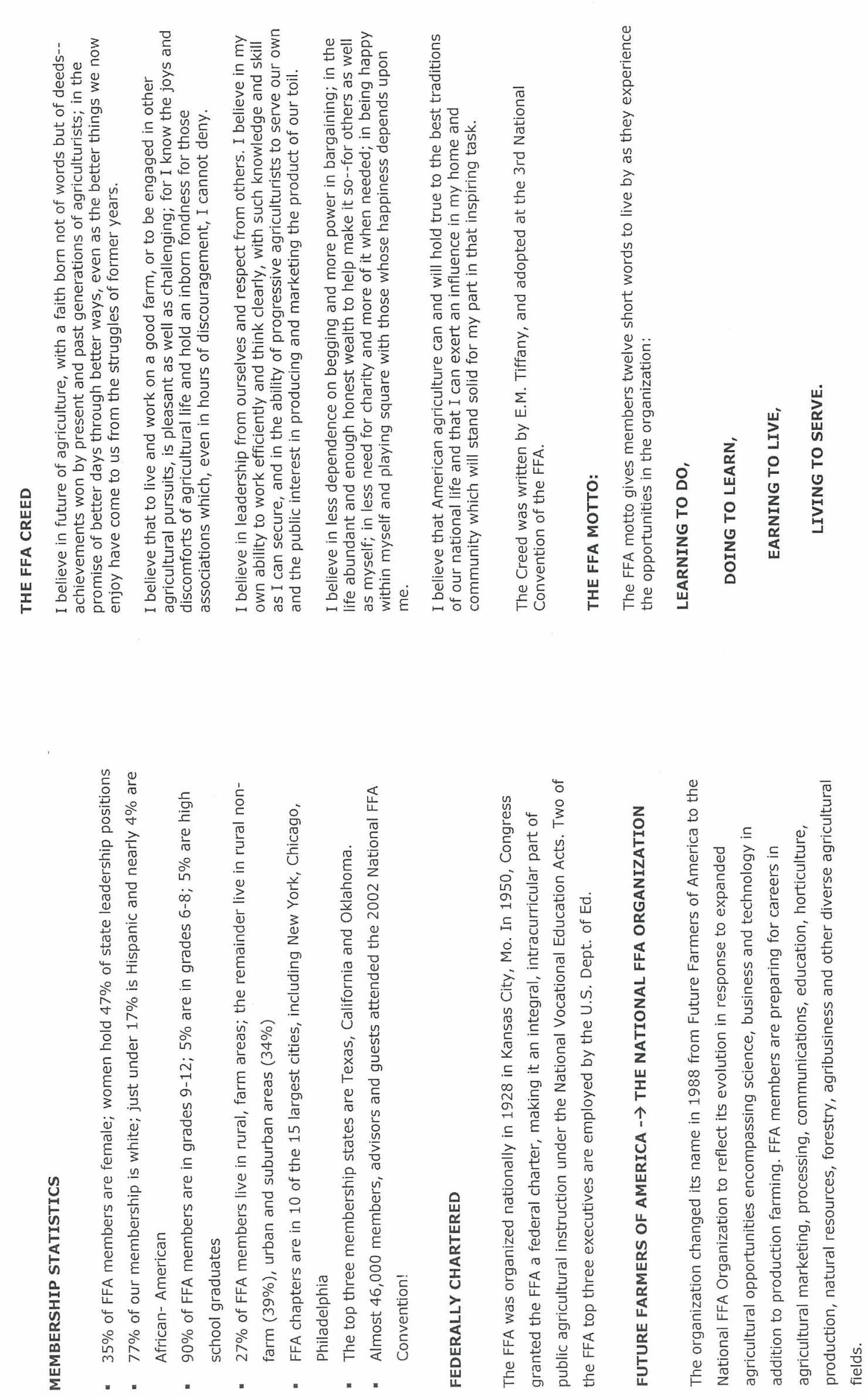

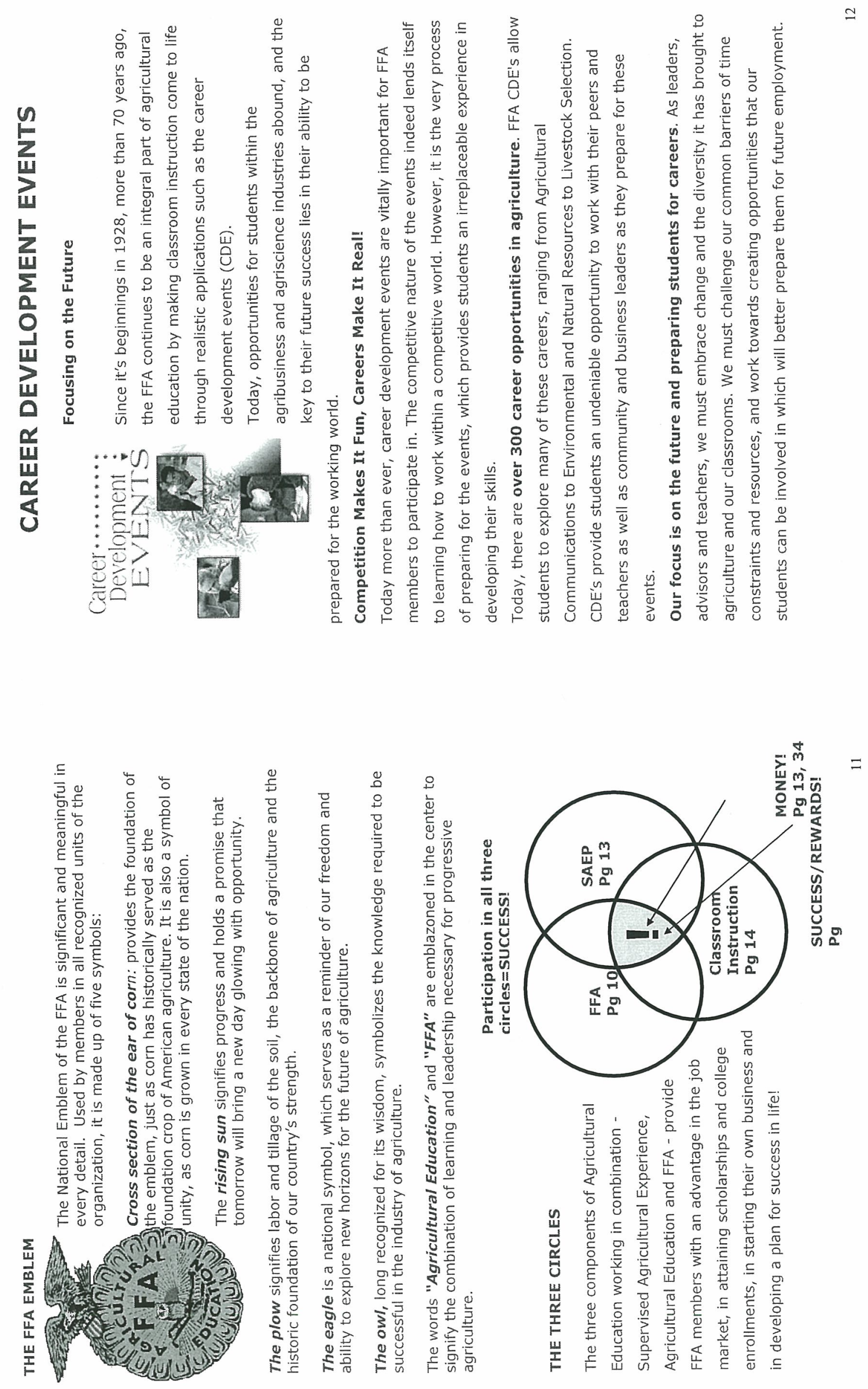

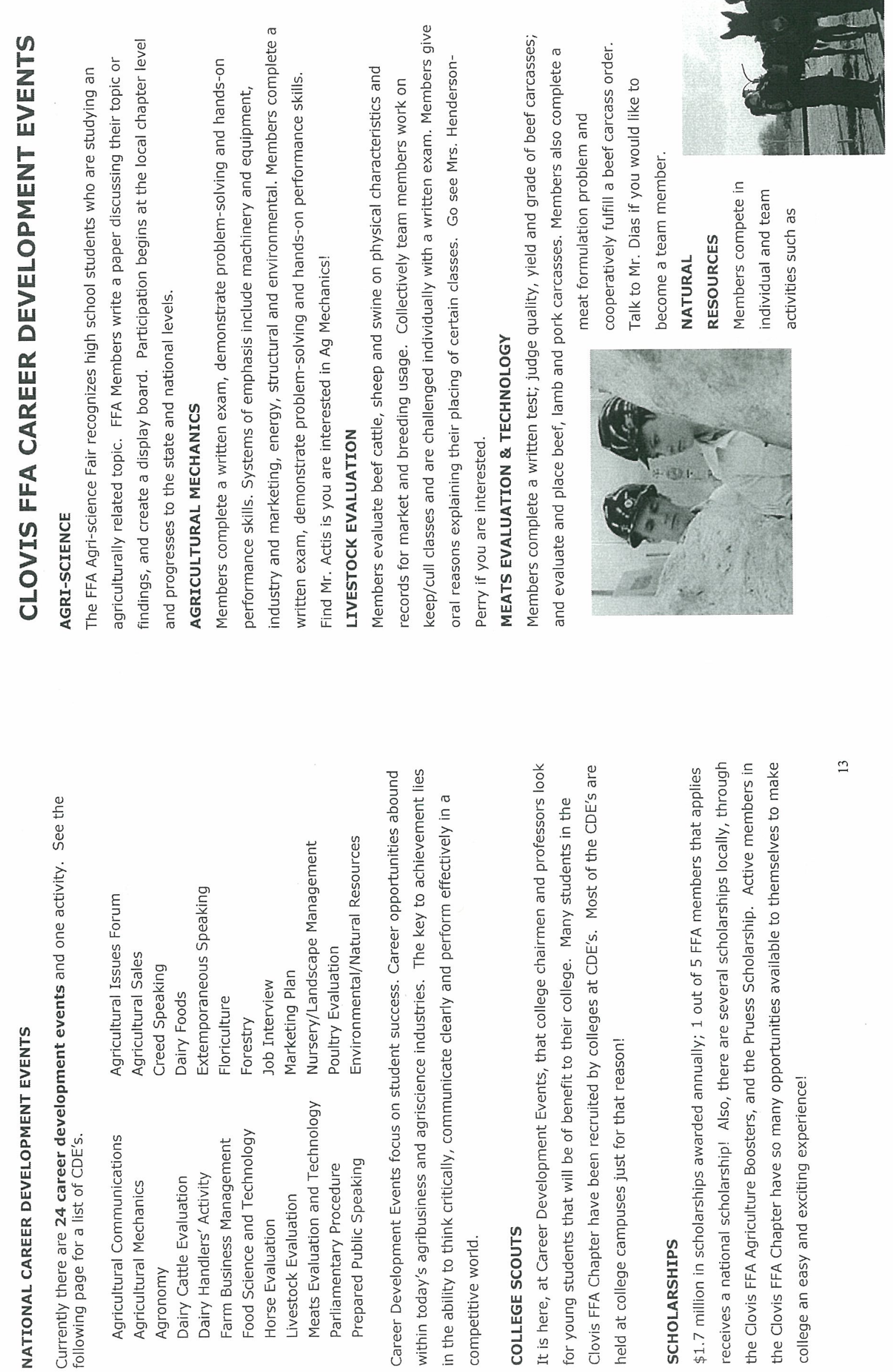

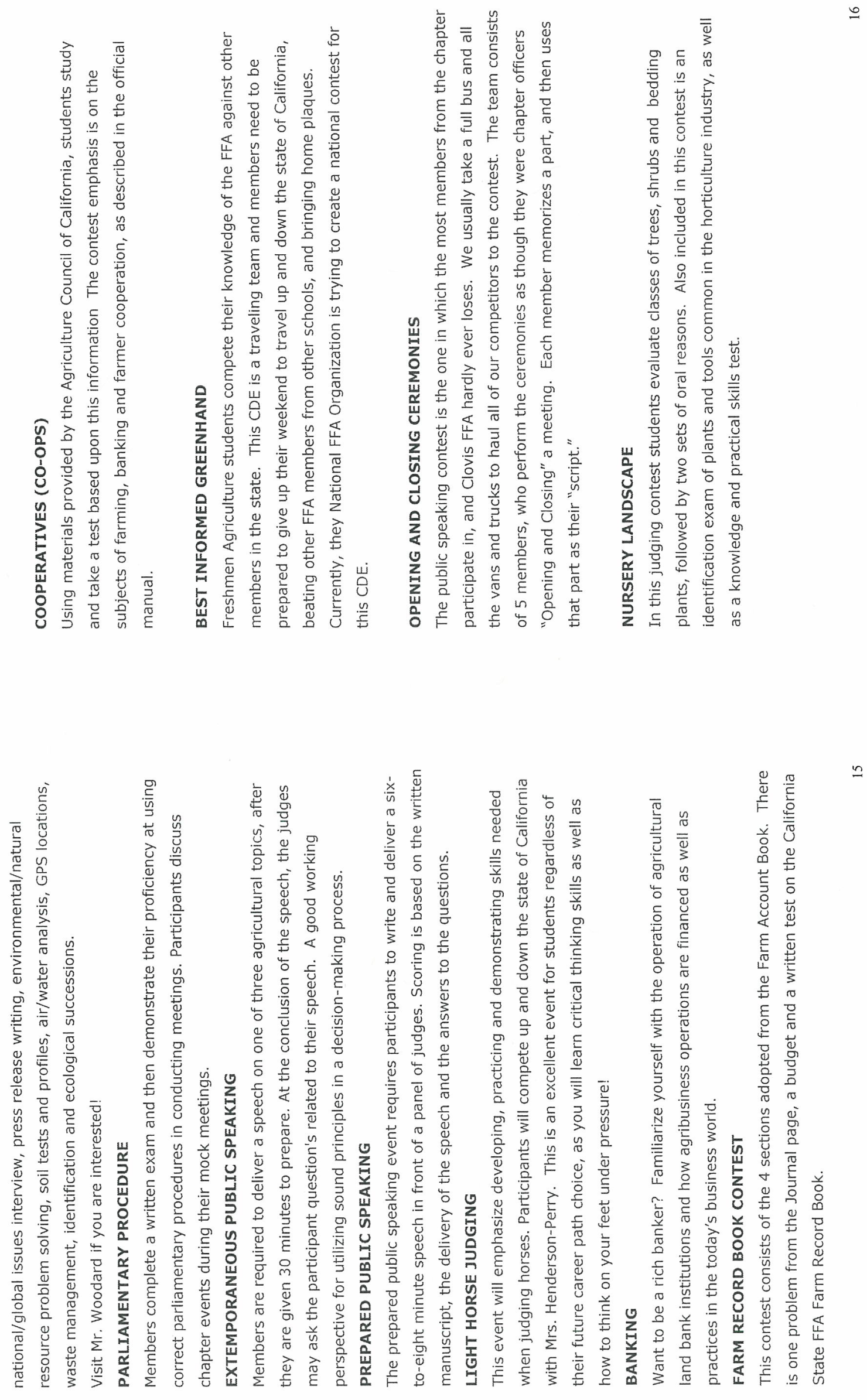

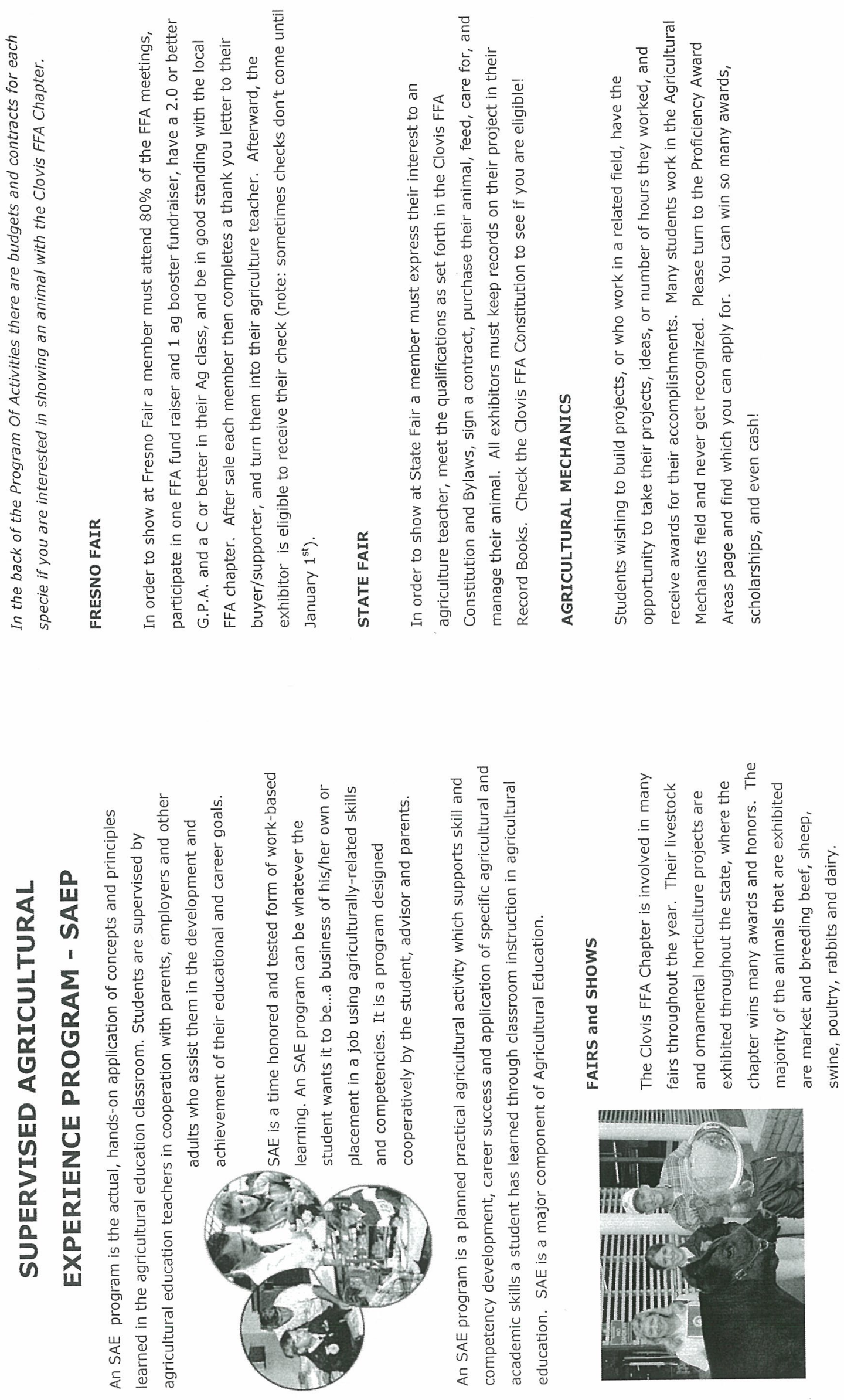

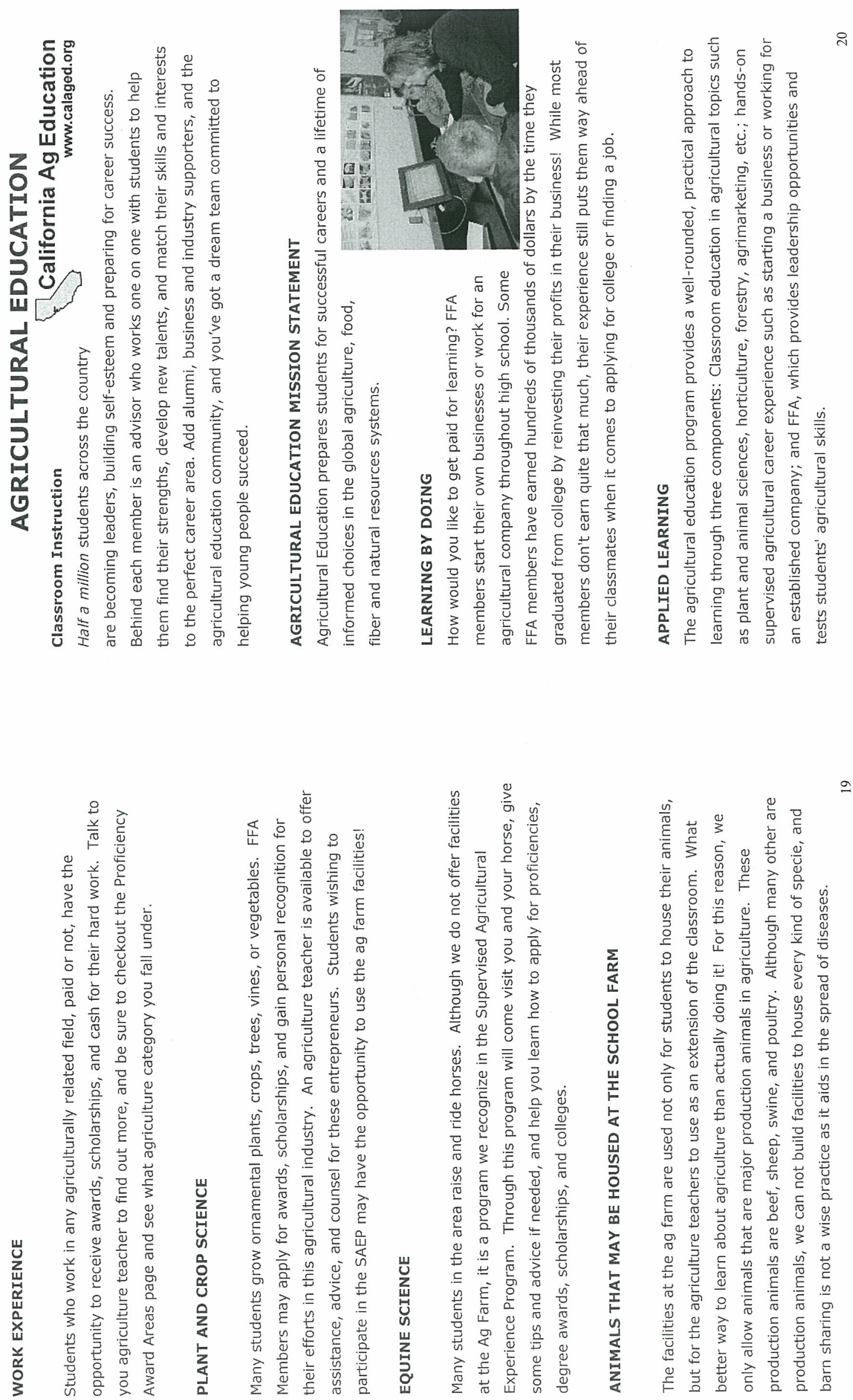

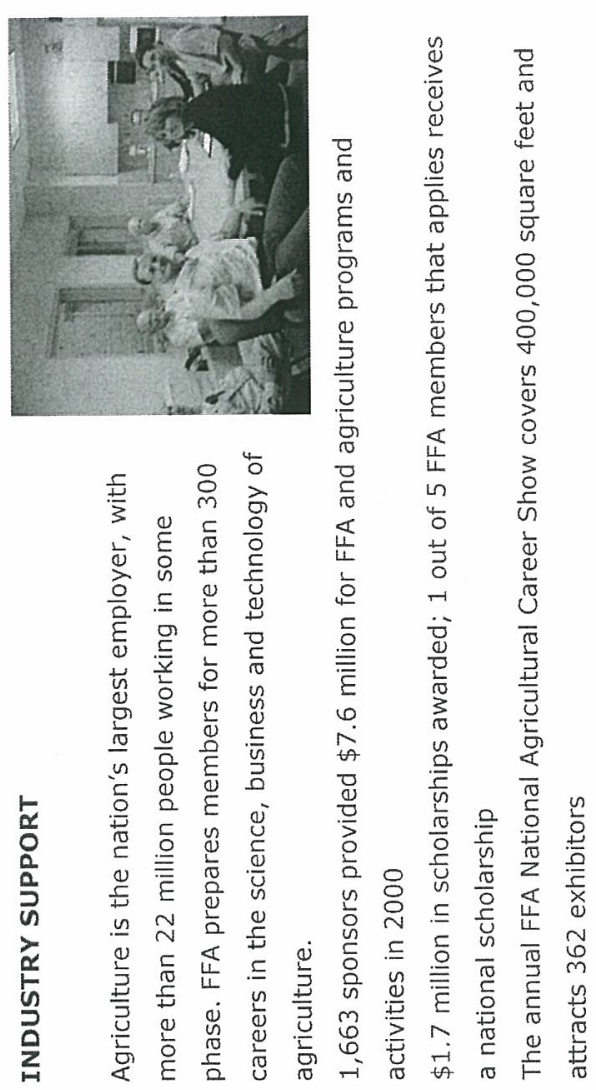
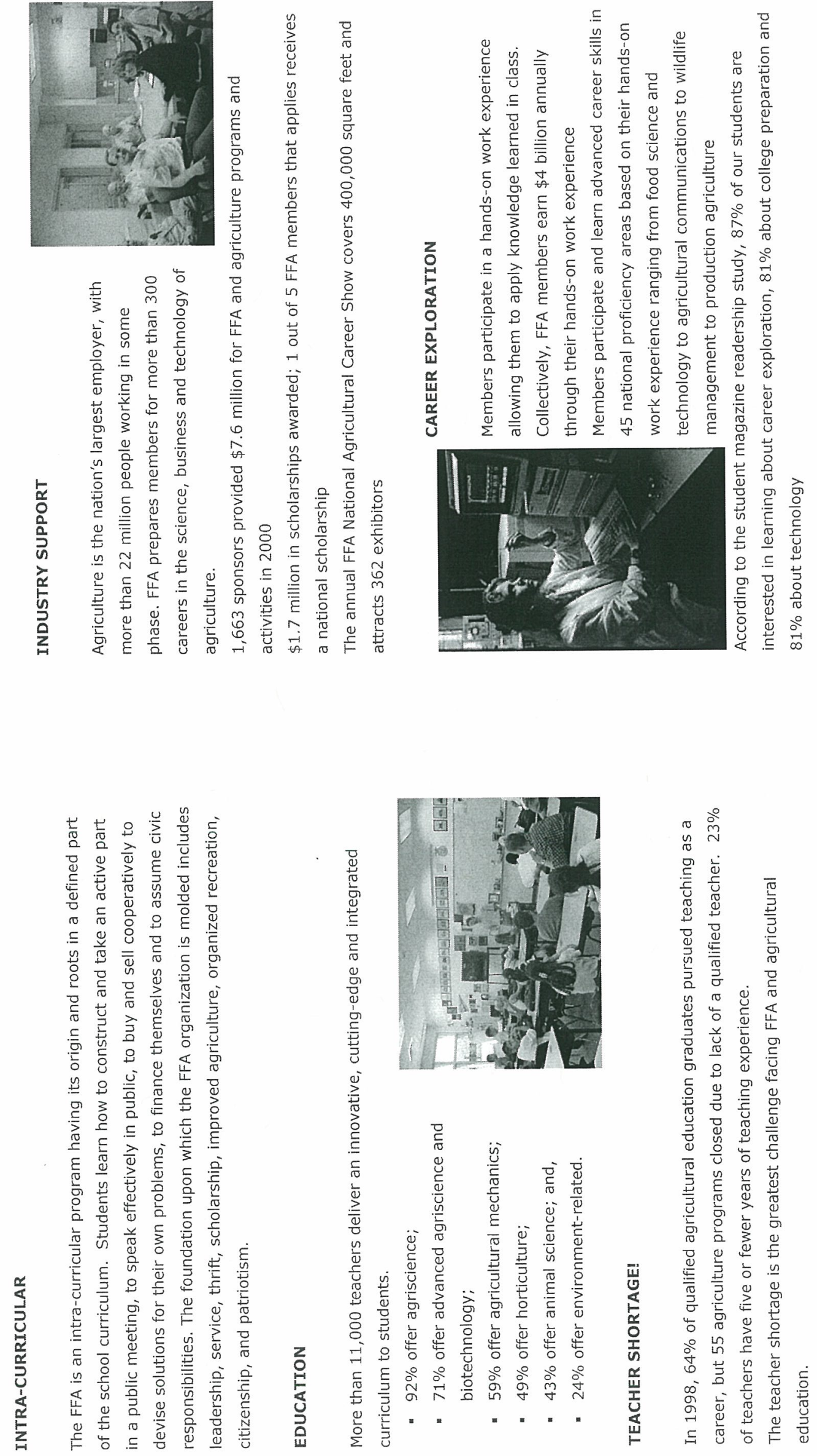

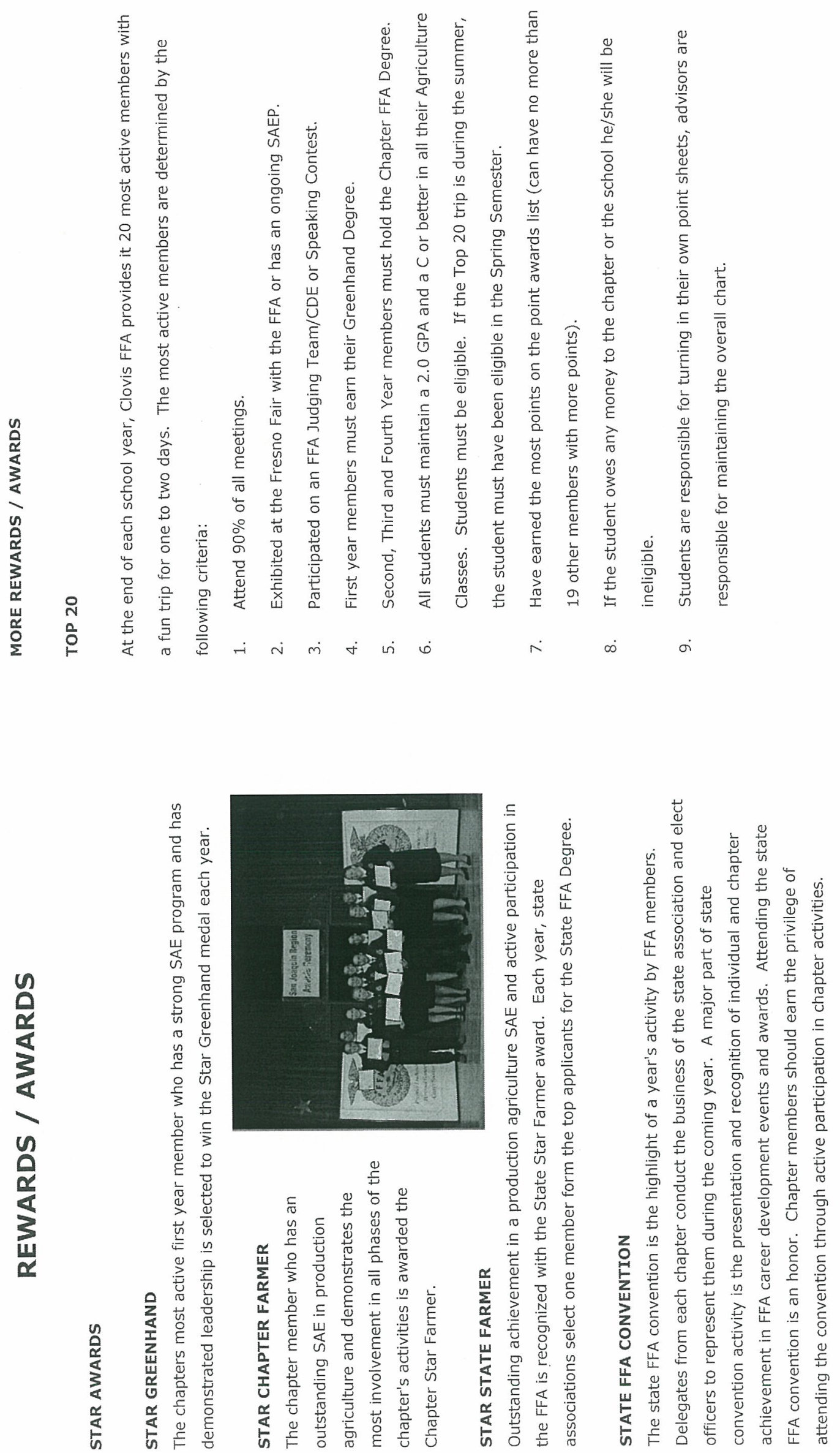

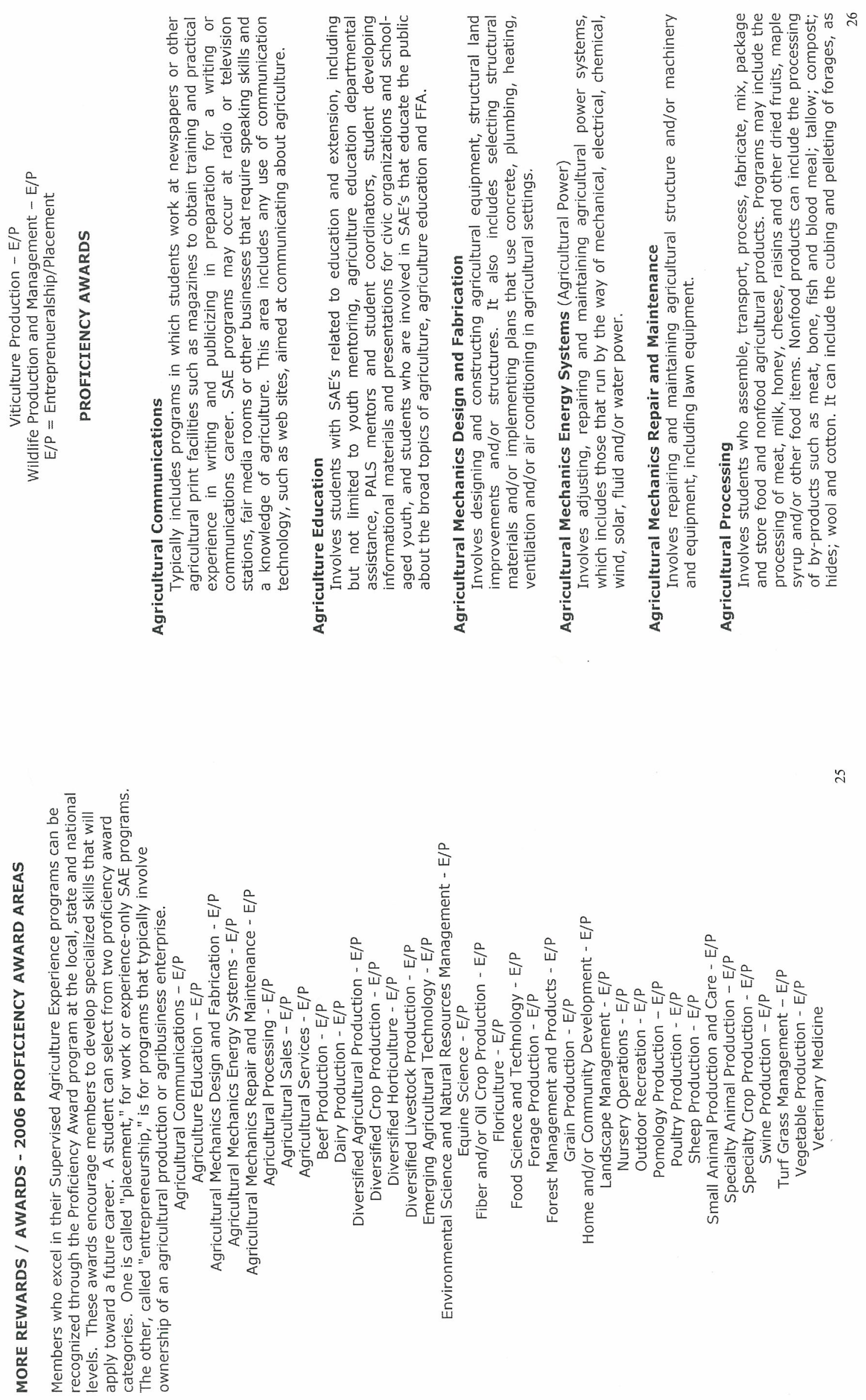


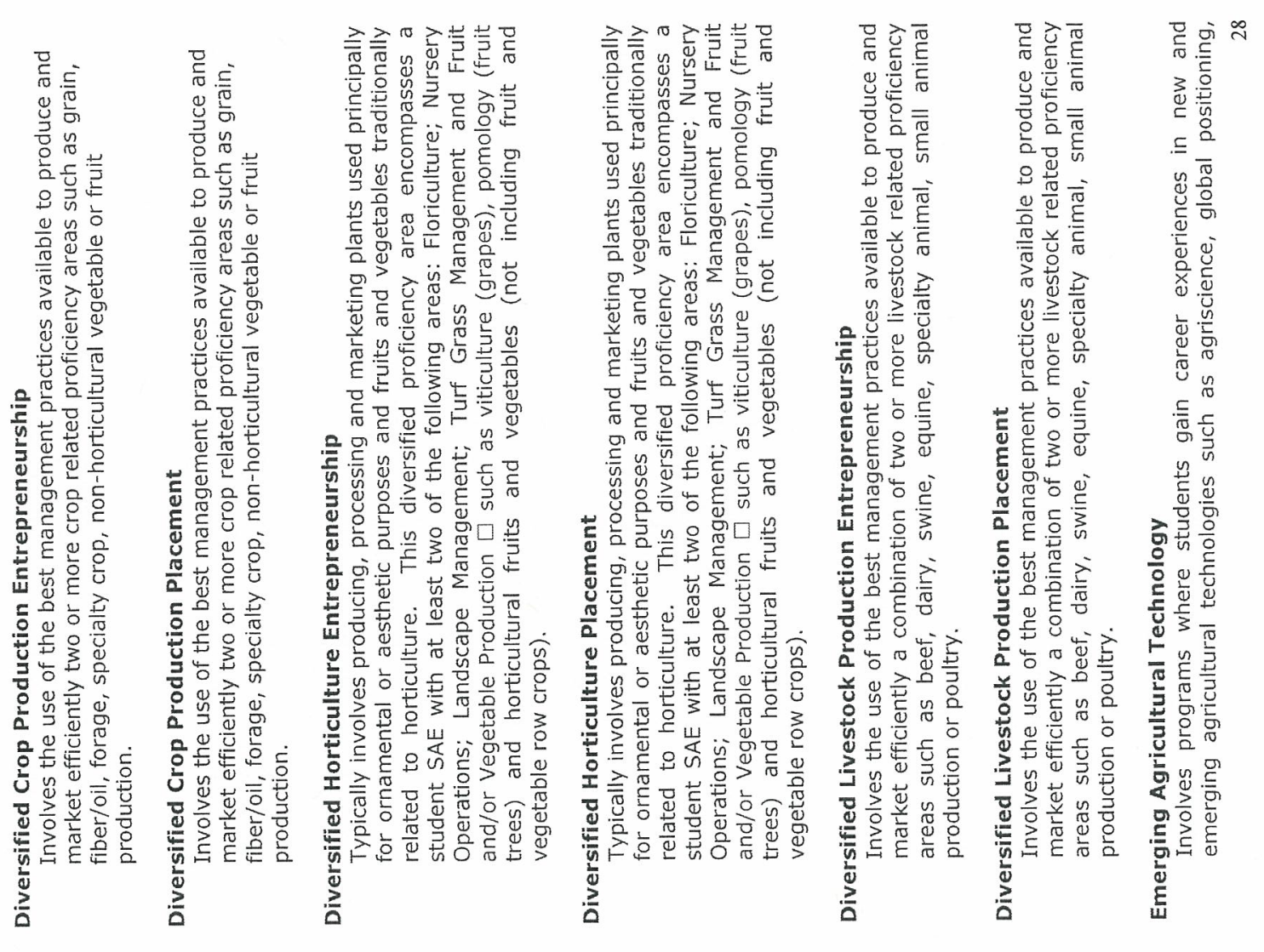

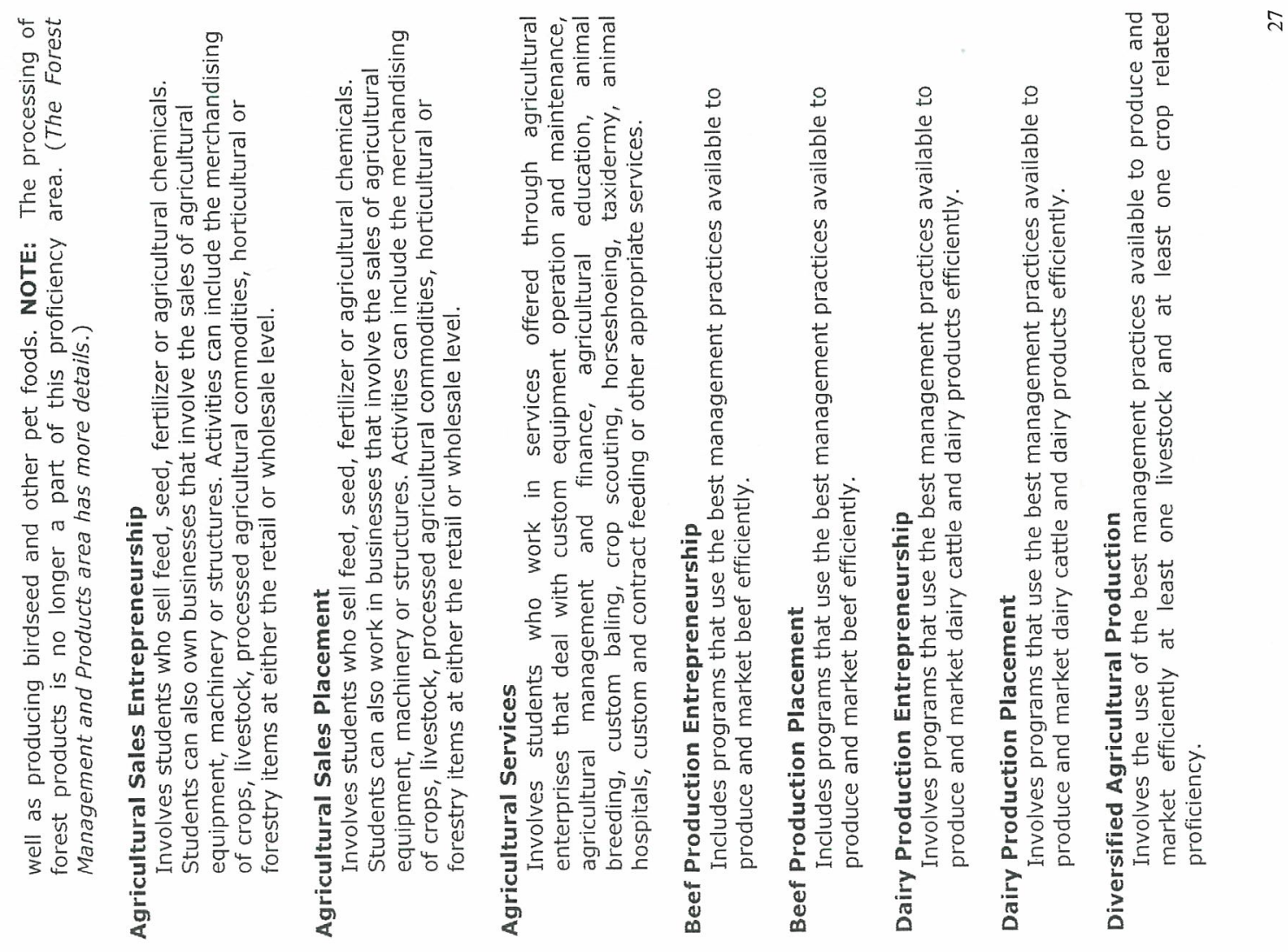



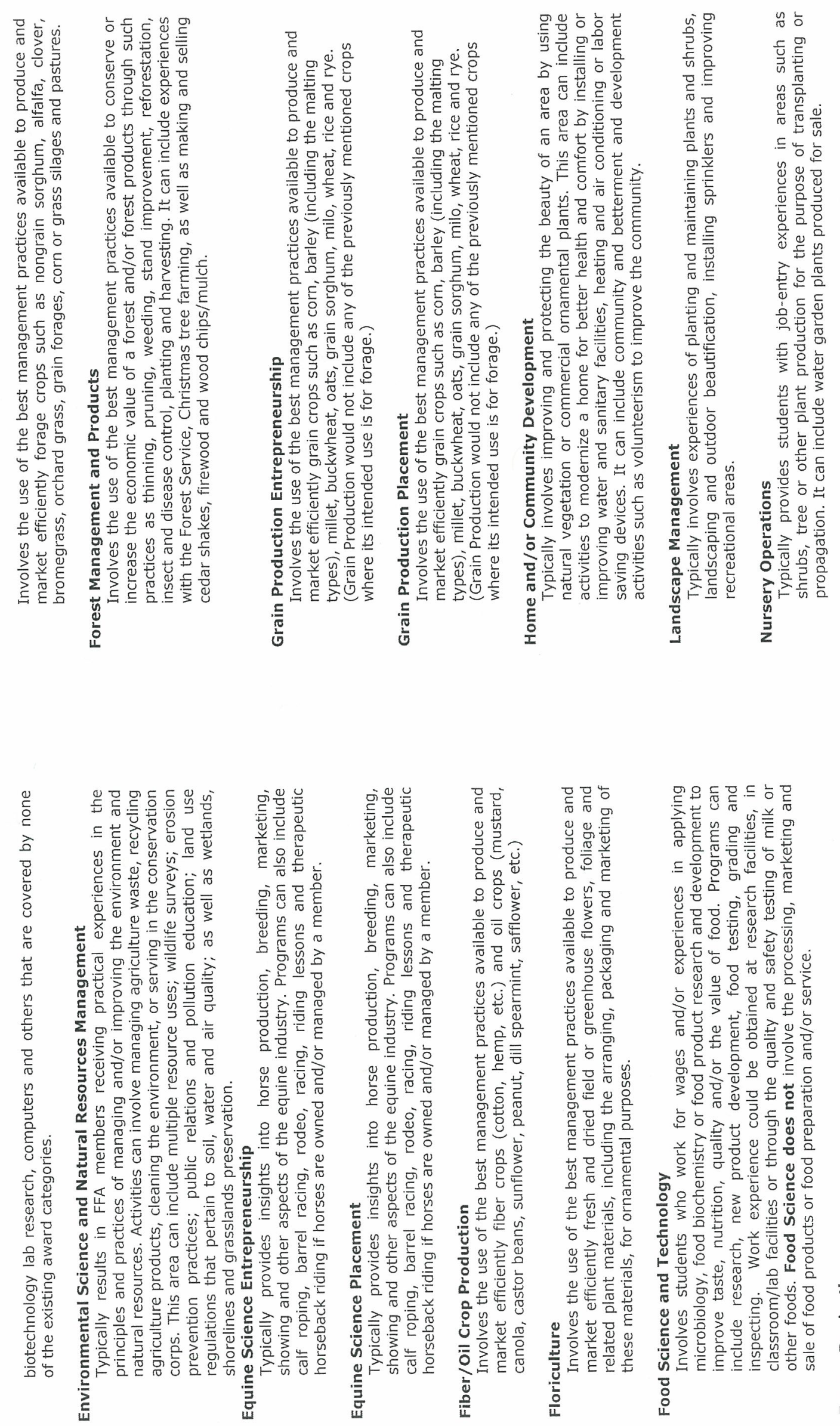

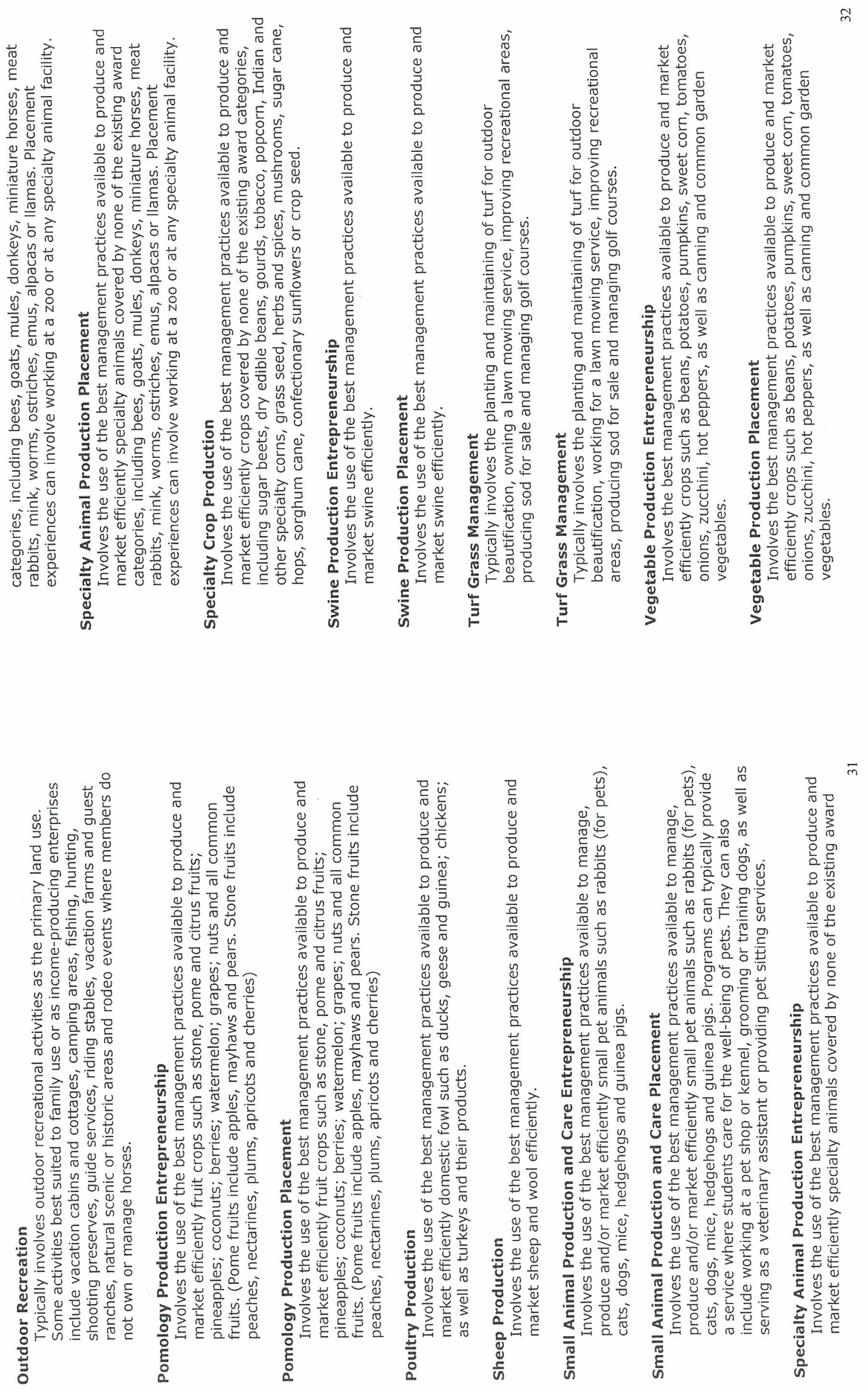

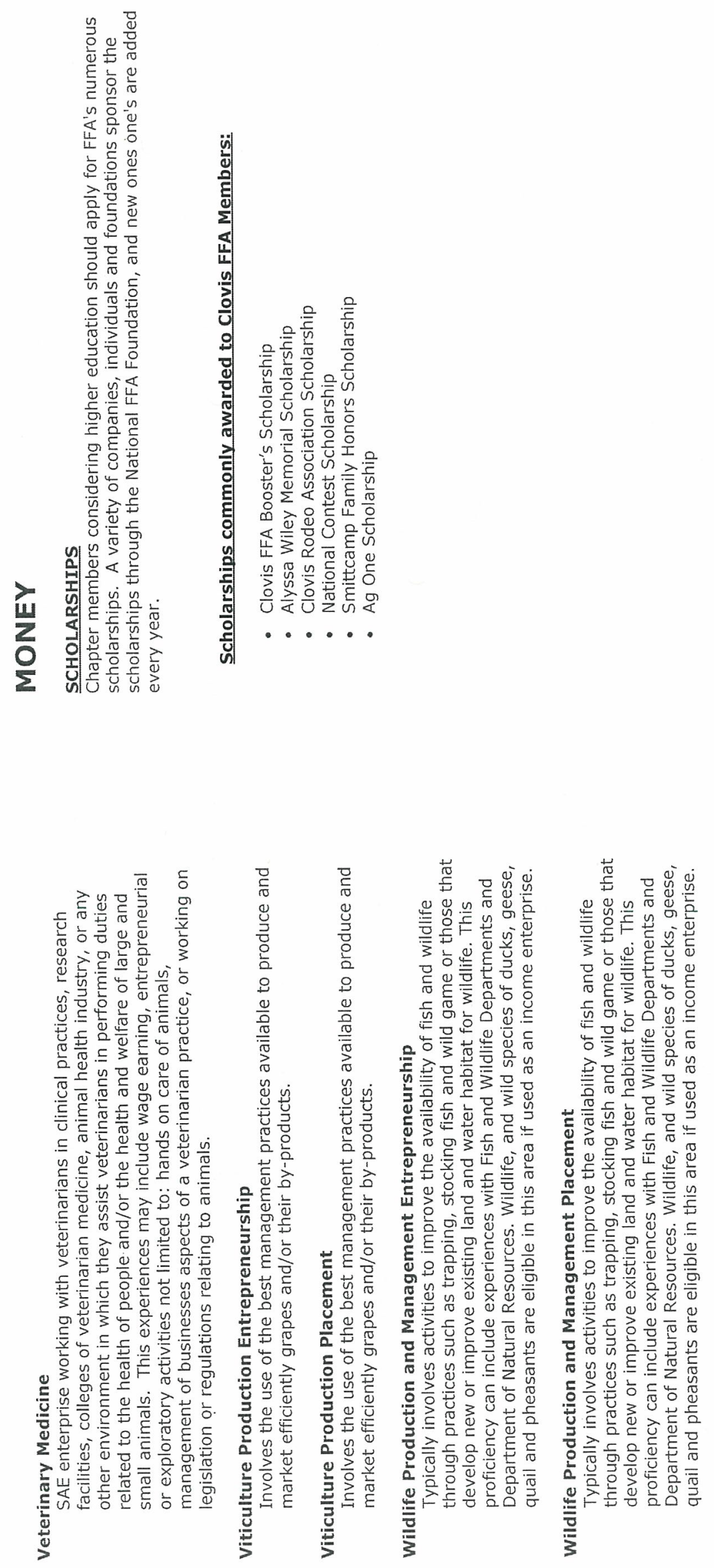

$m$ 

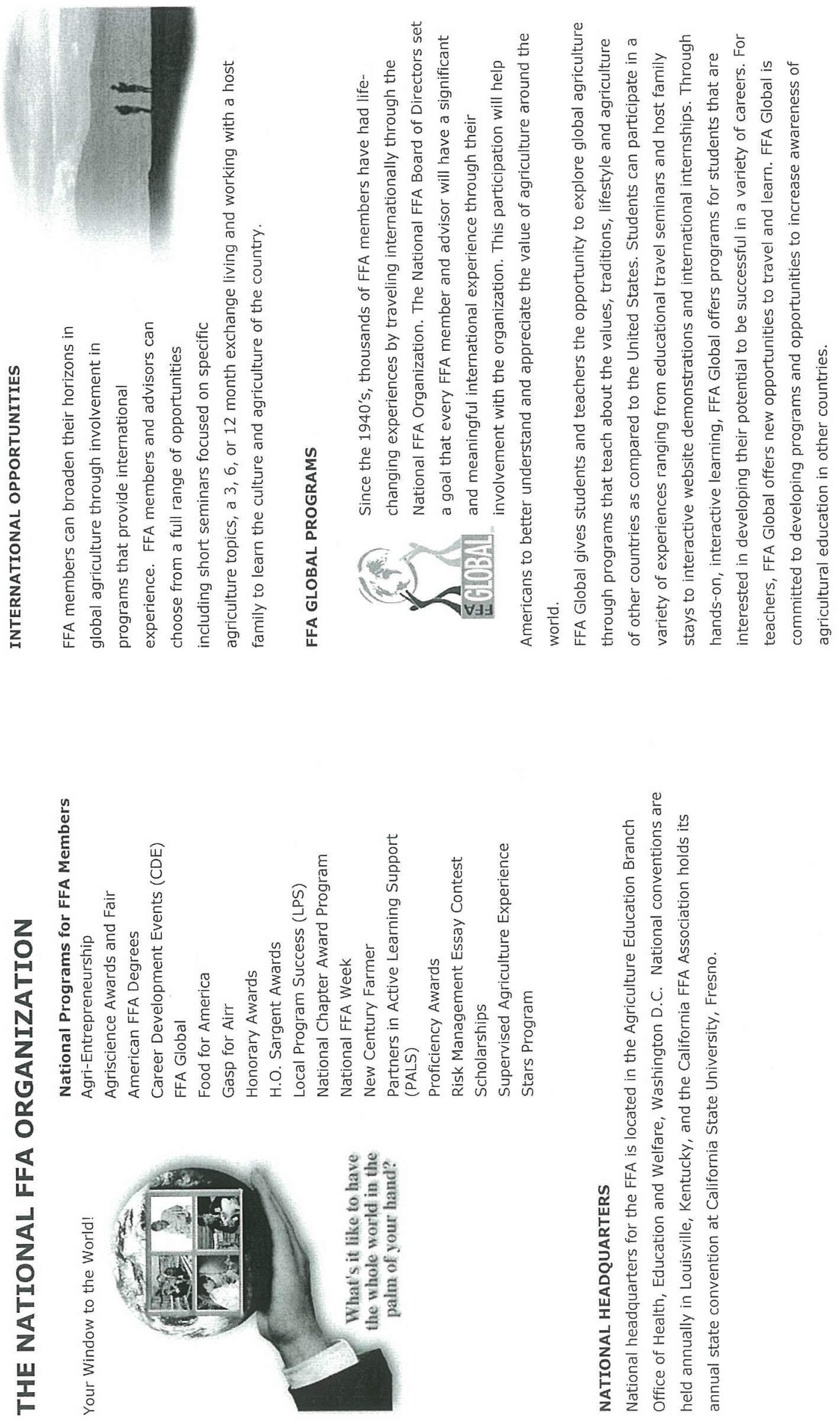

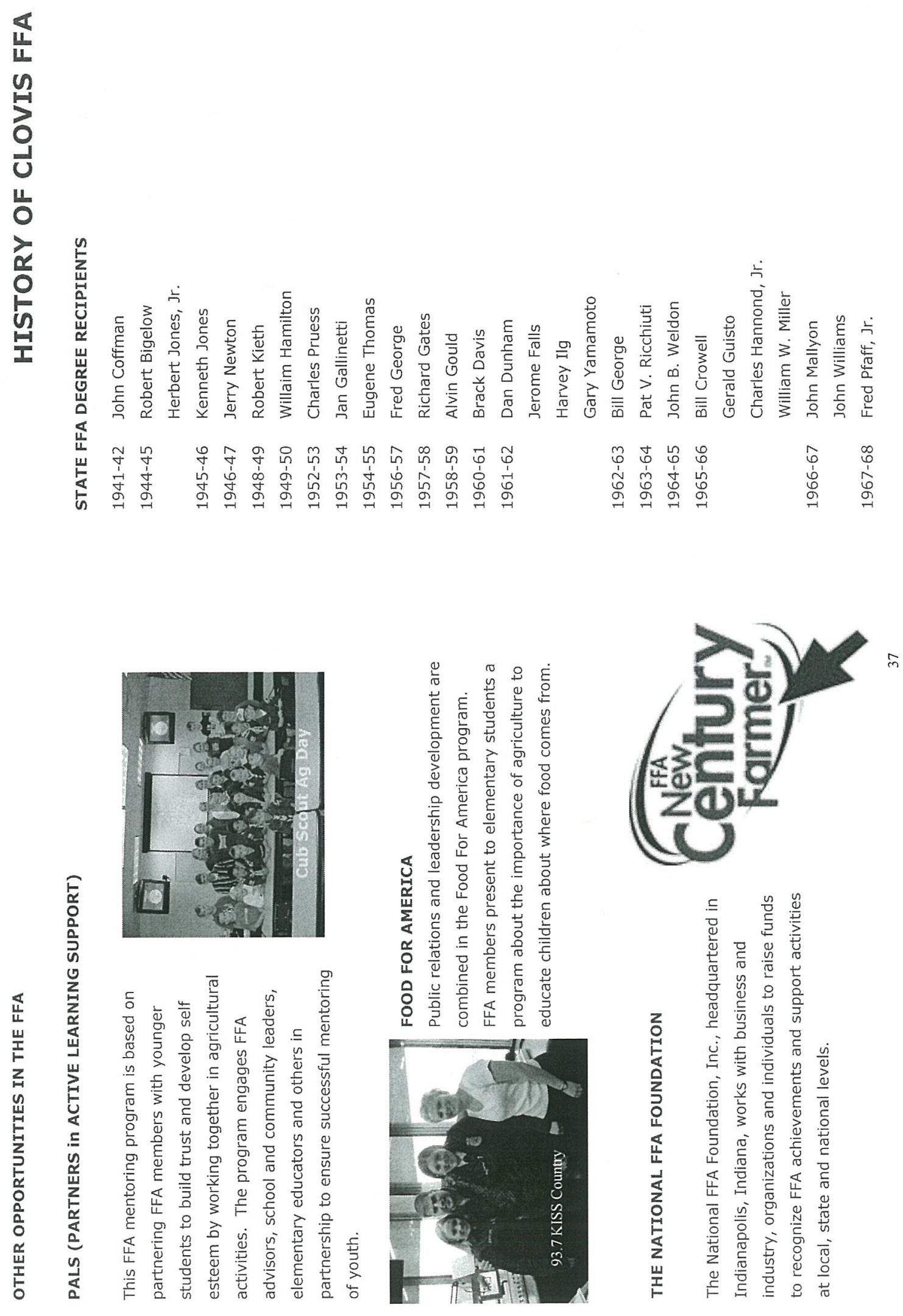

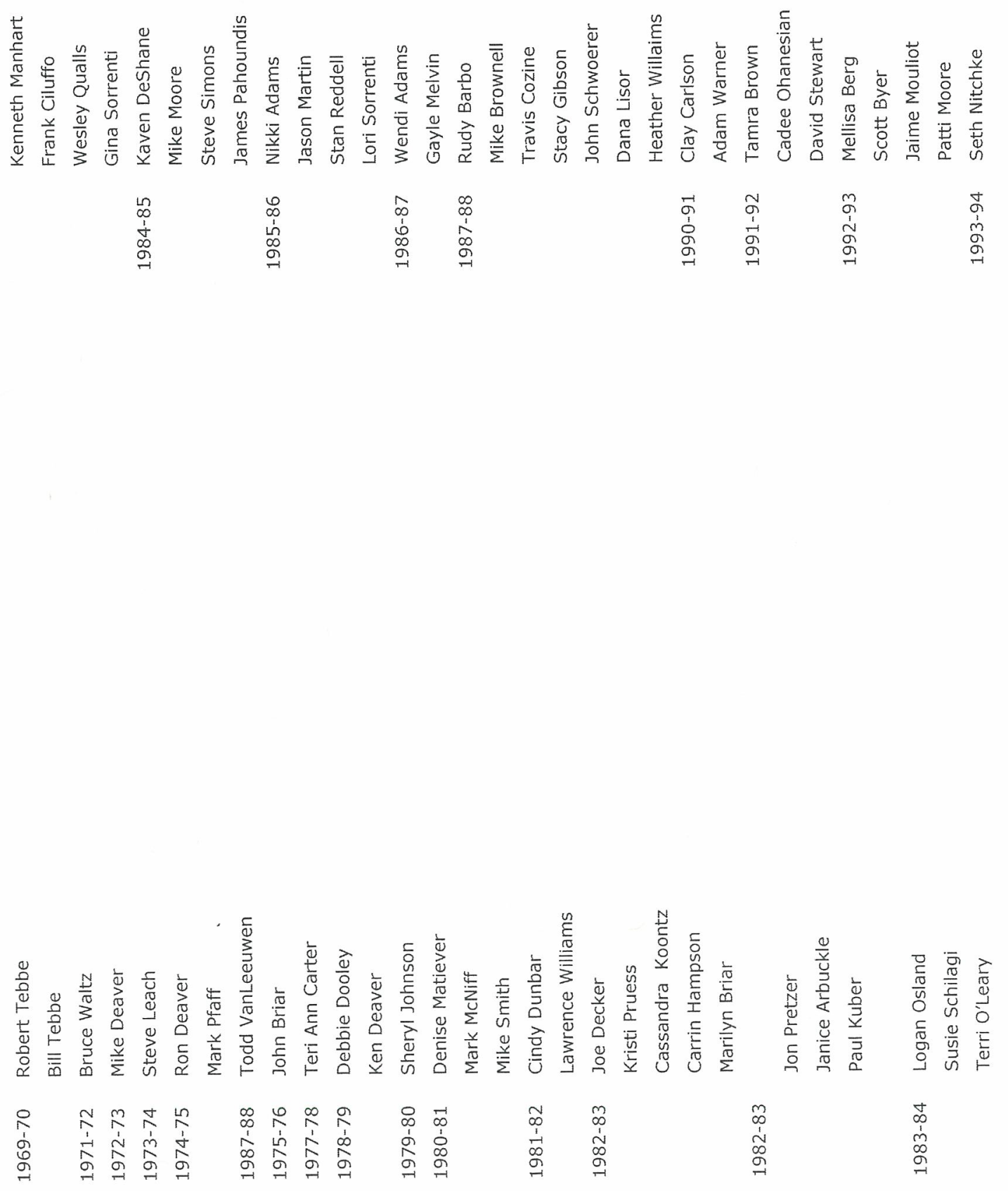

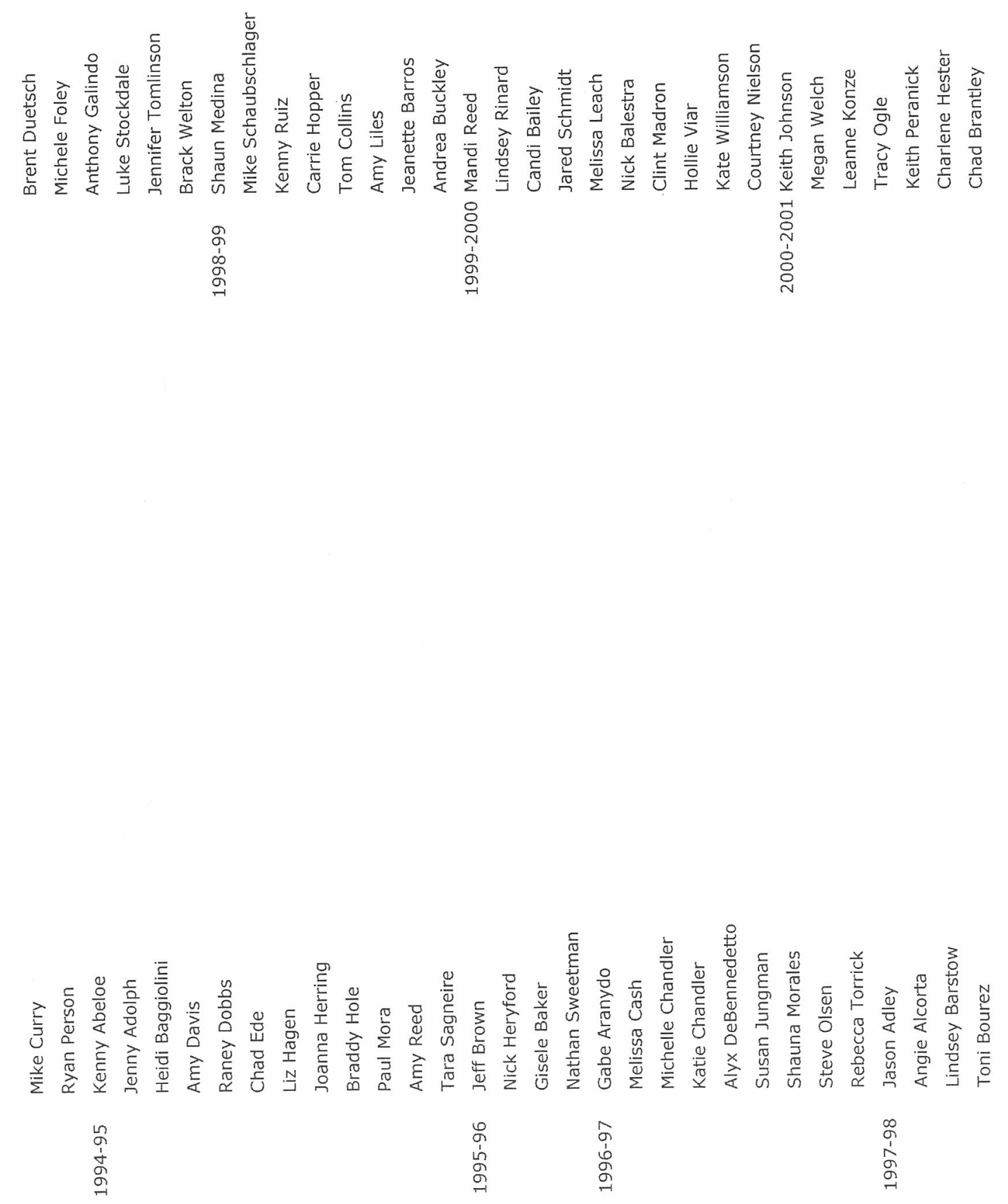

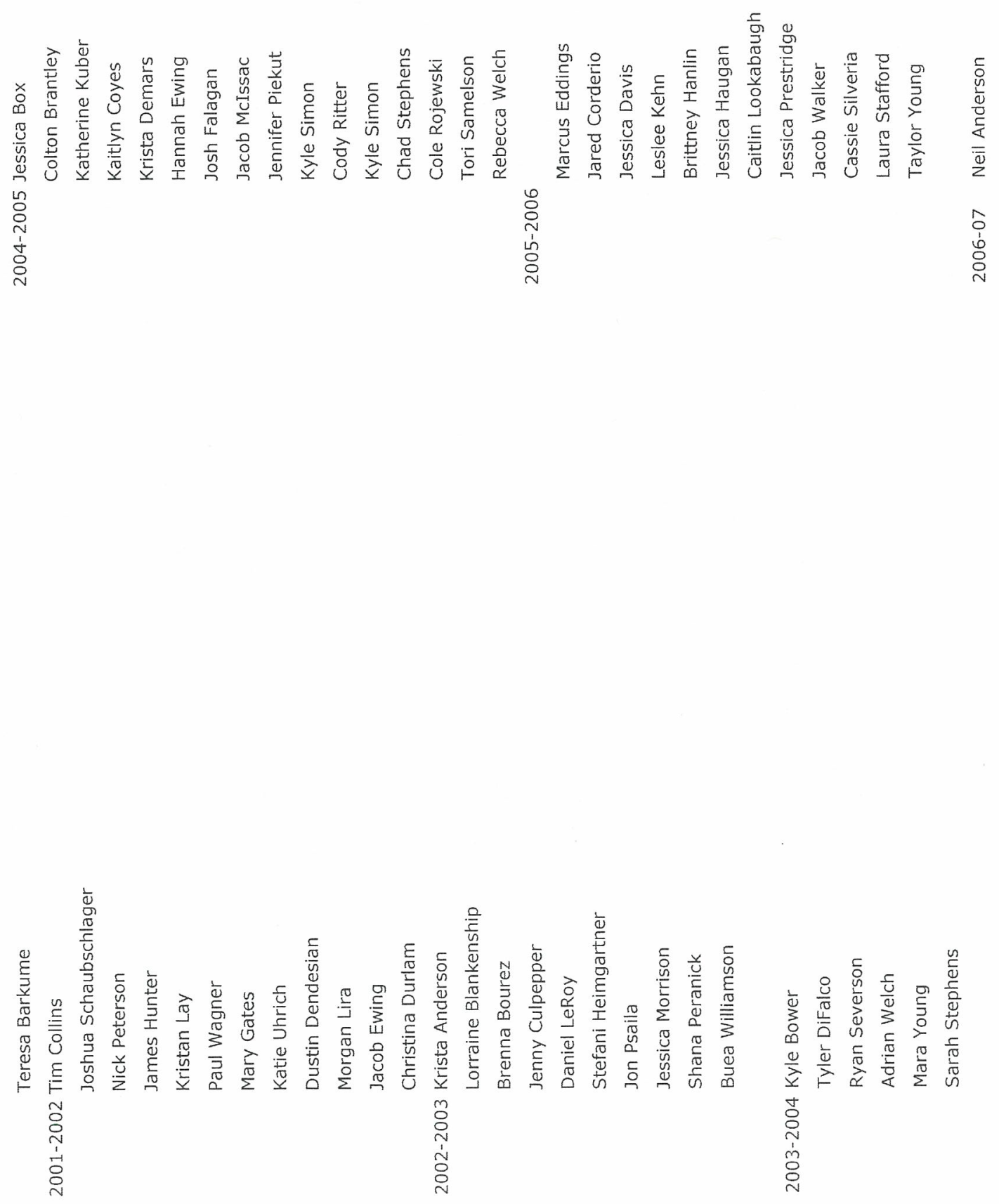

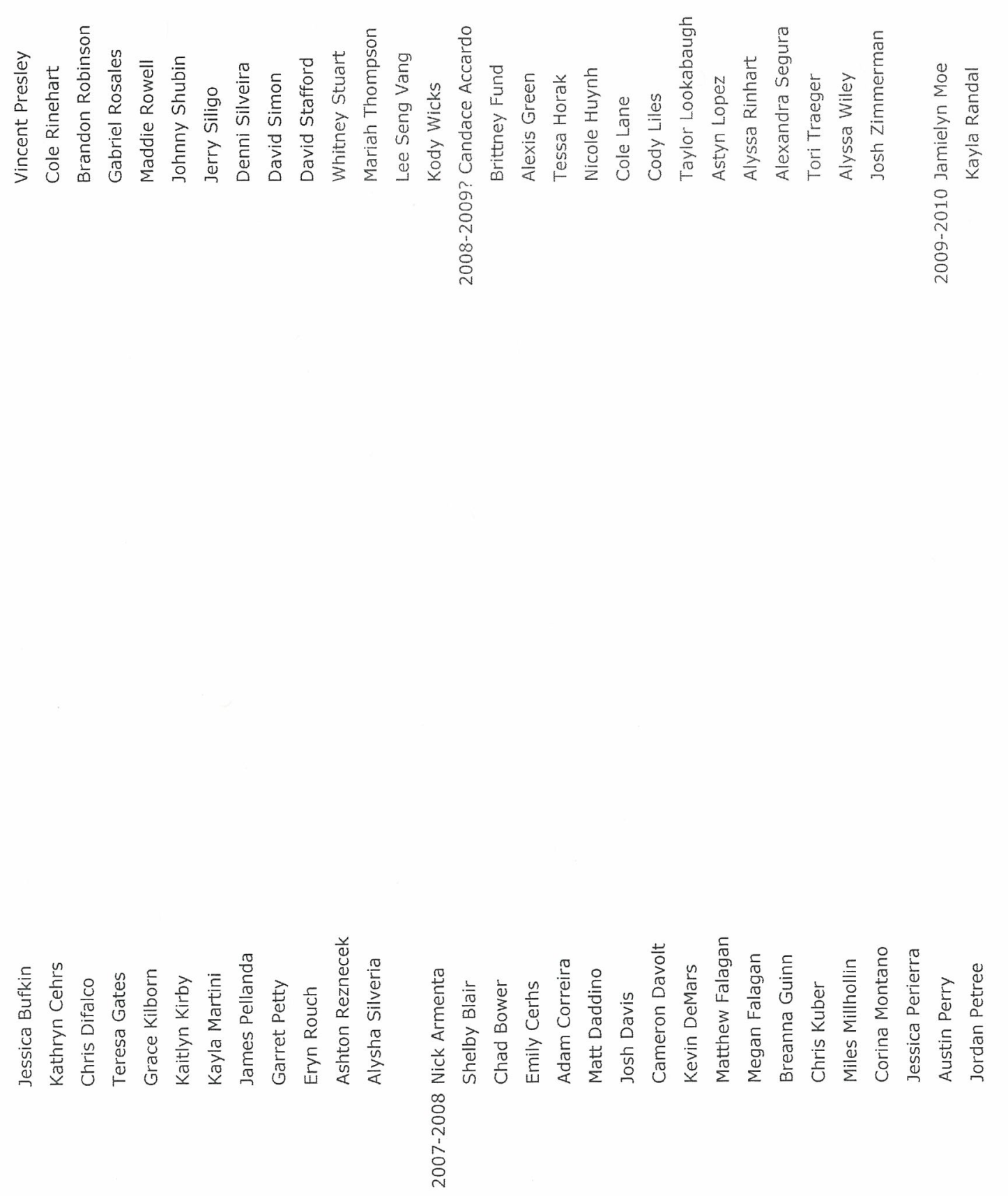

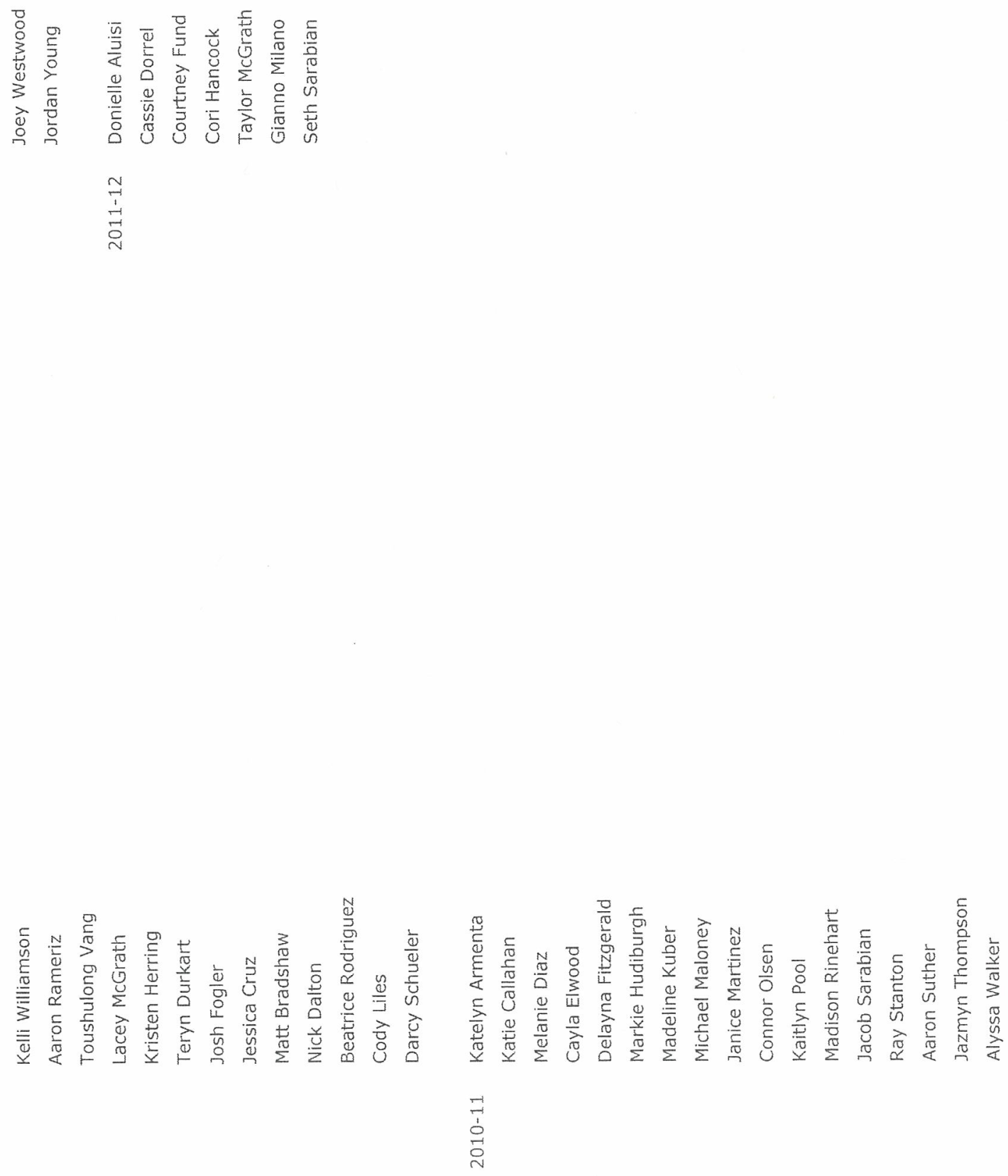


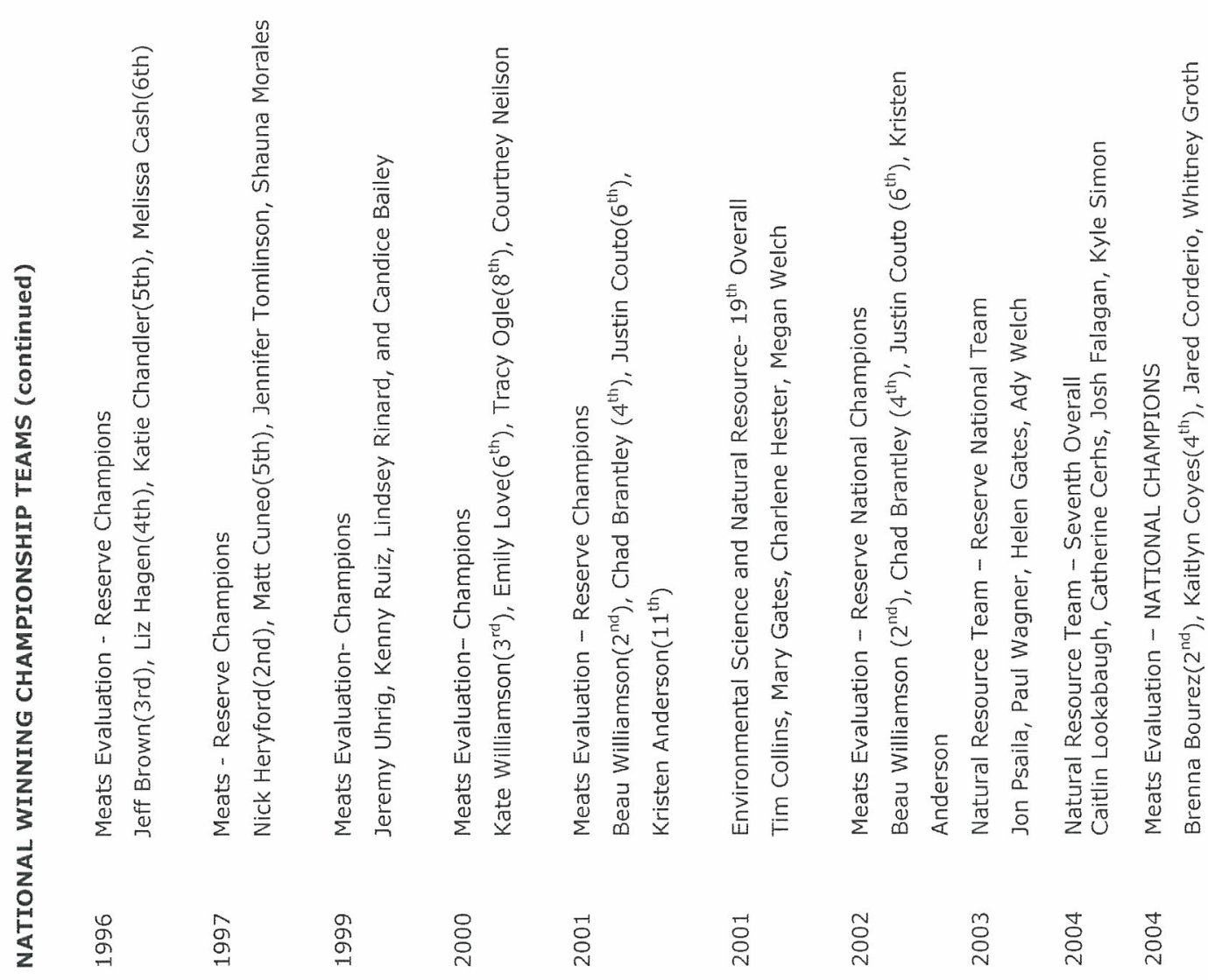

ถ 

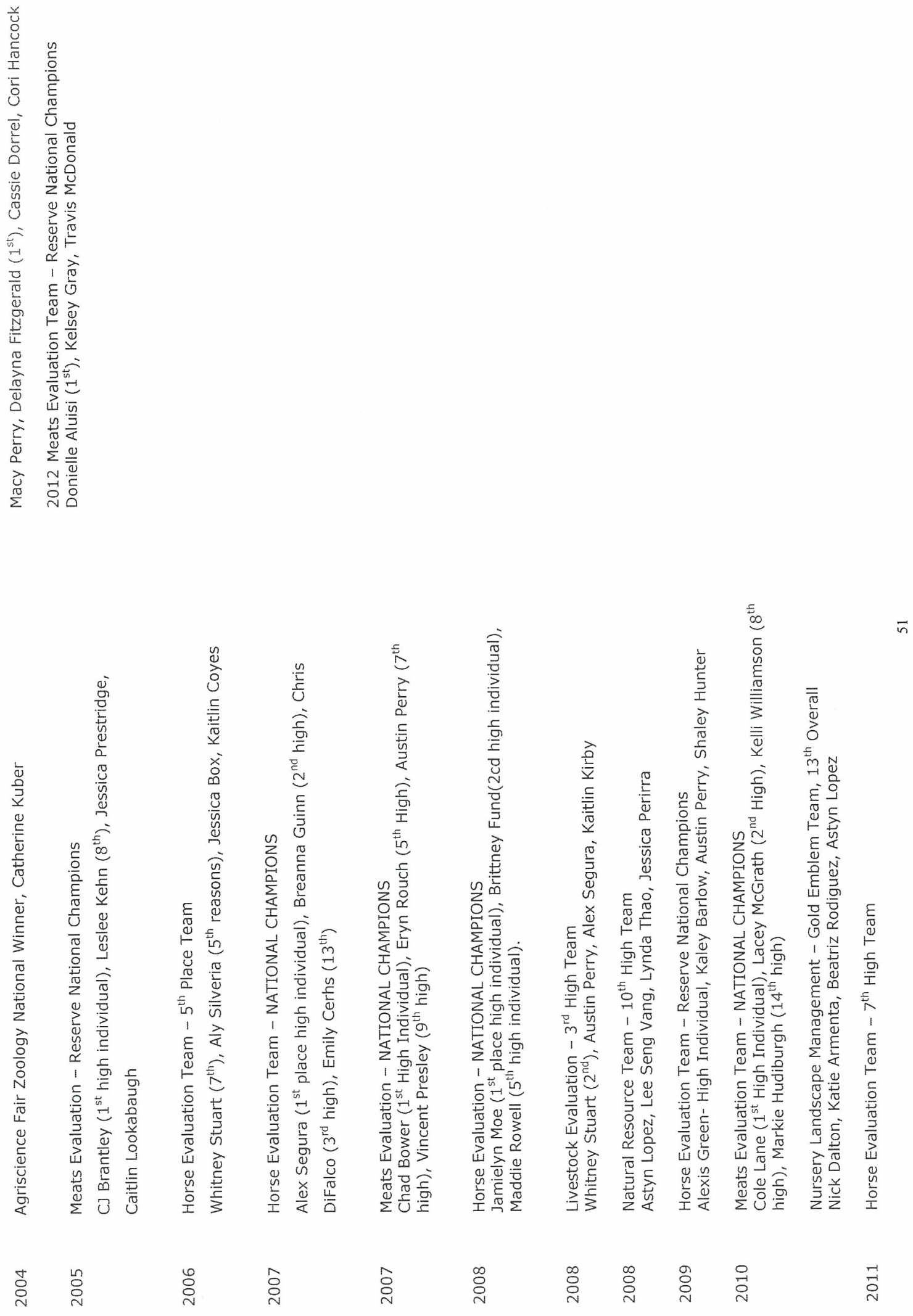

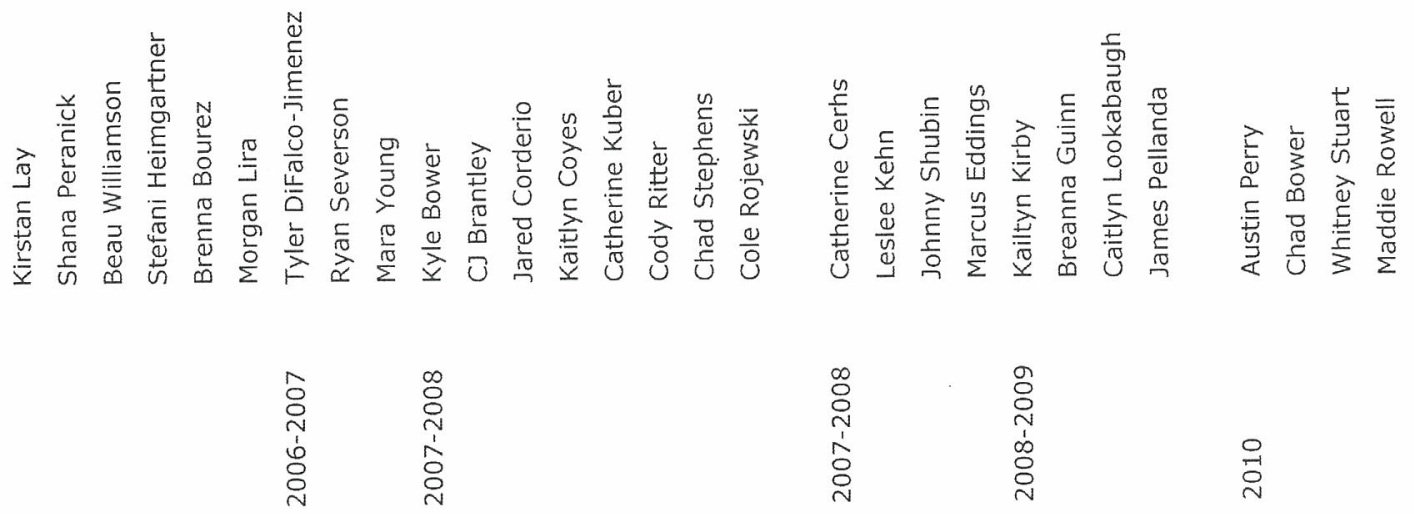

in

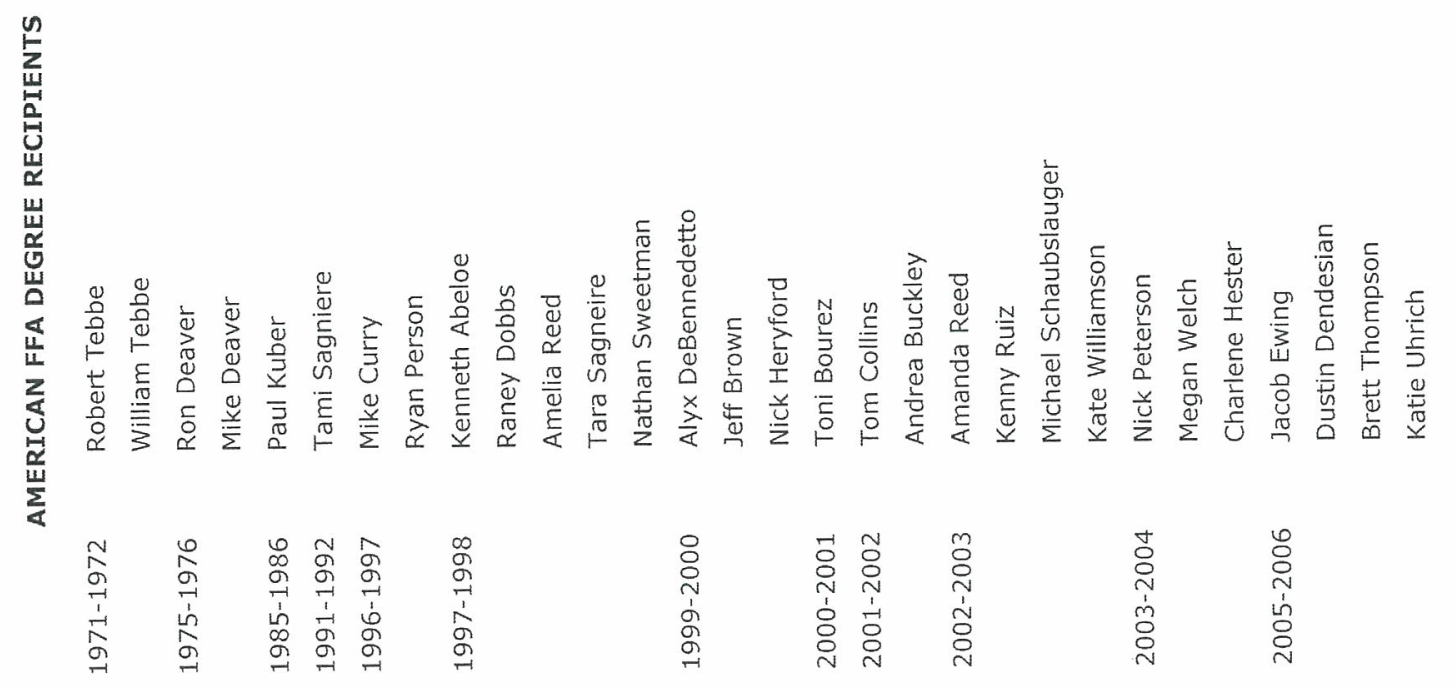



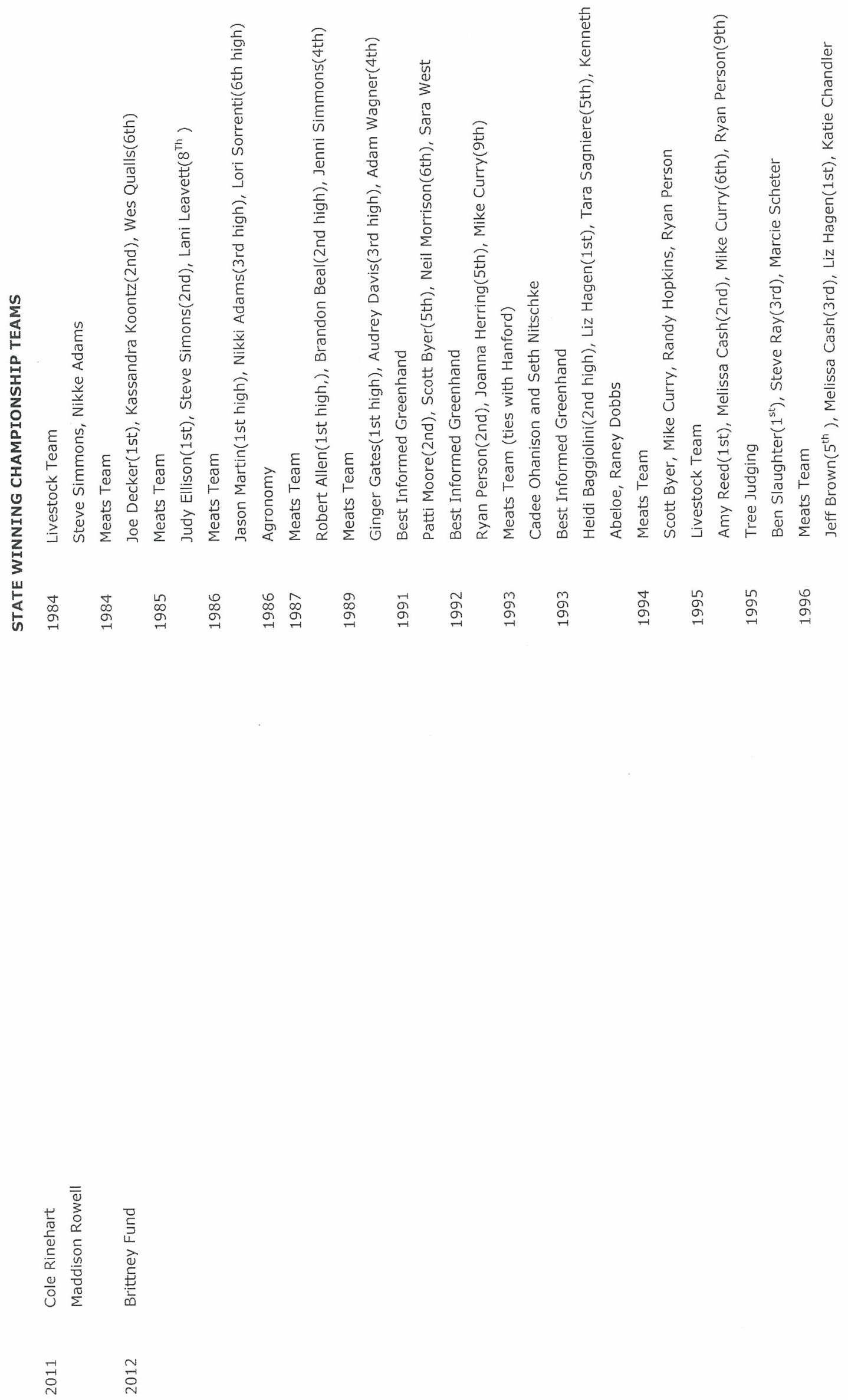


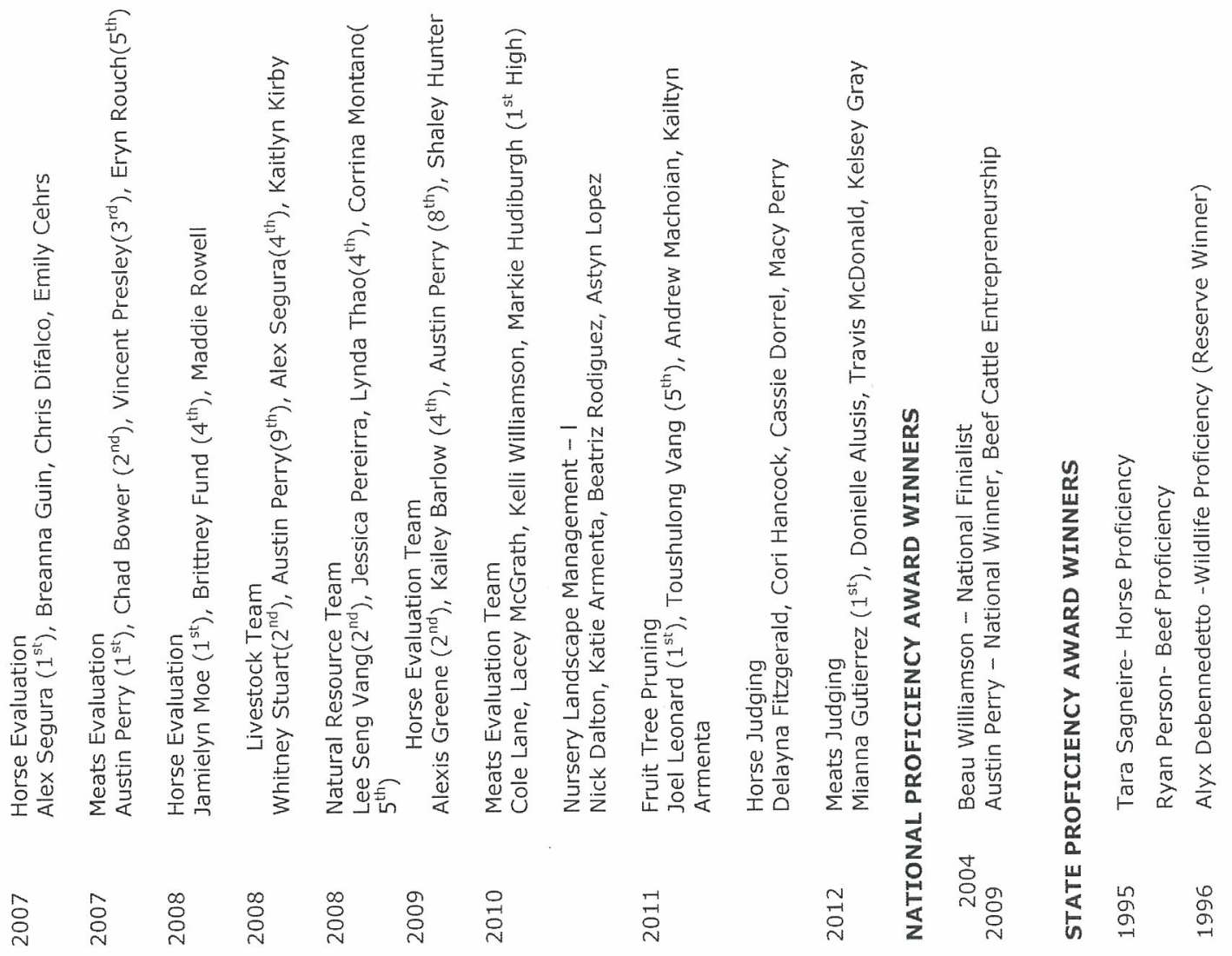

n

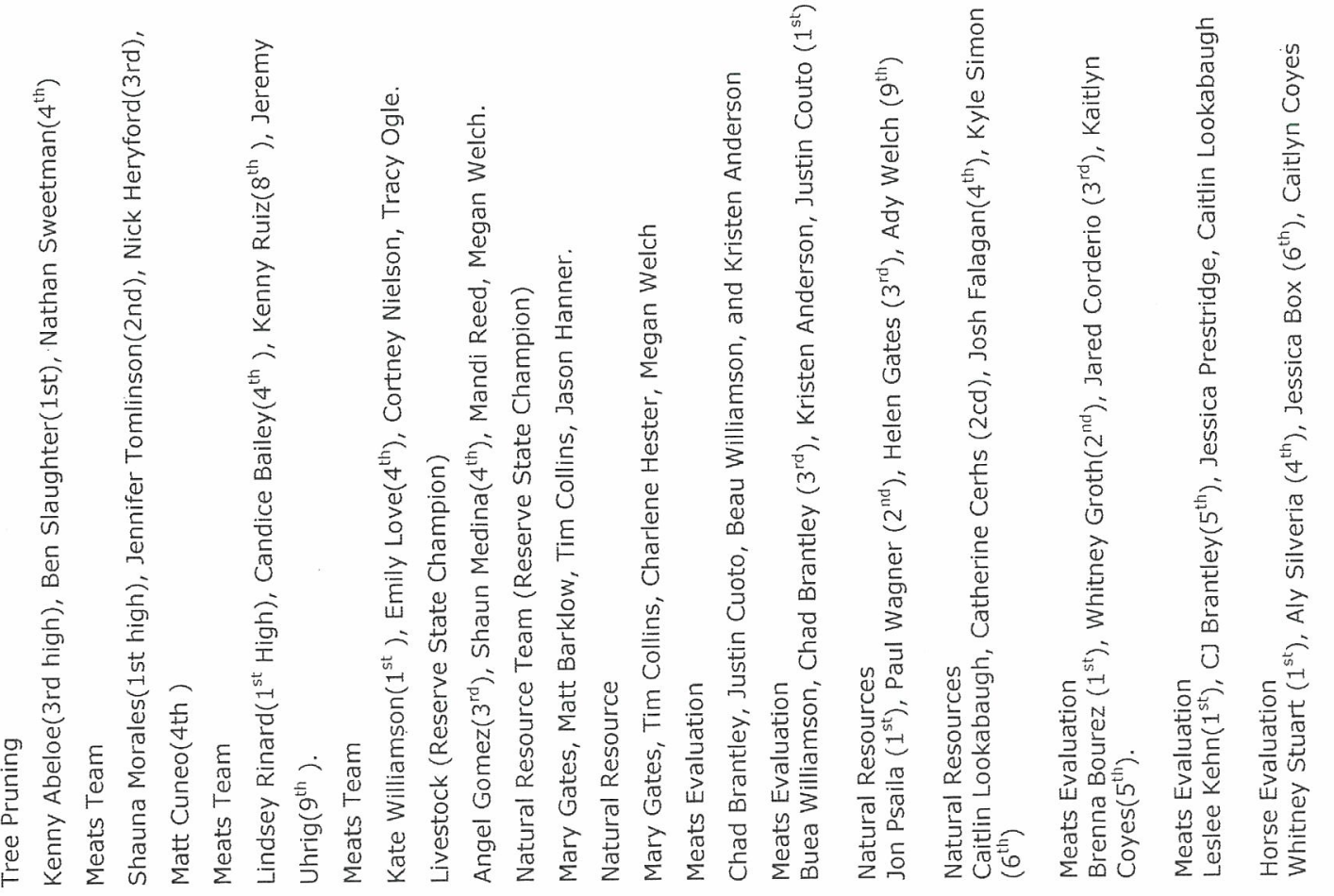

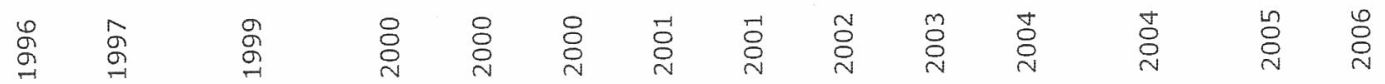



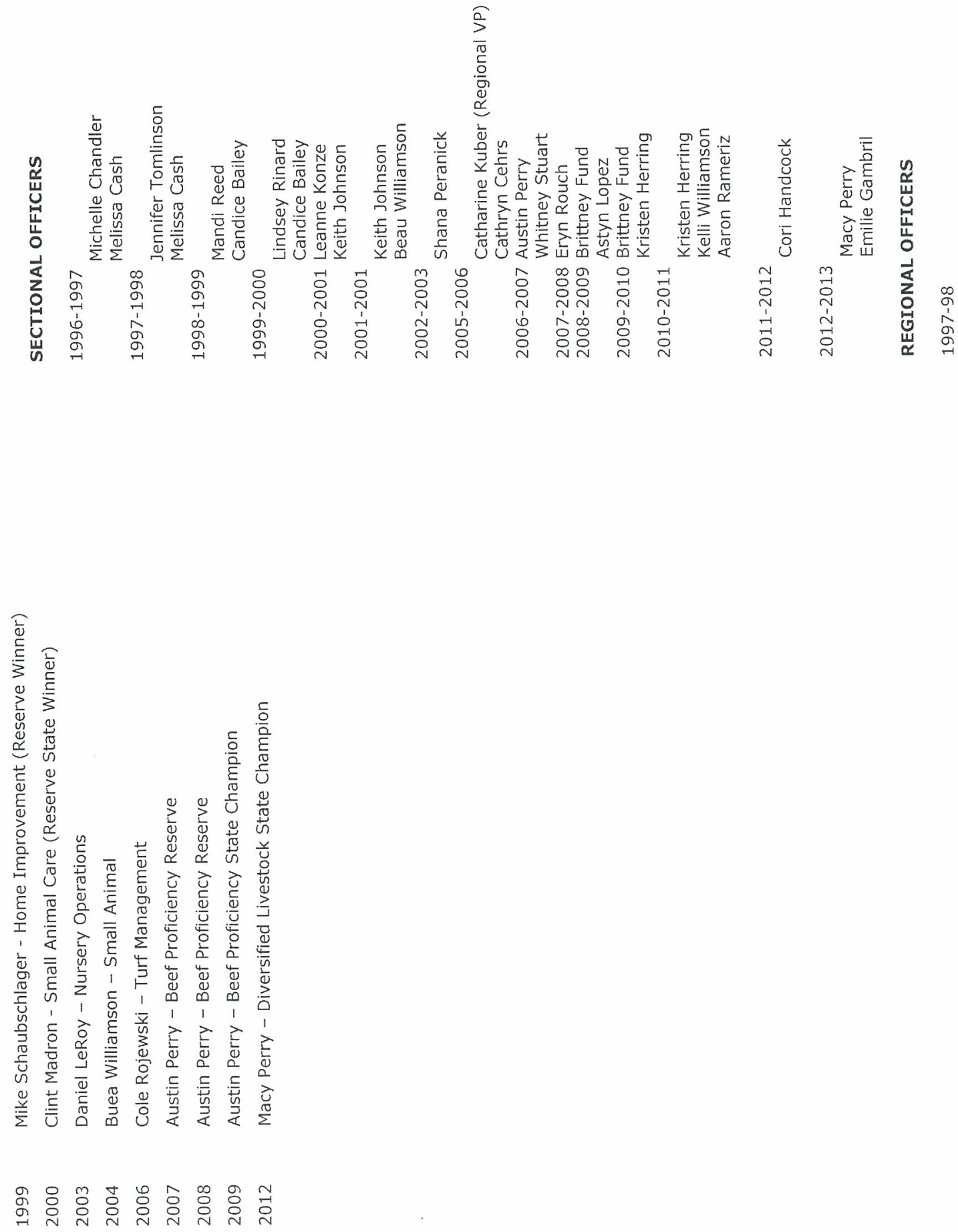


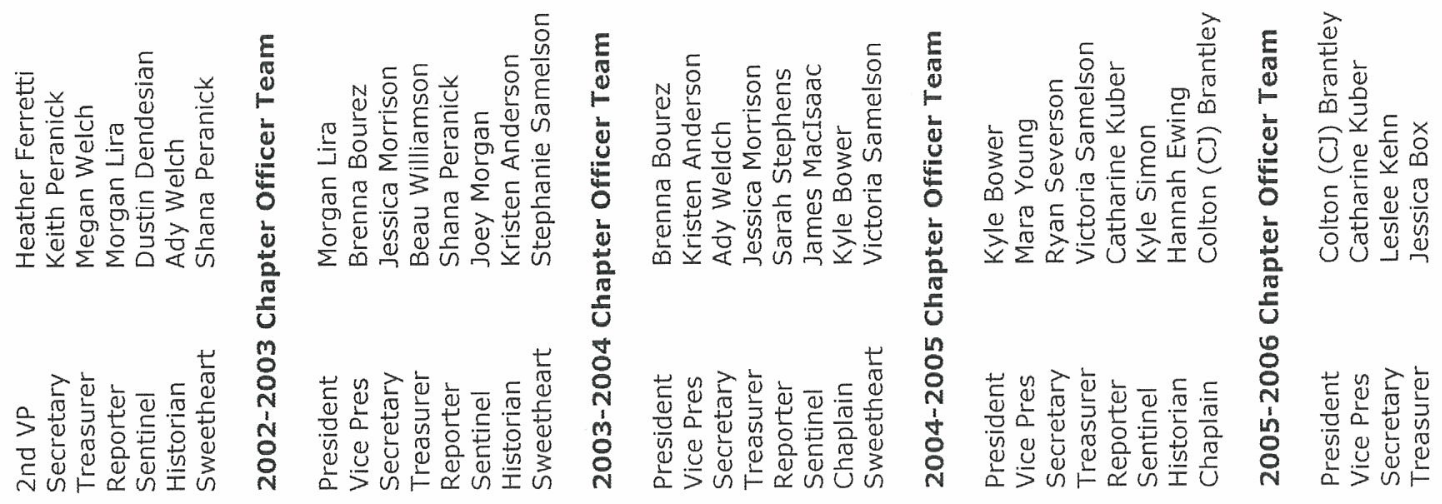

5

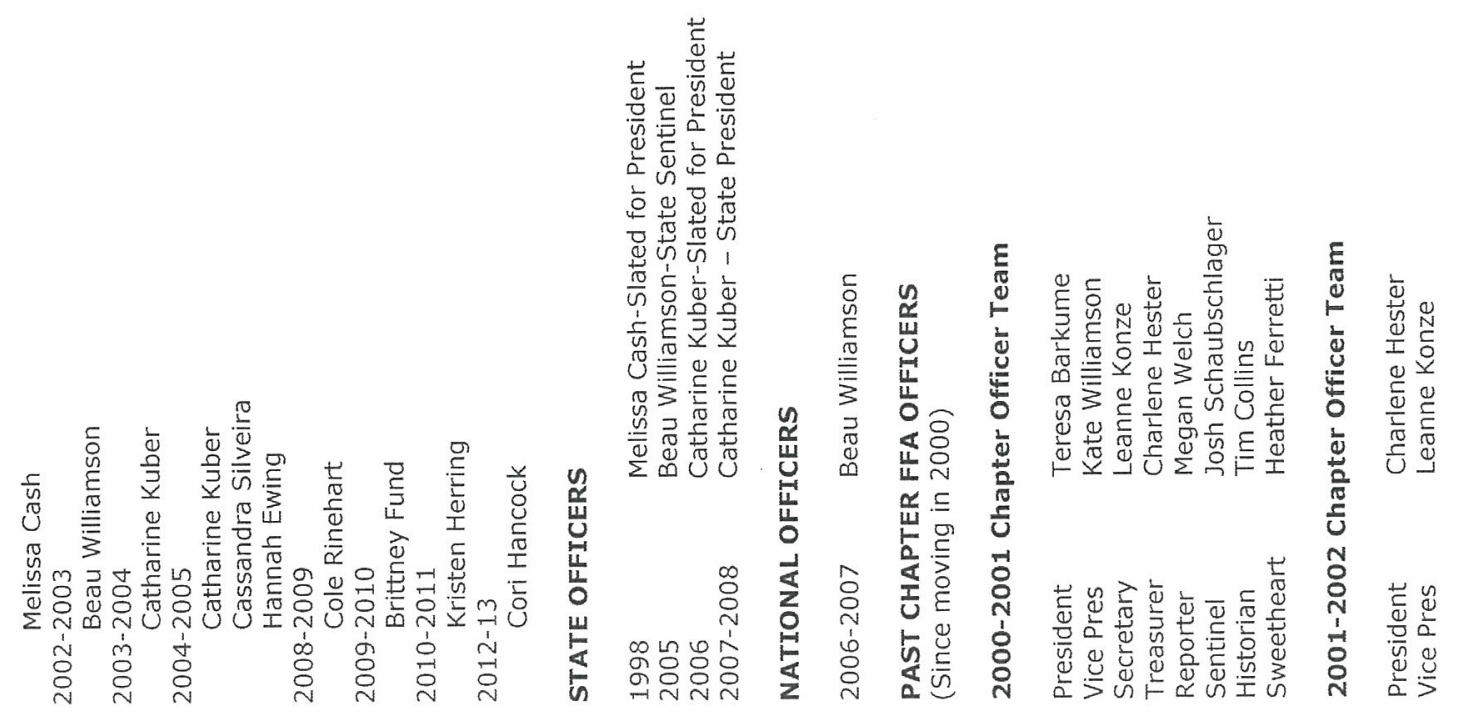



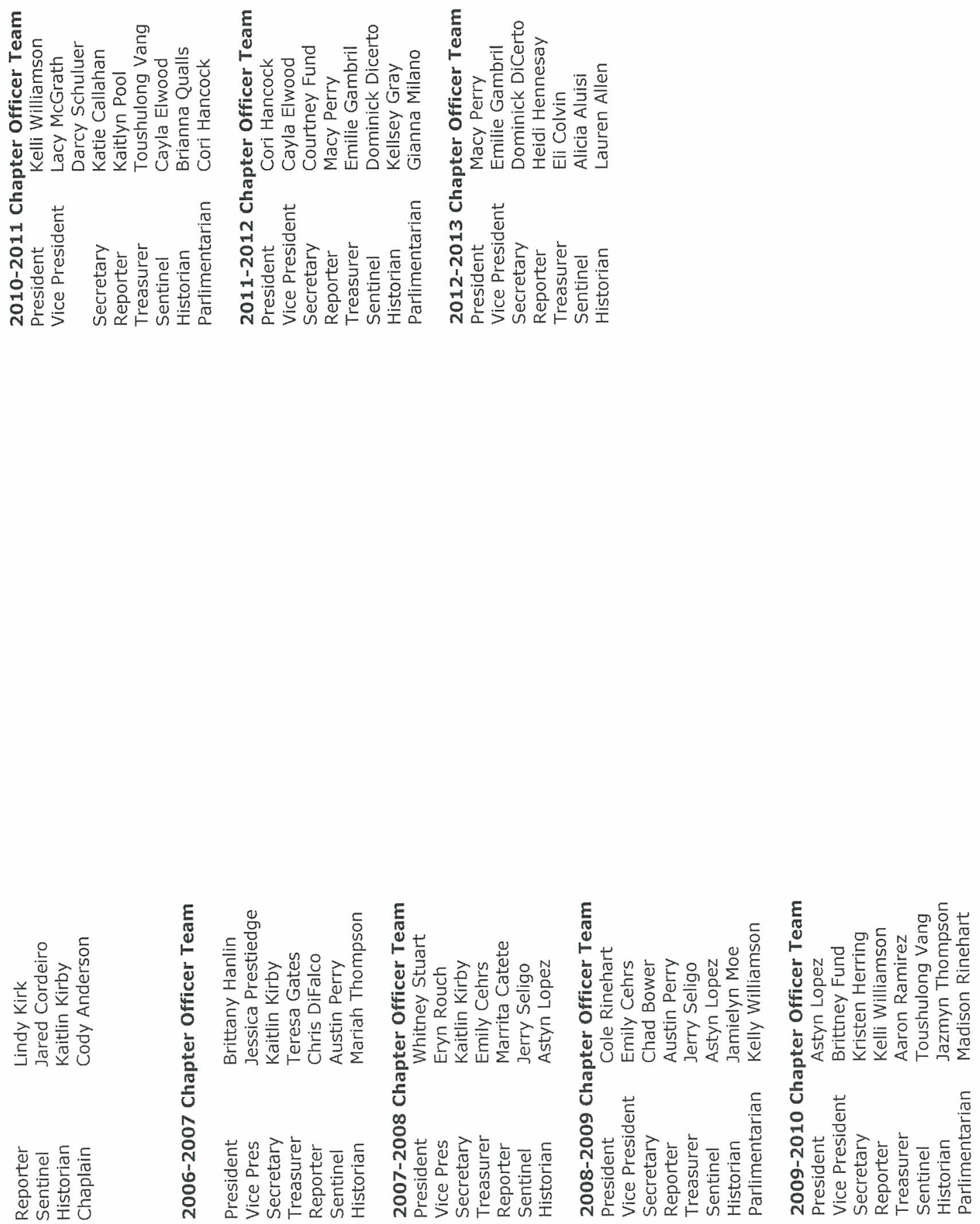

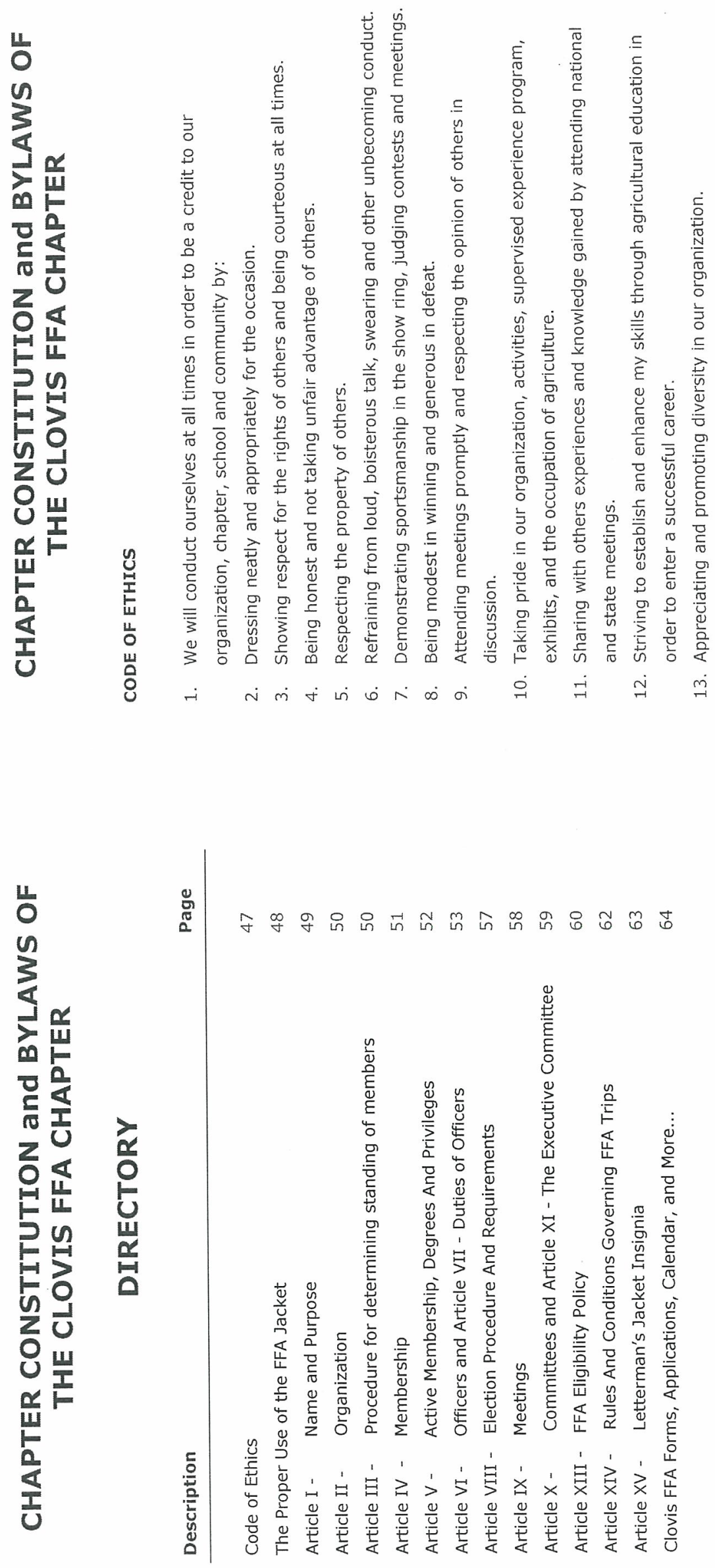


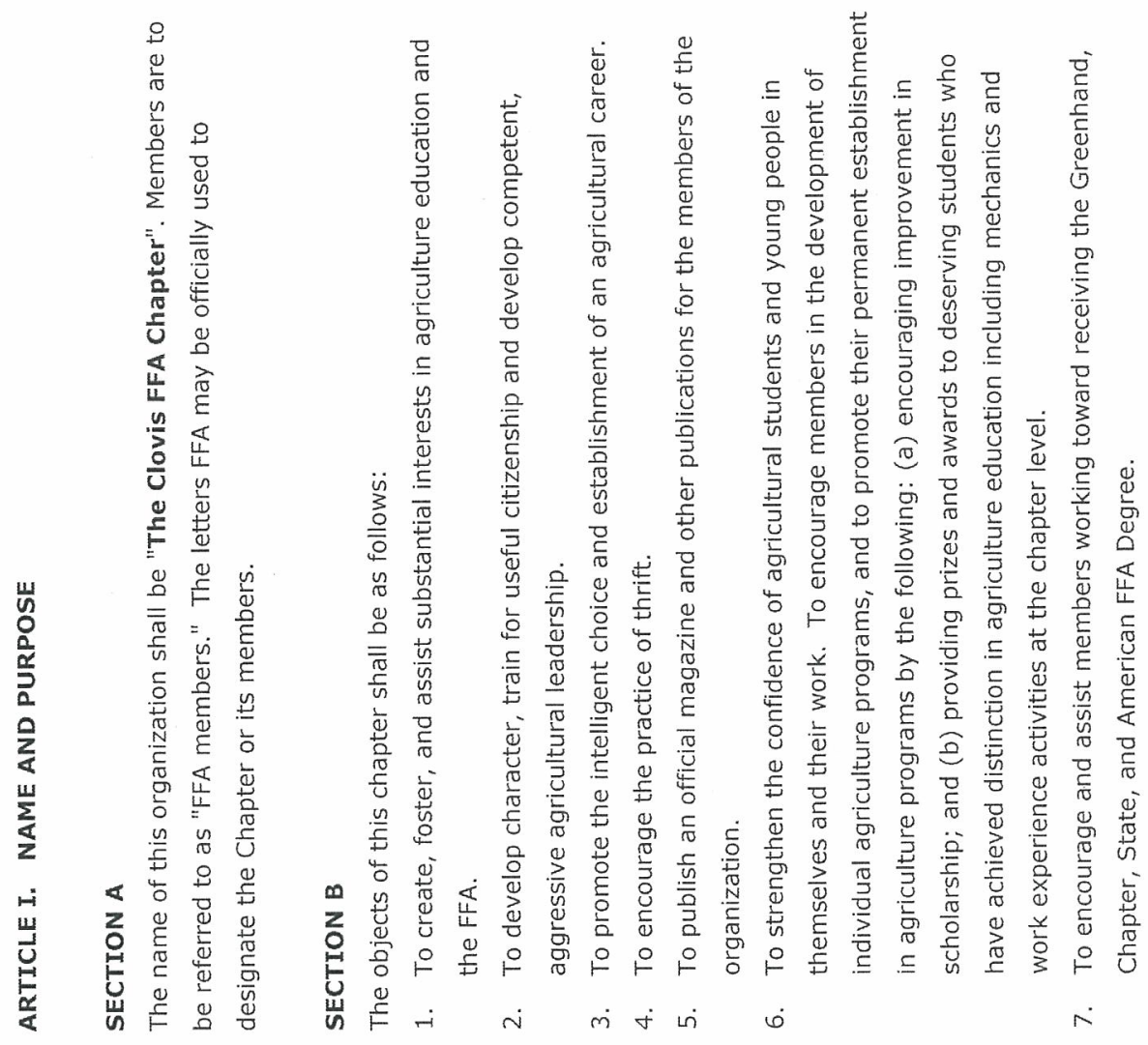

$\infty$

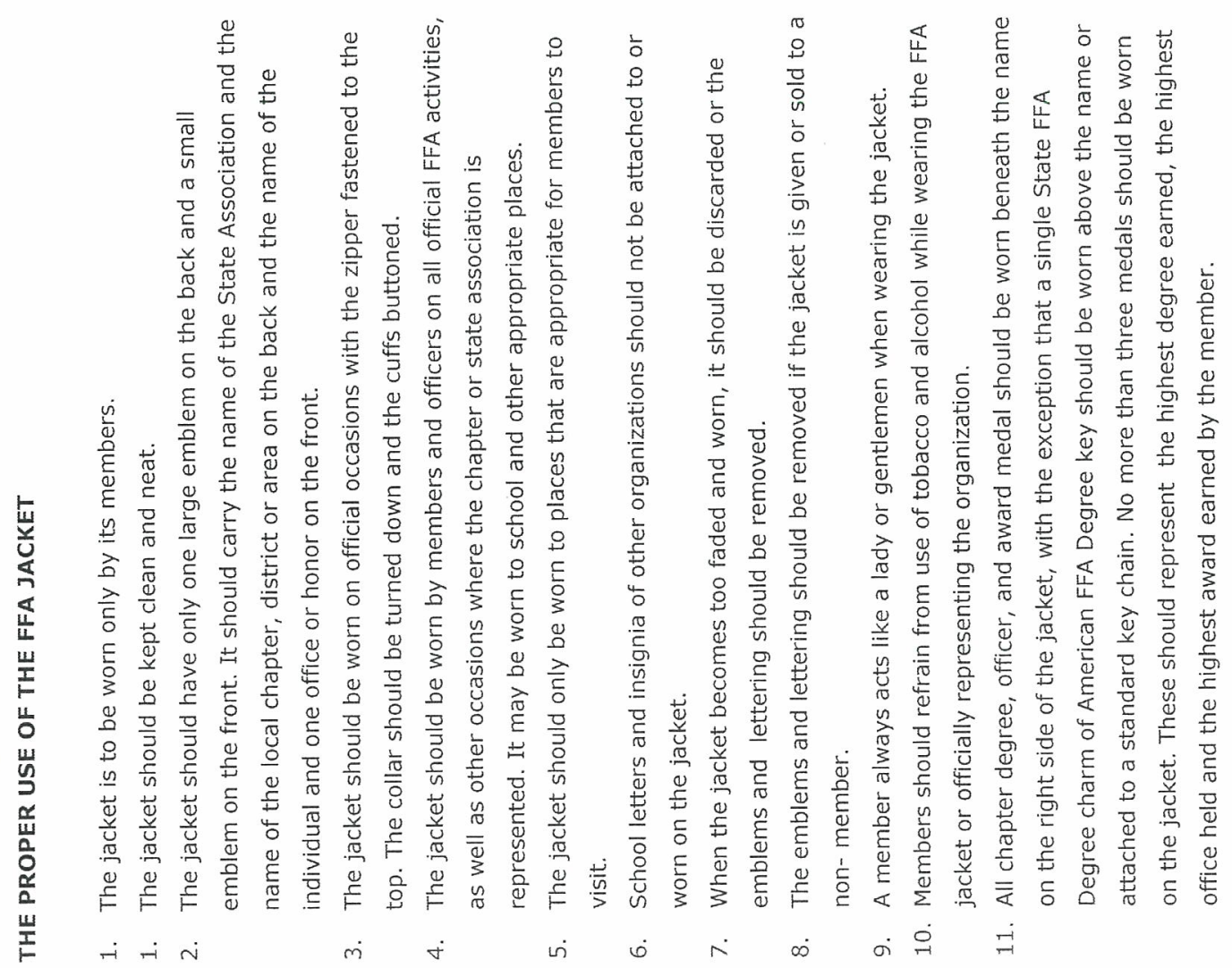




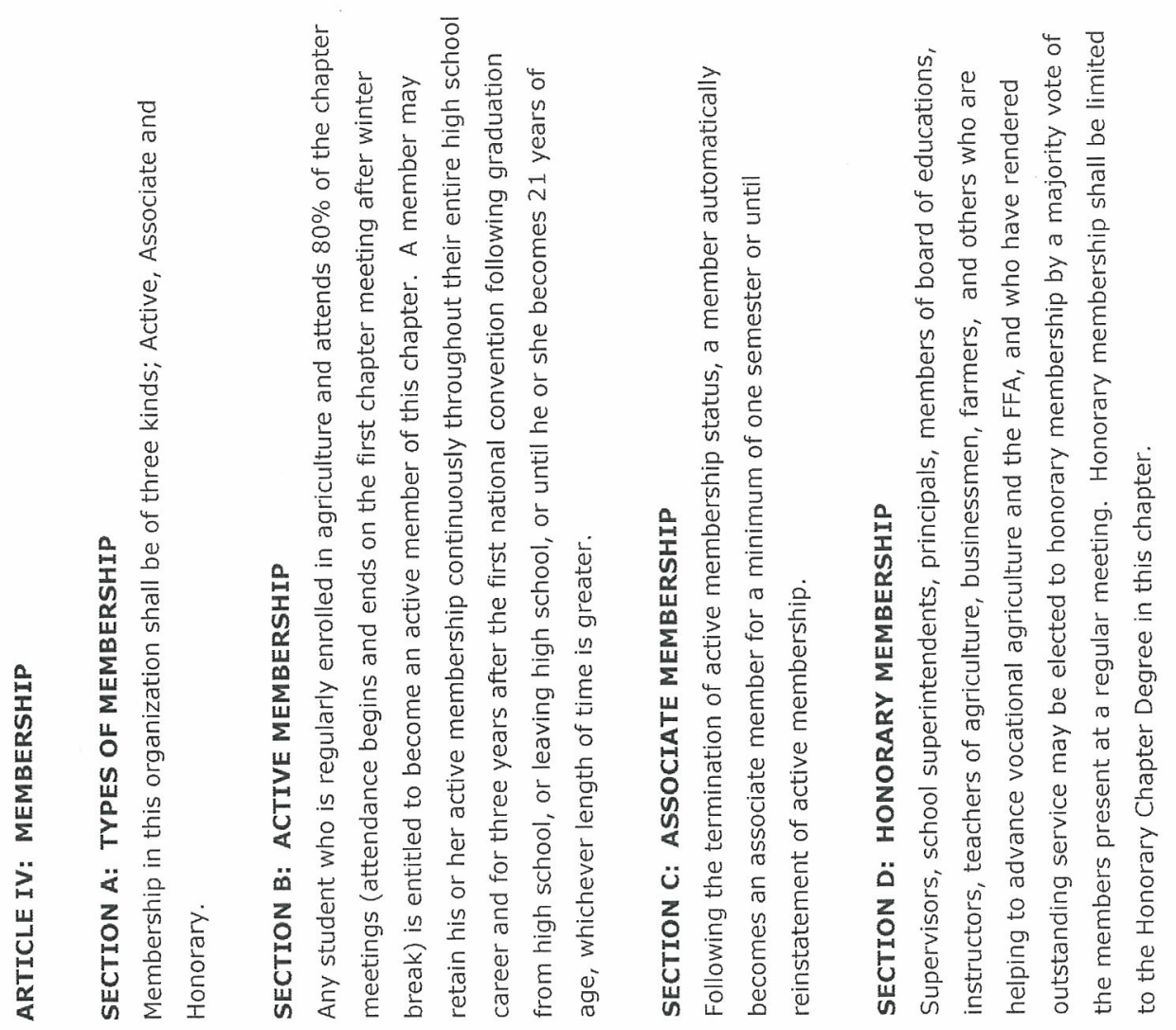

ㅇ

ㅇำ

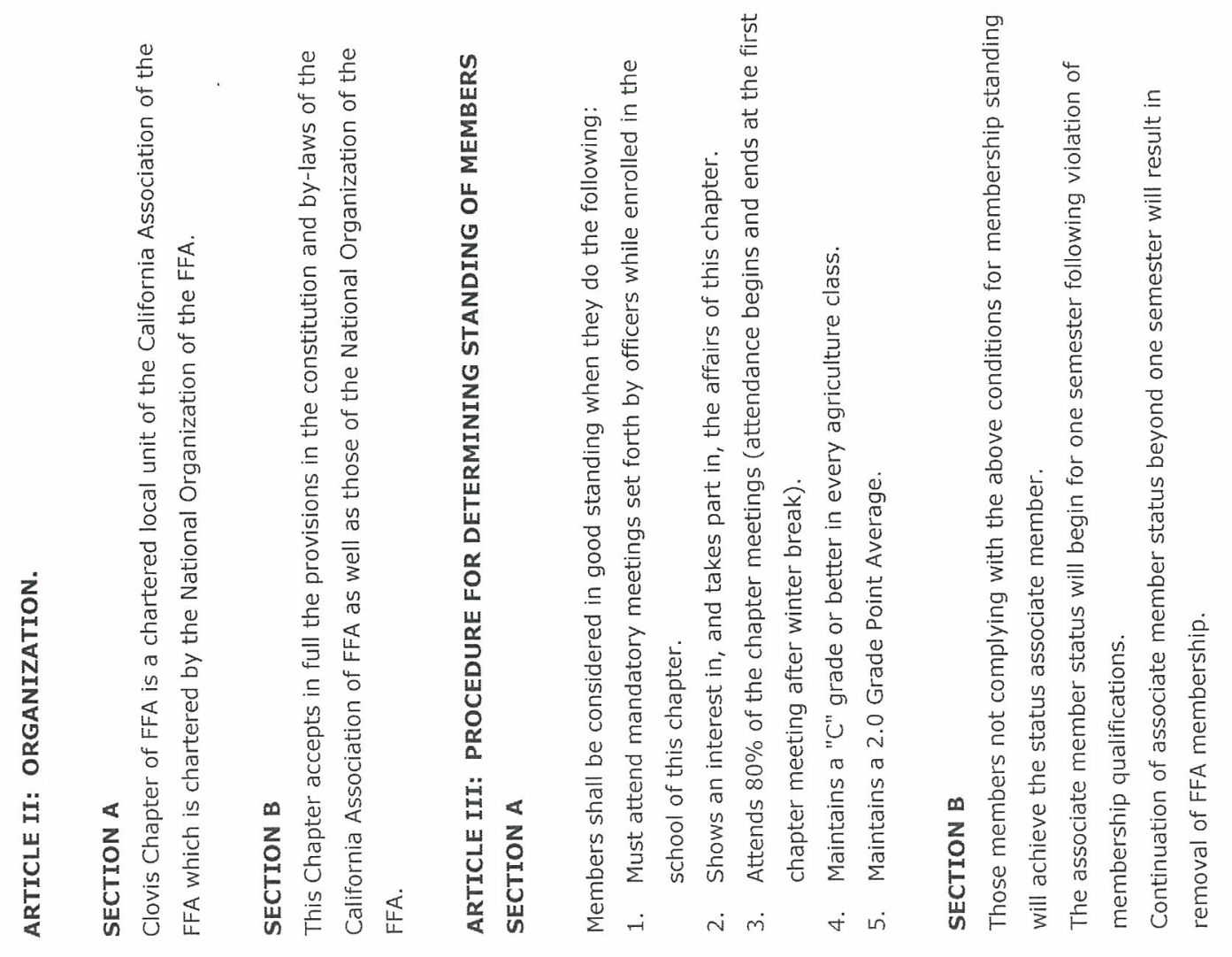



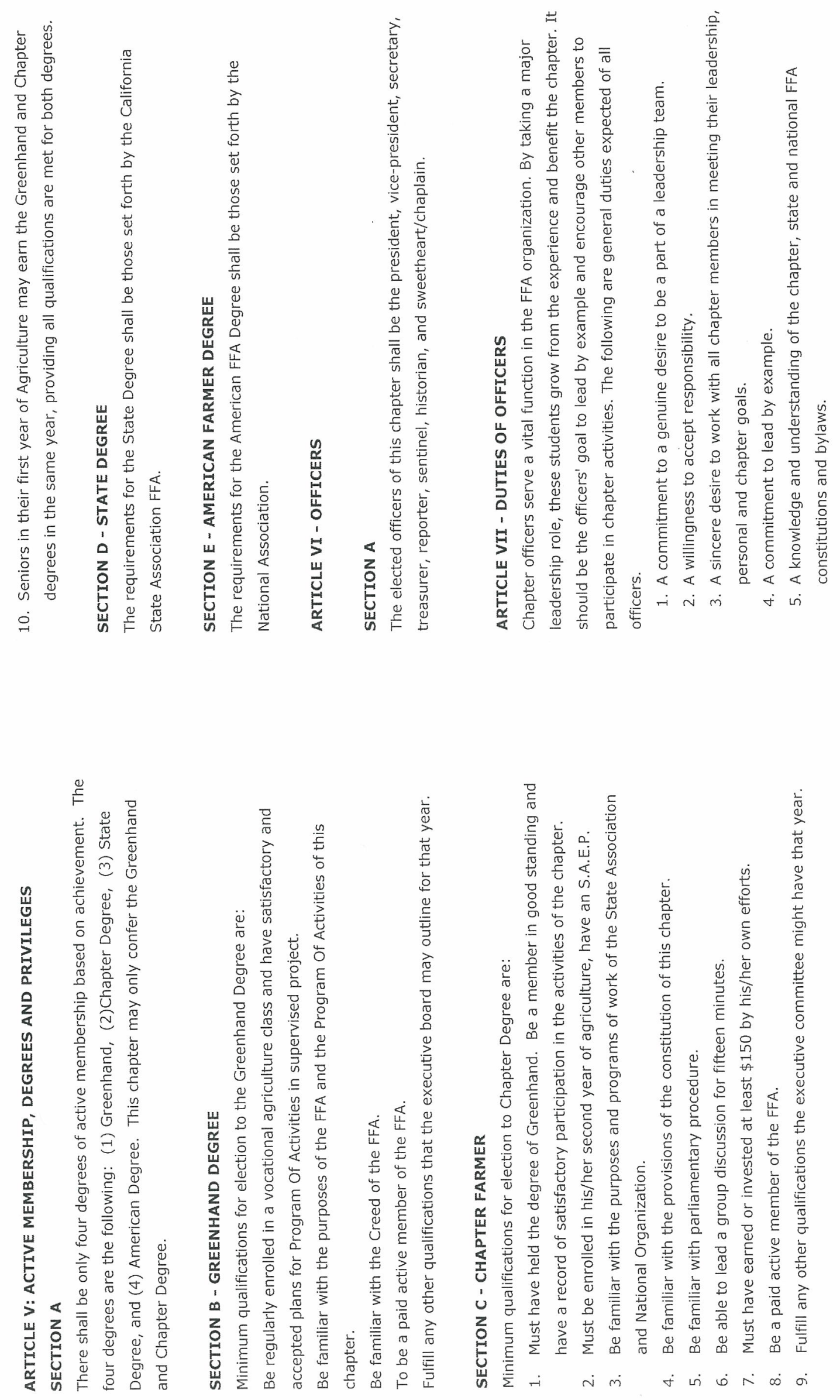

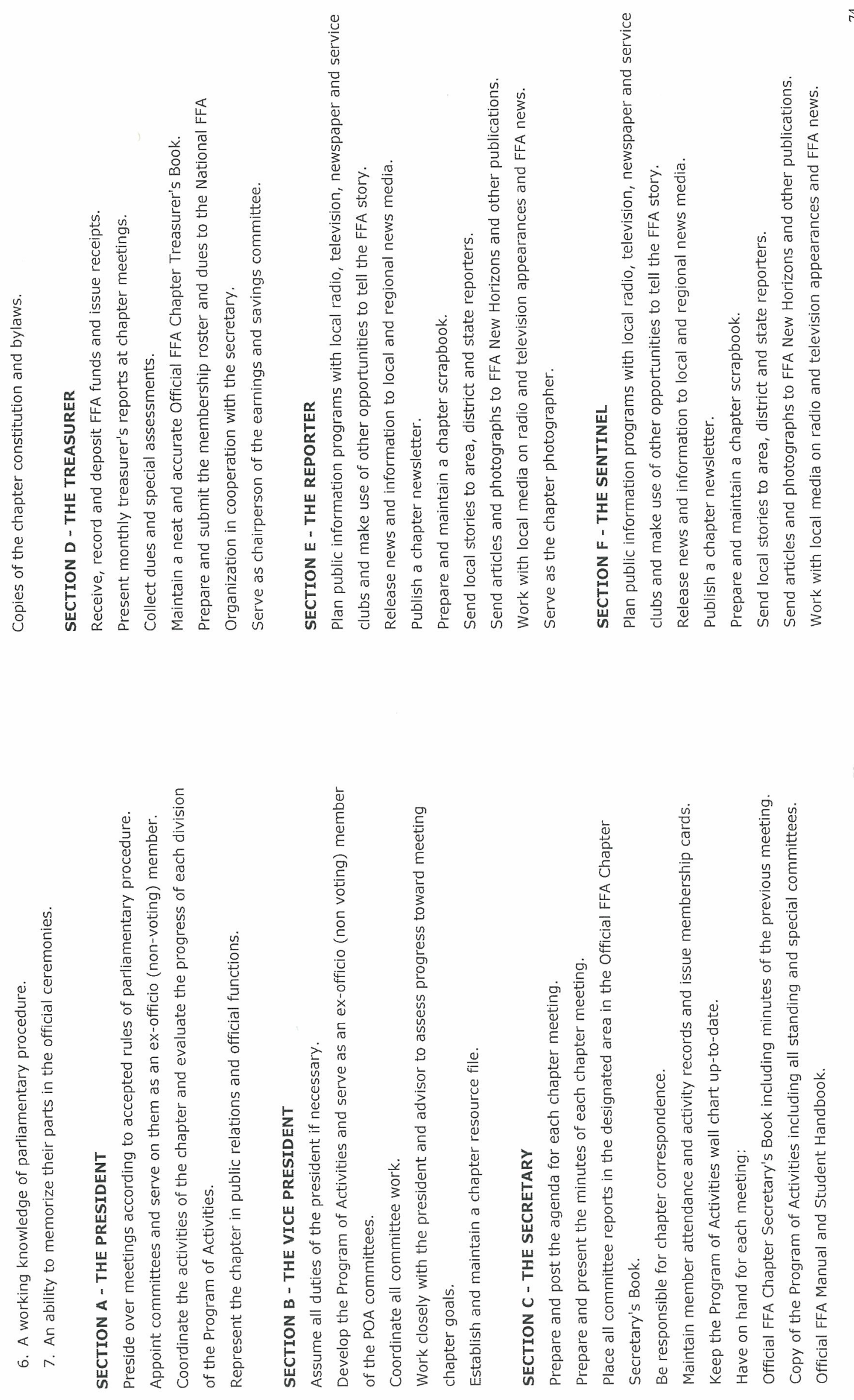


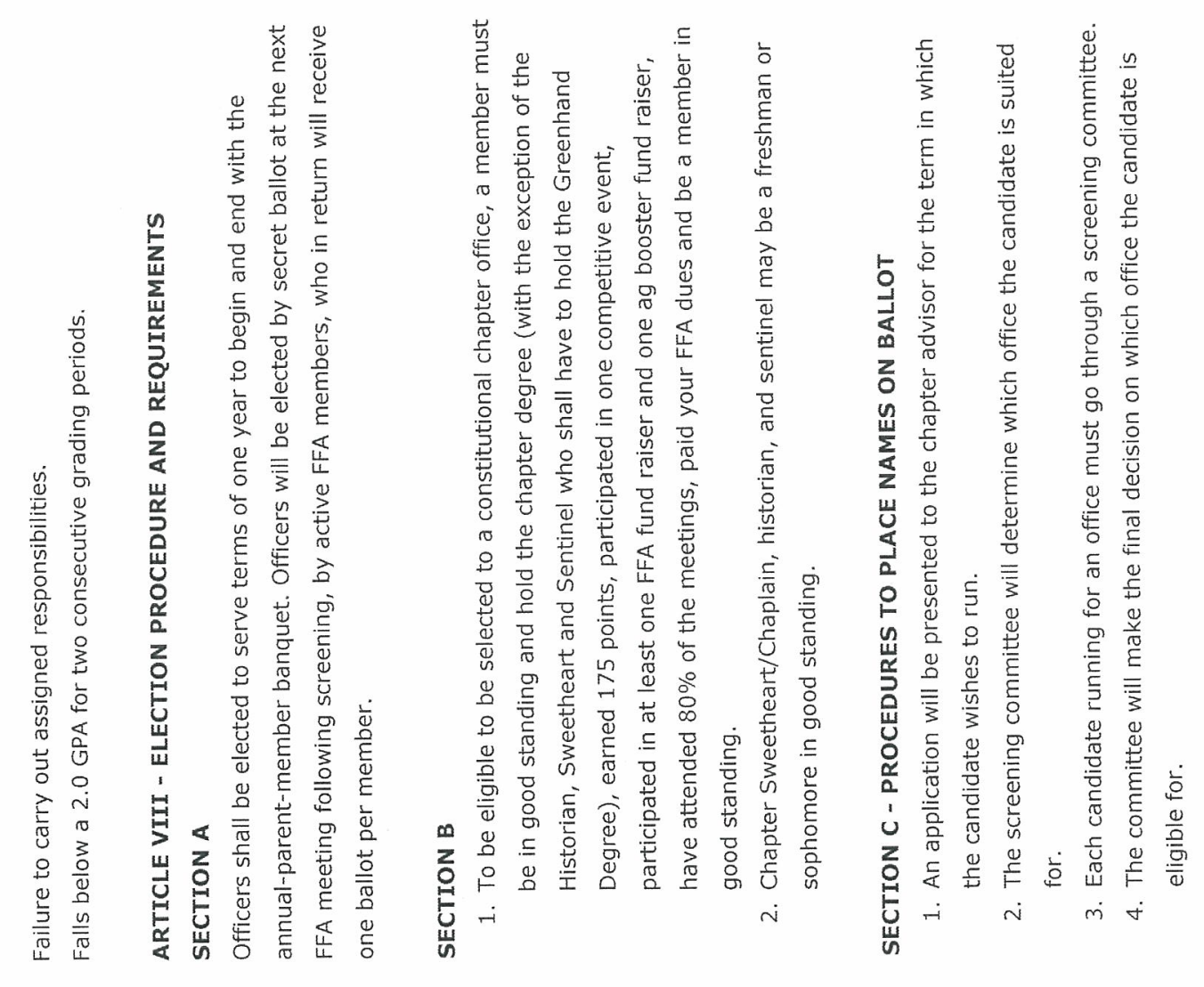

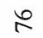

$n$
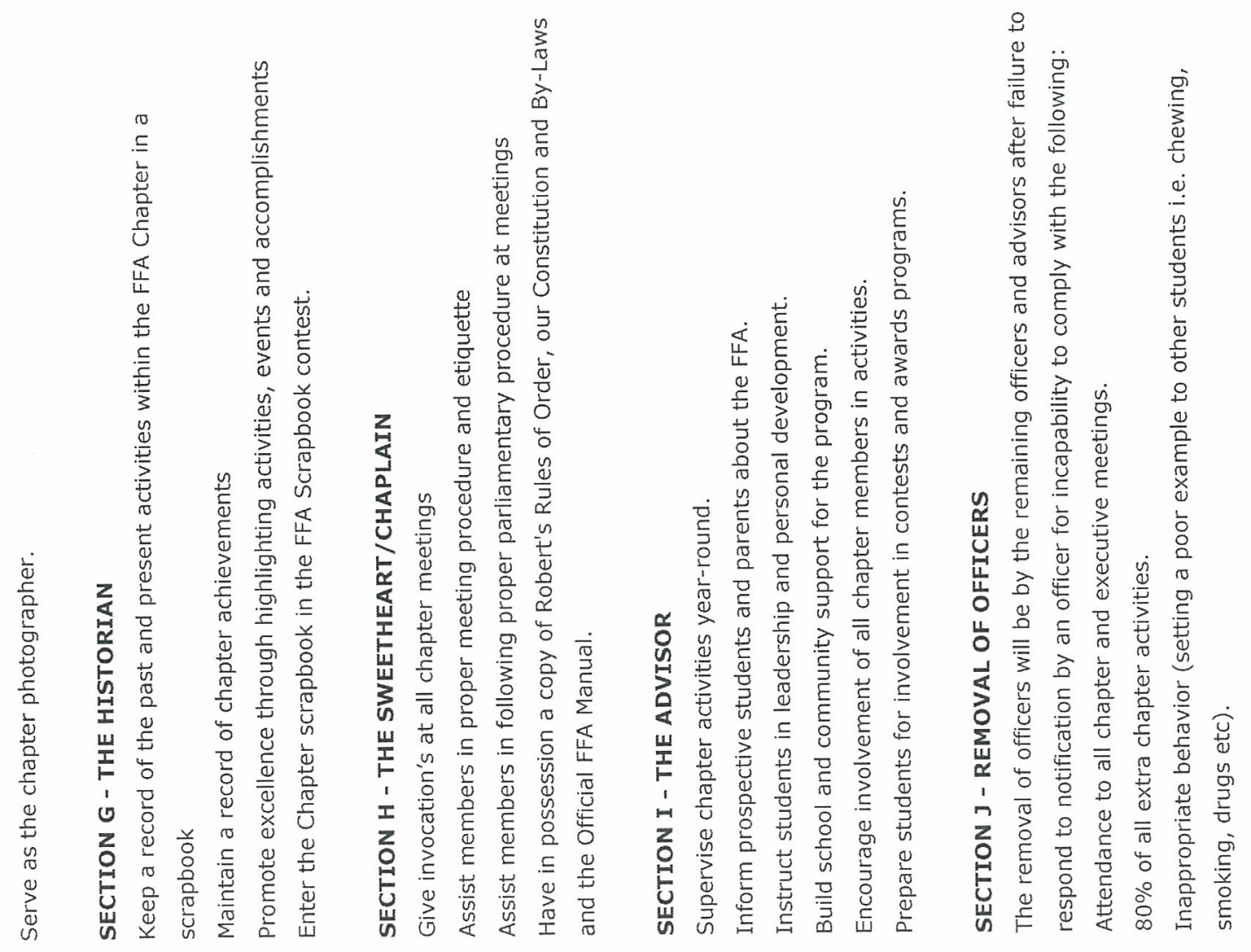

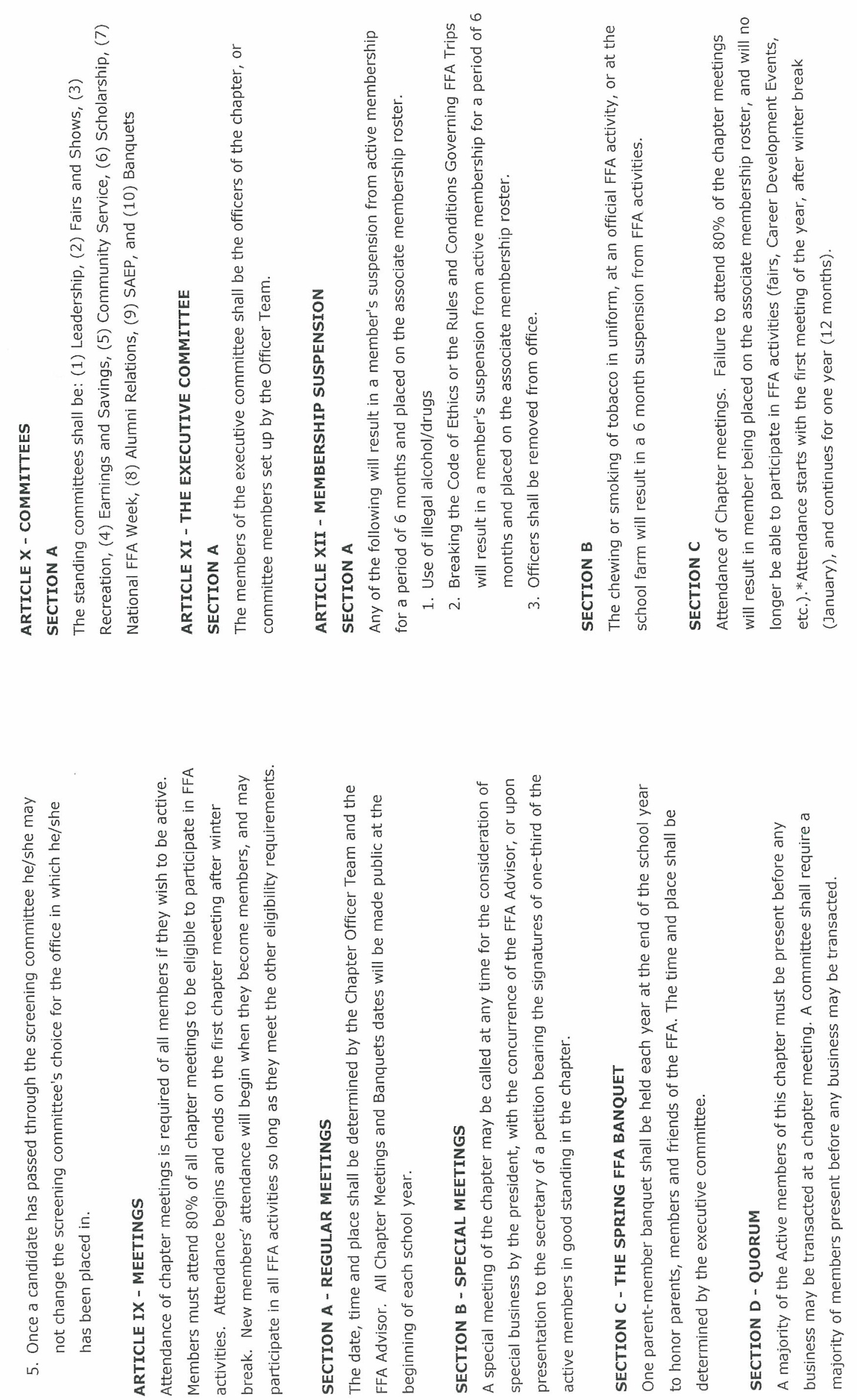


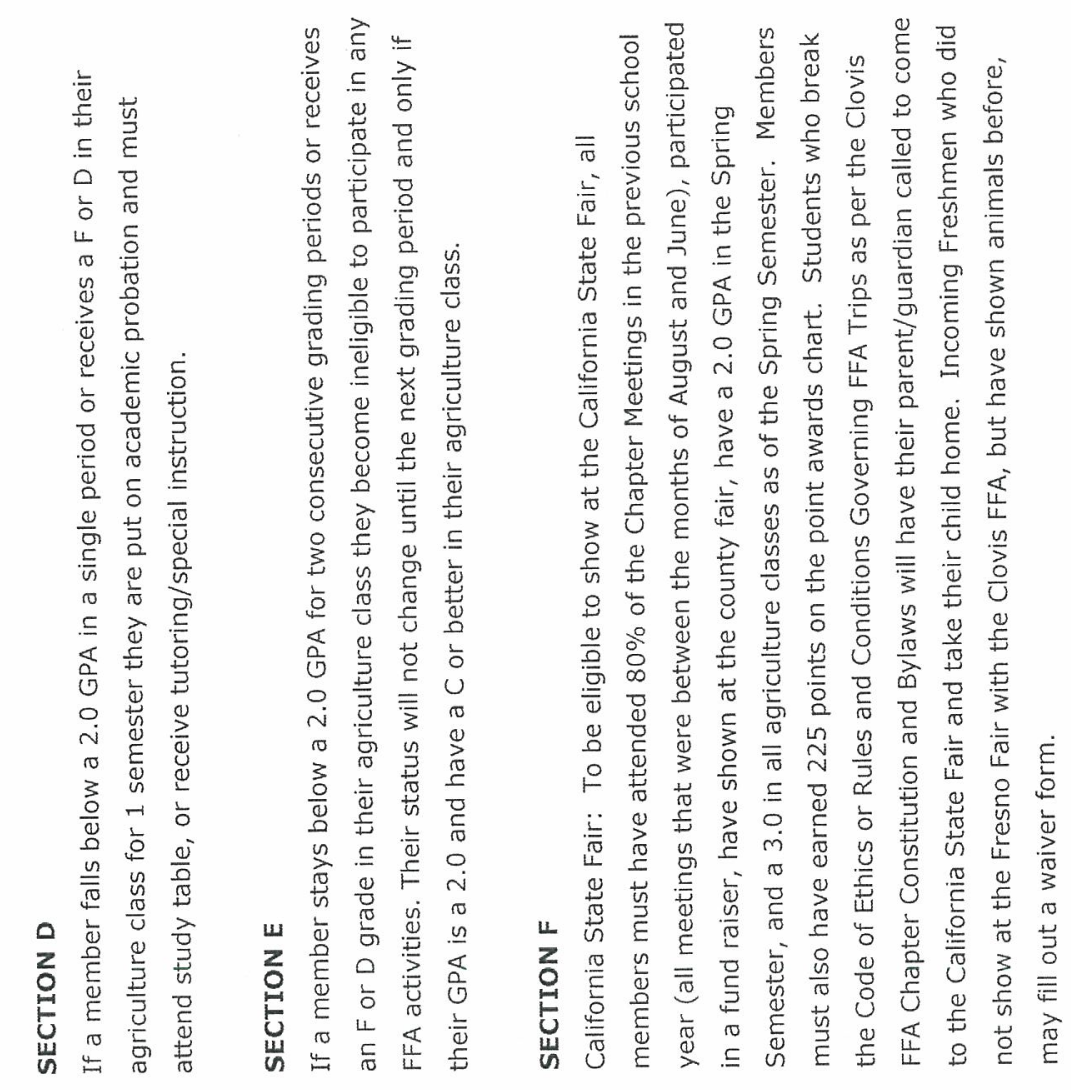

$\infty$

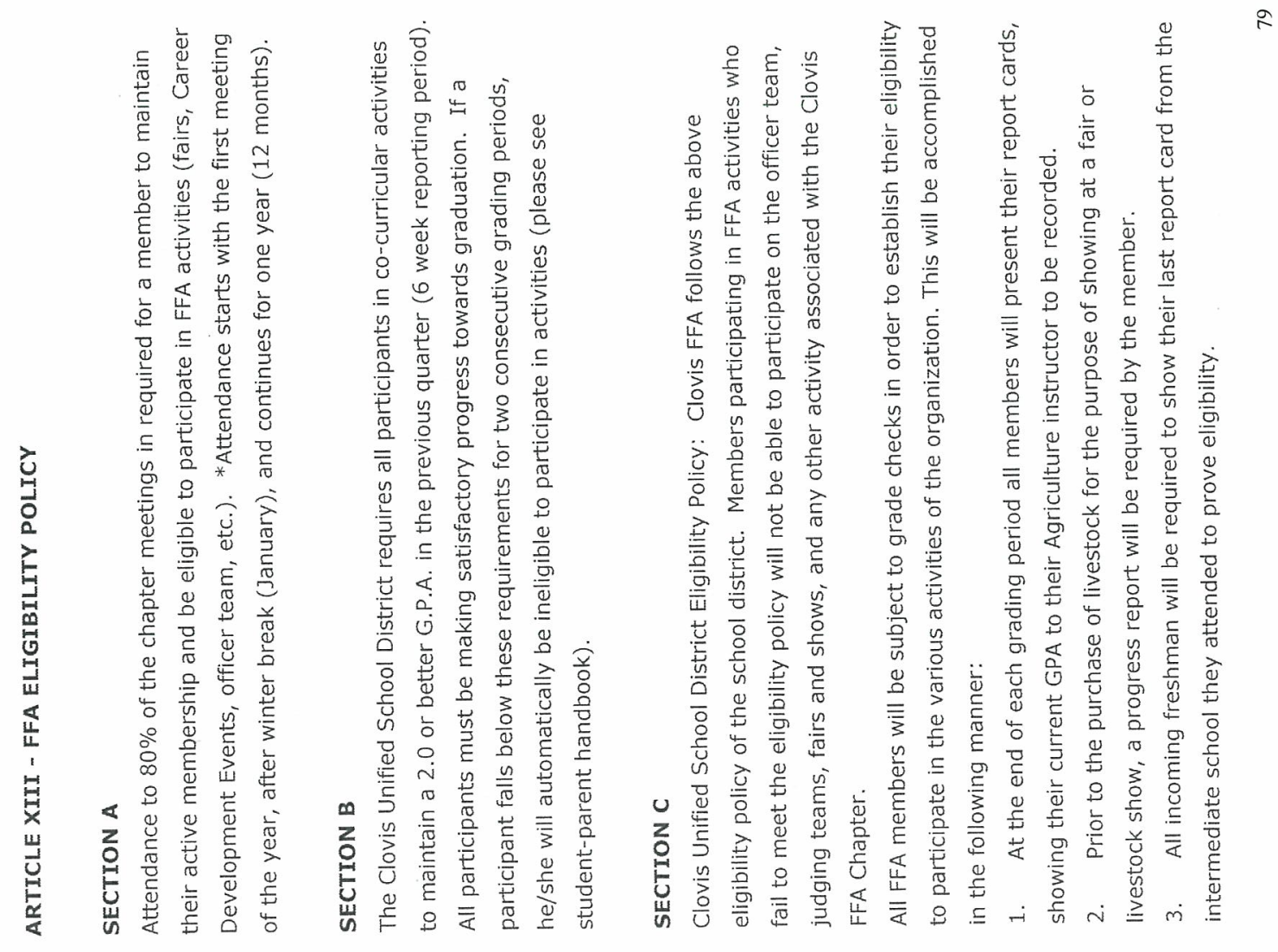




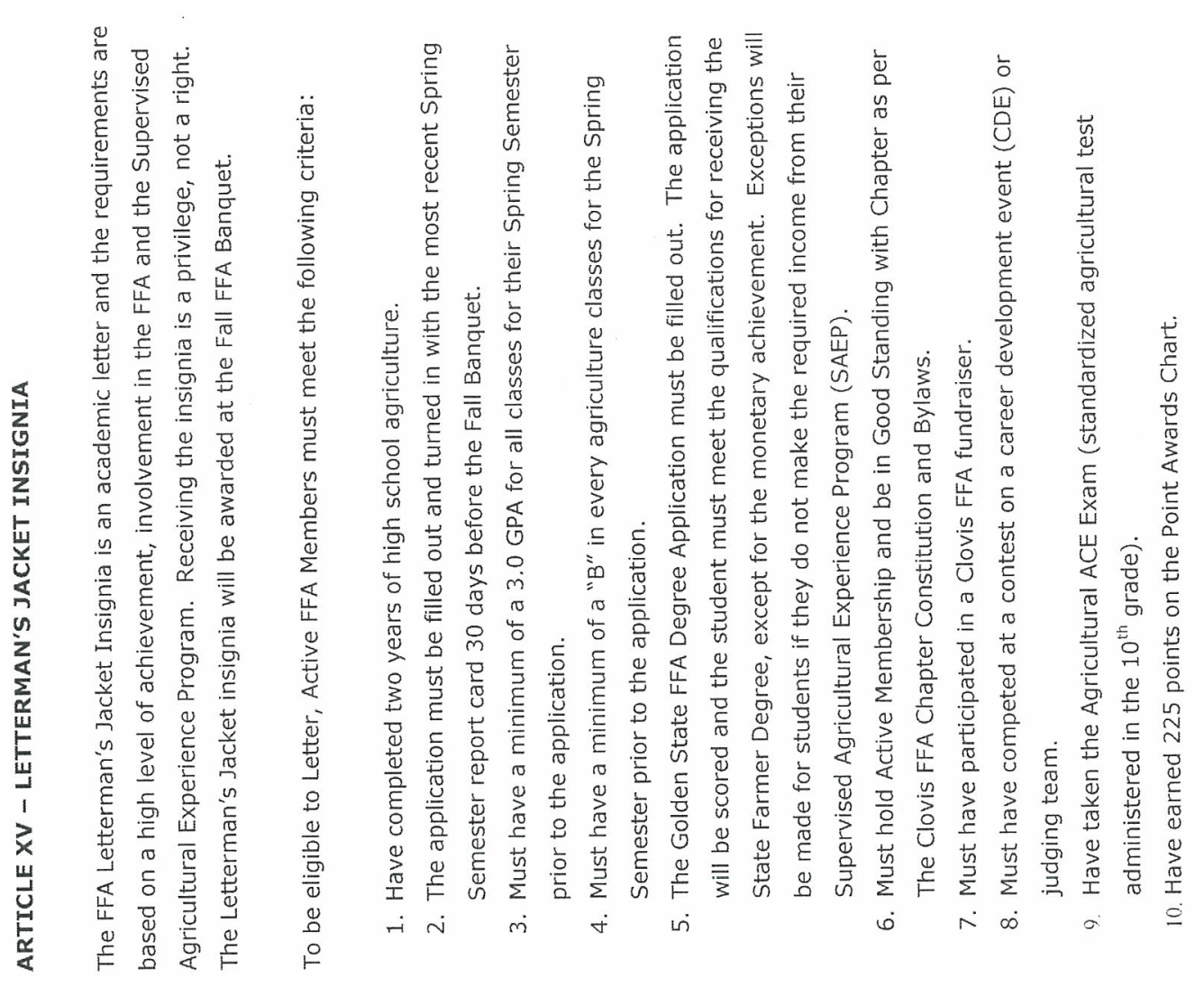

$\infty$

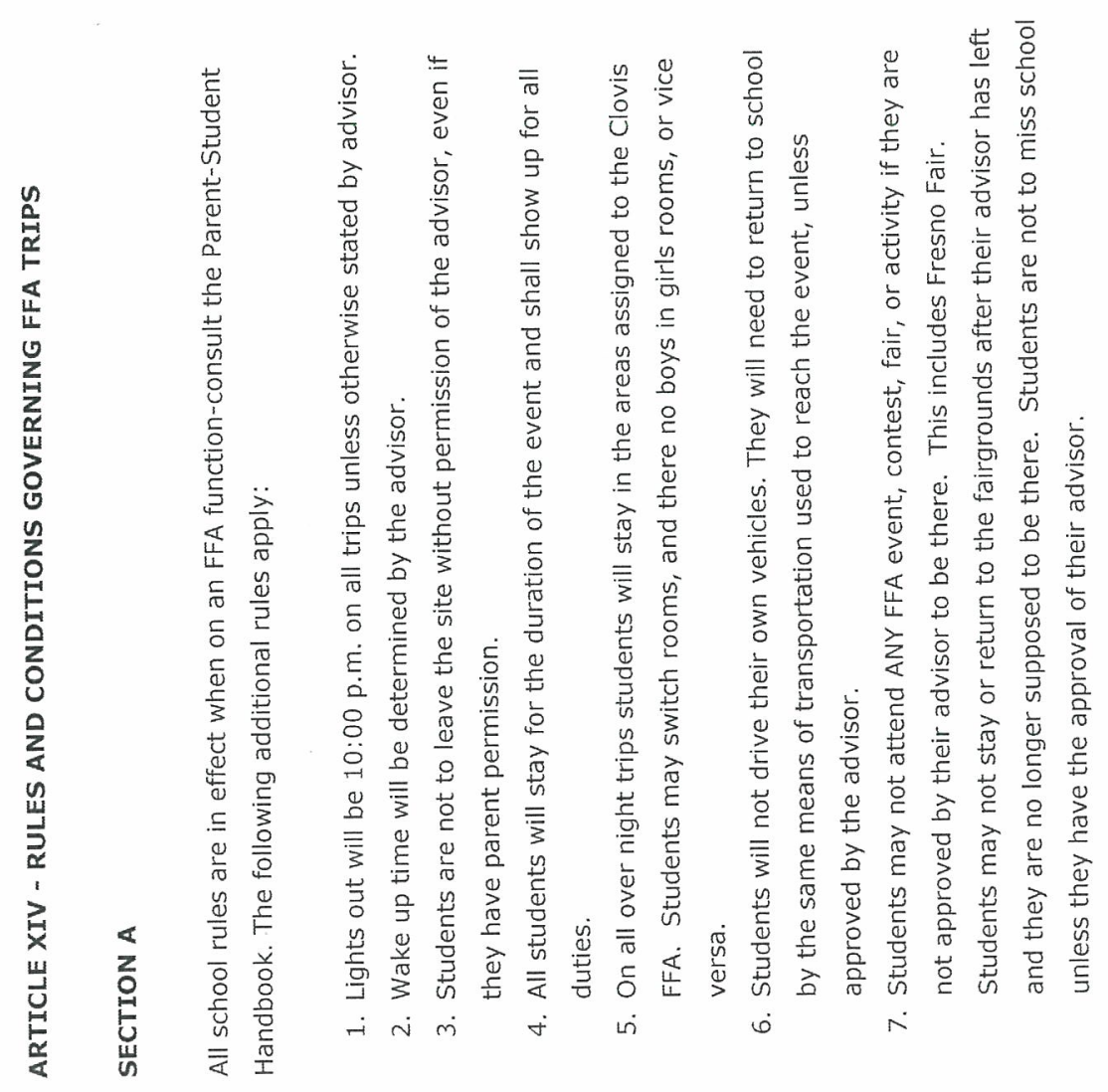




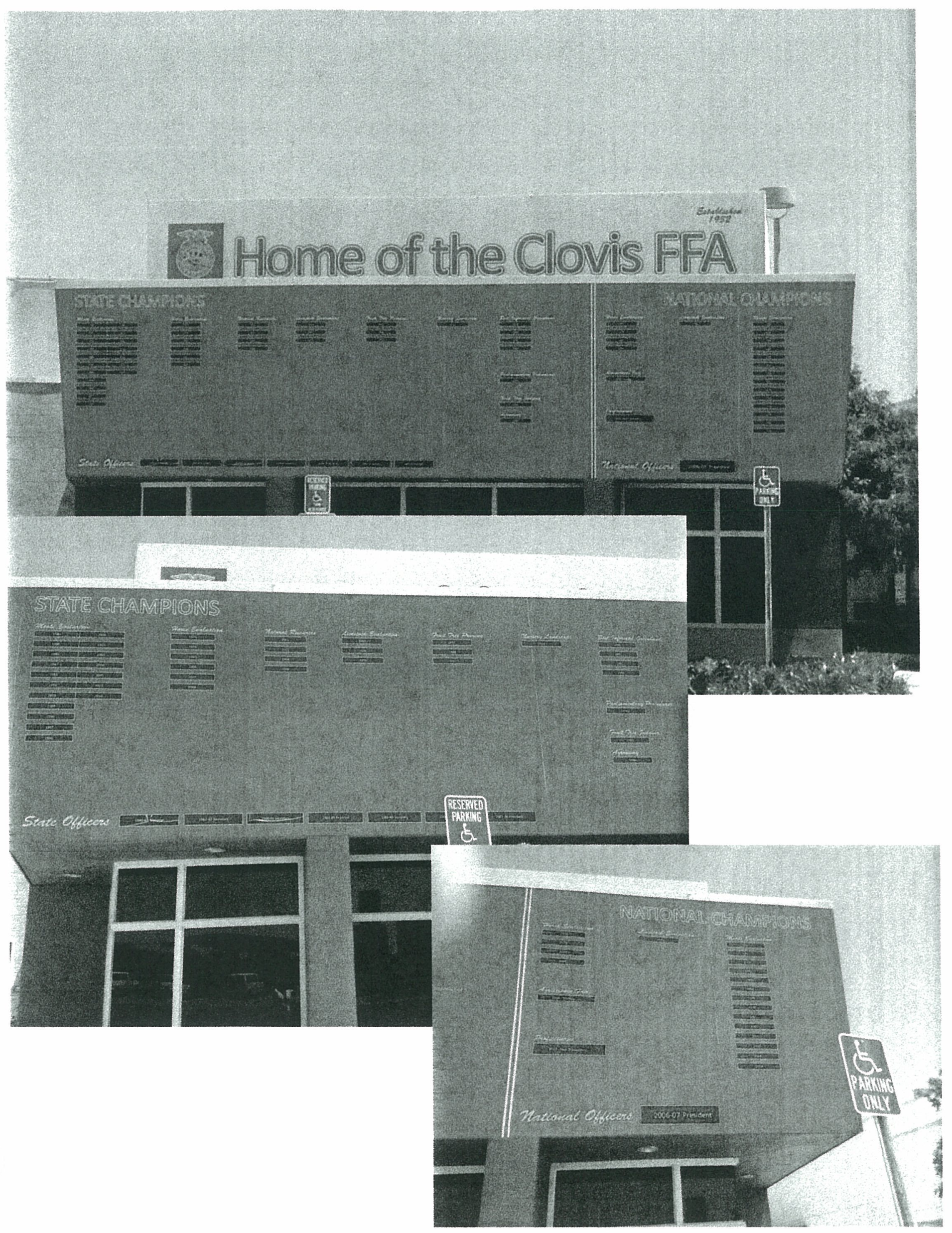




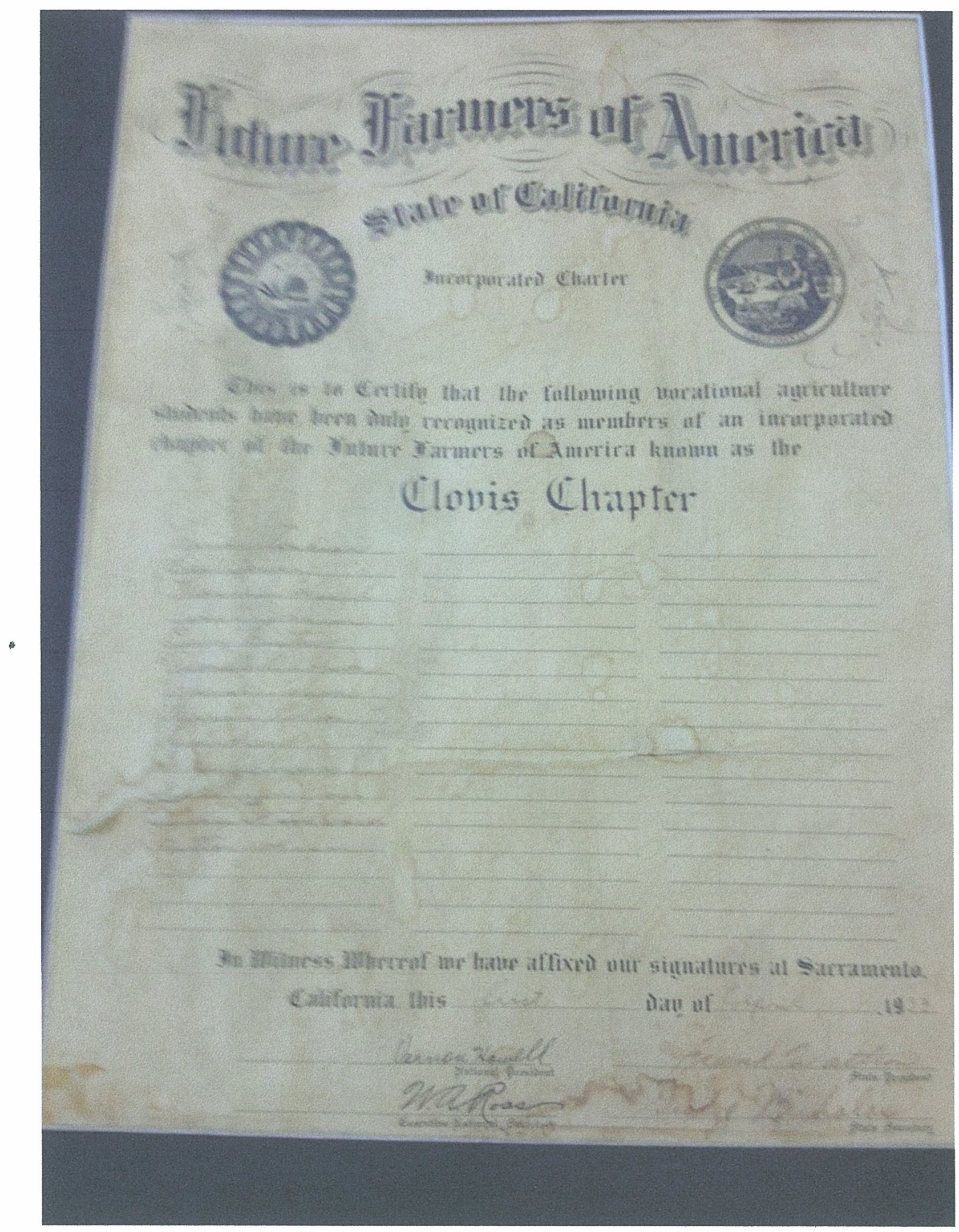


Regan Educational Center

McFarlane Coffman Ag Center Room 903

Mrs. Knight

jenniferknight@cusd.com

$559-327-4343$ or $559-327-4327$

\section{Course Information:}

Course work provides for the study of classification systems, anatomy, physiology, nutrition, reproduction, mitosis, meiosis, respiration, genetics, and health care of domesticated animals. Curriculum includes lab and fieldwork utilizing scientific methodology while reinforcing writing and presentation skills. Students will seek placement at a Community Classroom site (Vet Clinic or Animal Facility) during the second semester of instruction and will be expected to complete their class hours as an unpaid assistant or job shadow participant. This class is designed to meet EVERY day $6^{\text {th }}$ and $7^{\text {th }}$ period(s) to allow for increased instructional hours in the Fall and time for job site participation in the Spring semester.

\section{Suggested Materials:}

- 3 ring binder $1.5^{\prime \prime}$ to 2" (To use for Vet Science only)

- 8 tab dividers for vet science 3 ring binder

- Writing instrument (Pen or Pencil)

- Lined paper

- Composition or spiral notebook for work summaries (Spring Semester)

- Clear sheet protectors for portfolio materials (Spring Semester)

***If a student is unable to acquire the recommended materials the student should contact the teacher to obtain materials.

\section{Classwork and Participation:}

- Students are expected to participate actively in class by completing daily warm ups, taking notes, keeping resource materials organized, completing activities and labs, and doing homework.

Agriculture Education is a comprehensive program and requires students to participate in a Supervised Agriculture Experience Project component as well as FFA leadership activities and events.

\section{Homework and Projects:}

- Homework may be assigned 3-4 times a week.

- Students will complete several research projects.

- Students will complete a Resume and Cover Letter that they will use to find a community classroom location placement.

- A portfolio will be due as the Spring semester final project.

- Torn, crumpled, decorated, or sloppy work will not be accepted.

- Student work should have the students first and last name, course title, period, date, and assignment number in the top right corner.

\section{Late or make up work:}

- Late work will be accepted during the CURRENT unit of study for half credit. 
- Make up work is available for EXCUSED absences. It is the student's responsibility to collect make up work upon returning to class. Make-up school days are equal to number of days school days missed. This policy may change, depending on the particular situation.

\section{Testing Policy:}

- Missed tests must be made up within one week of student returning to school, an appointment should be made at lunch, break, before or after school.

- Students may re take a test once if they scored below 75\%. The max score that can be earned on a re take is $75 \%$

Grade Breakdown FALL SEMESTER:

- Tests \& Quizzes $30 \%$

- Class \& Homework $\quad 25 \%$

- Labs \& Projects $\quad 30 \%$

- Binders $10 \%$

- FFA and SAE $5 \%$

O SAE Credit by completion of FFA record book

FFA Points awarded by attending 1 activity for each 6 week grading period.

Grade Breakdown SPRING SEMESTER:

- Special Presentations $10 \%$

- Job Site Journal $10 \%$

- Final Portfolio $\quad 40 \%$

- Time Cards and Hours Worked 30\%

- Points can be deducted for:

- Not calling in absences or tardies

- Turning in time cards late

- Not meeting hour requirements

- Quality of work on Job Site

$10 \%$

○ (Instructor and Supervisor Evaluations)

\section{Behavior and Discipline:}

- Please refer to the student handbook. ALL CEHS and CUSD rules will be strictly enforced.

- Character Counts pillars and principals will also be a part of our classroom environment.

\section{Extra Credit and Incentives:}

- Incentive tickets will be awarded in class for exemplary participation, as a reward, or for displays of Character Counts.

- Tickets may be used for the following: (The 3 P's)

- (Points) Extra Credit Points at the end of a grading period

- (Potty) Exchange 1 ticket for a restroom trip

- (Prizes) Exchange for various prizes

- Other extra credit may be assigned as the instructor sees fit but is not frequently available, and will not replace the need to complete regular assignments. 


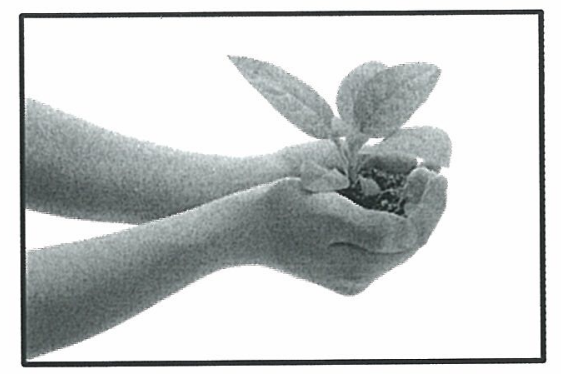

\section{Botany}

Clovis East High School

McFarlane Coffman Ag Center Room 903

Mrs. Knight

jenniferknight@cusd.com

$559-327-4343$ or $559-327-4327$

\section{Course Information:}

Botany is the Biology of plants, and will include concepts like plant classification, plant anatomy and physiology, growth principals, reproduction, soil science, biotechnology, and weed science and entomology.

\section{Suggested Materials:}

- 3 ring binder 1" to 1.5" (To use for Botany and leave in the classroom)

- 5 tab dividers for Botany 3 ring binder

- Writing instrument (Pen or Pencil)

- Lined paper to write notes or journal entries

***If a student is unable to purchase the recommended materials, the student should contact the teacher and materials will be provided.

\section{Classwork and Participation:}

- Students are expected to participate actively in class by completing daily warm ups, taking notes, keeping resource materials organized, completing activities and labs, and doing homework.

- Agriculture Education is a comprehensive program and requires students to participate in a Supervised Agriculture Experience Project component as well as FFA leadership activities and events.

\section{Homework and Projects:}

- Homework may be assigned 2-3 times a week.

- Students will complete an agriscience research project.

- Torn, crumpled, decorated, or sloppy work will not be accepted.

- Student work should have the students first and last name, course title, period, date, and assignment number in the top right corner.

Late or make up work:

- Late work will be accepted during the CURRENT unit of study for half credit. 
- Make-up work is available for EXCUSED absences. It is the student's responsibility to collect make up work upon returning to class. Make-up school days are equal to number of days school days missed. This policy may change, depending on the particular situation.

\section{Testing Policy:}

- Missed tests must be made up within one week of student returning to school, an appointment should be made at lunch, break, before or after school.

- Students may re-take a test once if they scored below $75 \%$. The max score that can be earned on a re take is $75 \%$

\section{Grade Breakdown:}

- Tests \& Quizzes

- Class \& Homework

- Labs \& Projects

- Binders

- FFA and SAE
$25 \%$

$25 \%$

$35 \%$

$10 \%$

$5 \%$

- SAE Credit by completion of FFA record book

- FFA Points awarded by attending 1 activity for each 6 week grading period.

\section{Behavior and Discipline:}

- Please refer to the student handbook. ALL CEHS rules will be strictly enforced.

- Character Counts pillars and principals will also be a part of our classroom environment.

\section{Extra Credit and Incentives:}

- Incentive tickets will be awarded in class for exemplary participation, as a reward, or for displays of Character Counts.

- Tickets may be used for the following: (The 3 P's)

- (Points) Extra Credit Points at the end of a grading period

- (Potty) Exchange 1 ticket for a restroom trip

- (Prizes) Exchange for various prizes

- Other extra credit may be assigned as the instructor sees fit but is not frequently available, and will not replace the need to complete regular assignments. 


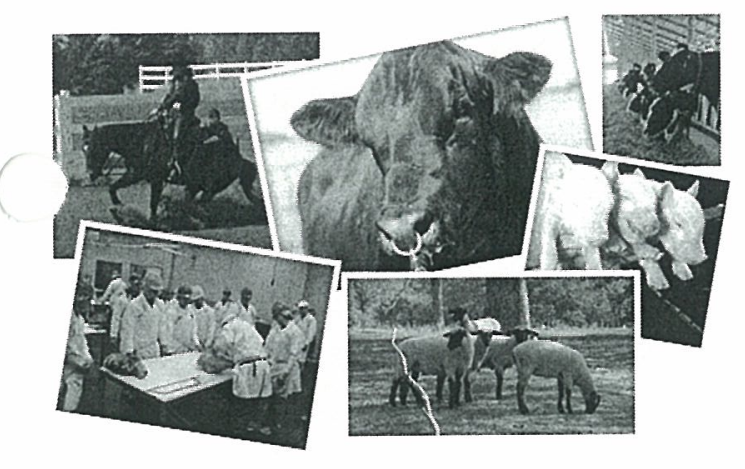

\section{Animal Science}

Clovis East High School

McFarlane Coffman Ag Center Room 903

Mrs. Knight

jenniferknight@.cusd.com

559-327-4343 or 559-327-4327

\section{Course Information:}

This course will provide the student with principals in Animal Science focusing on the areas of livestock (Mammalian) production, anatomy, physiology, reproduction, nutrition, respiration, and genetics. This course is intended to prepare those students who plan on majoring in Agriculture or Animal Sciences at a college or University.

\section{Suggested Materials:}

- 3 ring binder 1" to 1.5" (To use for Animal Science and leave in the classroom)

- 5 tab dividers for Animal science 3 ring binder

- Writing instrument (Pen or Pencil)

- Lined paper to write notes or journal entries

***If a student is unable to purchase the recommended materials, the student should contact the teacher and materials will be provided.

\section{Classwork and Participation:}

- Students are expected to participate actively in class by completing daily warm ups, taking notes, keeping resource materials organized, completing activities and labs, and doing homework.

Agriculture Education is a comprehensive program and requires students to participate in a Supervised Agriculture Experience Project component as well as FFA leadership activities and events.

\section{Homework and Projects:}

- Homework may be assigned 2-3 times a week.

- Students will complete an agriscience research project.

- Torn, crumpled, decorated, or sloppy work will not be accepted.

- Student work should have the students first and last name, course title, period, date, and assignment number in the top right corner.

\section{Late or make up work:}

- Late work will be accepted during the CURRENT unit of study for half credit.

- Make-up work is available for EXCUSED absences. It is the student's responsibility to collect make up work upon returning to class. Make-up school days are equal to number of days school days missed. This policy may change, depending on the particular situation. 


\section{Testing Policy:}

- Missed tests must be made up within one week of student returning to school, an appointment should be made at lunch, break, before or after school.

- Students may re-take a test once if they scored below $75 \%$. The max score that can be earned on a re-take is $75 \%$

\section{Grade Breakdown:}

- Tests \& Quizzes

$25 \%$

- Class \& Homework $\quad 25 \%$

- Labs \& Projects $\quad 35 \%$

- Binders $10 \%$

FFA and SAE

$5 \%$

- SAE Credit by completion of FFA record book

- FFA Points awarded by attending 1 activity for each 6 week grading period.

\section{Behavior and Discipline:}

- Please refer to the student handbook. ALL CEHS rules will be strictly enforced.

- Character Counts pillars and principals will also be a part of our classroom environment.

\section{Extra Credit and Incentives:}

- Incentive tickets will be awarded in class for exemplary participation, as a reward, or for displays of Character Counts.

- Tickets may be used for the following: (The 3 P's)

- (Points) Extra Credit Points at the end of a grading period

- (Potty) Exchange 1 ticket for a restroom trip

- (Prizes) Exchange for various prizes

- Other extra credit may be assigned as the instructor sees fit but is not frequently available, and will not replace the need to complete regular assignments.

\footnotetext{
The Clovis Unified School District does not discriminate on the basis of race, color, disability, or national origin in admission or access to and treatment of employment in its programs and activities as required by Title VI, Title IX and Section 504. Complaints: Contact Dr. Cheryl Rogers, Human Resources, (559) 327-9308. 


\begin{tabular}{|c|c|c|c|c|c|c|c|c|c|c|c|c|c|c|c|c|c|c|c|c|c|c|c|c|c|c|c|c|}
\hline ब্ & 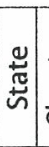 & 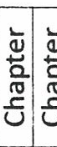 & 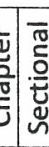 & 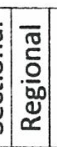 & 芯 & 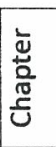 & & 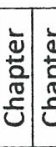 & & & ئ & & & ڤั & & 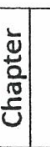 & $\frac{\pi}{\pi}$ & & 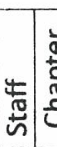 & & 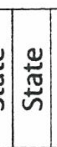 & & & 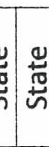 & 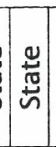 & & & \\
\hline 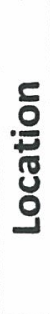 & 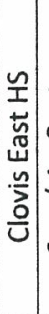 & 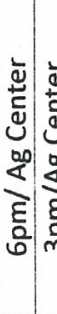 & 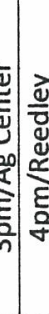 & 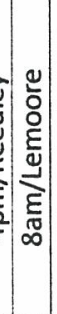 & 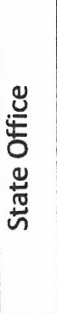 & 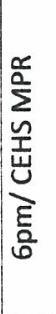 & & 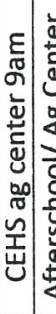 & 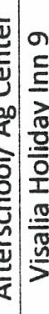 & & 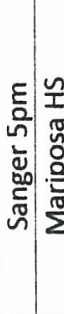 & ర్ & 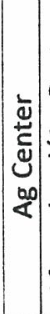 & 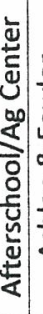 & & 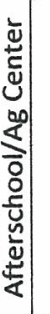 & 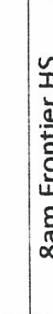 & 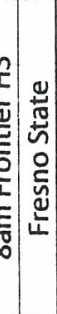 & 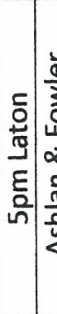 & & 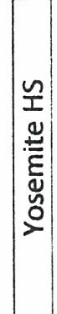 & & 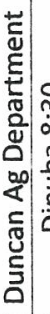 & & 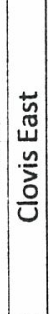 & & & 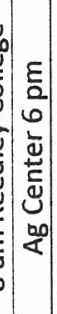 \\
\hline : & 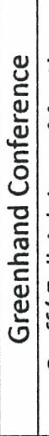 & 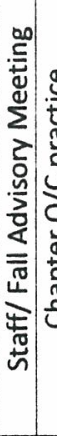 & 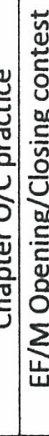 & 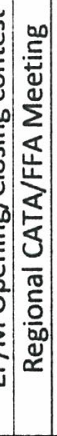 & 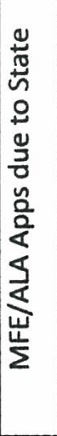 & 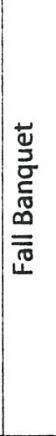 & & 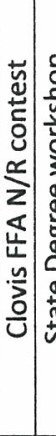 & & & 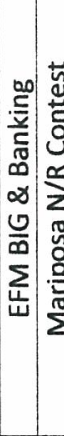 & 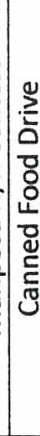 & 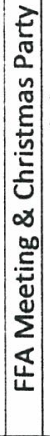 & 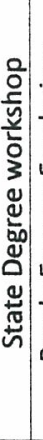 & & 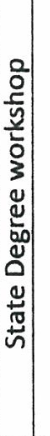 & & 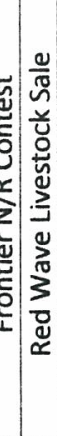 & 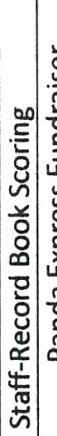 & 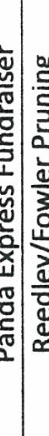 & 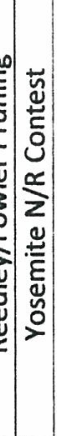 & 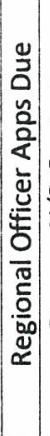 & 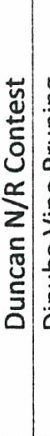 & 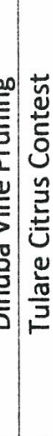 & 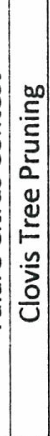 & 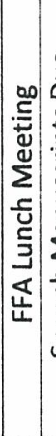 & 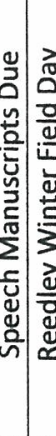 & 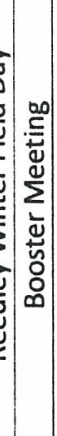 \\
\hline هั & $\stackrel{\emptyset}{-1}$ & $\stackrel{\infty}{=} \underset{z}{=}$ & & & $\stackrel{n}{\beth} \underset{\exists}{\exists}$ & $\underset{\exists}{\stackrel{2}{\xi}}$ & 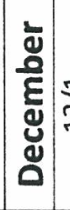 & 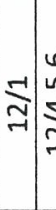 & : & & $\underset{\sim}{\stackrel{0}{\sim}} \underset{\sim}{\infty}$ & & 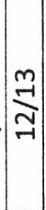 & $\begin{array}{l}\overrightarrow{7} \\
- \\
= \\
=\end{array}$ & & 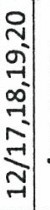 & 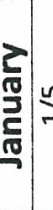 & & & & $\vec{y}$ & $\underset{\sim}{-}$ & 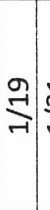 & & & $\underset{\sim}{\stackrel{N}{2}}$ & $\underset{J}{\mathbb{Z}}$ & \\
\hline
\end{tabular}

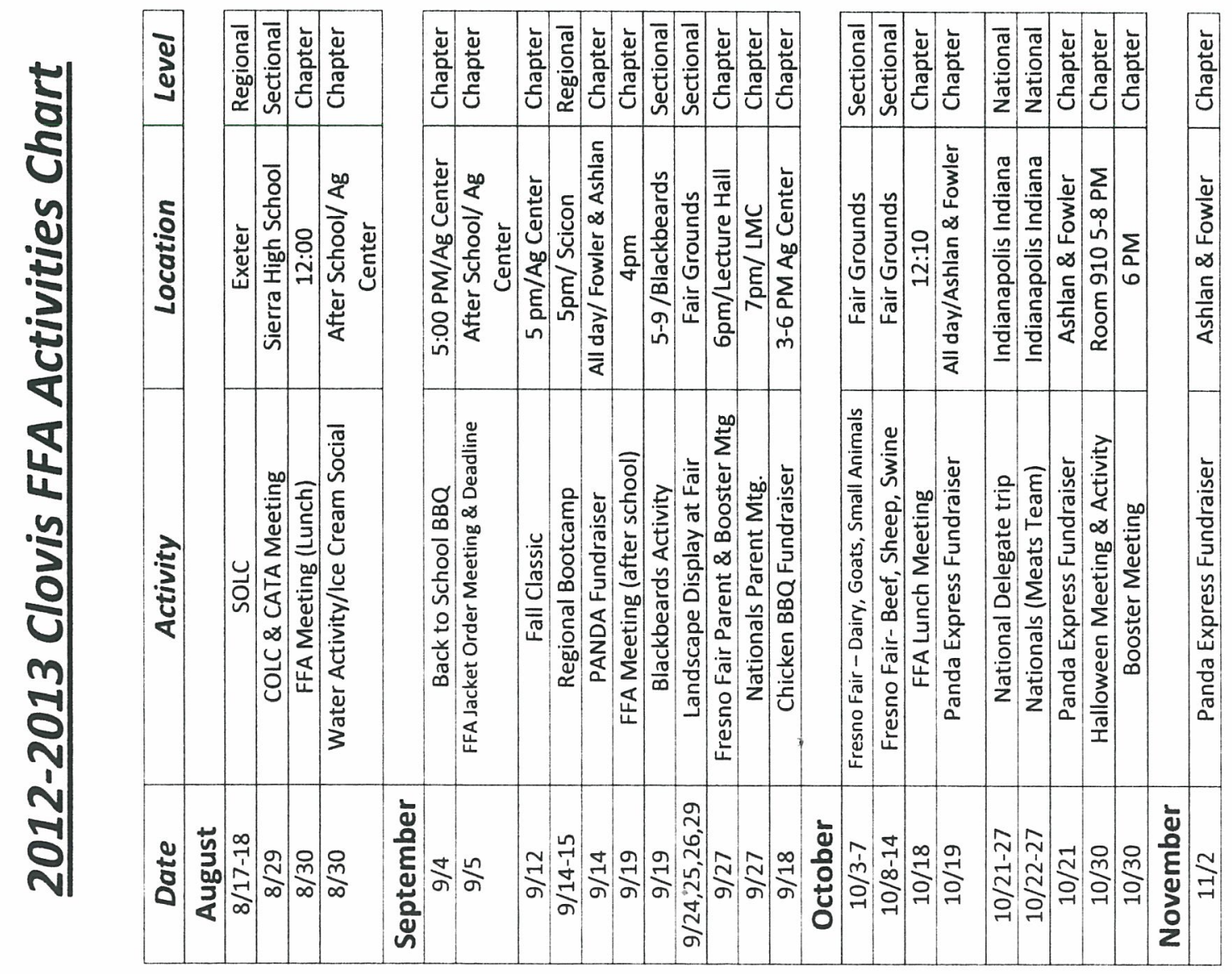




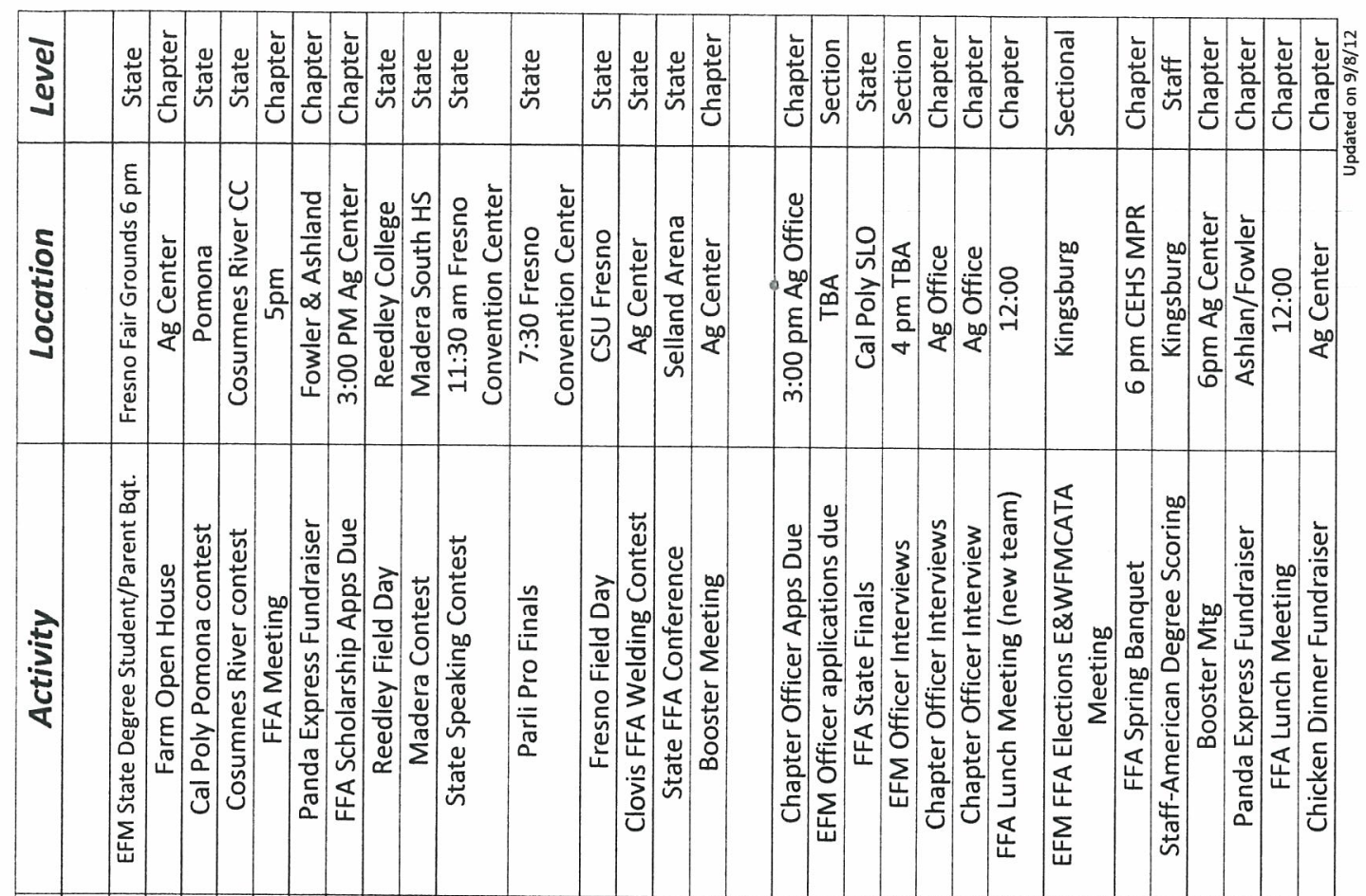

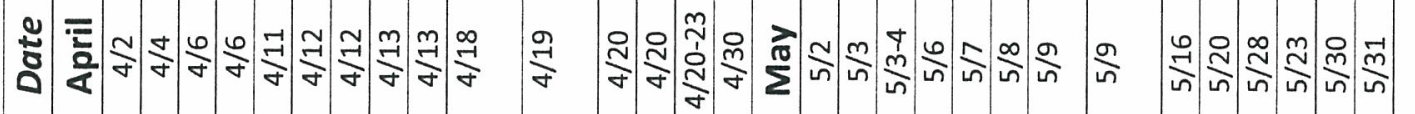

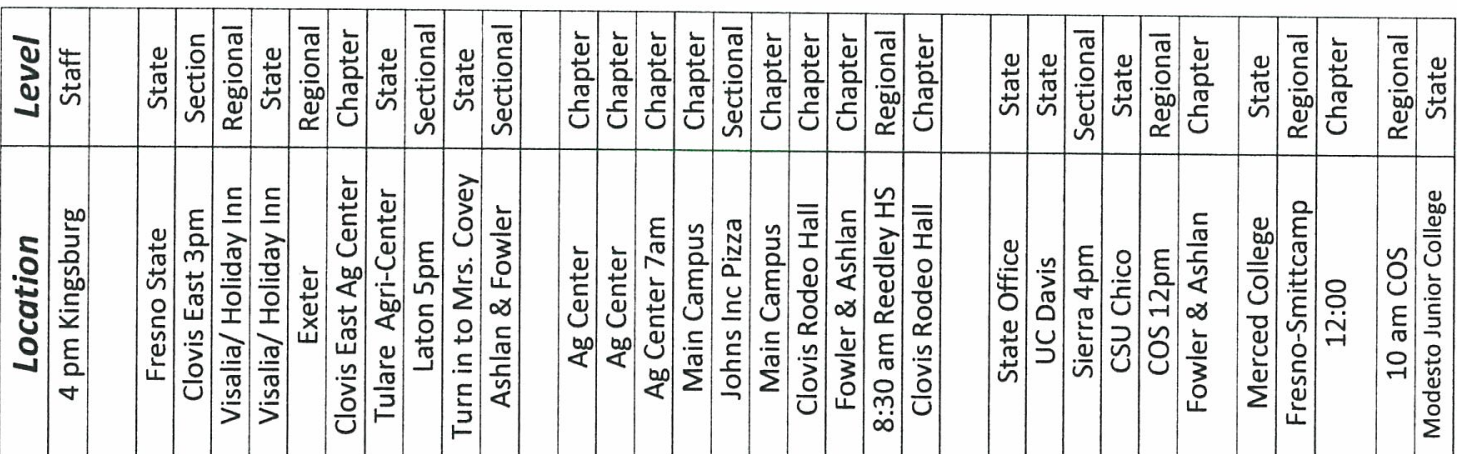

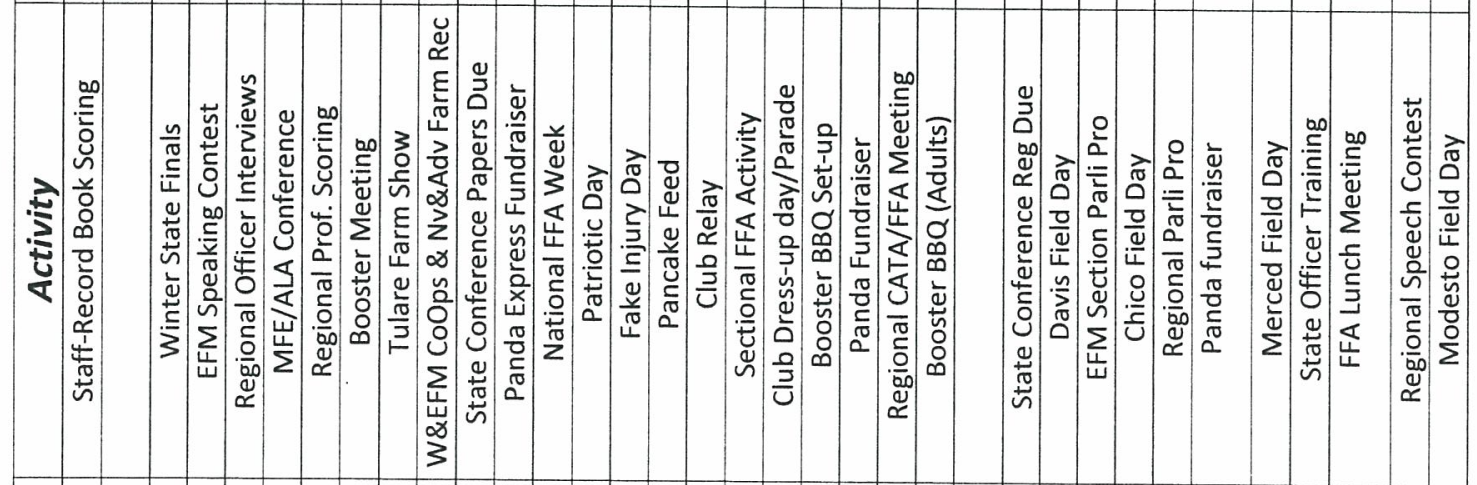

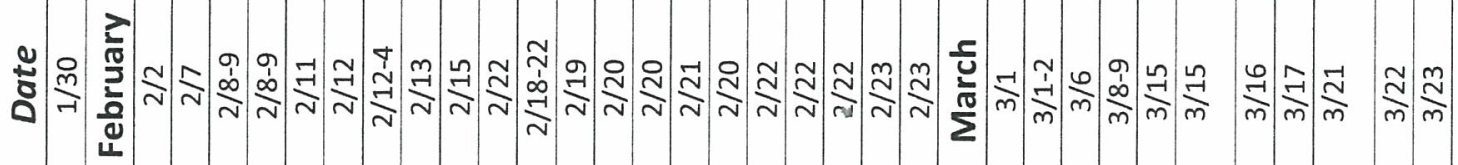


Clovis FFA Top 20 Award Application

Due to Mrs. Covey by Friday May 9th

\begin{tabular}{llll}
$N$ & Ge: & Grade: & Ag Teacher: \\
\hline & Point Value & Fall & Spring
\end{tabular}

\section{Officer}

1. Greenhand Officer

3

2.Exucitive Committee

3. Chapter Officer

4. Sectional or Regional Officer

\section{Leadership}

1. Officer Retreat

2. COLC

3. SOLC

4. ROLC

5.Regional Bootcamp

6. 13-14 Chapter Officer Applicant

7. Sectional Meeting

*Attend

*Run for Office

8. Regional Meeting

*Attend

*Run for Office

9.State Convention

* One Day

* 4 Day/delegate

*Special assignmnet

10. National Convention

*Conference

*Delegate

*National Team

11. Greenhand Conference

12. MFE

13. ALA

14. SLE

15. WLE

\section{3}

5

5

5

5

5

5

5

3

2

3

2

3

5

7

10

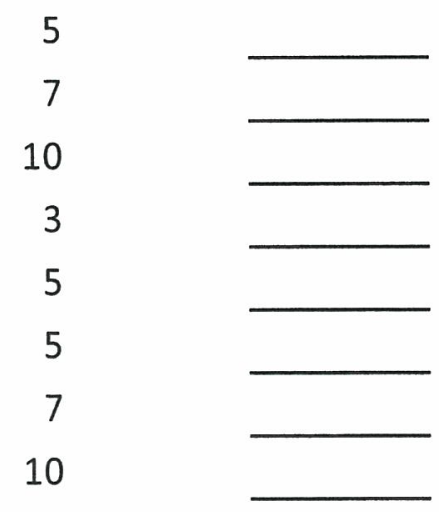

\section{Activities}

1. Sectional Activity

(Blackbeards/Johns Inc) 


\section{Clovis FFA Top 20 Award Application}

Due to Mrs. Covey by Friday May 9th

Point Value Fall

Spring

2. Chapter Activity

Fall Classic

\begin{tabular}{lll}
\multicolumn{1}{c}{ exibitor } & 5 & \\
work & 3 & \\
Halloween Party & 3 & \\
Winter Party (Movie) & 3 & \\
Spring Party (Movie) & 3 & \\
Water Day/lce cream & 3 & \\
FFA Week participant & 3 & \\
explain & 3 & \\
Booster BBQ helper & 3 & \\
Speaking contest helper & 3 & \\
Rodeo Snack bar helper & 3 & \\
Greenhand Initiation & 5 points per meeting \\
Chapter Meeting &
\end{tabular}

*List months :

3. FFA Community Service

$\begin{array}{lll}\text { Can Food Drive } & 5 & \\ \text { Farm Tours } & 5 & \end{array}$

4. Fresno Fair

Work buyer $\mathrm{BBQ}$

Ag Mech

3

Horticulture

3

Landscape Display

3

Small Animal pjt

3

Large animal pjt

5

Breeding Project

5

5

5. Chapter Banquet

with parent

10

without parent

5

6. Fundraiser

Panda 2 points each fundraiser

* List months :

Chicken Dinner

sell tickets ( 2 points per ticket)

Work

5 


\section{Clovis FFA Top 20 Award Application}

Due to Mrs. Covey by Friday May 9th

$$
\text { Point Value Fall }
$$

Spring

Toyota Truck Tickets

1 point per ticket sold

Tulare Farm Show

5

4. Individual Achievements

1. Greenhand Degree

3

2. Chapter Degree

5

3. State Degree

Recipient

Banquet

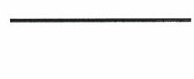




\section{Clovis FFA Top 20 Award Application}

Due to Mrs. Covey by Friday May 9th

Point Value Fall Spring

\begin{tabular}{lccc} 
7. Parli Pro & Sectional & 5 \\
& Regional & 10 & \\
& State & 15 & \\
Judging Team Member & 5 \\
Contest & 2 point each contest \\
List contest_ & \\
\hline & \\
\hline State FinalsTeam & 10
\end{tabular}

\section{SAE Project}

Project must be in your record books and signed off by your project area advisor

1.Large Market Animal Project

2. Large Breeding Animal Project

3. Small Market Animal Project

4. Small Breeding Animal Project

5. Other Animal Project

6. Orchard Project less than $25 \mathrm{hrs}$.

7. Orchard Project more than 25 hrs.

8. Greenhouse Pjt less than $25 \mathrm{hrs}$.

9. Greenhouse Pjt more than 25 hrs.

10. Ag Mech Project less than 25 hrs.

11. Ag Mech Pjt more than 25 hrs.

12. Work Exp. less than 10 hrs. a wk

13. Work Exp. more than $10 \mathrm{hrs}$ a wk

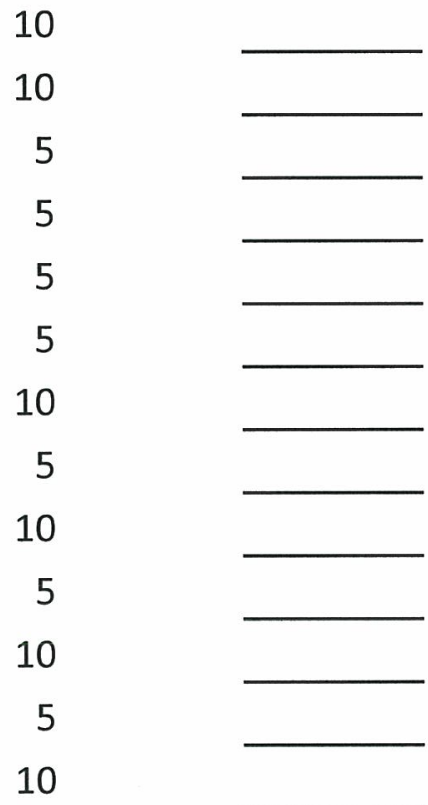

Must attach report card/zangle print out

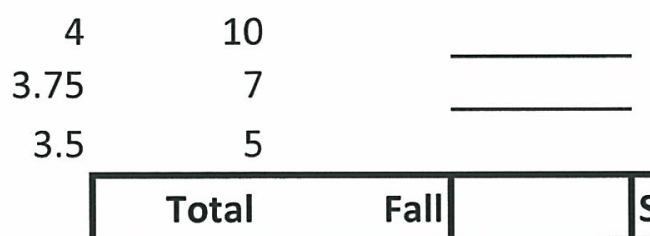




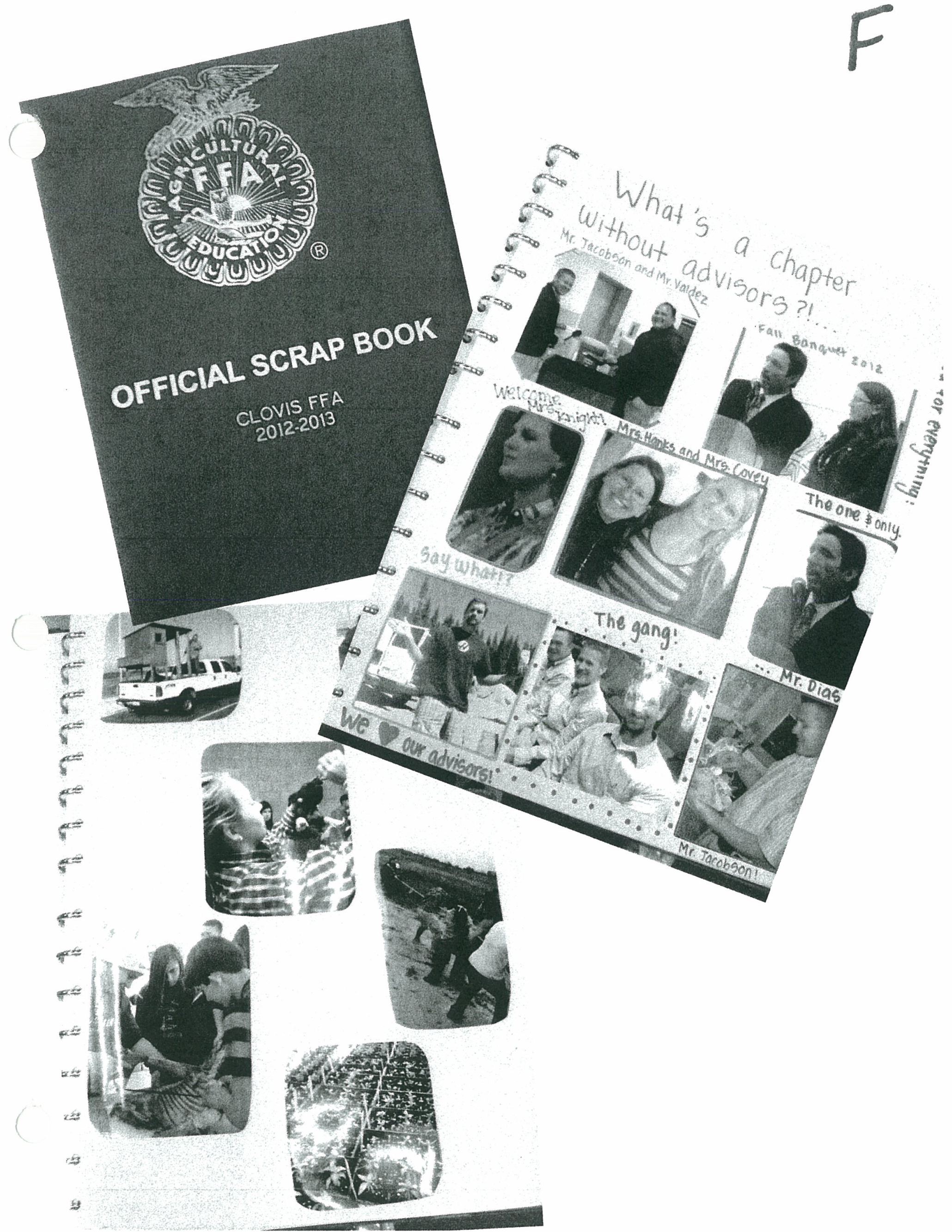




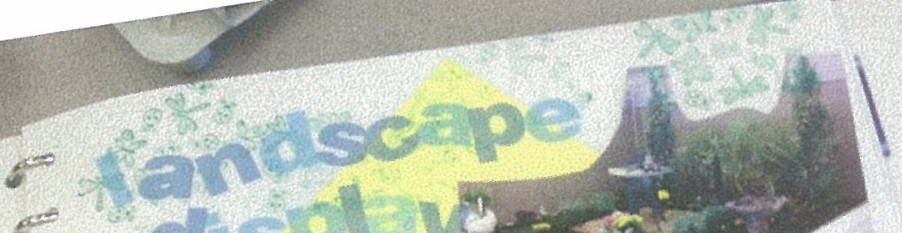
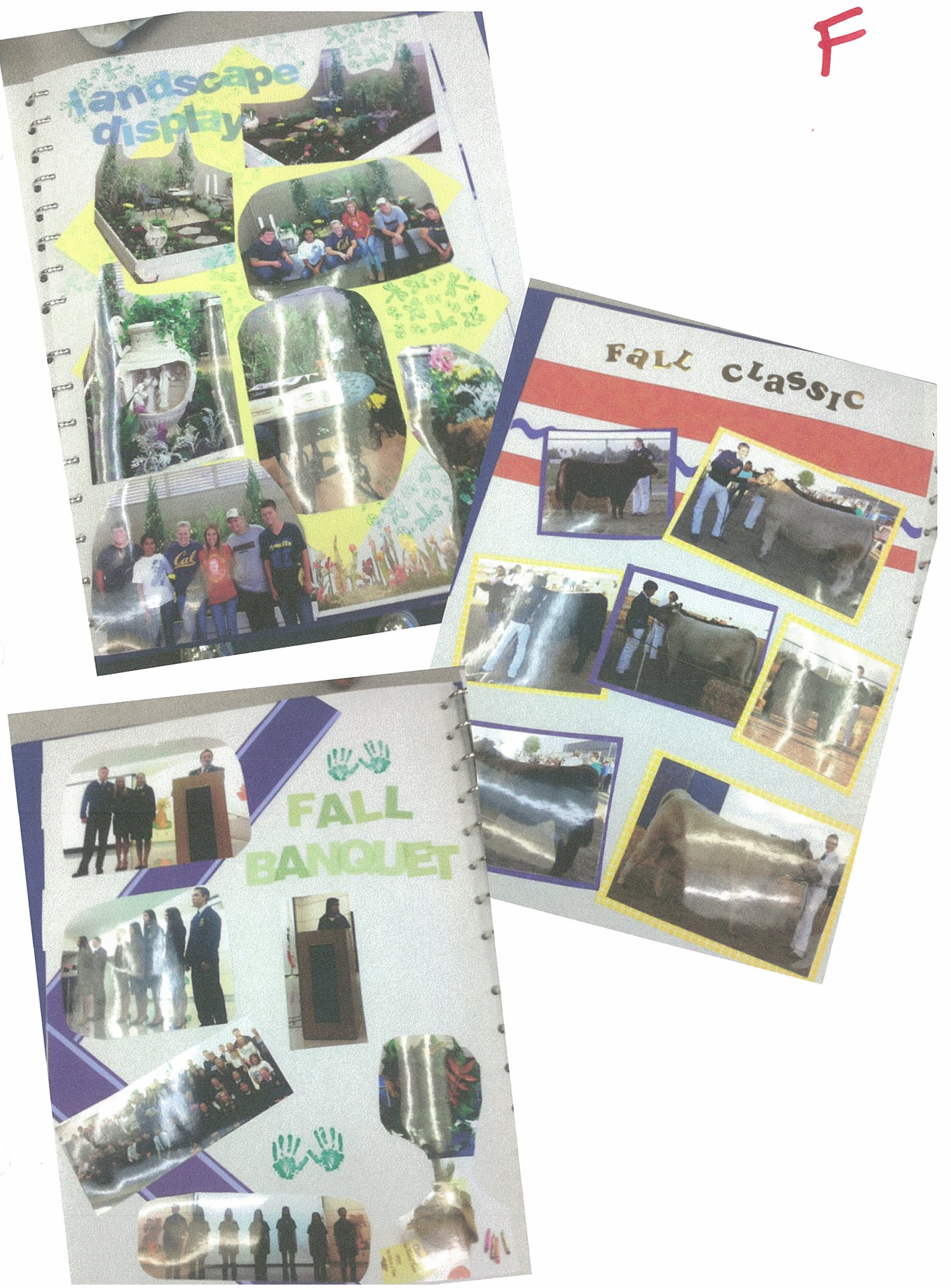
Today's Date: $1 / 24 / 13$

Name of Trip/Conference: UC Davis FFA Parlimentary City/State Traveling to: Davis, CA Procedure Contest and FFA Field Day

Dates of Trip/Conference (include travel days): Friday, March $1^{\text {st }} 2013$ to Saturday, March $2^{\text {nd }} 2013$

Number of Nights: 1

NOTE: Conference request for Out Of State trip submitted with trip request for board approval $\square$ yes Names of Teachers/Coaches Traveling: Jennifer Knight (Ag Teacher), Donald Vernon Clark (Volunteer) Number of Students Traveling: 12

Specific Hotel (include address, phone number (or website) and rate quoted):

Hotel 1: Comfort Suites I-80 155 Dorset Dr. Dixon, CA (707) $676-5000$

Rate 1: 99.00

Total Number of Rooms: 3
Hotel 2: Best Western Plus 1345 Commercial Way Dixon, CA 95620 (707) 678-0754

Rate 2: 105.00
Hotel 3: Comfort Suites UC Davis 1640 Research Park Drive Davis, CA 95618 (530) $297-1500$

Rate 3: 139.00 ***PLEASE INCLUDE BUS DRIVER ROOM IF APPLICABLE***

\section{Please attach room lists for students/teachers/coaches.}

Mode of Travel? Auto $\square$

If air travel needed:

Airline:

Rate Quoted:

Passenger Name as Appears Date of Birth DL/ID
Departure Info. (date \& time):

Air $\square$

Rental Car

ADDITIONAL INFORMATION (AS NEEDED):

Funding Source: ASB and Ag Boosters

Purchase Order No.:

For Area Office Use Only

Board Approved: 


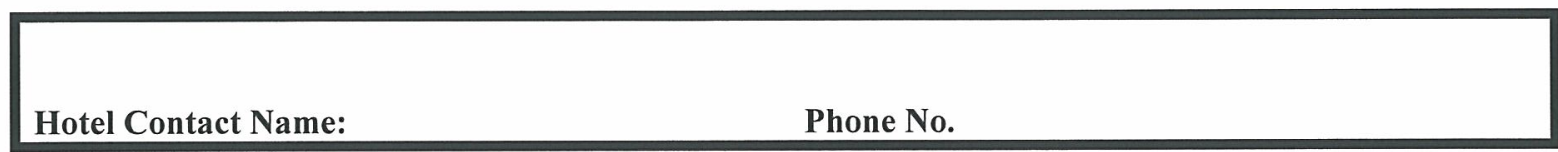

Incomplete forms will not be processed (-) 


\section{PARTicipation in Voluntary Field Trip FORM 3204-1}

\section{CONSENT, ASSUMPTION OF RISK AND EMERGENCY MEdiCAL AUTHORIZATION}

(This form must be completed for participation in all field trips conducted by the CUSD)

The following voluntary field trip has been authorized by (school): Clovis East High School
Overnight Trip:
$\square$ Yes
$\square$ No
Out-of-State Trip:
$\square$ Yes $\square$ No

Specific Location: Visalia Holiday Inn and Conference Center 9000 W Airport Drive

Description of Field Trip: Made For Excellence and Advanced Leadership Academy Conferences

$\operatorname{Day}(\mathrm{s}) /$ Date(s): Friday, Feb 8th and Saturday,Feb 9th Departure: 11:30am AM/PM Return: 2:00pm AM/PM

School Person in Charge: Jennifer Knight \& Aireal Covey Position: Ag Teacher

LUNCH

$\square$ Student will be at school during lunch

Participant should bring sack lunch/drink*

Other Bring Money for Fri and Sat Lunch. Other Meals Provided.

* See Authorization section

**Parent/Guardian Permission for Transporting Student in Private Vehicle is included.

A field trip fee (covering direct costs) in the amount of $\$ 100$ PREPAID will be collected.
METHOD OF TRANSPORTATION

$\square$ Walking

$\square$ School Bus

$\square$ Airplane (commercial)

The participant may be exposed to the following high risk activities during this field trip/activity: Dancing at Friday night activity

AUTHORIZATION: (Please return this form to the school person in charge listed above)

Participant Name:

Charter Bus

曰 Other Ag Vans

$\square$ Minor Student $\square$ Adult Student $\square$ Volunteer/Chaperone

I hereby authorize the above-named individual to participate in the field trip outlined above.

In the event of illness or injury, I do hereby consent to whatever x-ray examination, anesthetic, medical, surgical or dental diagnosis or treatment and hospital care and transportation considered necessary in the best judgment of the attending physician, surgeon, or dentist and performed under the supervision of a member of the medical staff of the hospital or facility furnishing medical or dental services. I further acknowledge that the District does not provide medical coverage for participants in this activity.

I fully understand that participants are to abide by all rules and regulations governing conduct.

$\square$ I acknowledge that although the field trip may not be considered a high-risk activity, participants may be exposed to the high-risk activity(ies) listed above during this trip.

$\square$ Special instructions regarding emergency medical treatment for the above-named individual are on file in the school office. (Please refer to the Emergency Card located in the school office.)

$\square$ *IF APPLICABLE: I need Campus Catering to provide a sack lunch for the above-named participant. (Students will be charged according to their status in the National School Lunch Program. Other participants will be charged the full amount.)

$\square$ I wish to volunteer as a chaperone and understand that I must also complete Form 9212-1, Volunteer Application, and meet the requirements of Board Policy No. 9212.

$\square$ I have read and completed the waiver on Page 2 .

Approval Signature (Parent or Guardian/Adult Student/Volunteer)

Medical Insurance Carrier (i.e., Blue Cross, Kaiser):

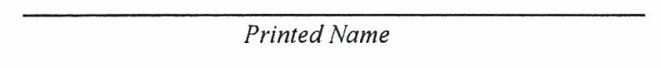
Policy Number:
Date

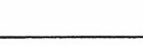

\begin{tabular}{c} 
Phone Number \\
\hline Phone Number \\
Page 1 of 2
\end{tabular}




\section{Participation in Voluntary Field Trip \\ FORM 3204-1 (continued)}

\section{WAiver OF Claims}

All adults and adult students taking part in a field trip and all parents/guardians of minor students taking field trips are required to sign a statement waiving such claims.

\section{Waiver by Parent/Guardian of Minor Student}

I certify that I am the parent/guardian of the student identified below. As provided for in California Education Code Section 35330, I agree to waive all claims against the Clovis Unified School District (District) and hold the District, its officers, agents and employees harmless from any and all liability or claims which may arise out of or in connection with my child's participation in this activity. This waiver shall not apply to any occurrences which may arise solely out of the negligence of the District, its employees or agents.

Student Name:

Parent/Guardian Signature:

Date:

\section{Waiver by Adult Student}

As provided for in California Education Code Section 35330, I agree to waive all claims against the Clovis Unified School District (District) and hold the District, its officers, agents and employees, harmless from any and all liability or claims, which may arise out of or in connection with my participation in this activity. This waiver shall not apply to any occurrences which may arise solely out of the negligence of the District, its employees or agents.

Printed Name:

Signature:

Date:

\section{Medical Authorization and Waiver by Adult Accompanying Student on Trip (Volunteer/Chaperone)}

As provided for in California Education Code Section 35330, I agree to waive all claims against the Clovis Unified School District (District) and hold the District, its officers, agents and employees, harmless from any and all liability or claims, which may arise out of or in connection with my participation in this activity. This waiver shall not apply to any occurrences which may arise solely out of the negligence of the District, its employees or agents.

In the event of illness or injury, I hereby consent to whatever x-ray examination, anesthetic, medical, surgical or dental diagnosis or treatment and hospital care and transportation considered necessary in the best judgment of the attending physician, surgeon, or dentist and performed under the supervision of a member of the medical staff of the hospital or facility furnishing medical or dental services. It is understood that the resulting expenses will be the responsibility of the participant.

Special medical instructions, if any:

Printed Name:

Signature:

Date: 
PURCHASE ORDER

and

CLAIM VOUCHER
Clovis East High School

2940 Leonard Ave.

Clovis, CA 93619

Voice: $559-327-4013$

Fax: $559-327-4190$
No. CE1213-376

Show this Purchase Order No. on Invoice and on outside of each Package and Carton

VENDOR:

43009941500994107249011100600

0101

1450 Herndon Ave.

Clovis, CA 93611
DELIVER TO:

Jennifer Knight

Clovis East High School

2940 Leonard Ave.

Clovis, CA 93619

BILL TO:

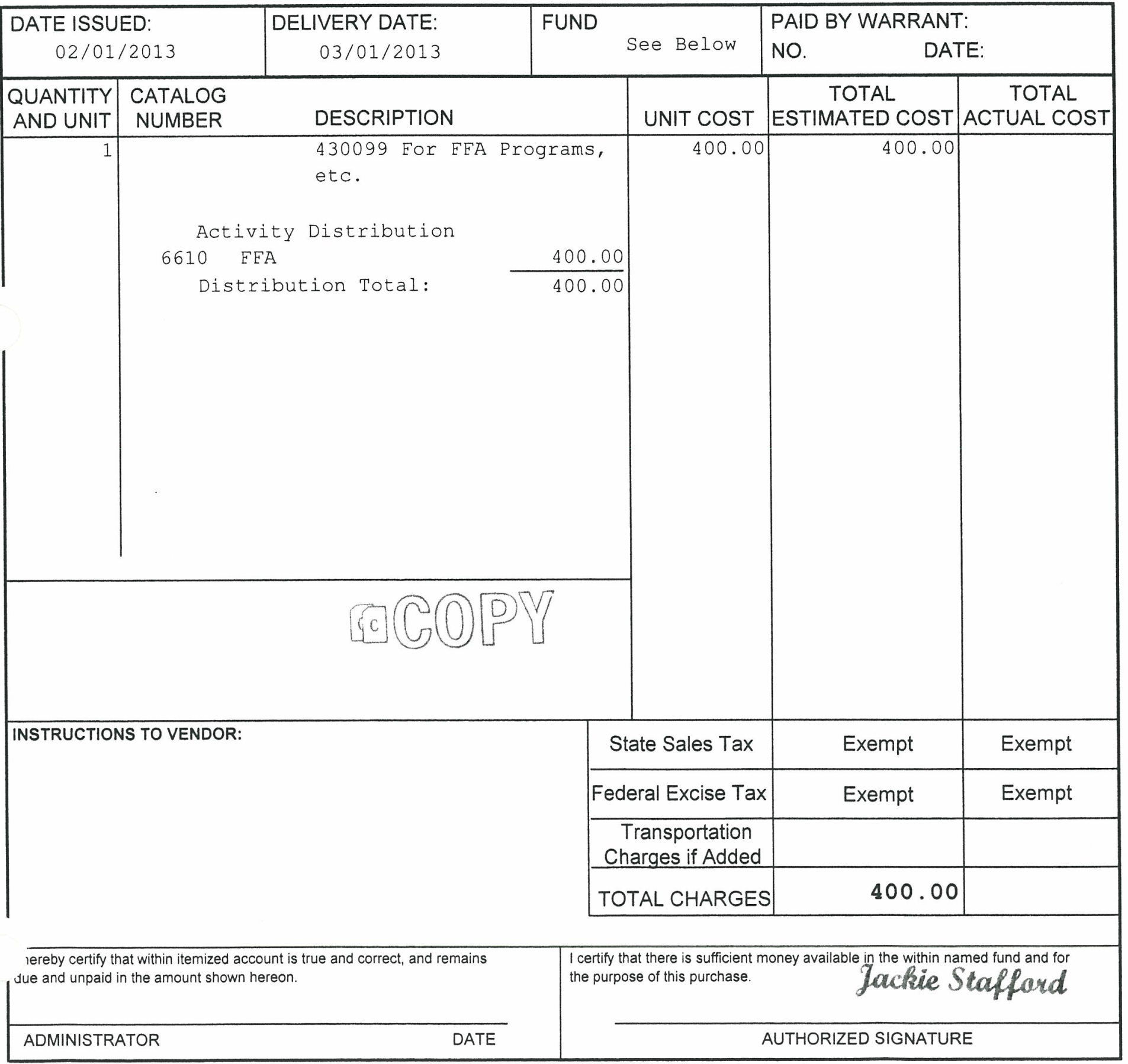




\section{CLOVIS EAST HIGH SCHOOL}

2012-2013

$* * * * * * * * * * *$ REMITTANCE REQUEST FOR PERSONAL REIMBURSEMENT***********

PAYMENT ON ESTABLISHED ASB PURCHASE ORDER NO. CE1213-

ASB ACCOUNT \# ORGANIZATION

DATE:

Original Receipts Submitted

Your Signature:

Your Learning Director's Signature:

\section{AMOUNT \$}

\section{CLOVIS EAST HIGH SCHOOL}

$\underline{2012-2013}$

$* * * * * * * * * * *$ REMITTANCE REQUEST FOR PERSONAL REIMBURSEMENT***********

PAYMENT ON ESTABLISHED ASB PURCHASE ORDER NO. CE1213-

ASB ACCOUNT \# ORGANIZATION

DATE:

Original Receipts Submitted

Your Signature:

Your Learning Director's Signature:

\section{AMOUNT \$}




\section{Quality Criteria Three}

\section{Practical Application of Agricultural Skills}

Practical application of Agriculture Skills is the goal that all good Ag Teachers should have for their students. At Clovis East we try to follow the learn by doing philosophy of Career Technical Education. We are fortunate to have a state of the art Agriculture Center for our students to engage in hands on learning along with the facilities to house numerous student projects.

When a student joins an agriculture class for the first time they will be introduced to Supervised Agriculture Experience by a lesson on SAE projects. This will often include a tour of the agriculture center where the students can see first-hand what type of projects our students have the opportunity to be involved with. They can ask questions and begin to think about what project area would interest them the most. Many of our Freshmen are ready to go their first year on an SAE project because they have had the opportunity to take an Exploring Agriculture Class their $7^{\text {th }}$ or $8^{\text {th }}$ grade year where they have already learned about and explored project ideas or options. Students who have not yet been exposed to SAE will need to conference with the Ag Teachers during the completion of their student data sheet do that a project area may be determined.

Because SAE participation and Record Book evidence is part of a student's grade in their Ag Class it is expected that all students earning an A grade in their Ag Class have completed a successful SAE project. Evidence of a successful project will be shown in a completed FFA Record Book. SAE credit will only be given if evidenced in the record book, and project visits recorded in the student's journal and on the Calendar then signed by their project supervisor. Advisors will keep records on all project visits, and our schools discipline triplicate form can be used to make recommendations or issue warnings or strikes per our project policies. We currently have a handbook for students with orchard projects, but are working as a staff to develop consistent farm and project policies.

The Ag Center does provide housing for cattle, sheep, swine, and rabbit projects, along with the shops and greenhouses that are used to grow/create projects. The Ag center 
also has opportunities for paid and unpaid internships on site, students can cooperate in the orchard management team where they tend the 11 acre orchard and sell the produce at the local Farmers Market. Profits from the sale of goods are paid to students based on the number of hours they log in their record books. I am currently working to establish a new unpaid position of a school sheep flock manager. After acquiring a set of school owned sheep, one or two students will manage them throughout the year and then receive a free lamb for the following year as their payment.

We also have students that after participation in the ROP Community Classroom program retain their placement over the summer for a paid or unpaid work experience project. These are mostly in the areas of Veterinary Science and Agriculture Mechanics.

Project visits are easily facilitated with our farm facility being on campus. For students who have projects on campus they must attend weekly meetings with their project supervisors. Those students with off campus projects should schedule monthly visits with their advisor. In today's era of technology, much of the communication with students about projects happens over text message. I know students always have their phones, and I have a permanent record in my phone of the contact made.

Our Agriculture Department has three vans, one truck, and two trailers that are kept here at the Ag Center for our use in making project visits. We can obtain fuel at the district office or we each have a Voyager card we can use to make fuel purchases at regular gas stations. Any expenses occurred by the teacher can be reimbursed by the Ag Department or the Ag Boosters as long as they have received prior approval from the Department Chair and the Secretary.

\section{Supporting Completion Materials}

Item A- Grade Book Print Out showing FFA/SAE Participation points earned.

Item B- Course Syllabi showing SAE as a graded component of agriculture classes. 
Item C- Print out of 2013 Market Lamb Workbook. Not much info in it now but this is what I use to manage the sheep projects all year long. I record all exhibitor and lamb info, keep track of paperwork, bills and payments made.

Item D- Sheep SAE Exhibitors Meeting Schedule. I provide the meeting schedule when a student signs up for the project, that way they can mark their calendars and communicate with their parents the schedule ahead of time.

Item E- Sheep Exhibitor 2012 Fresno Fair Information packet. This is what I gave to my Fair showmen last year in order to prepare our Chapter group for the Fresno Fair.

Item F- SAE Documentation. I have for the past 6 years of teaching have said I am going to create a nice project visit form, I have yet to make this happen but it is still on my to do list. I do however use many types of project documentation forms. The pictures on page F show the triplicate form I can use to document strikeable offences. It also shows the feeding sign in sheet and activity log that hangs on a clipboard on each lamb pen in the barn. I use this to monitor student feeding and project activity. The students then can use the activity logs to transfer hours and activities into their record book journals. I know I should have 10 project visit forms but I hate paper clutter and threw away all the old sign in sheets I had from last year.

Item G- Sheep Project Management. This shows how we keep our beef and sheep barn clean. We assign one day of barn duty to the students who keep their projects in the barn, they must clean the whole barn two times a day on the day they are assigned. We always try to maintain a clean facility and never know when a community or school board member may stop by. Student are also issued lockers so they can neatly store tack and feed needed for their SAE projects.

Item H- Agriculture Vehicle Fleet.

Item I- Project supervision and communicating using technology. I use texting to communicate with my students about their projects, I do this because they normally always have their phones and then we also create written records of our conversations. 
Item J- Fair Check Sign Off Sheet. This is the sheet I give to fair exhibitors after the Fresno Fair. They must complete the sign off sheet prior to winter break to receive their fair check.

Item K- Education Code stating that SAE is an important component of Ag Education.

\section{${ }^{* *}$ Ag Eg 539 Special Project}

- I realized a need for updated and more complete policies for Fair Exhibitors and students who kept their SAE projects at the school farm. Part of my Ag Ed 539 project was to create a new Farm Use Contract for sheep exhibitors and a new FFA Exhibitors contract for sheep showmen. See the new policies in the Ag Ed 539 project section. 


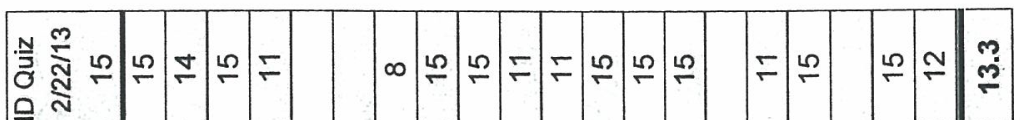

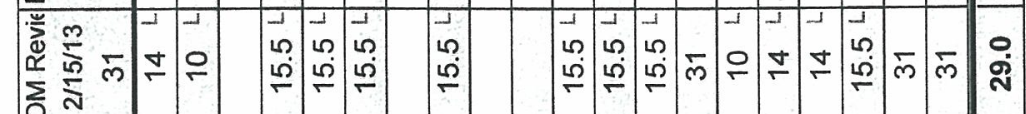

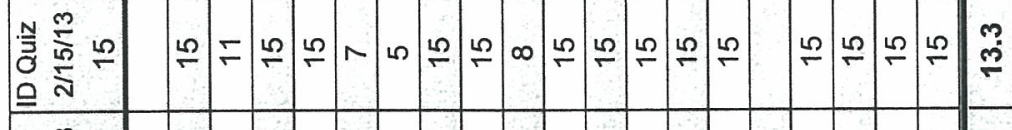

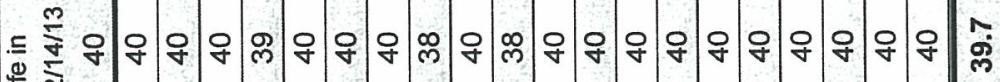
垔

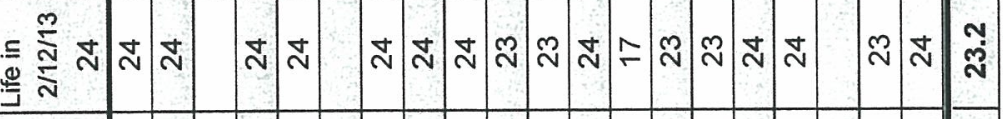

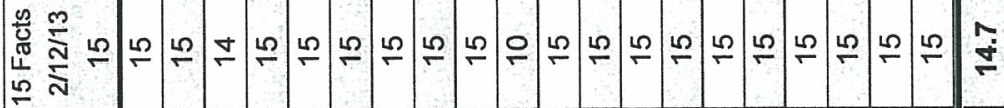

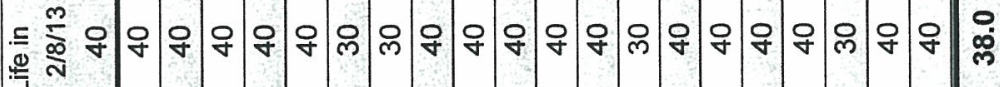

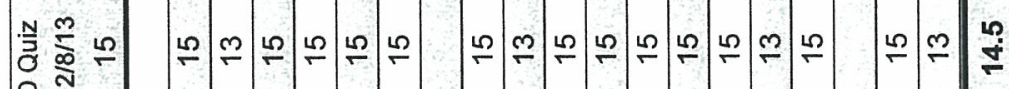
on

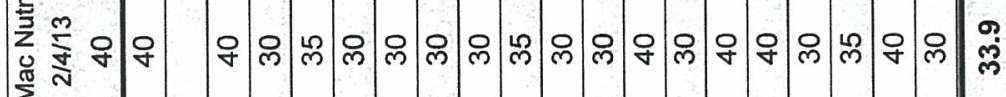
每

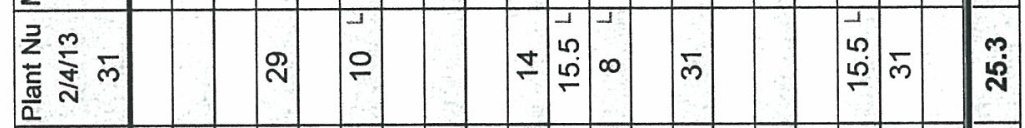

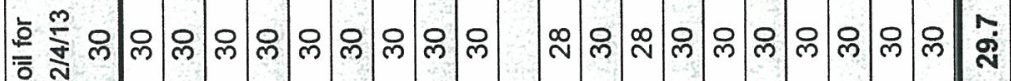
禹

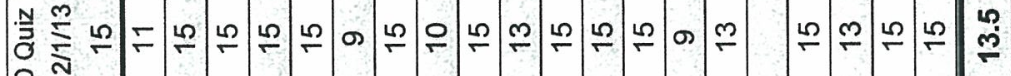
要

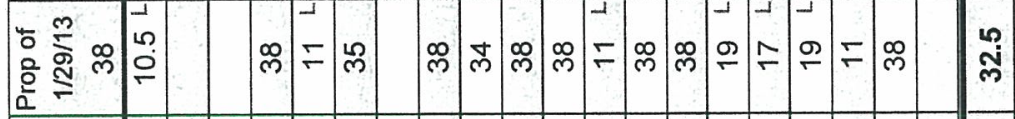

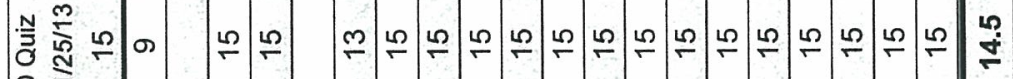
每

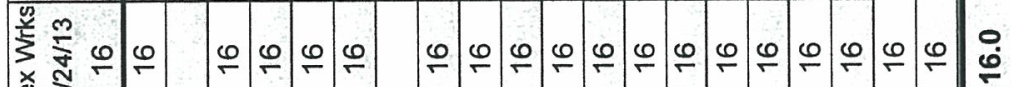
ج.

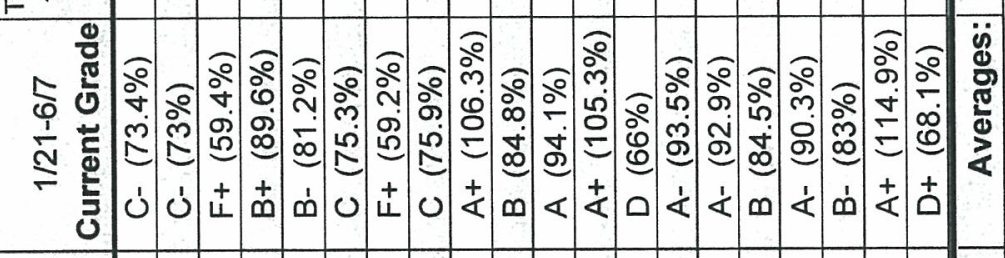

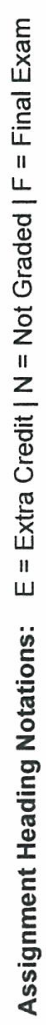




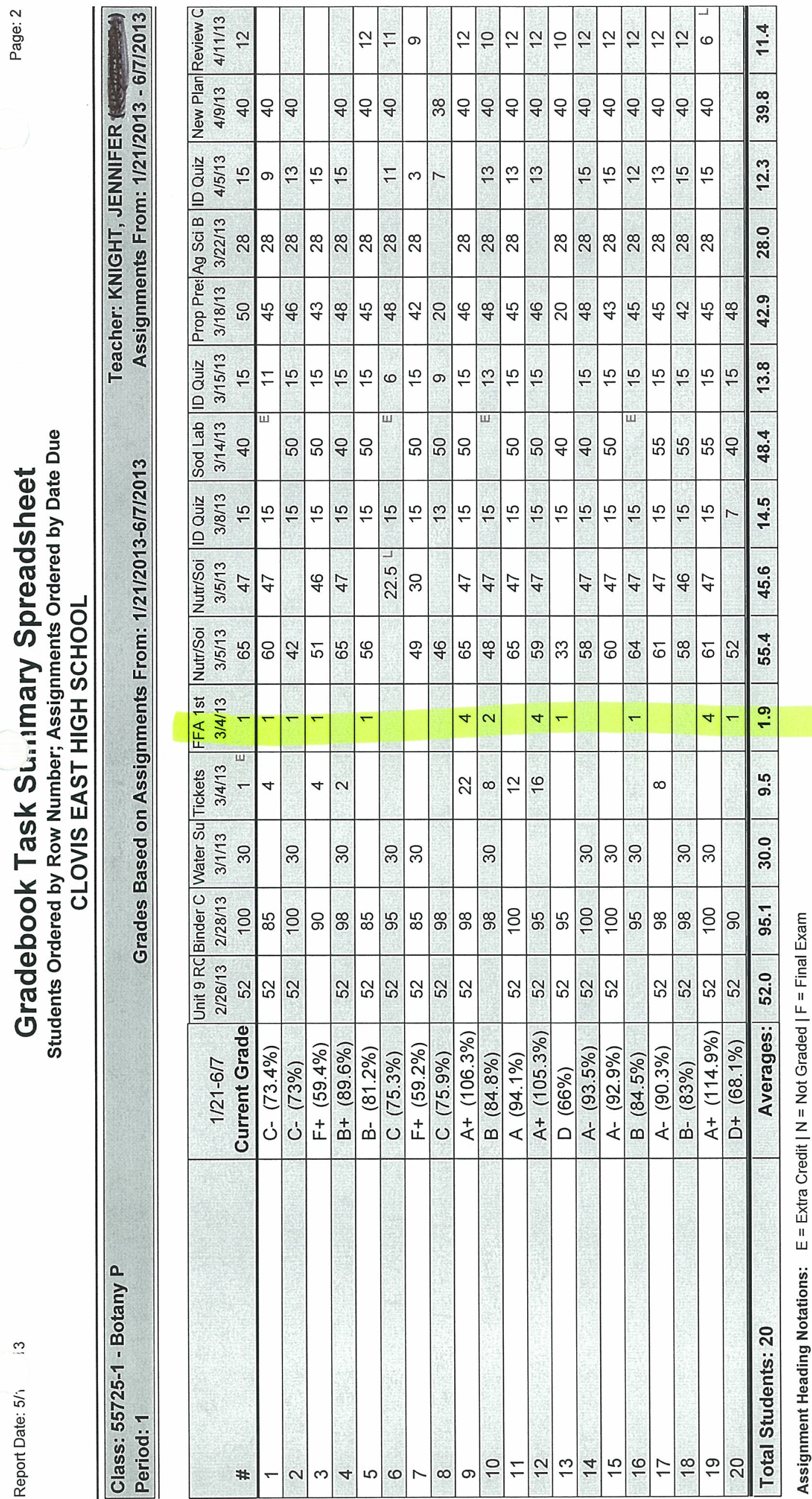




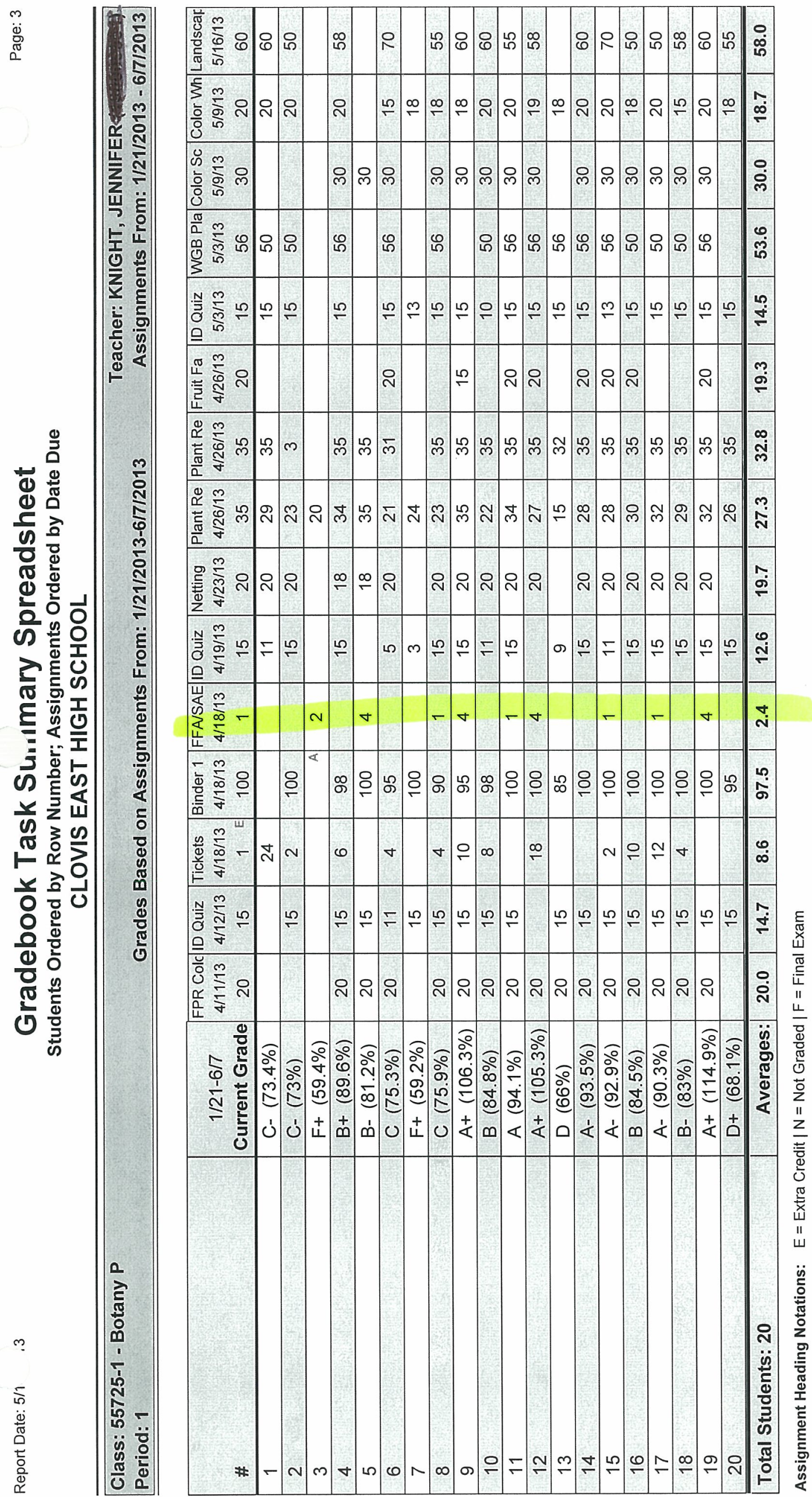




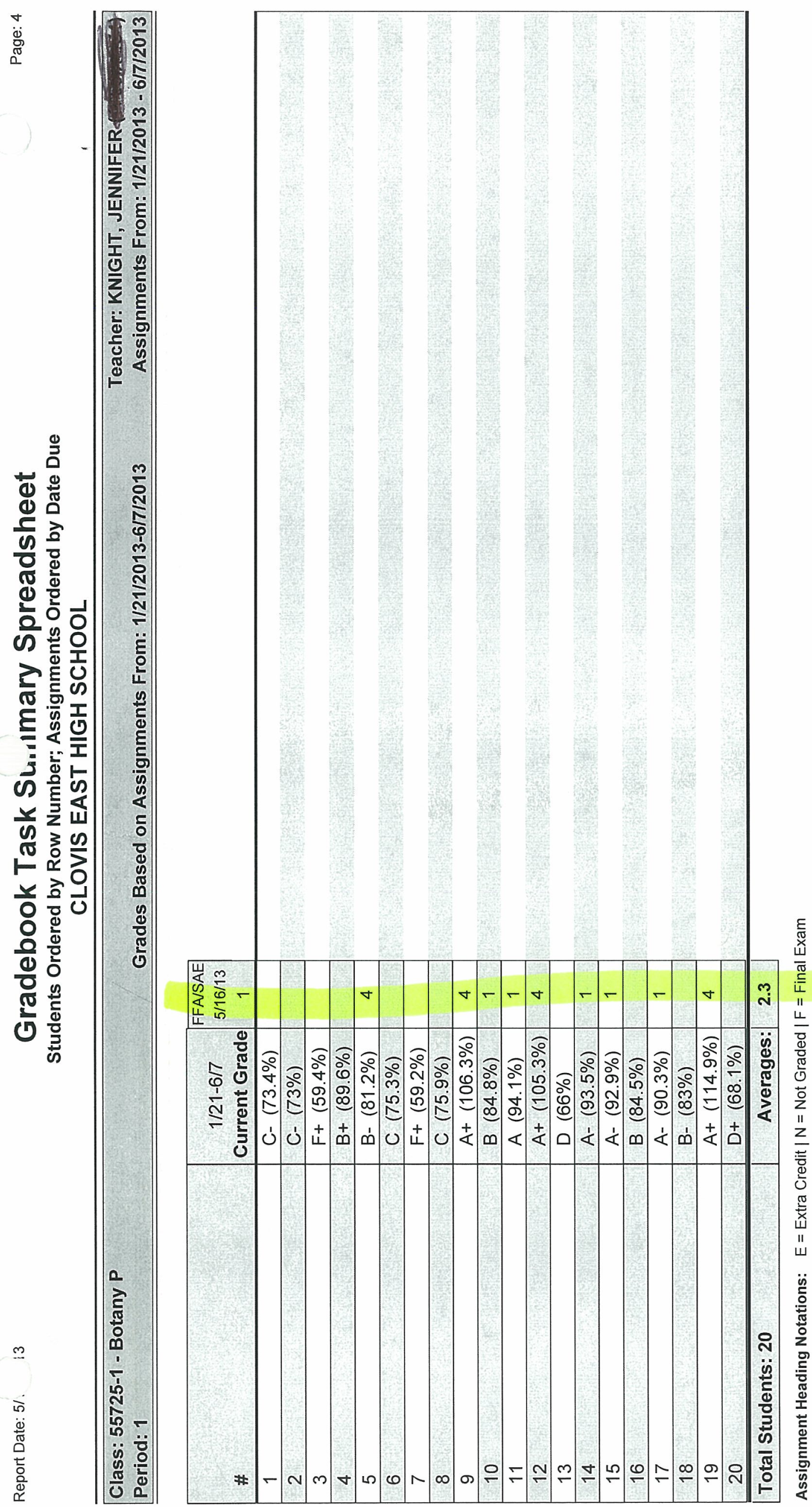



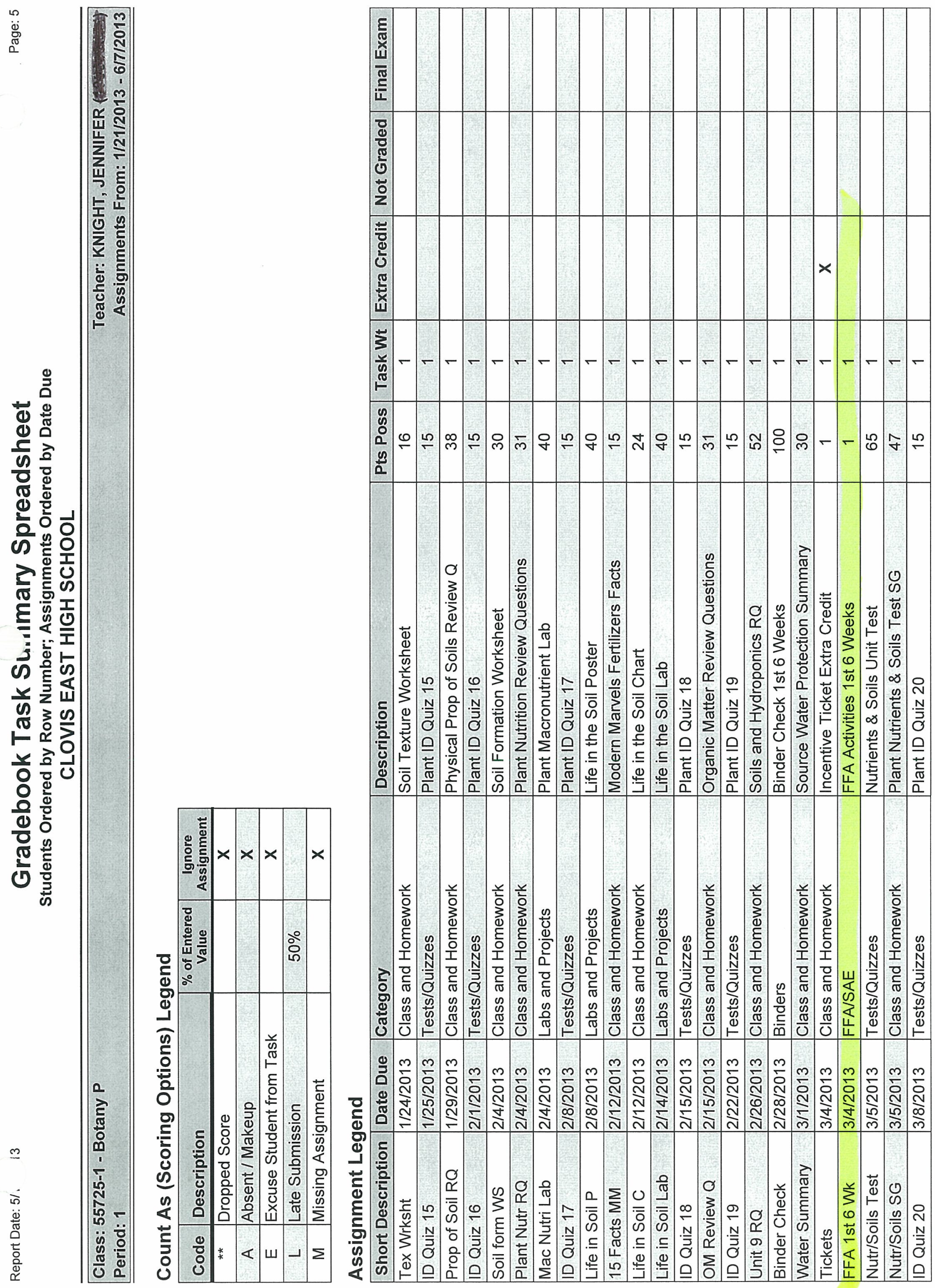


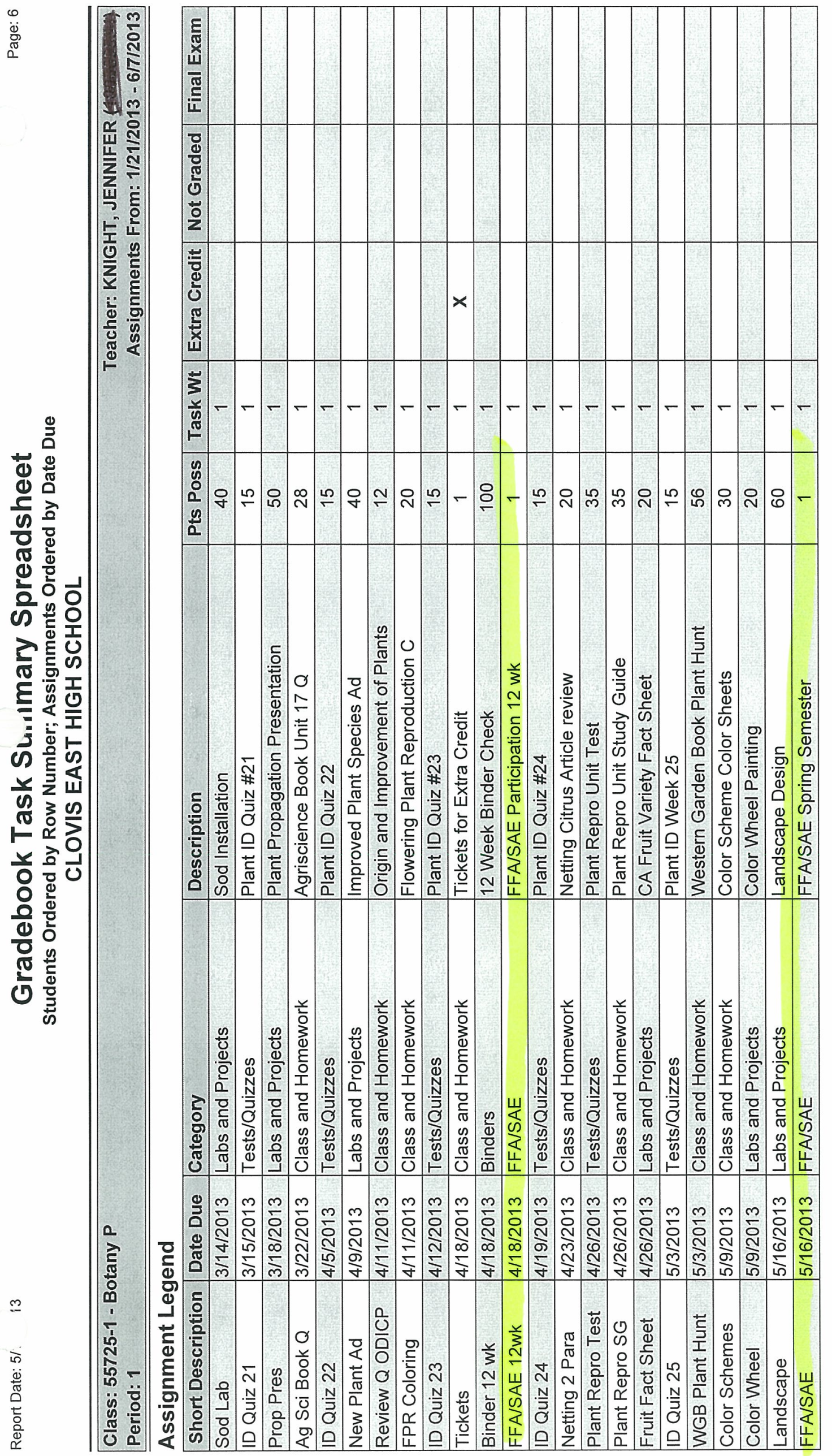




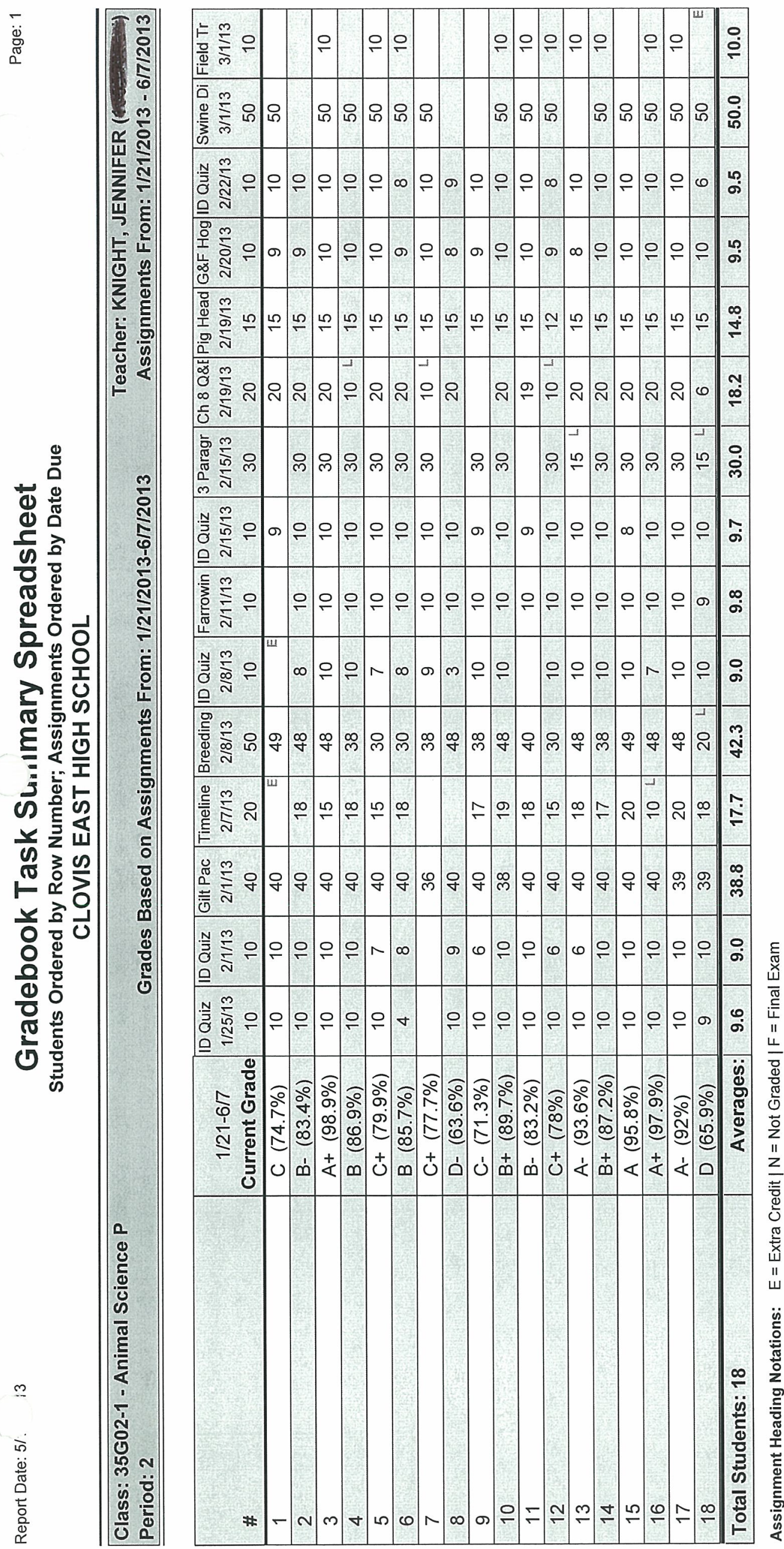




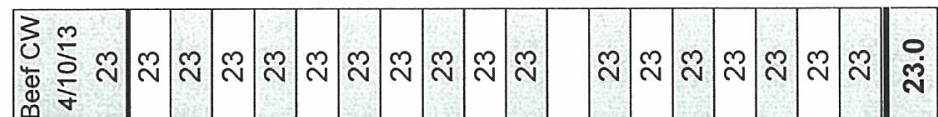

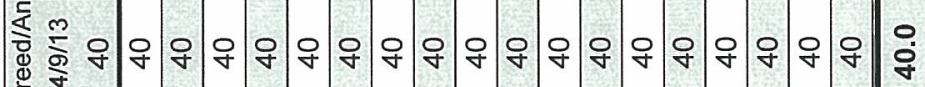

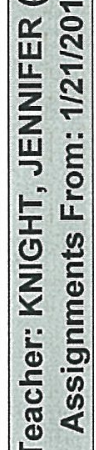

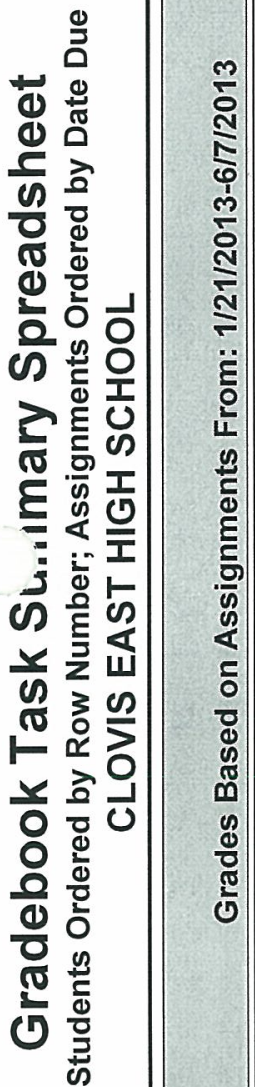

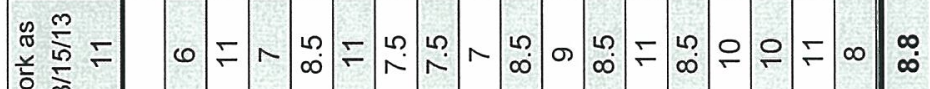

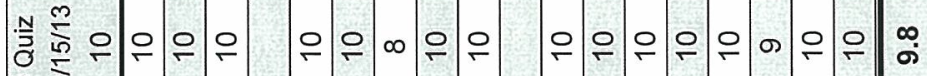
ㄷ

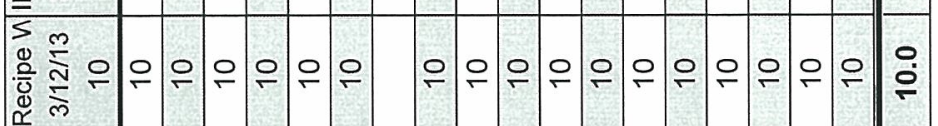

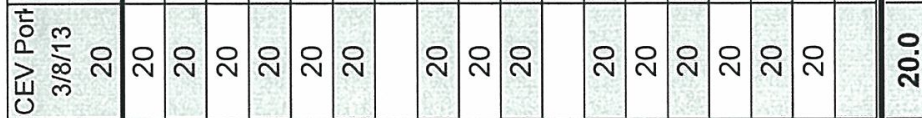

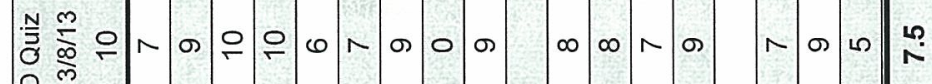

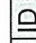

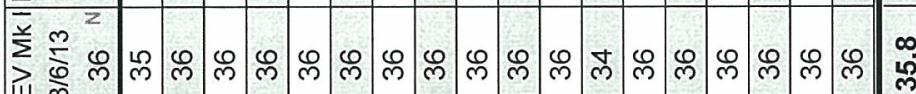
岂

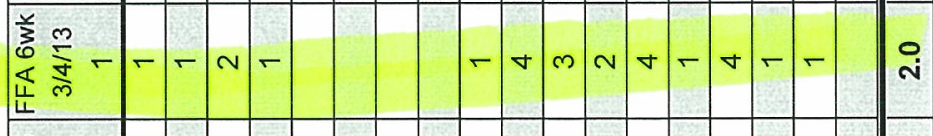

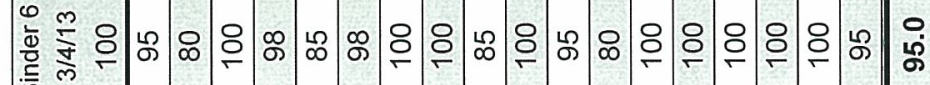
ले

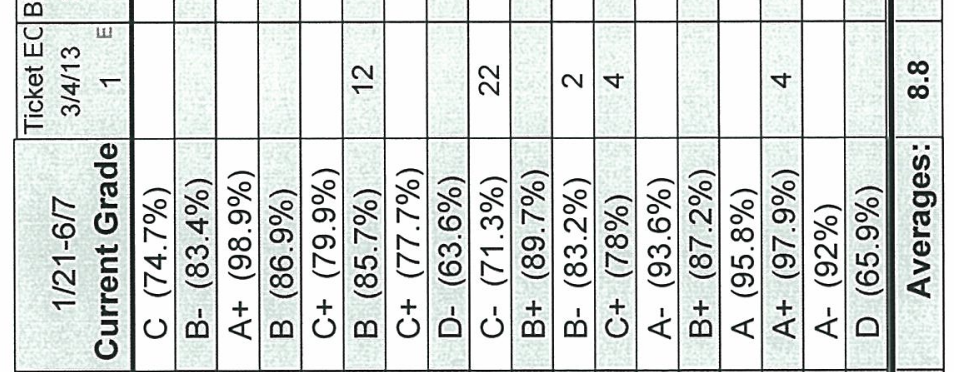




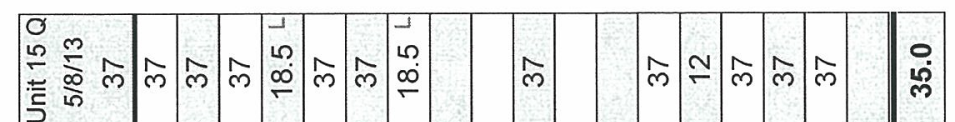

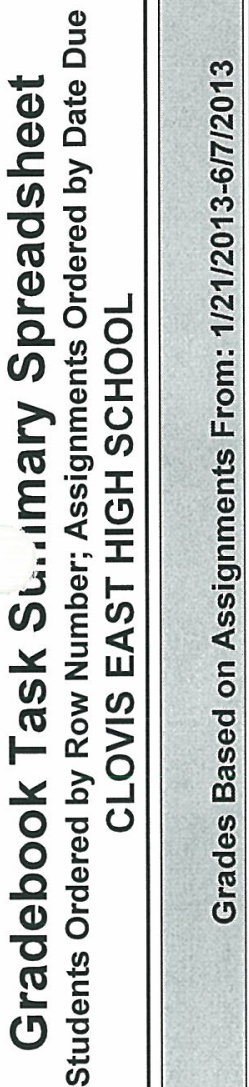

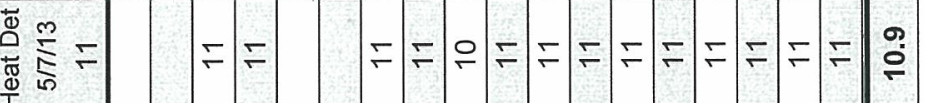

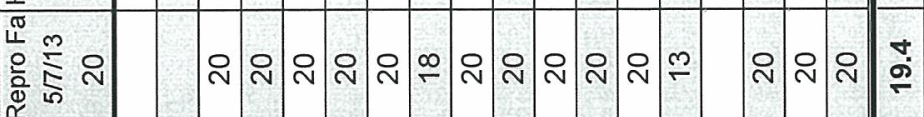

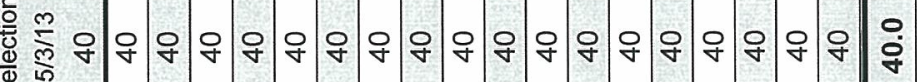

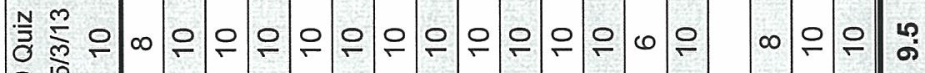

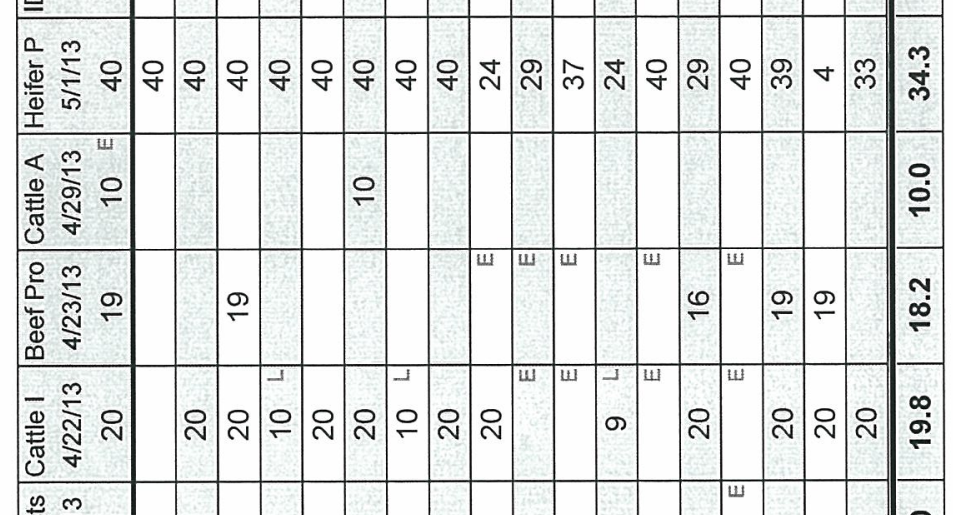

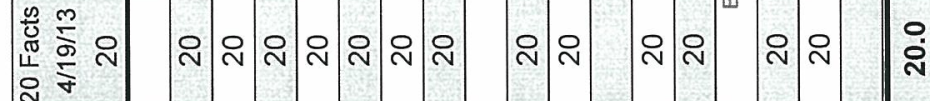
i

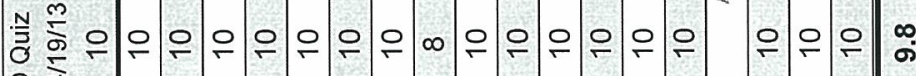

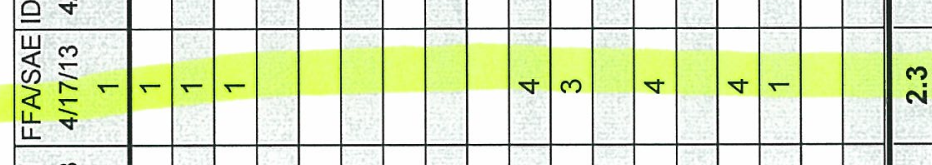

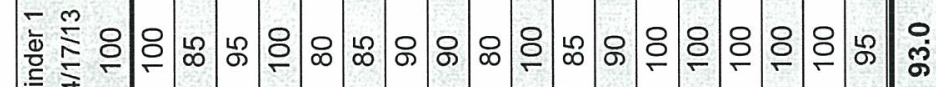
离

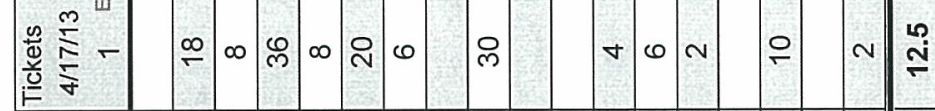
第紊

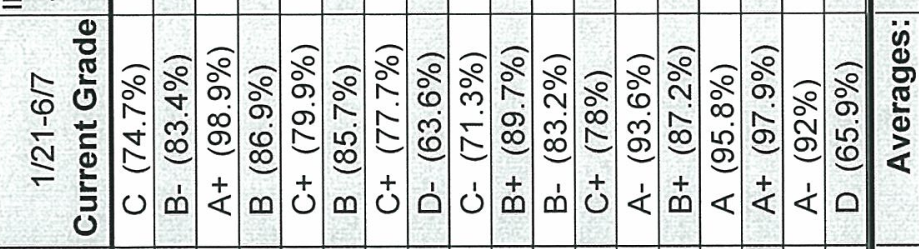

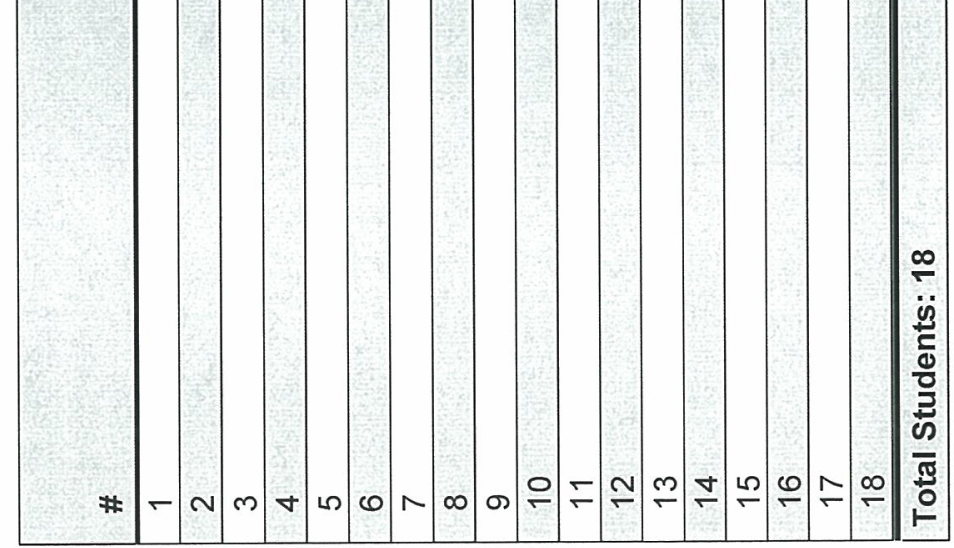



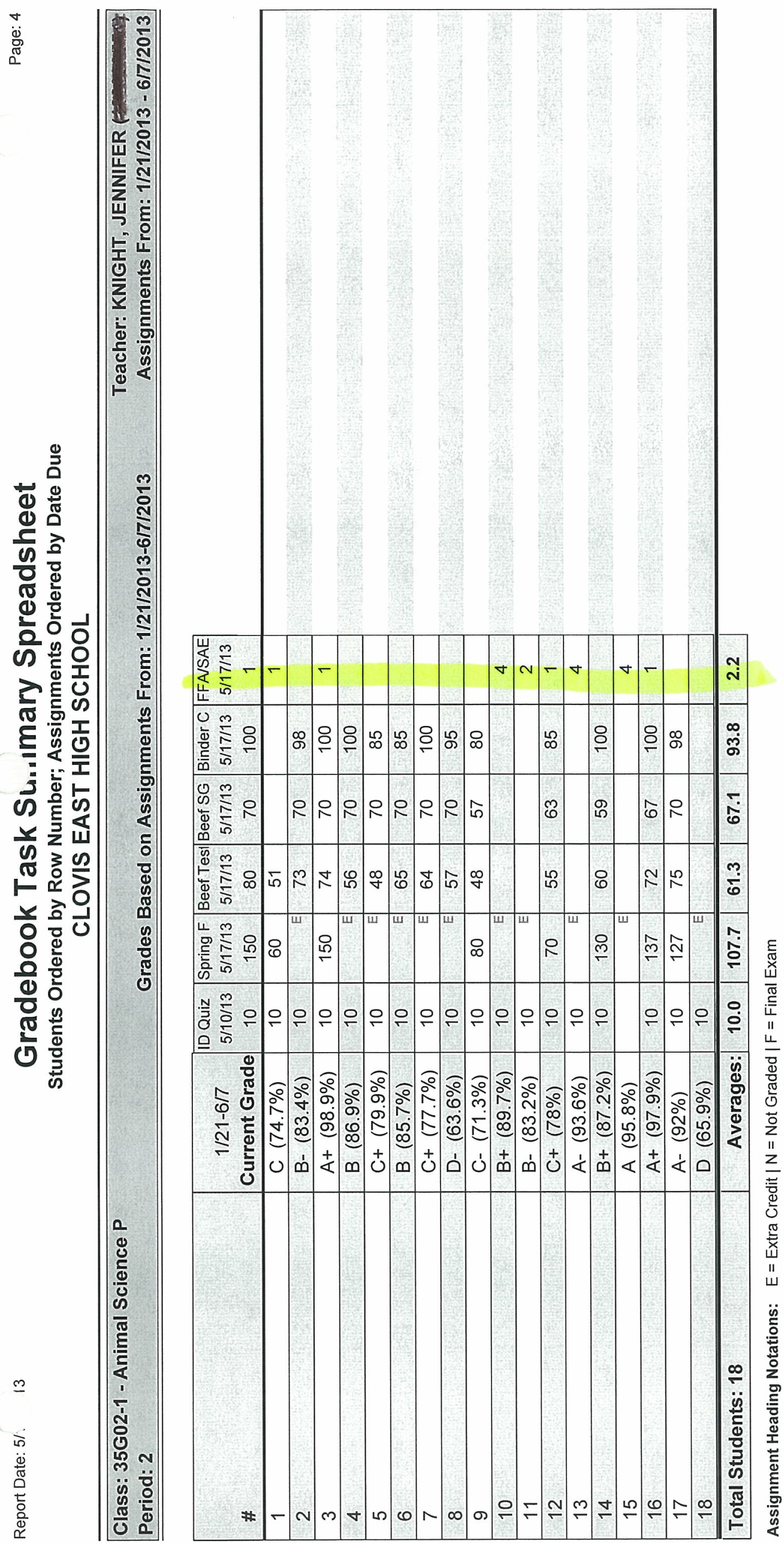

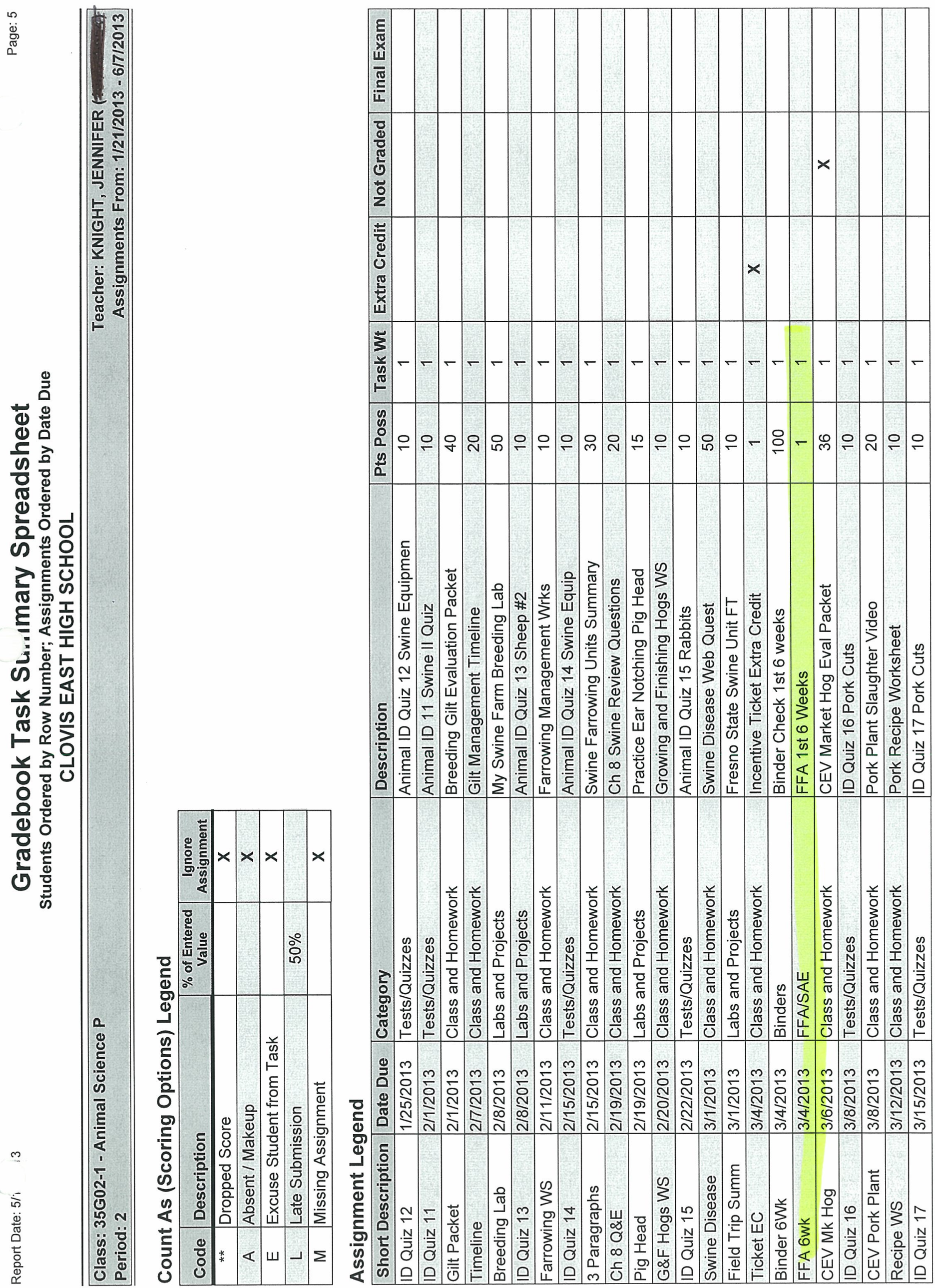


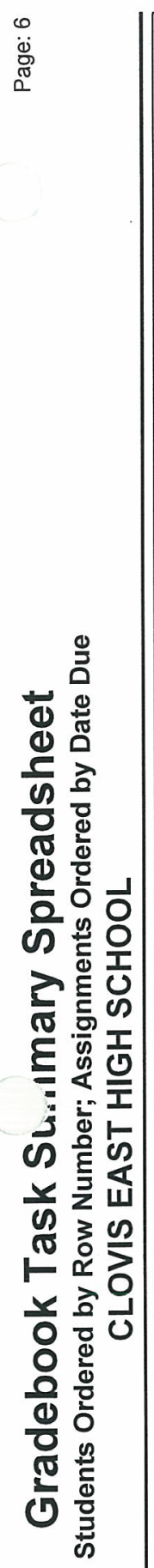

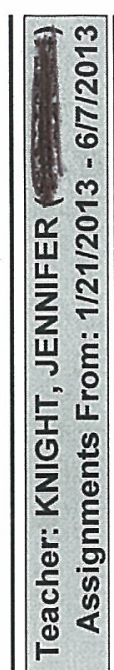

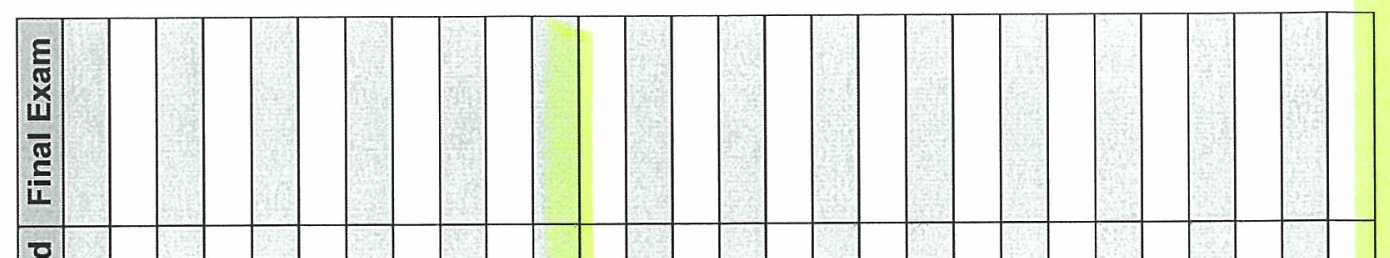

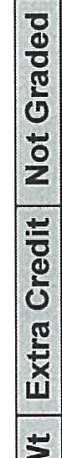

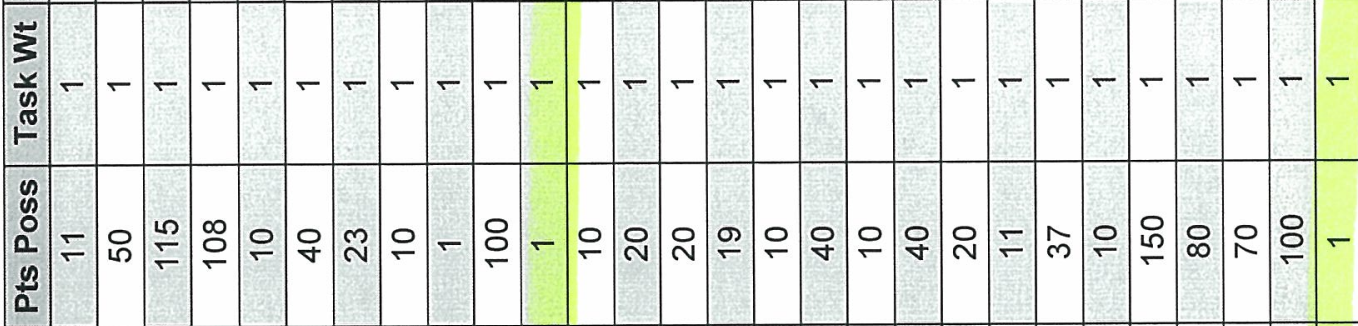

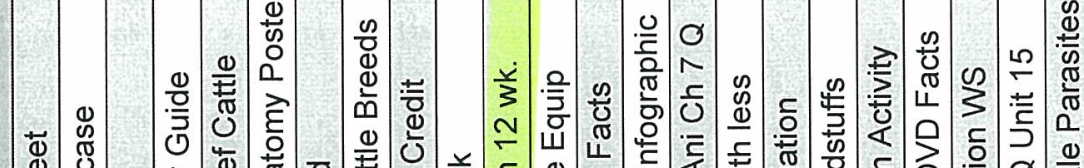

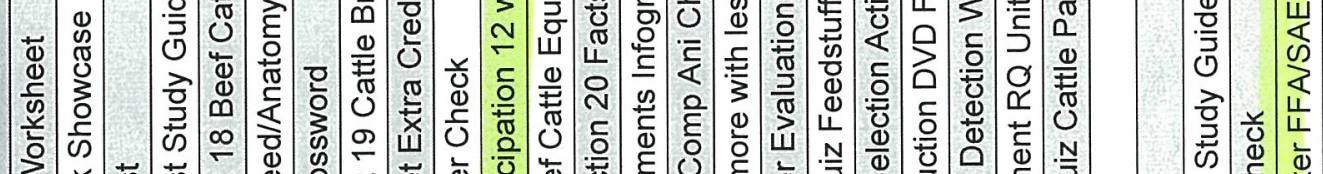

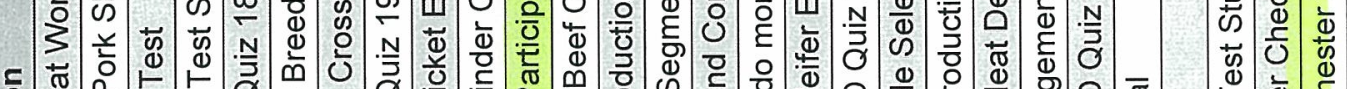
ᄃ。

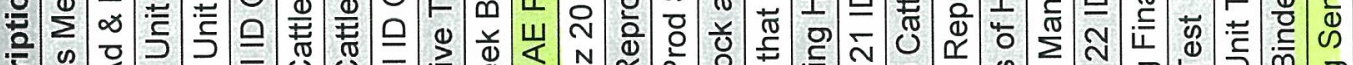

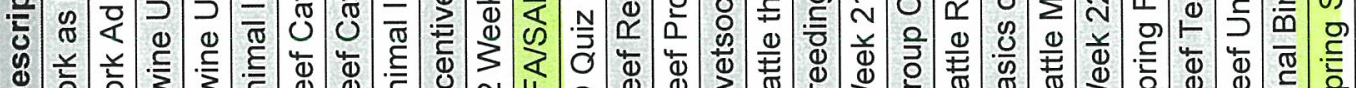
ఏ。

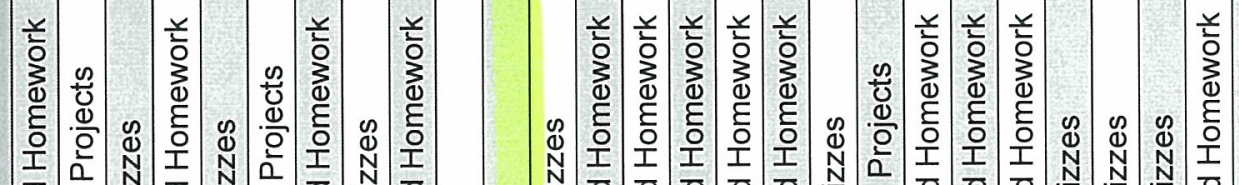

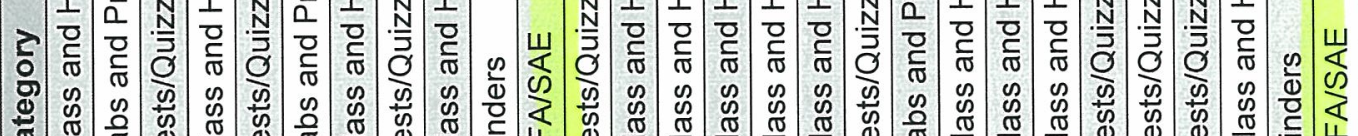

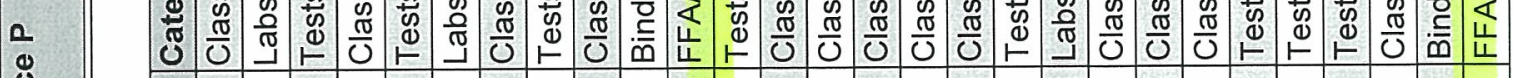

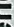

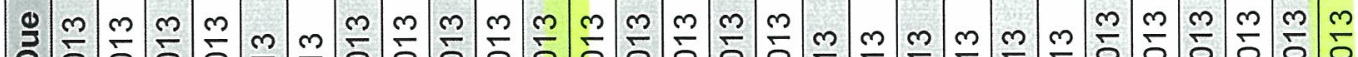

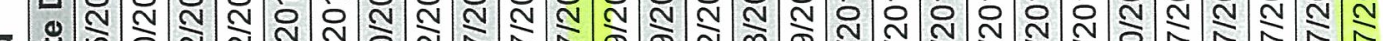
들

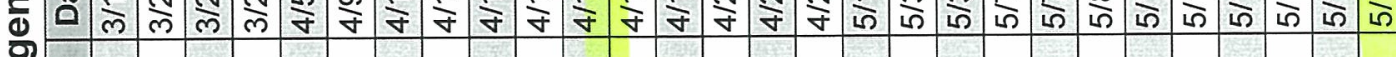




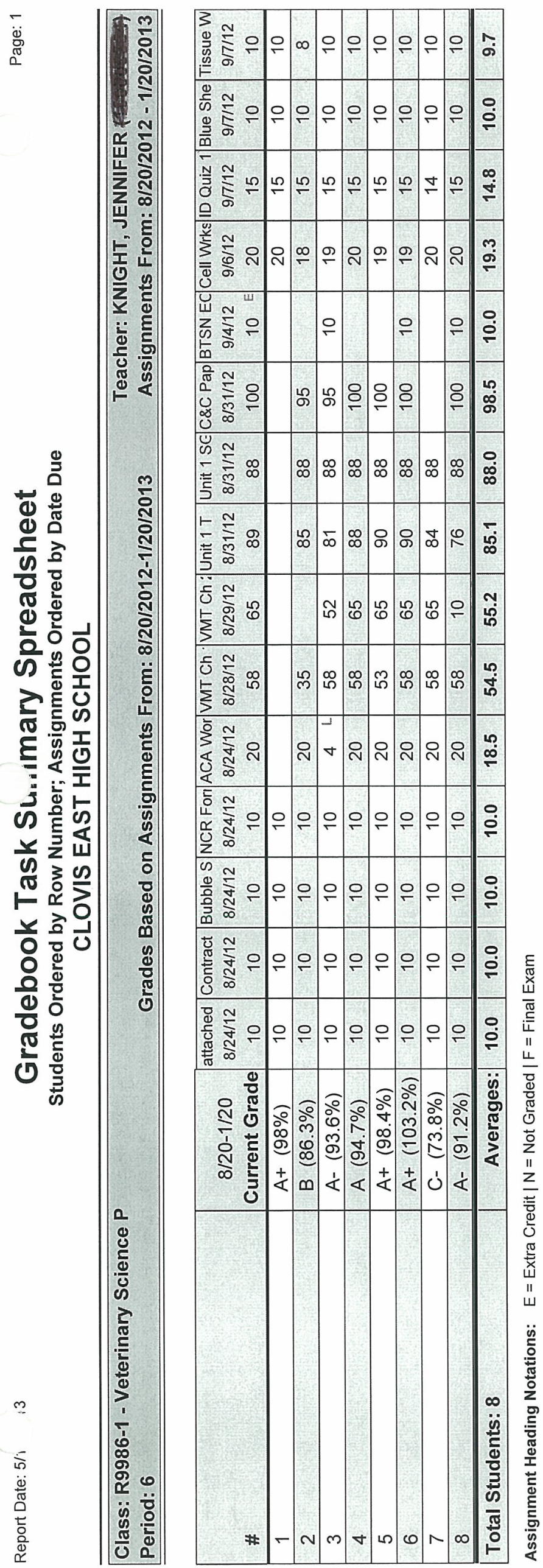




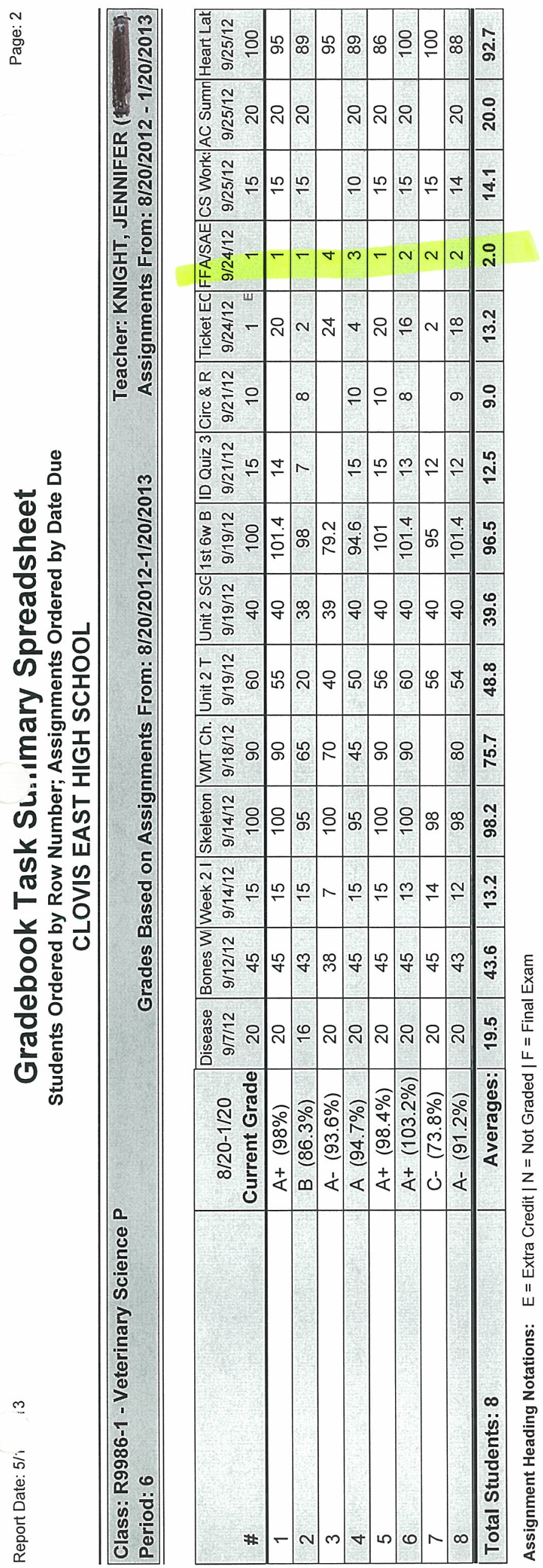




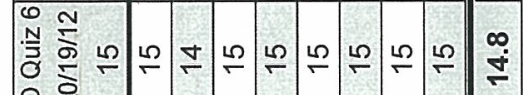

垔

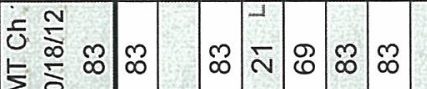

$\sum$

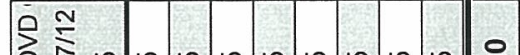

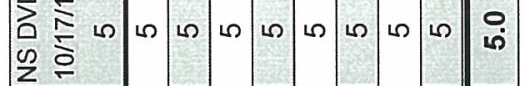

政

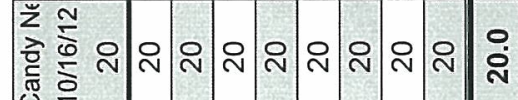

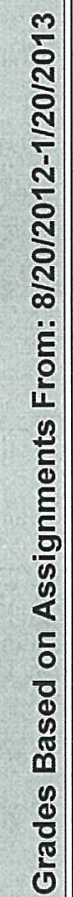

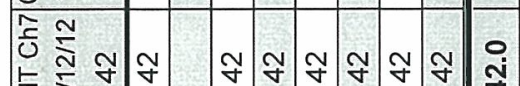

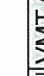

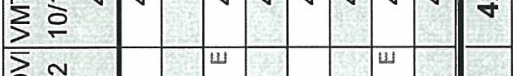

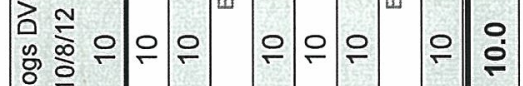

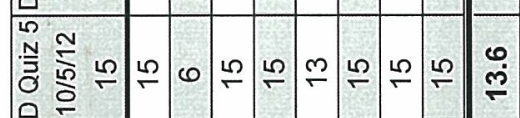

ఏ

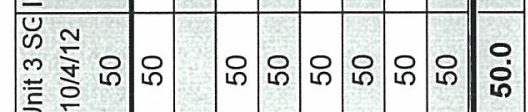

。

준이

तั क。

트

: 诖

के है

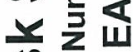

它合

당

즈을

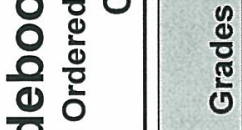

ชั

完

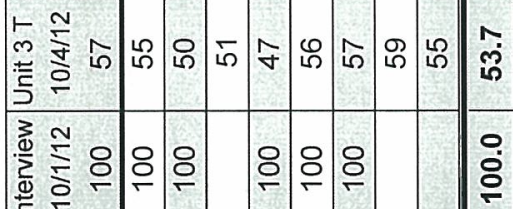

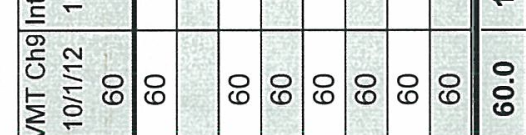

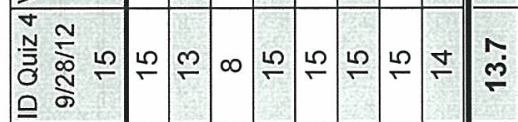

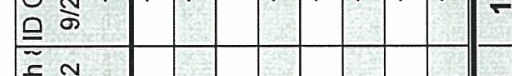

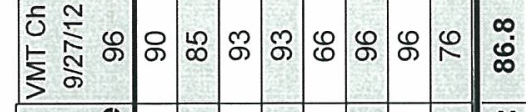

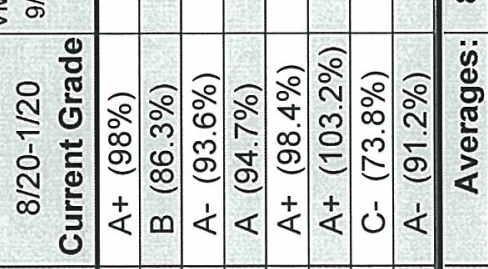

苞

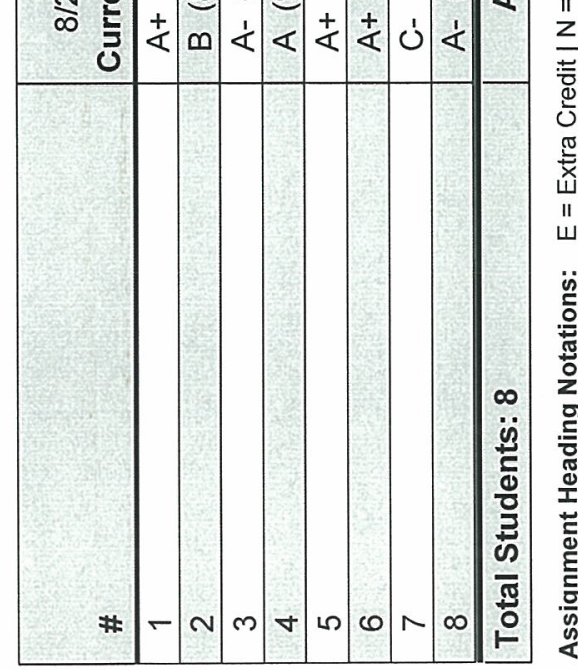


$\underset{\substack{0 \\ \dot{0}}}{\ddot{0}}$

\begin{tabular}{|c|c|c|c|c|c|c|c|c|}
\hline 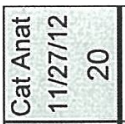 & 오 & 이 & 10 & & i্ & 오 & ำ & $\stackrel{\leftrightarrow}{\infty}$ \\
\hline 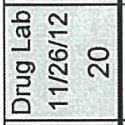 & & $\stackrel{\sim}{\sim}$ & 10 & & શ્ల & 오 & & $\stackrel{?}{\stackrel{?}{\sim}}$ \\
\hline
\end{tabular}

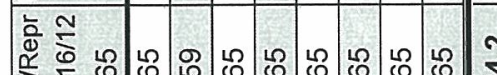

产采

궁

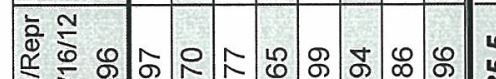

产总

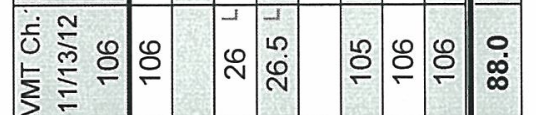

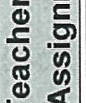

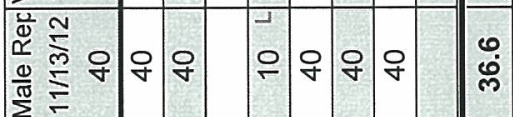

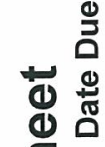

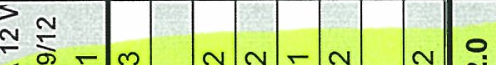

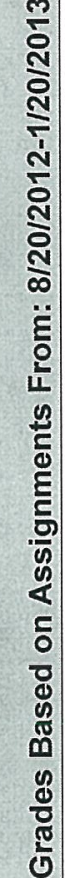

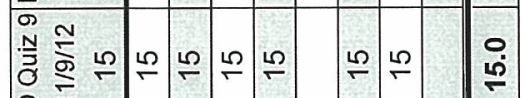

을

ชิ

(1) 인

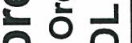

ผ ํㅗㄴ 우

글

तั

ह

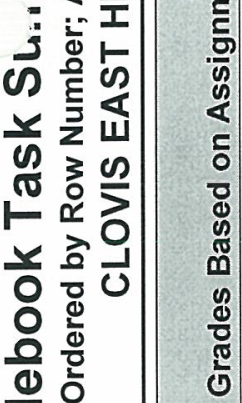

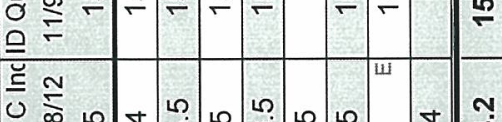

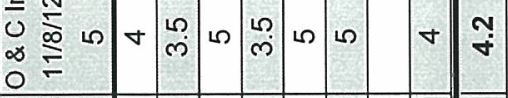

0

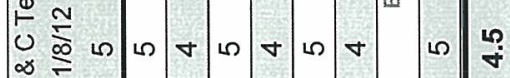

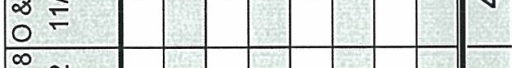

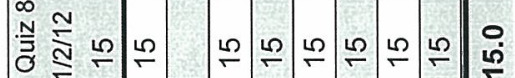

\section{으}

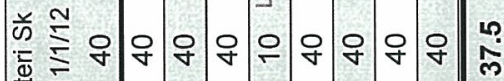

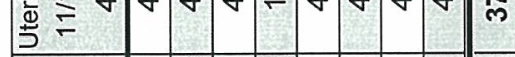

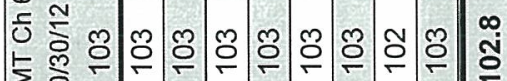

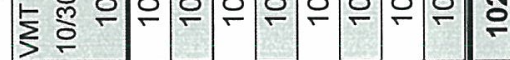

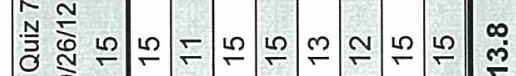

으

บ

$m$

㠃 竞

음

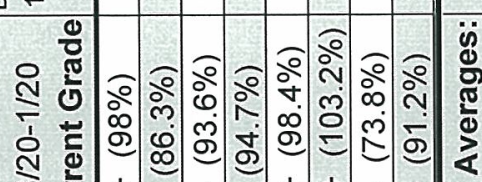

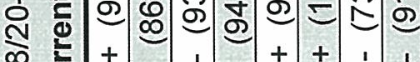

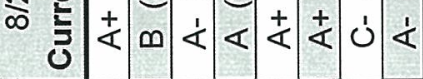




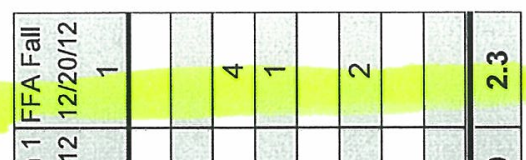

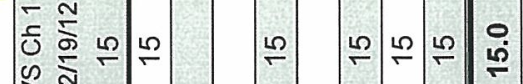

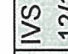

응

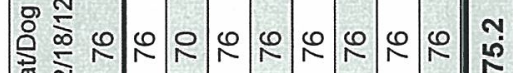

苋

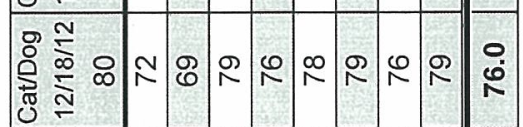

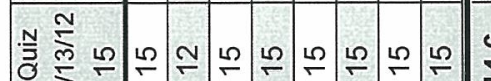

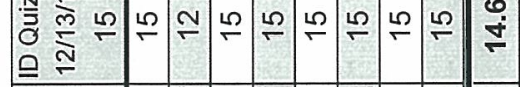

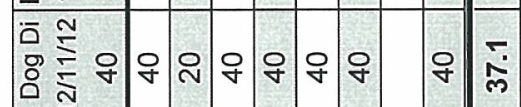

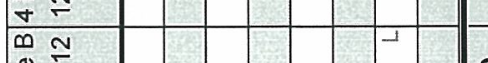

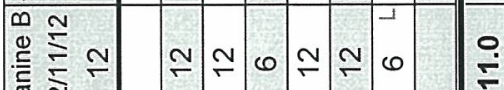

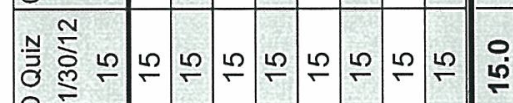

잉

Ч 으

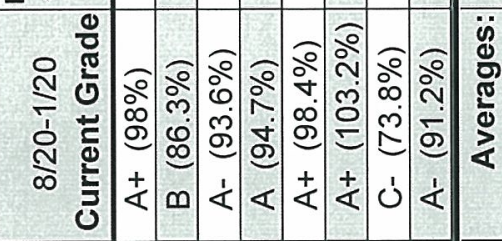




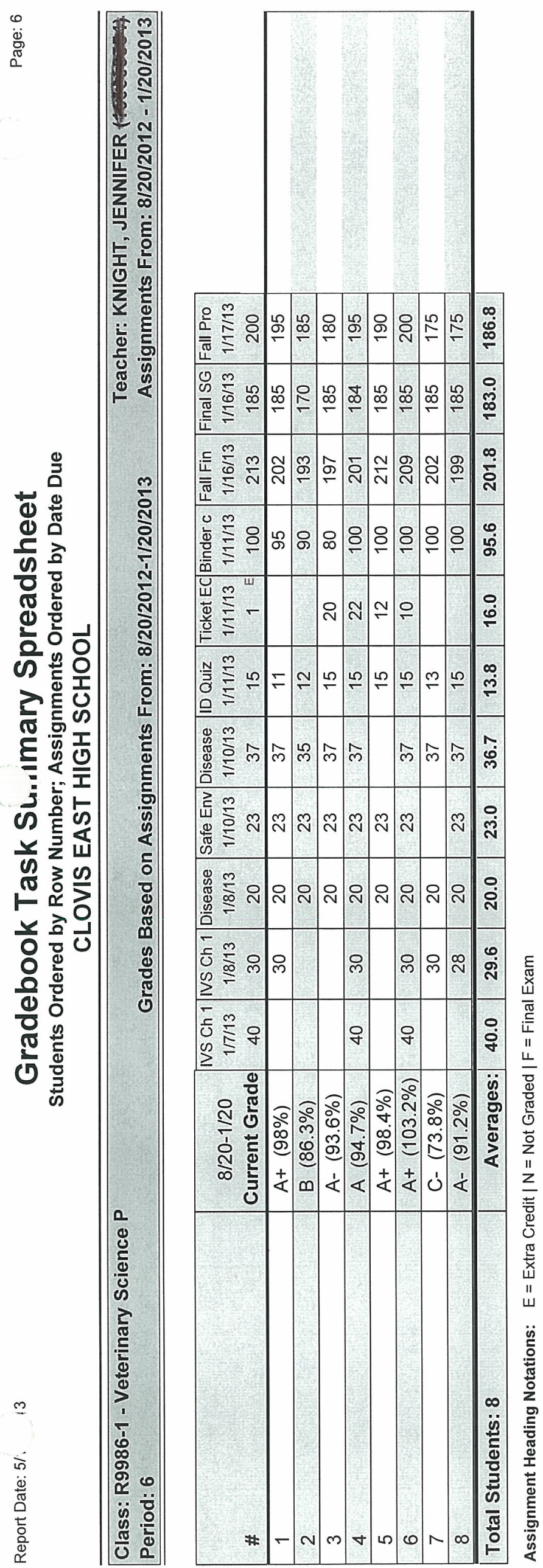




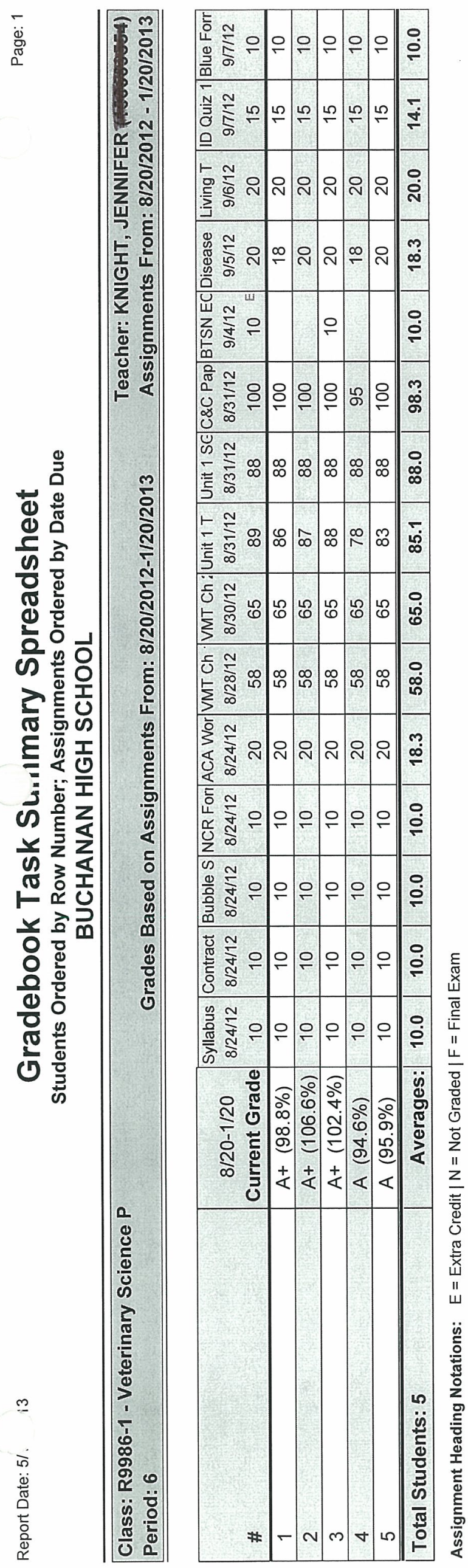




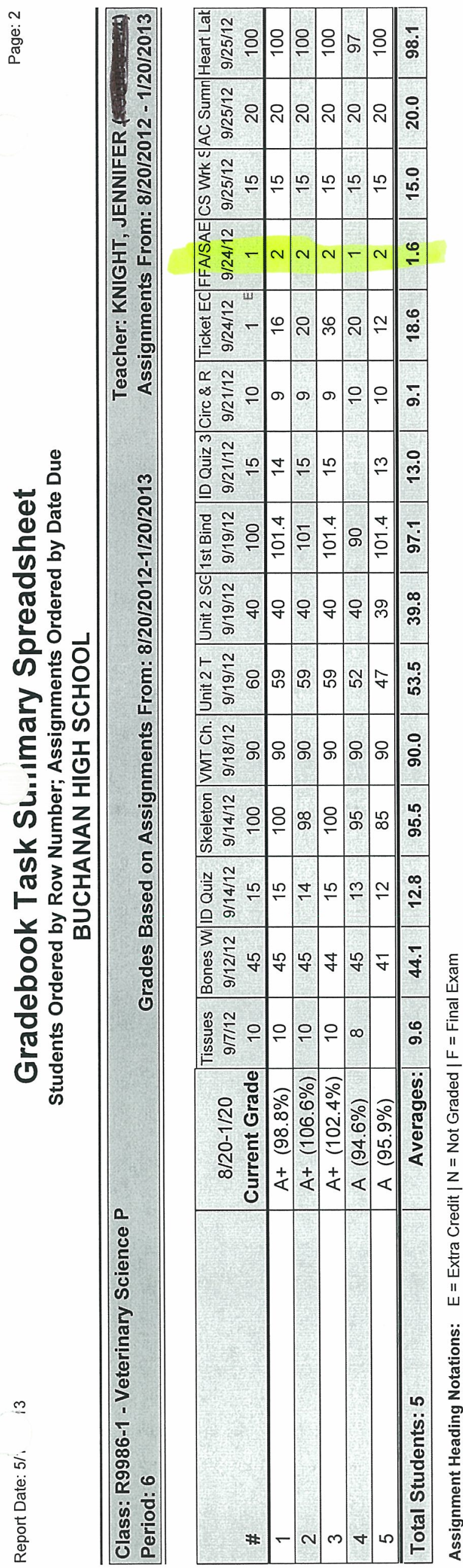




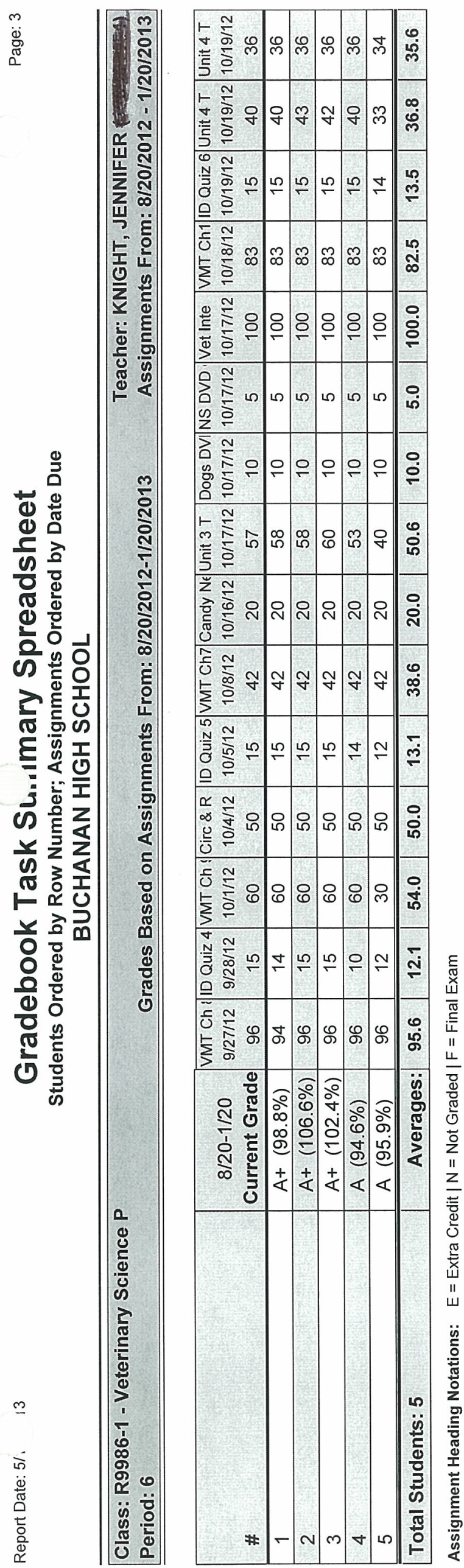




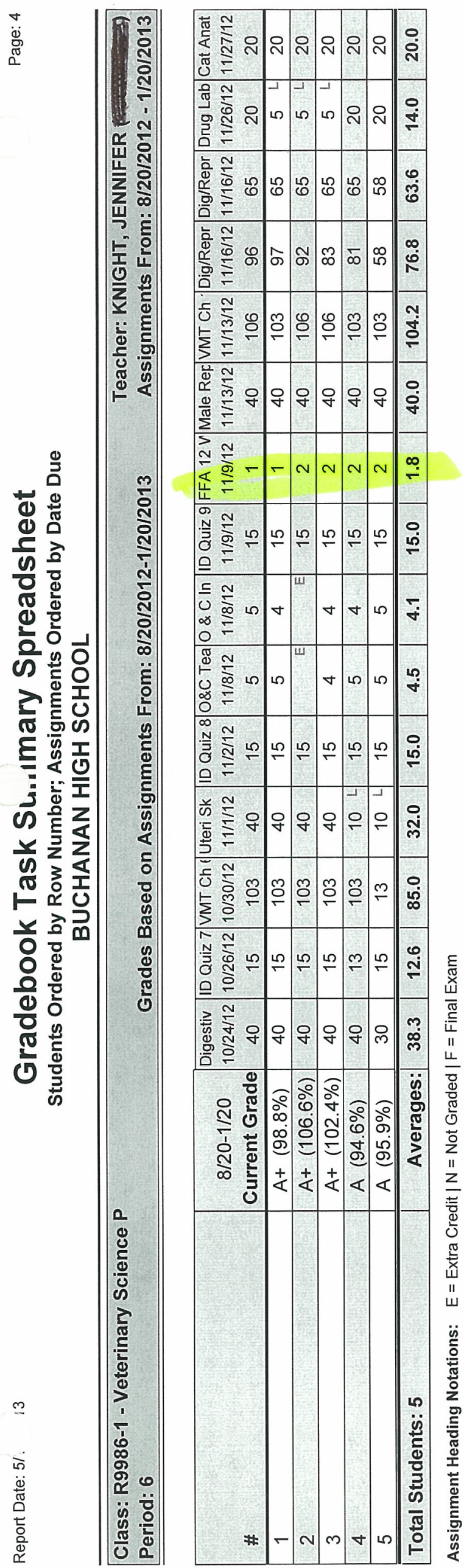




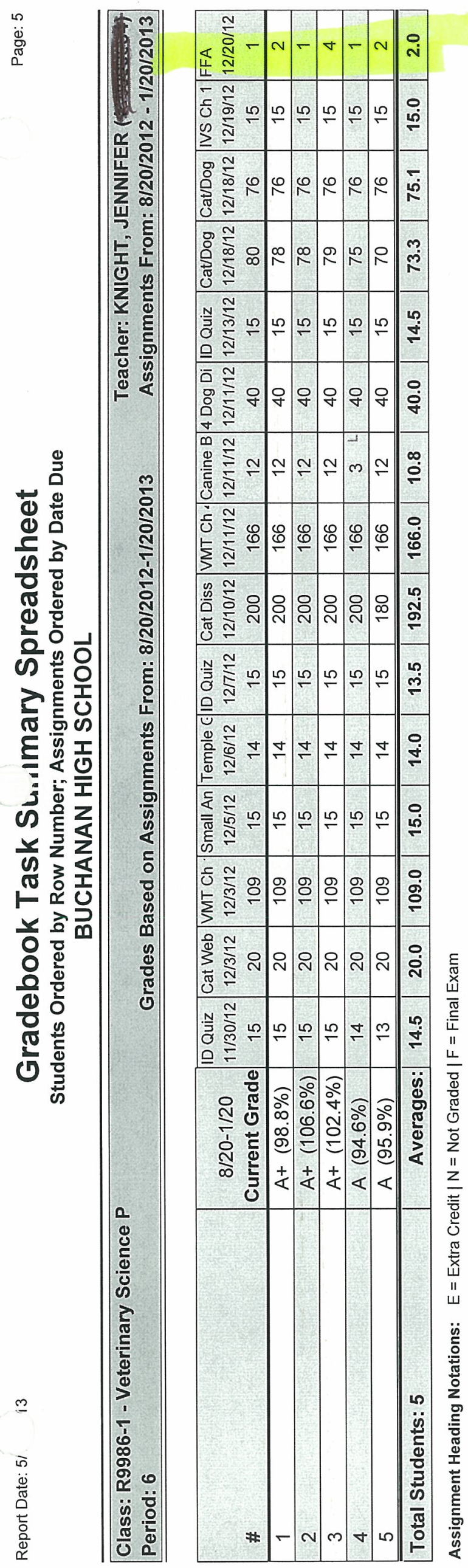




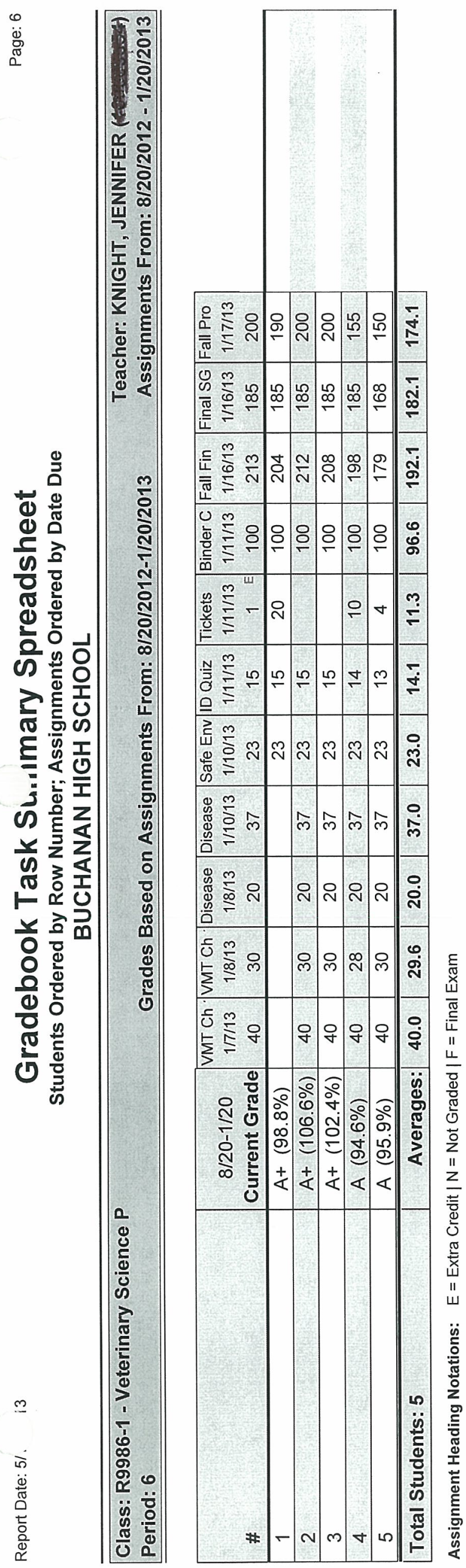




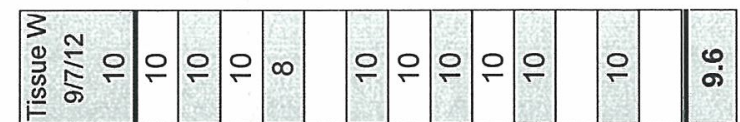

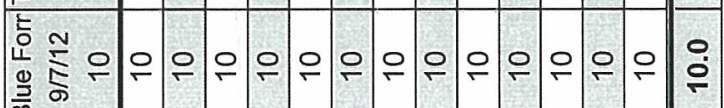
高

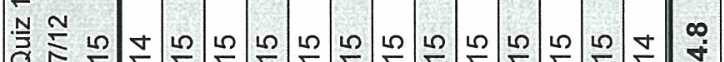

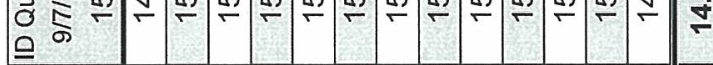
ำำ ㅇำ

点

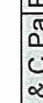

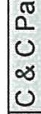

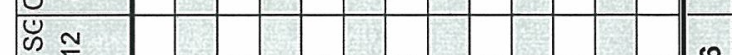

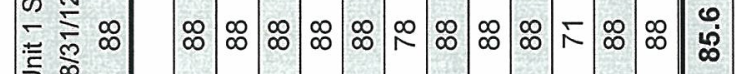
5 क

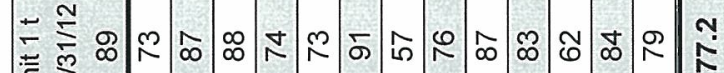
$\begin{array}{lll}2 & \infty \\ \infty & \infty & \infty\end{array}$

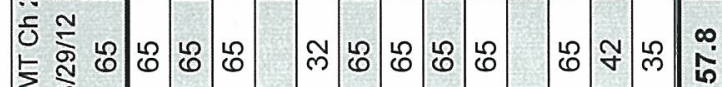

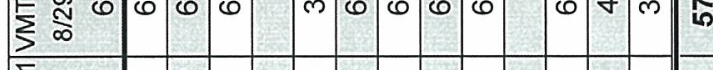

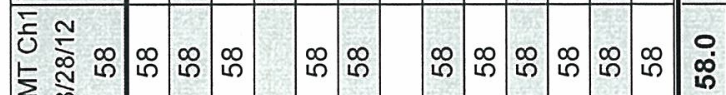
$\lambda \varepsilon$ 


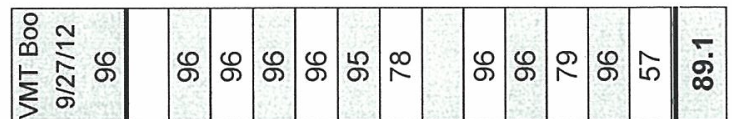

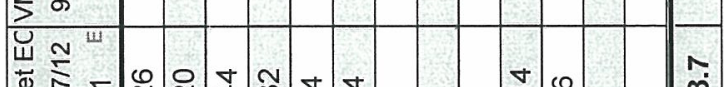

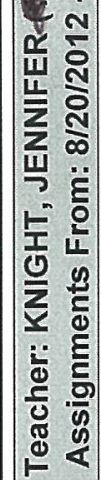

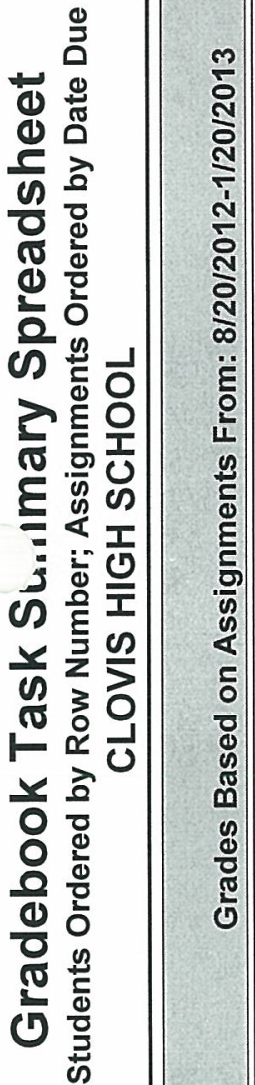

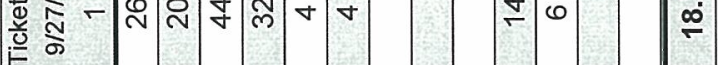

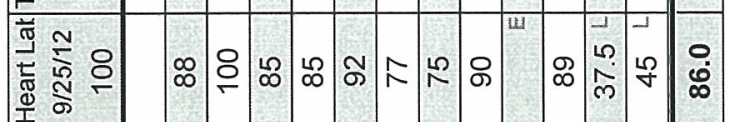

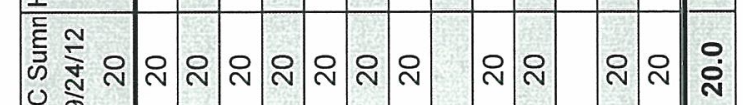
远告

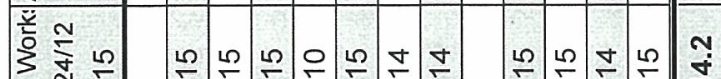

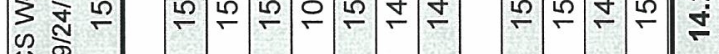

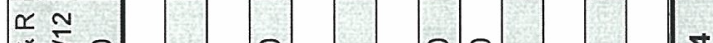

$\infty$ 长 ग)

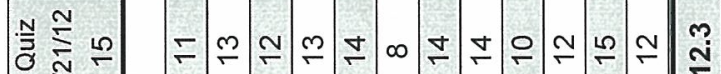

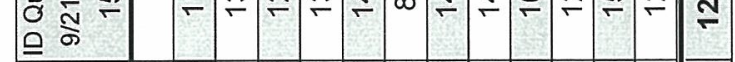

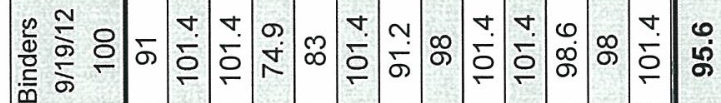
蒙帝

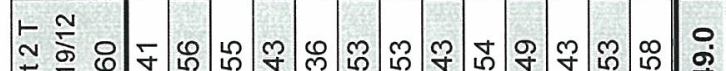

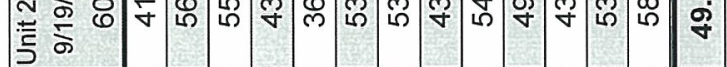

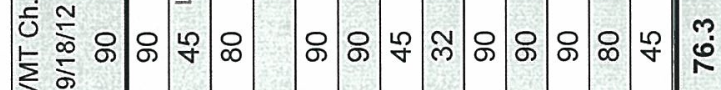

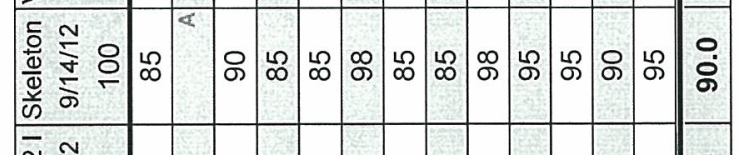

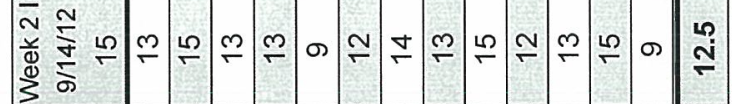

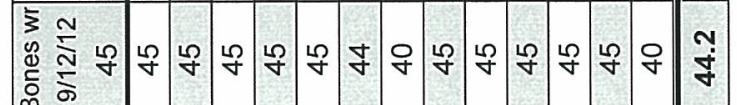

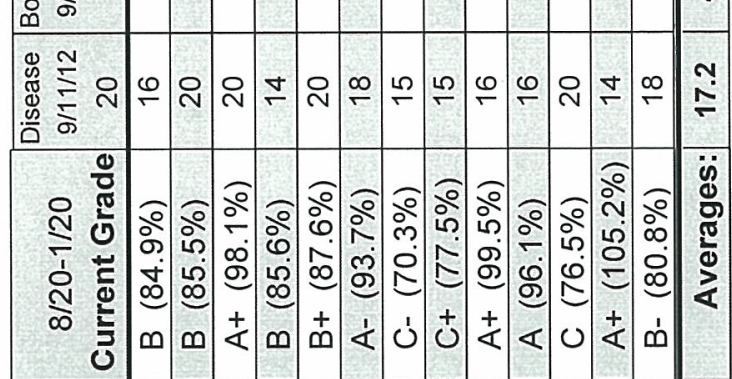

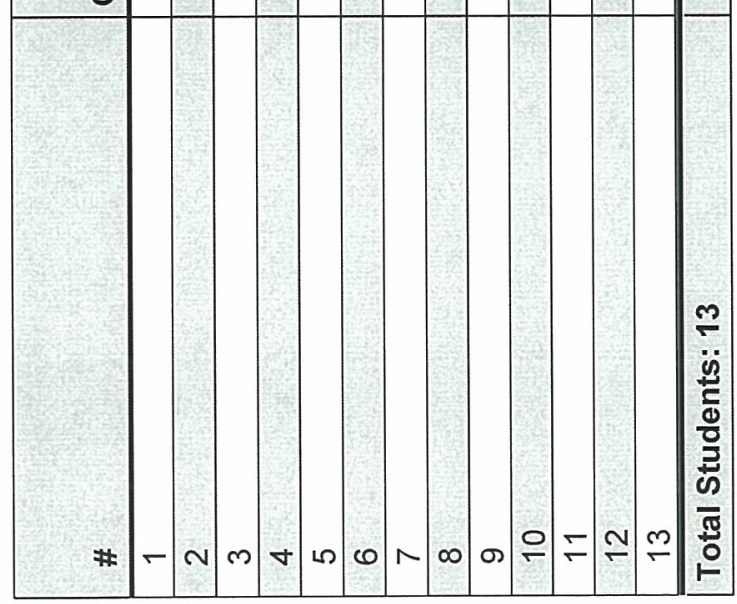




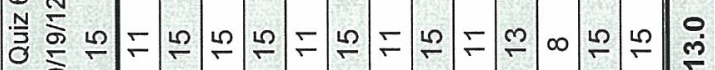
을

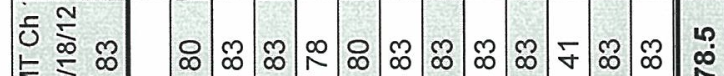

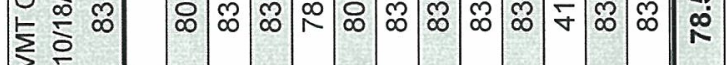

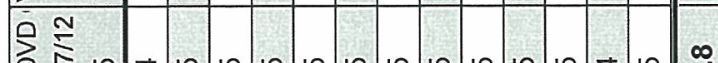

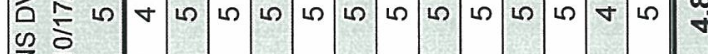

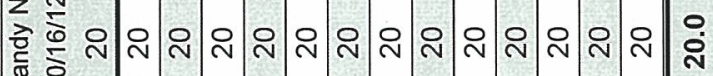
i

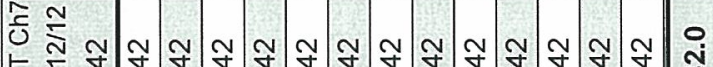

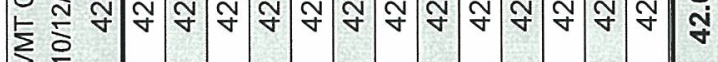

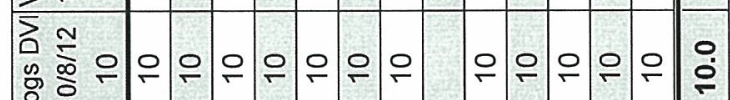
든

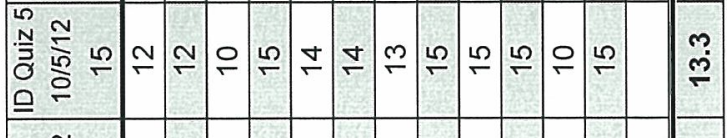

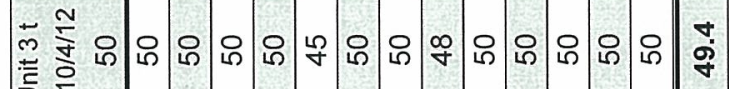
5

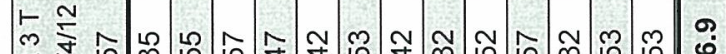

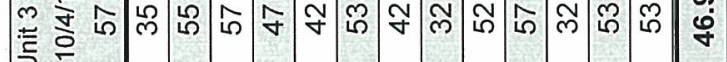

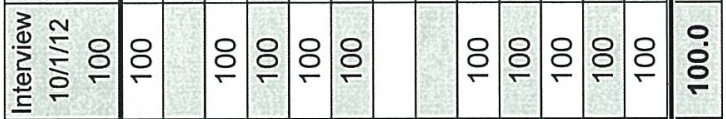

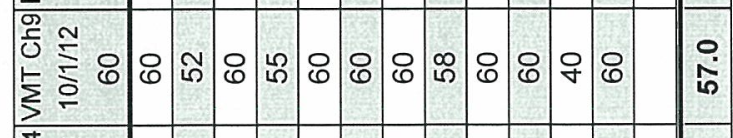

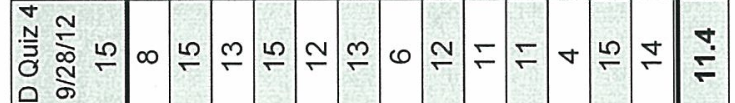
政

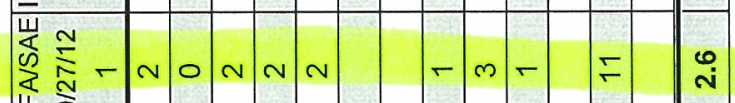
这
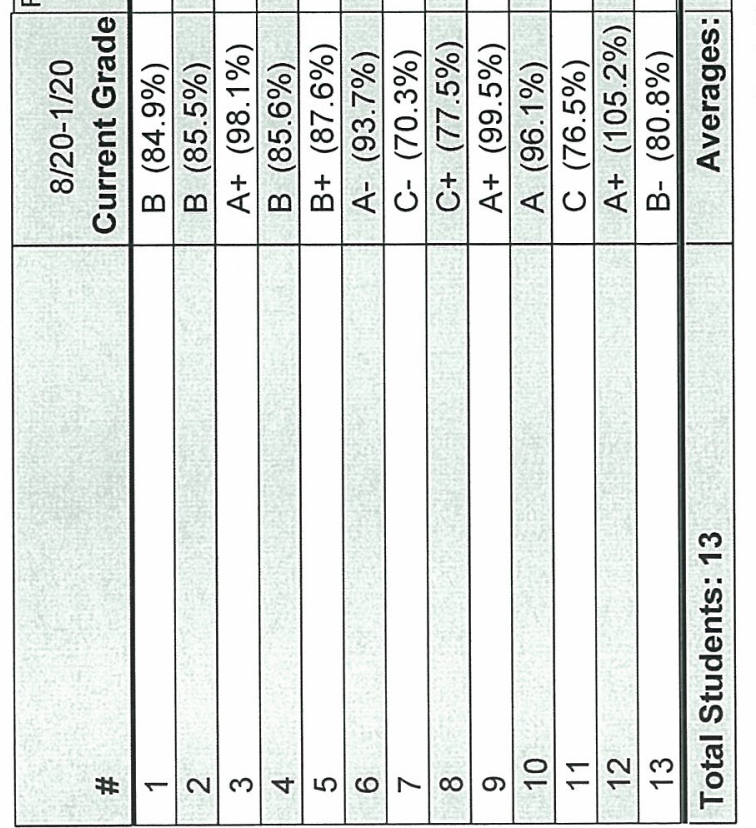

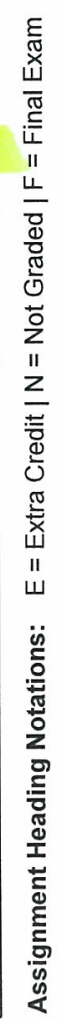




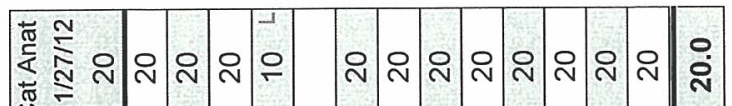
)

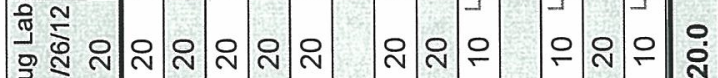
量

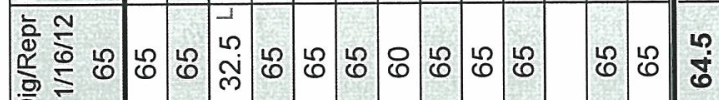
$\overline{0}=$

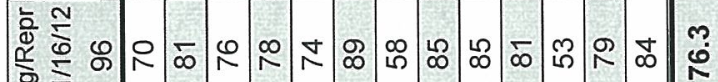
훙

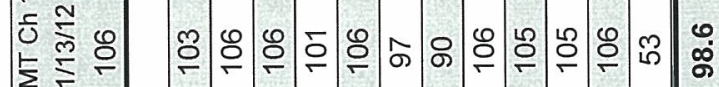

$\sum$

\begin{tabular}{|c|c|c|c|c|c|c|c|c|c|c|c|c|c|}
\hline 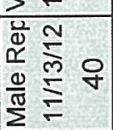 & 우 & 우 & 움 & 우 & & 워 & $\stackrel{\infty}{m}$ & & 우 & 움 & 웅 & 웅 & 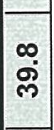 \\
\hline 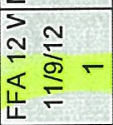 & - & - & $r$ & - & $r$ & & & & $n$ & $v \mid r$ & $-m$ & & $\stackrel{?}{?}$ \\
\hline 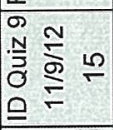 & $\stackrel{\sim}{\sim}$ & $\stackrel{2}{2}$ & 10 & $\stackrel{20}{-}$ & 10 & $\stackrel{20}{\sim}$ & $\stackrel{20}{\sim}$ & $\underset{\square}{\square}$ & $\stackrel{5}{\leftarrow}$ & $\frac{15}{5}$ & $\stackrel{2}{2}$ & $\stackrel{20}{\leftarrow}$ & $\stackrel{g}{\mathbb{2}}$ \\
\hline 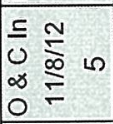 & w & 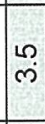 & $\checkmark$ & $\nabla$ & $\left|\begin{array}{l}n \\
m \\
m\end{array}\right|$ & $\nabla$ & $\begin{array}{l}10 \\
\dot{m}\end{array}$ & $\nabla \mid$ & 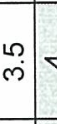 & $\nabla \mid \begin{array}{ll}n \\
m\end{array}$ & مُ & WI & $\stackrel{\infty}{\infty}$ \\
\hline 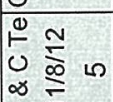 & w & $\stackrel{\sim}{\sim}$ & L? & 10 & $\begin{array}{l}10 \\
\dot{m}\end{array}$ & $\left|\begin{array}{l}n \\
m\end{array}\right|$ & $\begin{array}{lll}10 & 4\end{array}$ & in & $\nabla$ & $\nabla \mid \begin{array}{l}b \\
r\end{array}$ & 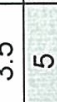 & 㝳 & F \\
\hline
\end{tabular}

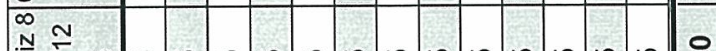

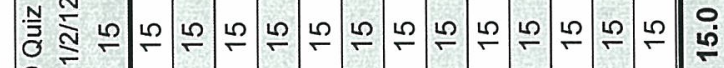

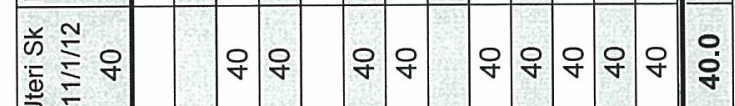

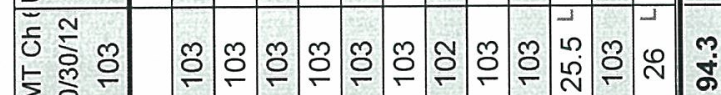

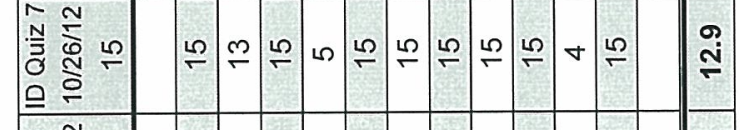

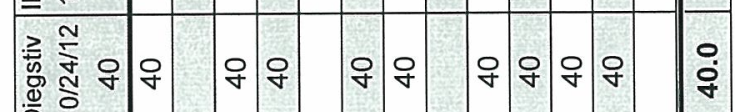




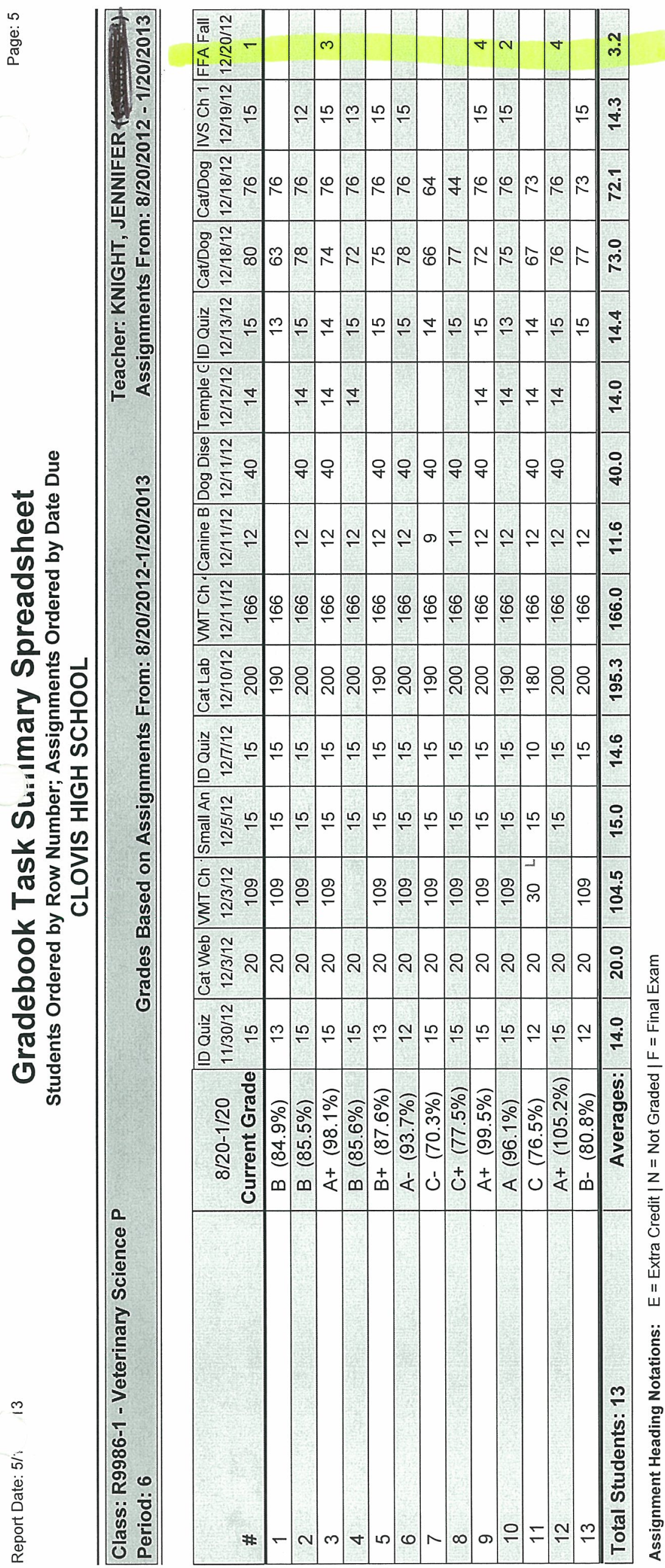




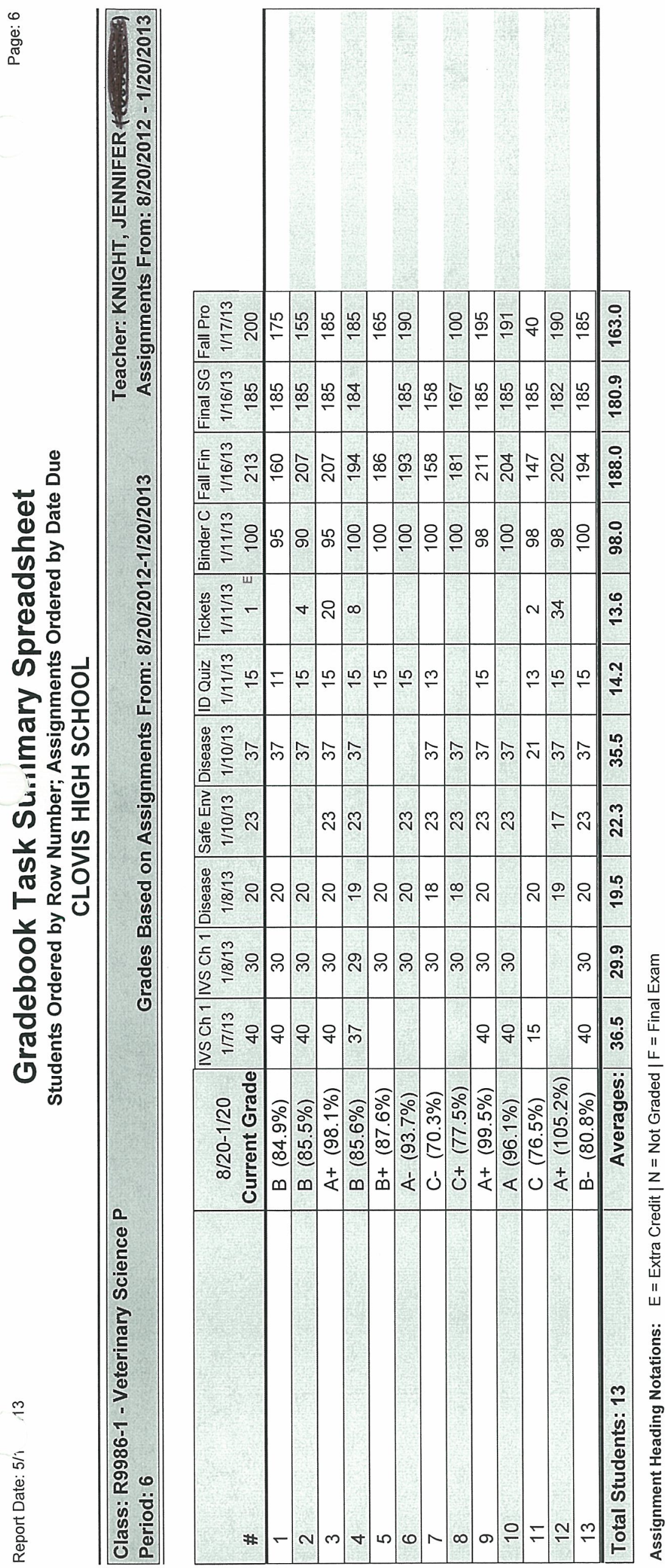




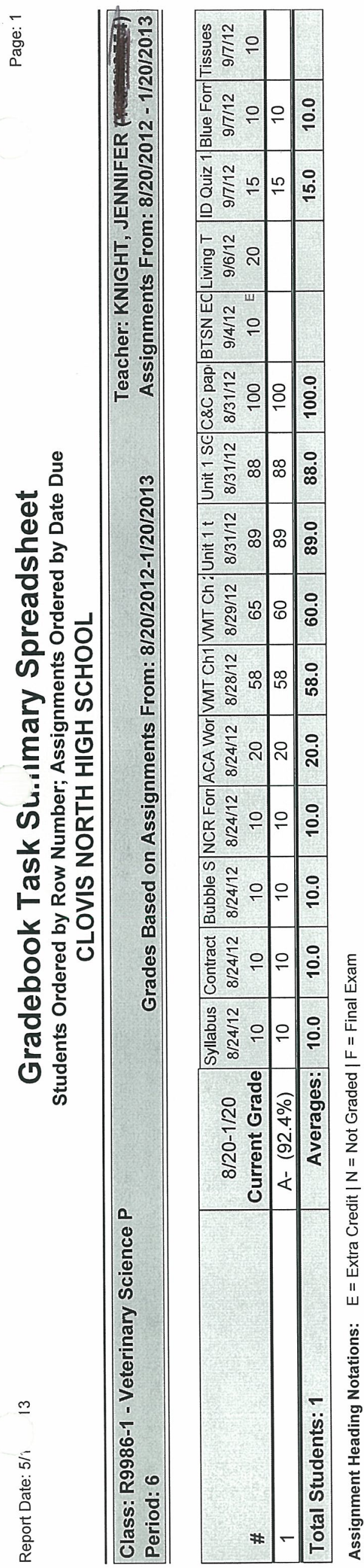




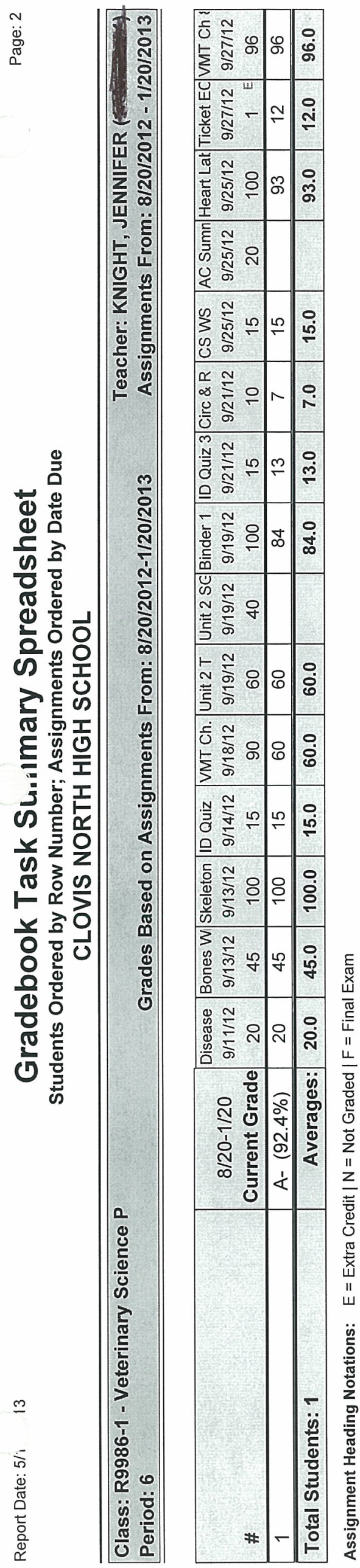




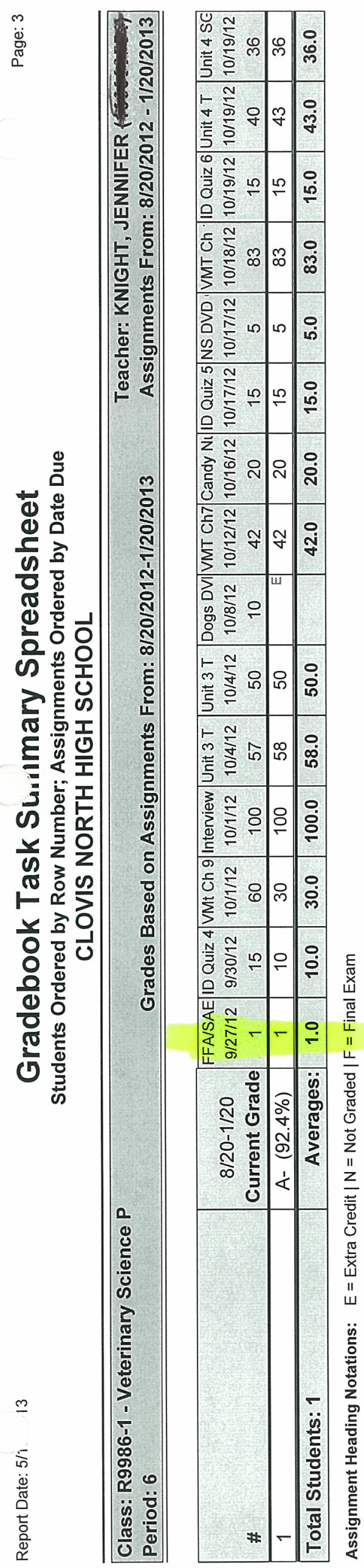




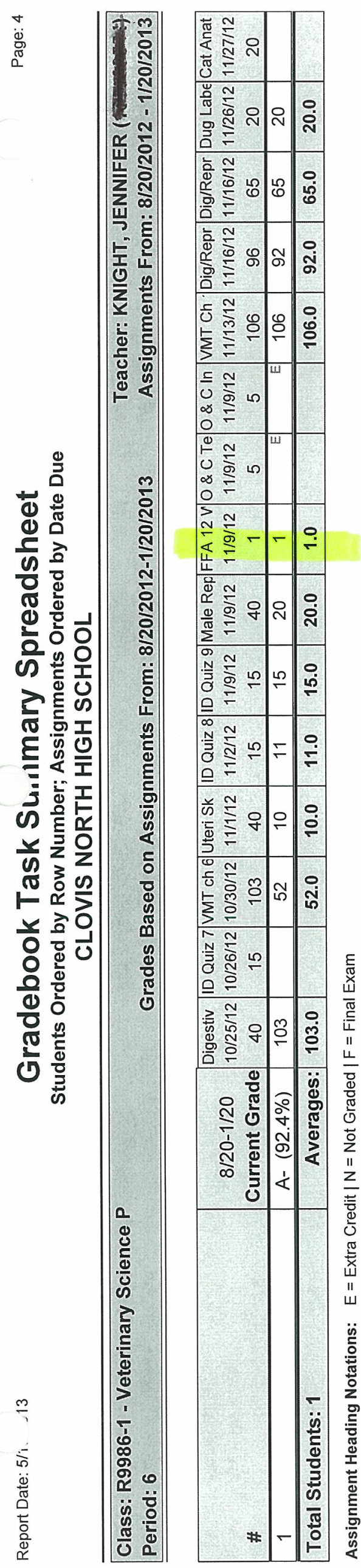




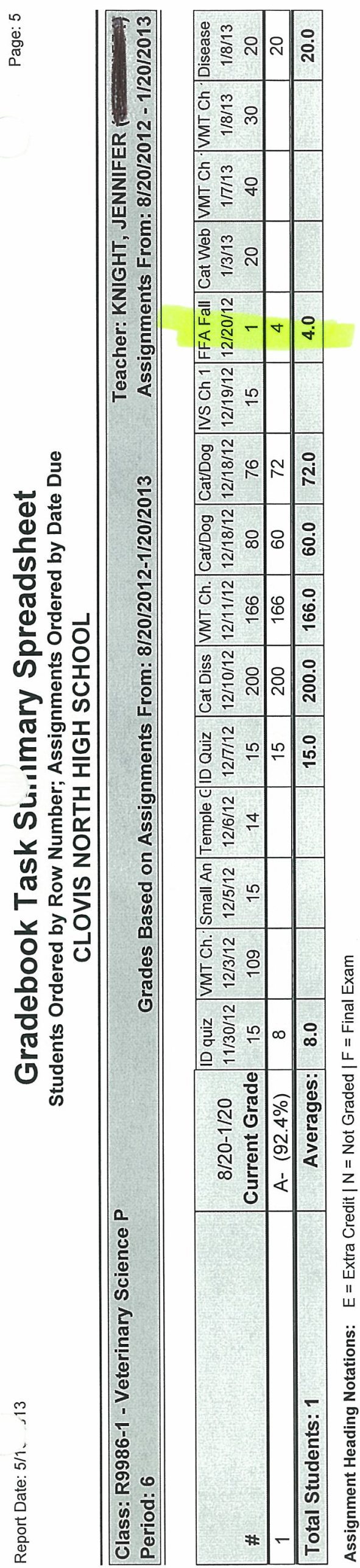




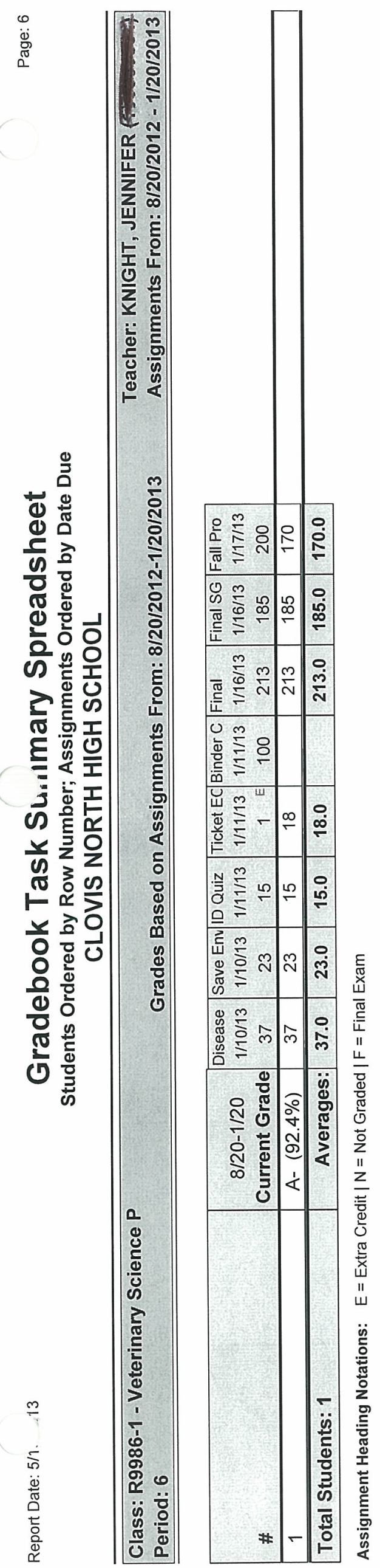




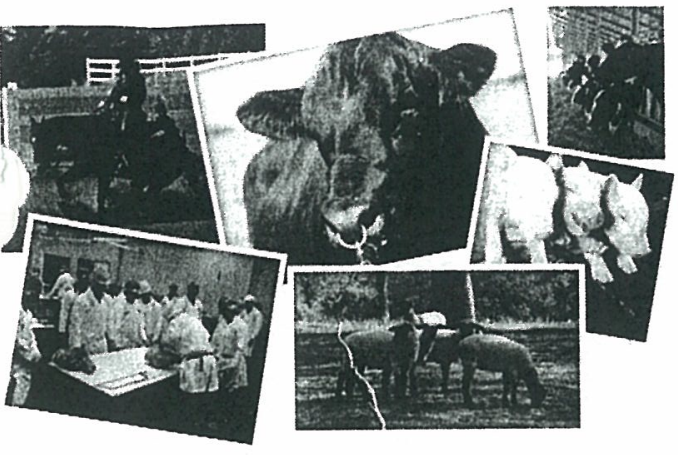

\section{Animal Science}

Clovis East High School

McFarlane Coffman Ag Center Room 903

Mrs. Knight

jenniferknight@cusd.com

$559-327-4343$ or $559-327-4327$

\section{Course Information:}

This course will provide the student with principals in Animal Science focusing on the areas of livestock (Mammalian) production, anatomy, physiology, reproduction, nutrition, respiration, and genetics. This course is intended to prepare those students who plan on majoring in Agriculture or Animal Sciences at a college or University.

\section{Suggested Materials:}

- 3 ring binder 1" to 1.5" (To use for Animal Science and leave in the classroom)

- 5 tab dividers for Animal science 3 ring binder

- Writing instrument (Pen or Pencil)

- Lined paper to write notes or journal entries

***If a student is unable to purchase the recommended materials, the student should contact the teacher and materials will be provided.

\section{Classwork and Participation:}

- Students are expected to participate actively in class by completing daily warm ups, taking notes, keeping resource materials organized, completing activities and labs, and doing homework.

- Agriculture Education is a comprehensive program and requires students to participate in a Supervised Agriculture Experience Project component as well as FFA leadership activities and events.

\section{Homework and Projects:}

- Homework may be assigned 2-3 times a week.

- Students will complete an agriscience research project.

- Torn, crumpled, decorated, or sloppy work will not be accepted.

- Student work should have the students first and last name, course title, period, date, and assignment number in the top right corner.

\section{Late or make up work:}

- Late work will be accepted during the CURRENT unit of study for half credit.

- Make-up work is available for EXCUSED absences. It is the student's responsibility to collect make up work upon returning to class. Make-up school days are equal to number of days school days missed. This policy may change, depending on the particular situation. 


\section{Testing Policy:}

- Missed tests must be made up within one week of student returning to school, an appointment should be made at lunch, break, before or after school.

- Students may re-take a test once if they scored below $75 \%$. The max score that can be earned on a re-take is $75 \%$

\section{Grade Breakdown:}

- Tests \& Quizzes $\quad 25 \%$

- Class \& Homework $\quad 25 \%$

- Labs \& Projects $\quad 35 \%$

- Binders $10 \%$

- FFA and SAE 5\%

- SAE Credit by completion of FFA record book

- FFA Points awarded by attending 1 activity for each 6 week grading period.

\section{Behavior and Discipline:}

- Please refer to the student handbook. ALL CEHS rules will be strictly enforced.

- Character Counts pillars and principals will also be a part of our classroom environment.

\section{Extra Credit and Incentives:}

- Incentive tickets will be awarded in class for exemplary participation, as a reward, or for displays of Character Counts.

- Tickets may be used for the following: (The 3 P's)

- (Points) Extra Credit Points at the end of a grading period

- (Potty) Exchange 1 ticket for a restroom trip

- (Prizes) Exchange for various prizes

- Other extra credit may be assigned as the instructor sees fit but is not frequently available, and will not replace the need to complete regular assignments. 


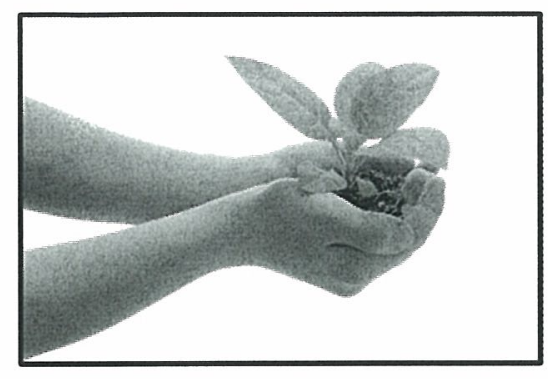

\section{Botany}

Clovis East High School

McFarlane Coffman Ag Center Room 903

Mrs. Knight

jenniferknight@cusd.com

$559-327-4343$ or $559-327-4327$

Course Information:

Botany is the Biology of plants, and will include concepts like plant classification, plant anatomy and physiology, growth principals, reproduction, soil science, biotechnology, and weed science and entomology.

Suggested Materials:

- 3 ring binder 1" to 1.5" (To use for Botany and leave in the classroom)

- 5 tab dividers for Botany 3 ring binder

- Writing instrument (Pen or Pencil)

- Lined paper to write notes or journal entries

*** If a student is unable to purchase the recommended materials, the student should contact the teacher and materials will be provided.

Classwork and Participation:

- Students are expected to participate actively in class by completing daily warm ups, taking notes, keeping resource materials organized, completing activities and labs, and doing homework.

- Agriculture Education is a comprehensive program and requires students to participate in a Supervised Agriculture Experience Project component as well as FFA leadership activities and events.

Homework and Projects:

- Homework may be assigned 2-3 times a week.

- Students will complete an agriscience research project.

- Torn, crumpled, decorated, or sloppy work will not be accepted.

- Student work should have the students first and last name, course title, period, date, and assignment number in the top right corner.

Late or make up work:

- Late work will be accepted during the CURRENT unit of study for half credit. 
- Make-up work is available for EXCUSED absences. It is the student's responsibility to collect make up work upon returning to class. Make-up school days are equal to number of days school days missed. This policy may change, depending on the particular situation.

\section{Testing Policy:}

- Missed tests must be made up within one week of student returning to school, an appointment should be made at lunch, break, before or after school.

- Students may re-take a test once if they scored below $75 \%$. The max score that can be earned on a re take is $75 \%$

\section{Grade Breakdown:}

- Tests \& Quizzes

- Class \& Homework

- Labs \& Projects

- Binders FFA and SAE

$25 \%$
$25 \%$
$35 \%$
$10 \%$
$5 \%$

- SAE Credit by completion of FFA record book

- FFA Points awarded by attending 1 activity for each 6 week grading period.

\section{Behavior and Discipline:}

- Please refer to the student handbook. ALL CEHS rules will be strictly enforced.

- Character Counts pillars and principals will also be a part of our classroom environment.

\section{Extra Credit and Incentives:}

- Incentive tickets will be awarded in class for exemplary participation, as a reward, or for displays of Character Counts.

- Tickets may be used for the following: (The 3 P's)

- (Points) Extra Credit Points at the end of a grading period

- (Potty) Exchange 1 ticket for a restroom trip

- (Prizes) Exchange for various prizes

- Other extra credit may be assigned as the instructor sees fit but is not frequently available, and will not replace the need to complete regular assignments.

\footnotetext{
The Clovis Unified School District does not discriminate on the basis of race, color, disability, or national origin in admission or access to and treatment of employment in its programs and activities as required by Title VI, Title IX and Section 504. Complaints: Contact Dr. Cheryl Rogers, Human Resources, (559) 327-9308.

Favor de llamar a la oficina para assistencia en espanol.

Thov tiv tauj peb lub hoob kas yog xav tau kev pab nrog lus Hmoob.
} 


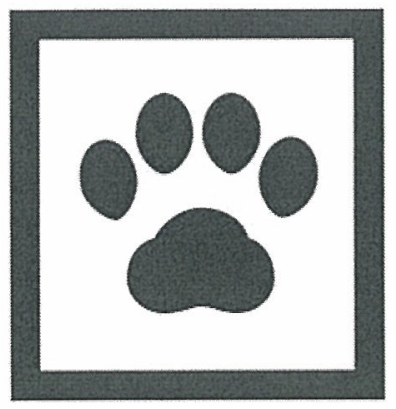

\section{Veterinary Science R.0.P}

Regan Educational Center

McFarlane Coffman Ag Center Room 903

Mrs. Knight

jenniferknight@cusd.com

559-327-4343 or 559-327-4327

\section{Course Information:}

Course work provides for the study of classification systems, anatomy, physiology, nutrition, reproduction, mitosis, meiosis, respiration, genetics, and health care of domesticated animals. Curriculum includes lab and fieldwork utilizing scientific methodology while reinforcing writing and presentation skills. Students will seek placement at a Community Classroom site (Vet Clinic or Animal Facility) during the second semester of instruction and will be expected to complete their class hours as an unpaid assistant or job shadow participant. This class is designed to meet EVERY day $6^{\text {th }}$ and $7^{\text {th }}$ period(s) to allow for increased instructional hours in the Fall and time for job site participation in the Spring semester.

\section{Suggested Materials:}

- 3 ring binder 1.5 " to 2" (To use for Vet Science only)

- 8 tab dividers for vet science 3 ring binder

- Writing instrument (Pen or Pencil)

- Lined paper

- Composition or spiral notebook for work summaries (Spring Semester)

- Clear sheet protectors for portfolio materials (Spring Semester)

***If a student is unable to acquire the recommended materials the student should contact the teacher to obtain materials.

\section{Classwork and Participation:}

- Students are expected to participate actively in class by completing daily warm ups, taking notes, keeping resource materials organized, completing activities and labs, and doing homework.

- Agriculture Education is a comprehensive program and requires students to participate in a Supervised Agriculture Experience Project component as well as FFA leadership activities and events.

\section{Homework and Projects:}

- Homework may be assigned 3-4 times a week.

- Students will complete several research projects.

- Students will complete a Resume and Cover Letter that they will use to find a community classroom location placement.

- A portfolio will be due as the Spring semester final project.

- Torn, crumpled, decorated, or sloppy work will not be accepted.

- Student work should have the students first and last name, course title, period, date, and assignment number in the top right corner.

Late or make up work:

- Late work will be accepted during the CURRENT unit of study for half credit. 
- Make up work is available for EXCUSED absences. It is the student's responsibility to collect make up work upon returning to class. Make-up school days are equal to number of days school days missed. This policy may change, depending on the particular situation.

\section{Testing Policy:}

- Missed tests must be made up within one week of student returning to school, an appointment should be made at lunch, break, before or after school.

- Students may re take a test once if they scored below $75 \%$. The max score that can be earned on a re take is $75 \%$

Grade Breakdown FALL SEMESTER:

- Tests \& Quizzes $\quad 30 \%$

- Class \& Homework $\quad 25 \%$

- Labs \& Projects $\quad 30 \%$

- Binders 10\%

- FFA and SAE $5 \%$

- SAE Credit by completion of FFA record book

- FFA Points awarded by attending 1 activity for each 6 week grading period.

Grade Breakdown SPRING SEMESTER:

- Special Presentations $10 \%$

- Job Site Journal $10 \%$

- Final Portfolio $\quad 40 \%$

- Time Cards and Hours Worked $\quad 30 \%$

- Points can be deducted for:

- Not calling in absences or tardies

- Turning in time cards late

- Not meeting hour requirements

- Quality of work on Job Site

○ (Instructor and Supervisor Evaluations)

\section{Behavior and Discipline:}

- Please refer to the student handbook. ALL CEHS and CUSD rules will be strictly enforced.

- Character Counts pillars and principals will also be a part of our classroom environment.

\section{Extra Credit and Incentives:}

- Incentive tickets will be awarded in class for exemplary participation, as a reward, or for displays of Character Counts.

- Tickets may be used for the following: (The 3 P's)

- (Points) Extra Credit Points at the end of a grading period

- (Potty) Exchange 1 ticket for a restroom trip

- (Prizes) Exchange for various prizes

- Other extra credit may be assigned as the instructor sees fit but is not frequently available, and will not replace the need to complete regular assignments.

The Clovis Unified School District does not discriminate on the basis of race, color, disability, or national origin in admission or access to and treatment of employment in its programs and activities as required by Title VI, Title IX and Section 504. Complaints: Contact Dr. Cheryl Rogers, Human Resources, (559) 327-9308. 

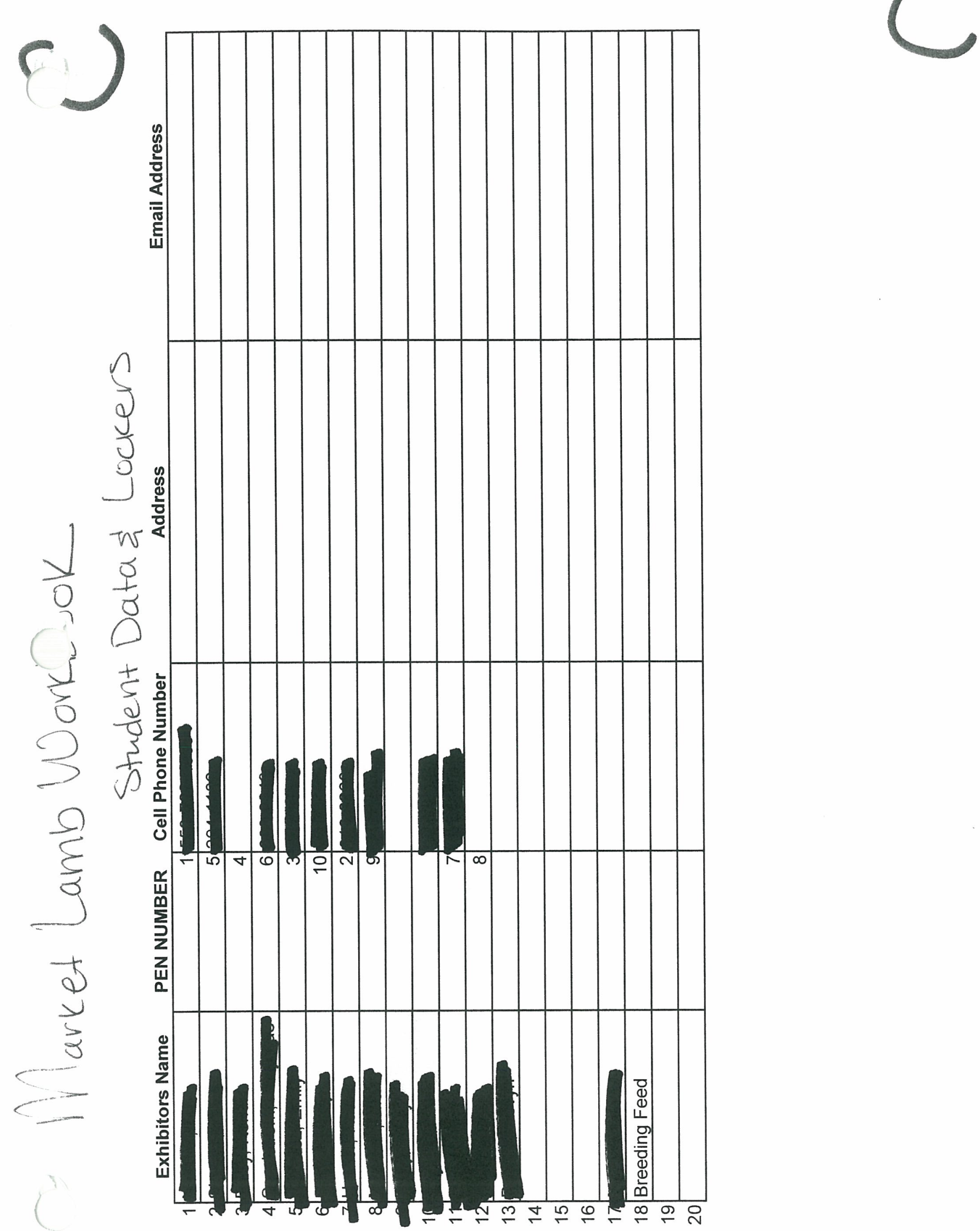


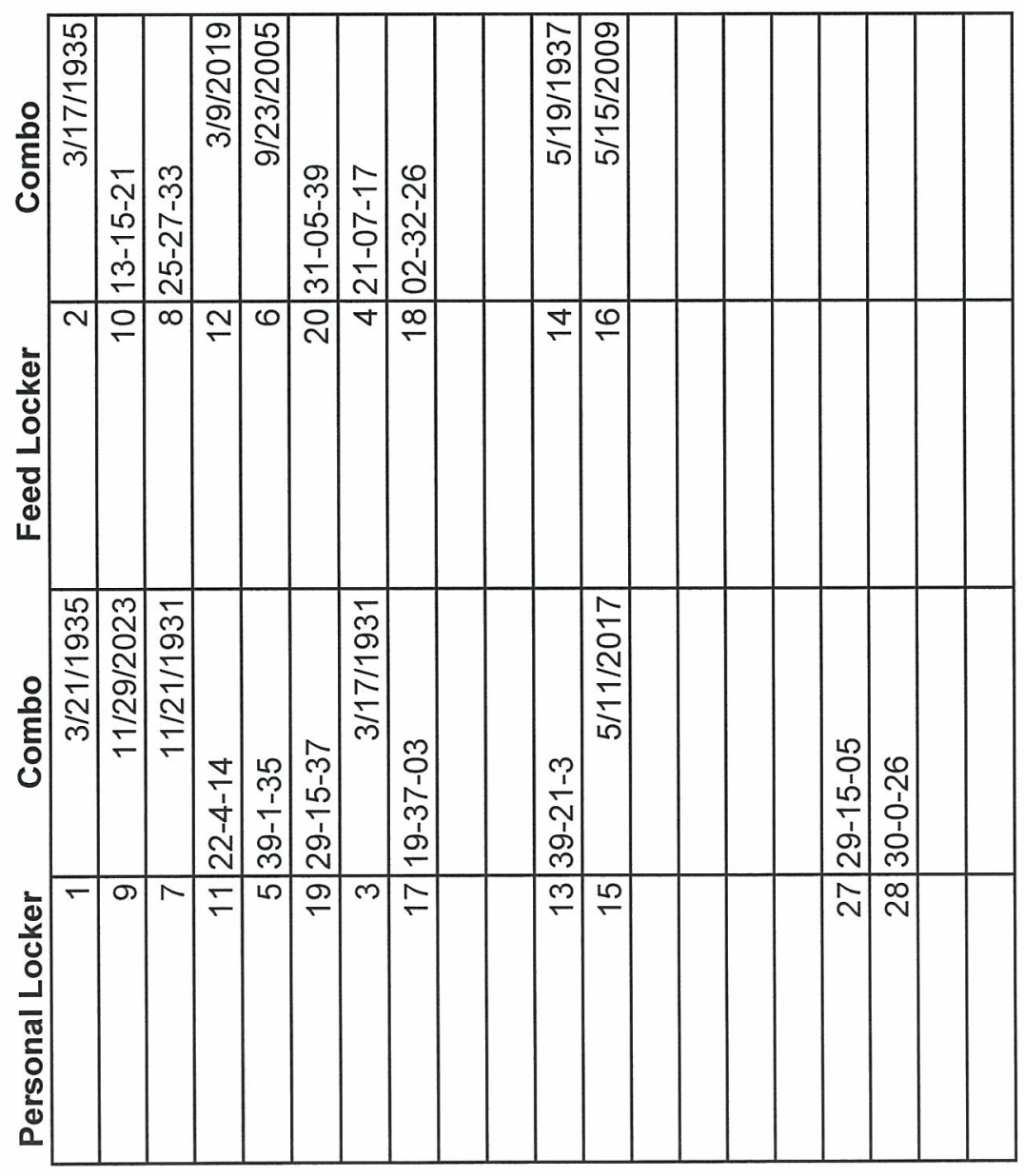


Lamb Info

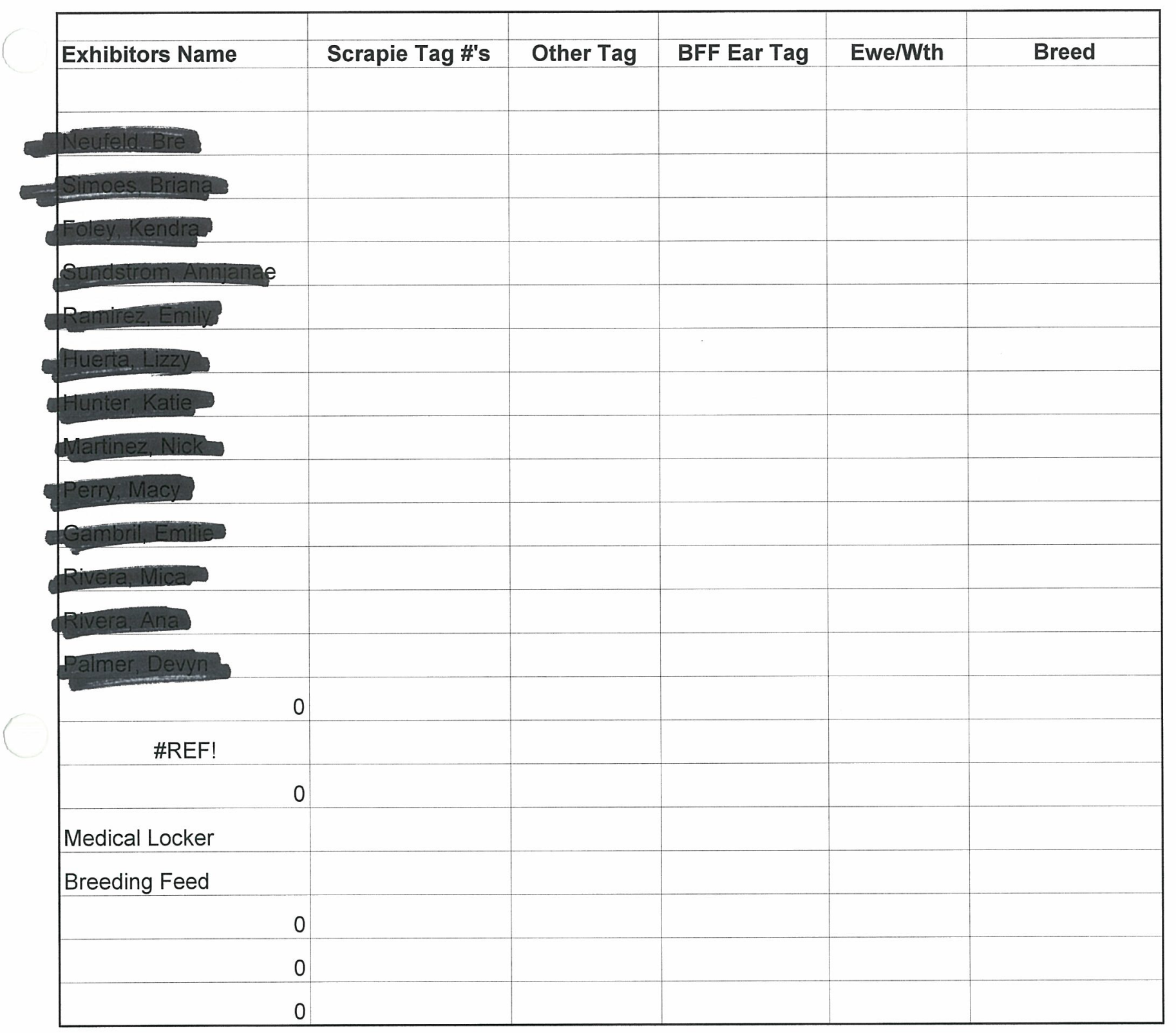




\begin{tabular}{|l|c|}
\hline Breeder & School Farm/Home \\
\hline & \\
\hline & \\
\hline & \\
\hline & \\
\hline & Home \\
\hline & \\
\hline & \\
\hline & \\
\hline & \\
\hline & \\
\hline & \\
\hline & \\
\hline & \\
\hline & \\
\hline & \\
\hline & \\
\hline & \\
\hline & \\
\hline & \\
\hline & \\
\hline & \\
\hline & \\
\hline
\end{tabular}




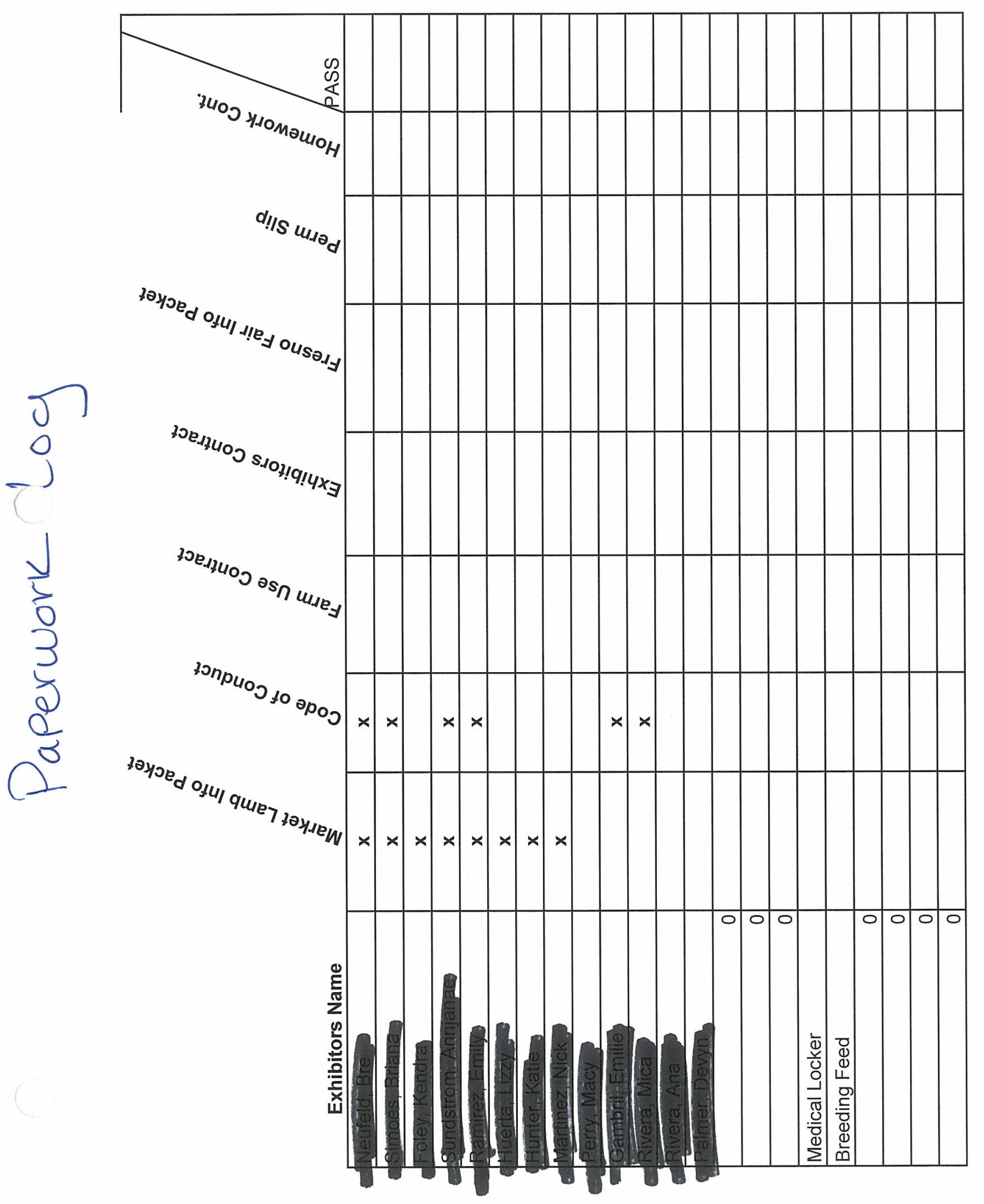




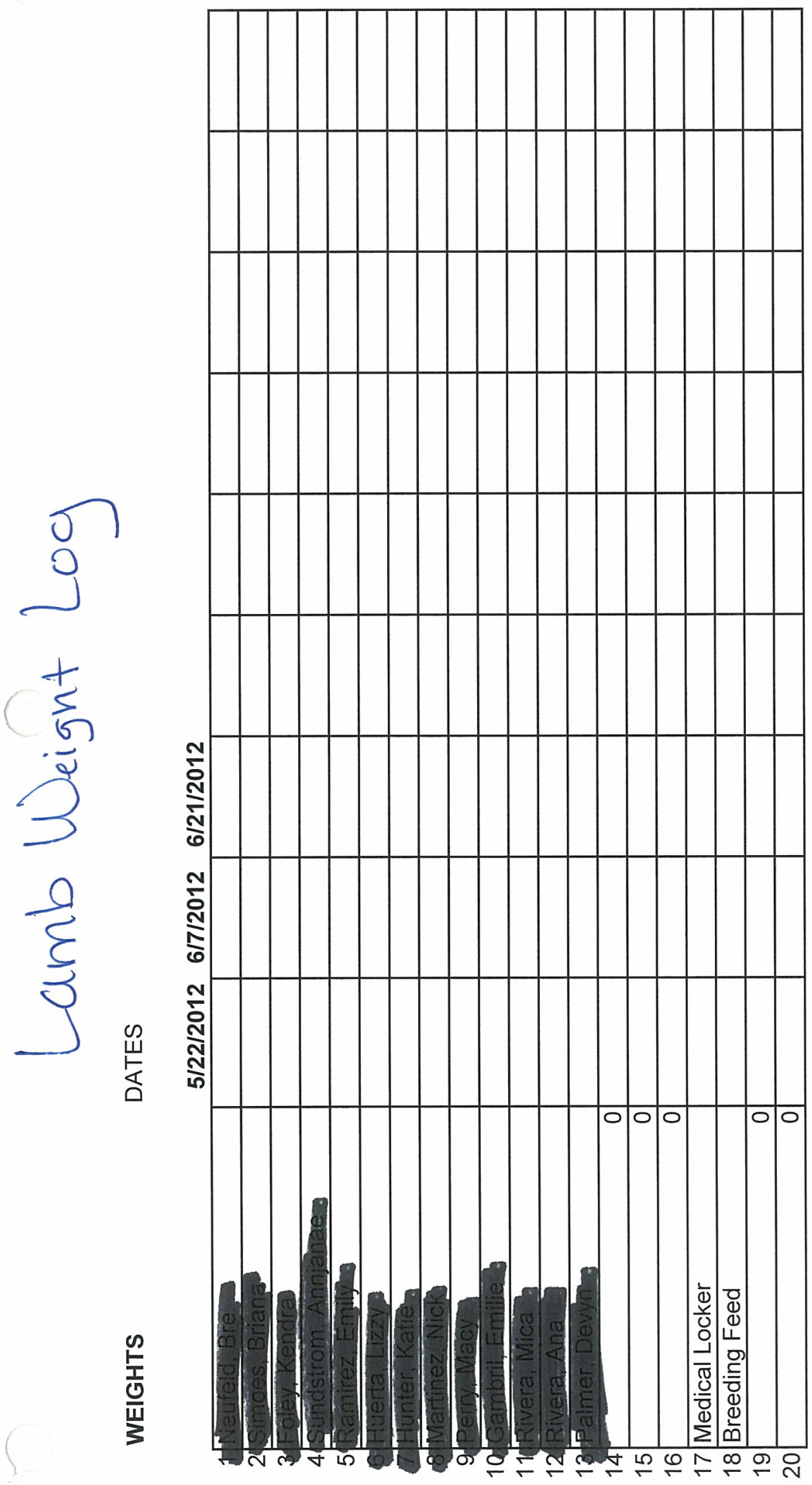




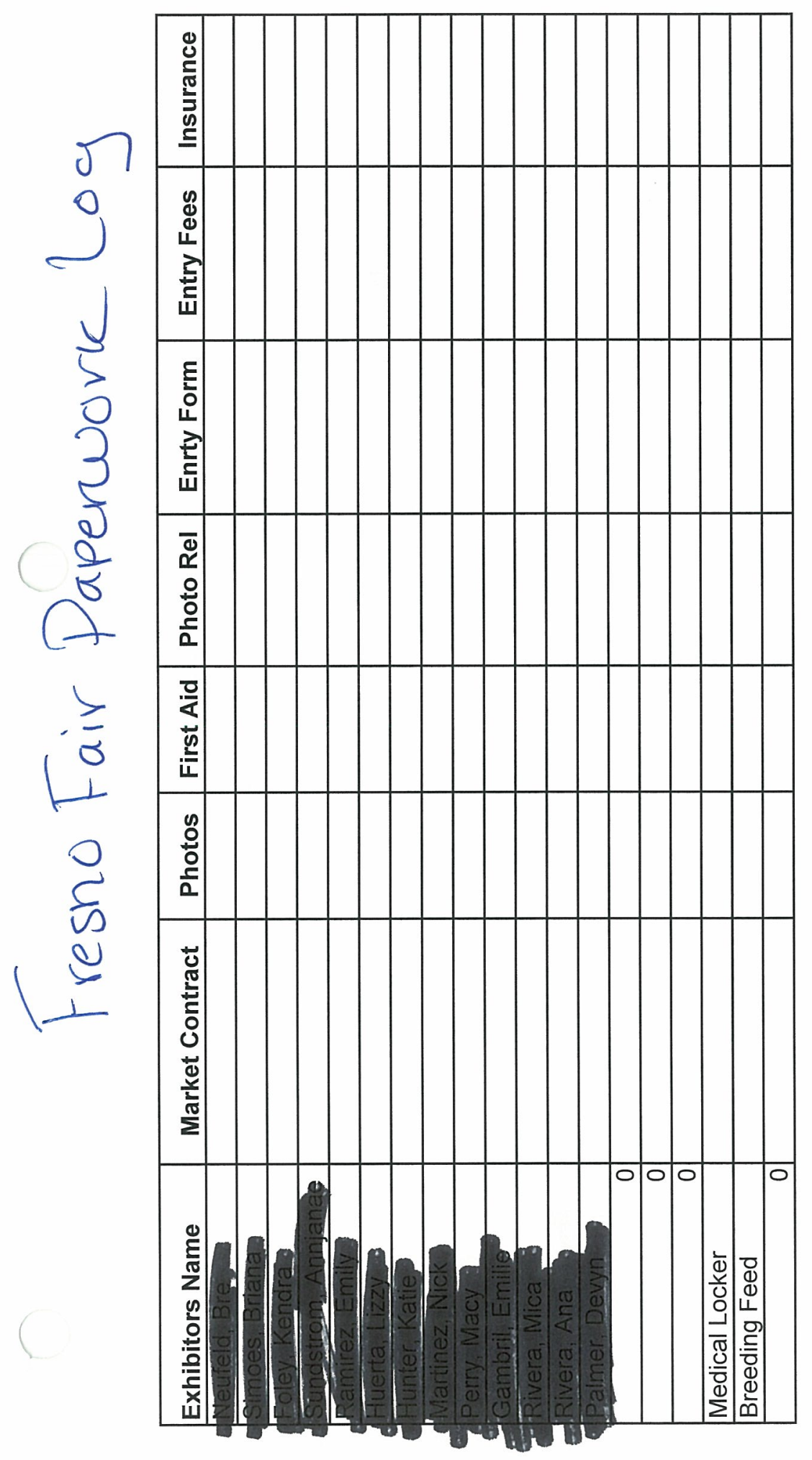




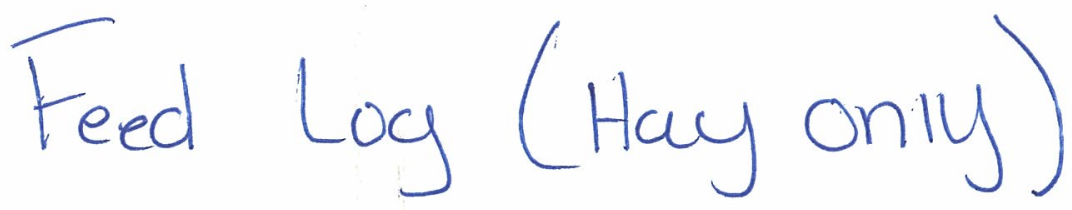

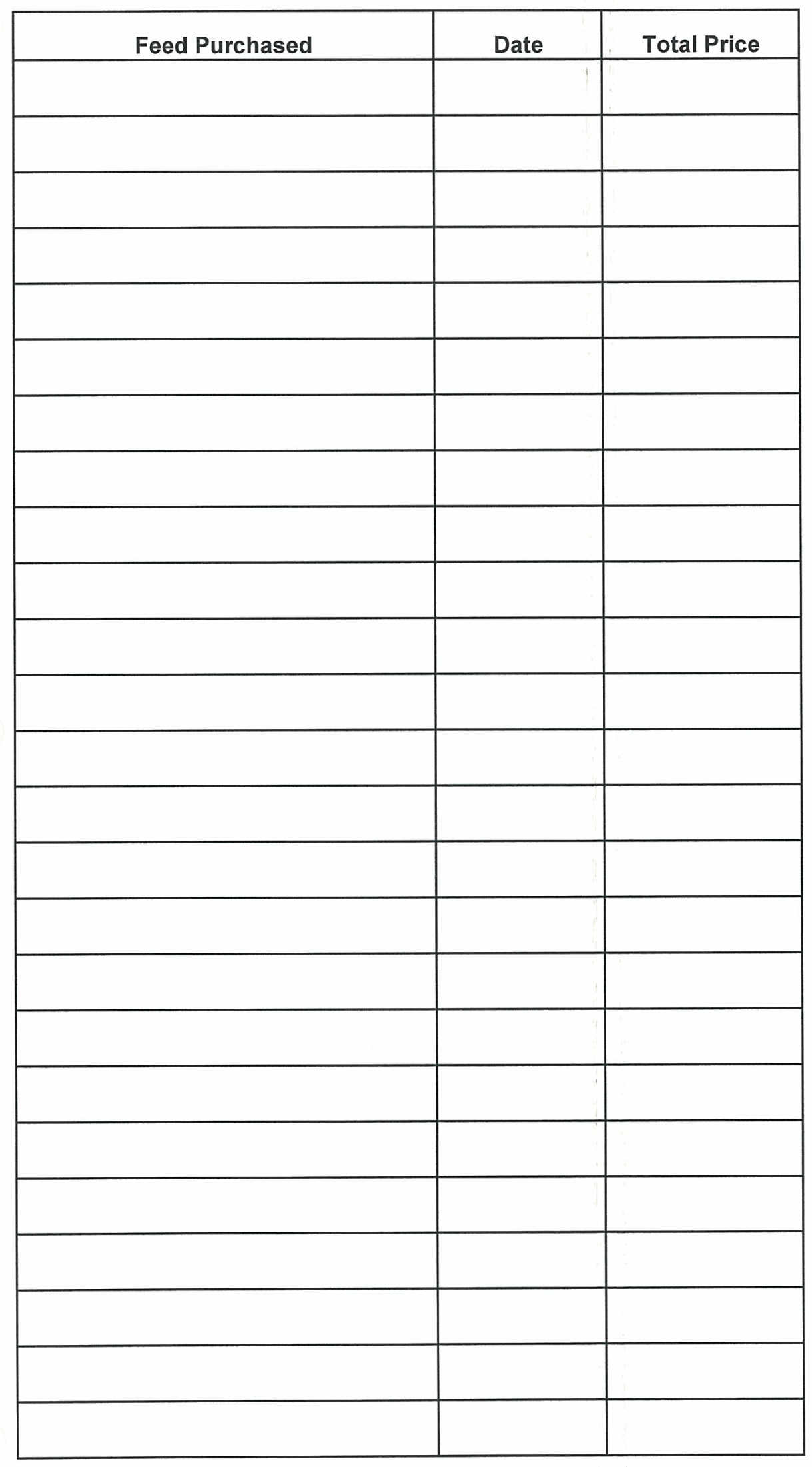




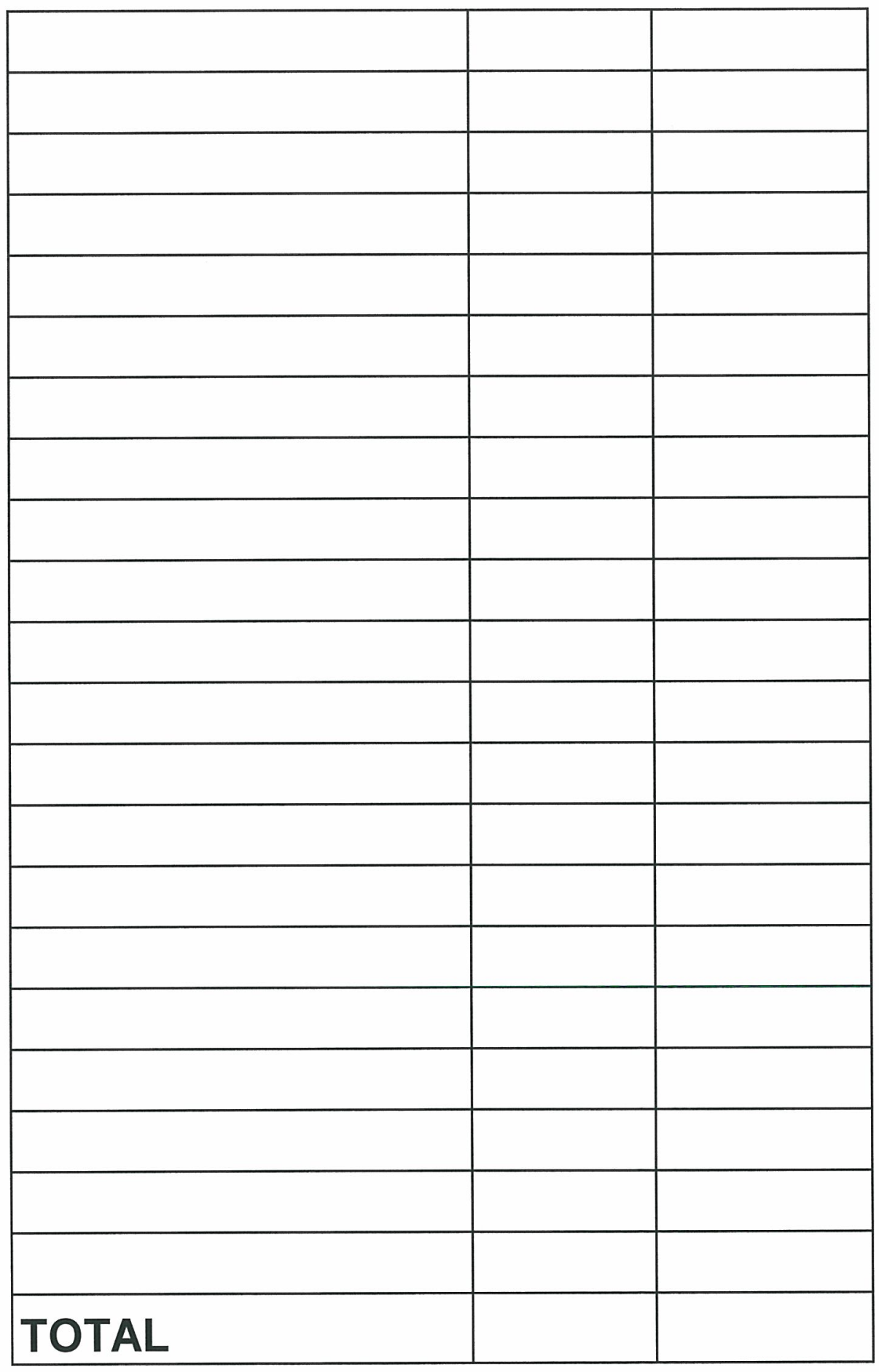




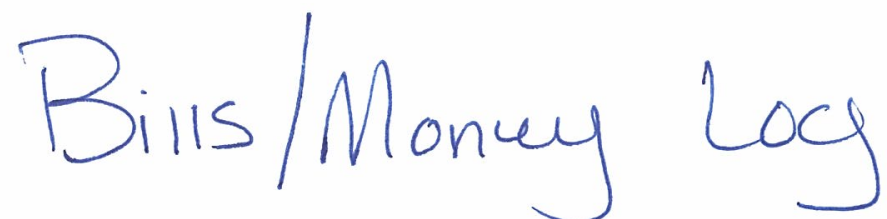

\begin{tabular}{|c|c|c|c|c|c|c|}
\hline Exhibitors Name & \multicolumn{2}{|l|}{ Balance } & Price of Lamb & \multirow[t]{2}{*}{ Feed Bill } & \multirow{2}{*}{$\begin{array}{l}\text { Fair Supplies } \\
\qquad 20.00\end{array}$} & \multirow{2}{*}{$\begin{array}{l}\text { Deposit } \\
\$ 250.00\end{array}$} \\
\hline & & $\$ 70.00$ & $\$ 300.00$ & & & \\
\hline & & $\$ 70.00$ & $\$ 300.00$ & & $\$ 20.00$ & $\$ 250.00$ \\
\hline 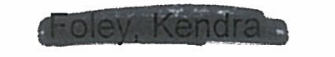 & & $\$ 70.00$ & $\$ 300.00$ & & $\$ 20.00$ & $\$ 250.00$ \\
\hline ndstrom. & ته & $\$ 70.00$ & $\$ 300.00$ & & $\$ 20.00$ & $\$ 250.00$ \\
\hline Mirez, En & & $\$ 20.00$ & $\$ 300.00$ & & $\$ 20.00$ & $\$ 300.00$ \\
\hline arta? iz & & $\$ 320.00$ & $\$ 300.00$ & & $\$ 20.00$ & $\$ 0.00$ \\
\hline if & & $\$ 20.00$ & $\$ 300.00$ & & $\$ 20.00$ & $\$ 300.00$ \\
\hline intunez, NI & & $\$ 70.00$ & $\$ 300.00$ & & $\$ 20.00$ & $\$ 250.00$ \\
\hline erry, Macy & & $\$ 20.00$ & $\$ 0.00$ & & $\$ 20.00$ & $\$ 0.00$ \\
\hline ambril,Emilie & & $\$ 20.00$ & $\$ 0.00$ & & $\$ 20.00$ & $\$ 0.00$ \\
\hline ivera Mic: & & $\$ 20.00$ & $\$ 0.00$ & & $\$ 20.00$ & $\$ 0.00$ \\
\hline & & $\$ 20.00$ & $\$ 0.00$ & & $\$ 20.00$ & $\$ 0.00$ \\
\hline almer, Devyan & & $\$ 20.00$ & & & $\$ 20.00$ & \\
\hline & 0 & $\$ 20.00$ & & & $\$ 20.00$ & \\
\hline & 0 & $\$ 20.00$ & & & $\$ 20.00$ & \\
\hline & 0 & $\$ 20.00$ & & & $\$ 20.00$ & \\
\hline Medical Locker & & $\$ 20.00$ & & & $\$ 20.00$ & \\
\hline Breeding Feed & & $\$ 0.00$ & & & & \\
\hline & 0 & $\$ 0.00$ & & & & \\
\hline & 0 & $\$ 0.00$ & & & & \\
\hline & 0 & $\$ 0.00$ & & & & \\
\hline & 0 & & & & & \\
\hline & 0 & & & & & \\
\hline
\end{tabular}




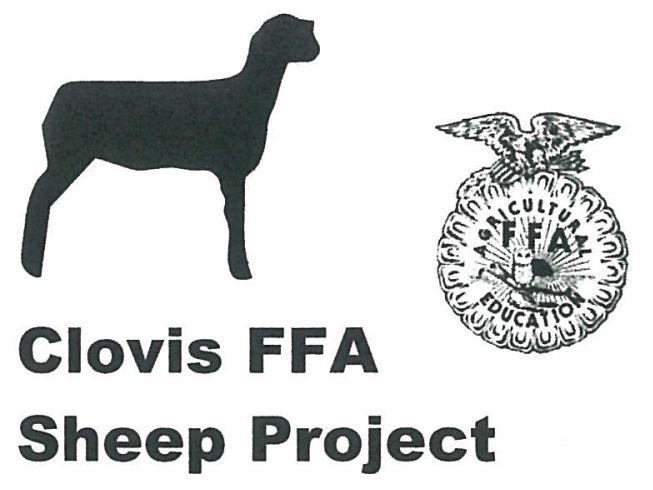

Sheep Exhibitor

Dates and Times

Highlighted are Meetings that are required for Exhibitors keeping their animals at home.

\section{(MUST ATTEND ONE OF THESE TWO Friday MEETINGS)}

Friday, May $10^{\text {th }} 3: 00-4: 30 \mathrm{pm}$ Room 903, Record Book Start Up Farm and Exhibitors Contract Lamb Care

\section{CHOOSE ONE}

Friday, May $17^{\text {th }} 3: 00-4: 30 \mathrm{pm}$ Room 903

Record Book Start Up

Farm and Exhibitors Contract Lamb Care

Monday, May $20^{\text {th }}$ - After School PICKING LAMBS (Take Ownership) Balance owed DUE

Wednesday, May $29^{\text {th }}-$ Sheep Meeting After School

Monday, June $3^{\text {rd }} 3: 00 \mathrm{pm}$ Meeting and Practice

Tuesday, June $11^{\text {th }} 6: 30 \mathrm{pm}$ Meeting and Practice
Wednesday, June $19^{\text {th }} 6: 30 \mathrm{pm}$ Meeting and Practice

Thursday, June $27^{\text {th }} 6: 30 \mathrm{pm}$

Tagging and Photos for Fair Contracts

Monday, July $8^{\text {th }} 6: 30 \mathrm{pm}$ Fair Entries and Contracts

Tuesday, July $9^{\text {th }} 6: 30 \mathrm{pm}$ MONDATORY Fresno Fair

Exhibitors Meeting

Fresno Fair Grounds 6:30pm

Monday, July $15^{\text {th }} 6: 30 \mathrm{pm}$

Meeting and Practice

Monday, July $22^{\text {nd }} 6: 30 \mathrm{pm}$

Record Book work

Saturday, July $27^{\text {th }} 8: 00 \mathrm{am}-12$ noon

Optional Rate of Gain Weigh in at the Fresno Fair Grounds

Sunday, July $28^{\text {th }}$ (Not a Meeting) ALL FAIR ENTRIES AND CONTRACTS DUE to Fairgrounds

Monday, July $29^{\text {th }} 6: 30 \mathrm{pm}$ Meeting and Practice

Monday, August $5^{\text {th }} 6: 30 \mathrm{pm}$ Meeting and Practice

Monday, August $12^{\text {th }} 6: 30 \mathrm{pm}$ Meeting and Practice

Monday, August $26^{\text {th }} 3: 00 \mathrm{pm}$ Meeting and Practice

Wednesday, September $4^{\text {th }}$ 3:00pm

Fresno Fair Planning Meeting Fair Plans and Displays 
Monday, September $9^{\text {th }} 3: 00 \mathrm{pm}$

Meeting and Practice

Shearing for Fall Classic

Tuesday, September $10^{\text {th }}$

Shearing for Fall Classic

Wednesday, September $11^{\text {th }}$

FALL CLASSIC SHOW at CEHS

Monday, September $16^{\text {th }} 3: 00 \mathrm{pm}$

Record Book work

** Fresno Fair Parent Meeting DBD

Possible Dates 9-19 or 9-26

MANTOTORY FOR ALL

EXHIBITORS

Monday, September $23^{\text {rd }} 3: 00 \mathrm{pm}$

Meeting and Practice

Wednesday, September $25^{\text {th }}$

3:00pm

Work on Fair Displays and Prep

Equipment

Friday, September $27^{\text {th }} 3: 00 \mathrm{pm}$

Work on Fair Displays and Prep

Equipment

Monday, September $30^{\text {th }} 3: 00 \mathrm{pm}$

Meeting and Practice

SHERING DATES, SIGN UP FOR

ONE OR THE OTHER

Saturday, October $5^{\text {th }} 8: 00 \mathrm{am}$

Shearing for Fair

Sunday, October $6^{\text {th }} 8: 00$ am

Shearing for Fair

Monday, October $7^{\text {th }}$

Group of 3-4 Students

Fresno Fair Haul In

Eveyone else report after school
Tuesday, October $8^{\text {th }}$

At Fair all Day

Weigh In of Lambs

Wednesday, October $9^{\text {th }}$

At Fair all Day

Market Lamb Show

Thursday, October $10^{\text {th }}$

At Fair all Day

Lamb Showmanship

Friday, October $11^{\text {th }}$

Skeleton Crew of 2 Students

Everyone else reports to Fair after school

Saturday, October $12^{\text {th }}$

At Fair all day

Junior Livestock Auction

Sunday, October $13^{\text {th }}$

Morning Feeding Crew

Evening Feeding Crew

Monday, October $14^{\text {th }}$

Haul Out Crew after school

Monday, October $21^{\text {st }} 3: 00 \mathrm{pm}$

Fresno Fair Clean Up

Record Book work

Fair Check Sign Off Sheets 


\section{Big Times are Back Passport to FUN}

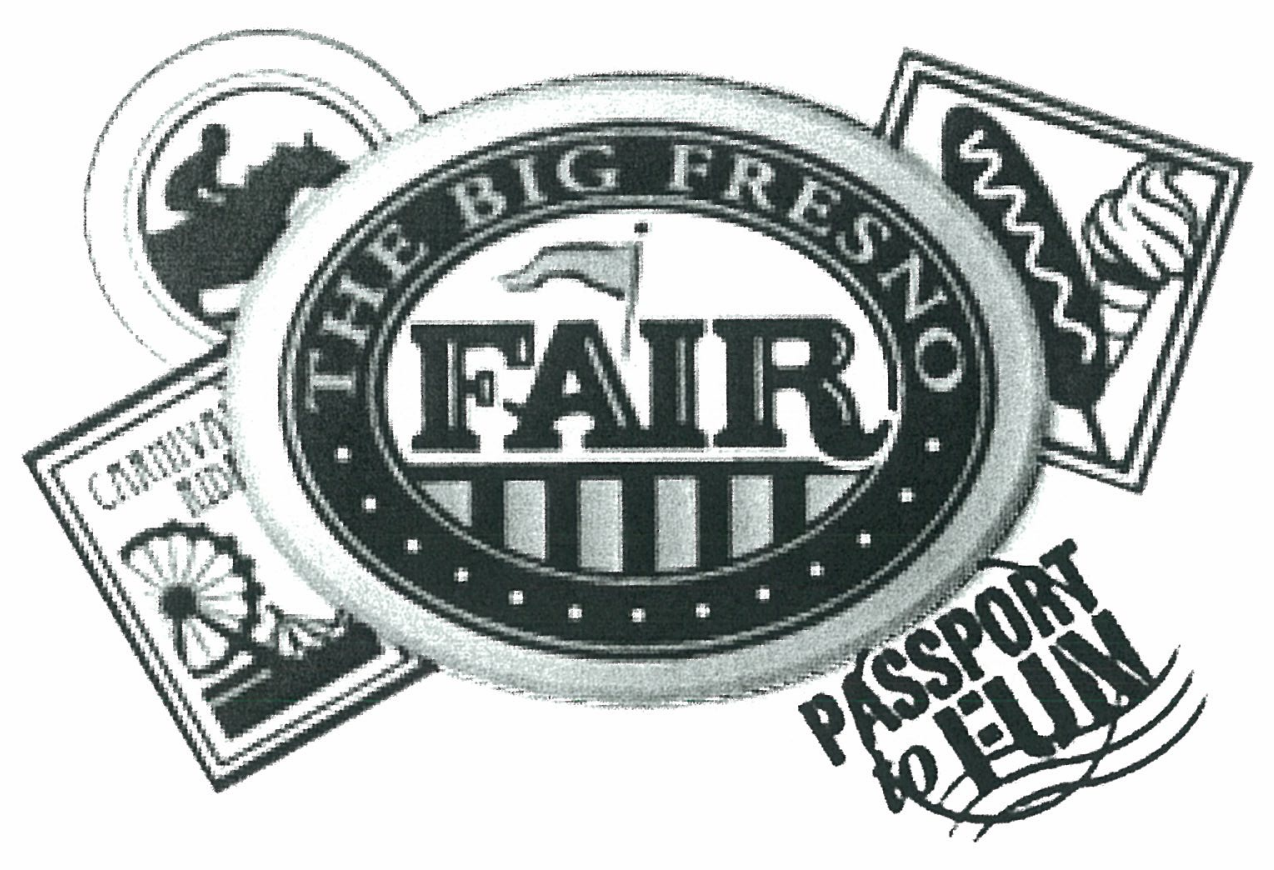

Clovis FFA Sheep Exhibitors 2012 


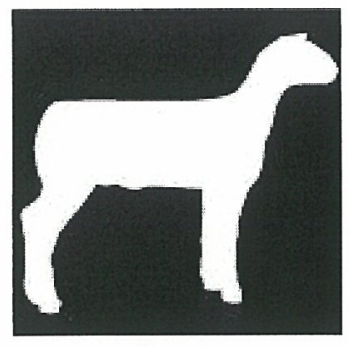

\section{Sheep Exhibitors}

\section{Fresno Fair Information}

- This is a school activity and all school rules will apply.

- You need to let your teachers know ahead of time that you will be missing class. Get your work before fair so that you can work on it after the show.

- If you are not on haul in crew on Monday you need to go to school. Also if you are not on skeleton crew on Friday you need to go to school! It is not ok to be out on independent study because when you go on independent study you MAY NOT participate in school activities like the fair. Also if you just choose to stay home and sleep it makes our Ag Department and FFA look bad in the eyes of the campus administration and other teachers.

- You will need your own ride to and from the fair. Ag Teachers will not be able to transport you. Being picked up and dropped of late may result in your loss of privileges to show the following year!

- If you plan on riding with another student or their parents you will need to bring a note from your parents saying it is ok.

- Most of the days we will leave the fair about 7:30pm. You will need to get a ride home from the fair because I don't have a way to transport 30 students at one time. An adult will have to check you out. DO NOT leave without checking out.

- Just because your parent wants go home early does not mean you can leave, you are committed to be at the barn for the times scheduled below.

-We are at the fair to exhibit livestock so don't expect to go to the midway.

- While at the fair you are expected to complete barn duty and stay in the sheep area during your assigned time.

- Make sure you have your buyers lined up.

- You will not lie in the pen with your animals while at the fair. 
- Care of your animal is your responsibility, don’t expect Mrs. Knight or your parents to do everything for you.

- We will all work together as a team, follow instructions from Mrs. Knight, and help each other out!

- I do not know exactly what time you will show! It depends on the size of the classes and the speed of the judge.

- IT IS MANDATORY THAT YOU ARE THERE ACCORDING TO THE SEHEDULE BELOW, NOT PARTICIPATAING OR CHOOSING TO NOT SHOW UP WILL RESULT IN STRIKES AND POSSIBLY LOOSING THE PRIVLEGE OF SHOWING WITH OUR GROUP NEXT YEAR.

\section{What Clovis FFA Will Provide with \$10 Fair Fee:}

- Alfalfa Hay for feeding

- Water Buckets

- Shavings for the Pen Bedding

- Clippers

- Sharpening of Clipper Blades

- Sheep Stands

- Show supplies

- Bright Lights Shampoo

- Freshen Up

- Black Hoof Paint

- Cool Blue

o Silk 


\section{Fair Supplies YOU Need to Bring}

- Two Towels for Each Show Day

- One Clean one to dry lamb

- One that you don't mind getting dirty to clean Ears \& Armpits

- $\quad$ FFA Show Uniform (Wed, Thurs, Sat.)

- CLEAN WHITE PANTS (you may need two pair because you may not have time to wash them between Wednesday and Thursday).

- White Button Up Shirt

- Belt (Black or Brown) NO Spikes, so Skulls or weird belt buckles.

- Boots or work Footwear (Black or Brown)- No Tennis Shoes

- FFA Jacket- Can barrow one if you don't have your own

- FFA Scarf or Tie

- Garment Bag to Keep Uniform Clean- If you lay it on anything it will get dirty.

**** DO NOT FORGET ANY PART OF YOUR UNIFORM! I do not have extra stuff to loan you, if you forget it your parent will have to bring it for you. You will not be allowed to show if you do not have your uniform; you don't show, you don't sell!

- Lamb Halter

○ To Tie Lamb up when washing and drying

- Shampoo for lamb washing

- Grain to feed your lamb

- Only bring what you need for the week, consider sharing with a friend who is feeding the same feed.

- Tack Boxes

- If you feel you must bring a tack box then you can bring one reasonably sized one.

- PLEASE do NOT bring a ton of "stuff" the more things we have in the isles the less room we have to work and it also looks messy for herdsmanship.

- Donation for Food or Sack Lunch

- You will be at fair during lunch and dinner times

- Wednesday \& Thursday Breakfast will be served to exhibitors. (For Free)

- The Ag Boosters will provide meals: breakfast, lunch, and dinner during the week for a donation of $\$ 25$ per student per week.

- You can pack food \& Snacks but you can't eat them in the barn.

- You can also purchase food at the Fair

**** If you or your parents get caught eating in the barn the Fair can kick you out of the show! Please only eat in the livestock exhibitors food tent.

$* * * *$ Whatever you bring please pack it neatly and in a small backpack, small plastic tub or tack box. We do not have tons of room in the sheep barn for storing your items. Also our barn gets judged on cleanliness and too much clutter is not good. 


\section{Pre-Fair Schedule:}

People who have their animals at home need to attend the Saturday and Sunday Practice prior to the Fair. Everyone must attend one of the Fair Display Board meetings. Those who keep animals at home should also talk to Mrs. Knight about their arrangements for Haul in.

Monday, September $24^{\text {th }}$

Tuesday, September $25^{\text {th }}$

Wednesday, September $26^{\text {th }}$

Thursday, September $27^{\text {th }}$

Monday, October $\mathbf{1}^{\text {st }}$

Tuesday, October $2^{\text {nd }}$

Wednesday, October $3^{\text {rd }}$

Friday, October $5^{\text {th }}$

Saturday, October $6^{\text {th }}$

8:00am-12 Noon

Sunday, October $7^{\text {th }} 2012$

11:00am- 4:00pm
Practice After School (Everyone)

Work on Fair Display Boards After School (Choose 1)

Practice 7am (Everyone)

Work on Fair Display Boards After School (Choose 1)

MANDATORY PARENT MEETING 6pm

Practice After School (Everyone)

Work on Display Boards after school (Choose 1)

Practice 7am (Everyone)

Shearing After School for Fair (Those on List)

1.

2.

3.

4.

5 .

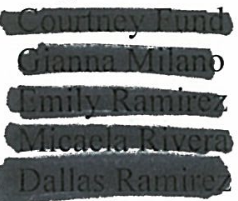

Shearing for Fair/ Working on Displays and Equipment (Practice) (EVERYONE)

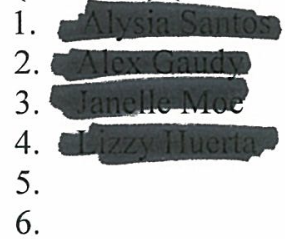

Shearing for Fair/ Loading tack and Equipment (EVEYONE)

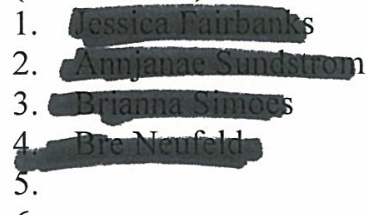

6.

\section{$\underline{\text { Fresno Fair Schedule }}$}

Monday, October $8^{\text {th }}$

Time To Be Determined

Haul In Crew Meet at Farm

Haul In Crew 
Tuesday, October $9^{\text {th }}$ 6:30am- 7:30pm
Be at Fair Grounds- Weigh In, Showmanship practice Touch Up and Clean Lambs Everyone

Wednesday, October $10^{\text {th }}$ 6:30am - 7:30pm

FFA Market Lamb Show - (Need Uniform)

Show starts at 8am and FFA Shows First Everyone

Thursday, October $11^{\text {th }}$

7:00am- 7:30pm

FFA Sheep Showmanship - (Need Uniform)

Show starts at 8 am $4-\mathrm{H}$ shows first.

Friday, October $12^{\text {th }}$ 7:00am- 7:00pm

\section{Skeleton Crew at Sheep Barn ALL DAY}

Skelton Crew-

1 .

2.

3.

3:00pm- 7:00pm Come to Fair after School

Paint Branding of Animals for Sale

Feed and Clean- All other exhibitors

Saturday, October $13^{\text {th }}$

7:30 am- 7:00pm

Livestock Auction Day- (Need Uniform)
Auction Begins at 8:30am

Everyone

*** You MUST be at the Fair ALL DAY SATURDAY. You may not leave after you are done selling your animal!

Sunday, October $14^{\text {th }}$

Last day of Fair- Care of animals until haul out

Everyone in Shifts on Sunday

7:00am-11:00am Shift 1

1.

2.

3.

4.

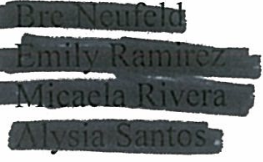

11:00am-3:00pm Shift 2

1.

2.

3.

4.

5.

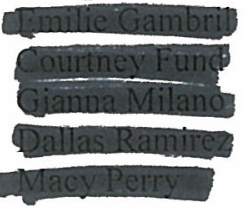


3:00pm-7:00pm Shift 3

1.

2.

3.

4.

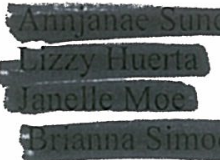

7:00pm-10:30pm Shift 4 HAUL OUT

1.

2.

3.

4.

5.

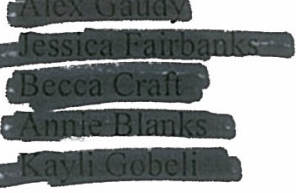

I have read and understand the fair schedule and rules.

Student Name Printed

Parent Name Printed
Student Signature

Parent Signature

Parent Cell Phone \#

The Clovis Unified School District does not discriminate on the basis of race, color, disability, or national origin in admission or access to and treatment of employment in its programs and activities as required by Title VI, Title IX and Section 504. Complaints: Contact Dr. Cheryl Rogers, Human Resources, (559) 327-9308.

Favor de llamar a la oficina para assistencia en espanol.

Thov tiv tauj peb lub hoob kas yog xav tau kev pab nrog lus Hmoob. 


\section{Clovis FFA Sheep Barn Duty Schedule:}

Tuesday, October $9^{\text {th }}$

6:30am-8:30am

1.

2.

8:30am-10:30am

1.

2.

10:30am-12:30pm

1.

2.

$12: 30 \mathrm{pm}-2: 30 \mathrm{pm}$

1.

2.

2:30pm-4:30pm

1.

2.

4:30-6:30pm

1.

2.

Wednesday, October $10^{\text {th }}$

6:30am-8:30am

1.

2.

8:30am-10:30am

1.

2.

10:30am-12:30pm

1.

2.

$12: 30 \mathrm{pm}-2: 30 \mathrm{pm}$

1.

2.

2:30pm-4:30pm

1.

2.

4:30-6:30pm

1.

2. 
Thursday, October $11^{\text {th }}$

7:00am-8:30am

1.

2.

8:30am-10:30am

1.

2.

10:30am-12:30pm

1.

2.

12:30pm-2:30pm

1.

2.

$2: 30 \mathrm{pm}-4: 30 \mathrm{pm}$

1.

2.

4:30-6:30pm

1.

2.

Friday, October $12^{\text {th }}$

4:30-6:30pm

1.

2.

Saturday, October $13^{\text {th }}$

8:30am-10:30am

1.

2.

10:30am-12:30pm

1.

2.

$12: 30 \mathrm{pm}-2: 30 \mathrm{pm}$

1.

2.

2:30pm-4:30pm

1.

2.

4:30-6:30pm

1.

2. 


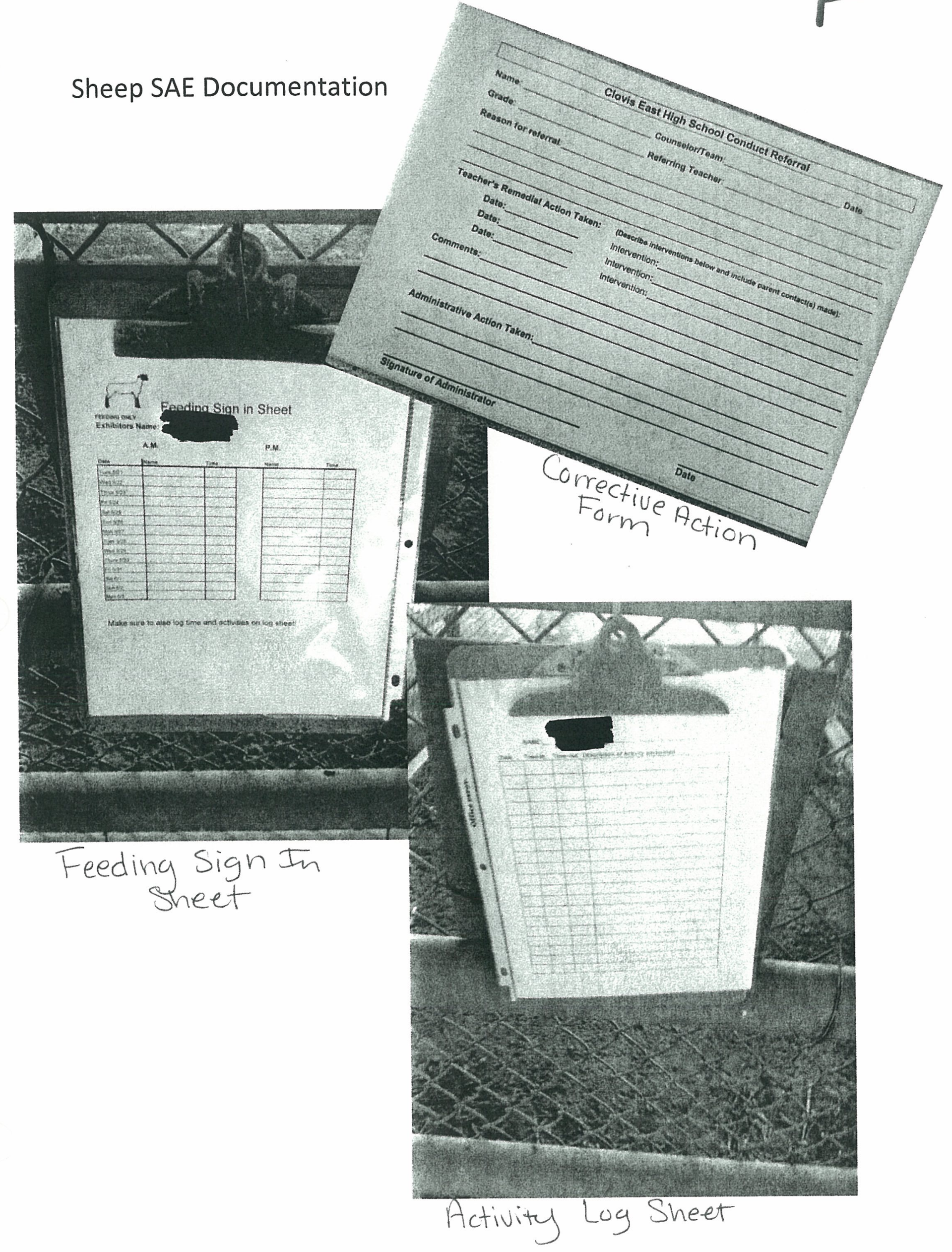




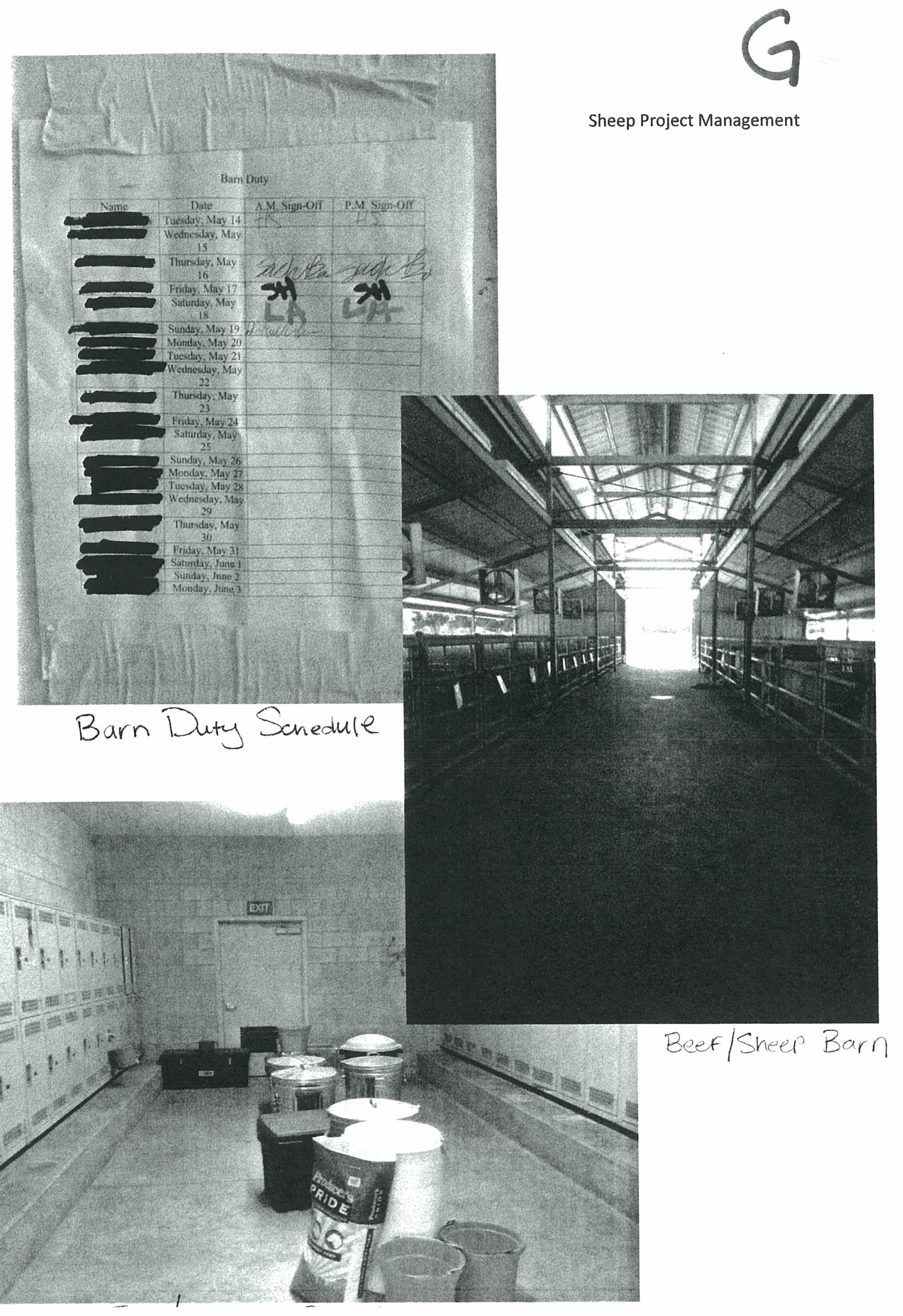




\section{Ag Vehicle Fleet}

\section{$H$}

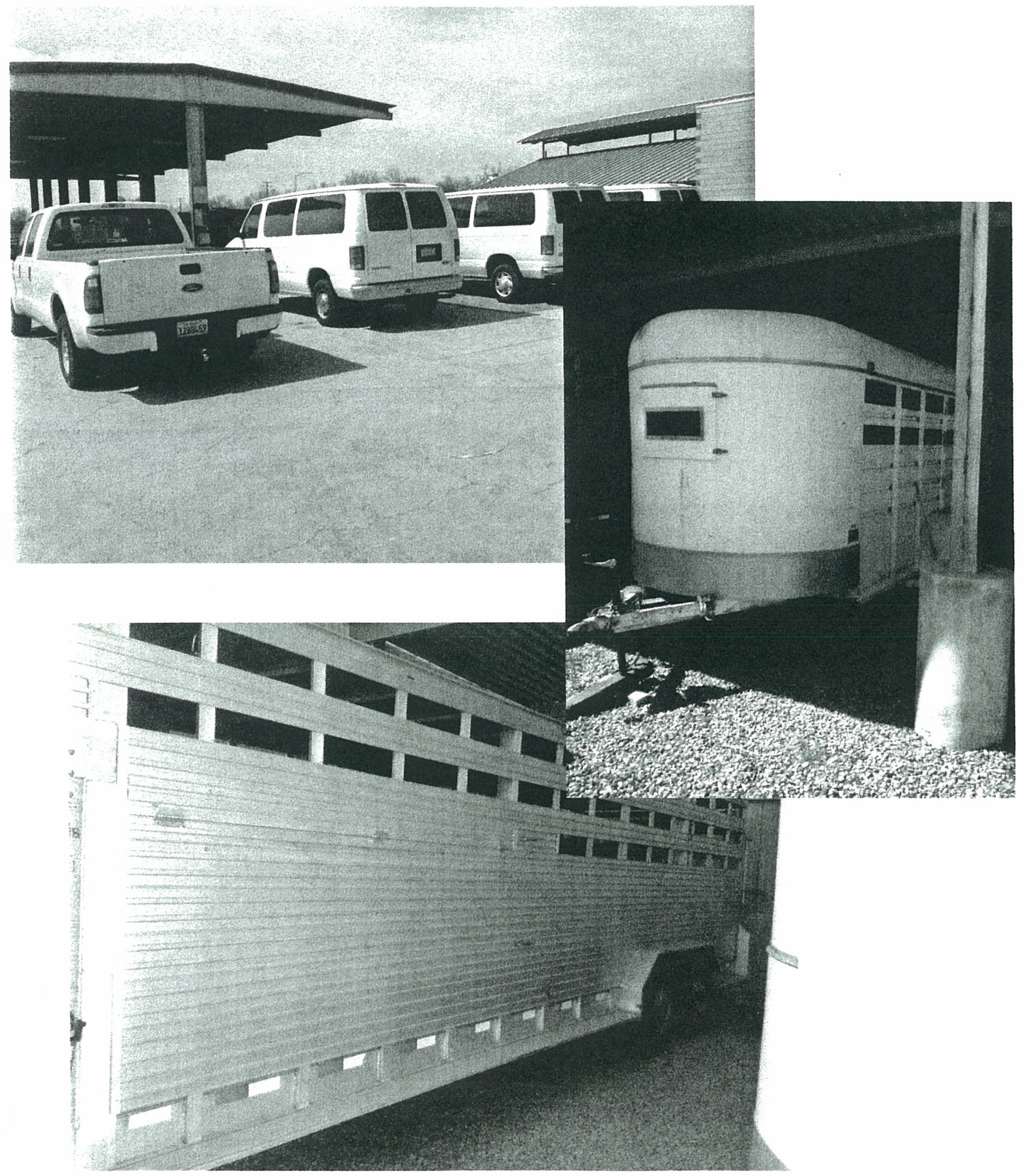




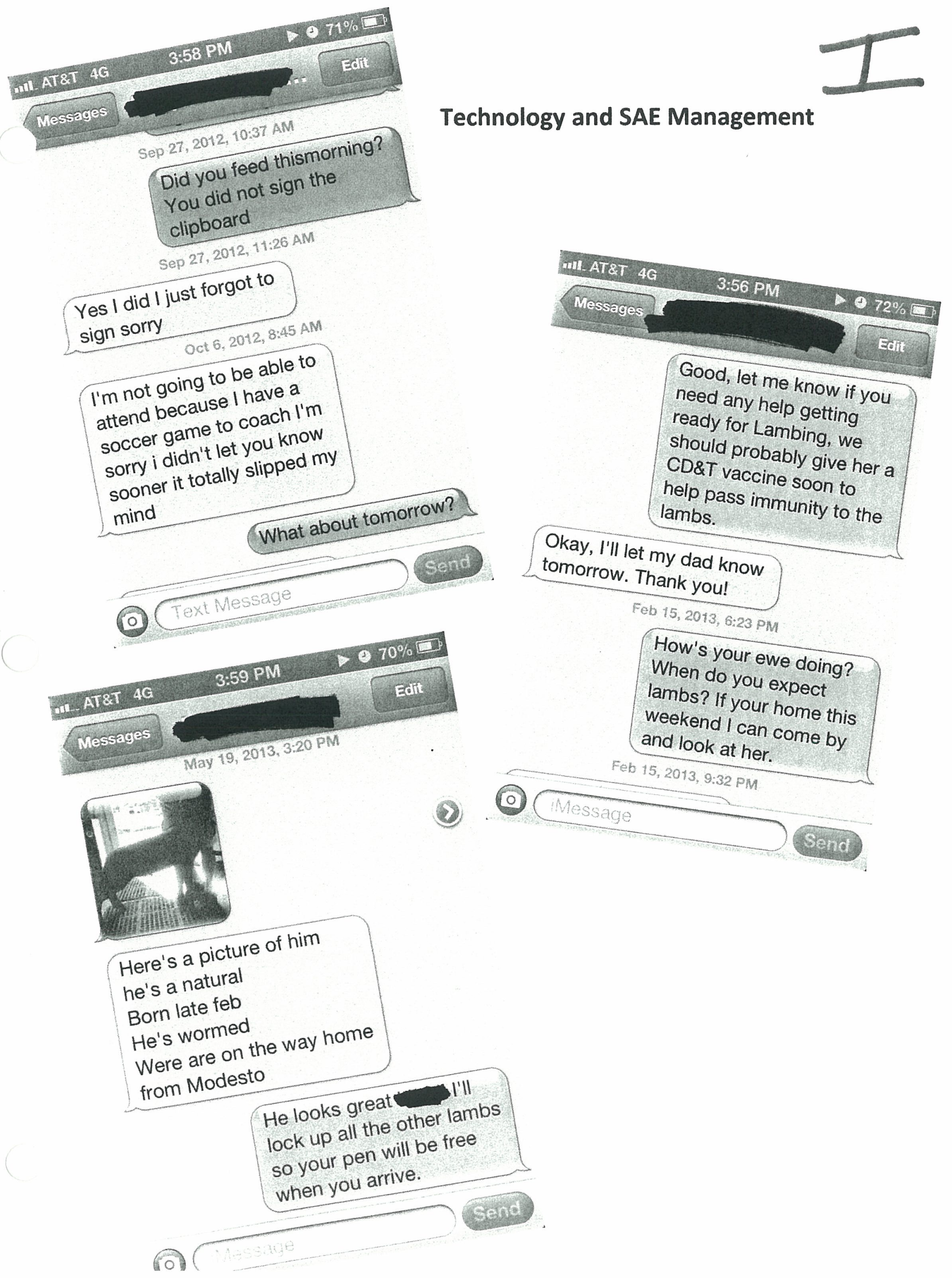




\section{Sheep Exhibitor Project Completion Sign Off Sheet}

NAME OF EXHIBITOR

\begin{tabular}{|c|c|c|}
\hline Item to be Completed & Date Completed & Advisor Signature \\
\hline $\begin{array}{l}\text { Clean Pen used at Farm- If you kept } \\
\text { your animal at the farm. }\end{array}$ & $10-22-12$ & \\
\hline $\begin{array}{l}\text { Clean out Locker and Remove Lock- } \\
\text { For students who kept animals at } \\
\text { Farm }\end{array}$ & 10.22 .12 & \\
\hline $\begin{array}{l}\text { Two Hours Farm Clean-Up-If you } \\
\text { kept your Animal at the Farm }\end{array}$ & $10 \cdot 22 \cdot 12$ & \\
\hline $\begin{array}{l}\text { Thank you letter to Buyer-Show } \\
\text { original to Mrs. Knight BEFORE you } \\
\text { mail it out. }\end{array}$ & $11-13-12$ & \\
\hline $\begin{array}{l}\text { Thank you letter(s) to Bump Bidders- } \\
\text { Show Original(s) to Mrs. Knight prior } \\
\text { to mailing out. }\end{array}$ & $11-13-13$ & \\
\hline $\begin{array}{l}\text { Thank letter to the Fresno Fair- Show } \\
\text { Original(s) to Mrs. Knight prior to } \\
\text { mailing out. }\end{array}$ & $11-1312$ & \\
\hline $\begin{array}{l}\text { Thank you letter to Ag Boosters- } \\
\text { Give Original Letter to Mrs. Knight }\end{array}$ & $11-13-12$ & \\
\hline $\begin{array}{l}\text { Record Book Up to Date- If this was } \\
\text { the end of your project(s) for the year } \\
\text { books must be closed out. }\end{array}$ & $10 / 23 / 12$ & \\
\hline $\begin{array}{l}\text { Degree Application Completed- If } \\
\text { you qualify for the Greenhand, } \\
\text { Chapter, State or American FFA } \\
\text { Degree YOU MUST SUBMIT A } \\
\text { COMPLETED APPLICATION WITH } \\
\text { YOUR RECORD BOOK(S) }\end{array}$ & $11-13-12$ & \\
\hline $\begin{array}{l}\text { All Bills/Fees to Clovis FFA Paid in } \\
\text { Full. }\end{array}$ & $10-22-12$ & \\
\hline
\end{tabular}




\section{EDUCATION CODE SECTION 52450-52454}

32454. (a) The curriculum of school districts that choose to participate in the state program of agriculturall career technical ducation shall include all of the following components:

(1) Organized classes in the study of agricultural science and technology.

(2) A student-supervised occupational experience program in aquiculture.

(3) A program of leadership, organization, and personal development.

(b) Student learning activity developed to supplement these components shatl be considered curricular and shall contribute to the grade of the participating student when those activities are integral to assisting the student to achieve the career objective of. the clasis or course.

It is the intent of the Legislature that opportunities are provided for teachers to be employed on a 12-month basis in order to maintain smpervised occupational experience on a year-round basis for students ensolled in agricultural career technical programs. 


\section{Quality Criteria Four}

\section{Qualified and Professional Personnel}

There are six full time agriculture teachers at Clovis East High School, all of which have an Agriculture Teaching Credential. Four of the six teachers also hold a Masters Degree in Agriculture Education. Instructors are assigned courses to teach based on their experience and strengths in agriculture. All teachers have been awarded with an outstanding teacher award by the CATA and we have had four teachers named as Teachers of Excellence.

Each year we complete a chart of responsibilities for the Ag Staff which states what each teacher will be responsible for, projects they will advise, farm areas they will care for, FFA activities they will attend, and what Professional Development and CATA events they plan to participate in. All Ag Staff attend a minimum of three professional development seminars or meetings annually. These include but are not limited to Regional road shows, State CATA Conference, Agri Skills seminars, Regional CATA Meetings, Vision 2013, California DELTA conferences, and Educating for Careers Conference. Many of our staff are asked to be presenters at various professional events, as well as lead school wide professional development sessions. Above and beyond general professional development our Ag Staff remain active in the Agriculture Community serving on boards and belonging to other agricultural associations and groups. Many of our staff are personally involved in production agriculture.

Our Ag Staff meets weekly for a department meeting where we can discuss the activities for the upcoming weeks and create action plans for things needing to be done. We can also voice any concerns we may have and our Department Chair will carry them forward to our Learning Director. Our Department Chair also keeps a detailed account of all meetings and sends us via email the minutes shortly after the conclusion of each meeting. One of our biggest challenges is that we just never seem to have enough time to discuss everything we would like to, there is always just so much going on.

Our Ag Staff is also involved in the professional development and Professional Learning Communities on Campus for general education teachers. We attend staff meetings 
with all school staff and are a part of the Science PLC. Mrs. Covey is an important part of the Biology PLC and has contributed to the science curriculum greatly, and Mr. Woodard is the head of the Earth Science PLC group on campus. Our Ag Teachers are respected on campus as master science teachers and experts in our field.

Our Ag staff also does our part in training our future agriculture educators. We consistently have one to two student teachers on campus from Fresno State, who are working on obtaining their teaching credential. As an Ag Staff we see the investment of our time in these students as an investment in the future of agriculture. We know how difficult it can be for new teachers and we don't want them to give up or get discouraged so we provide them with as much support and resources as possible in order to help them be successful.

Our Ag Staff at Clovis East are Professional people who are always striving to do the right thing. We have the support of our community and parents because of our commitment to professionalism and a quality program. This is as simple as showing up to work dressed to impress, even though we could just wear jeans and boots we don't. We take pride in setting a professional example for our students. We also are cautious of how we represent our program in our personal lives by using social media appropriately and behaving ourselves at community social functions. Clovis Unified only hires the best, and we are proud of this and will show it in everything that we do.

\section{Supporting Completion Materials}

Item A- Copies of my teaching credentials.

Item B- Sub Plans. I have included two copies of Sub Plans that I have recently used this spring. They include comments from the subs.

Item C \& D- Agriculture Department Meeting Agenda. Mr. Woodard types notes into these during the meetings and emails them out to us later that day. He also includes our 


\section{Ag Ed 539 Teacher Internship Report}

Agriculture Department Administrator and her Secretary so they know what is going on also.

Item E- Ag Staff Chart of responsibilities.

Item F- Ag Staff Picture

Item G- List of Professional Development for the 2012-2013 School Year

Item H-Professional and Personal Goals. Created in the summer of 2012 for AGED 520 Class.

Item I- Letter to Administration (Stephanie Hanks) thanking her for time off this year for Professional Development, and skills gained from my training and experiences.

Item J-Thank you letter from Mr. Cummings for presenting at the New Professionals Conference.

Item K- Copy of my CATA Membership Card 

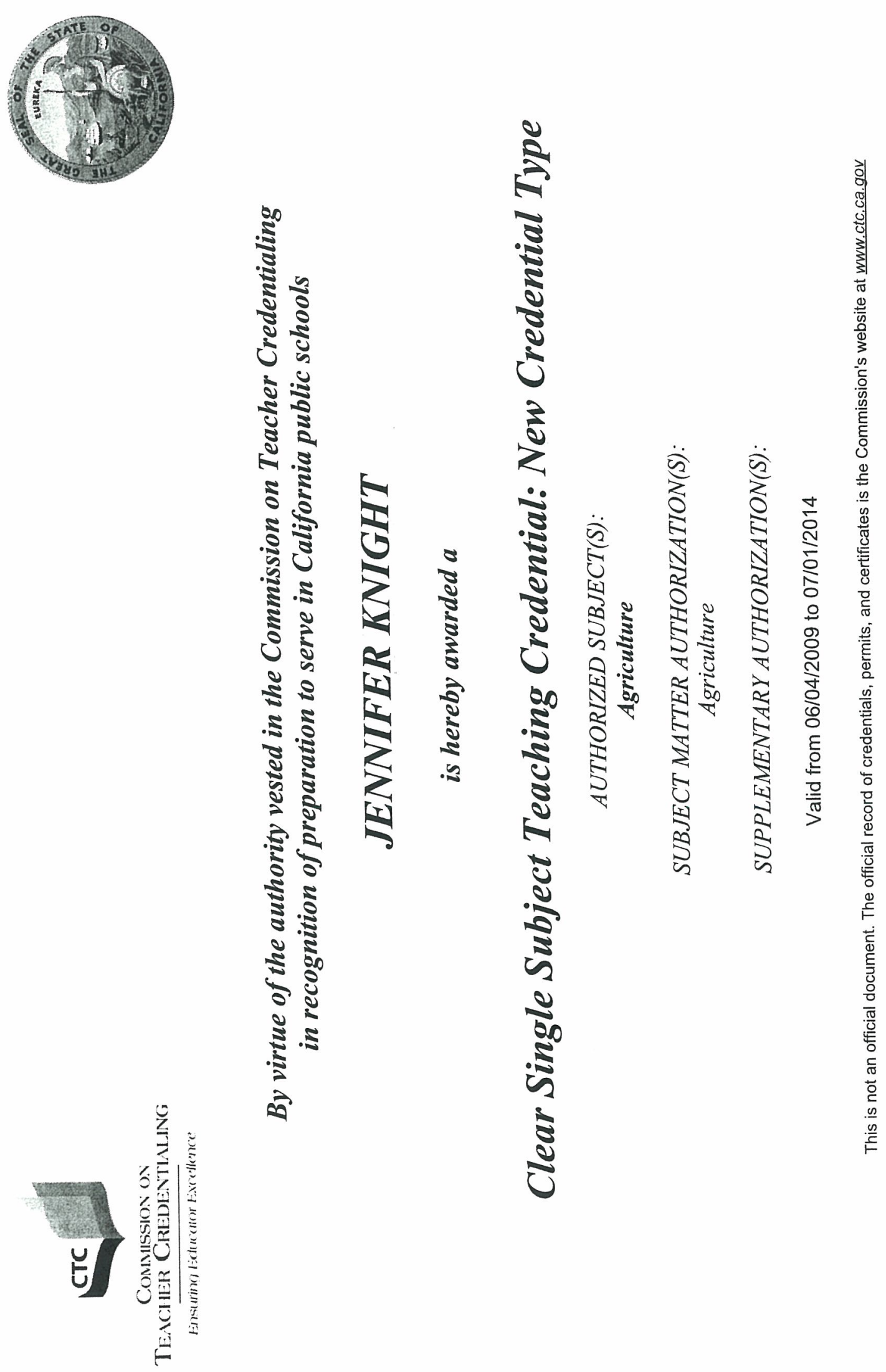

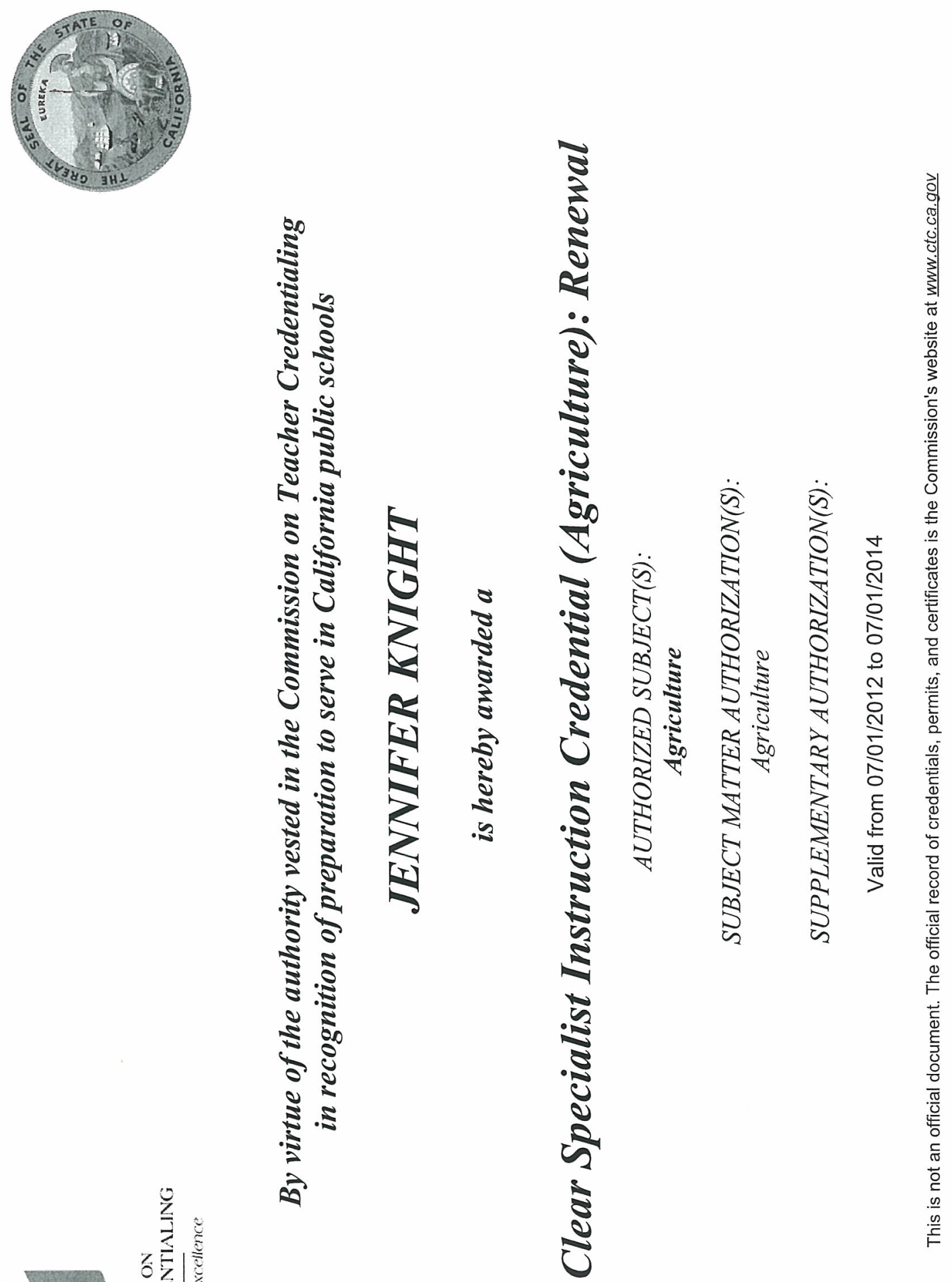
Sub Lesson Plans

Mrs. Knight's Agriculture Courses (Room 903)

Contact \# for Any Questions (559) 765-9580

Tuesday, April 30 2013

Class Schedule - Regular Schedule

Period 1 Botany

Period 2 Animal Science

Period 4 Exploring Ag $\left(7^{\text {th }} \& 8^{\text {th }}\right.$ Graders $)$

Period 6\& 7 ROP Vet Science

General Classroom Procedures:

- When students enter the classroom they are to get their notebooks or Binders out and begin working on Daily Warm Ups.

- Students should begin working on their Daily Warm Up at the beginning of class.

- Please take attendance on the rosters I have left for you each day. I will have to enter attendance each evening, so please leave these rosters for me to use.

- Students must raise their hand to ask a question or get out of their seats.

- IPod and CD Players are never allowed!

- Restroom use is a privilege, and they will need to give you 1 Yellow or Blue ticket to be able to use the restroom. Please ask them for a ticket when they ask to use the restroom, no ticket, no restroom!

- Class will not be dismissed unless students clean up their surrounding area, and put all supplies away.

- If students have work to turn in they can place it in the black wire basket on the West counter.

- Feel free to write students up on referrals to the SRC or leave notes on who caused problems. I have left forms out for this purpose.

- NOTE: ROP Vet does not meet in class today, they are all out on Job Sites.

Note: If it gets Warm, Push the small round button 8 times to run the $A C$. It will run for 4 Hours at a time Lower the temp with the Arrow buttons Afc unit is located to the right of the Phone Near the East Dour. 


\section{$\underline{\text { Period 1: Botany }}$}

1. Write on the dry erase board (the eastern most side) just like listed below:

Today's Lesson Topic: Plant Selection and the Western Garden Book

Learning Objective: Learn how to use the Western Garden Book as a plant selection tool.

Mind Moover: What things should you consider when selecting a plant for the landscape?

Assignment:

- Western Garden Book Plant Hunt (Due at end of Class)

$\sqrt{2}$ When students arrive to class they should begin on their daily warm ups.

$\sqrt{3}$. Take attendance while they are working on their daily warm up.

4. Have students share their responses to the mind mover. Give 1 blue ticket to the students who share answers with the class.

5. Pass out the Western Garden Book plant hunt assignment, students may work in pairs with the Western Garden Books to complete the assignment. One paper per student due at the end of the period.

6. Make sure they clean up and put books away at the end of the period. 


\section{Period 2: Animal Science}

1. Write on the dry erase board (the eastern most side) just like listed below:

Today's Lesson Topic: Breeding Heifer Evaluation

Learning Objective: Identify the major factors to consider when selecting a beef heifer. Analyze and interpret performance records for a sample judging class.

Mind Moover: How can EPD data be helpful when selecting heifers?

Assignment: Video Quiz Packet (Due Today)

2. When students arrive to class they should begin on their daily warm ups.

L3. Take attendance while they are working on their daily warm up.

4 . Have students share their responses to the mind mover. Give 1 blue ticket to the students who share answers with the class.

15. Pass out the video quiz packet. Instruct them to complete the video quizzes and answer the questions during the video.

6. Put on the VHS titled Livestock Judging, breeding heifer evaluation. The projector and VCR should be all set up and ready to go, just push the power button on the projector and turn on the VHS player and insert the tape. Pull down the screen over the white boards.

V. If there is time after the VHS, then you can go over the packet answers with the class. I have provided the answer key for you to use.

$\sqrt{8 .}$ Video quiz packets are due at the end of class. 


\section{Period 4: Exploring Ag:}

ฟ. Write on the dry erase board (the eastern most side) just like listed below:

Today's Lesson Topic: Beef Harvest Video

Learning Objective: Take a virtual field trip of the Excel Beef Slaughter Plant to see how beef cattle are harvested for meat.

Mind Moover: Does the sight of blood bother you?

\section{Assignment:}

- Beef Slaughter Video Quizzes (Due after Video)

2. When students arrive to class they should begin on their daily warm ups.

3. Take attendance while they are working on their daily warm up. Have them respond to the mind mover. Give one blue ticket to those who respond.

4. Inform them that they will watch the beef cattle harvest video today. If they find it too much to handle they may put their head down, but inform them it shows exactly what we have already discussed in class and does NOT have lots of blood.

15. Pass out the Video quiz paper and put on the VHS titled Excel Beef Plant: Slaughter.

6. If there is time remaining after the video go over the video quiz answers with the students. Have them turn in their video quizzes at the end of the period.

17. Make sure students put away notebooks and supplies before they leave for lunch. 
Period 6 \& 7: ROP Vet Science:

Will not meet in class today, they are on Job Sites.

Comments/Feedback:

Sub Name:

Please leave comments here:

H. Mrs. Knight,

You have great classes! Lesson plans were followed of and went smoothly; even the Beef slaughter before lunch (0)

AII handout were collected; 99\%: of students in period 1 did not finish but they were working hard and quietly through out the period.

In Period two, I stopped the video periodically to check for un der standing; they have the last questions to finish...

Thank You! Plea are feel free to text me if you need a sub. I am a science and

chemistry teacher... finally have a job next Heart. But, if you need help, feel free to text! Thank you,

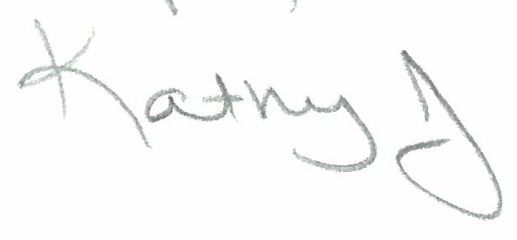




\section{Sub Lesson Plans \\ Mrs. Knight's Agriculture Courses (Room 903) \\ Contact \# for Any Questions (559) 765-9580}

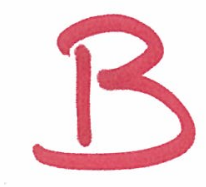

Friday, May $3^{\text {rd }} 2013$

Class Schedule - Regular Schedule

Period 1 Botany

Period 2 Animal Science

Period 4 Exploring $\mathrm{Ag}\left(7^{\text {th }} \& 8^{\text {th }}\right.$ Graders $)$

Period 6\& 7 ROP Vet Science

\section{General Classroom Procedures:}

- When students enter the classroom they are to get their notebooks or Binders out and begin working on Daily Warm Ups.

- Students should begin working on their Daily Warm Up at the beginning of class.

- Please take attendance on the rosters I have left for you each day. I will have to enter attendance each evening, so please leave these rosters for me to use.

- Students must raise their hand to ask a question or get out of their seats.

- IPods and CD Players are never allowed!

- Restroom use is a privilege, and they will need to give you 1 Yellow or Blue ticket to be able to use the restroom. Please ask them for a ticket when they ask to use the restroom, no ticket, no restroom!

- Class will not be dismissed unless students clean up their surrounding area, and put all supplies away.

- If students have work to turn in they can place it in the black wire basket on the West counter.

- Feel free to write students up on referrals to the SRC or leave notes on who caused problems. I have left forms out for this purpose.

- NOTE: ROP Vet does not meet in class today, they are all out on Job Sites. 


\section{$\underline{\text { Period 1: Botany }}$}

1. Write on the dry erase board (the eastern most side) just like listed below:

Today's Lesson Topic: Plant ID Quiz \&

Plant Selection and the Western Garden Book Cont...

Learning Objective: Test my knowledge of the five plants of the week. Learn how to use the Western Garden Book as a plant selection tool.

Mind Moover: What do you find challenging about using the Western Garden Book?

\section{Assignment:}

- Finish Western Garden Book Plant Hunt (Due at end of Class)

2. When students arrive to class they should begin on their daily warm ups.

3. Take attendance while they are working on their daily warm up.

4. Have students share their responses to the mind mover. Give 1 blue ticket to the students who share answers with the class.

5. Inform them that they have $5 \mathrm{~min}$ to review for their plant ID Quiz. After $5 \mathrm{~min}$ pass out the plant ID quiz, you will need to show them the plant ID Power Point for questions 6-10 (plant ID) It is saved on the desktop of the laptop on the projection cart.

6. Once they have all finished the Plant ID quiz, then have them switch papers and grade each other's test. I have provided the key so you can guide them through this process. Collect the graded tests.

7. Pass out the Western Garden Book plant hunt assignment that they began on Tuesday. Students may work in pairs with the Western Garden Books to complete the assignment. One paper per student due at the end of the period.

8. Make sure they clean up and put books away at the end of the period. 


\section{Period 2: Animal Science}

1. Write on the dry erase board (the eastern most side) just like listed below:

Today's Lesson Topic: Feed stuffs ID Quiz \& Technology in Beef Cattle Reproduction

Learning Objective: Test my knowledge of beef cattle feedstuffs on today's

quiz. Capture facts pertaining to the estrus cycle of cattle and technology used in reproduction.

Mind Moover: How can technology help us in breeding better cattle?

\section{Assignment:}

- ID Quiz

- 20 Facts from Technology in Reproduction DVD

2. When students arrive to class they should begin on their daily warm ups.

3. Take attendance while they are working on their daily warm up.

4. Have students share their responses to the mind mover. Give 1 blue ticket to the students who share answers with the class.

5. Inform students that they have five minutes to review their beef cattle feedstuffs before today's quiz.

6. Have them close their binders, and then pass out the quiz. They will need to see slides on the ID Power Point for numbers 6-10. The Power Point will be saved on the desktop of the laptop left on the projector cart.

7. After all students have finished the quiz, then have them switch papers to grade the quizzes. Read the answer key aloud so they can grade the tests. Collect the graded tests.

8. Inform students that they will be watching a DVD on Beef Cattle Reproductive Technologies. They are to record 20 facts they learn from the video as their assignment.

- Place the DVD in the laptop and it should pop up with an autoplay option.

- Click on Technology in Beef Reproduction

- Then click on Introduction. It should play through the whole section on reproductive technology.

- Students should record facts during the video

- Collect Facts at the end of the period. 


\section{Period 4: Exploring Ag:}

1. Write on the dry erase board (the eastern most side) just like listed below:

Today's Lesson Topic: Beef Unit Study Guide

Learning Objective: Review key concepts from the Beef Unit by completing the beef unit study guide for next week's test.

Mind Moover: What has been the most interesting thing you have learned about cattle during this unit?

\section{Assignment:}

- Beef Cattle Unit Study Guide (Due Monday)

2. When students arrive to class they should begin on their daily warm ups.

3. Take attendance while they are working on their daily warm up. Have them respond to the mind mover. Give one blue ticket to those who respond.

4. Inform them that they will be working on a study guide for the beef cattle unit test today. They can work with a partner and use their notebooks to complete the study guide. Tell them it is due on Monday and the test will be on Tuesday.

5. Make sure students put away notebooks and supplies before they leave for lunch. 
Period 6 \& 7: ROP Vet Science:

Will not meet in class today, they are on Job Sites.

Comments/Feedback:

Sub Name: Stan Gibrandf $5-3-13$

Please leave comments here:

All of the classes wire well braved

* payed on task e. A pleasure!

If you need me again dart hesitate to confect me cell: $709-5865$

$$
\text { a.c. : 213502 }
$$

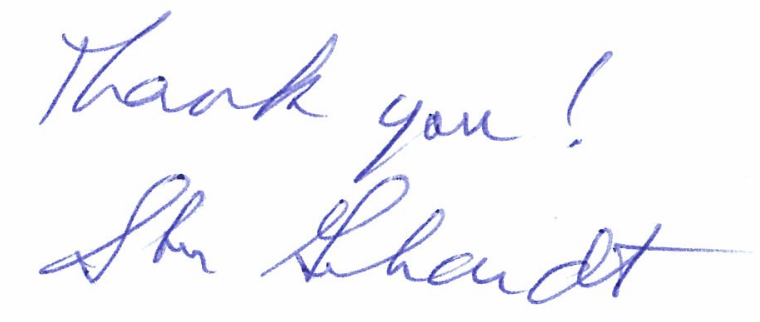

Thank You! 


\section{AGRICULTURE DEPARTMENT WEEKLY MEETING AGENDA}

ATE

$$
\text { April 18, } 2013
$$

\section{ITEMS FOR DEPARTMENT} CONSIDERATION

1) FFA Report

a) Officer Applications due MAY th $^{\text {th }}$

b) Chapter Officer Interviews

i) Tuesday and Wednesday $7^{\text {th }}$ and $8^{\text {th }}$

ii) Aireal, Jennifer, Kevin

2) Top 20 Trip June $10^{\text {th }}$

a) Forms due May $10^{\text {th }}$

3) Curriculum

a) Kevin working on Revise of Ag Econ class to UC

4) American Degree Workshops May 7 and 8

a) Aireal will make a list of American Candidates and send out to advisors

b) Ken, Steve, David after school

5) Faculty Senate Information

6) Advisory Committee

7) FARMS Leadership Program

a) May 21- Steve

b) Final Presentations - 5 Points

б) Ag Booster Report

a) Kevin Call Bryan Hereford Re: District Budget

b) Kevin and Chuck Dement work on Swine facility

c) Ken Call A1 Re: Shade Structure

d) Jennifer Call Mendez re: Portable Panels

9) Transportation

10) Fund Raiser Requests for 2013-14

a) Aireal will submit our norms

11)Farm Manager Report

12) McFarlane Memorial Funds - No action has been taken. Kevin will talk with McFarlane family to set up a meeting

13) School Sheep Flock

a) We will contact Dr. Perry at Fresno State re range management and pasture rotation.

b) Sheep off for rest as of 4-29.

14) State finals Cal Poly
a) Grade Checks and Permission slips on Monday
b) Subs called
c) Rooming Lists

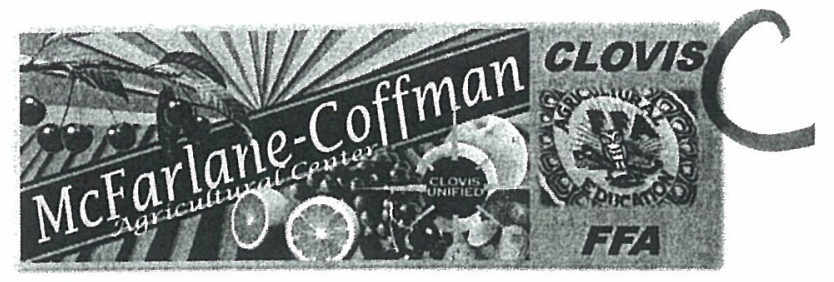

i) Comfort Inn and Suites - Lamp Lighter 5-3 San Luis Obispo

d) Transportation

i) Ag Mechanics - Truck @8AM

ii) Welding -Van 120 @8AM

iii) Livestock 219 @8 AM

iv) Nursery Landscape - Aireal personal Vehicle @8AM

v) Meats - DO Van @8AM

vi) Specialty Animals - 245 @8AM CALENDAR ITEMS May

5/2 Chapter Officer Apps Due

5/3 EFM Officer Applications due

5/3-4 FFA State Finals Cal Poly SLO

5/6 EFM Officer Interviews at Clovis

5/7 Chapter Officer Interviews TUE.

5/8 Chapter Officer Interviews WEDN.

5/9 FFA Lunch Meeting (new team)

5/9 EFM FFA/CATA Meeting

5/14 Booster Meeting 6:15

5/16 FFA Spring Banquet

5/20 Staff-American Degree Scoring

5/28 Booster Mtg.

5/23 Panda Express Fundraiser

5/30 FFA Lunch Meeting

5/31 Chicken Dinner Fundraiser June 


\title{
Department Meeting 5-09-2013
}

\section{Kevin Woodard}

\author{
Sent: $\quad$ Thursday, May 09, 2013 8:04 AM \\ To: $\quad$ Ken Dias; Steve Gambril; Jennifer Knight; Aireal Covey; David Valdez \\ Cc: Stephanie Hanks; Dana Garrison \\ Attachments: Department Meeting 5-09-2 1.doc (165 KB)
}




\begin{tabular}{|c|c|c|c|c|c|c|c|}
\hline & Description & Woodard & Dias & Knight & Covey & Valdez & Gambril \\
\hline \multicolumn{8}{|c|}{ PROJECT SUPERVISION } \\
\hline Agricultural Mechanics & & & & & & $x$ & $\mathrm{x}$ \\
\hline Beef & & & $x$ & & & & \\
\hline Canine & & & $x$ & & & & \\
\hline Crops & & & & & & & $x$ \\
\hline Dairy & & $\mathrm{x}$ & & & & & \\
\hline Goats & & $x$ & & & & & \\
\hline Horses & & & $x$ & & & & \\
\hline Horticulture/Landscape & & & & & $x$ & & \\
\hline Poultry & & & $x$ & $\mathrm{x}$ & & & \\
\hline Rabbits & & $x$ & & & & & \\
\hline Sheep & & & & $\mathrm{x}$ & & & \\
\hline Swine & & $x$ & & & & & \\
\hline Work Experience & & $x$ & $\mathrm{x}$ & $\mathrm{x}$ & $\mathrm{x}$ & $x$ & $x$ \\
\hline
\end{tabular}

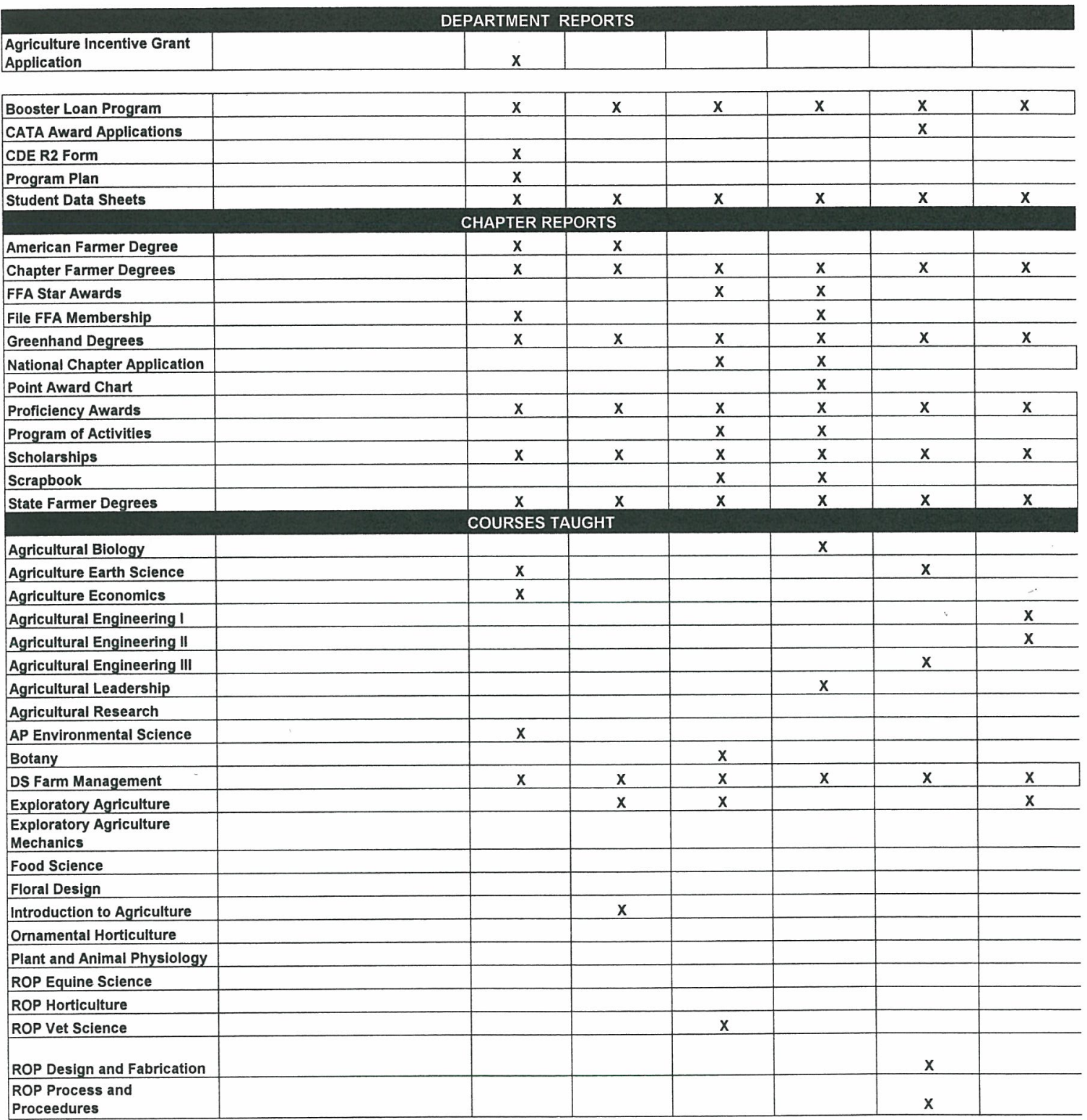




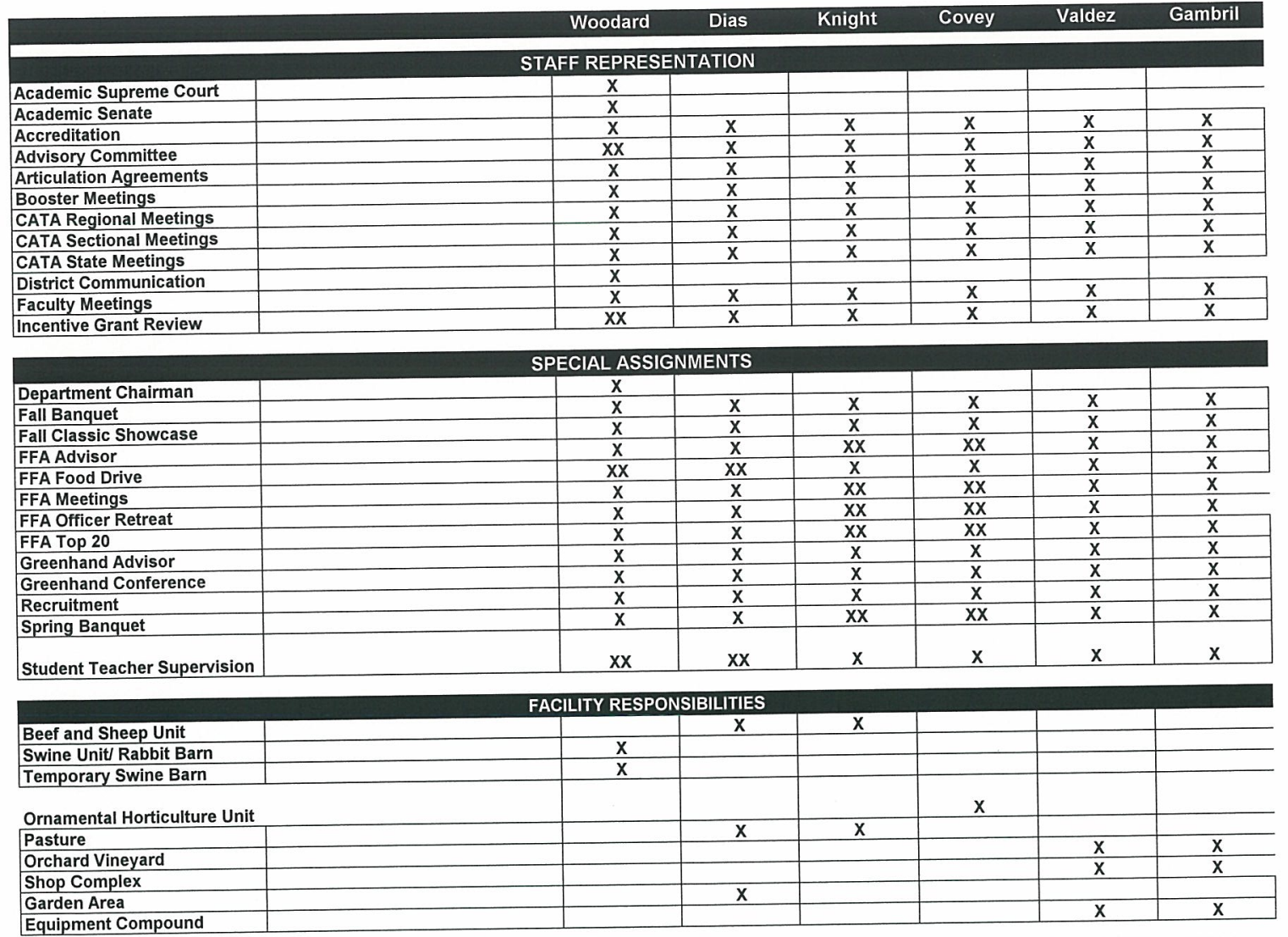

\begin{tabular}{|c|c|c|c|c|c|c|c|}
\hline \multirow{2}{*}{\multicolumn{8}{|c|}{$\begin{array}{l}\text { FFA, Advanced Leadership } \\
\text { Conference }\end{array}$}} \\
\hline & & & & & & & \\
\hline \multicolumn{8}{|l|}{$\begin{array}{l}\text { Conference } \\
\text { FFA, Chapter Officer } \\
\text { Leadership Conference }\end{array}$} \\
\hline \multicolumn{8}{|l|}{$\begin{array}{l}\text { Leadership Conference } \\
\text { FFA, Made for Excellence } \\
\text { Conference }\end{array}$} \\
\hline \multicolumn{8}{|l|}{$\begin{array}{l}\text { Conference } \\
\text { FFA, National Convention, } \\
\text { American Degrees }\end{array}$} \\
\hline \multicolumn{8}{|l|}{\begin{tabular}{|l} 
American Degrees \\
FFA, National Finals \\
\end{tabular}} \\
\hline \multicolumn{8}{|l|}{\begin{tabular}{|l|} 
FFA, National Finals \\
FFA, Regional Officer \\
Leadership Conference
\end{tabular}} \\
\hline \multicolumn{8}{|l|}{$\begin{array}{l}\text { Leadership Conference } \\
\text { FFA, Sacramento Experience } \\
\text { Conference }\end{array}$} \\
\hline \multicolumn{8}{|l|}{ FFA, Sectional Bowl-a-thon } \\
\hline \multicolumn{8}{|l|}{$\begin{array}{l}\text { FFA, Sectional Bowl-a-thon } \\
\text { FFA, Sectional Officer } \\
\text { Leadership Conference }\end{array}$} \\
\hline \multicolumn{8}{|l|}{$\begin{array}{l}\text { Leadership Conference } \\
\text { FFA, State Leadership } \\
\text { Conference }\end{array}$} \\
\hline FFA, World Ag Expo & & & & & & & \\
\hline Field Day, Arbuckel & & & & & & & $\mathrm{x}$ \\
\hline \multicolumn{8}{|l|}{\begin{tabular}{|l|} 
Field Day, Arbuckel \\
Field Day, CSU Bakersfield \\
\end{tabular}} \\
\hline \multicolumn{8}{|l|}{ Field Day, Cal Poly Pomona } \\
\hline \begin{tabular}{|l} 
Field Day, Chico State \\
\end{tabular} & & $x$ & $\mathrm{x}$ & $x$ & $\mathrm{X}$ & $\mathbf{x}$ & $\mathrm{X}$ \\
\hline Field Day, Exeter & & & & & & $\mathrm{x}$ & \\
\hline Field Day, Fowler & & & & & & $\mathrm{x}$ & \\
\hline Field Day, Fresno State & & $\mathrm{X}$ & $x$ & $\mathrm{x}$ & $\mathrm{x}$ & $\mathrm{X}$ & $\mathrm{X}$ \\
\hline \multicolumn{8}{|l|}{\begin{tabular}{|l|} 
Field Day, Fresno State \\
Field Day, Mendota \\
\end{tabular}} \\
\hline Field Day, Merced & & $\mathrm{X}$ & & $\mathrm{X}$ & $x$ & & \\
\hline Field Day, Modesto & & $\mathrm{x}$ & & & & & \\
\hline Field Day, Monrovia Nursery & & & & & $\mathrm{x}$ & & \\
\hline Field Day, Reedley College & & $\mathrm{X}$ & & $\mathrm{X}$ & $\mathrm{x}$ & & $x$ \\
\hline $\begin{array}{l}\text { Field Day, State Finals, Cal } \\
\text { Poly SLO }\end{array}$ & & $x$ & $\mathrm{x}$ & $x$ & $x$ & $\mathrm{x}$ & $x$ \\
\hline $\begin{array}{l}\text { Field Day, State Finals, } \\
\text { Fresno State }\end{array}$ & & & $x$ & & & & \\
\hline $\begin{array}{l}\text { Field Day, State Finals, } \\
\text { Reedley College }\end{array}$ & & $x$ & & & & & \\
\hline Field Day, UC Davis & & $\mathrm{X}$ & $x$ & $\mathrm{X}$ & $\mathrm{x}$ & & $\mathrm{X}$ \\
\hline AQHA World Horse Show & & & & $\mathrm{X}$ & & & \\
\hline \multicolumn{8}{|l|}{\begin{tabular}{|l|} 
AQHA World Horse Show \\
Show, California State Fair \\
\end{tabular}} \\
\hline Show, Great Western & & & & & & & \\
\hline Show, Red Wave Classic & & & $\mathrm{x}$ & & & & \\
\hline Show, The Big Fresno Fair & & $\mathrm{x}$ & $\mathrm{x}$ & $\mathrm{X}$ & $\mathrm{x}$ & $\mathrm{x}$ & $x$ \\
\hline
\end{tabular}



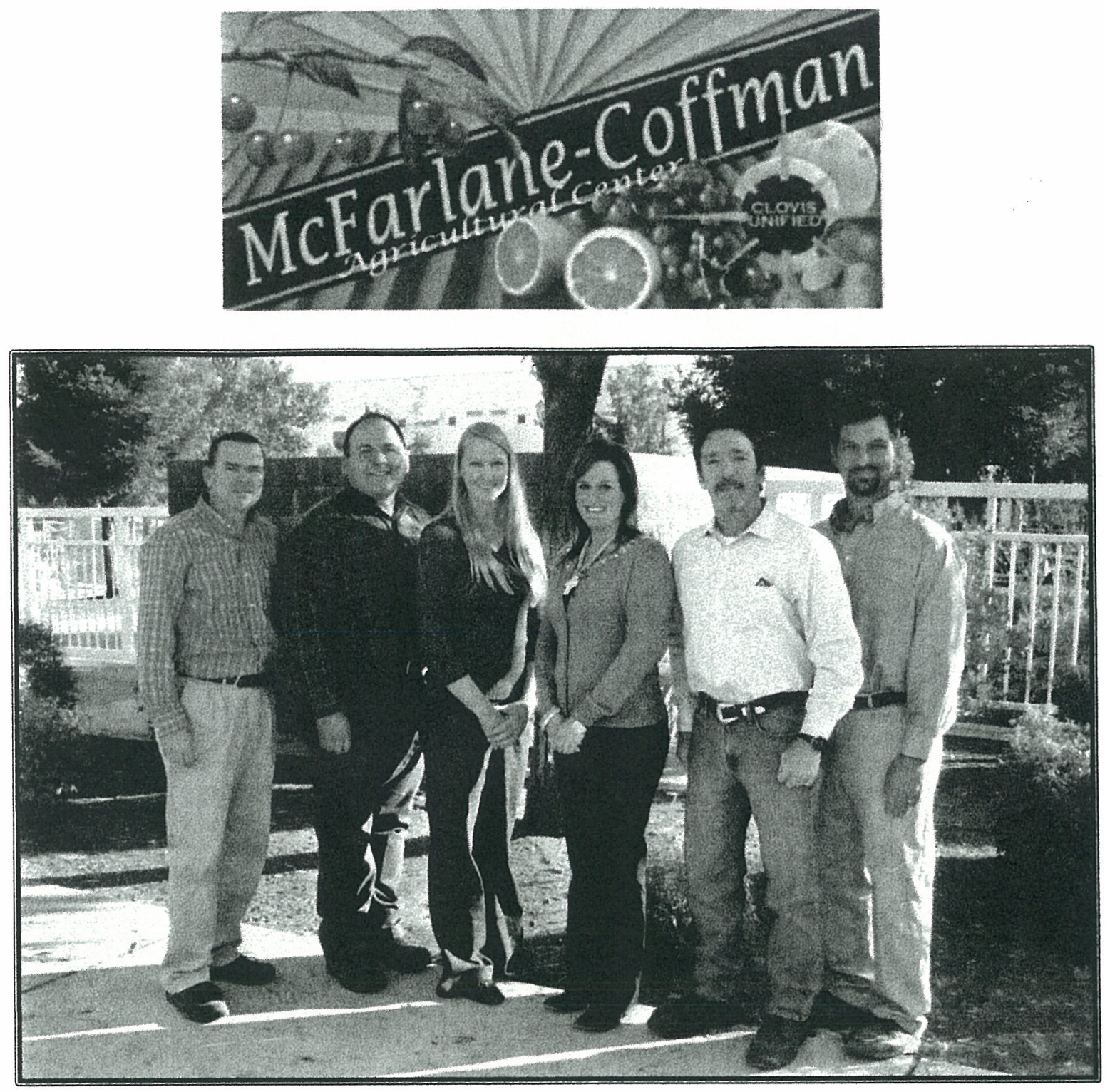

2012-2013 Clovis East Agriculture Teachers

Left to Right

Kevin Woodard-MS Ag Education

David Valdez-MS Ag Education

Aireal Covey-MS Ag Education

Jennifer Knight- BS Ag Education

Ken Dias- Single Subject Ag Education

Stave Gambril- MS Ag Education 


\section{Professional Development Activities 2012-2013}

Jennifer A Knight

\section{Past Year's Participation}

\begin{tabular}{|c|c|c|}
\hline Activity & Level/Organization & Date \\
\hline $\begin{array}{l}\text { CATA Summer } \\
\text { Conference }\end{array}$ & CATA State & $6 / 17-6 / 202012$ \\
\hline $\begin{array}{l}\text { Agriskills Workshop } \\
\text { Presenter }\end{array}$ & CATA State & $6 / 22 / 12$ \\
\hline $\begin{array}{l}\text { Dear Beginning Teacher } \\
\text { Professional } \\
\text { development }\end{array}$ & CUSD & $8 / 8 \& 8 / 92012$ \\
\hline CATA Fall Meeting & Section & $8 / 29 / 12$ \\
\hline $\begin{array}{l}\text { Regional Boot Camp } \\
\text { Workshop Presenter }\end{array}$ & Regional FFA & $9 / 14 / 12$ \\
\hline $\begin{array}{l}\text { ROP Professional } \\
\text { Development } \\
\text { Conference }\end{array}$ & Regional ROP & $9 / 26 / 12$ \\
\hline $\begin{array}{l}\text { New Professionals } \\
\text { Conference Speaker }\end{array}$ & CATA State & $11 / 15 / 12$ \\
\hline $\begin{array}{l}\text { Fall CATA Regional } \\
\text { Meeting }\end{array}$ & CATA Region & $11 / 17 / 12$ \\
\hline CATA Road Show & CATA Region & $12 / 3 / 12$ \\
\hline Spring Regional Meeting & CATA Region & $2 / 23 / 13$ \\
\hline $\begin{array}{l}\text { Ag Staff Professional } \\
\text { Development Day }\end{array}$ & CUSD Department & $3 / 12 / 13$ \\
\hline ROP Advisory Night & ROP Regional & $4 / 23 / 13$ \\
\hline $\begin{array}{l}\text { CATA Sectional Planning } \\
\text { Meeting }\end{array}$ & CATA Section & $5 / 9 / 13$ \\
\hline $\begin{array}{l}\text { Elected CATA Sectional } \\
\text { Secretary }\end{array}$ & $\begin{array}{l}\text { CATA East Fresno Madera } \\
\text { Section }\end{array}$ & $5 / 9 / 13$ \\
\hline
\end{tabular}


AGED 520

Professional Development Plan

Jennifer Knight

\begin{tabular}{|c|c|c|}
\hline Goals & Timeline & Action Plan \\
\hline Finish Master's Degree (Personal) & Winter 2012 & $\begin{array}{l}\text { Set up meeting with committee chair } \\
\text { to go over action plan. }\end{array}$ \\
\hline $\begin{array}{l}\text { Join Professional Organizations } \\
\text { (Professional) }\end{array}$ & Spring 2013 & $\begin{array}{l}\text { California Women in Agriculture, } \\
\text { American Meat Science Association, } \\
\text { NAAE }\end{array}$ \\
\hline Start a Family (Personal) & Fall 2013 & Not sure \\
\hline Purchase our first home (Personal) & Fall 2014 & Continue saving for down payment \\
\hline $\begin{array}{l}\text { Integrate Agriculture into every } \\
\text { Biology standard based lesson } \\
\text { (Professional) }\end{array}$ & Fall 2014 & $\begin{array}{l}\text { Continue finding new activities to use } \\
\text { in each lesson. }\end{array}$ \\
\hline $\begin{array}{l}\text { Have a National Winning Meats } \\
\text { Judging Team (Professional) }\end{array}$ & Fall 2013 & $\begin{array}{l}\text { Work my team hard this year!! They } \\
\text { can do it! }\end{array}$ \\
\hline $\begin{array}{l}\text { Finish re doing sheep barn pens } \\
\text { (Professional) }\end{array}$ & Summer 2012 & $\begin{array}{l}\text { Continue scheduling work days and } \\
\text { plugging away at the project }\end{array}$ \\
\hline $\begin{array}{l}\text { Hold a State CATA Office } \\
\text { (Professional) }\end{array}$ & 2017 & $\begin{array}{l}\text { Currently Section Secretary, continue } \\
\text { moving up through the chairs. }\end{array}$ \\
\hline $\begin{array}{l}\text { Be nominated as a Teacher of } \\
\text { Excellence (Professional) }\end{array}$ & 2020 & Keep doing a great job as a teacher \\
\hline $\begin{array}{l}\text { Take some college courses in } \\
\text { Microbiology and Food Science } \\
\text { (Professional) }\end{array}$ & 2017 & Not sure how to squeeze this in??? \\
\hline
\end{tabular}




\title{
Clovis East High School
}

\author{
2940 LEONARD AVENUE, CLOVIS, CA 93619
}

Jennifer A. Knight

Clovis East High School Agriculture Department

2940 Leonard Avenue

Clovis, CA 93619

559-765-9580 Cell

jenniferknight@cusd.com

\section{Dear Mrs. Stephanie Hanks (LD)}

I would just like to personally thank you for allowing me to participate in various professional development events this 2012-2013 school year. I realize that classroom instruction is best when the teacher of record is present and while I aim to be present on a regular basis I also know the value of collaboration and learning with fellow professionals. By providing me with sub coverage I have been able to participate in some exciting and helpful professional opportunities this year.

I was a presenter at the CATA new professionals conference this year, and what a great experience it was. Because I was on the presenting side of this event it really challenged me in new ways and made me reflect on the best practices I use in my classroom on a daily basis. These types of challenges I know create growth and confidence in myself which will transfer over into the classroom.

I also appreciate you allowing me to be absent due to the completion of my Master's Degree. I want to be regarded as a professional in this arena, and want to join my fellow teaching partners in holding this degree. The entire process of completion has prompted me to complete various reviews and creation of new Ag and FFA policies, as well as curriculum projects that will greatly benefit our Clovis Unified students.

Thank you again for allowing me to participate in professional development this year and letting me become a real example to our students of what is means to be a lifelong learner.

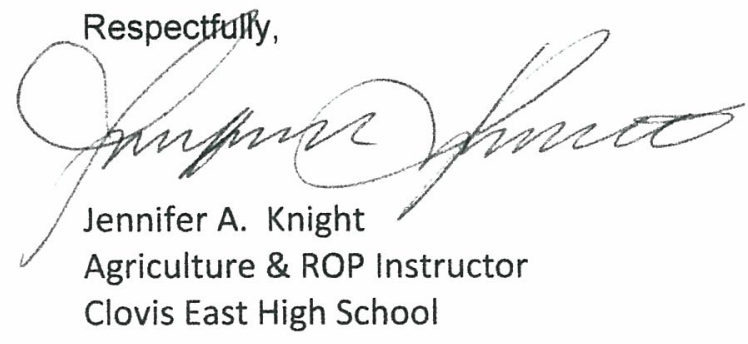




\section{CAL POLY}

SAN LUIS OBISPO

Agricultural Education \& Communication Department

College of Agriculture, Food is Environmental sciences

Tel $305-756-2803$

Fax 805-756-2799

November 20, 2012

Jennifer Knight

Agriculture Department

Clovis East High School

10621 E. Fieldstone Ave.

Clovis, CA 93619-4618

Dear: Jennifer,

Thank you for presenting at the 2012 New Professionals Institute. Your presentation "Bring Out the Best in Students" was very well received by your peers. The information in your workshop and the added material you provided were both creative and timely.

Thank you again for taking time from your busy schedule and I hope we can call on you again for this or other workshops. Happy Holidays to you and your family!

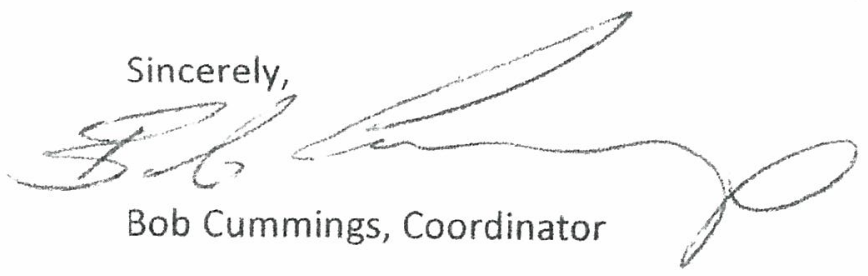




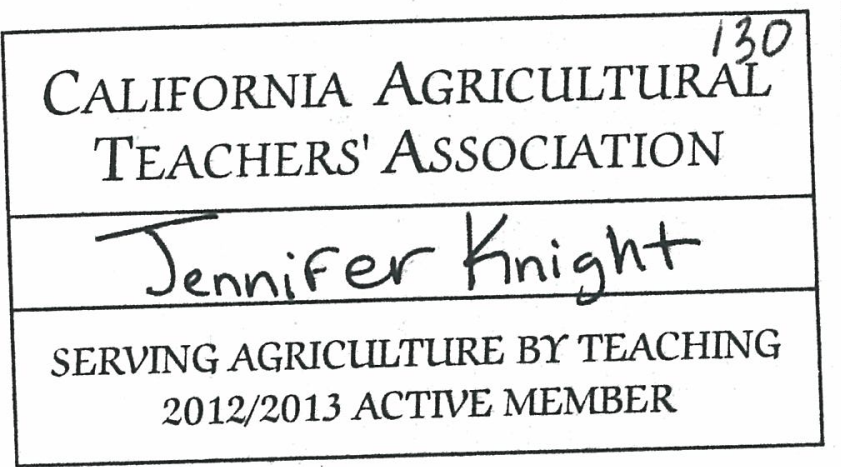




\section{Quality Criteria Five}

\section{Facilities, Equipment \& Materials}

The agriculture program for Clovis Unified is fortunate to have one of the newest and most advanced high school facilities in the Nation. The McFarlane-Coffman Agriculture Center was established in August of 2000 to serve students from throughout Clovis Unified School District. Currently, the facility consists of two traditional classrooms, two science laboratory classrooms, three engineering classrooms, and a biotechnology facility. The Agriculture Engineering Facility consists of six separate shops, each outfitted with state of the industry equipment. The Farm includes animal units for beef, sheep, swine, and rabbits. Four acres of irrigated pasture houses cattle, sheep, and horse projects. The 4,800 sq./ft. Greenhouse facility supports year around student plant projects. The Crop Science Laboratory includes eleven acres that consists of numerous plots growing apples, citrus, vines, tree fruit, and nut crops.

Agriculture Staff, Students, District Personnel, and a full time Farm Manager all help maintain this massive facility. Recently our Ag Staff was tasked with the process of listing out everything that is done/performed/needed for each unit and who is expected to do it, what time of the year is needs done and how much time it takes. This is still under construction but helps show who bears the responsibility for each task, that way when a community member has a question about why this person does or does not do this or that task on the farm we have a chart that can be referenced by any staff or district personnel. This also will help us develop a job description for our Farm Manager position. One of our biggest challenges is that some community members think we need to spend more class time working on the farm and we have to try to balance the Farm with FFA, SAE, CDE Teams, and standards based instruction with benchmarks and pacing. Having a clear responsibilities schedule of tasks for the farm will help us in managing it correctly and show the community who is responsible for what specific tasks.

When things need repaired or replaced on the Farm Facility we are to contact our Department Chair, and he will advise on if we need to address it and pay for it out of 
Incentive Grant funds or if we can use the District grounds personnel to handle it. If the District is to be used we will submit a work order. There are also signs posted in the farm buildings that state for any complaints or facility concerns to be immediately reported to the project area supervisor. The Clovis Unified School District also follows all local, State, and Federal regulations regarding The Americans with Disabilities Act. Our facilities meet or exceed these standards.

Students who use the Ag Center facilities for SAE projects are expected to sign and agree to follow the Ag Center Farm Use Contract. This contract is currently under review by our Ag Staff and we are working to create one standardized general policy for the Ag Center to be placed in a policies handbook. Each species or project area will have additional rules that will be added to the Ag Center policy handbook. Students who keep projects at the farm are issued lockers to securely store personal project related items. Storage rooms exist on the farm facility to store all equipment needed for livestock projects, and coolers to store produce from orchard and garden projects.

All science classrooms have adequate storage with cabinets surrounding the perimeter of the classroom. They also have numerous sinks, gas and air outlets for lab activities. A separate storage/Prep room is attached to each science classroom, upon my arrival at Clovis East the science storage room that I share with Mr. Woodard was a total mess; over Christmas Break we teamed up to clean, organize, and inventory the room; it now looks great and is functional again. The shop/engineering rooms are small but the shops themselves have adequate space for storing equipment. Mr. Gambril designed and installed tool storage cages in the woodshop that hold all necessary tools for woodworking. In the fabrication shop organization and storage can be an issue and our two shop teachers are working on improving storage and organization for that area.

We also have two extra classrooms which we have converted into special use rooms. One room attached to the shop is the AUTOCAD computer lab equipped with new computers for shop students to work with design and layout. The other extra classroom is called the Blue and Gold room, and is our FFA Officers room. This room is used to hold our weekly Executive committee meetings and store our FFA materials. 
As far as technology equipment goes we are fortunate to have access to many different things. Our District issues a laptop to all teachers to use in the classroom and at home. All classrooms have a media cart with a LCD projector and document camera. The Ag Department also owns 6 SMART boards and two sets of SMART response systems, the only down side to the SMART boards is that our classrooms are so big and set up in a way where the SMART boards are almost too small to see in the large classrooms. We do all have access to email, and Google sites for webpage creation and document sharing. Our district also does not block or filter websites on teacher computers, so if we need to use a You Tube video we can at our professional discretion. Students also have access to laptop carts that we have purchased through Ag Incentive Specialized Grants and through ROP funds. I was also lucky enough to have ROP purchase an I Pad for me to use and a new Color Printer this year.

\section{Supporting Completion Materials}

Item A- Agriculture Center Photo

Item B- Ag Center Facilities Map

Item C- Beef And Sheep Barn Pictures

Item D- Swine Barn Pictures

Item E- Ag Park and Greenhouse Pictures

Item F- Orchard and Row Crop Pictures

Item G- Shop Pictures

Item H- Classroom, Ag Office, and Computer Lab

Item I- District/Department Budget Process

Item J- My "Wish List" 
Item K- Current Year's Ag Department Budget 


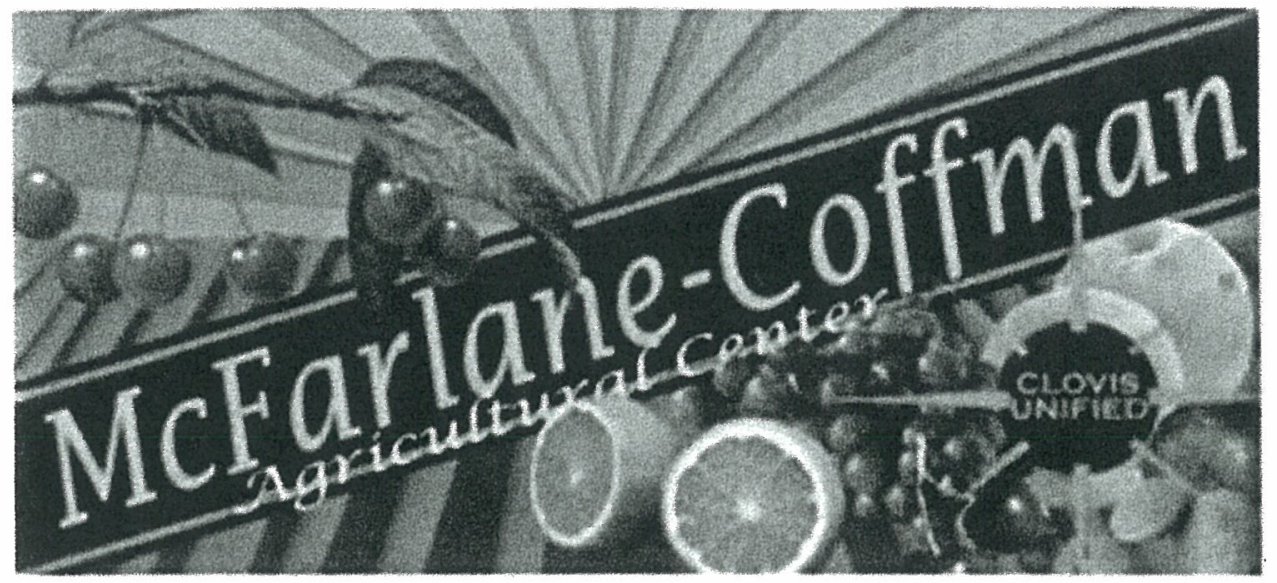

\section{Agriculture Center}

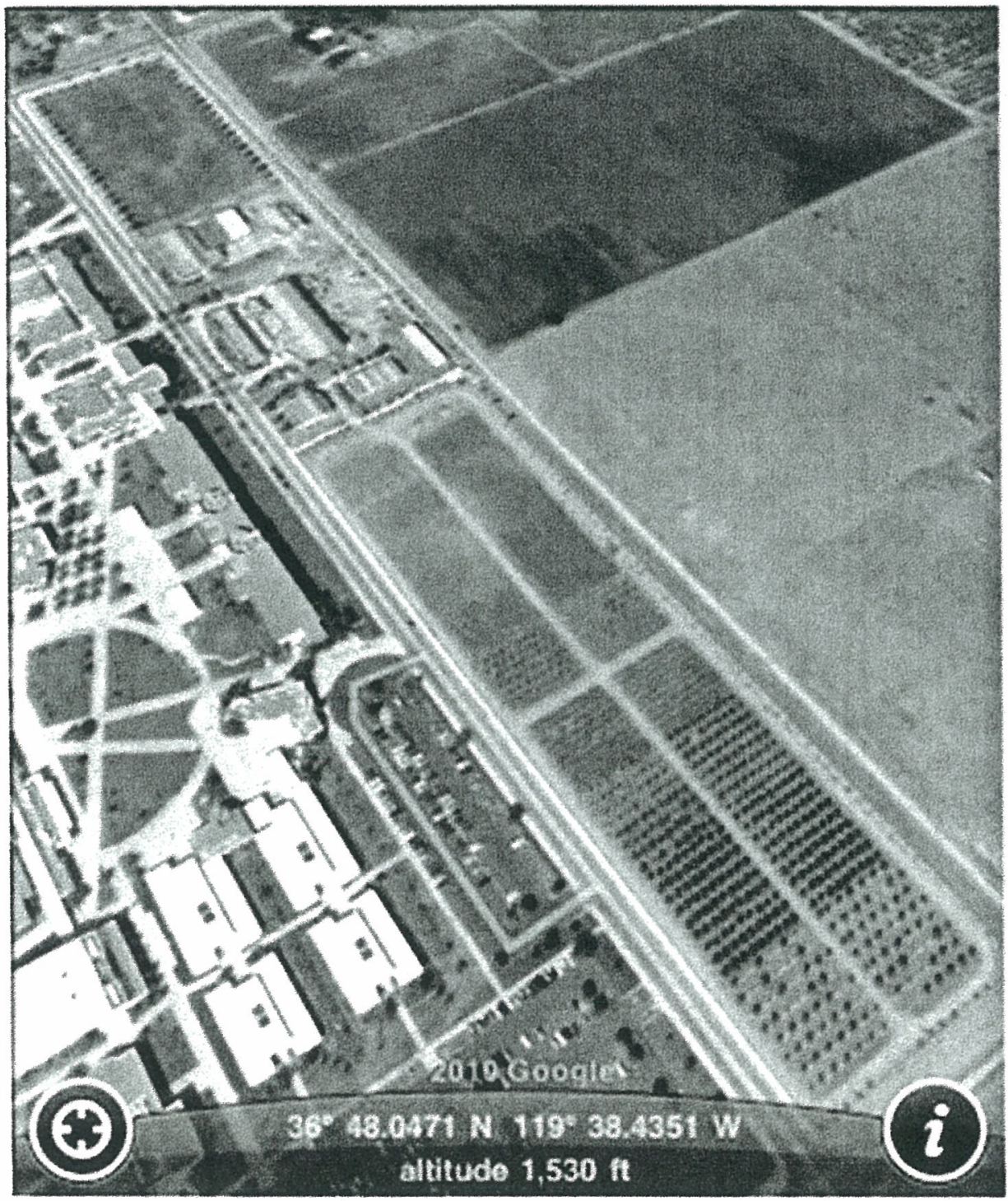




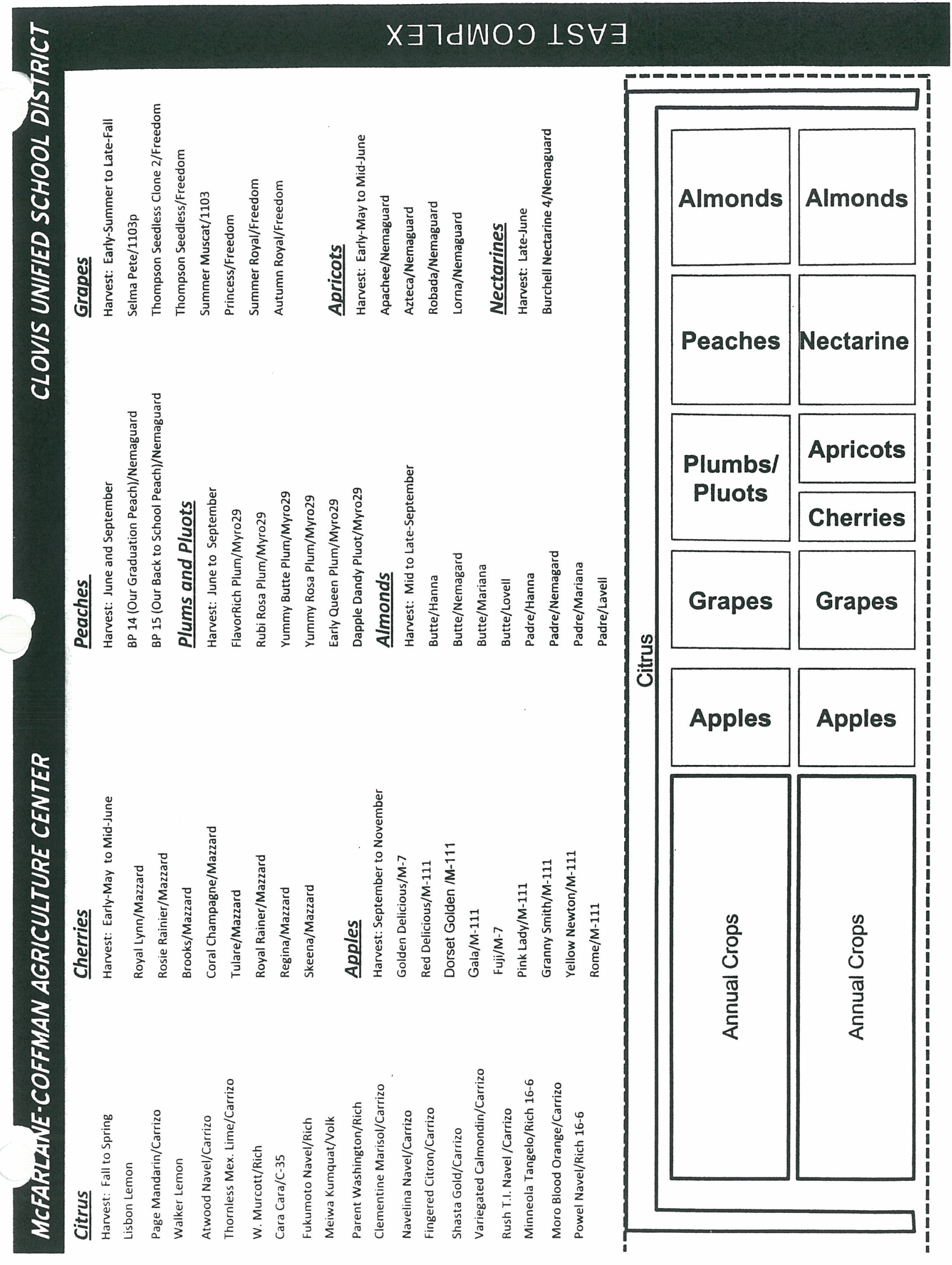




\section{WEST COMPLEX}
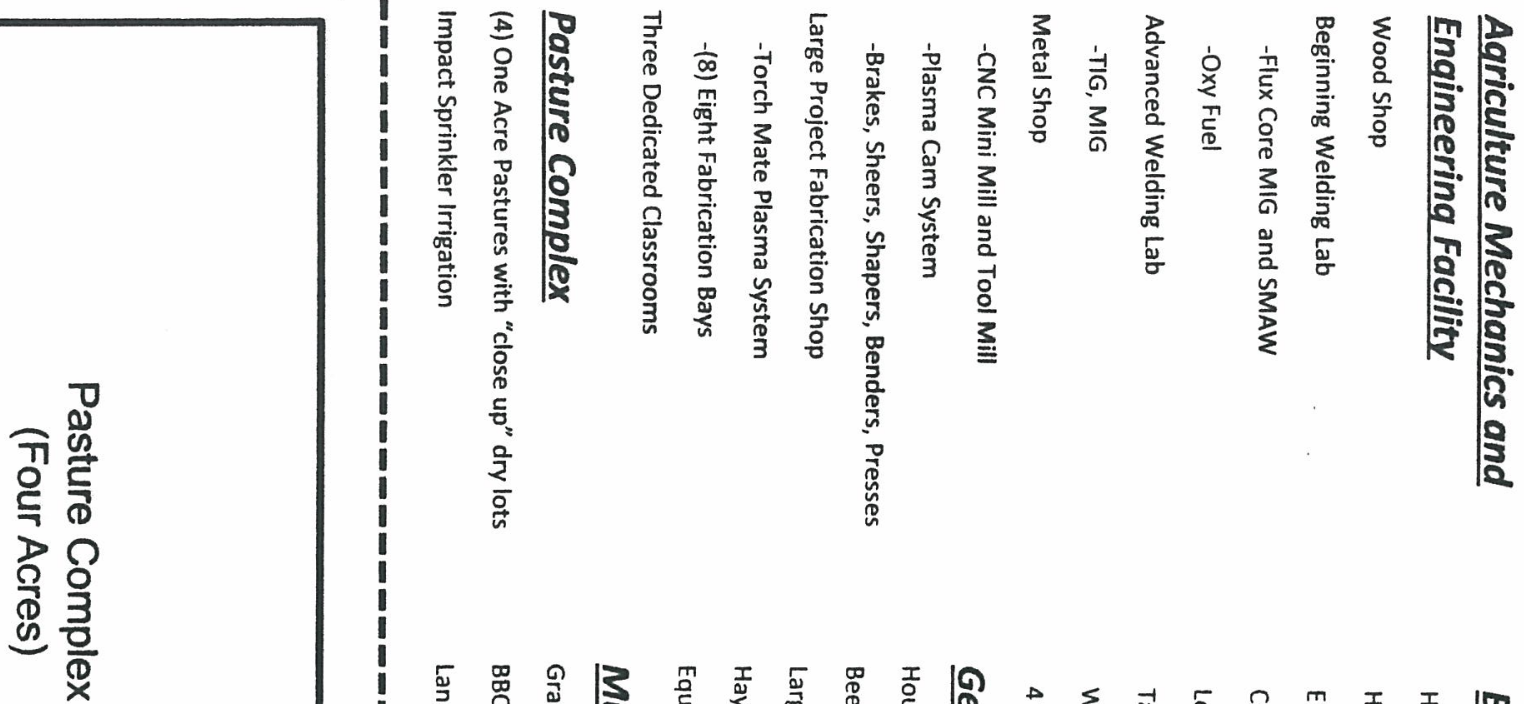

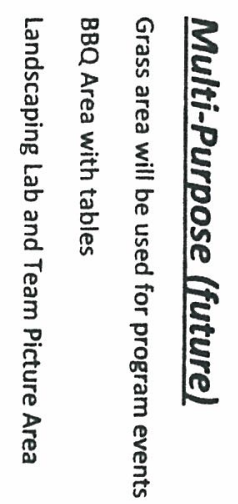
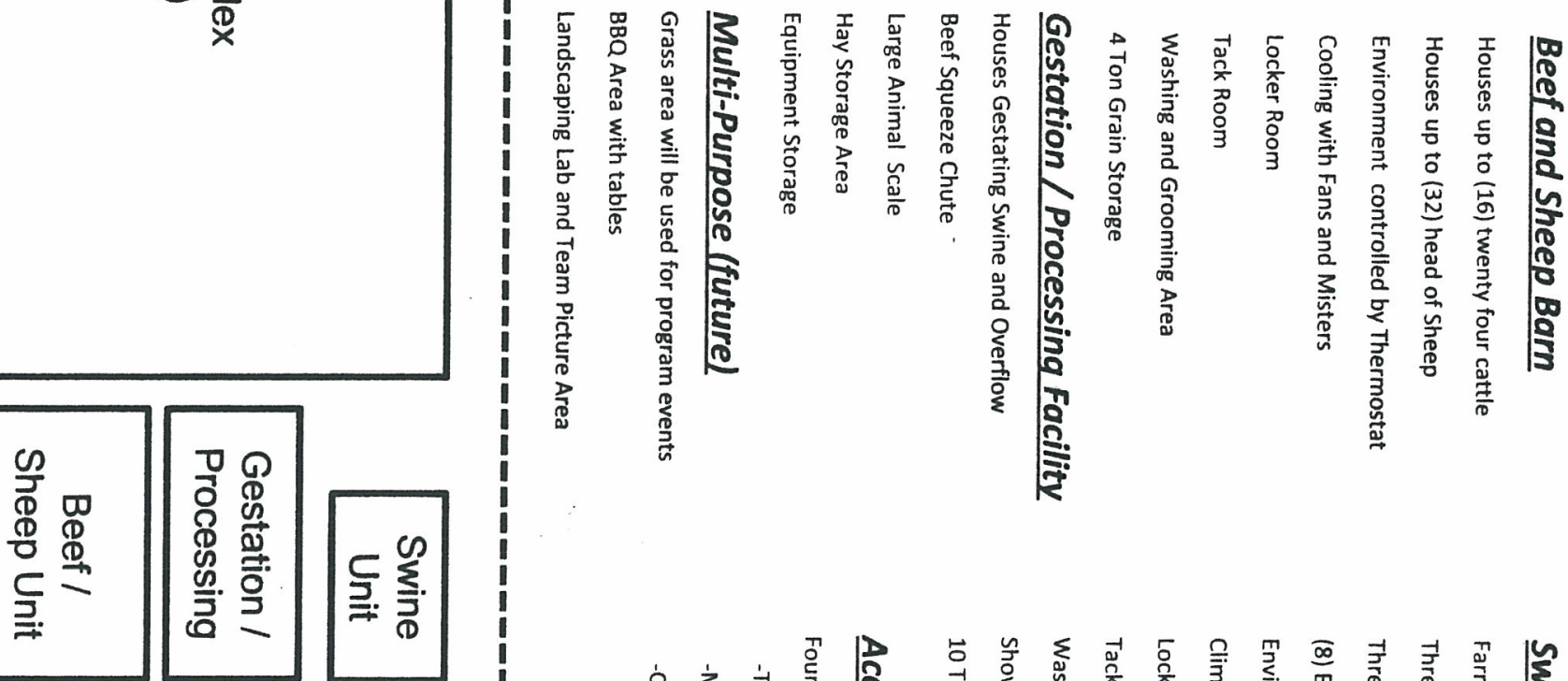
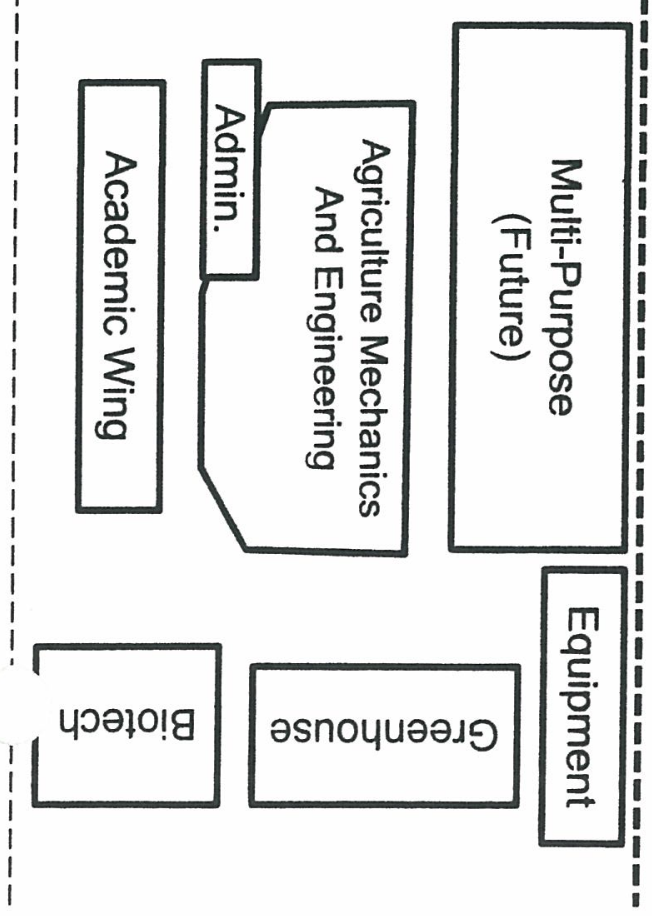

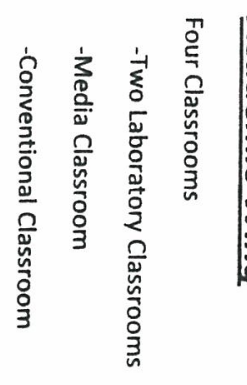

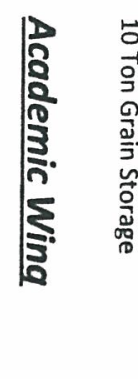
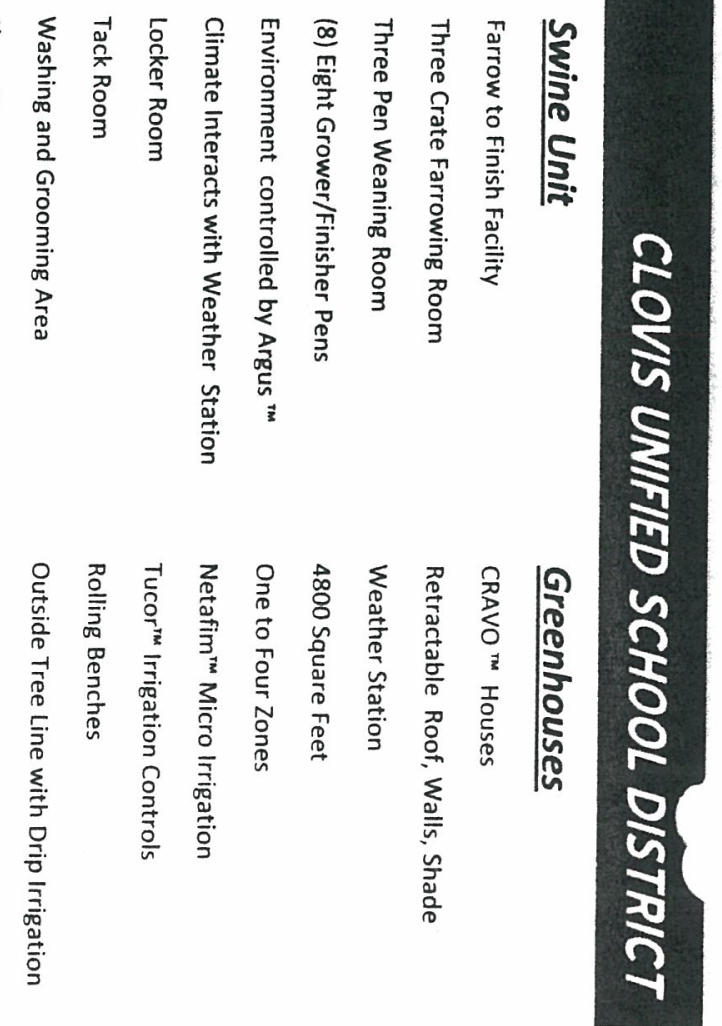
Beef and Sheep Barn

\section{Dry Lots and Pastures}
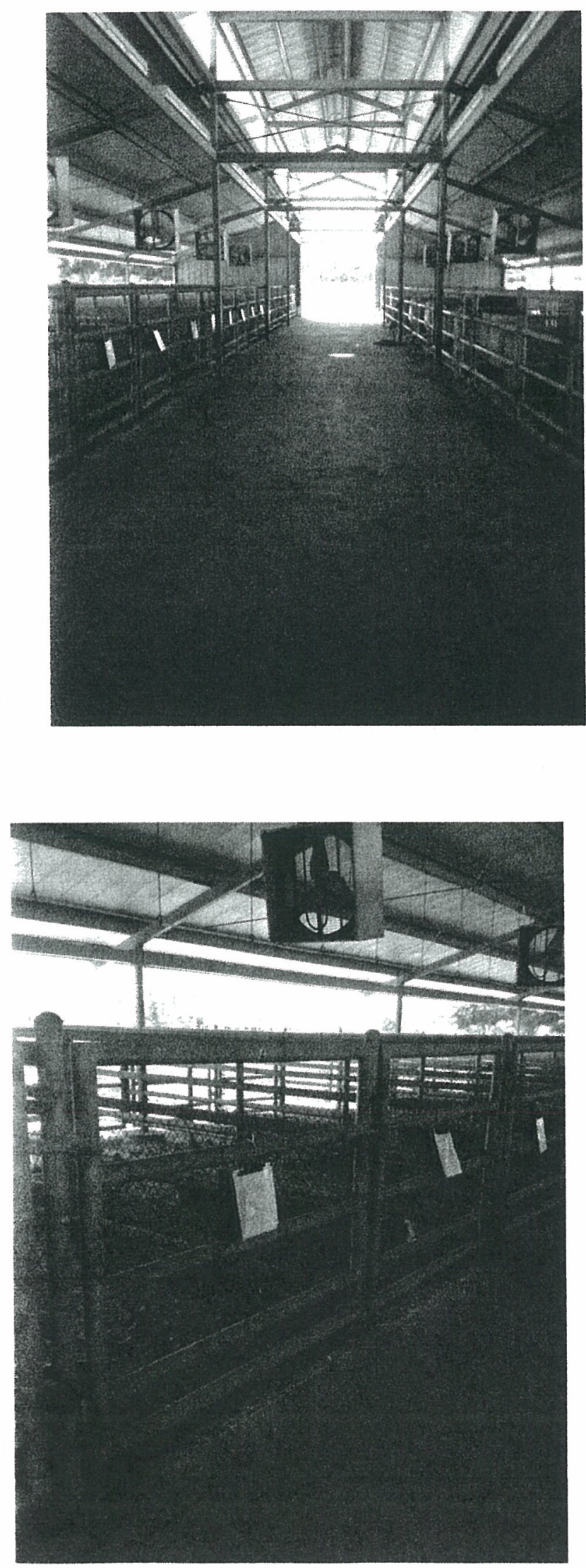
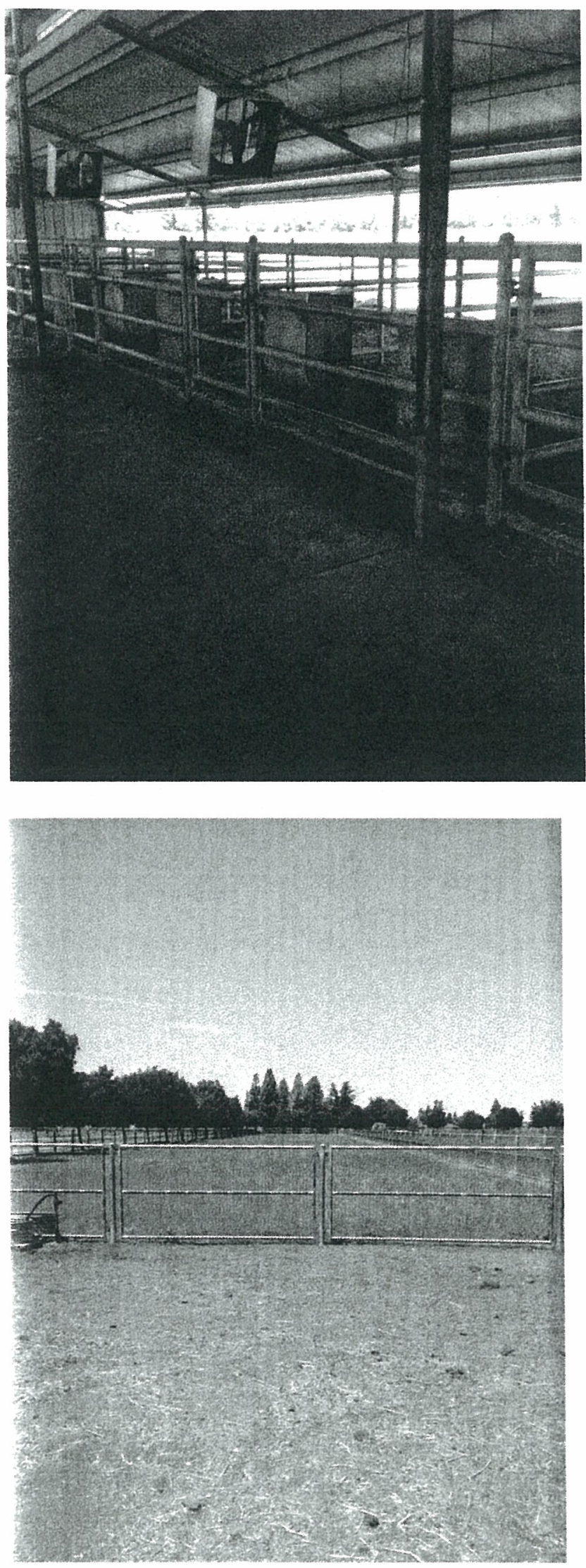
Swine Barn

Farrrowing House \& Rabbit Room

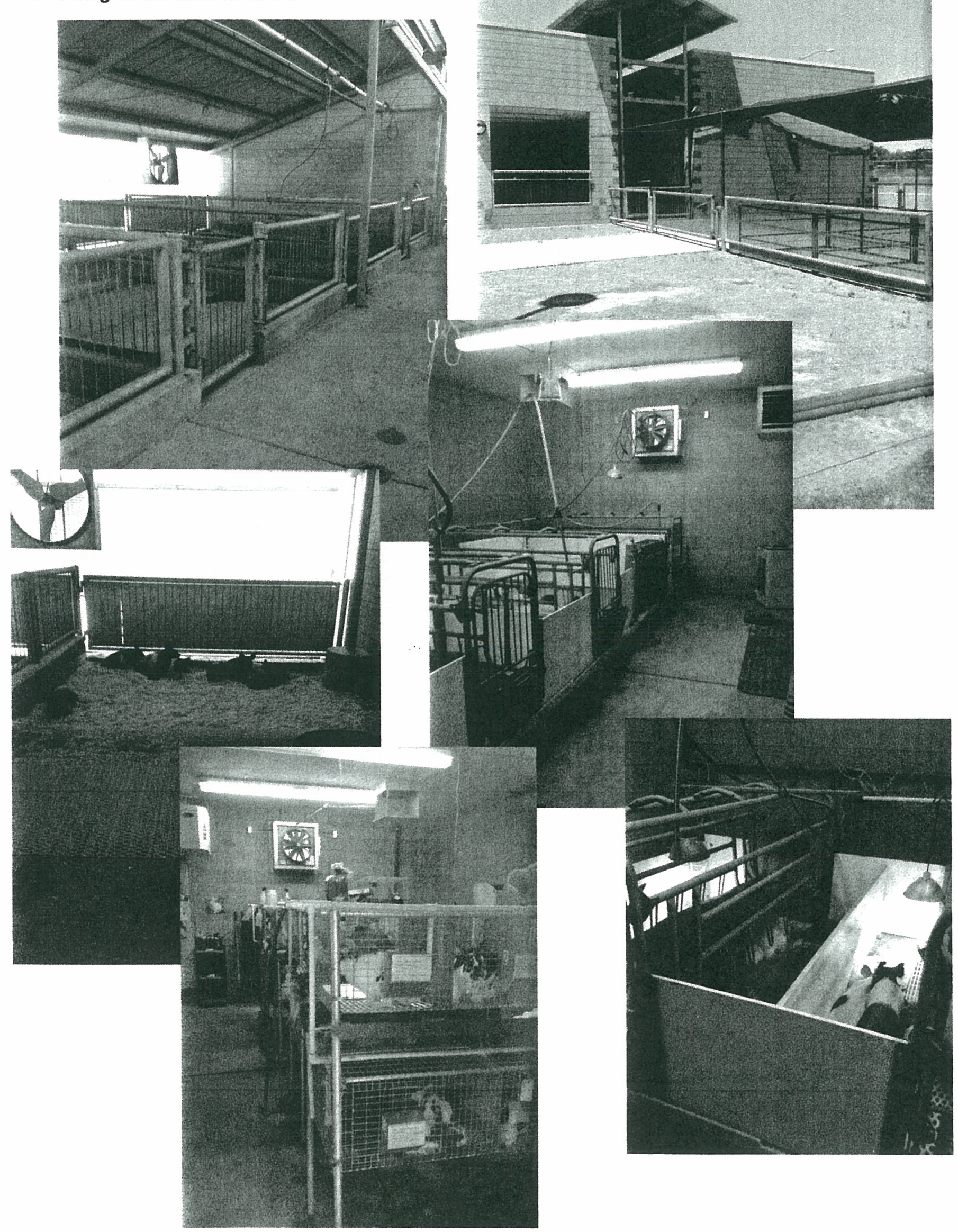




\section{New Ag Park}

\section{Greenhouses}

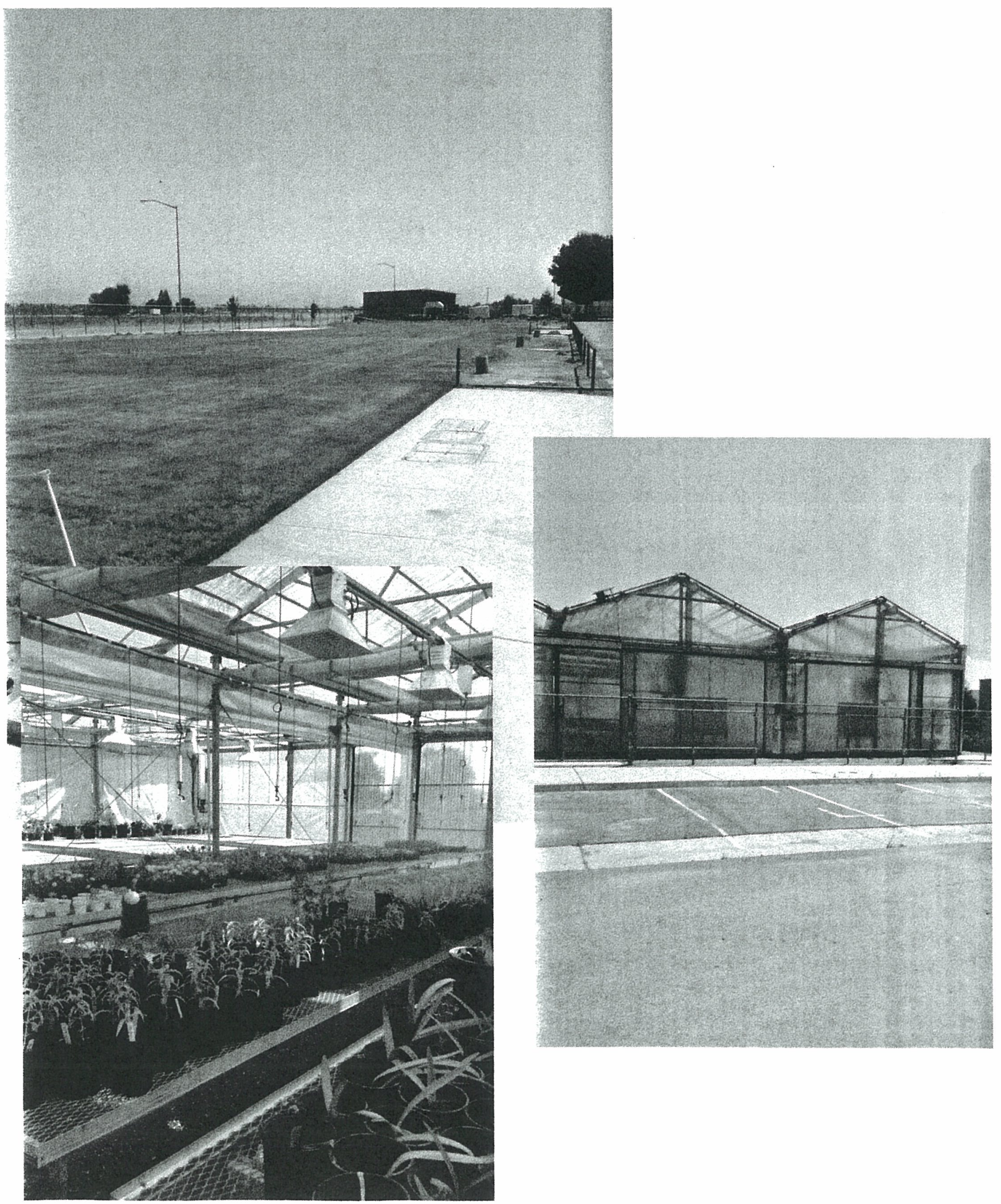




\section{Orchard and Row Crops}

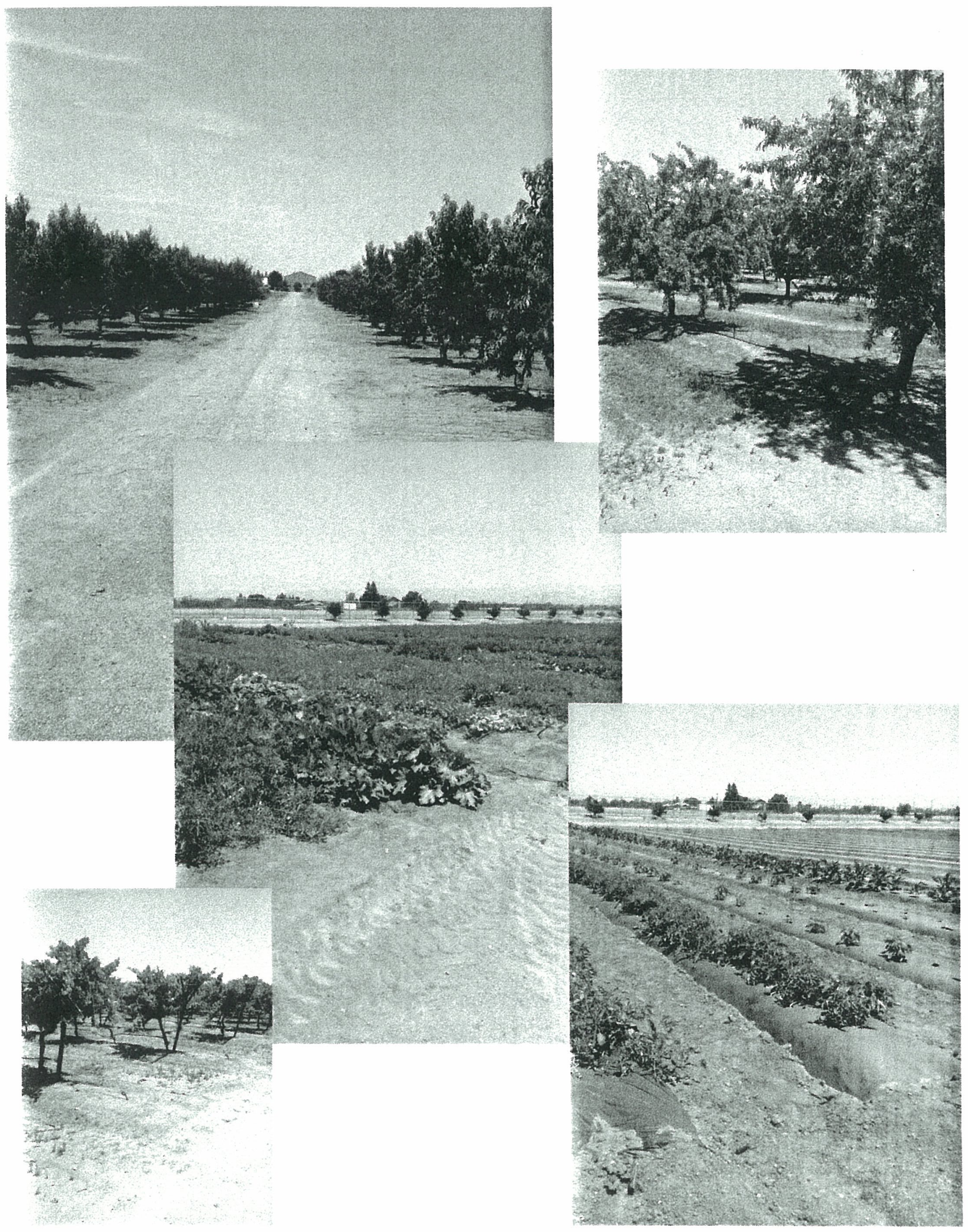




\section{Shops}

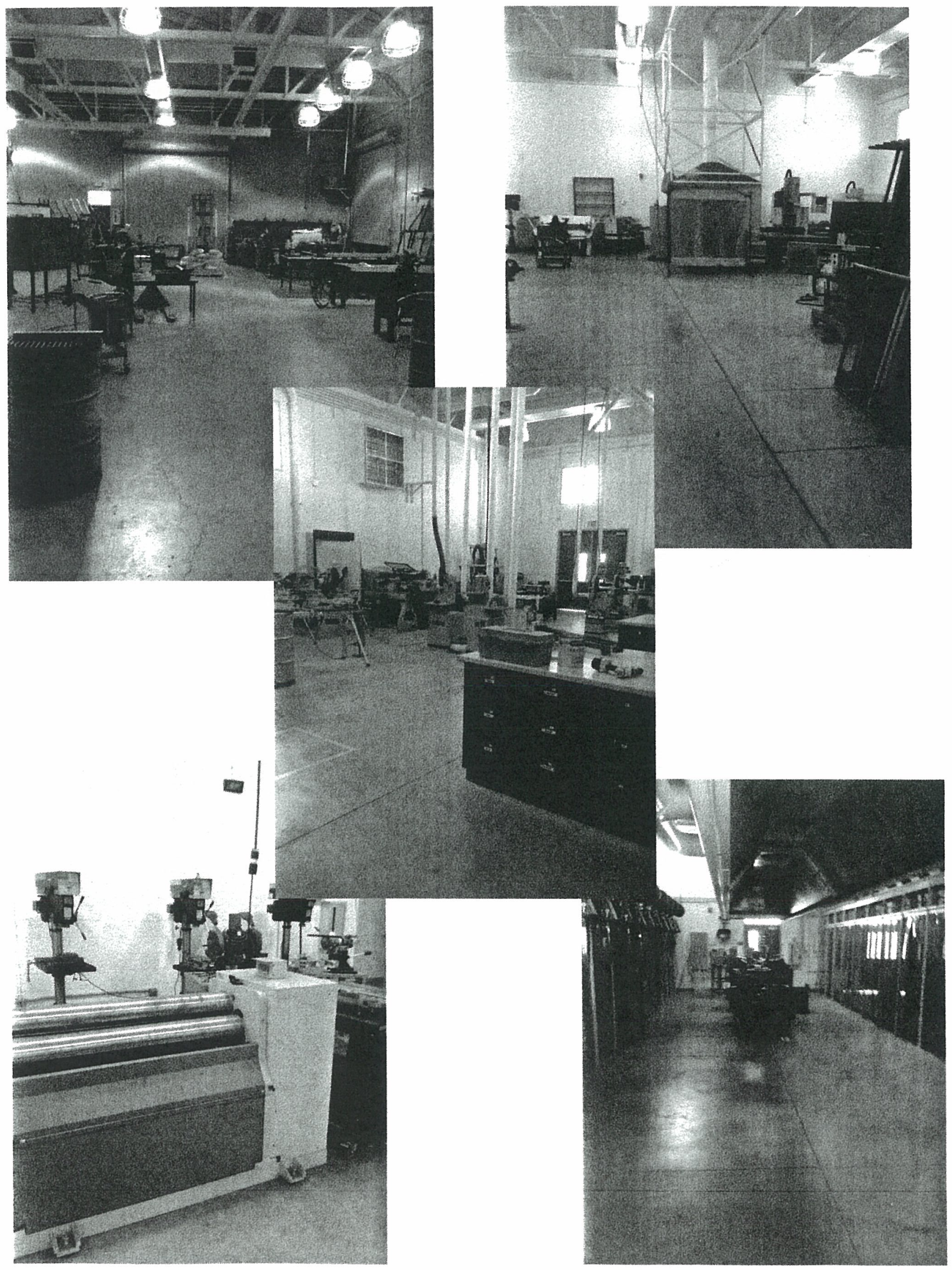




\section{Ag Office, My Classroom, AUTOCAD Computer Lab}

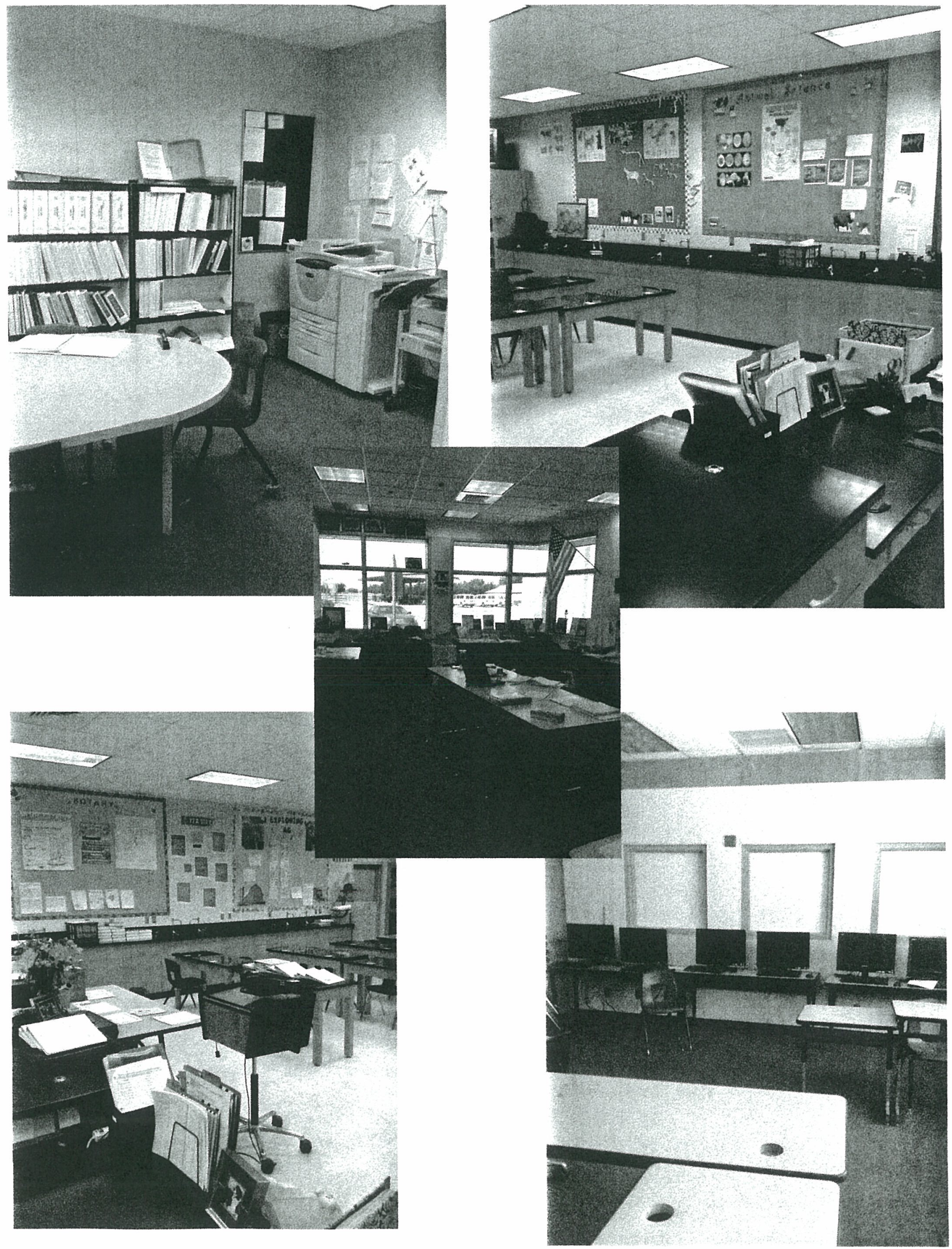




\section{District/Department Budget Process}

The Clovis Unified School District office maintains the Agriculture department's budget. The Clovis East Agriculture Department is responsible for submitting all purchase orders, travel requests and transportation needs to the district office. The budget included funds from the Agriculture Incentive Grant and Perkins Funds.

Reports are received on a monthly basis to the Department head, who keeps track of all expenditures and funds available. We have a binder for each account and keep a copy of purchase orders, receipts, and invoices in the binders. To Open a Purchase Order, you get approval from the Department Chair, then ask Dana our Learning Directors Secretary to create the PO. Once the items are received then you can fill out a payment request form and send it to Dana for payment. Dana is very helpful with any of our Ag Department needs; she can handle most of the financial paperwork for you if you just send her an email telling her what you need.

At the end of the year, all accounts are closed out and purchase orders are signed off. A report of expenditures is completed to best determine changes in the budget for the following year. The report is also used to compare with our proposal to see if we stayed within our budget from the previous year. It is sent to the State for approval. 


\section{Equipment and Materials Wish List}

\section{Sheep Barn}

- (4) Metal hanging hay feeders

- (4) Portable shade structures for Pastures

- Permanent Shade structures for dry lot pens

- Hay Storage area (Structure)

- Portable Panels

- Sheep Head Clippers and Blades for Ewes

- Truck Topper to haul sheep without big trailer

\section{Classroom}

- Autoclave

- Surgical tools for Vet Science

- Updated Edition of Modern Livestock and Poultry Production Book

- 15 New laptops 

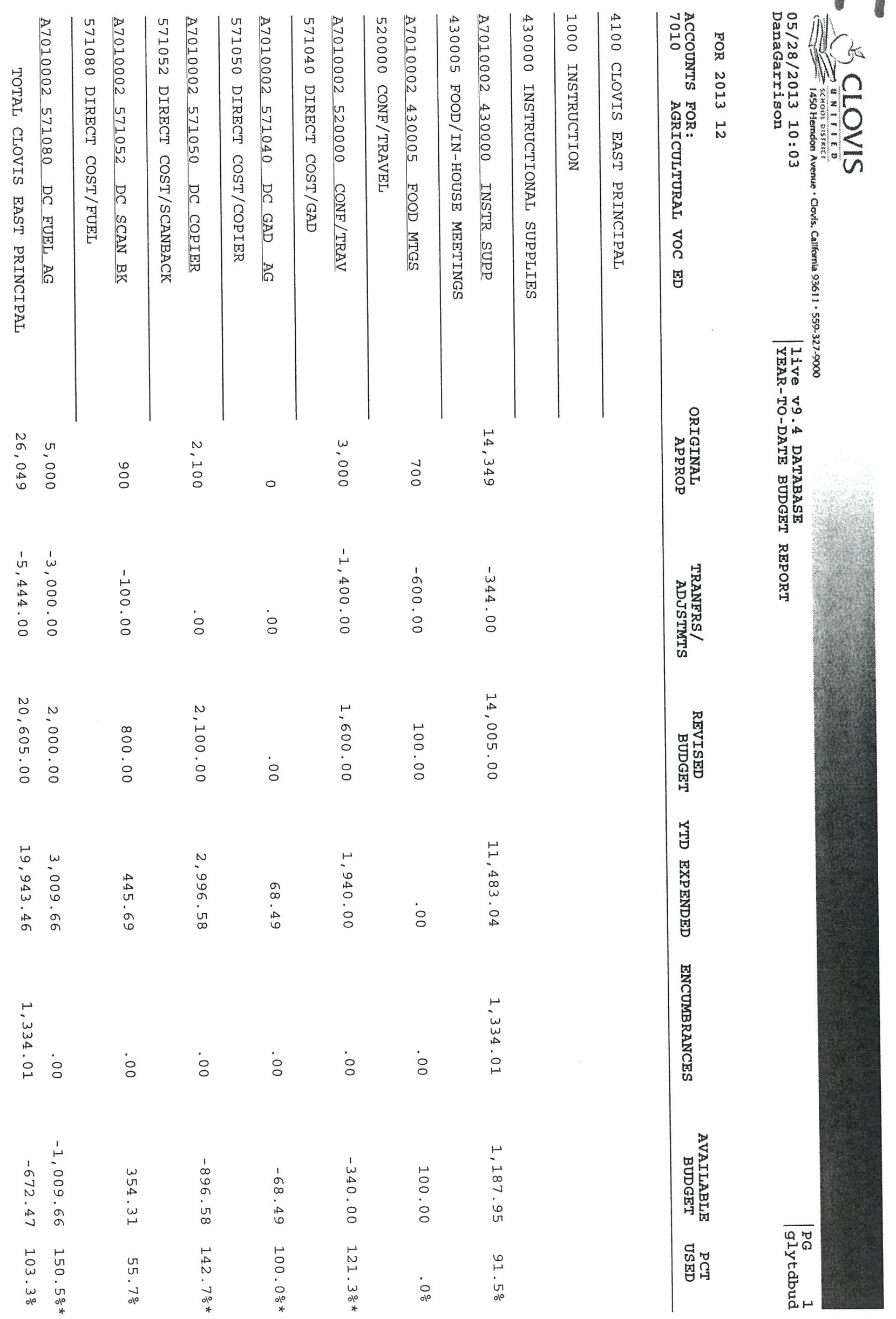


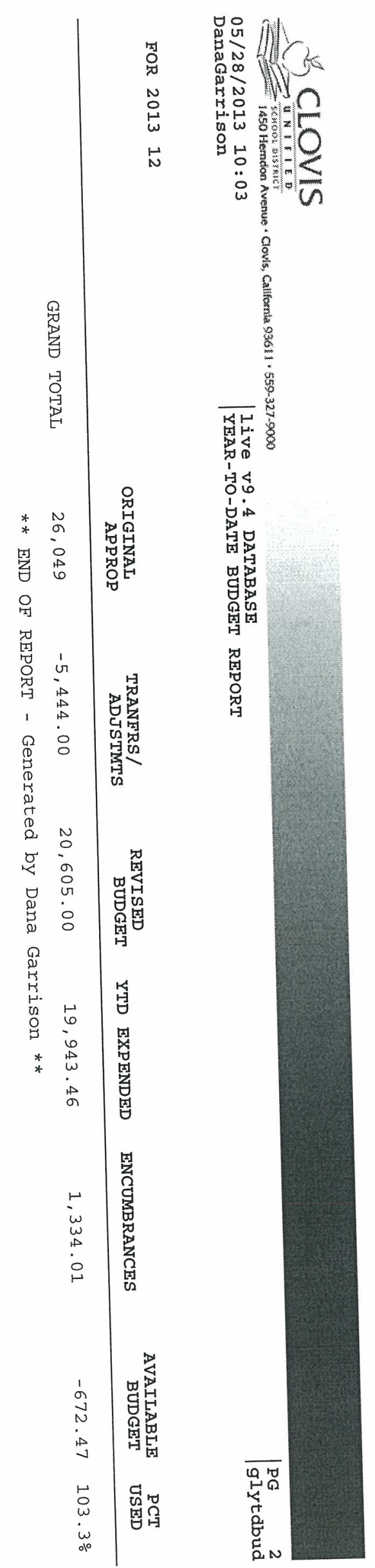




\section{Quality Criteria Six}

\section{Community, Business, and Industry Involvement}

Clovis Unified strongly believes in community support, and that is one of the reasons that our District is so successful. Our District embraces the concept of Advisory Committees and how they can provide a connection to today's industry. Our Agriculture Advisory Committee consists of community members, agriculture industry professionals, postsecondary teachers and staff, and district staff and administration. We keep a roster of all Advisory members and update it twice a year following each meeting.

The Advisory Committee meets twice a year at the Ag Center; invitations and agendas are sent out to our members prior to the meeting. At our meetings we cook and serve dinner to our members and meet as a general assembly. During the dinner time we also have FFA members present special highlights showing the successes of our program since the last meeting. After the general assembly time we break into pathway or industry sector groups. We currently have groups for Animal/Vet Science, Ag Mechanics, Plant Science and Horticulture, and Environmental Science. One improvement we are striving to make next year is to separate the Plant group into Horticulture and Floral and Plant and Crop Science. Minutes are taken for the general assembly as well as all break out groups.

The role of our Ag Advisory Committee is to provide our Ag Staff and School Administration with guidance and support in order to ensure a quality program and well trained students. With our community there always remains a large focus on the maintenance of our Ag Center; that things look presentable, equipment is operational, and all safety standards are being met. Our Committee is very passionate about Agriculture and takes great pride in our program and our Ag Center facility. Recommendations by our Advisory Committee are carried forward to the District Administration for consideration. It is also common for some of our members to call our Area Superintendent any time they have a concern about how things are being ran at the Ag Center.

One challenge that we always face is recruitment of new members. Some of our advisory members have served for many years and are still of great value to us, however 
we need to get fresh new eyes coming in every so often to keep us up to date with the current and modern agriculture industry. We have discussed as a Staff and proposed to the Advisory Members the idea of some by laws that would set term limits or term commitment times so we can be assured that when they sign up they are making a commitment, but do not feel obligated to stay forever. Each Ag teacher is responsible for recruiting new members especially in their industry sector focus area. This year I am attempting to recruit 4 new members for the Animal/Vet Science sector.

One really neat thing that we do for our Advisory Committee is host an Open House Night. This is a special night where we give them two tickets for themselves and two tickets to give to someone that they know in the Ag Industry who is currently not on our committee. We have the whole Ag Center up and running that evening with students doing demonstrations in all areas, Beef Cattle being fit in the barn, Vet Science kids checking vital signs on live animals, rabbit showmanship, transplanting in the greenhouse, tree pruning in the orchard, welding in the shops and much more. We also serve a nice dinner and have special FFA presentations made by students during the dinner. This is a very fun evening and a chance for use to show off all the great things we are doing with our program. It also opens the door for us to meet new industry professionals and possible add them to our committee.

Another aspect of community involvement is our Ag Booster Club. The boosters operate as a complete separate entity from our Ag Program or Advisory Committee with the main focus of raising money for our program and our FFA students. We have many parents of students who are active members of our boosters club, along with parents of student who graduated long ago, and past FFA members who are yet to have students in the program. It is exciting to see that we have people who are willing to support our program even though they do not have kids in program. Our Ag Booster Club is one of the most successful in our area, raising over $\$ 40,000$ a year for our program. They use these funds to purchase equipment we need for the farm, pay for entries at FFA contests, help fund conferences and travel costs for students, and just provide assistance on about anything we ask for. We are very fortunate that when we have a need and bring it to their attention the answer is always yes. 


\section{Supporting Completion Materials}

Item A- Ag Advisory Committee Roster

Item B- Functions and Duties of the Advisory Committee

Item C- Ag Advisory Committee Bylaws

Item D- Ag Advisory Meeting Agendas

Item E- Ag Advisory Minutes 


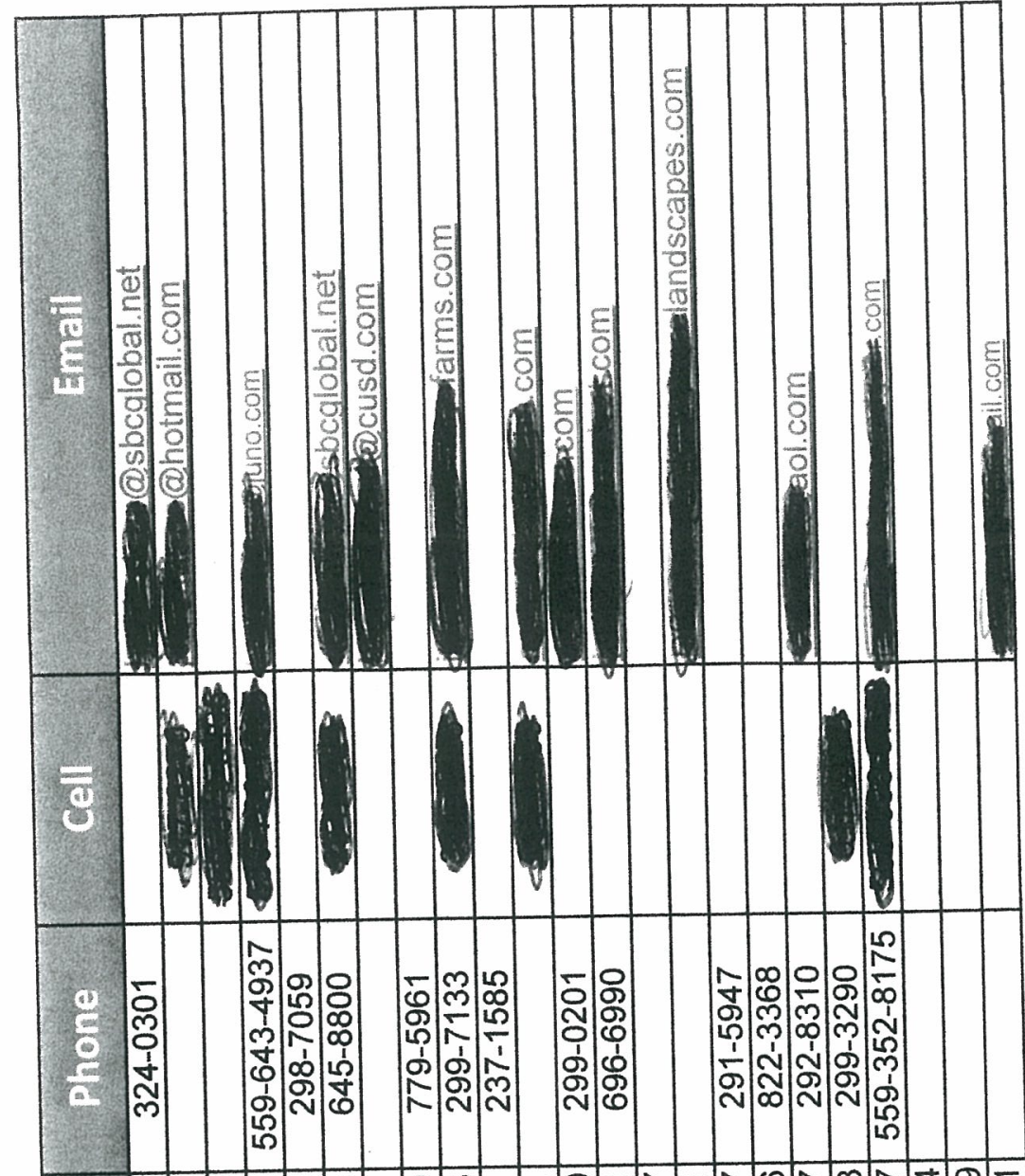

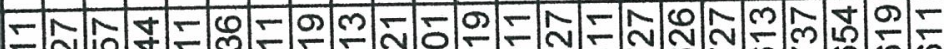

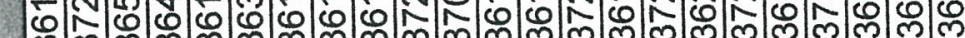

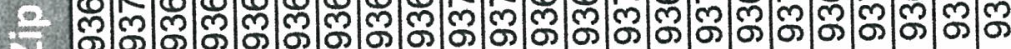

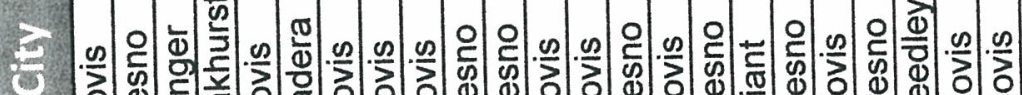

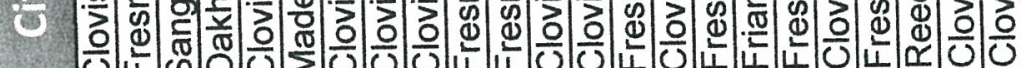

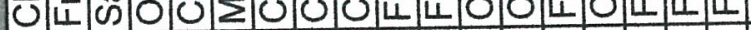

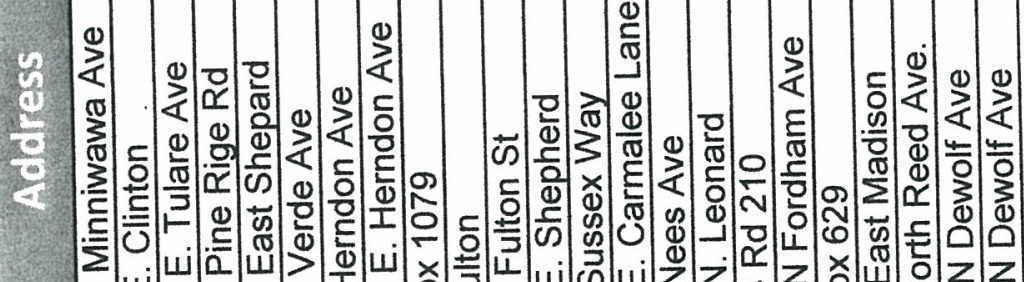

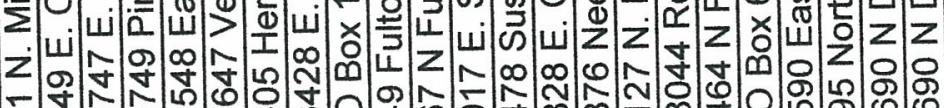
ơ

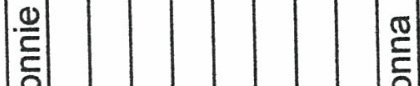

t.

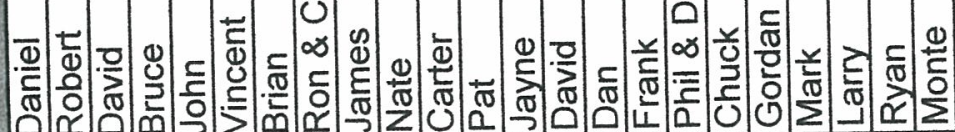

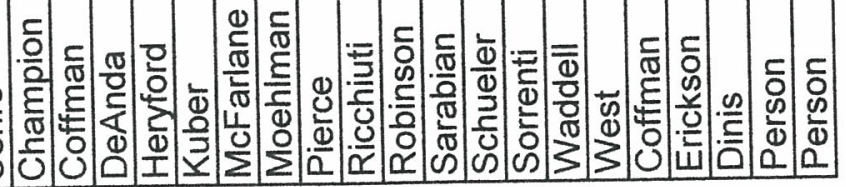




\section{Functions and Duties of Advisory Committees}

1. Help to determine what type of Agricultural Education Tech Prep program is offered.

2. Assist the teacher(s) in finding suitable work stations (internships, work-study, cooperative learning, partnerships) for students in both production agriculture and agriindustry occupations.

3. Help the instructor establish curriculum that has a hands-on, technological approach.

4. Help attract and encourage qualified/capable students into the Agricultural Education Tech Prep program.

5. Help in recruiting and providing opportunities for special-needs students.

6. Help to evaluate the effectiveness of the Ag Tech Prep program. Guidelines for evaluation should be developed cooperatively with the advisory committee, administration, school board, and the Agricultural Education Unit of the California Department of Education and/or Chancellors Office California Community Colleges. ( $\Lambda$ ssessment and certification tools will be made available.)

7. Help gain support for legislation and appropriations.

8. Help the teacher(s) develop a list of capable resource persons for use as speakers, and/or judges for both in-school and out-of-school tests and contests.

9. Help obtain sponsors for appropriating funds for awards, scholarships, or needed equipment and supplies that are useful in carrying out classroom activities and F.F.A. or other youth programs.

10. Help unify the activities of the Agricultural Education Tech Prep program with those of other groups and agencies interested in agriculturc.

11. Assist the teacher in determining skills needed for particular jobs at entry, technical and professional levels so that he/she may be included in the instructional program.

12. When appropriate, serve as resource person to instructor visiting work place learning sites of students and participating in classroom instruction or demonstrations and accompanying or hosting field trips. 
13. Study and make recommendations on problems presented to it by the school board on which further information is needed.

14. Provide the teacher with technical assistance and keep him/her aware of new developments in the agricultural industry.

15. Provide current resources to develop and maintain an Ag library of visual aids, magazines, and books concerning agriculture and agricultural occupations.

16. Serve as speakers at civic clubs, open houses, and career days to tell the story of school-industry cooperation.

17. Identify current standards for new equipment.

18. Assist in procuring opportunities to upgrade the teacher's technical skills and knowledge. 


\section{Ag Advisory Meeting Bylaws}

It is important that correct procedures and rules be established and clearly understood by committee members, school administrative staffs, and the board of education. These rules should be

decided upon by the committee with assistance from the school. All correspondence should be sent to administrators and advisory committee members. Items to be considered are:

\section{Number of meetings}

1.1 Must meet regularly and often enough to carry out their assignment.

1.2 Monthly or bi-monthly meetings are usually the most desirable.

1.3 Minimum number is two per year.

1.4 Practical number is between three and eight per year.

1.5 Necessity should always determine the exact number.

1.6 Often the most valuable advice comes from busy individuals.

1.7 Better to have fewer well planned, well attended meetings.

\section{Selection of Officers}

2.1 Generally a chairperson, vice chairperson, and recorder are sufficient.

2.2 Chairperson should be a lay person elected by the committee.

2.3 It is usually best that the agriculture teacher serves as recorder and general consultant.

\section{Length of Service by Committee Members}

3.1 Three-year terms are recommended.

3.2 At formation meeting members draw for one, two, or three year terms to provide for continuity of membership.

3.3 Individual preferences in length of service need to be considered.

3.4 Limitation should be placed on reappointments.

3.5 Nominees should be submitted to board of trustees for approval. 


\section{Length and Place of Meetings}

4.1 For efficient and effective use of time, the agenda for each meeting must be well planned.

4.2 Ample meeting notice of 10 days to 2 weeks is recommended.

4.3 Copy of agenda, minutes from previous meeting, and any reading material requiring action should be sent in advance of meeting date.

4.4 Two-hour meetings, held at a time and date chosen by the committee, are recommended.

4.5 The meeting place should provide a conference table in a quiet environment.

4.6 Usually the agricultural department of the school provides the best meeting site, allowing members to become familiar with facilities of the department.

\section{Filling Committee Vacancies}

5.1 Vacancies which occur because of term completion or other reasons should be filled by nomination from the advisory committee, teacher, superintendent, department head, or principal, and approved by the board of education.

5.2 The committee may be asked for suggestions.

5.3 A committee should not be permitted to choose its own replacements. 5.3.1 This would be self perpetuating.

5.3.2 May become unrepresentative and unduly independent of the school administration.

5.4 Rules of procedure should indicate that if a committee member misses meetings repeatedly without reason, the position be declared vacant by the chairperson, and the school board so notified.

6. Distribution of Minutes: All committee members, the vocational education director, the principal/president, and the regional supervisor.

7. Making Decisions: Currently many organizations operate by consensus approval of agenda items. When consensus cannot be reached or decorum is in question, refer to Robert's Rules of Order. 


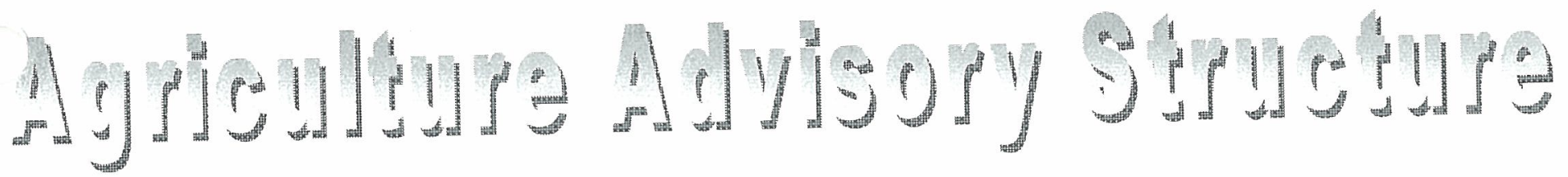

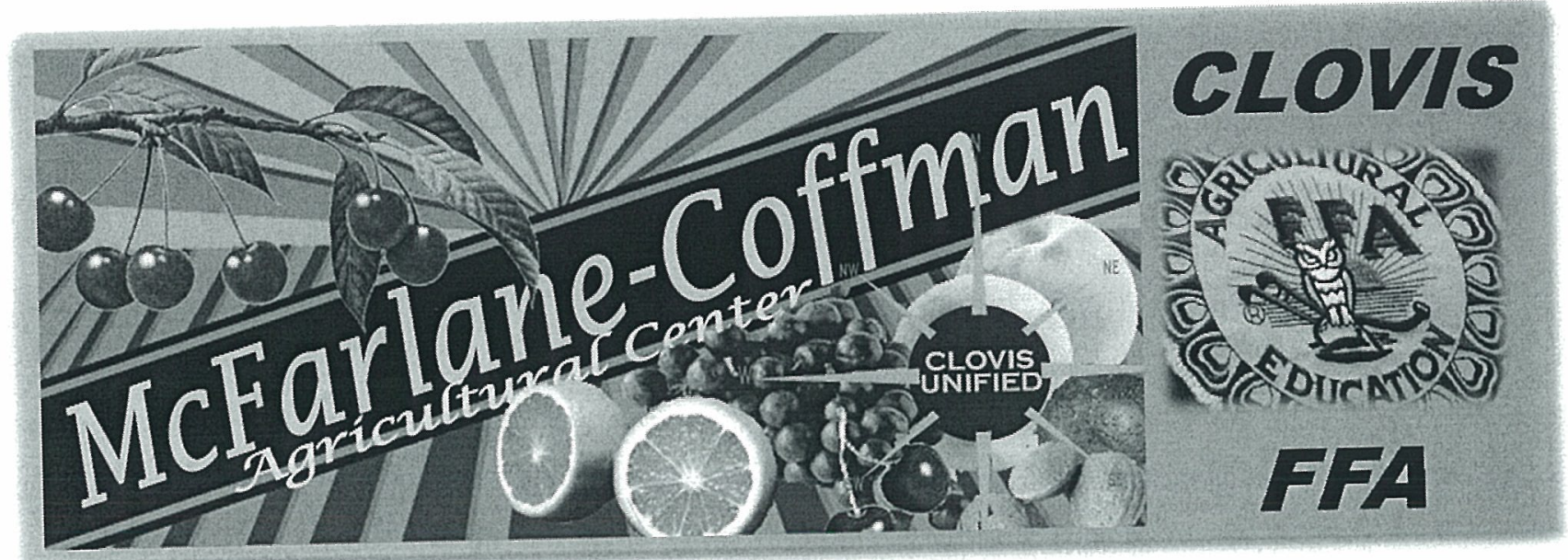

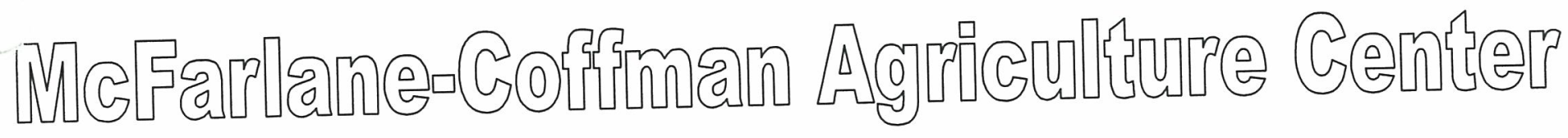




\section{Instructions for the McFarlane-Coffman Agricultural Education Advisory Committees}

1. We welcome you on behalf of the Agriculture Staff, Administration and Clovis Unified School District governing board.

2. You have been selected to be a member of this advisory committee by the Agriculture Department Chairman in support of the majority of the Agriculture Department faculty.

3. The current roster of this committee will be submitted for approval by the Clovis Unified School Board of Trustees.

4. While you are not a policy making body, you serve advisory to the faculty of the McFarlane-Coffman Agriculture Center and through channels, to the principal, superintendent, and board. We need your expertise in this area.

5. The Clovis Unified School District is interested in the best possible Agricultural Education program. We need to know what is "ideal" for this program from the standpoint of the community. Please bear in mind that our final decisions must be compatible with available funds and state and local rules and regulations.

6. You will be a working committee. Students \& school staff expects to benefit from your work.

7. Purpose of this Advisory Committee:

7.1 Review and evaluate existing programs, courses of study, facilities, equipment.

7.2 Propose new programs and/or courses when needed based on solid data for this community.

7.3 Help revise existing programs, suggest changes or deletions, and develop educational specifications for the programs. (For use in building the program and planning for equipment and facilities.)

7.4 Help develop building plans; review architects plans, etc., where new buildings are being proposed. 7.5 Help point out changes needed for the future in your area of interest - Keep the program up to date. 7.6 Help in placement and in evaluating performance of our Agricultural Education students at your school, college or workplace.

8. You will be a "helping group" (as well as advisory) to the instructors, as the program is progresses.

9. This committee serves at the pleasure of the school board and may be dissolved at any time by board action.

WE NEED YOUR HELP. WE APPRECIATE YOUR WILLINGNESS TO GIVE IT AND BE OF SERVICE TO YOUR SCHOOL. 


\section{Operation of Advisory Committee}

It is important that correct procedures and rules be established and clearly understood by committee members, hool administrative staffs, and the school board. These rules should be decided upon by the committee with assistance from the school. All correspondence should be sent to administrators and advisory committee members. Items to be considered are:

\section{Number of meetings}

1.1 The Agriculture Advisory will hold its general meeting twice per year (Fall and Spring)

1.2 Special meetings may be called by the Advisory Board with the approval of the Agriculture

Department Chair.

1.3 Necessity will always determine the exact number.

1.4 Better to have fewer well planned, well attended meetings.

\section{Selection of Officers}

2.1 A Chairperson and Vice Chairperson will be selected by the committee membership.

2.2 Committee member receiving the greatest number of votes will serve as Chairperson.

2.3 Committee member receiving the second greatest number of votes will serve as Vice Chairperson.

2.3 The Current Agriculture Department Chairman will serve as the Recording Secretary.

\section{Length of Service by Committee Members}

3.1 Members will be placed on a Graduated Service Schedule. (See Attached)

3.2 Each year one-third of the committee membership will end their term.

3.3 Upon formation of Graduated Service Schedule members will draw for service placement (1,2,or 3 years)

3.3 When a committee member ends their term they may be asked to continue their service to the program.

3.2 If a committee member continues their service they will be placed at year three on the graduated service schedule.

3.5 Nominees shall be submitted to board of trustees for approval.

\section{Length and Place of Meetings}

4.1 For efficient and effective use of time, the agenda for each meeting must be well planned.

4.2 Ample meeting notice of 10 days to 2 weeks prior to each meeting will be the goal.

4.3 Copy of agenda, minutes from previous meeting, and any reading material requiring action should be sent in advance of meeting date.

4.4 Two-hour meetings, held at a time and date chosen by advisory board as advised by the Agriculture department staff

4.5 Unless otherwise noted, meetings will take place at the McFarlane-Coffman Agriculture Center.

\section{Filling Committee Vacancies}

5.1 Vacancies which occur because of term completion or other reasons should be filled by nomination from the advisory committee, teacher, superintendent, department head, or principal, and approved by the board of education.

5.2 The committee may be asked for suggestions.

5.3 A committee member is not permitted to choose their own replacements.

5.4 Rules of procedure should indicate that if a committee member misses meetings repeatedly without reason, the position be declared vacant by the chairperson, and the school board so notified.

\section{Distribution of Minutes:}

6.1 Minutes will be distributed to all committee members, CTE/ROP Director, the principal, school board representative and the Regional Supervisor for Agriculture Education(CDE).

\section{Making Decisions:}

Currently many organizations operate by consensus approval of agenda items. When consensus cannot be reached or decorum is in question, refer to Robert's Rules of Order. 
CUSD - GOVERNING BOARD
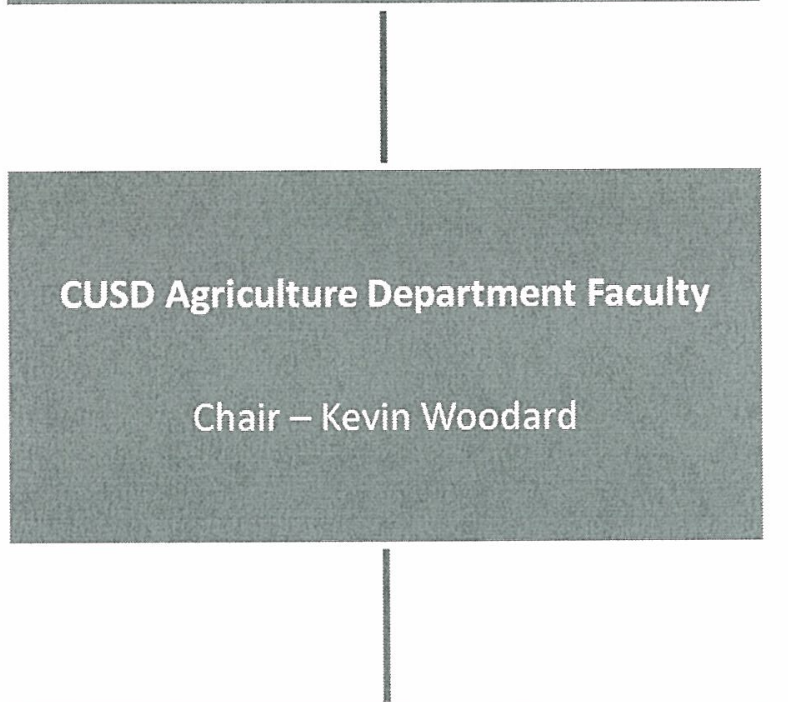

Agriculture Advisory Board

Chair

Vice Chair

Secretary - Kevin Woodard

Animal Science Committee

Chair -

Secretary - Jennifer Knight

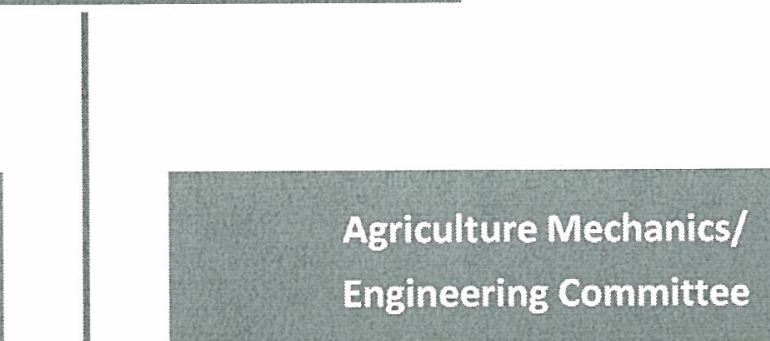

Chair -

Secretary - David Valdez

\section{Environment/}

Natural Resources Committee

Plant Science Committee

\section{Chair-}

Chair -

Secretary - Kevin Woodard

\section{Secretary - Steve Gambril}




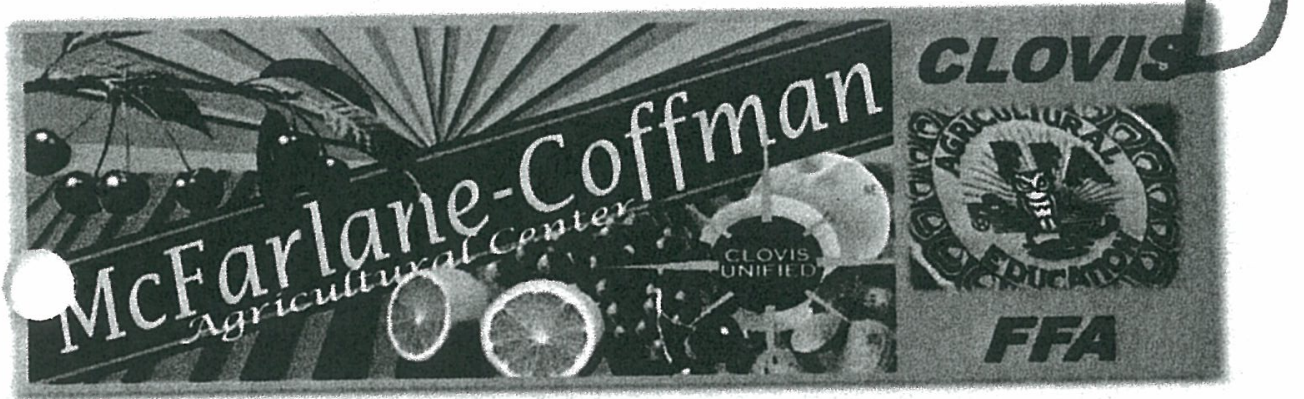

\section{Spring Advisory Meeting and Industry Open House}

\section{Thursday, April $4^{\text {th }} 2013$}

You and a guest are invited to represent your industry at the Spring Advisory Committee Meeting and Industry Open-House for the McFarlane-Coffman Agriculture Center at Clovis East High School.

When: Thursday, April $4^{\text {th }}$ from 6:00 PM to 8:00 PM

6:00 PM Welcome

6:15 PM Dinner/Conversation

6:45 PM Program Update

7:00 PM Facility Tour

Where:McFarlane-Coffman Agriculture Center Clovis East High School 2940 Leonard Avenue, Clovis, CA

Purpose: Create industry relationships that will foster agricultural experiences and opportunities for our students.

Please RSVP with the contact information below. Be sure to indicate your "Ticket Number" and "number of attendees".

RSVP To: Kevin Woodard

by Monday April $1^{\text {st }} \quad$ (559)327-4324

kevinwoodard@cusd.com

Ticket No. 0250 


\section{Fall Agriculture Advisory Meeting}

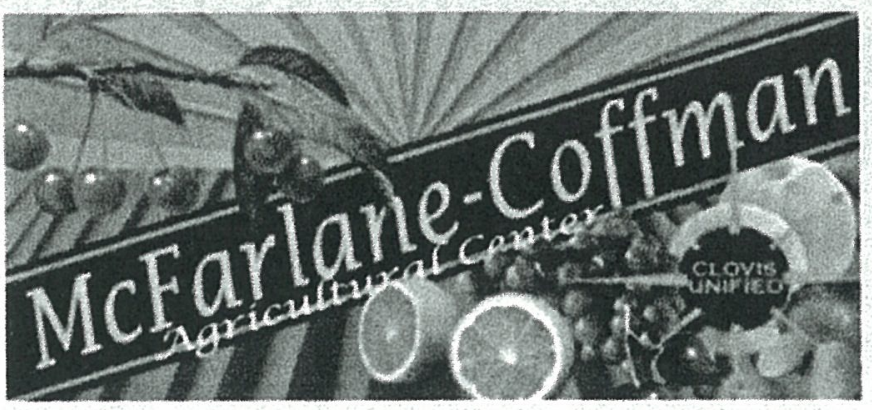

\section{Dinner Is Served}

- Smoked Turkey

- Ham

- Dressing

- Mashed Potatoes

- Corn

- Green Beans

- Pie for Dessert

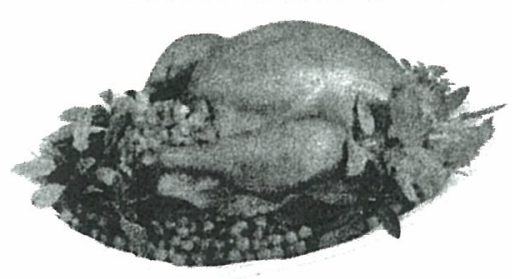




\section{Agenda}

- Dinner

- Welcome

- Introductions

- Program Update

- Advisory Committee Structure

- Committee Breakout Sessions

\section{Introductions}




\section{FFA Update}

- NATIONAL

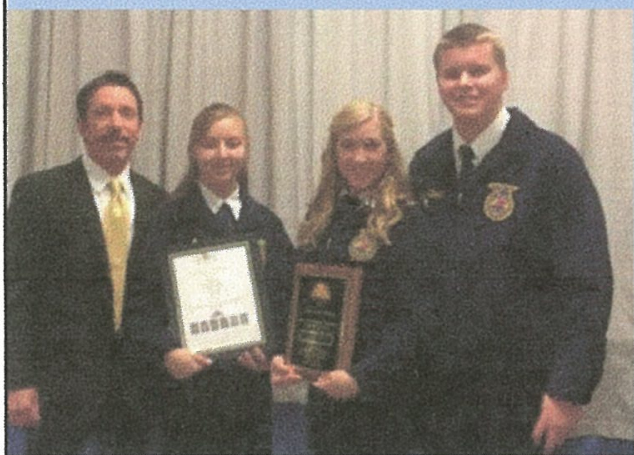
CONVENTION

- Reserve National Champions

- Donielle Aluisi $1^{\text {st }}$ Place

- Travis McDonald

- Kelsey Gray

- California Delegates

- Emily Gambril

- Macey Perry

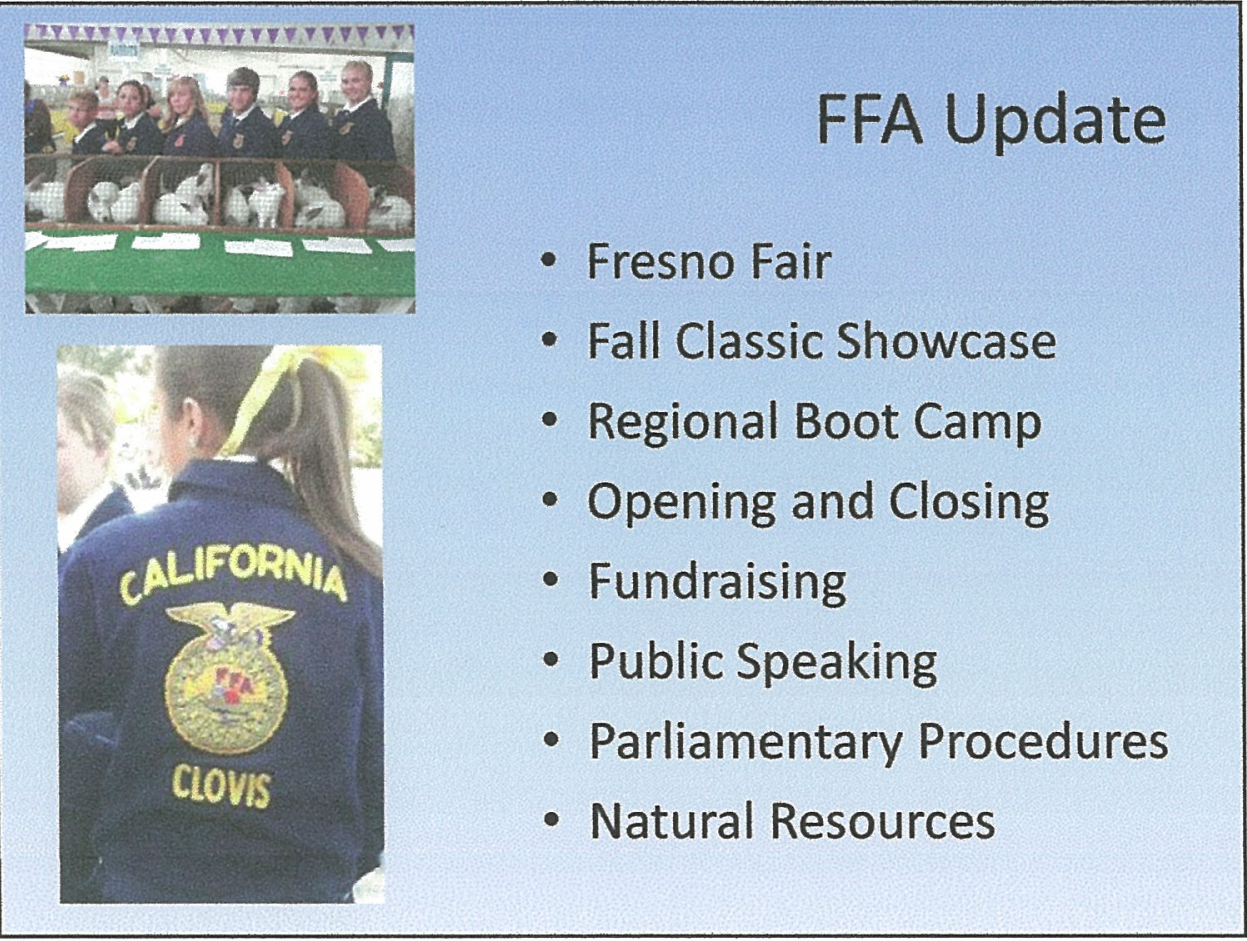



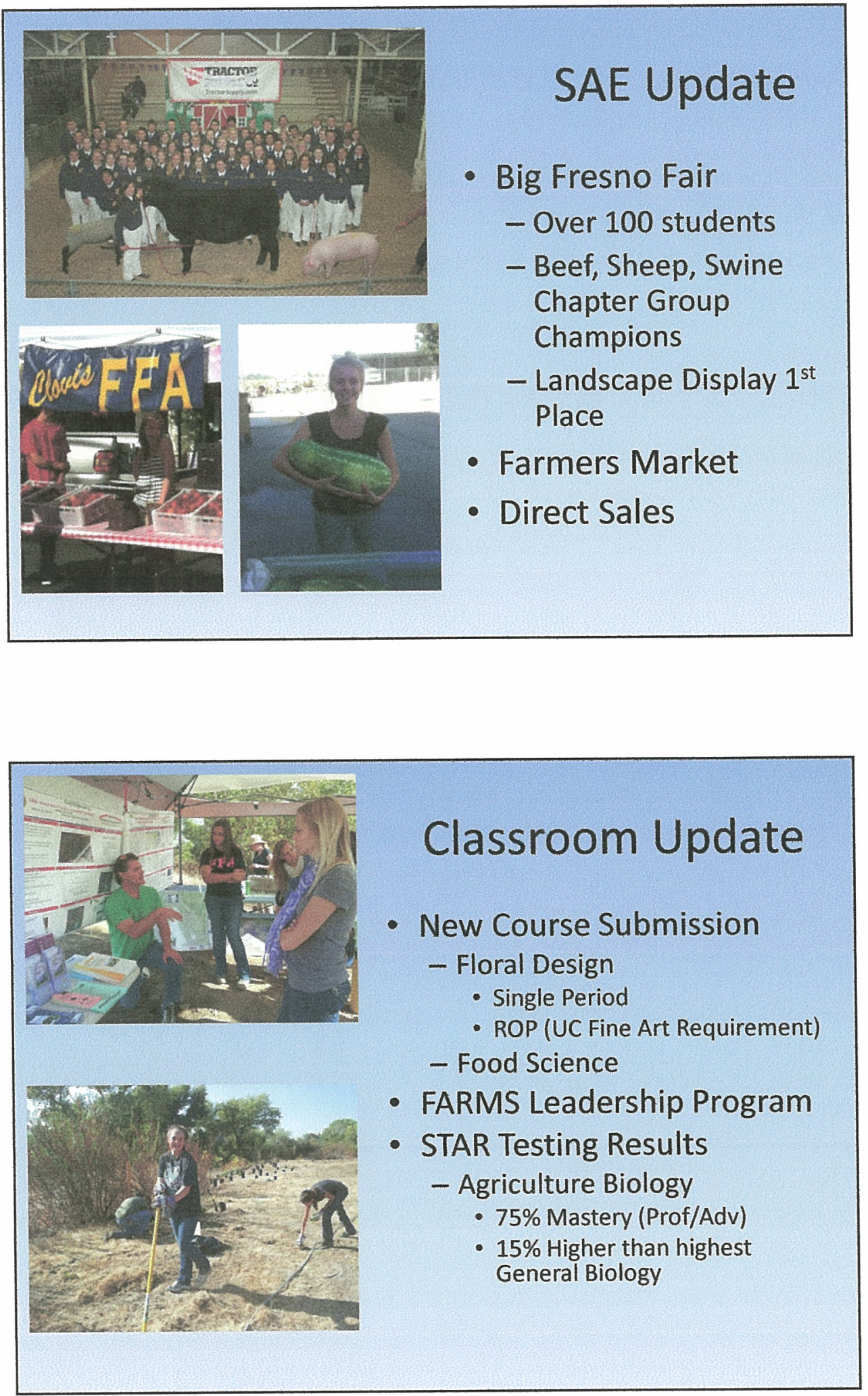


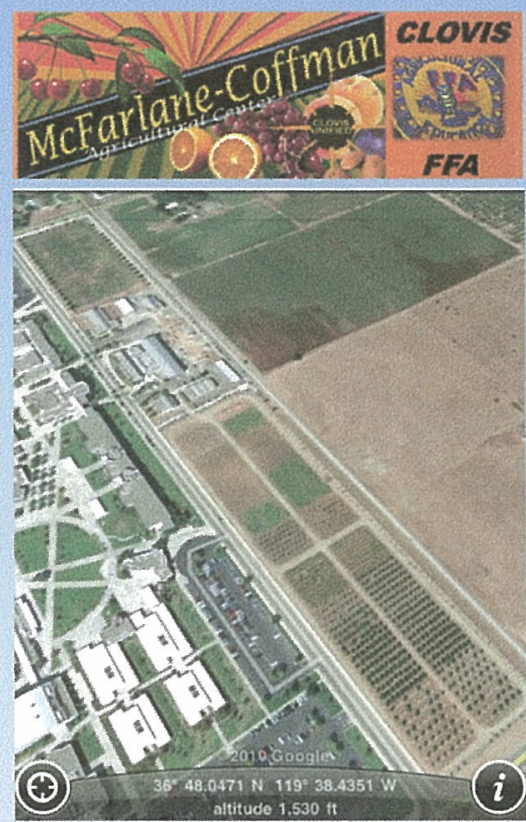

\section{Farm and Facilities Update}

- On Farm Production

- Student Projects

- Ag Center Landscape Project

- Booster Club Donations

- Gator and Mower

\section{Program Enrollment History}

\begin{tabular}{|c|c|c|c|c|c|c|c|c|}
\hline Year & 2012 & 2011 & 2010 & 2009 & 2008 & 2007 & 2006 & 2005 \\
\hline Enrollment 9-14 & 583 & 604 & 589 & 685 & 663 & 633 & 694 & 637 \\
\hline Enrollment 9-12 & 551 & 567 & 569 & 665 & 636 & 614 & 667 & 617 \\
\hline Enrollment 7-8 & 350 & 274 & 328 & 315 & 302 & 329 & 297 & 319 \\
\hline $\begin{array}{l}\text { Number of } \\
\text { Teachers }\end{array}$ & 6 & 6 & 6 & 6 & 6 & 6 & 6 & 6 \\
\hline $\begin{array}{l}\text { Number of } \\
\text { Sections }\end{array}$ & 31 & 32 & 27 & 32 & 32 & 32 & 32 & 32 \\
\hline $\begin{array}{l}\text { Number of } \\
\text { Students / } \\
\text { Teacher }\end{array}$ & 150 & 140 & 150 & 163 & 156 & 157 & 161 & 156 \\
\hline $\begin{array}{l}\text { Ave. Students / } \\
\text { Section }\end{array}$ & 29.1 & 26.3 & 33.2 & 30.6 & 29.3 & 29.5 & 30.1 & 29.3 \\
\hline $\begin{array}{l}\text { Active Program } \\
\text { Completers } \\
\text { (APCs) }\end{array}$ & 37 & 37 & 20 & 20 & 27 & 19 & 27 & 20 \\
\hline Seniors & 122 & 123 & 117 & 158 & 165 & 109 & 110 & 86 \\
\hline Juniors & 135 & 137 & 173 & 211 & 135 & 143 & 108 & 134 \\
\hline Sophomores & 104 & 107 & 114 & 176 & 188 & 174 & 184 & 134 \\
\hline Freshman & 190 & 200 & 165 & 120 & 148 & 189 & 266 & 263 \\
\hline $\begin{array}{l}\text { Intermediate } \\
\text { Students }\end{array}$ & 350 & 274 & 328 & 315 & 302 & 329 & 297 & 319 \\
\hline
\end{tabular}




\section{Advisory Committee Restructuring}

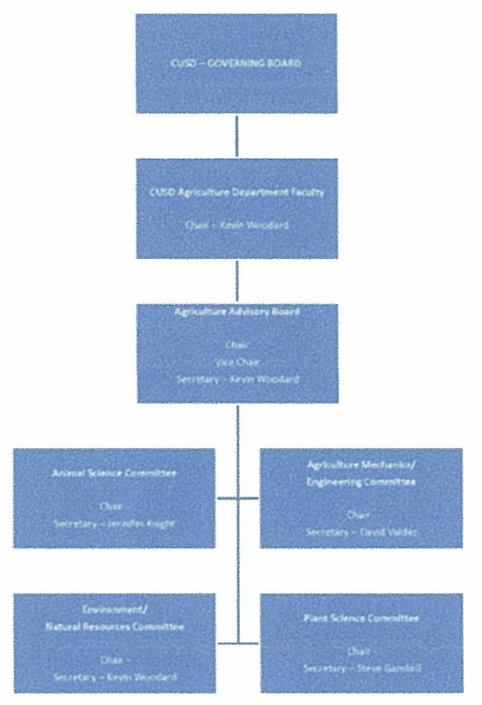

- Instructions for Advisory Committee Members

- Operation of Advisory Committee

- Advisory Organizational Chart

- Advisory Member Information Card

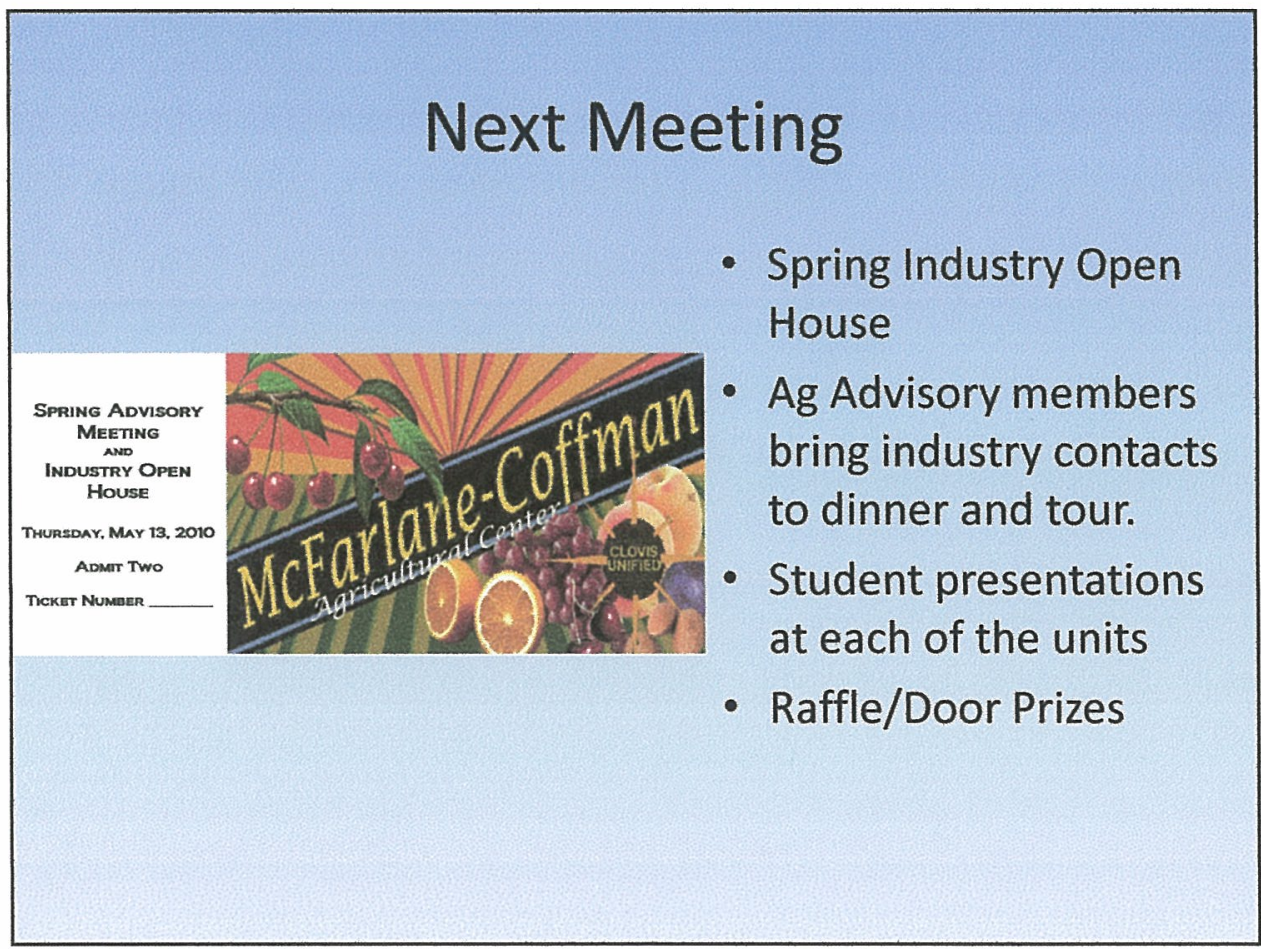




\section{Committee Breakout Sessions}

- Animal Science

- Jennifer Knight

- Ken Dias

- Room 903

- Environmental Science

- KevinWoodard

- Room 904
- Plant Science

- Steve Gambril

- Aireal Covey

- Room 907

- Ag Mechanics

- David Valdaz

- Room 905 
Date:

April, $23^{\text {rd }} 2013$

Class: ROP Veterinary Science

School/District: Clovis East High School / Clovis Unified School District

Teacher (s): Jennifer Knight

Business and Industry Members Present (full name, title, and business name): Ryan Person

Business Members Absent:

All other ROP Vet Teachers and Advsiors

Educators, Ad Hoc members, and others present (non-voting membership): Jennifer Knight

I. Purpose of Meeting

The purpose of the Advisory Committee is to provide up-to-date information on current trends and technologies, and to make recommendations for the development and improvement of the program. To review all course content and surveys.

II. Introductions

All advisors and participants in the meeting were introduced.

III. Meeting Called to Order Jennifer called the meeting to order at $6: 40 \mathrm{pm}$.

IV. Review of 2012 Follow-up Survey Course Summary Wondering why Clovis East ROP did not have any survey data reported? The data reported only reflected a small portion of the total student population. Maybe a web survey would be more effective than the telephone survey.

\section{Labor Market Information}

A. Current Trends and Emerging Technologies

a. Jobs in relation to Food Animals and Food Safety are in high demand

b. There is a current shortage of Large Animal Vets

i. Large Universities are offering 5 year programs that include a DVM along with an MBA. This is due to the fact that may large cooperate farms and large livestock owners need to staff vets with an understanding of the business side of the industry.

ii. In order to prepare our Vet students for this aspect of the industry we will encourage them to take Animal Science as a Junior to learn more about the livestock industry before enrolling in Vet Science their Senior year.

c. Animal Genetics

i. Students need to be strong in applied sciences

ii. Animal reproductive technologies is a fast growing field

1. Embryo Transfer

2. Artificial Insemination

a. AI technologies are even being applied to the wildlife and sport hunting sector today.

\section{Review Industry Certifications}

This course currently does not have an industry certification. We would like to explore the option of an agreement with College of the Sequoias once they get their Vet Tech program running. Once they can acquire the necessary funding they will be an accredited Vet Tech Program. 


\section{Internships/ Employment/Field Trip Suggestions}

Explore the option of taking the class to U.C. Davis for a tour and an introduction to the Vet School.

VIII. Suggestions and Recommendations

Explore requirements for admission to various vet schools. Research a new school called Ross University, may be easier to gain admission as they build their program. Mrs. Knight also talked about going on a job shadow with a few vets over the summer to gain hands on training herself.

The Advisory night would have been much more helpful and beneficial if other ROP Vet Science teachers and advisors would have shown up. I was embarrassed that I and my one advisor were the only ones present at this meeting.

\section{Recommendations to Support Continuing the Program}

Ryan moved to support continuing the program, and Jennifer seconded the motion. All agreed to approve support for continuing the program.

X. Next Meeting The Fall advisory meeting will be a Clovis East Ag Center, date yet to be scheduled.

XI. Adjournment

The meeting adjourned at 7:40 pm. 
Date: November 8, 2012

Class: ROP Veterinary Science

School/District: Clovis East High School / Clovis Unified School District

Teacher (s): Jennifer Knight

Business and Industry Members Present (full name, title, and business name): Ryan Person, John Coffman, Connie Kuber, Ken Dias

Business Members Absent:

Educators, Ad Hoc members, and others present (non-voting membership): Jennifer Knight, Amber Sawyer and Cody Jacobson, student teachers for Jennifer, Beth Lederach, CUSD ROP

\section{Purpose of Meeting}

The purpose of the Advisory Committee is to provide up-to-date information on current trends and technologies, and to make recommendations for the development and improvement of the program.

\section{Introductions}

All advisors and participants in the meeting were introduced.

\section{Meeting Called to Order} Beth called the meeting to order at 7:45 pm.

\section{Review and Approval of Minutes}

All advisors reviewed and studied the fall 2011 minutes. Connie moved to approve the minutes, and Ryan seconded the motion. All agreed to approve the fall 2011 minutes.

\section{Curriculum Review}

\section{A. Status of Previous Recommendations}

Ryan remembered that Susan had trouble placing her students in community classroom facilities. Advisors brainstormed more places that students might be able to work next semester.

\section{B. Course Description}

All advisors reviewed the course description and agreed that it was a great course description.

\section{Job Titles}

The course descriptions are adequate. Dairy herding lab technician would also be relevant to this career area.

D. Course Outline and Hours of Instruction

The course outline is thorough and looks comprehensive to the advisors. Connie was a substitute teacher for Susan Henderson-Perry, and she is always amazed at the depth and breadth of study that the vet students complete. Ryan said the course looks complete and rigorous, and Connie agreed.

E. Training Plans for Community Classroom/CVE Jennifer described her plans for community classroom for next semester. Jennifer has 28 students to place in community classroom facilities. She shared that she really has to speed through the curriculum in order to prepare students for the community classroom experience, but she wants them to be ready to work in the various settings. Students have a variety of abilities according to Jennifer, so she really needs to differentiate instruction in order to prepare them for working in the community classroom facilities. 


\section{F. Instructional Materials \\ Jennifer is content with the materials she has. ROP has been helpful in providing the instructional supplies she needs. Jennifer discussed the textbook she uses, and she showed the advisors a new textbook that would be helpful for her students. She hopes to be able to get a new textbook for next year.}

\section{G. Equipment} Jennifer has all the equipment that she needs to teach the class successfully. She is still finding things in the classroom from the previous teacher. For now, she has what she needs.

\section{Articulation/UC Approval}

This course is currently UC-approved with a-g status. This class should become articulated with Reedley and Fresno City College, and we will work on this with Ryan who teaches at Reedley College.

VII. Labor Market Information and Future Trends

The advisors discussed the possible jobs for the future and what the possibilities would be for students getting a career in veterinary science. There is a shortage of vets in the large animal industry. Ryan said that schools and colleges are accepting students for large animal study-this has become a real focus. Connie said food safety is an important field of study for Jennifer's students, things like biohazard study and pharmaceutical companies. Ryan reiterated that there is definitely a demand for large animal vets.

\section{Placement/Continuing Education}

Students will need to get a four-year college degree, and some students could become a Vet Technician at a two-year school. Connie said there are master's programs available to students who want to go beyond the four years. Ryan shared that a vet must be trained in five years, but they may also get an MBA concurrently, which would make them highly marketable. College of the Sequoias is a school to watch because they are getting the Vet Tech program in the near future because of Prop 30 passing. This would be a great option for students to become trained in 2-3 years.

IX. Suggestions and Recommendations

The advisors recommended that students get as much experience as possible with a wide range of animals. Connie asked about summer work/experience as somewhat of a prerequisite for the course. Jennifer might be able to have a summer "reading packet" as a way to increase knowledge before students arrive in her classroom. The ROP Summer Intensive has a different focus-but the summer reading requirement would help Jennifer have better prepared students. Connie and Ryan said they would try to find guest speakers for her class. Jennifer asked her advisors for magazines and journals that students could read and annotate, in preparation for Common Core State Standards, and Connie agreed to bring journals and reading sources for her students. The advisors will help her with getting the students placed in community classroom settings. They gave several names for contacts with community classroom possibilities.

X. Recommendations to Support Continuing the Program Ryan moved to support continuing the program, and Connie seconded the motion. All agreed to approve support for continuing the program.

XI. Next Meeting The spring advisory meeting is at Clovis North High School on April 23, 2013, 6:00 pm.

\section{Adjournment}

The meeting adjourned at 8:30 pm. 


\section{Quality Criteria Seven}

\section{Career Guidance}

Students are encouraged to select a career pathway in their ninth or tenth grade year, then in their eleventh and twelfth grade years they will receive advanced instruction in their selected career pathway. Because of the unique structure of our Ag Program in a multi high school district we may not have students all four years, we commonly get students for upper level electives like ROP their Junior and Senior years only. Another challenge we face with pathway and career selection is that because we have students from each of the five Clovis Unified high schools, which means working with many different counseling staff all with a different level of knowledge about our program. We do attempt to provide a yearly refresher course for our District counseling staff.

All students do complete the career planning portion of the Student Data sheet in class each year with guidance from their Ag teacher. Based on a student's career interest we can pre counsel them about what sequence of courses they should take in our Ag Program. In all Ag classes we teach a careers and job skills lesson or unit. In our pathway specific electives like ROP students will create resumes, cover letters, and professional portfolios. They will also participate in mock job interviews. One aspect of our ROP courses is that all students participate in community classroom for the second semester. They will have to use their job seeking skills to interview and be placed at an actual job site.

Another way we expose our students to careers in agriculture is by inviting guest speakers into our classes and taking industry field trips. Each year representatives from trade schools, community colleges, State Universities, and Businesses are invited to visit our program and inform our students about the opportunities for careers in their field, or postsecondary education needed for their career goal. We also utilize field trips like visiting the Animal Units at Fresno State or attending the World Ag Expo as a tool to promote knowledge and career opportunities. We can also not discount our participation in CDE Field Days as a means to expose students to various postsecondary options. 
Clovis Unified has also previously entered into articulation agreements with Reedley College and College of the Sequoias. These agreements have been maintained through the Fresno County Office of Education and the Regional Occupation Program (ROP) within our District. Students completing our ROP courses in Vet Science and Welding receive a $2+2$ certification which carries course credit upon completion of 12 units of coursework at either institution. I however cannot find the paperwork for this agreement.

\section{Supporting Completion Materials}

Item A- Student Data Sheets

Item B- CUSD High School Educational 4 year Plan, showing our A-G approved agriculture courses. 


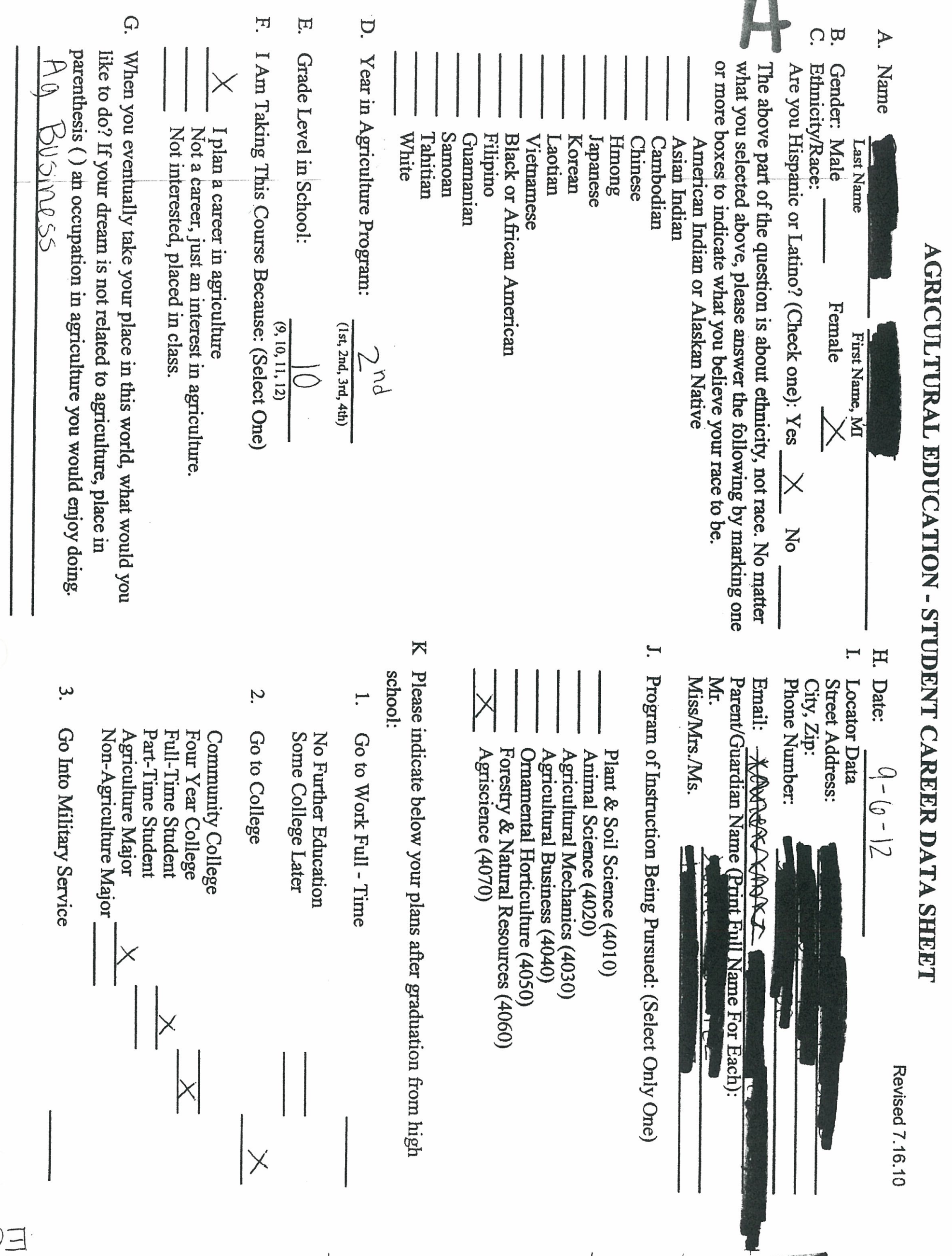




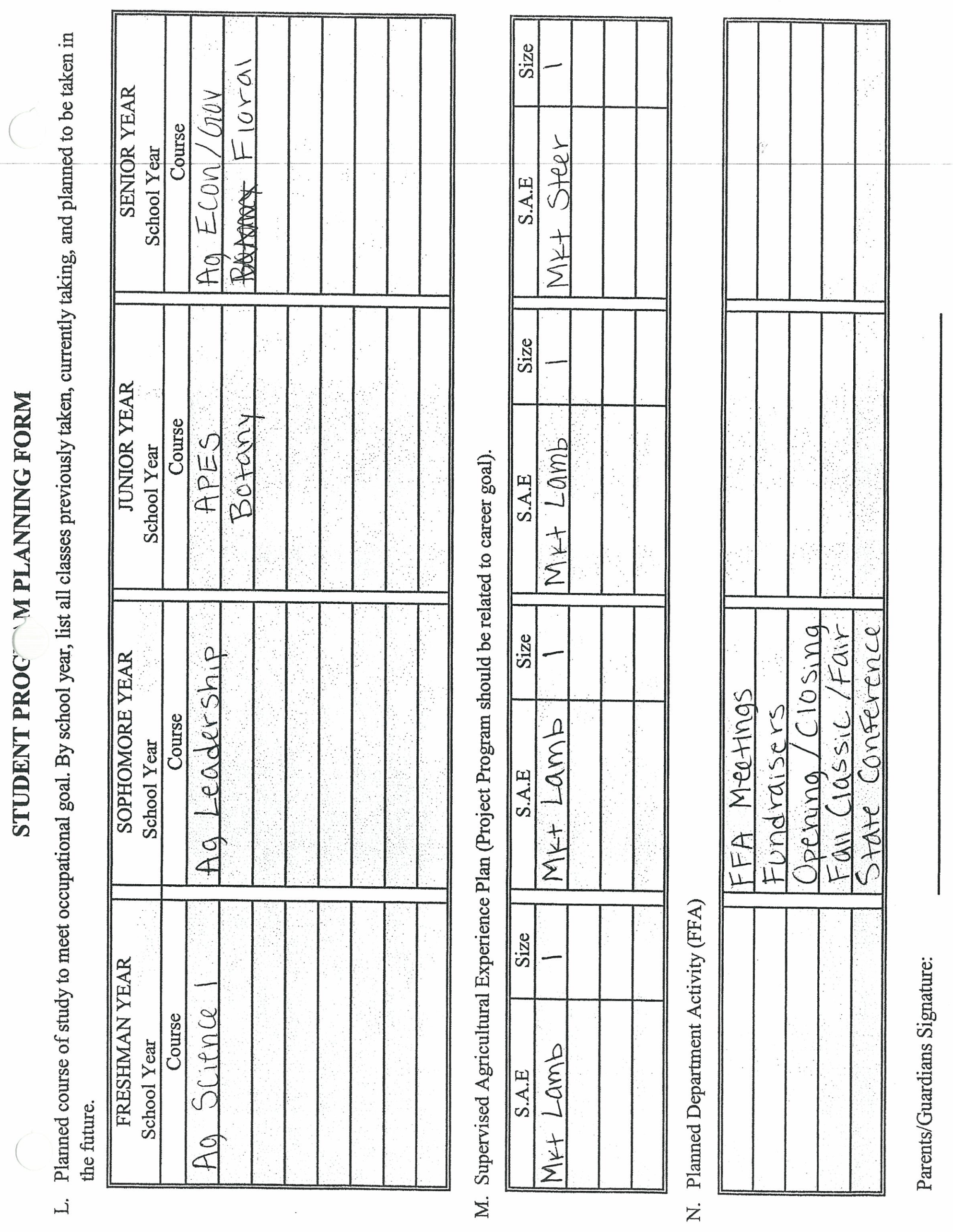




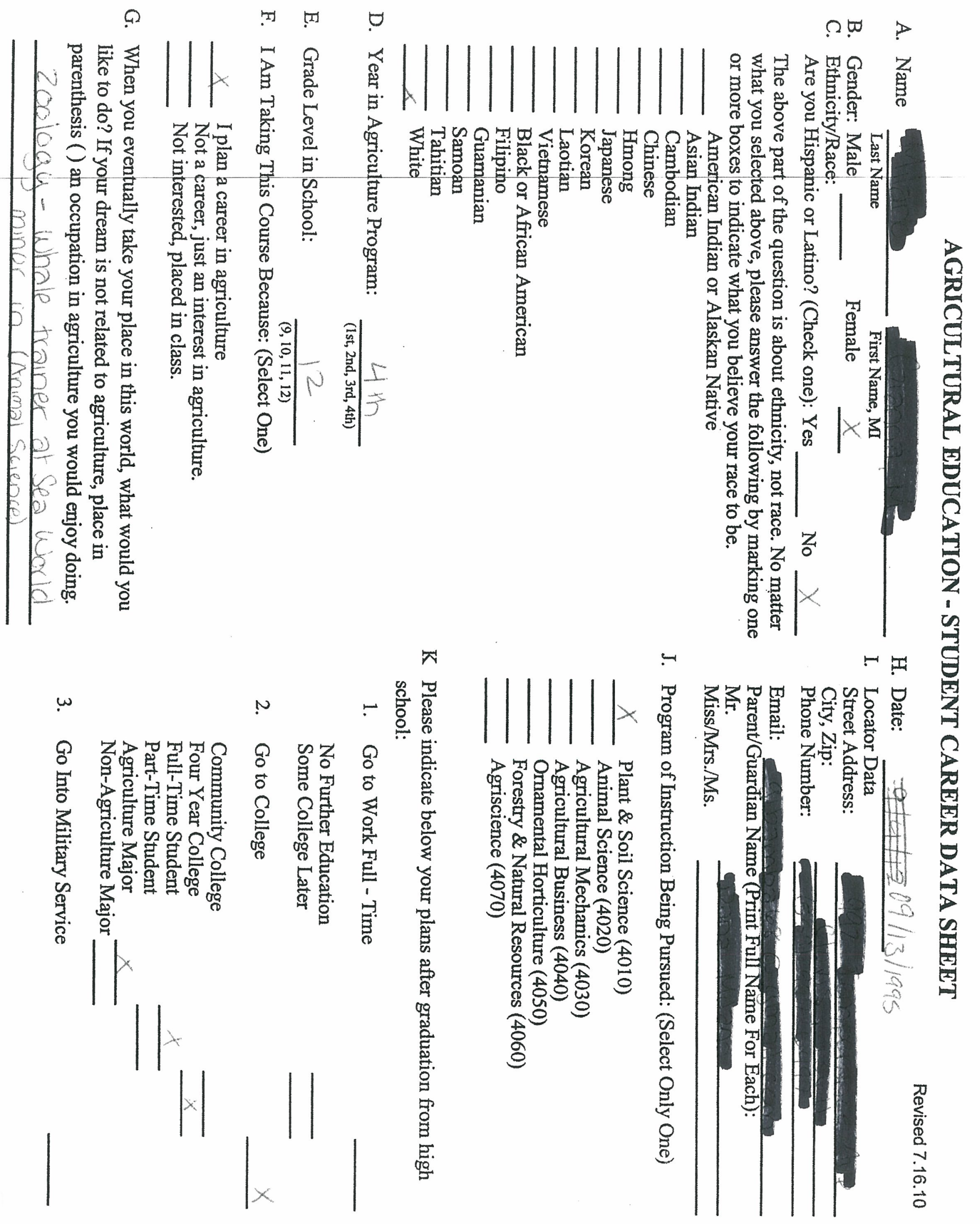




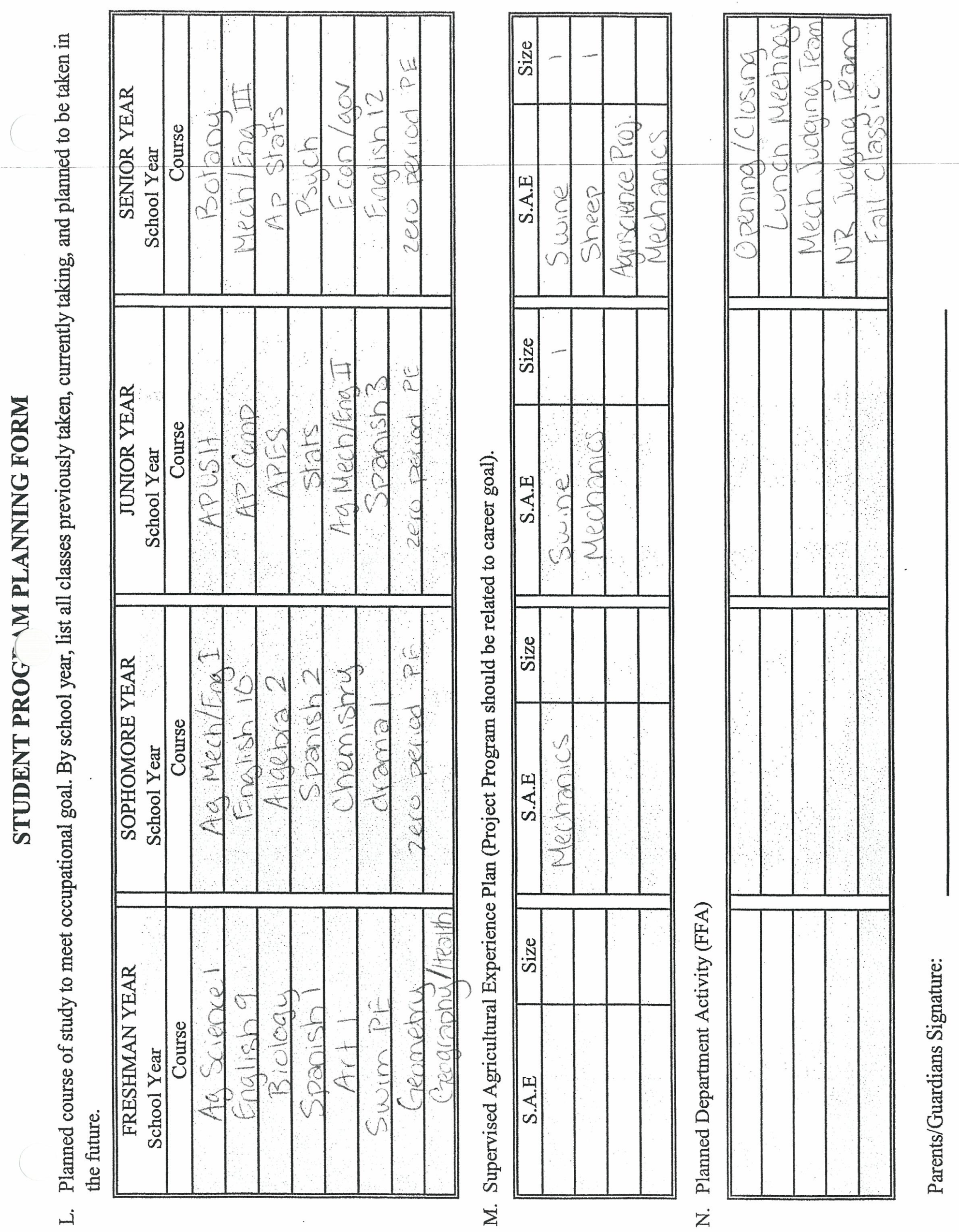




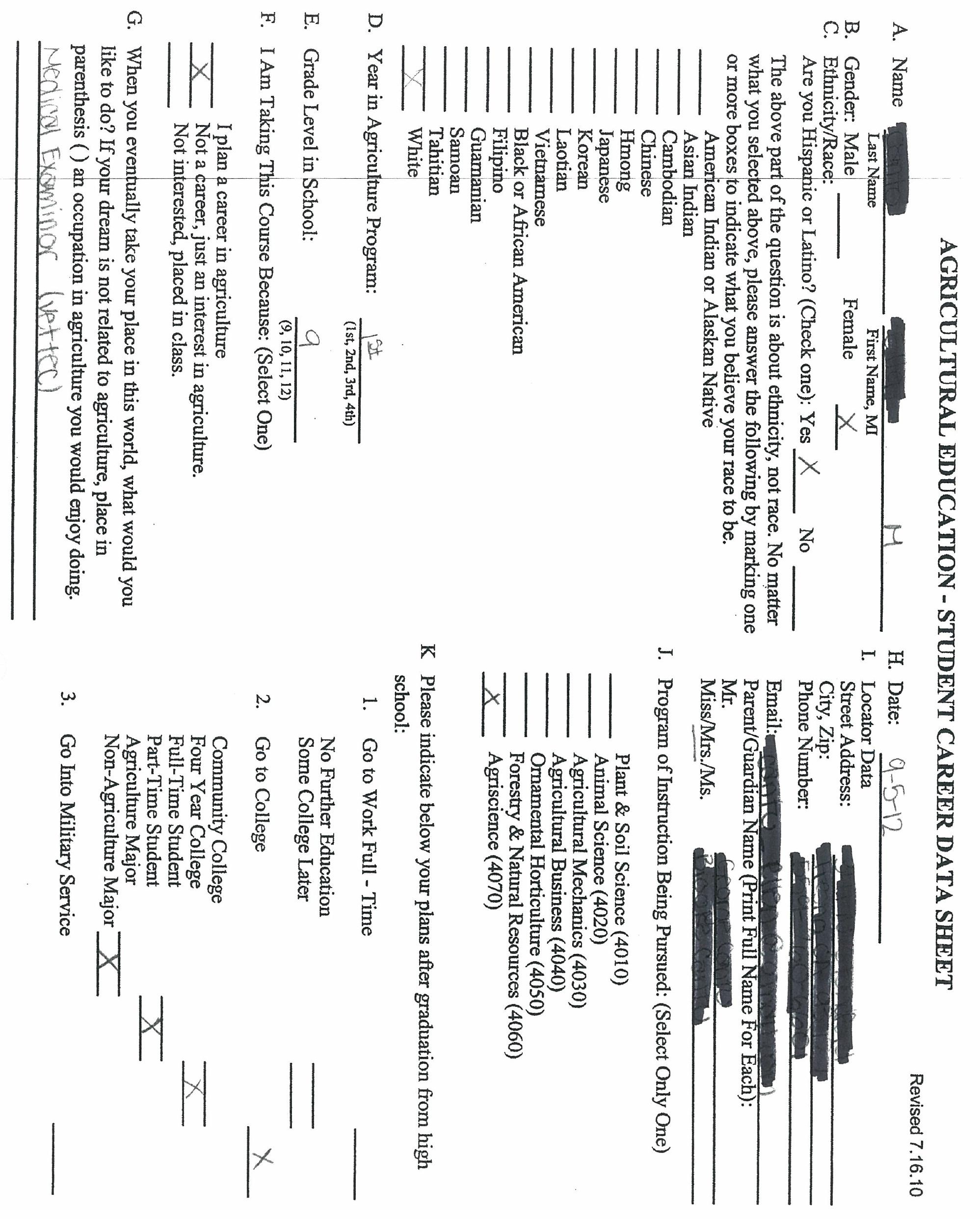



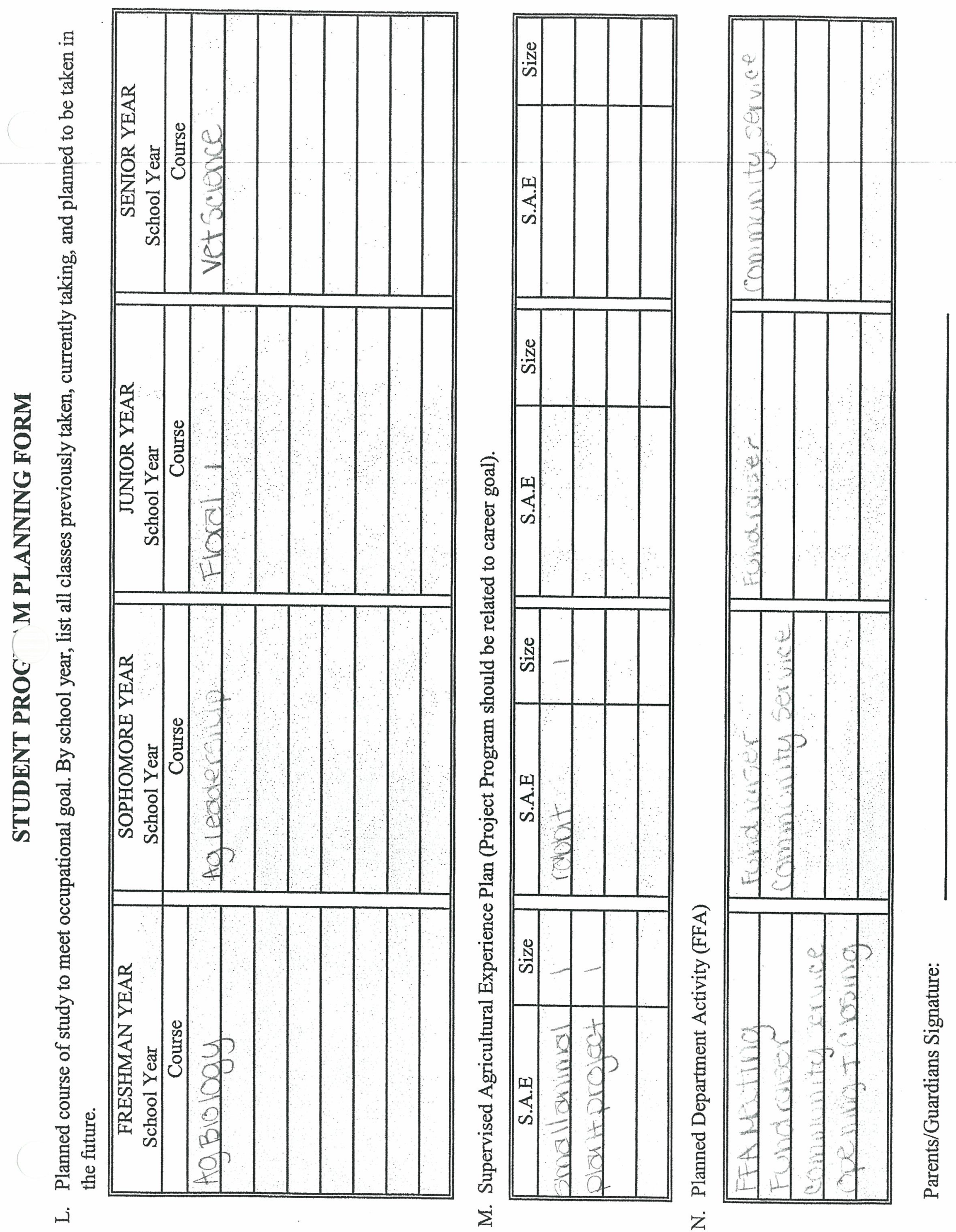


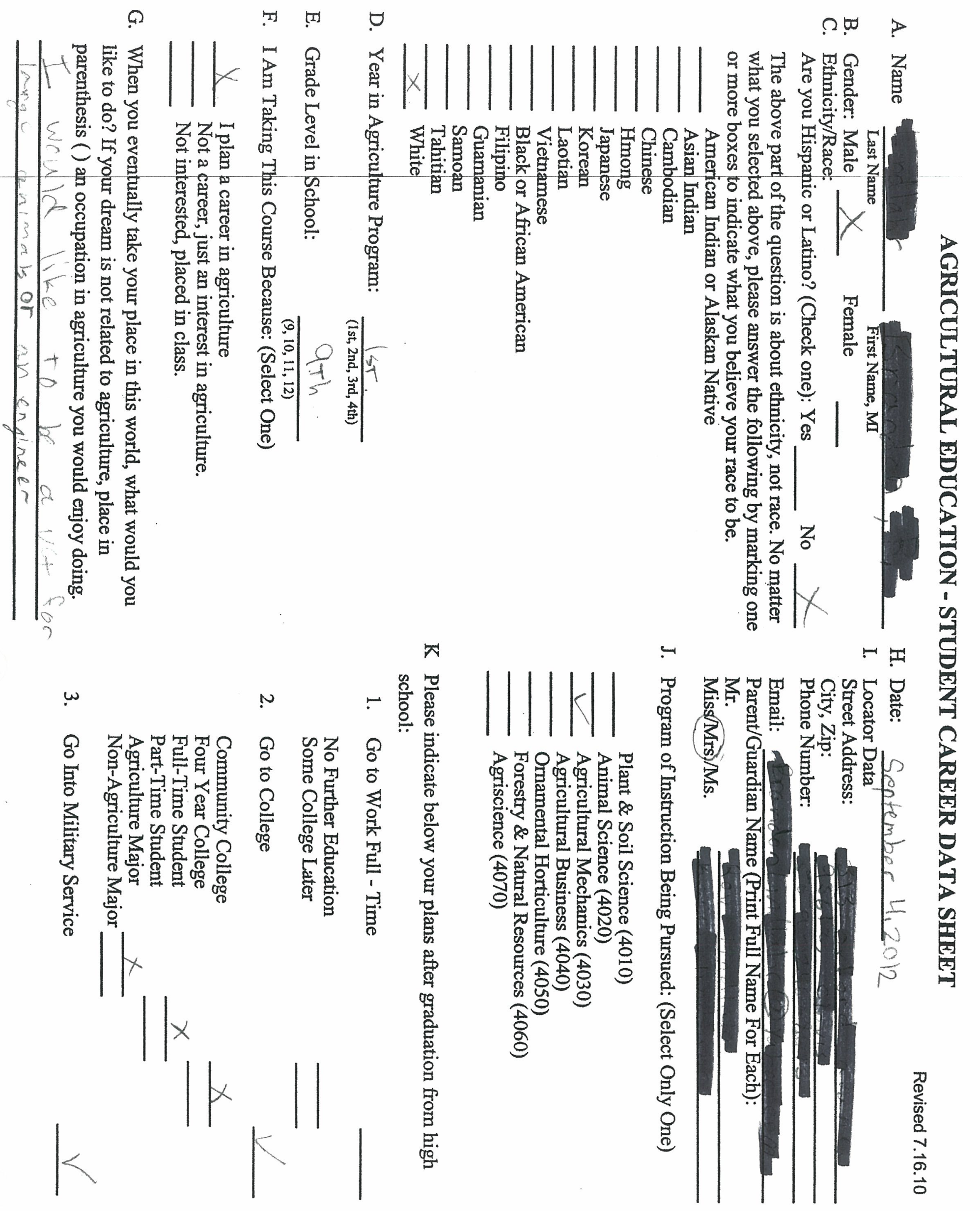




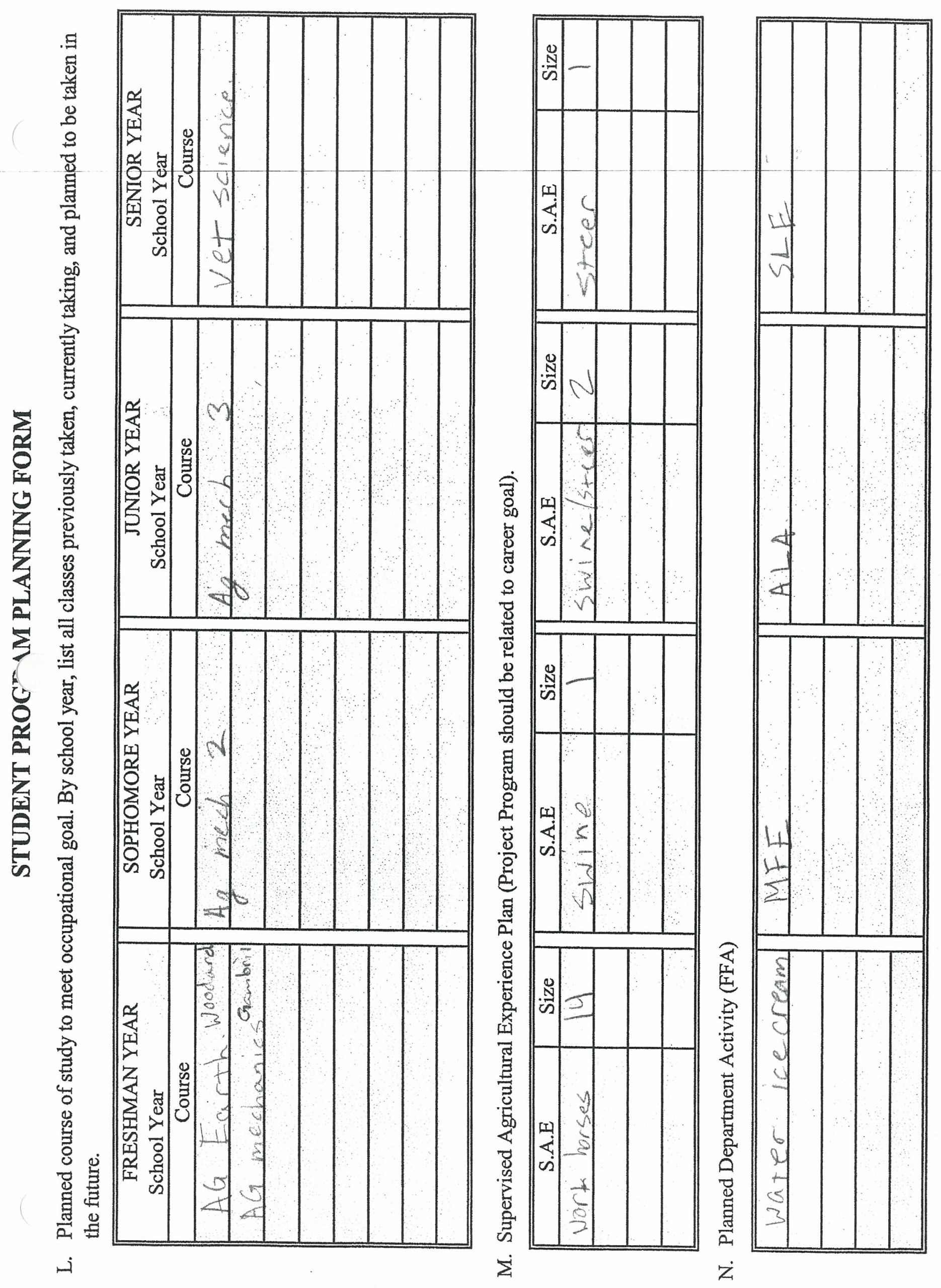

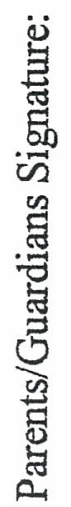




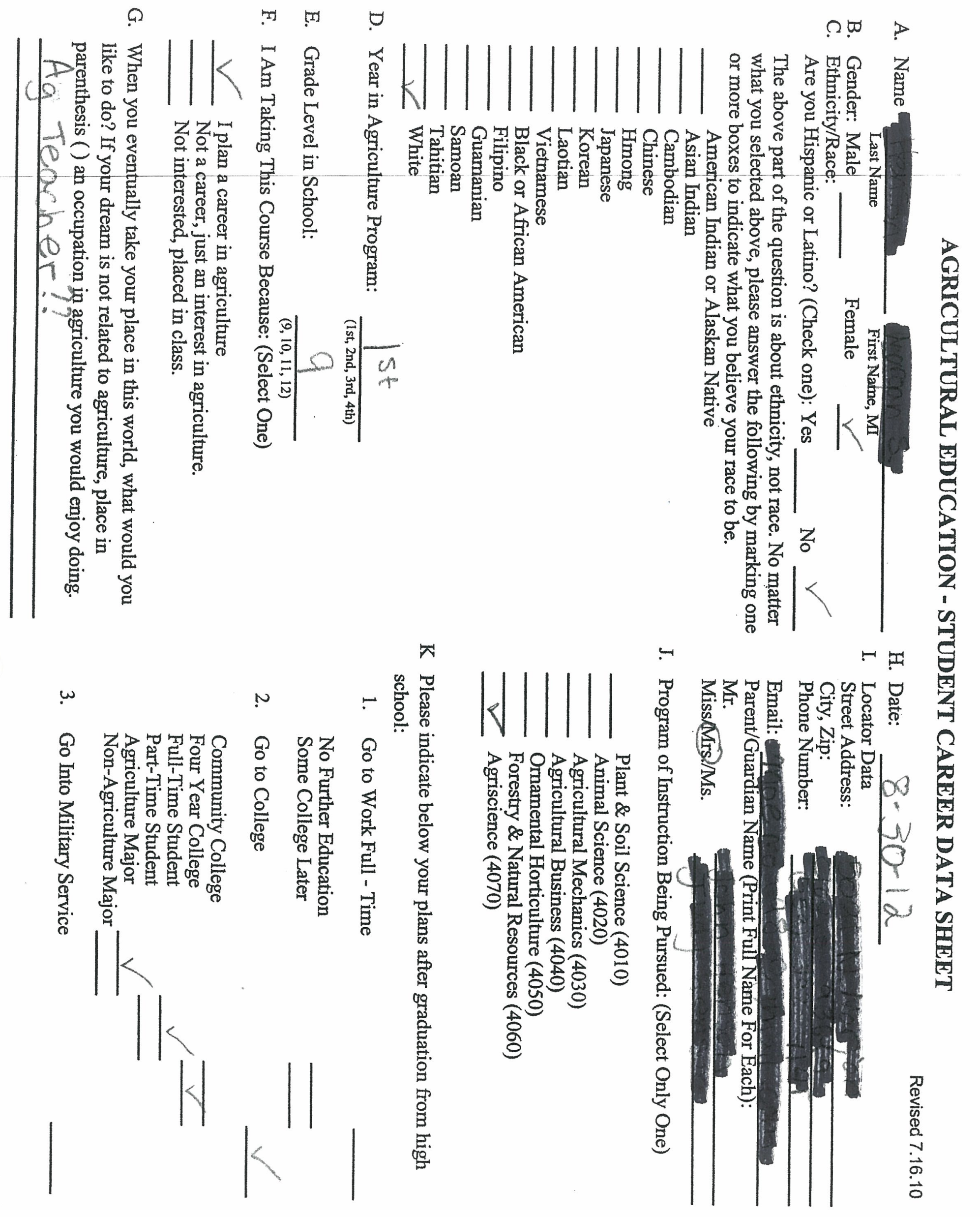




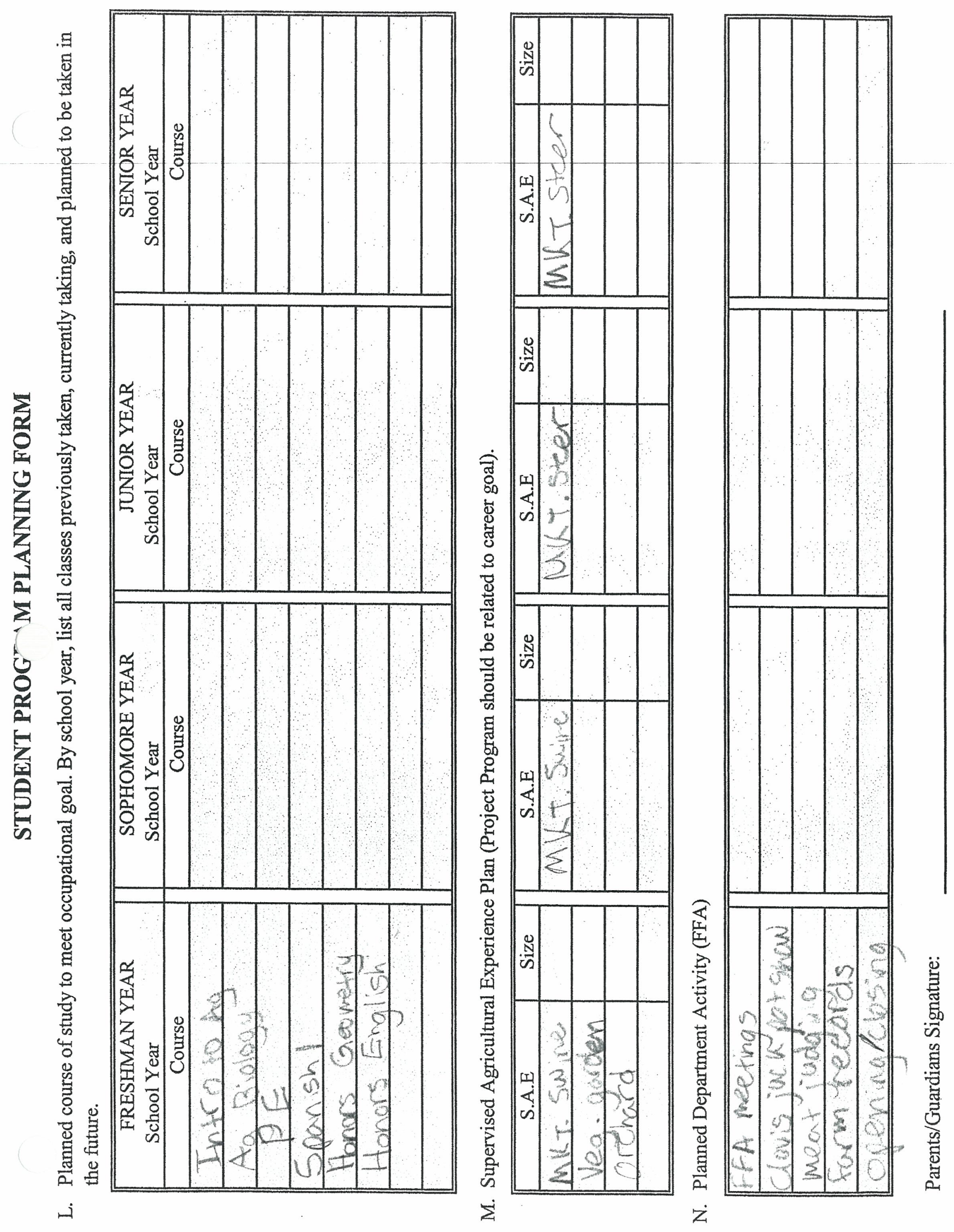




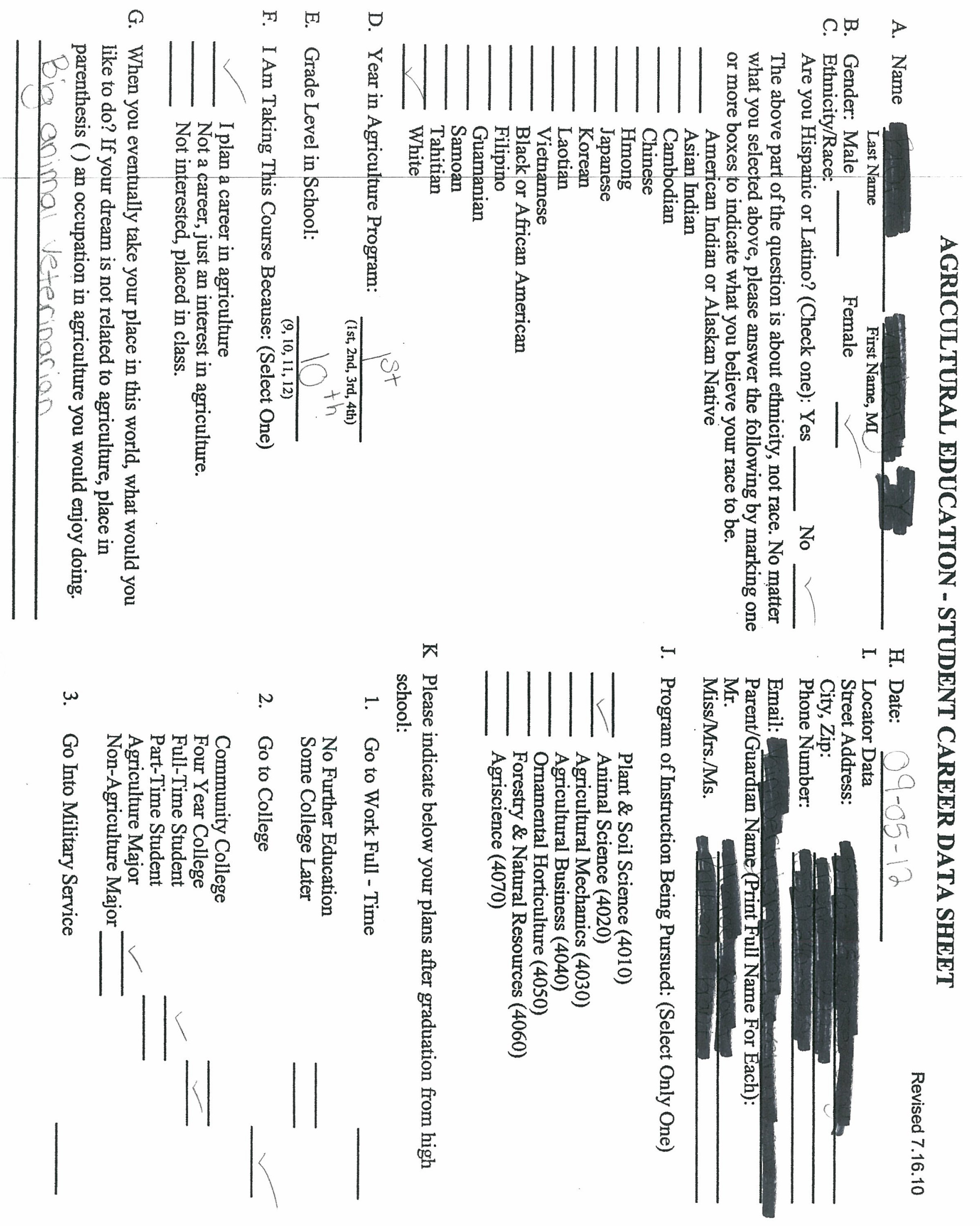




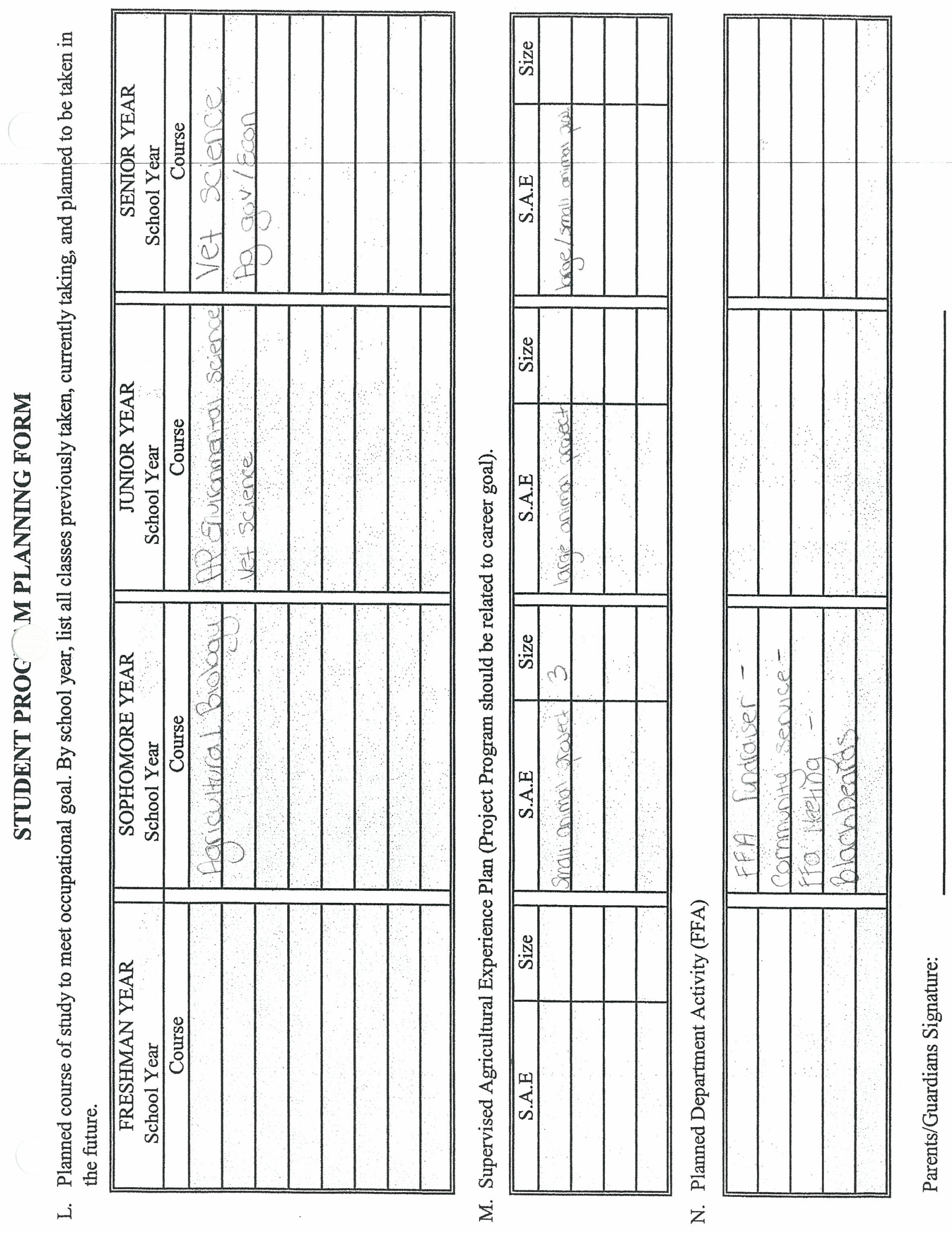




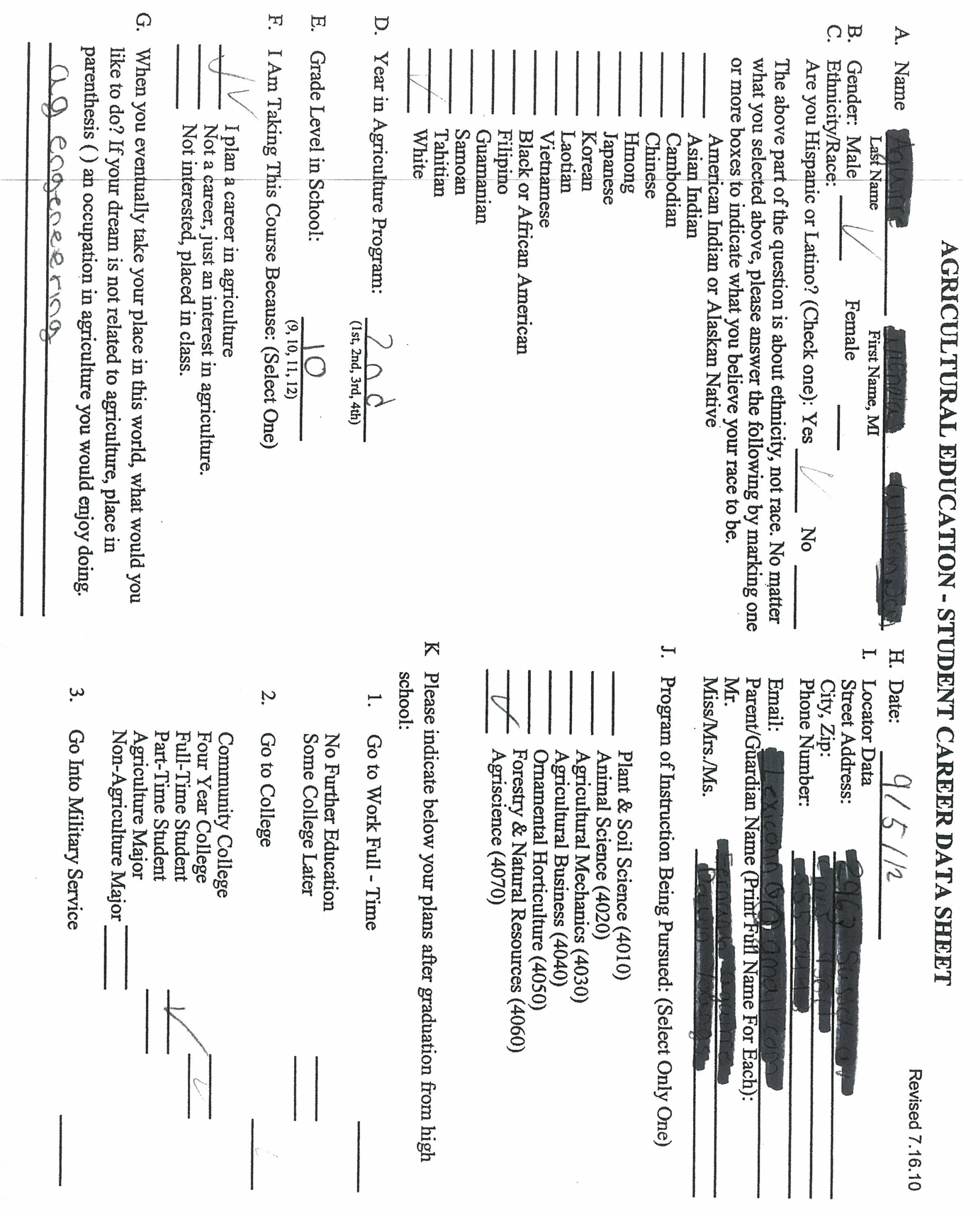




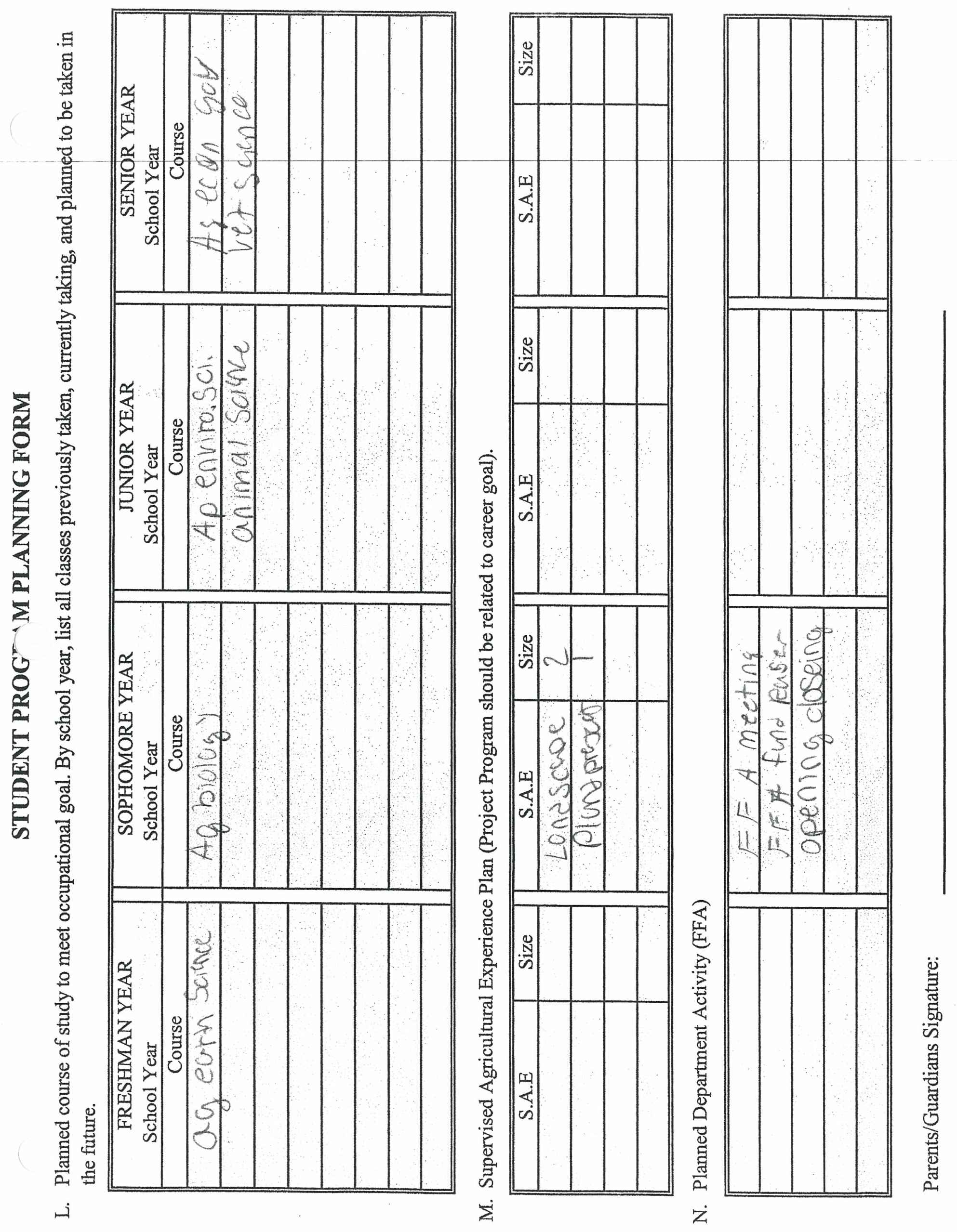




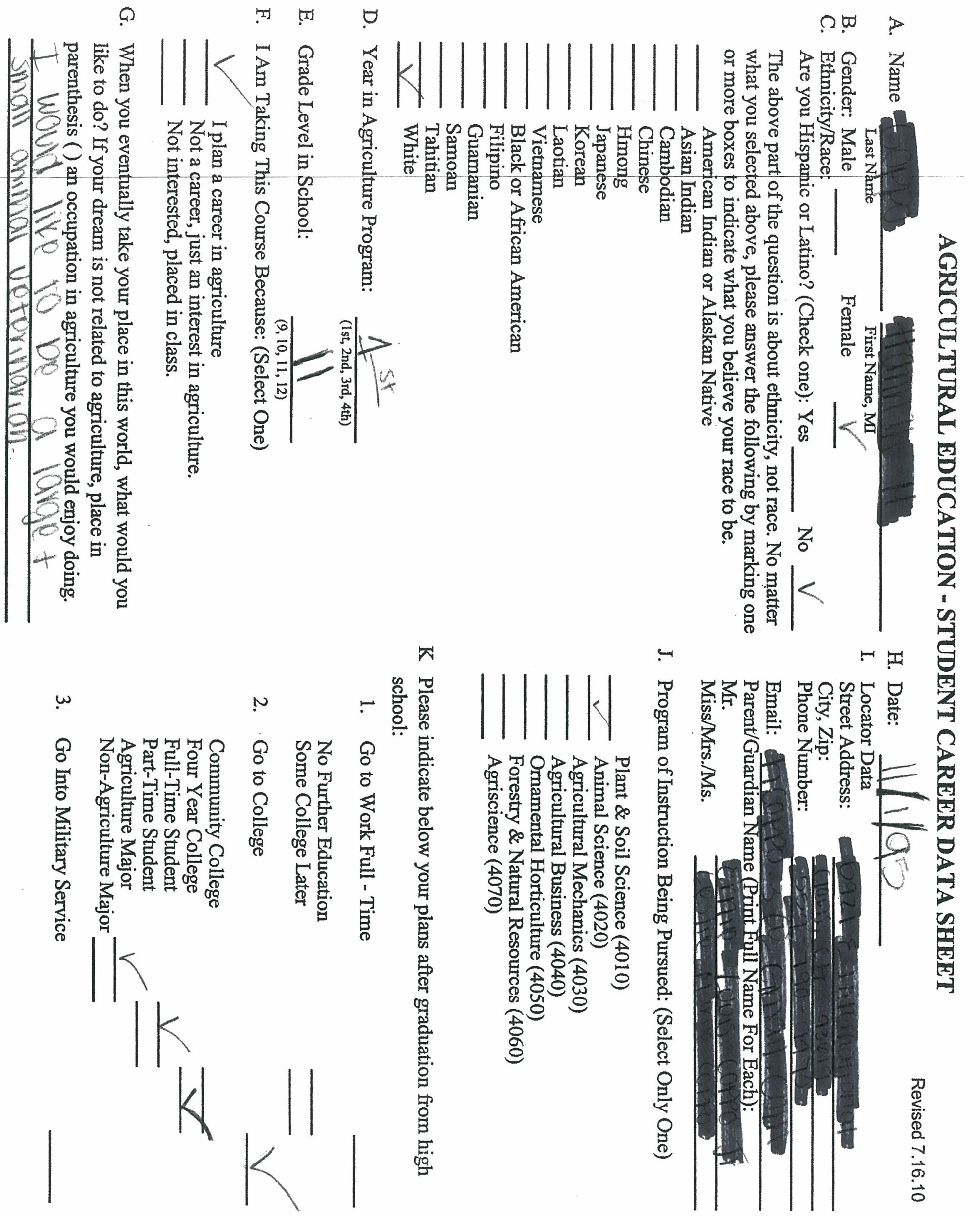




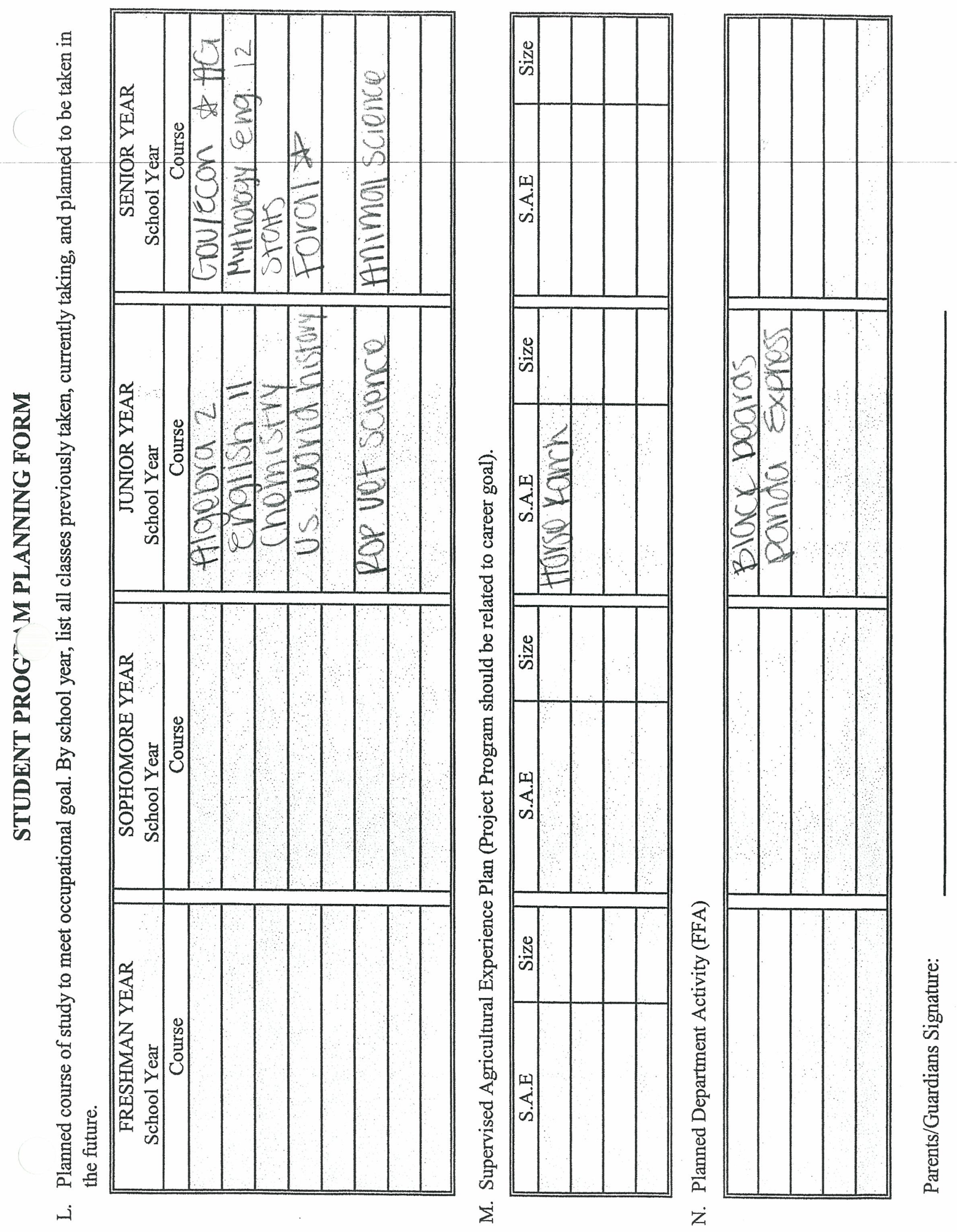




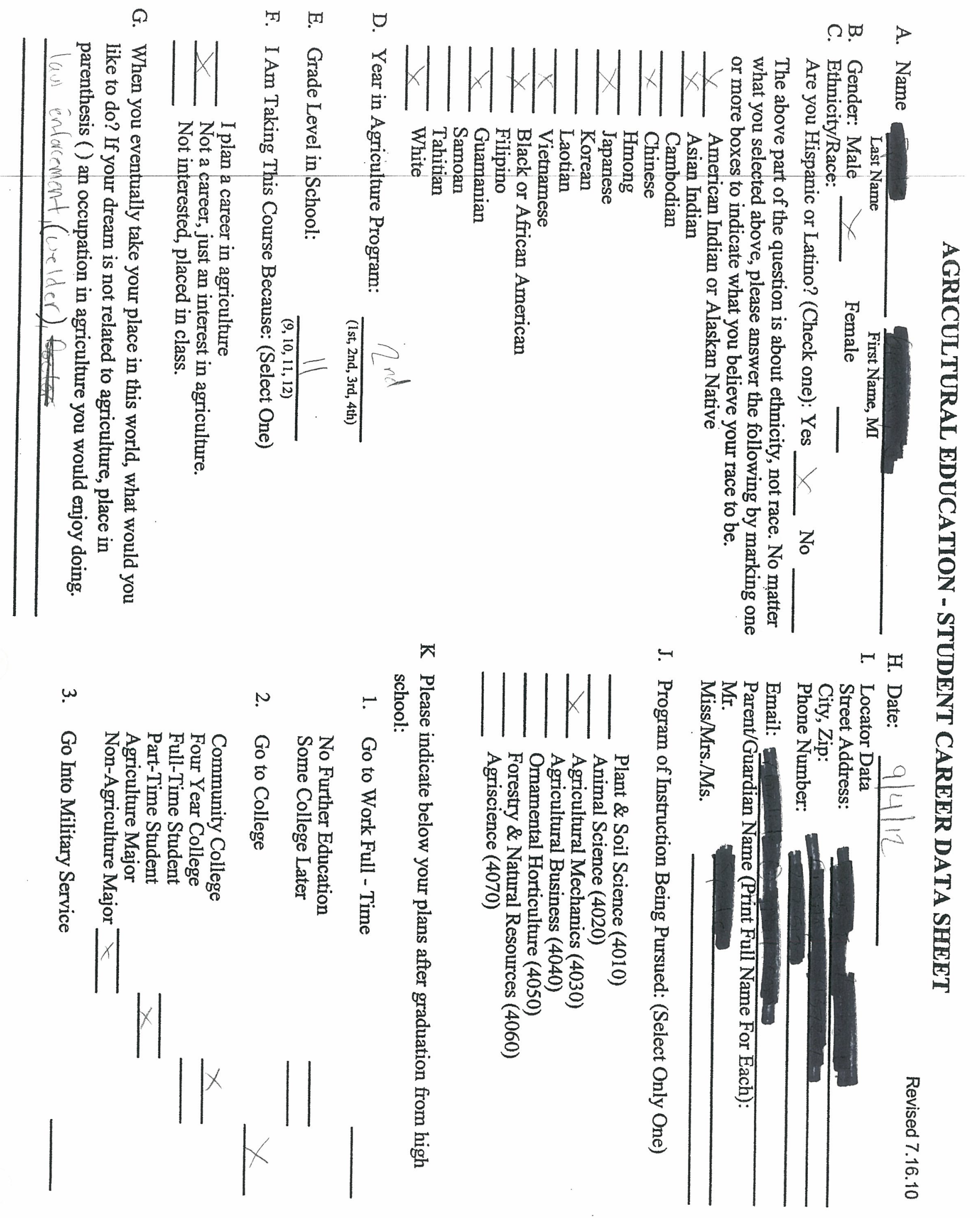




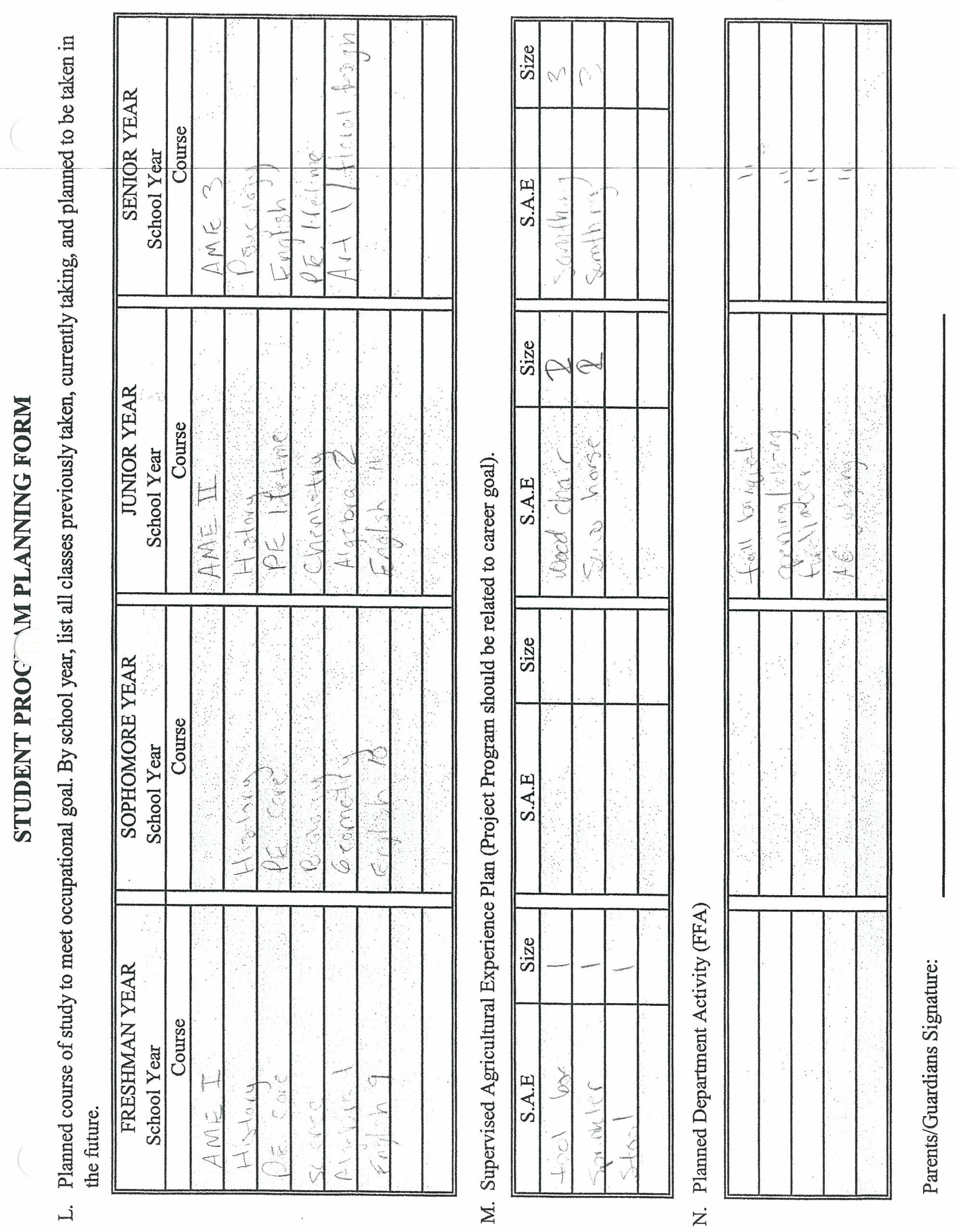




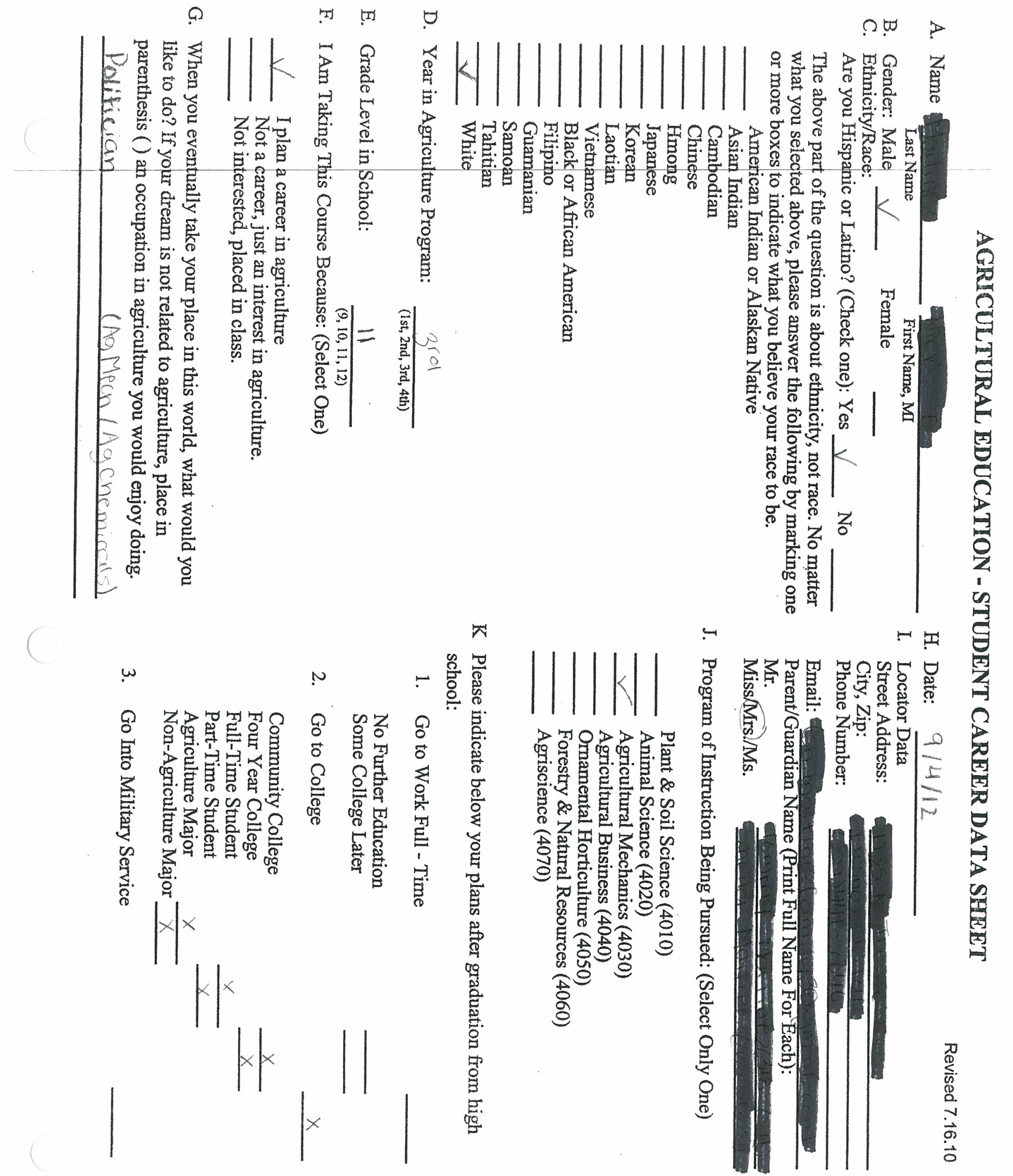




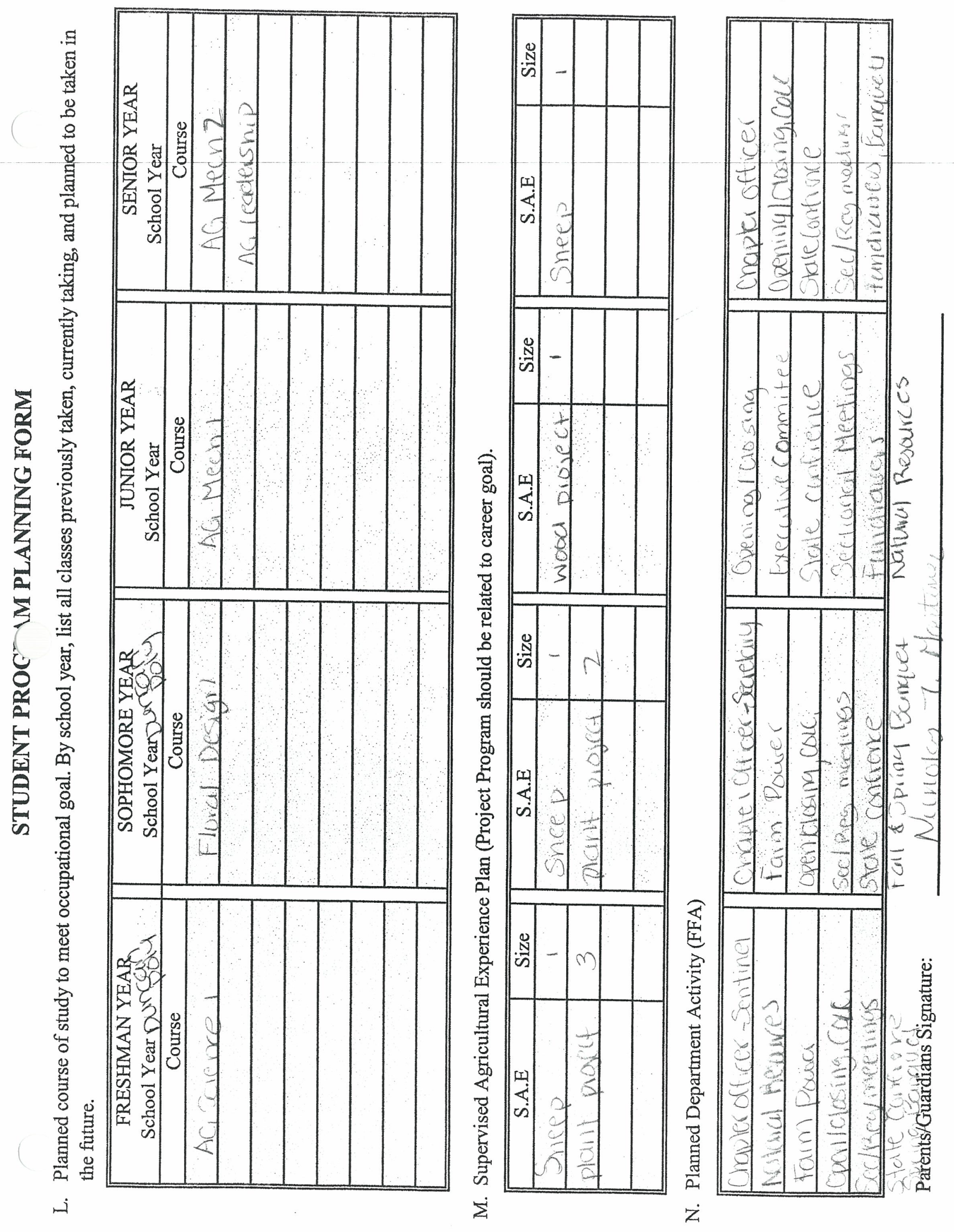




\section{CUSD High School Educational 4-Year Plan}

\begin{tabular}{|c|c|c|}
\hline & For CUSD Diploma & For UC/CSU \& CUSD Diploma \\
\hline Core Subject Area & Credit Requirements & Credit Requirements \\
\hline English & 8 Semesters $/ 40$ Credits & 8 Semesters $/ 40$ Credits \\
\hline Mathematics & 4 Semesters/20 Credits & 6 Semesters/30 Credits through Algrabra 2 \\
\hline Science & 4 Semesters/20 Credits & 4 Semesters $/ 20$ Credits \\
\hline History/Social Science & 7 Semesters/35 Credits & 7 Semesters/35 Credits \\
\hline \multirow{2}{*}{$\begin{array}{l}\text { CUSD Academic Breadth } \\
\text { Includes Foreign Language \& Visual Preforming Arts }\end{array}$} & \multirow{2}{*}{4 Semesters/20 Credits } & \begin{tabular}{l|l} 
& 4 Semesters/20 Credits (Course Specific) \\
\end{tabular} \\
\hline & & \begin{tabular}{|l|l} 
Visual Preforming Arts & 2 Semesters $/ 10$ Credits \\
\end{tabular} \\
\hline Health $\quad$ - & 1 Semesters/5 Credits & 1 Semesters/5 Credits \\
\hline Physical Education & 8 Semesters $/ 40$ Credits & 8 Semesters/40 Credits \\
\hline Pathway Courses & 4 Semesters/20 Credits & 4 Semesters/20 Credits \\
\hline Speech & Embedded in English & Embedded in English \\
\hline Core Required Credits & 200 Credits & 200 Credits \\
\hline Electives & 8 Semesters/40 Credits & 8 Semesters/40 Credits \\
\hline Total Possible Credits & 240 Credits $^{*}$ & 240 Credits $^{*}$ \\
\hline
\end{tabular}

The above table lists the required credits by content area for a student to graduate from Clovis Unified School District and receive a CUSD diploma (center column), and also an example of the courses that would satisfy the credit and UC/CSU course requirements for a CUSD diploma. There are many class/course options available based upon student and ability (see tables below). ALL STUDENTS MUST PASS CAHSEE AS WELL to graduate.

${ }^{*}$ Clovis East High School has 305 total possible credits

\begin{tabular}{|c|c|c|c|c|c|}
\hline \multicolumn{6}{|c|}{ Example of 4 Year CUSD High School Graduation Plan } \\
\hline Subject Area & Grade 9 & Grade 10 & Grade 11 & Grade 12 & Credits \\
\hline English & English 9 & English 10 & English Elective & English Elective & 40 \\
\hline Mathematics & Algebra & Geometry & & & 20 \\
\hline Science & $\begin{array}{c}\text { Life Science } \\
\text { with Lab }\end{array}$ & $\begin{array}{c}\text { Physical Science } \\
\text { with Lab }\end{array}$ & & & 20 \\
\hline $\begin{array}{l}\text { History/Social } \\
\text { Science }\end{array}$ & $\begin{array}{l}\text { World Geography } \\
\text { \& Cultures } \\
\text { (1 semester) }\end{array}$ & World History & U.S. History & $\begin{array}{l}\text { Government } \\
\text { \& Economics }\end{array}$ & 35 \\
\hline $\begin{array}{l}\text { CUSD } \\
\text { Academic Breadth }\end{array}$ & \multicolumn{4}{|c|}{ Foreign Language or Visual Preforming Arts are spaced over the 4 years } & 20 \\
\hline Health & \multicolumn{4}{|c|}{1 semester of Health to be taken anytime during the 4 years } & 5 \\
\hline Physical Education & P.E. 9 & P.E. 10 & P.E. 11 & P.E. 12 & 40 \\
\hline Pathway Courses & \multicolumn{4}{|c|}{ Pathway Courses are spaced over the 4 years } & 20 \\
\hline Speech & \multicolumn{4}{|c|}{ Speech is incorporated into English classes throughout the 4 years } & \\
\hline Electives & \multicolumn{4}{|c|}{ Electives are spaced over the 4 years } & 40 \\
\hline & & & & tal Possible Credits & 240 \\
\hline
\end{tabular}

\begin{tabular}{|c|c|c|c|c|c|}
\hline Subject Area & Grade 9 & Grade 10 & Grade 11 & Grade 12 & Credits \\
\hline English & English $9(\mathrm{P})$ & English $10(\mathrm{P})$ & English Elective $(\mathrm{P})$ & English Elective $(\mathrm{P})$ & 40 \\
\hline Mathematics & Algebra $1(\mathrm{P})$ & Geometry $(\mathrm{P})$ & Algebra 2 & $\begin{array}{l}4^{\text {th }} \text { year Math }(\mathrm{P}) \\
\text { (recommended) }\end{array}$ & 20 \\
\hline Science & Science (P) & Science (P) & $\begin{array}{l}3^{\text {rd }} \text { Science }(\mathrm{P}) \\
\text { (reconmended) }\end{array}$ & & 20 \\
\hline History/Social Science & $\begin{array}{l}\text { World Geography } \\
\& \text { Cultures }(\mathrm{P}) \\
(1 \text { semester })\end{array}$ & World History (P) & U.S. History (P) & $\begin{array}{l}\text { Government } \\
\text { \& Economics }(\mathrm{P})\end{array}$ & $\begin{array}{l}\text { UC/CSU - } 20 \\
\text { CUSD - } 35\end{array}$ \\
\hline Foreign Language & Foreign Language(P) & Foreign Language( $\mathrm{P})$ & $\begin{array}{c}3^{\text {rd }} \text { year Foreign } \\
\text { Language } \\
\text { (recormmended) }\end{array}$ & & 20 \\
\hline Visual \& Preforming Arts & \multicolumn{4}{|c|}{ Visual Preforming Arts are spaced over the 4 years } & 10 \\
\hline Health & \multicolumn{4}{|c|}{1 semester of Health to be taken anytime during the 4 ycars } & 5 \\
\hline Physical Education & P.E. 9 & P.E. 10 & P.E. 11 & P.E. 12 & 40 \\
\hline Pathway Courses & \multicolumn{4}{|c|}{ Pathway Courses are spaced over the 4 years } & 20 \\
\hline Speech & \multicolumn{4}{|c|}{ Speech is incorporated into English classes throughout the 4 years } & \\
\hline Electives & \multicolumn{4}{|c|}{ Electives are spaced over the 4 years } & $\begin{array}{l}\text { UC/CSU }-10 \\
\text { CUSD - } 40\end{array}$ \\
\hline & & & & Total Possible Credits & 240 \\
\hline
\end{tabular}




\section{A - G Requirements 2009 - 2010}

-Visual/Performing Arts

idvanced Animation/Special Effects AB

Advanced Studies in Media Communication Design AB

Advanced Three-Dimensional Art Studies AB

Three-Dimensional Design $2 \mathrm{AB}$

Architectural Design

Art $1 \mathrm{AB}$

Art $2 A B$

Art $3 \mathrm{AB}$

Art and Design $A B$

Art History $A B$

Art \& History of Fashion $1 \mathrm{AB}$

Beginning Band $\mathrm{AB}$

Broadcast Communications $A B$

Intermediate Band $A B$

Advanced $B$ and $A B$

Jazz Band $A B$

Beginning Orchestra $A B$

Advanced Orchestra $A B$

Ceramics $1 \mathrm{AB}$

Ceramics $2 A B$

Ceramics $3 \mathrm{AB}$

Chamber Singers AB

Computer Graphics \& Page Design $A B$

Concert Choir $\mathrm{AB}$

Dance $1 \mathrm{AB}$

Dance $2 \mathrm{AB}$

Dance Repertory $A B$

Drama $1 \mathrm{~A}$

ama $2 A B$

rama $3 \mathrm{AB}$

Drama $4 \mathrm{AB}$

Digital Photography $A B$

Folklorico Dance IAB

Folklorico Dance IIAB

Folklorico Dance IIIAB

Graphic Design $A B$

Handbells $1 \mathrm{AB}$

Handbells 2AB

Advanced Handbells $\mathrm{AB}$

Mixed Choir $1 \mathrm{AB}$

Mixed Choir 2AB (max. 2 years allowed)

Mixed Choir $3 A B$ (max. 2 years allowed)

Mixed Choir $4 \mathrm{AB}$ (max. 2 years allowed)

Multicultural Studio Art $1 \mathrm{AB}$

Multimedia Communication Design \& Production

Music Theory AB (AP)

Music Theory \& Technology $A B$

Performing Arts $A B$

Photography $1 \mathrm{AB}$

Photography $2 \mathrm{AB}$

Photography $3 \mathrm{AB}$

Soprano Alto Choir $1 A B$

Soprano Alto Ensemble $A B$

Studio Art AB (AP)

Tenor/Bass Choir AB

Theatre Production AB

Videography $\mathrm{AB}$

ocal Ensemble $1 \mathrm{AB}$

cal Ensemble 2AB (max. 2 years allowed)

cal Ensemble $3 A B$ (max. 2 years allowed)

Vocal Ensemble $4 \mathrm{AB}$ (max. 2 years allowed)

\section{G-Electives}

Advanced Topics in Medical Research AB

Advanced Topics in Medicine $1 \mathrm{AB}$

Agriculture $2 \mathrm{AB}$

Agriculture $3 \mathrm{AB}$

Agriculture Mechanical Engineering I**

Agriculture Mechanical Engineering II**

Agriculture Mechanical Engineering III**

Architectural Design $1 \mathrm{AB}$

Biochemistry $A B$

Dance 2 Technical Theater Arts AP $(P)^{* *}$

Economics A

Economics of Marketing and Advertising

Engineering I

Engineering II

Environmental Field Studies

Environmental Research \& Technology

Forensics AB

Forensic Research \& Technology AB

Global Economics and Finance

Journalism $A B$

Journalism and Publishing $A B$

Law \& Order and Public Policy

Microeconomics A (AP)

Macroeconomics **

Modern American Economy

Money and Banking

Physics \& Technology AB

Plant \& Animal Physiology $A B$

Playwriting $A B$

Product Development $1 \mathrm{AB}$

Psychology A

Psychology AB (AP)

Robotics and Electronics IAB

Sports Medicine

Sociology A

Sociology of Media AB

Technology of Emergency Management Systems AB

Veterinary Science

**Pending UC Approval 


\section{A - G Requirements 2009 - 2010}

- History/Social Science

imerican Government A

American Government A (AP)

European History AB (AP)

U.S. History $A B$

U.S. History $A B(A P)$

World Geography and Cultures A

World History $A B$

World History $A B(\mathrm{H})$

World History AB (AP)

\section{B - English/Language Arts}

America in Change/Literature AB

American Literature A

Bible as Literature A

British Literature \& Composition AB

College Composition \& American Lit. AB

Contemporary Cultural Literature \& Composition $\mathrm{AB}$

Contemporary Issues In Literature A

Creative Composition \& Literature $\mathrm{AB}$

Dramatic Literature $A B$

Education Careers $\mathrm{AB}$

English $9 \mathrm{AB}$

English $9 A B(H)$

English $10 \mathrm{AB}$

English $10 \mathrm{AB}(\mathrm{H})$

English $11 \mathrm{AB}$

English $12 \mathrm{AB}$

English Lang. \& Composition $\mathrm{AB}$ (AP) glish Literature \& Composition AB (AP)

ythology \& Science Fiction AB

erforming Arts $\mathrm{AB}$

Publishing Management $A B$

Theatre Production $A B$

World Literature \& Composition $1 \mathrm{AB}$

Yearbook Publication AB

\section{C - Mathematics}

Algebra $1 \mathrm{AB}$

Algebra $C D$

Algebra $2 \mathrm{AB}$

Algebra $2 \mathrm{AB}(\mathrm{H})$

Advanced Algebra CD

Advanced Mathematics AB

Advanced Mathematics Analysis AB (HP)

Calculus AB (AP)

Calculus BC (AP)

Differential Calculus $A B$

Geometry $1 A B$

Statistics AB (AP)

Statistics and Probability $A B$

\section{D - Laboratory Science}

Agricultural Biology AB

Advanced Science Topics AB

Anatomy \& Physiology AB

Bioengineering I

Biology $\mathrm{AB}$

Biology AB (H)

ology $A B(A P)$

tany $\mathrm{AB}$

nemistry $A B$

Chemistry $A B(H)$

Chemistry $A B(A P)$
Core Science $2 \mathrm{AB}$

Environmental Science AB (AP)

Geology $\mathrm{AB}$

Physics $A B$

Physics AB (AP)

Physics C AB (AP)

Zoology $A B$

\section{E - Foreign Language}

Chinese $1 \mathrm{AB}$

Chinese $2 A B$

Chinese $3 \mathrm{AB}$

Chinese $4 \mathrm{AB}$

French $1 A B$

French $2 A B$

French $3 A B$

French $4 \mathrm{AB}$

French Language $A B(A P)$

French Literature $A B(A P)$

German $1 A B$

German $2 A B$

German $3 \mathrm{AB}$

German $4 A B$

German Language AB (AP)

Hmong $1 \mathrm{AB}$

Hmong $2 A B$

Hmong $3 A B$

Spanish $1 \mathrm{AB}$

Spanish $2 A B$

Spanish $3 A B$

Spanish $4 \mathrm{AB}$

Spanish for Spanish Speakers $1 A B$

Spanish for Spanish Speakers $2 A B$

Spanish Language AB (AP)

Spanish Literature AB (AP) 


\section{ROP Career Pathways Course}

GRICULTURE:

$\mathrm{Ag}$ Welding Process and Procedures

Equine Science

Veterinary Science $(P)$

ART/FINE ARTS:

Architectural Engineering Design (P)

Art and Design (P)

Digital Photography (P)

Graphic Design (P)

Interior Design

Videography

Music Technology (P)

Theatre Production (Eng 11/12 credit) (P)

BUSINESS

Small Business Management
ENGLISH:

Education Careers (Eng.11/12 credit) (P)

Theatre Production (Eng.11/12 credit) (P)

Publication Manaagement (Eng 11/12 credit

(P)

Yearbook Publication (Eng. 11/12 credit) (P)

FAMILY AND HUMAN SERVICES

Careers and Children

HEALTH:

Education Careers (Eng 11/12 credit) (P)

Emergency Medicine

Health Careers

Principles of Athletic Training

Sports Medicine
INDUSTRIAL TECHNOLOGY:

$\mathrm{Ag}$ Welding Process and Procedures Architectural Drafting CAD

Engine Technology

Computer \& Networking Technology

Video Security Design

PUBLIC SERVICES:

Criminal Investigation

Criminal Justice

\section{SCIENCE:}

Emergency Medicine

Equine Science

Health Careers

Principles of Athletic Training

Sports Medicine

Veterinary Science (P)

\section{ROP COURSE OFFERINGS 2008/2009}

Credit and Certification Information

\begin{tabular}{|c|c|c|c|c|c|c|c|c|c|}
\hline \multirow{3}{*}{ ROP Class Name } & \multicolumn{6}{|c|}{ Credit Information } & \multicolumn{3}{|c|}{ Certification / College Credit } \\
\hline & \multirow{2}{*}{$\begin{array}{l}\text { CUSD } \\
\text { Elective } \\
\text { Credit }\end{array}$} & \multicolumn{4}{|c|}{ UC Approved College Prep } & \multirow[b]{2}{*}{$\begin{array}{c}\text { Total Credits } \\
\text { Offered }\end{array}$} & \multirow[b]{2}{*}{$\begin{array}{l}\text { Training for } \\
\text { Industry } \\
\text { Cerrificarion }\end{array}$} & \multirow{2}{*}{$\begin{array}{l}\text { Community } \\
\text { College } \\
\text { Articulation }\end{array}$} & \multirow[b]{2}{*}{ Uni-Track* } \\
\hline & & $\begin{array}{l}\text { English } \\
11 \text { or } 12\end{array}$ & Lab Science & $\begin{array}{c}\text { Visual or } \\
\text { Performing } \\
\text { Arts }\end{array}$ & Elective & & & & \\
\hline Ag Welding Process and Procedures & 20 & & & & & 20 & $\checkmark$ & & \\
\hline Architectural Engineering Design & 20 & & & & & 20 & & & \\
\hline rt and Design (P) & & & & 20 & & 20 & & & \\
\hline Engine Technology & 20 & & & & & 20 & & $\checkmark$ & \\
\hline Careers with Children & 20 & & & & & 20 & $\checkmark$ & $\checkmark$ & \\
\hline Computer and Networking Technology & 20 & & & & & 20 & $\checkmark$ & $\checkmark$ & \\
\hline Criminal Investigation & 20 & & & & & 20 & & $\checkmark$ & \\
\hline Criminal Justice & 20 & & & & & 20 & & $\checkmark$ & $\checkmark$ \\
\hline Digital Photography (P) & & & & 20 & & & & & \\
\hline Education Careers - with English (P) & 10 & 10 & & & & 20 & & $\checkmark$ & $\checkmark$ \\
\hline Emergency Medicine & 20 & & & & & 20 & $\checkmark$ & & \\
\hline Equine Science & 20 & & & & & 20 & & & \\
\hline Graphic Design (P) & & & & 20 & & 20 & & & $\checkmark$ \\
\hline Health Careers & 30 & & & & & 20 & $\checkmark$ & & \\
\hline Health and Wellness Careers & 20 & & & & & 20 & & & \\
\hline Interior Design & & & & 20 & & 20 & $\checkmark$ & & \\
\hline Multimedia Communication (P) & & & & 20 & & & & & \\
\hline Music Technology $(\mathrm{P})$ & & & & 20 & & 20 & & & \\
\hline Performing Arts $(\mathrm{P})$ (with English - $\mathrm{P}$ ) & & 10 & & 10 & & & & & \\
\hline Principles of Athletic Training & 20 & & & & & 20 & & & \\
\hline Small Business Management & 20 & & & & & 20 & & & \\
\hline Sports Medicine / Fitness & 20 & & & & & 20 & $\checkmark$ & & $\checkmark$ \\
\hline Veterinary Science (P) & & & & & 20 & 20 & & $\checkmark$ & \\
\hline Video Security Design & 20 & & & & & 20 & $\checkmark$ & & \\
\hline earbook Publication (with English - P) & 10 & 10 & & & & 20 & & & \\
\hline
\end{tabular}

${ }^{*}$ Uni-Track is a collaborative program between California State University, Fresno and local school districts allowing capable high school students to receive both high school graduation credit and CSUF (transferable credit) at the same time. Students register at their home high schools to receive credit for approved CSUF Uni-Track classes by completing a CSUF registration form and paying the $\$ 20$ registration fee. 


\section{Quality Criteria Eight}

\section{Program Promotion}

Numerous promotional pieces are produced by the Ag Department each year. Since 2000 when the Ag Center was established the school of Agriculture Science and Technology has created many brochures and handouts. Each teacher and advisor also creates material specific to their CDE Teams and project areas for promotion. ROP also has brochures that highlight our two ROP courses.

Promotion of our program is a year round activity taking place on many levels. On site promotion is done through flyers displayed on FFA Walls in the classrooms and bulletin boards outside each teacher's door. These flyers are created by teachers, Ag Leadership team members, Committees, or the Chapter Officers. We make a point to keep all bulletin boards refreshed and updated so only pertinent information is displayed. We also submit announcements to be read over the PA system each morning; we announce meetings and results from contests and events. Great stories of success are also submitted to CUSD Today, our districts newsletter as well as emailed to all district employees. In Clovis Unified competition is a way of life so anytime we can highlight our successes it promotes drive in our students and others. We also have our Ag department chair and our FFA reporter update the Clovis FFA website weekly, here students can see pictures and results from past FFA events and gain access to permission slips and event information.

Program promotion and recruitment is focused on primary grades as well as junior high school students. We begin introducing the primary grades to our Ag Center by inviting them for a one hour farm tour; we have students here weekly in the spring for this popular tour. Children and their teachers will learn about production agriculture, and get to participate in many different hands on activities. We usually see over 3,000 kids each spring from this activity. At the early secondary level we offer Exploratory Agriculture classes to our students at our primary feeder school Reyburn Intermediate. Reyburn is part of the Regan Educational Complex and right across the street from our Ag Center. Over 300 Junior High students take an Exploratory Ag class each year, and are introduced to SAE's 
and FFA opportunities that will be available to them as $9^{\text {th }}$ graders. Because of these two forms of recruitment we are always fill up courses quickly and typically end up with more students than we can handle. We have discussed other recruitment options but are afraid to do more because we have plenty of students as it currently is. Also because our program has a reputation for success in the community many students in the District are already aware that our program exists.

We are fortunate to have a highly successful Ag Boosters Club that exists to raise money and help assist in funding of student projects. Each year our Ag Boosters provide a project loan program that provides students with "seed money" through an interest free loan. The loans are payable at the time students fair or first income check is received. Other assistance is often granted for large conferences like National FFA Convention, students who are seeking assistance for conference or FFA activities will be asked to write a letter and make a presentation at the Boosters Meeting in order to receive assistance.

One area where our program recruitment could be improved is at our non-feeder schools in the district. Because we are unique and serve all Clovis Unified there may be students who would love to be a part of the Agriculture Program but don't fully understand the opportunities it presents for them. I know that I have had juniors from other CUSD High Schools that have said they wished they had known about the Agriculture Program and FFA sooner. I'm not really sure on the best way to improve in this area but staring with educating the other site counselors is a start.

\section{Supporting Completion Materials}

Item A- School of Agriculture Science and Technology Pamphlet

Item B- Recruitment Brochure Samples

Item C- Incoming Freshmen Ag Class Flyer

Item D \& E- Sheep Meeting Flyer and Greenhand Conference Flyer. These are examples of the types of flyers we post on the bulletin boards around the Ag Center. 

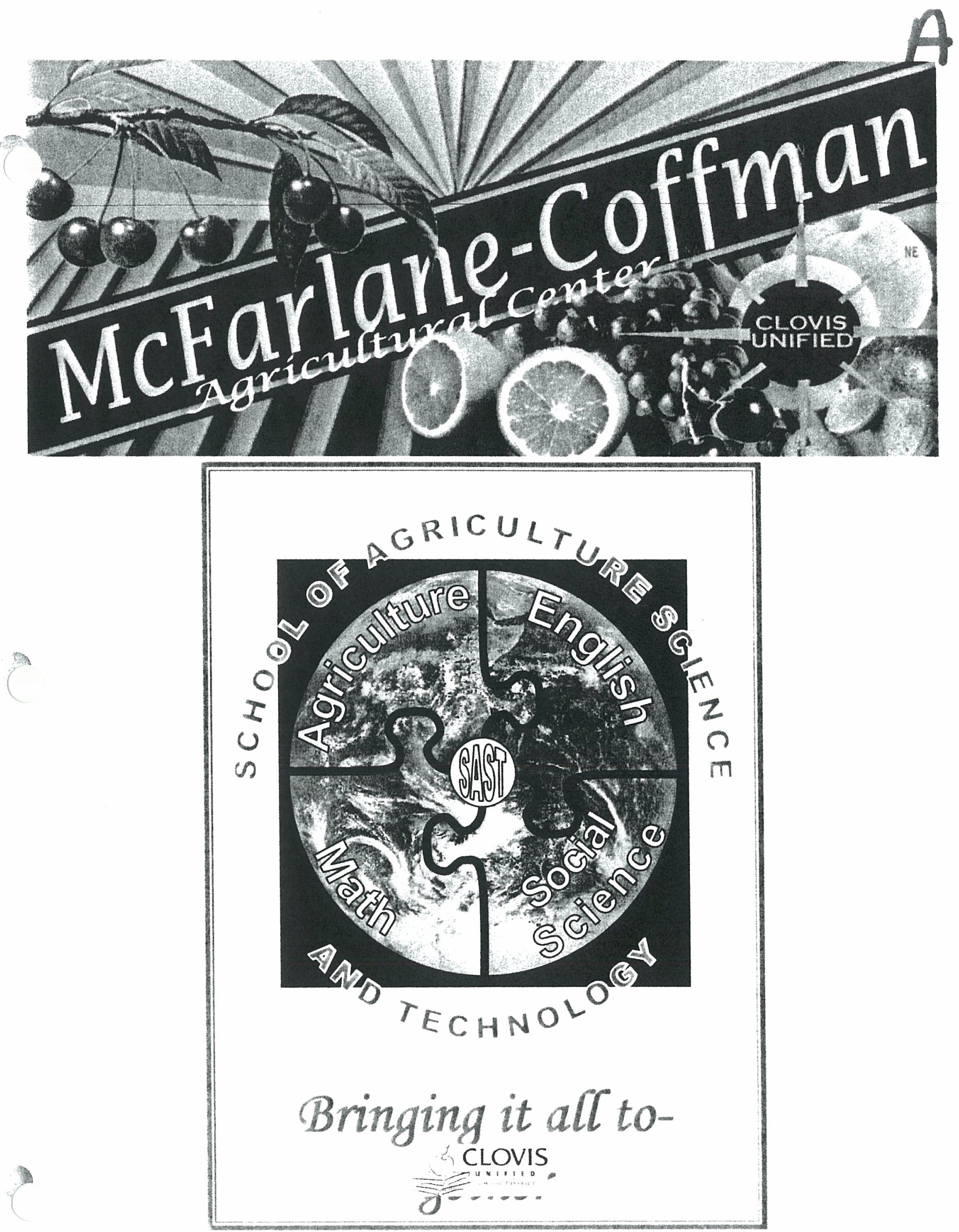
Introduction to Agricultural Science:

While using plants and animals as the main topic of discussion, students will be learning about science and completing their Science Graduation Requirement.

\section{*Earth Science}

This is a hands on Earth Science class that allows students to learn by doing while completing their Physical Science Graduation Requirement. Agriculture Earth Science gets the same credit as a Science 1 class.

\section{Agricultural Biology}

This is a hands on biology class that allows students to learn by doing while completing their UC Lab Science Requirement for college. Agricultural Biology gets the same college credit as a regular Biology class.

\section{AP Environmental Science}

This is an exploration into the issues surrounding our environment. APES allows students to learn by doing while completing their UC Physical Lab Science Requirement for college. APES gets the same college credit as a Chemistry class.

\section{Agricultural Leadership}

This course integrates agriculture instructional strategies with English language arts standards. This course will increase awareness and understanding of the industry of agriculture. Students in this class will be the agricultural ambassadors for the McFarlane-Coffman Agriculture Center.

\section{Botany}

Meets UC Elective credit

This class is an advance level plant science class examining a deeper level of plant science.

\section{Ornamental Horticulture}

This elective class is perfect for the hands-on learner. Students learn how to propagate plants through the many ways of today's agriculturalists and grow them in either the growth chamber, grow room, greenhouse, or 11 acre farm.

\section{Plant and Animal Physiology}

Meets UC Elective credit

This class is an accelerated agriculture class for students serious in learning more about plants and animals.

\section{Advanced Animal Science}

This class addresses the advanced science of animals and animal systems. Great for anyone interested

\section{ROP Veterinary Science}

This class exposes and trains students in the career of a veterinarian or a vet technician. Students will learn about the health, nutrition, and well being of animals. This class utilizes the Community Classroom Program and students will be placed at veterinary clinics the second semester to fully expose them to the career at a Vet or Vet Tech.

Previous Ag Science based class recommended!

\section{Agricultural Mechanics \& Engineering 1, 2 \& 3:}

Students will learn how to Arc and MIG weld, as well as learn carpentry skills, electrical wiring, computer aided drafting, plumbing, and much more! Ag Engineering II is UC Elective Credit Approved.

\section{ROP Welding and Engineering}

Interested in welding and woodworking? After taking Ag Engineering I and II, what else is there? Come and learn in the huge Ag mechanics shop about Arc Welding, MIG Welding, Gas Welding, Woodworking, Construction, Electrical Wiring, and much more! This class utilizes the Community Classroom Program and students will be placed at job sites to learn first hand about related careers.

\section{ROP}

Any class that is designated as ROP is for Juniors and Seniors. These classes are after lunch every day. Students must be 16 years old to take them. 


\section{McFarlane-Coffman Agriculture Center}

The McFarlane-Coffman Agriculture Center is a comprehensive, state of the art facility offering students real world hands on experiences in Animal Science, Plant Science, Agricultural Engineering, and Environmental/Natural Resources. Our Students come from a diverse population ranging from those with a family involved in agriculture to others experiencing agriculture for the first time.

Our Curriculum is driven by industry partnerships and aligned to state academic standards with the goal of making a personal connection between kids and content.

Our Technology rich environment allows students to develop and refine industry level skills while being prepared for post secondary education. Students use the same technology tools found in businesses and universities.

Dur Projects are based around student interest in their chosen agriculture discipline and are strengthened by their involvement in the FFA which is the largest student leadership organization in the United States.

Our Connection to the Agriculture Industry gives our students opportunities to be a part of the global powerhouse that is California Agriculture.

Our Facilities Opened in August of 2000 , our program was known as the Central Valley Applied Agriculture and Technology Center. A bipartisan effort by Senator Charles Poochigian and then Lieutenant Governor Cruz Bustamonte the goal of the project was to develop regional center that would serve as a model for career and technical education throughout the state. Since that time our program has pursued and received additional local, state, and federal funding to further develop the program and its facilities. In 2003 the centers' name was changed to reflect the relentless commitment of two local agriculture leaders who have been the driving force behind building and achieving the vision of this magnificent facility.

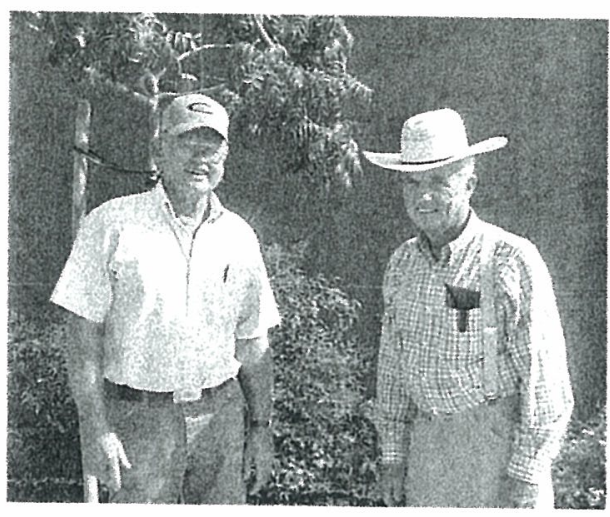

John Coffman and Bill McFarlane.

Longtime advisory committee members and namesakes of the McFarlane-Coffman Agriculture Center

\section{Why should you enroll your child in Agriculture}

The McFarlane-Coffman Agriculture center has served as a model for agricultural education at local, state, national and international level. We have been visited by many political figures such as Congressman George Radanovich, former US Secretary of Agriculture Ann Venemin and former VicePresident Dick Cheney. Countless tours and presentations have been made to representatives from educational institutions such as Iowa State, University of California and California Polytechnic University, San Luis Obispo. Annually the center serves as a student teaching site for credential students from Fresno California State University, Fresno. Additionally, our staff has presented to agriculturalists and agriculture educators from Mexico, China and Costa Rica. Our students also organize "Farm Tours" for over 2,000 elementary school students each year. These hands-on interactive tours give a new meaning to the slogan "California Grown".
Since its inaugural year the center has grown to serve nearly 700 high school and 350 seventh-eighth grade students from throughout Clovis Unified School District with a focus on Premier Leadership, Personal Growth and Career Success.

Benefits of Career Academics

A reduction in drop-out rates; an increase in students applying to college; an increased involvement in youth development activities; assistance to all students to prepare them for future careers; help students relate academics to career opportunities; help students understand the importance of education as they plan career goals; make academy students higher achievers; and give all students a "sense of belonging."

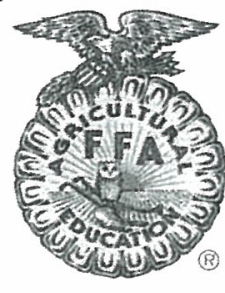



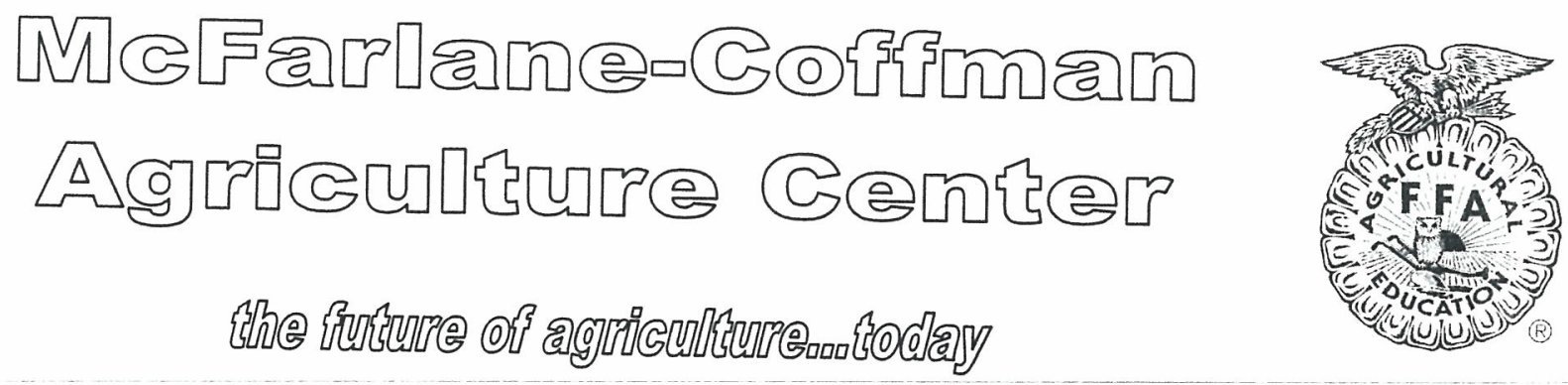

The McFarlane-Coffman Agriculture Center is a comprehensive, state of the art facility offering students real world hands on experiences in Animal Science, Plant Science, Agricultural Engineering, and Environmental/Natural
Resources. Our Students come from a diverse population ranging from those with a family involved in agriculture to others
experiencing agriculture for the first time.

Our Curriculum is driven by industry partnerships and aligned with state standards with the goal of making a personal Our Technology rich environment allows students to develop and refine industry level skills while preparing them for
post secondary education.

Our Projects are based around student interest in their chosen agriculture discipline and are strengthened by their involvement in the FFA which is the largest student leadership organization in the United States Our Connection to the Agriculture Industry gives our students opportunities to be a part of the global powerhouse that

Opened in August of 2000, our program was known as the Central Valley Applied Agriculture and Technology Center. A bi-partisan effort by Senator Charles Poochigian and then Lt. Governor Cruz Bustamonte the centers goal was to develop regional center that would serve as a model for career and technical education throughout the state. Since that time our program has pursued and received additional local, state, and federal funding to further develop the program and its facilities. In 2003 the centers' name was changed to reflect the relentless commitment of two local agriculture leaders who have been the driving force behind building and achieving the vision of this magnificent facility.

The McFarlane-Coffman Agriculture center has served as a model for agricultural education at local, state, national and international level. We have been visited by many political figures such as Congressman George Radanovich, former US Secretary of Agriculture Ann Venemin and former Vice-President Dick Cheney. Countless tours and presentations have been made to representatives from educational institutions such as lowa State, University of site for credentialifornia Polytechnic University, San Luis Obispo. Annually the center serves as a student teaching agriculturalists and agriculture educators frolifornia State University, Fresno. Additionally, our staff has presented to Farm" tours for elementary school students from thro, China and Costa Rica. Our students also organize "Day on the meaning to the slogan "California Grown".

Since its inaugural year the center has grown to serve nearly 700 high school and 350 seventh-eighth grade students from throughout Clovis Unified School District with a focus on Premier Leadership, Personal Growth and Career
Success.

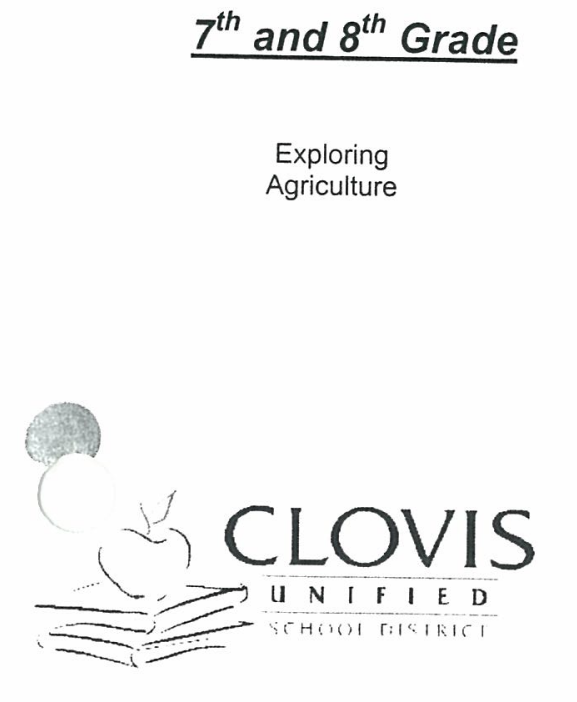

\section{Course Offerings}

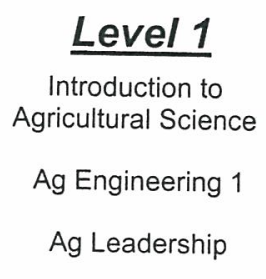

\section{Level 1}

ral Science

Ag Leadership

\author{
Level 2 \\ Plant and Animal \\ Physiology (P) \\ Ag Engineering 2 \\ (P) \\ AP Environmental \\ Science (AP) \\ Agricultural Biology \\ (P)
}

\section{Level 3 and 4}

$\begin{array}{ccc}\begin{array}{c}\text { Agricultural } \\ \text { Leadership }\end{array} & \begin{array}{c}\text { Ag Welding and } \\ \text { Construction }\end{array} & \text { Ag Leadership } \\ \begin{array}{c}\text { Veterinary } \\ \text { Science }(P)\end{array} & \begin{array}{c}\text { Ornamental } \\ \text { Horticulture }\end{array} & \text { Equine Science } \\ \text { Agricultural } & \begin{array}{c}\text { Advanced } \\ \text { Agricultural } \\ \text { Biotechnology }\end{array} & \text { Botany (P) } \\ & \text { Research } & \end{array}$

Ag Engineering 3 Ag Engineering 4

Ag Leadership 


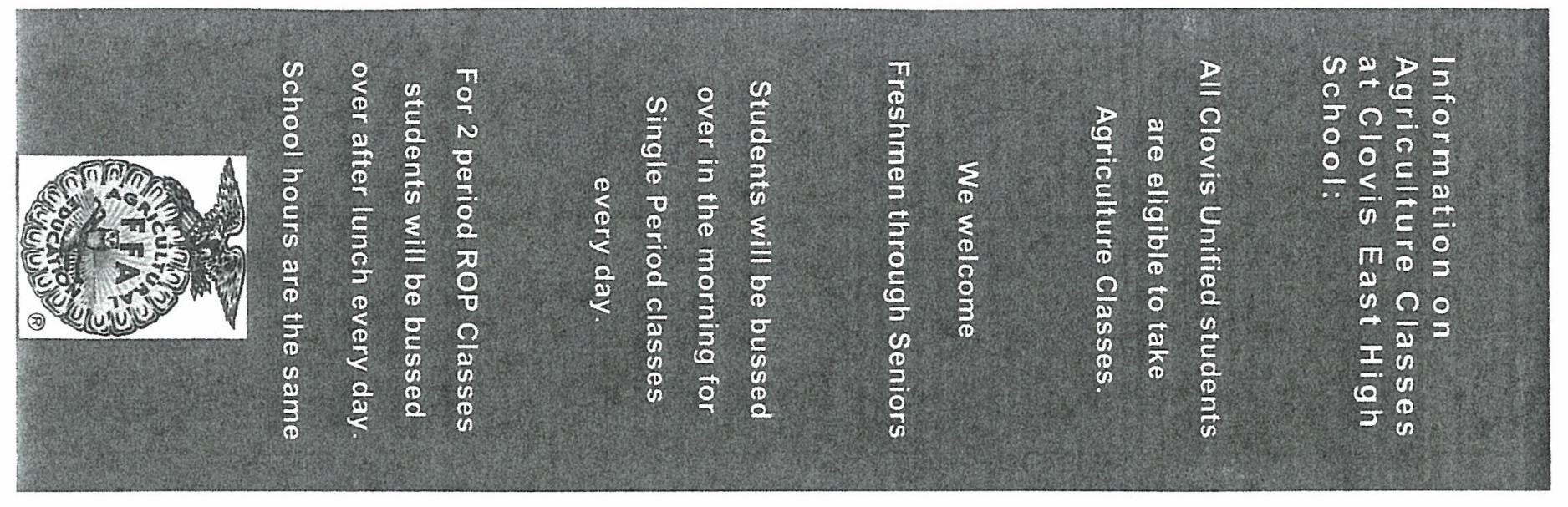

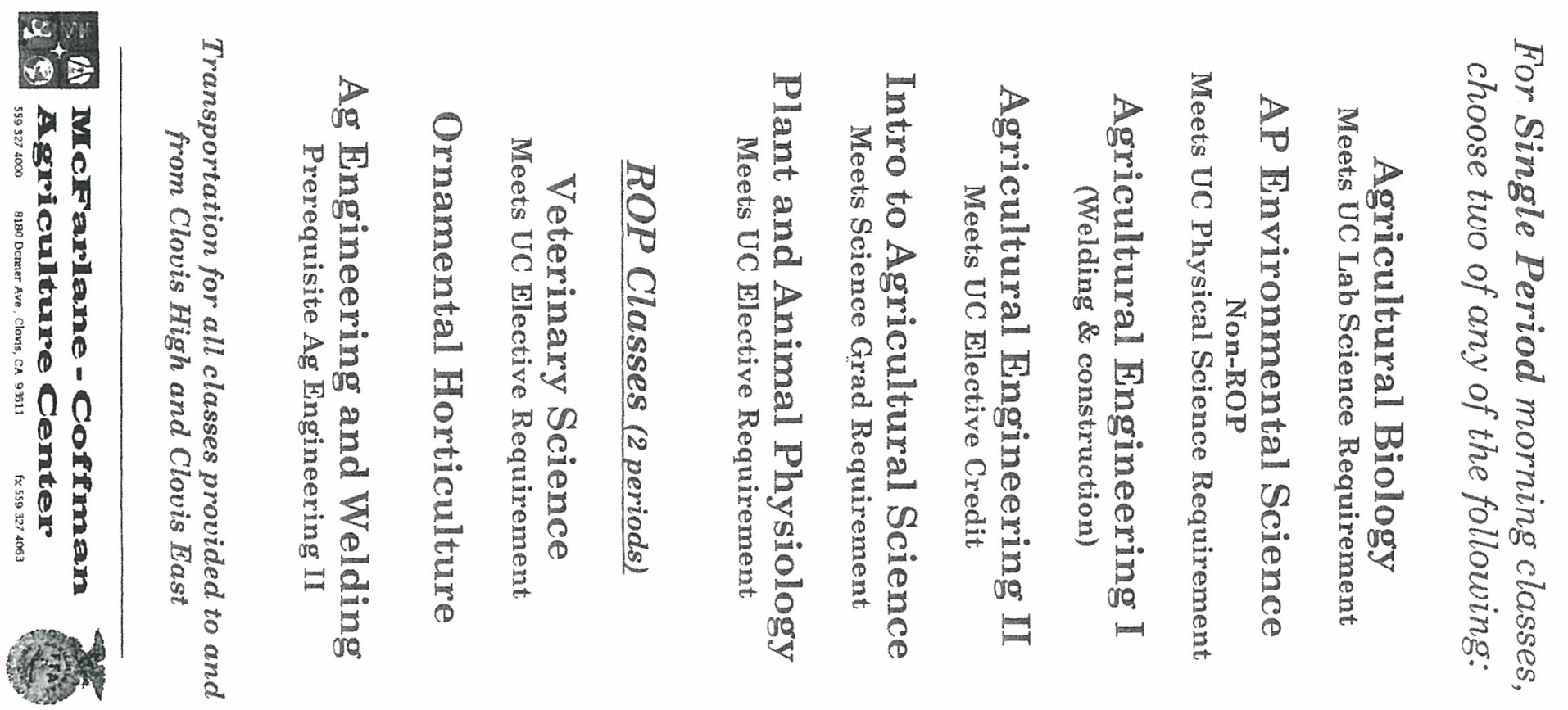

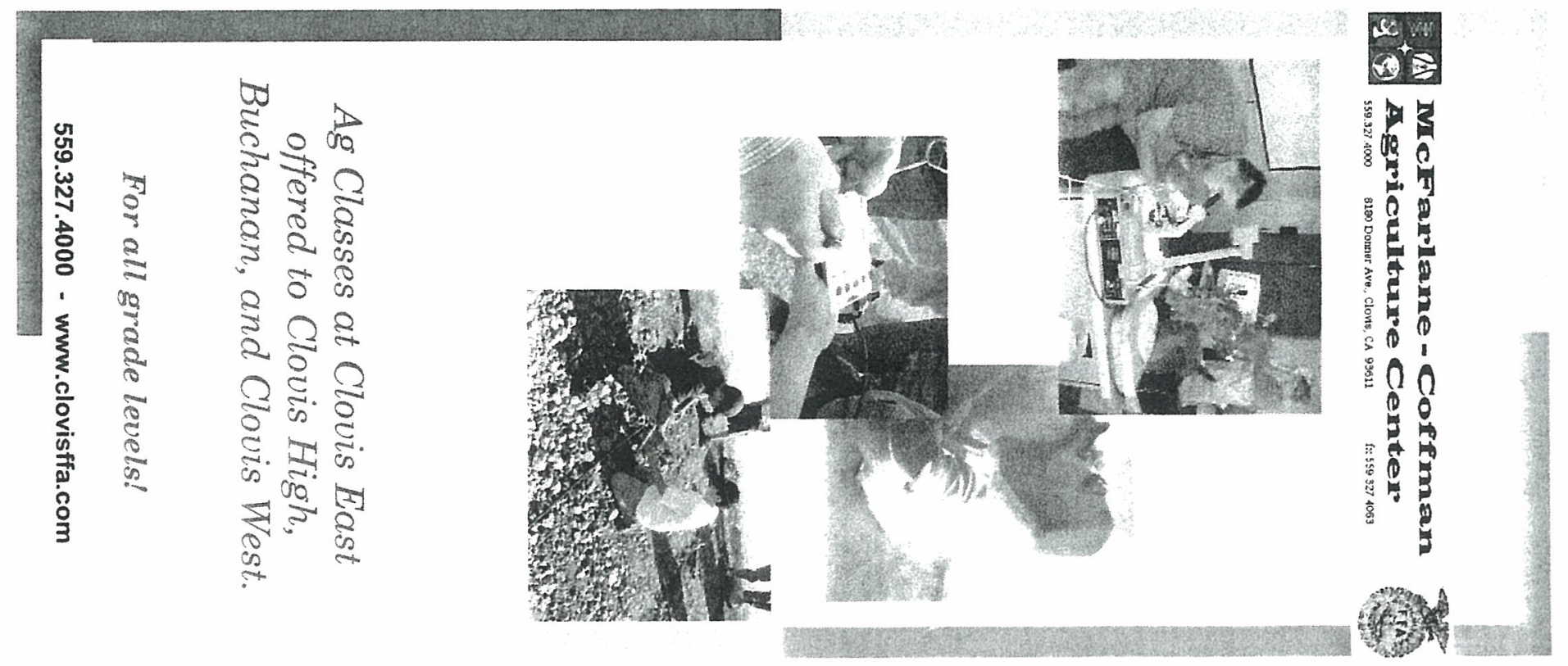



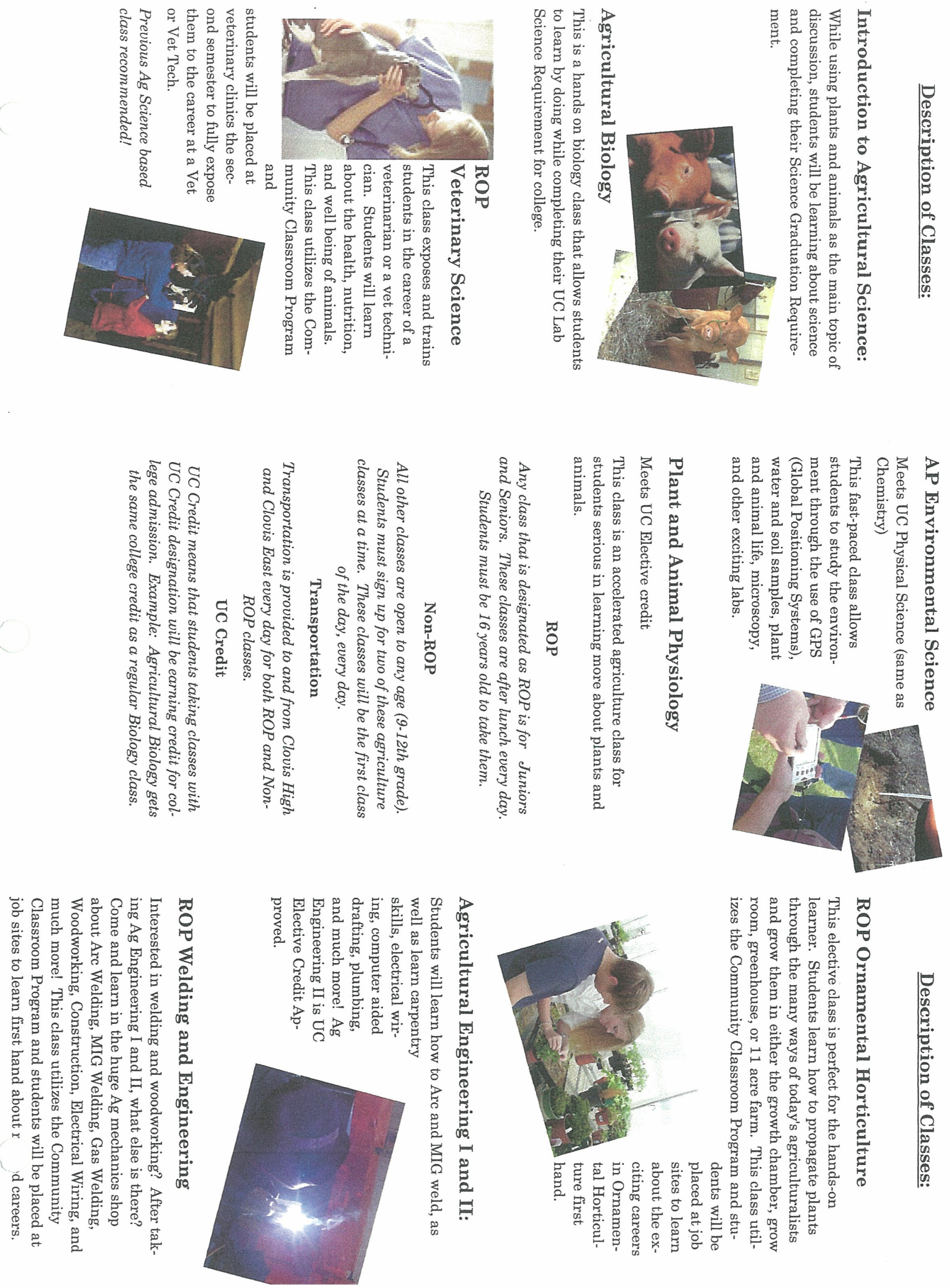

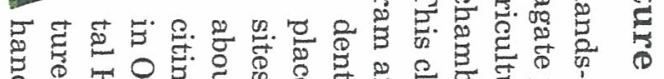

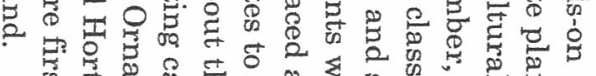

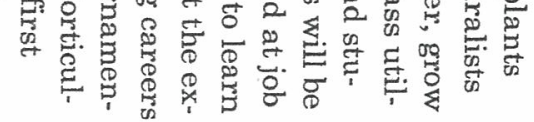



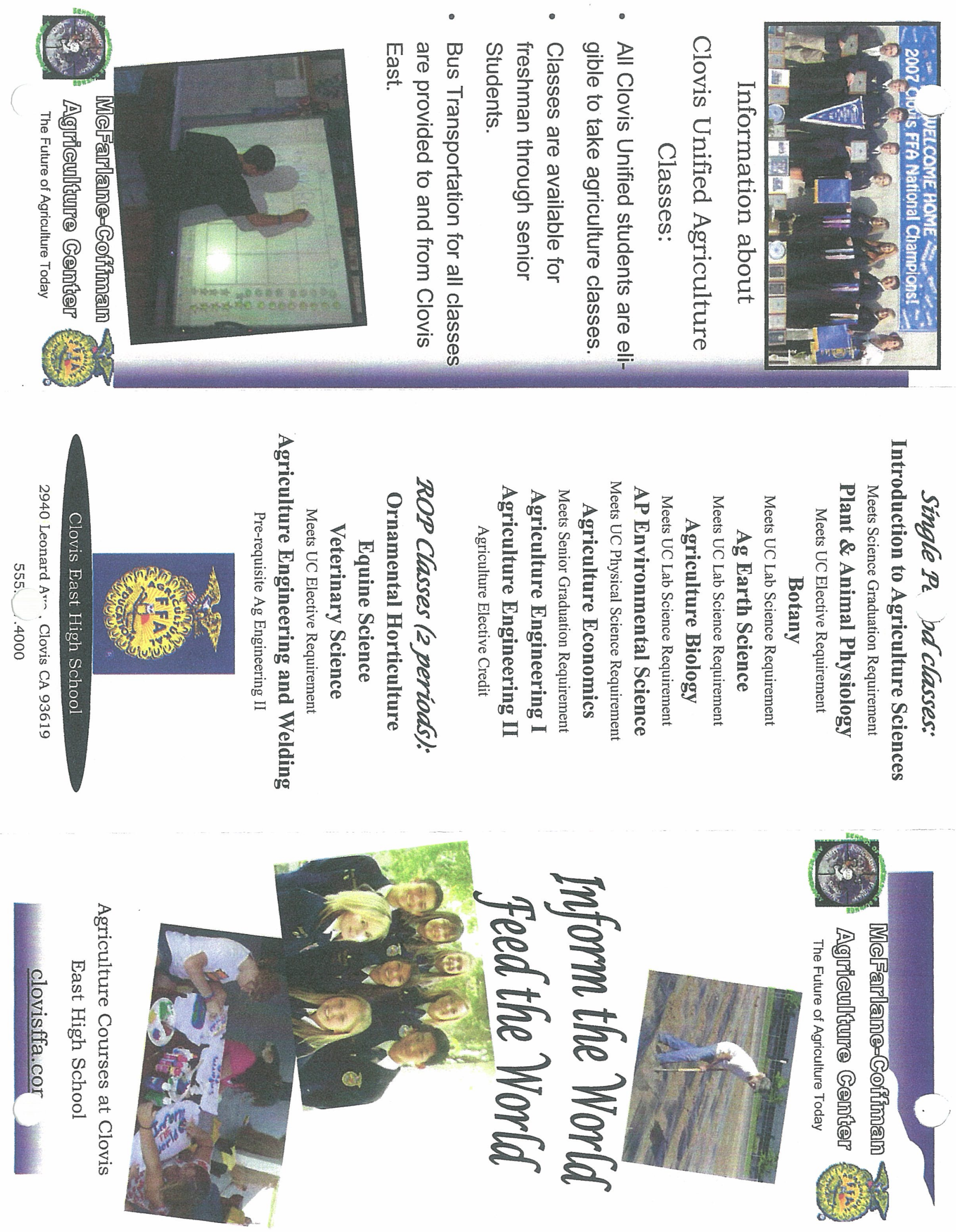

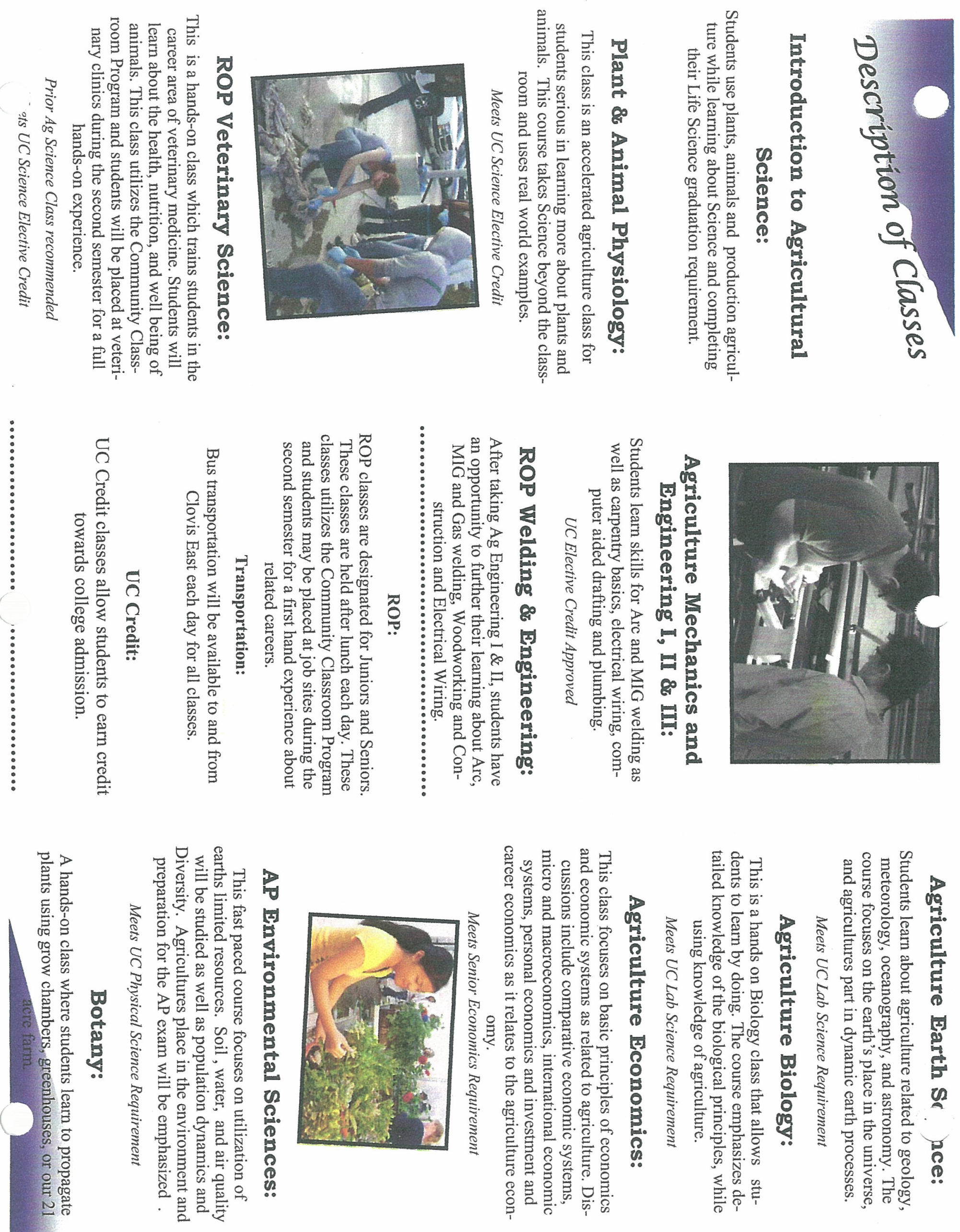

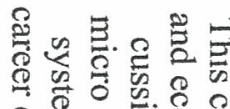

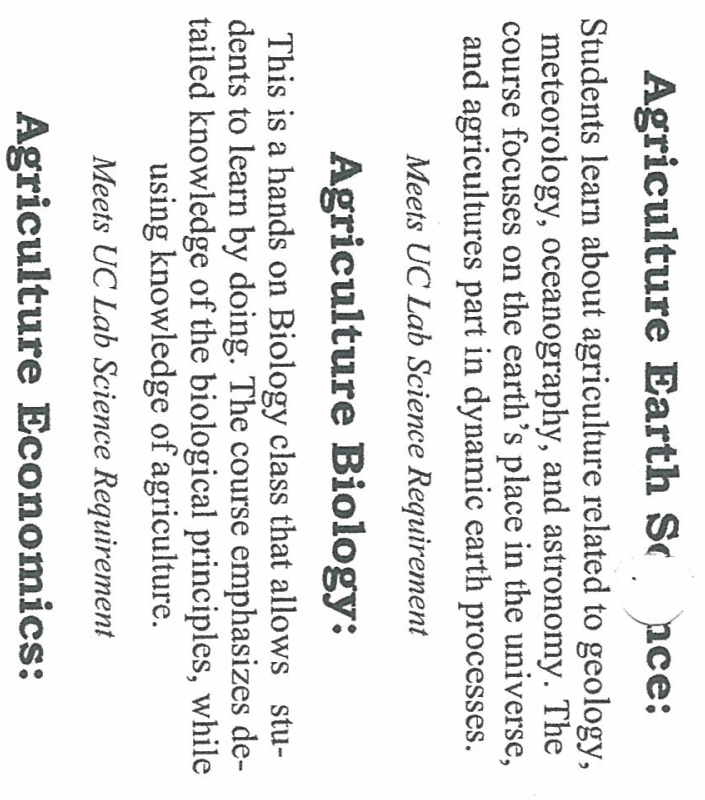



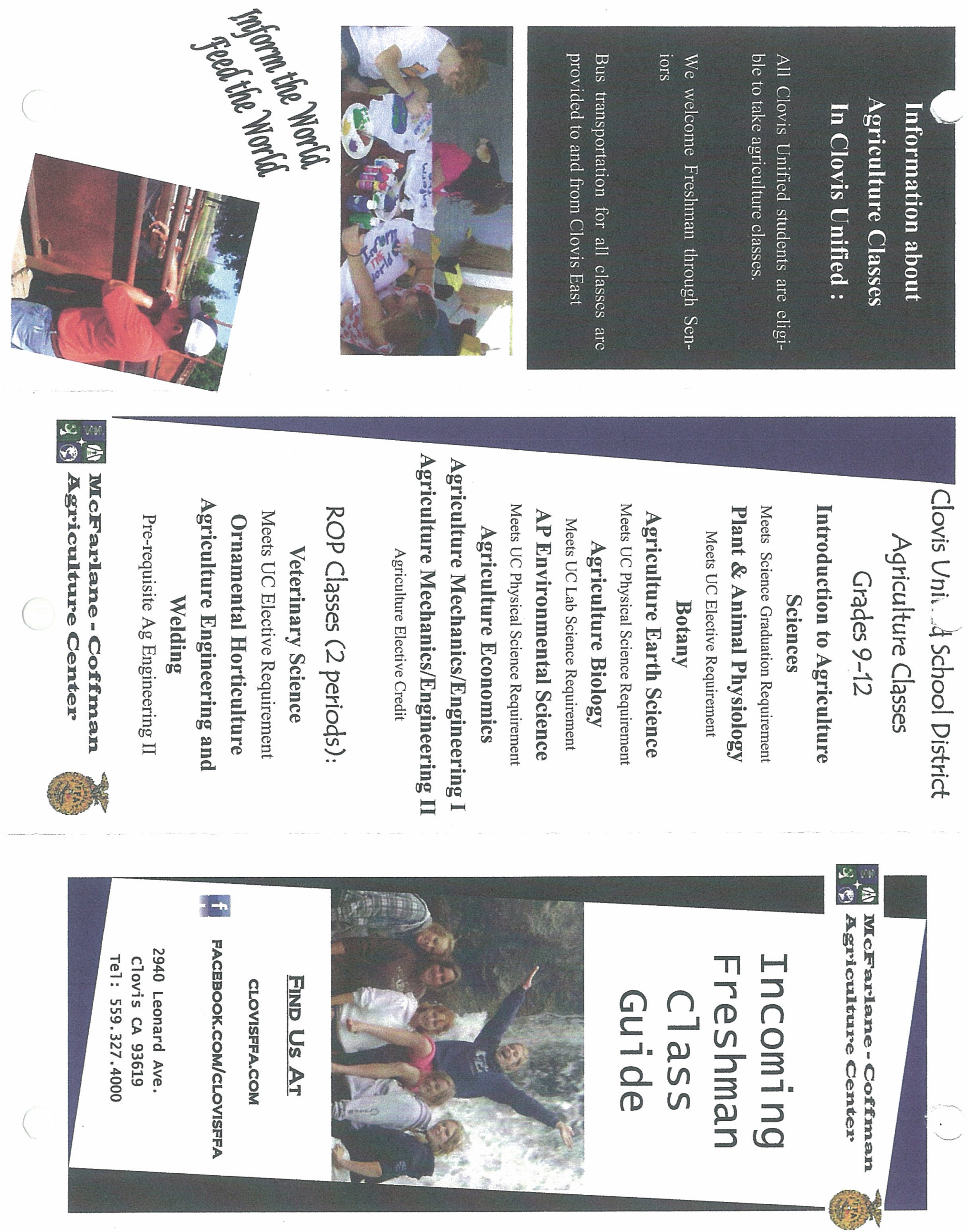

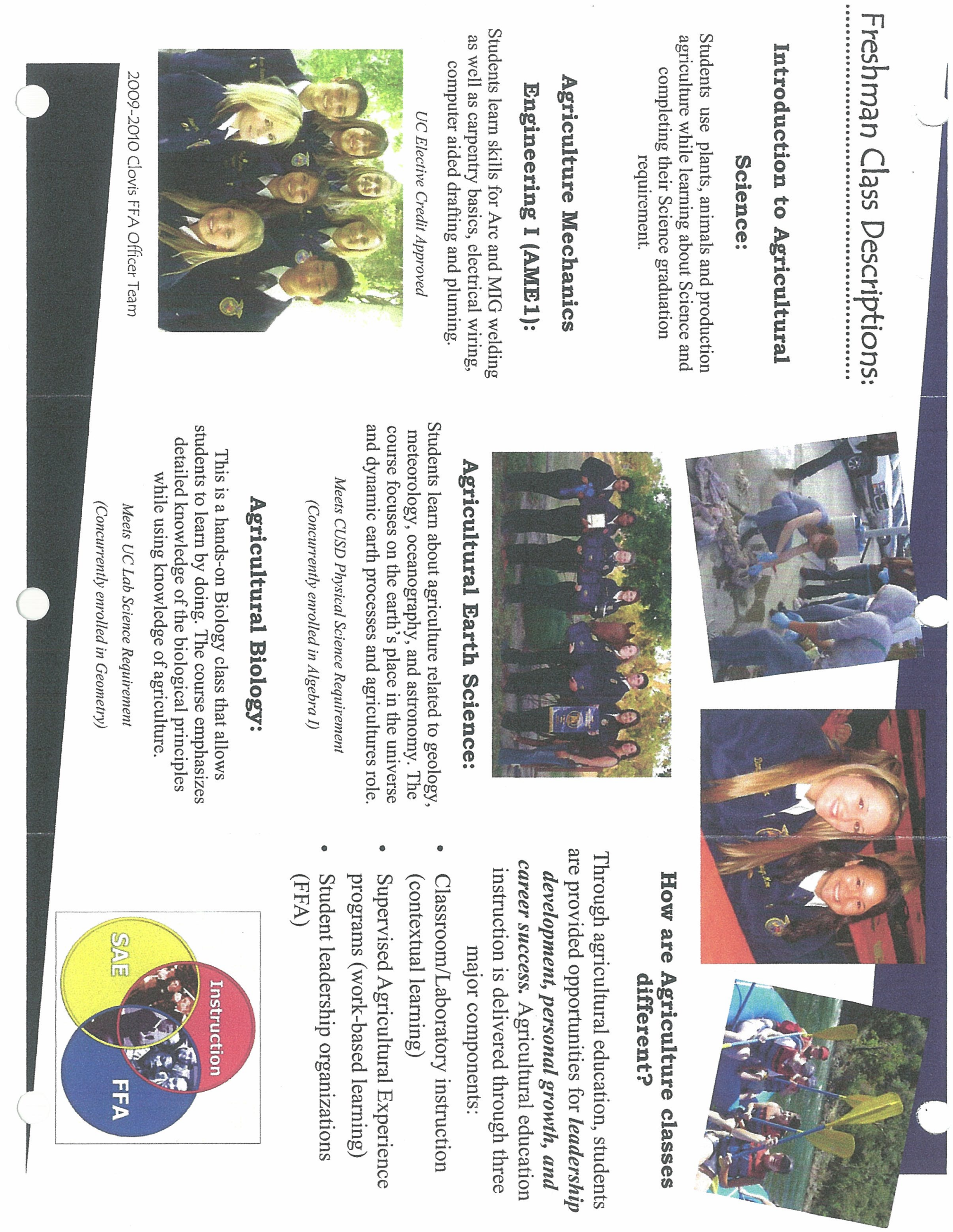


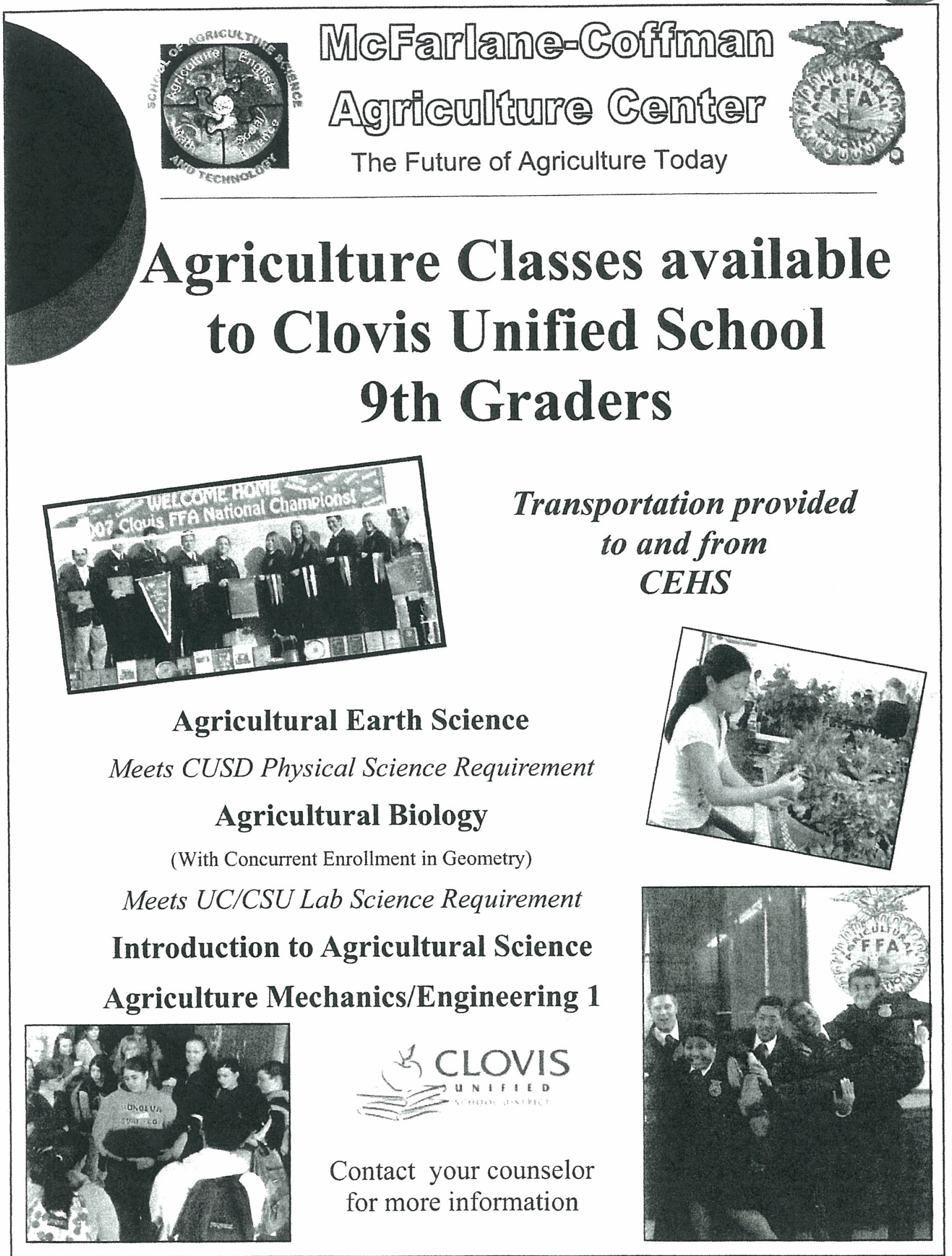




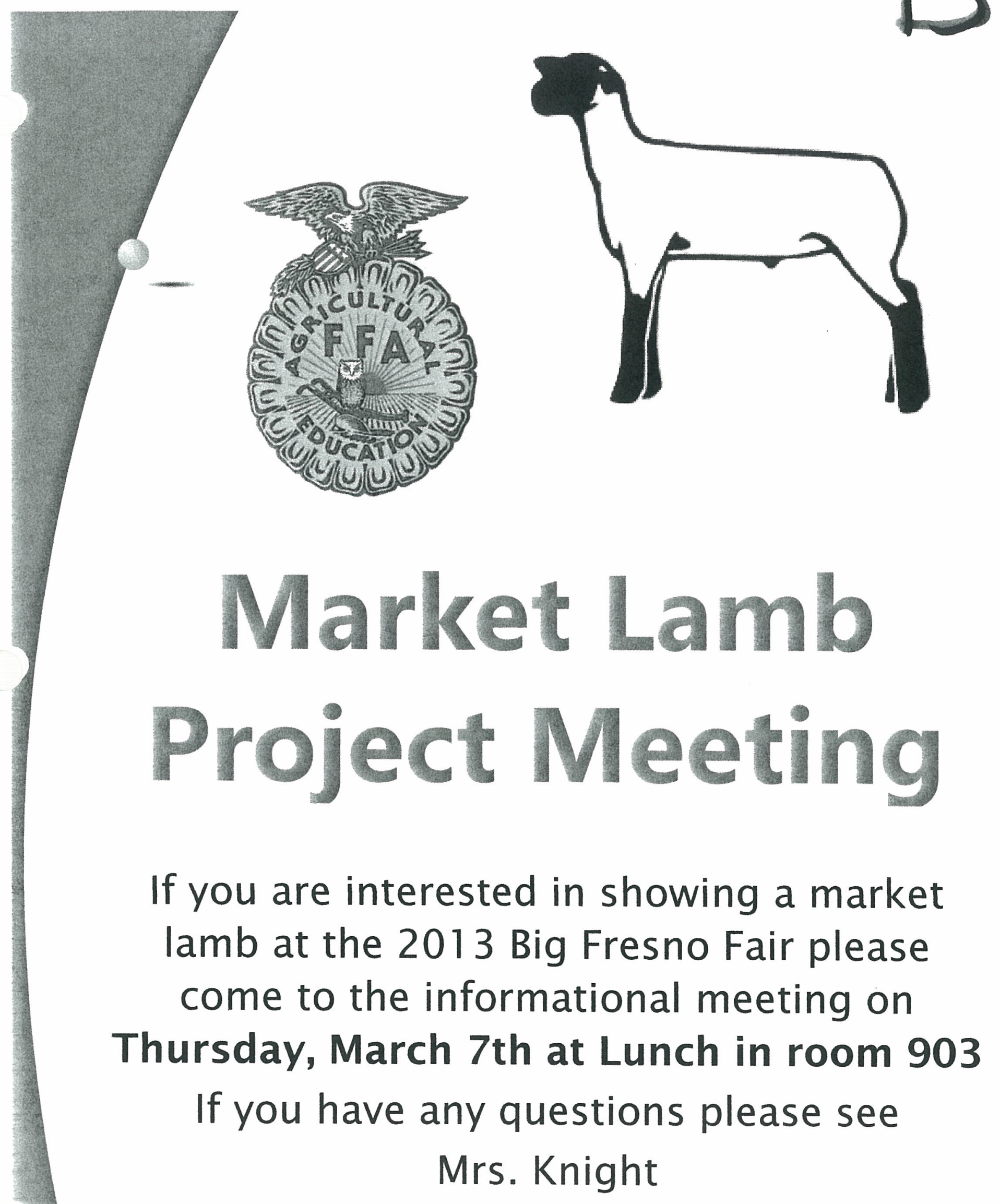




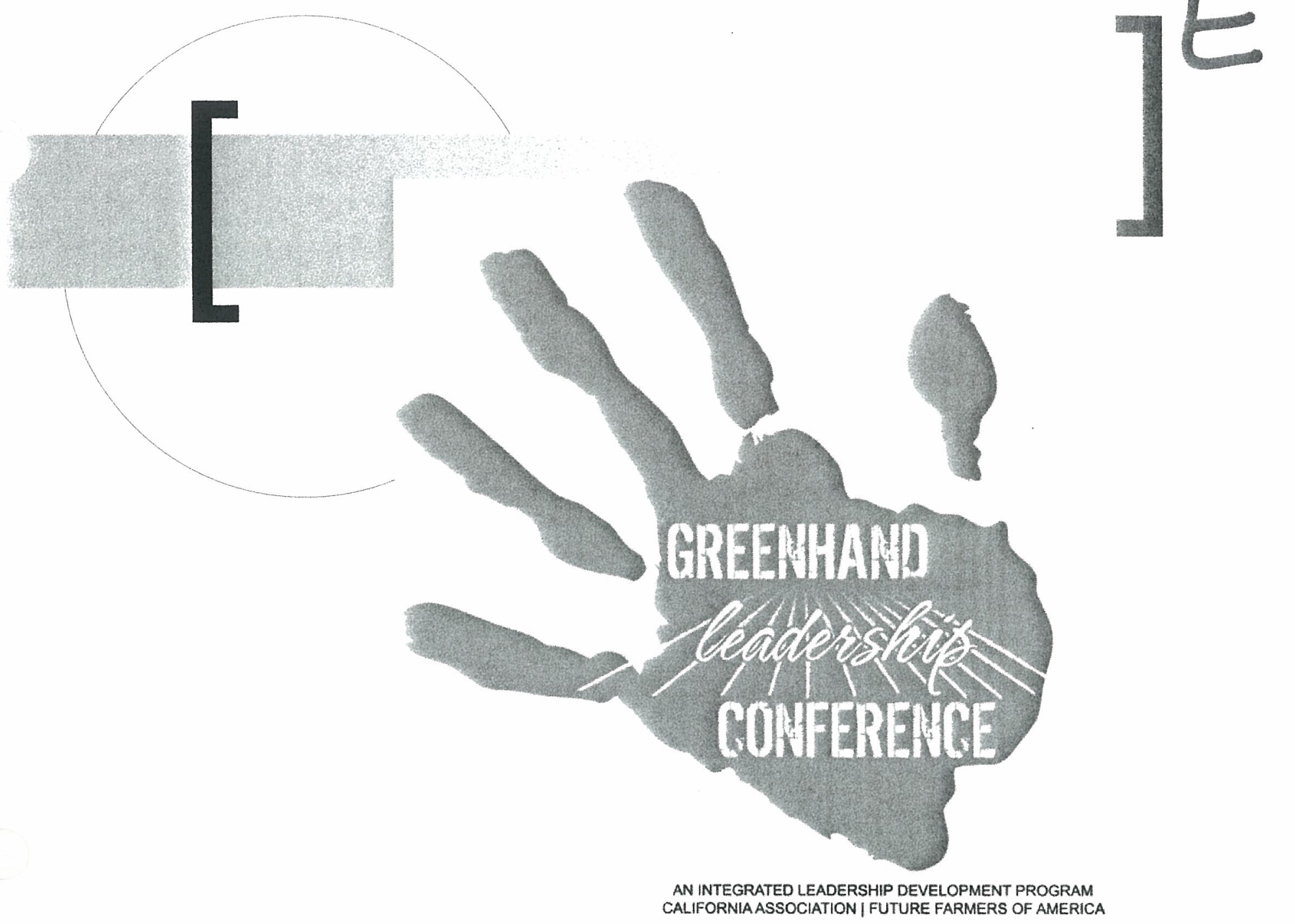

\section{GRENHAND LEADERSHIP CONFERENCE}

This conference is for Freshmen Only.

Sign up for an amazing day where you can meet other 9th grade FFA members. Learn how you can get more involved in the FFA and become an active part of this dynamic organization. Registration is limited to only 14 Spots! First Come First Serve! Turn in your permission slip with $\$ 30$ to Mrs. Knight ASAP. 


\section{Quality Criteria Nine}

\section{Program Accountability and Planning}

The Clovis East Comprehensive Program Plan is complete and we continue to submit all required paperwork and reports on time to our Regional Supervisor. The chart of responsibilities, equipment acquisition schedule, FFA program of work, and Advisory Committee rosters are updated annually and submitted by the deadline. A copy of our Program Plan is in a binder in our Ag Office where it is easily accessible to all teachers. Annual assessment of our Ag department's effectiveness by ourselves, our Learning Director, and our Advisory Committee allows us to focus on areas of needed growth.

We have several methods of communicating with graduates and obtaining follow up. We do send a survey out by mail to our graduates, but also have a Facebook page they can like and then send in answers to follow up questions that we may have. We also frequently have graduates attend the Ag Booster BBQ and will get them to fill out a new and updated contact card at that time. I think we could improve our graduate follow up by creating an online survey and emailing it to our graduates and posting it on the Clovis FFA webpage.

A department budget is also generated annually that describes the intended uses for VEA/Perkins funding as well as Ag Incentive Grant funds. Funds are allocated per course for supplies and also for FFA and Leadership activities. Conferences, Department supplies, copies, and sub costs tend to top the list of expenditures.

Each year student retention is analyzed. We continue to focus on retention of students and the numbers of program completers. Sometimes our numbers can be misleading because of the nature of our multi High School enrollment. Many of our upper division electives and ROP classes attract non Ag students from other campuses that are upper classmen. Sometimes our number of ninth graders is low, but this is partially due to the fact that we reduced to a six period day and with students wanting to meet UC/CSU entrance requirement they simply don't have room in their schedule for Ag during their ninth and tenth grade years. Our Ag Science courses like Ag Biology and Ag Earth Science are vital to keeping our ninth and tenth grade numbers up. With the addition of UC 
approved Floral Design as fine art, this will also help our Ag students meet requirements within our program.

\section{Supporting Completion Materials}

Item A- Pictures of the Agriculture Permanent Student Files

Item B- Ag Department Student Record Sheet, designed to keep in files with student data sheets.

Item C- Previously used Graduate follow up survey. As a graduate myself, I have taken a survey monkey online survey that was emailed to me or linked on the former Clovis FFA Facebook Page.

Item D- Former Clovis FFA Facebook Page, used to keep in touch with graduates and help facilitate the graduate follow up survey.

Item E- CUSD Agriculture Program Completer

Item F- Graduate Follow-up Report 2012

Item G- R-2 Report 2012-2013

Item H- Comprehensive Program Plan ** Parts of this are also included in other sections of this 539 binder. 


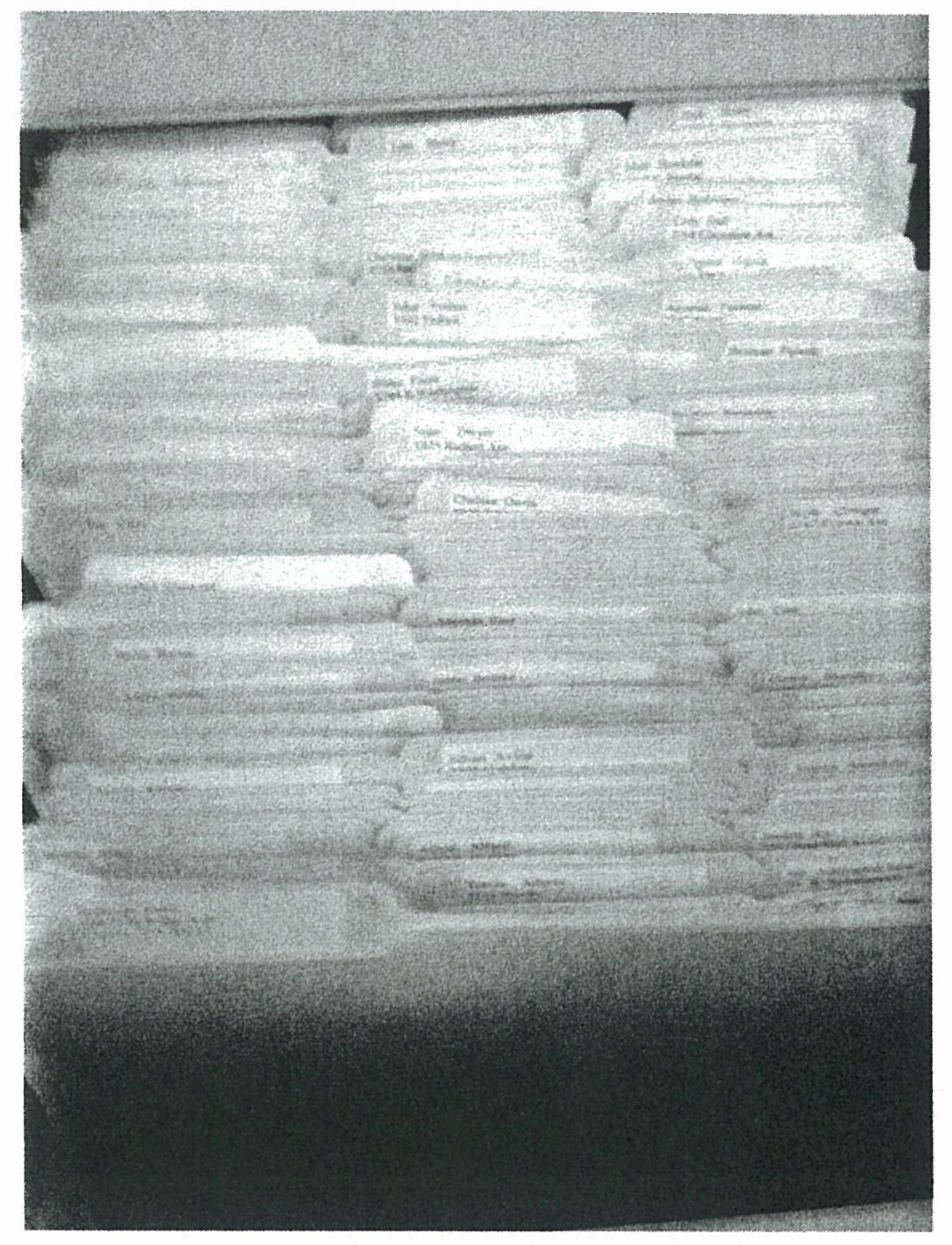

\section{Ag Department}

Permanent Records
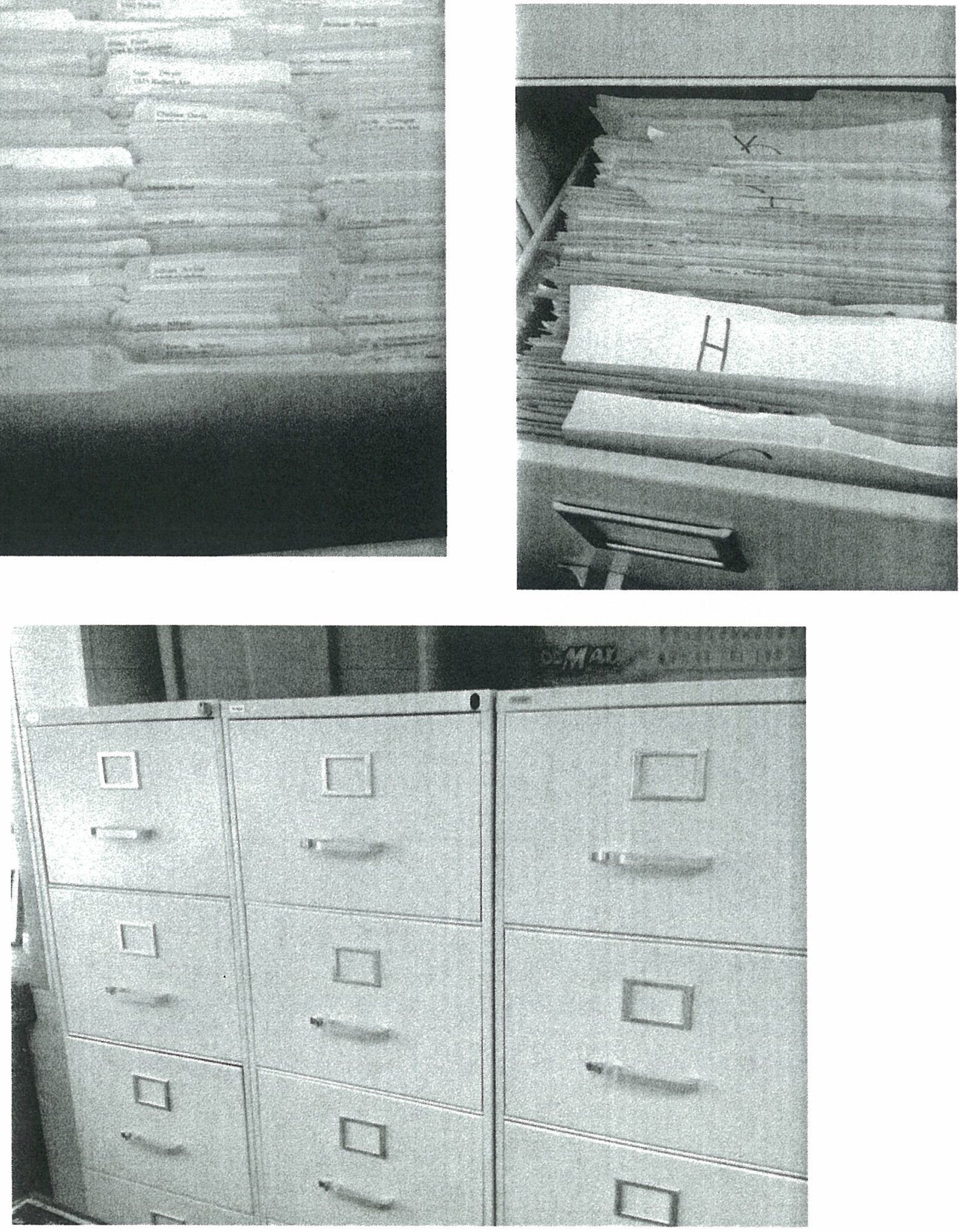


\section{Clovis East High School Agricultural Department}

CUMULATIVE RECORD FOR:

Student's name Date of Birth

Address Phone

(Box or street number) (City) (Zip)

Name of Parent / guardian

Student Occupational Goal

RECORD OF AGRICULTURAL ENROLLMENT

\begin{tabular}{|l|l|l|l|}
\hline Year & Course Name & Teacher & Grade \\
\hline & & & \\
\hline & & & \\
\hline & & & \\
\hline & & & \\
\hline & & & \\
\hline & & & \\
\hline & & & \\
\hline & & & \\
\hline
\end{tabular}

FFA DEGREES HELD

\begin{tabular}{|l|l|}
\hline DEGREE & Date Conferred \\
\hline Greenhand & \\
\hline Chapter & \\
\hline State & \\
\hline American & \\
\hline
\end{tabular}

FFA OFFICES HELD

\begin{tabular}{|c|c|c|c|c|c|c|}
\hline \multirow[b]{2}{*}{ Year } & \multirow[b]{2}{*}{ Office } & \multicolumn{4}{|c|}{ Level } & \multirow{2}{*}{ Nat'I } \\
\hline & & Chapt & S S & Reg. & State & \\
\hline & & & & & & \\
\hline & & & & & & \\
\hline & & & & & & \\
\hline & & & & & & \\
\hline & & & & & & \\
\hline & & & & & & \\
\hline
\end{tabular}


FFA COMMITTEE WORK

\begin{tabular}{|l|l|l|}
\hline Year & $\begin{array}{l}\text { Committee } \\
\text { Name }\end{array}$ & $\begin{array}{l}\text { Member=M } \\
\text { Chairman=C }\end{array}$ \\
\hline & & \\
\hline & & \\
\hline & & \\
\hline & & \\
\hline & & \\
\hline & & \\
\hline & & \\
\hline
\end{tabular}

FFA CONTEST PARTICIPATION

\begin{tabular}{|l|l|l|l|l|}
\hline \multirow{2}{*}{$\begin{array}{l}\text { School } \\
\text { Year }\end{array}$} & Name of Contest & \multicolumn{3}{|c|}{ Placing } \\
\hline & & Sec./Reg. & State & Nat'l \\
\hline & & & & \\
\hline & & & & \\
\hline & & & & \\
\hline & & & & \\
\hline & & & & \\
\hline & & & & \\
\hline & & & & \\
\hline & & & & \\
\hline
\end{tabular}

SCHOLARSHIPS RECEIVED

\begin{tabular}{|l|l|}
\hline Name of Schoalrship & Amount \\
\hline & \\
\hline & \\
\hline & \\
\hline
\end{tabular}

SCHOOL ACTIVITY PARTICIPATION

\begin{tabular}{|l|l|l|}
\hline $\begin{array}{l}\text { School } \\
\text { Year }\end{array}$ & Activity & $\begin{array}{l}\text { Responsibility/ } \\
\text { Placing }\end{array}$ \\
\hline & & \\
\hline & & \\
\hline & & \\
\hline & & \\
\hline & & \\
\hline & & \\
\hline & & \\
\hline
\end{tabular}


COMMUNITY \& OTHER NON-SCHOOL ACTIVITIES

\begin{tabular}{|l|l|l|}
\hline $\begin{array}{l}\text { School } \\
\text { Year }\end{array}$ & \multicolumn{1}{|c|}{ Activity } & $\begin{array}{l}\text { Responsibility/ } \\
\text { Placing }\end{array}$ \\
\hline & & \\
\hline & & \\
\hline & & \\
\hline & & \\
\hline & & \\
\hline & & \\
\hline
\end{tabular}

\section{RECORD OF SAE}

\begin{tabular}{|c|c|c|c|c|c|}
\hline Type & Scope & & Hrs & Net & Net \\
\hline First Year & Quantity & Unit & Labor & Income & Worth \\
\hline 1 & & & & & \\
\hline 2 & & & & & \\
\hline 3 & & & & & \\
\hline 4 & & & & & \\
\hline 5 & & & & & \\
\hline Second Yea & & & & & \\
\hline 1 & & & & & \\
\hline 2 & & & & & \\
\hline 3 & & & & & \\
\hline 4 & & & & & \\
\hline 5 & & & & & \\
\hline Third Year & & & & & \\
\hline 1 & & & & & \\
\hline 2 & & & & & \\
\hline 3 & & & & & \\
\hline 4 & & & & & \\
\hline 5 & & & & & \\
\hline Fourth year & & & & & \\
\hline 1 & & & & & \\
\hline 2 & & & & & \\
\hline 3 & & & & & \\
\hline 4 & & & & & \\
\hline 5 & & & & & \\
\hline Fifth Year & & & & & \\
\hline 1 & & & & & \\
\hline 2 & & & & & \\
\hline 3 & & & & & \\
\hline 4 & & & & & \\
\hline
\end{tabular}




\section{Graduate Follow-up}

Name:

Address:

Phone:

1. What are you doing at the present time?

Attending school

Full-time

Part-time

In the military

Homemaker

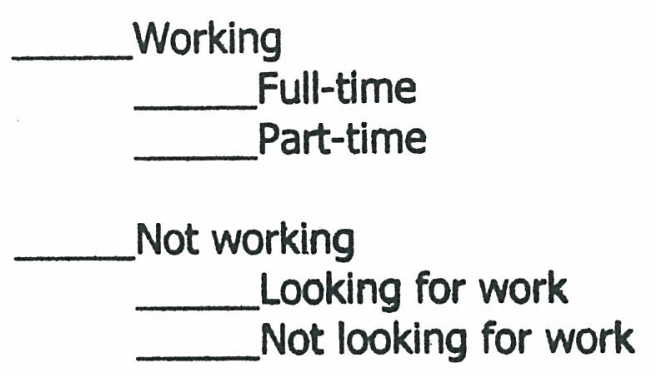

Other

2. In what type of business or industry are you employed?

3. What is your job title or job description?

4. Which statement best applies to your present occupation?

I am using most of the skills I learned in the vo-ag program at

I am using some of the skills I learned in the vo-ag program at

I am not using any of the skills I learned in the vo-ag program at Re CEHS

5. What type of school are you currently attending?

High school

4-year college

Adult education
Trade/technical school

Private business school

Other

6. What is your major course of study? 
7. How would you rate the training received in the RHS vo-ag program?

Excellent Good Fair Poor

8. How do you rate the career guidance and counseling you received in vo-ag? Excellent Good Fair Poor

\section{FFA}

1. Please check the following areas you feel are valuable components of FFA.

Officer and committee chairman experience Judging contests Advanced degree and proficiency awards Participation in chapter activities, working with others Livestock raising, shows, fairs, etc. Other-please describe

2. What were the most valuable aspects of the SOEP (supervised projects)? Learning skills related to future ag employment Development of responsibility Learning record keeping Other-please describe

3. Please rate the facilities and equipment used at RHS for the vo-ag program:

Facilities:

Equipment:
Overcrowded Modern

Modern Well-maintained $\quad$ Pdequate amount of equipment for all students in class Well-maintained $\quad$ Pdequate amount of equipment for all students in class Adequate space provided Out-of-date

Out-of-date Well-maintained $\quad$ Pdequate amount of equipment for all students in class

Other-please describe

Please note any suggestions you have for improving the Instructional Program, including the following areas: classroom, shop, greenhouse, school farm, etc; FFA; SOEP (supervised projects); teaching methods used; facilities/equipment. 


\section{mul. AT\&T 4G 3:27 PM $>063 \% \square$

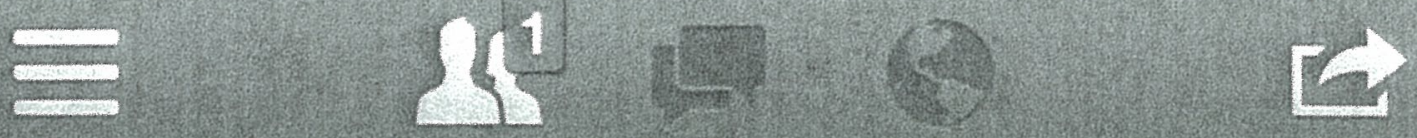

reantitarchy Former Clovis Ffa

May 3, 2011 ,

Please respond to our questionnaire!!!

2 comments

Like Comment $\rightarrow$ Share

Fortherchnt Former Clovis Ffa changed their profile picture.

April 29, 2011 解

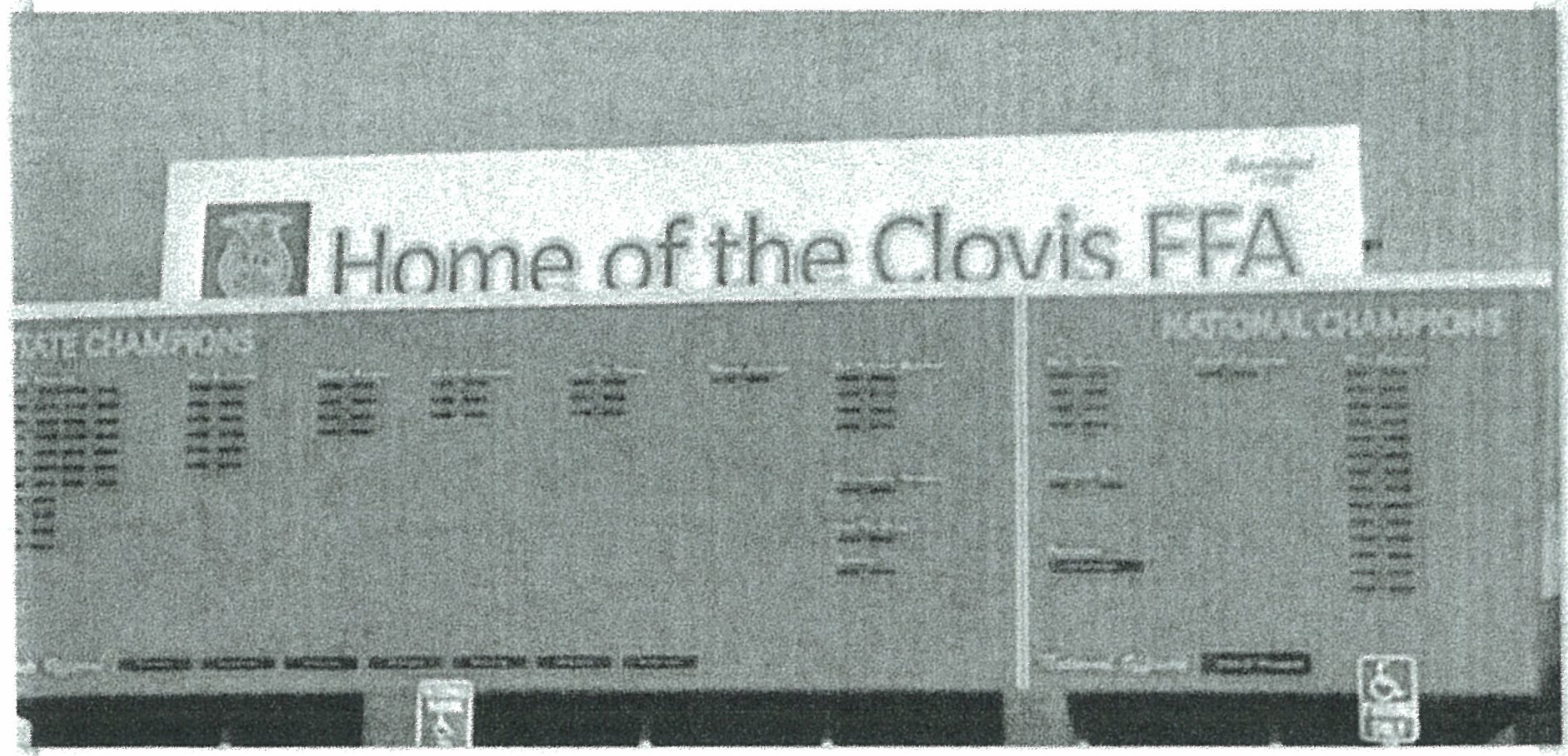




\title{
CUSD Agriculture Program Completer
}

\author{
Guidelines for Program Completers
}

- Successfully completed three years in the agriculture program

- Participated actively as an FFA Member

- Attend one FFA activity every 6 weeks

- Participated in the SAE Program

- Had a project maintained in the FFA Record book for three years.

\section{Recognition:}

- Program completing seniors will be recognized at the Spring FFA Awards Banquet and will be presented with a special gift. (Graduation Tassel)

- Students may also "letter" in FFA and receive a patch for their letterman's jacket by completing the following listed above and also receiving the State FFA Degree. 


\section{Graduate Follow-up Report}

Filing Year $=2012$

Clovis East Clovis

2940 Leonard Avenue

Clovis, CA 93619

Printed: 5/24/2013 3:56:23 PM

\begin{tabular}{|l|l|}
\hline Total Seniors (Year=2011 ): & 122 \\
\hline $\begin{array}{l}\text { Total Seniors having completed } \\
\text { 3 or more years of Ag Instruction: }\end{array}$ & 23 \\
\hline \begin{tabular}{l} 
Program Completer Status \\
\hline Two Year College Ag Major
\end{tabular} & 3 \\
\hline Two Year College Non-Ag Major & 8 \\
\hline Four Year College Ag Major & 4 \\
\hline Four Year College Non-Ag Major & 2 \\
\hline Employed - Fulltime Ag Job & 2 \\
\hline Military & 1 \\
\hline Location or Position Unknown & 3 \\
\hline
\end{tabular}

Site developed and maintained by the 
Select a school: $<$ Select a School $\gg$ $\mathrm{GO}>$

Data for Year: 2012-2013

\title{
School:
}

\author{
Clovis \\ Clovis East HS \\ 2940 Leonard Avenue \\ Clovis, CA 93619
}

\section{Wobsto}

Teachers: 6

Courses Offered:

\begin{tabular}{|c|c|c|c|}
\hline Type & Course & Enrollment & H.S. Grad Credit UC Credit \\
\hline Ag Biology & Ag Biology & 32 & Life Science \\
\hline Ag Biology & Ag Biology & 38 & Life Science \\
\hline Ag Biology & Ag Biology & 41 & Life Science \\
\hline Ag Biology & Ag Biology & 35 & Life Science \\
\hline Ag Mechanics & Ag Mech \& Eng 3 & 10 & Other \\
\hline Ag Mechanics & Ag Mech 1 & 26 & Other \\
\hline Ag Mechanics & Ag Mech 1 & 36 & Other \\
\hline Ag Mechanics & Ag Mech 1 & 31 & Other \\
\hline Ag Mechanics & Ag Mech 2 & 23 & Other \\
\hline Ag Mechanics & Ag Mech 2 & 20 & Other \\
\hline Ag Mechanics & Ag Welding and Construction & 25 & Other \\
\hline Ag Mechanics & Ag Welding and Construction & 25 & Other \\
\hline Agriscience I & Ag Science 1 & 24 & Other \\
\hline Animal Science & Animal Science & 25 & Other \\
\hline Animal Science & Vet Science & 27 & Other \\
\hline Animal Science & Vet Science & 27 & Other \\
\hline Forestry/NR & Ag Earth Science & \multicolumn{2}{|c|}{35 Physical/Earth Sci. } \\
\hline Forestry/NR & Ag Earth Science & \multicolumn{2}{|c|}{26 Physical/Earth Sci. } \\
\hline Forestry/NR & Ag Earth Science & \multicolumn{2}{|c|}{36 Physical/Earth Sci. } \\
\hline Forestry/NR & AP Environmental Science & \multicolumn{2}{|c|}{37 Physical/Earth Sci. } \\
\hline Forestry/NR & AP Environmental Science & \multicolumn{2}{|c|}{32 Physical/Earth Sci. } \\
\hline Forestry/NR & AP Environmental Science & \multicolumn{2}{|c|}{36 Physical/Earth Sci. } \\
\hline Other Ag & Ag Exploration & 29 & Does Not Meet \\
\hline Other Ag & Ag Exploration & 34 & Does Not Meet \\
\hline Other Ag & Ag Exploration & 18 & Does Not Meet \\
\hline Other Ag & Ag Exploration & 28 & Does Not Meet \\
\hline Other Ag & Ag Exploration & 36 & Not Entered \\
\hline Other Ag & Ag Exploration & 32 & Not Entered \\
\hline Other Ag & Ag. Econ/Government & 21 & History/Gov't \\
\hline \multirow[t]{2}{*}{ Plant/Soil Science } & Botany & \multicolumn{2}{|c|}{22 Physical/Earth Sci. } \\
\hline & TOTAL & 867 & \\
\hline
\end{tabular}


FFA Students by Pathway:

\begin{tabular}{lr} 
Pathway & Count \\
\hline Ag Bus Mgt & 19 \\
Ag Mech. & 141 \\
Agriscience & 217 \\
An. Science & 112 \\
Forestry/NR & 77 \\
O.H. & 6 \\
Plant/Soil Sci. & 25 \\
& 597
\end{tabular}

FFA Students by Grade Level:

$\begin{array}{lr}\frac{\text { Grade Level }}{9} & \text { Count } \\ 9 & 147 \\ 10 & 151 \\ 11 & 164 \\ 12 & 107 \\ 13 & 10 \\ 14 & 12 \\ 15 & 5 \\ 16 & 1 \\ \text { Total } & 597\end{array}$

FFA Students by Years in Ag:

\begin{tabular}{lr} 
Years in Ag & Count \\
\cline { 2 - 2 } 1 & 388 \\
2 & 121 \\
3 & 43 \\
4 & 17 \\
5 & 10 \\
6 & 12 \\
7 & 5 \\
8 & 1 \\
Total & 597 \\
Average Years & 1.7
\end{tabular}

Freshman Persistance:

Cohort Year: 2009-2010

\begin{tabular}{lrr} 
Years in Ag Completed & & Count \\
\cline { 2 - 3 } 1 & 65 & $54 \%$ \\
2 & 25 & $21 \%$ \\
3 & 13 & $11 \%$ \\
4 & 17 & $14 \%$ \\
Freshman Cohort Students & 120 & \\
Average Years Completed & 1.9 &
\end{tabular}

Ed Data provides demographic data for schools in California. To view this data click on the link.

View Ed Data 
Site developed and maintained by the California FFA Association. 


\section{Clovis East HS}

\section{R2 Student Report}

Year:2012

Gender

\begin{tabular}{|l|l|l|l|}
\hline Schnum & ProgName & Male & Female \\
\hline 152 & Ag Bus Mgt & 10 & 9 \\
\hline 152 & Ag Mech. & 132 & 9 \\
\hline 152 & Agriscience & 105 & 112 \\
\hline 152 & An. Science & 32 & 80 \\
\hline 152 & Forestry/NR & 35 & 42 \\
\hline 152 & O.H. & 4 & 2 \\
\hline 152 & Plant/Soil Sci. & 18 & 7 \\
\hline
\end{tabular}

\section{Hispanic}

\begin{tabular}{|l|l|l|}
\hline ProgName & Hispanic & Non-Hispanic \\
\hline Ag Bus Mgt & 2 & 17 \\
\hline Ag Mech. & 55 & 86 \\
\hline Agriscience & 64 & 153 \\
\hline An. Science & 38 & 74 \\
\hline Forestry/NR & 27 & 50 \\
\hline O.H. & 2 & 4 \\
\hline Plant/Soil Sci. & 6 & 19 \\
\hline
\end{tabular}

\section{Race*}

\begin{tabular}{|l|l|l|l|l|l|l|l|}
\hline ProgName & White & Black & Hispanic & Americian Indian & Asian & Native Hawaiian/Pacifc Island & 2 or more \\
\hline Ag Bus Mgt & 12 & 1 & 0 & 1 & 1 & 0 & 0 \\
\hline Ag Mech. & 96 & 6 & 0 & 9 & 9 & 1 & 15 \\
\hline Agriscience & 118 & 7 & 0 & 8 & 8 & 1 & 58 \\
\hline An. Science & 81 & 3 & 0 & 10 & 10 & 2 & 7 \\
\hline Forestry/NR & 49 & 4 & 0 & 3 & 3 & 5 & 1 \\
\hline O.H. & 2 & 0 & 0 & 2 & 2 & 0 & 1 \\
\hline Plant/Soil Sci. & 10 & 3 & 0 & 3 & 3 & 1 & 0 \\
\hline
\end{tabular}

\section{Grade Level}

\begin{tabular}{|l|r|r|r|r|r|r|r|r|r|}
\hline Year In Ag & Grade9 & Grade10 & Grade11 & Grade12 & Grade13 & Grade14 & Grade15 & Grade16 & Total \\
\hline 1 & 147 & 61 & 114 & 66 & 0 & 0 & 0 & 0 & 388 \\
\hline 2 & 0 & 90 & 14 & 17 & 0 & 0 & 0 & 0 & 121 \\
\hline 3 & 0 & 0 & 36 & 7 & 0 & 0 & 0 & 0 & 43 \\
\hline 4 & 0 & 0 & 0 & 17 & 0 & 0 & 0 & 0 & 17 \\
\hline 5 & 0 & 0 & 0 & 0 & 10 & 0 & 0 & 0 & 10 \\
\hline & & & & & & & & &
\end{tabular}




\begin{tabular}{|l|r|r|r|r|r|r|r|r|r|}
6 & 0 & 0 & 0 & 0 & 0 & 12 & 0 & 0 & 12 \\
\hline 7 & 0 & 0 & 0 & 0 & 0 & 0 & 5 & 0 & 5 \\
\hline 8 & 0 & 0 & 0 & 0 & 0 & 0 & 0 & 1 & 1 \\
\hline Total & 147 & 151 & 164 & 107 & 10 & 12 & 5 & 1 & 597 \\
\hline \multicolumn{1}{|r|}{ Total 9-12 } & 569 \\
\hline
\end{tabular}

\section{Freshman Persistance:}

Cohort Year: 2009-2010

\begin{tabular}{|l|r|r|}
\hline Years in Ag Completed & Count & Percent \\
\hline 1 & 65 & $54 \%$ \\
\hline 2 & 25 & $21 \%$ \\
\hline 3 & 13 & $11 \%$ \\
\hline 4 & 17 & $14 \%$ \\
\hline Freshman Cohort Students & 120 & \multicolumn{1}{|l}{} \\
\cline { 1 - 2 } Average Years Completed & 1.9 & \multicolumn{1}{|l}{} \\
\cline { 1 - 2 } &
\end{tabular}

*Prior to 2010 Hispanic is listed as a race.

Printed: 5/24/2013 3:57:13 PM

Site developed and maintained by the California FFA Association. 


\section{R2 Teacher Information Clovis East HS,Clovis Year: 2012}

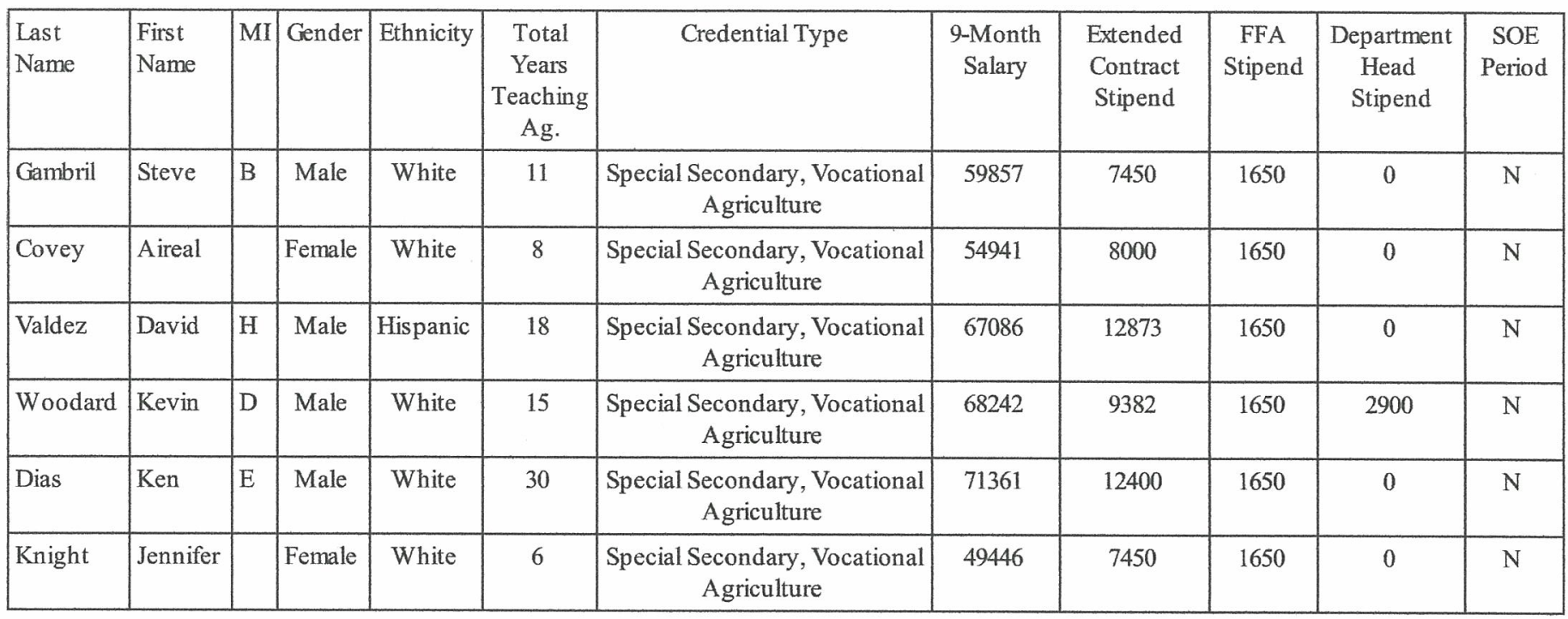

\begin{tabular}{|l|l|l|l|l|l|}
\hline Covey, Aireal & Course Title & Enrollment & Type \\
\hline Schedule & Period & Beginning Time & Crep & 0 & Prep \\
\hline 1 & 1 & $7: 50$ & Ag Biology & 32 & Ag Biology \\
\hline 1 & 2 & $8: 50$ & Ag Leadership & 27 & SAE \\
\hline 1 & 3 & $9: 58$ & Ag Biology & 38 & Ag Biology \\
\hline 1 & 4 & $11: 00$ & Ag Biology & 41 & Ag Biology \\
\hline 1 & 6 & $12: 42$ & Ag Biology & 35 & Ag Biology \\
\hline 1 & 7 & $1: 44$ & & & \\
\hline
\end{tabular}

\begin{tabular}{|l|l|l|l|l|l|}
\hline Dias, Ken & \multicolumn{5}{l|}{$\mid$} \\
\hline Schedule & Period & Beginning Time & Course Title & Enrollment & Type \\
\hline 1 & 1 & $7: 50$ & Ag Science 1 & 24 & Agriscience I \\
\hline 1 & 2 & $8: 52$ & Ag Exploration & 29 & Other Ag \\
\hline 1 & 3 & $9: 58$ & Ag Exploration & 34 & Other Ag \\
\hline 1 & 4 & $11: 00$ & Ag Exploration & 18 & Other Ag \\
\hline 1 & 6 & $1: 44$ & Ag Exploration & 28 & Other Ag \\
\hline 1 & 7 & $1: 44$ & Prep & 0 & Prep \\
\hline
\end{tabular}

\begin{tabular}{|l|l|l|l|l|l|}
\hline \multicolumn{6}{|l|}{ Gambril, Steve } \\
\hline Schedule & Period & Beginning Time & Course Title & Enrollment & Type \\
\hline 1 & 1 & $7: 50$ & Ag Mech 2 & 20 & Ag Mechanics \\
\hline 1 & 2 & $8: 52$ & Ag Mech 1 & 26 & Ag Mechanics \\
\hline 1 & 3 & $9: 58$ & Ag Mech 1 & 36 & Ag Mechanics \\
\hline 1 & 4 & $11: 00$ & Ag Mech 1 & 31 & Ag Mechanics \\
\hline 1 & 6 & $12: 42$ & Ag Mech 2 & 23 & Ag Mechanics
\end{tabular}




\begin{tabular}{|l|l|l|l|l|l|}
\hline 1 & 7 & $1: 44$ & Ag Exploration & 32 & Other Ag \\
\hline
\end{tabular}

\begin{tabular}{|l|l|l|l|l|l|}
\hline \multicolumn{6}{|l|}{ Knight, Jennifer } \\
\hline Schedule & Period & Beginning Time & Course Title & Enrollment & Type \\
\hline 1 & 1 & $7: 50$ & Botany & 22 & Plant/Soil Science \\
\hline 1 & 2 & $8: 52$ & Animal Science & 25 & Animal Science \\
\hline 1 & 3 & $9: 58$ & Ag Exploration & 36 & Other Ag \\
\hline 1 & 4 & $11: 00$ & Prep & 0 & Prep \\
\hline 1 & 6 & $12: 42$ & Vet Science & 27 & Animal Science \\
\hline 1 & 7 & $1: 44$ & Vet Science & 27 & Animal Science \\
\hline
\end{tabular}

\begin{tabular}{|l|l|l|l|l|l|}
\hline \multicolumn{2}{|l|}{ Valdez, David } \\
\hline Schedule & Period & Beginning Time & Course Title & Enrollment & Type \\
\hline 1 & 1 & $7: 50$ & Ag Earth Science & 35 & Forestry/NR \\
\hline 1 & 2 & $8: 52$ & Ag Mech \& Eng 3 & 10 & Ag Mechanics \\
\hline 1 & 3 & $9: 58$ & Prep & 0 & Prep \\
\hline 1 & 4 & $11: 00$ & Ag Earth Science & 26 & Forestry/NR \\
\hline 1 & 6 & $12: 42$ & Ag Welding and Construction & 25 & Ag Mechanics \\
\hline 1 & 7 & $1: 44$ & Ag Welding and Construction & 25 & Ag Mechanics \\
\hline
\end{tabular}

\begin{tabular}{|l|l|l|l|l|l|}
\hline \multicolumn{6}{|l|}{ Woodard, Kevin } \\
\hline Schedule & Period & Beginning Time & Course Title & Enrollment & Type \\
\hline 1 & 1 & $7: 50$ & Ag. Econ/Government & 21 & Other Ag \\
\hline 1 & 2 & $8: 52$ & AP Environmental Science & 36 & Forestry/NR \\
\hline 1 & 3 & $9: 58$ & Ag Earth Science & 36 & Forestry/NR \\
\hline 1 & 4 & $11: 00$ & AP Environmental Science & 32 & Forestry/NR \\
\hline 1 & 6 & $12: 42$ & AP Environmental Science & 37 & Forestry/NR \\
\hline 1 & 7 & $1: 44$ & Prep & 0 & Prep \\
\hline
\end{tabular}

Printed: 5/24/2013 4:12:30 PM

Site developed and maintained by the California FFA Association. 


\section{COMPREHENSIVE PROGRAM PLAN}

\section{TABLE OF CONTENTS}

* See votes as many of these irems

A. Job Market Description are in other sections of the 537 Binder

B. Targeted Occupations

C. Total Program Goals and Objectives

D. Program(s) Description of Included Courses, SOE and Leadership Development

E. Program and/or Course Subject Matter Content Outline

F. Program Completion Standards

G. Description of Facilities and Major Equipment

1. Five (5) Year Facility and Equipment Acquisition Schedule

(I) Staff Assignments

J) FFA Program of Activities - See In Section 2 Item A

K. School and/or Department Policies Pertaining to: - See In Program of Activities - Student Eligibility to Participate in Out-of-Class Activities Section 2 Item A

- Leadership Development Integration into Prngram

- SOE Integration into Program and other Policies

L. Proficiency Standards for Program Completers

M. Teacher Data Sheet for each Teacher

N. Roster of Agriculture Advisory Committee - See In Section 7 Item A

O. Advisory Committee Minutes- See In Section 7 Item E

P. Current Year Budget - see in section 5 Item K

Q. Signed Articulation Agreement and/or Evidence of Articulation

R. Graduate Follow-up System - see in Section 9 Item Cs D

S. List of Active Placement Sites -

T. Recruitment Activities and Materials - see in section 8 Item A, B, SC

U. Staff In-service Record

V. Staff Minutes-See In section 4 Item CiD

W. Department Inventory

$\mathrm{X}$. 


\section{JOB MARKET DESCRIPTION}

\section{Agriculture}

Clovis California is located in North Eastern Fresno County. Clovis's primary

agriculture industries come in the form of Citrus and Stone Fruit production as well as Forage crops Such as Alfalfa. Much of Clovis's agricultural ground is broken into small (5-20 acres) family farms producing specialty crops. Fresno County's 1996 agriculture production value was 3.3 Billon dollars, ranked \#1 in the state of California. Grapes ( $20 \%$ of the state total), cotton ( $1 / 3$ state total), poultry ( $28 \%$ state total) and processed tomatoes ( $1 / 3$ state total) are ranked as Fresno Countys leading crops.

Fresno County's agriculture is made up of $36.10 \%$ fruit \& nut, $20.85 \%$ livestock \& poultry, $20.82 \%$ vegetable crops, $20.30 \%$ field crops, $0.84 \%$ seed crops, $0.51 \%$ nursery, $0.35 \%$ industrial and $0.24 \%$ apiary.

\section{Fresno County's 10 Leading Crops (1996) Crop Dollar Value}

Grapes 555,341,000

Cotton 533,284,000

Poultry $357,906,000$

Tomatoes $257,177,000$

Milk 199,302,000

Almonds 134,104,000

Peaches $120,008,000$

Garlic 118,048,000

Cattle \& Calves 117,802,000

Nectarines 109,200,000

Top Ten Total $\$ 2,502,172,000$

\section{Industrial Potential}

Clovis has a number of industrial sites currently available in various locations. Including sites along the new Freeway 168.

The central California location and available transportation facilities create favorable conditions for industrial growth.

\section{Top Ten Manufacturers}

1. Pelco

2. Wawona Frozen Foods

3. Anlin

4. MiRancho Tortillas

5. Valley Chrome Plating Inc.

6. Apollo

7. Premier Offset Printing

8. Precision Plastics

9. Ano-Tech Metal Finishing

10. Western Products 


\section{Top Ten Non-Manufacturing Employers}

1. Clovis Unified School District

2. City of Clovis

3. Clovis Community Hospital

4. Wal-Mart

5. Gottschalks

6. Costco

7. SaveMart

8. U.S. Forest Service

9. Target

10. Bingham Toyota

\section{Fresno County}

Clovis is located in Fresno County. Fresno County is situated in the heart of the Central San Joaquin Valley between the Kings and San Joaquin Rivers, 189 miles southeast of San Francisco and 221 miles northwest of Los Angeles.

\section{The Region}

Fresno (a Spanish word meaning Ashtree) County was founded in 1872 when the Central (later known as the Southern) Pacific Railroad built a station. The area soon developed into a rich agricultural center when irrigation was introduced in the 1880 s.

Fresno County currently has a population of 761,900 . The County is approximately 6,017 square miles. 
The town of Clovis is located in Fresno County, in the San Joaquin Valley, the heartland of California's agriculture. Agriculture in the San Joaquin Valley is very large and diversified. During 1982, agricultural production in the San Joaquin totaled 7.46 billion dollars. San Joaquin Valley's principal crops include grapes, cotton lint and seed, cattle and calves, milk, almonds and tomatoes.

Fresno is the leading county in the state and the U.S. in gross cash receipts to growers. Fresno County's 1.85 billion dollars in receipts in 1982 had an additional economic impact on the county in excess of 5.5 billion dollars. Forty-five of the top fifty crops in California are produced commercially in Fresno County, illustrating the diverse nature of its agriculture.

Agricultural job opportunities for Clovis High School Vo Ag students are high in demand for crop farms and livestock ranches. In Component II is a list of agriculture job occupations for Clovis Vo Ag. graduates. 


\section{CGP \\ Top Agricultural Related Occupations}

\section{Occupational Information}

\begin{tabular}{|c|c|c|c|c|}
\hline Occupational Titles & $\begin{array}{l}\text { Beginning } \\
\text { Salary* }\end{array}$ & $\begin{array}{l}\text { Average } \\
\text { Salary* }\end{array}$ & Top Salary* & $\begin{array}{l}\text { Occupational } \\
\text { Outlook }\end{array}$ \\
\hline $\begin{array}{l}\text { Agricultural } \\
\text { Engineer }\end{array}$ & $\begin{array}{l}\$ 37,884 \text { a year } \\
\text { with bachelor's } \\
\text { degree }\end{array}$ & $\begin{array}{l}\$ 55,007 \mathrm{a} \\
\text { year }\end{array}$ & $\begin{array}{l}\$ 67,558 \text { or more a } \\
\text { year }\end{array}$ & $\begin{array}{l}\text { moderately good to } \\
\text { strong prospects }\end{array}$ \\
\hline Agronomists & $\begin{array}{l}\$ 28,000 \text { a year } \\
\text { depending on } \\
\text { degree of } \\
\text { education and } \\
\text { employer }\end{array}$ & $\$ 39,593$ & $\begin{array}{l}\$ 48,456 \text { or more a } \\
\text { year }\end{array}$ & $\begin{array}{l}\text { Increase } 10 \% \text { to } \\
20 \% \text { through the } \\
\text { year } 2006\end{array}$ \\
\hline Animal Scientist & $\begin{array}{l}\$ 25,777 \text { a year } \\
\text { with a } \\
\text { bachelor's } \\
\text { degree }\end{array}$ & $\begin{array}{l}\$ 37,247 \text { a } \\
\text { year for } \\
\text { federal } \\
\text { employees }\end{array}$ & $\begin{array}{l}\$ 45,749 \text { with a } \\
\text { doctoral degree } \\
\text { who work in } \\
\text { research, } \\
\text { marketing, and } \\
\text { development for } \\
\text { industry }\end{array}$ & $\begin{array}{l}\text { Increase } 10 \% \text { to } \\
20 \% \text { through the } \\
\text { year } 2005\end{array}$ \\
\hline Auctioneers & $\$ 39,418$ a year & $\$ 48,661$ & $\begin{array}{l}\$ 55,960 \text { or more a } \\
\text { year }\end{array}$ & $\begin{array}{l}\text { the auction } \\
\text { business is } \\
\text { growing and } \\
\text { skilled auctioneers } \\
\text { are always in } \\
\text { demand }\end{array}$ \\
\hline Biochemists & $\begin{array}{l}\$ 39,950 \text { a year } \\
\text { depending on } \\
\text { degree of } \\
\text { education and } \\
\text { employer }\end{array}$ & $\$ 57,062$ & $\begin{array}{l}\$ 72,471 \text { or more a } \\
\text { year }\end{array}$ & $\begin{array}{l}\text { Increase } 21 \% \text { to } \\
35 \% \text { through } 2006\end{array}$ \\
\hline Biologist & $\begin{array}{l}\$ 27,351 \text { a year } \\
\text { depending on } \\
\text { degree of } \\
\text { education }\end{array}$ & $\begin{array}{l}\$ 39,192 \mathrm{a} \\
\text { year }\end{array}$ & $\begin{array}{l}\$ 49,378 \text { or more a } \\
\text { year }\end{array}$ & $\begin{array}{l}\text { expected to } \\
\text { increase } 21 \% \text { to } \\
35 \% \text { through the } \\
\text { year } 2006\end{array}$ \\
\hline Botanists & $\$ 27,191$ a year & $\$ 38,299 \mathrm{a}$ & $\$ 47,102$ & increase $21 \%$ to \\
\hline
\end{tabular}




\begin{tabular}{|c|c|c|c|c|}
\hline & $\begin{array}{l}\text { depending on } \\
\text { degree of } \\
\text { education and } \\
\text { employer }\end{array}$ & $\begin{array}{l}\text { year } \\
\text { depending } \\
\text { on employer }\end{array}$ & & $\begin{array}{l}35 \% \text { through the } \\
\text { year } 2006\end{array}$ \\
\hline Chemical Engineers & $\begin{array}{l}\$ 46,606 \text { a year } \\
\text { depending on } \\
\text { degree of } \\
\text { education }\end{array}$ & $\begin{array}{l}\$ 64,371 \text { a } \\
\text { year }\end{array}$ & $\begin{array}{l}\$ 80,330 \text { or more a } \\
\text { year for } \\
\text { experienced } \\
\text { management } \\
\text { positions }\end{array}$ & $\begin{array}{l}\text { expected to } \\
\text { increase } 10 \% \text { to } \\
20 \%\end{array}$ \\
\hline Chemists & $\begin{array}{l}\$ 34,830 \text { a year } \\
\text { depending on } \\
\text { employer }\end{array}$ & $\begin{array}{l}\$ 45,418 \text { a } \\
\text { year } \\
\text { depending } \\
\text { on degree of } \\
\text { education } \\
\text { and industry }\end{array}$ & $\begin{array}{l}\$ 53,729 \text { a year } \\
\text { depending on } \\
\text { industry }\end{array}$ & $\begin{array}{l}\text { expected to } \\
\text { increase } 10 \% \text { to } \\
20 \% \text { through } 2006\end{array}$ \\
\hline Occupational Titles & $\begin{array}{l}\text { Beginning } \\
\text { Salary* }\end{array}$ & $\begin{array}{l}\text { Average } \\
\text { Salary* }\end{array}$ & Top Salary* & $\begin{array}{l}\text { Occupational } \\
\text { Outlook }\end{array}$ \\
\hline Counselors & $\$ 28,624$ a year & $\begin{array}{l}\$ 35,342 \text { a } \\
\text { year }\end{array}$ & $\begin{array}{l}\$ 40,645 \text { a year } \\
\text { with depending on } \\
\text { years of experience }\end{array}$ & $\begin{array}{l}\text { expected to } \\
\text { increase } 10 \% \text { to } \\
20 \% \text { through the } \\
\text { year } 2006\end{array}$ \\
\hline $\begin{array}{l}\text { Credit Analyst } \\
\text { (Banking) }\end{array}$ & $\begin{array}{l}\$ 22,147 \text { a year } \\
\text { with a } \\
\text { bachelor's } \\
\text { degree }\end{array}$ & $\$ 33,557$ & $\$ 41,648$ a year & $\begin{array}{l}\text { expected to } \\
\text { increase } 10 \% \text { to } \\
20 \% \text { through the } \\
\text { year } 2006\end{array}$ \\
\hline Dog Groomers & $\$ 15,839$ & $\$ 19,549$ & $\$ 22,486$ a year & $\begin{array}{l}\text { expected to } \\
\text { increase } 21 \% \text { to } \\
35 \% \text { through the } \\
\text { year } 2006\end{array}$ \\
\hline $\begin{array}{l}\text { Electricians, } \\
\text { Maintenance }\end{array}$ & $\$ 32,013$ & $\$ 41,568$ & $\$ 49,178$ & $\begin{array}{l}\text { employment rate is } \\
\text { expected to grow } \\
\text { more slowly than } \\
\text { the average rate for } \\
\text { all other } \\
\text { occupations } \\
\text { through the year } \\
2006\end{array}$ \\
\hline $\begin{array}{c}\text { Environmental } \\
\text { Engineers }\end{array}$ & $\begin{array}{l}\$ 35,108 \text { a year } \\
\text { depending on } \\
\text { degree of } \\
\text { education }\end{array}$ & $\begin{array}{l}\$ 52,711 \text { a } \\
\text { year }\end{array}$ & $\begin{array}{l}\$ 68,561 \text { or more a } \\
\text { year }\end{array}$ & excellent \\
\hline Fence Erectors & $\begin{array}{l}\$ 16,581 \text { for } \\
\text { unskilled }\end{array}$ & $\begin{array}{l}\$ 20,461 \text { for } \\
\text { skilled }\end{array}$ & $\begin{array}{l}\$ 23,539 \text { for } \\
\text { supervisors }\end{array}$ & good \\
\hline Florists (Floral & $\$ 18,037$ or & $\$ 24,714$ for & $\$ 30,148$ a year & expected to \\
\hline
\end{tabular}




\begin{tabular}{|c|c|c|c|c|}
\hline Designers) & $\begin{array}{l}\text { more } \\
\text { depending on } \\
\text { education }\end{array}$ & managers & & $\begin{array}{l}\text { increase } 21 \% \text { to } \\
35 \%\end{array}$ \\
\hline Occupational Titles & $\begin{array}{c}\text { Beginning } \\
\text { Salary* } \\
\end{array}$ & $\begin{array}{l}\text { Average } \\
\text { Salary* }\end{array}$ & Top Salary* & $\begin{array}{c}\text { Occupational } \\
\text { Outlook }\end{array}$ \\
\hline Food Scientists & $\begin{array}{l}\text { \$29,637 a year } \\
\text { depending on } \\
\text { degree of } \\
\text { education and } \\
\text { employer }\end{array}$ & $\begin{array}{l}\$ 36,585 \text { a } \\
\text { year or more } \\
\text { depending } \\
\text { on years of } \\
\text { experience }\end{array}$ & $\$ 42,069$ a year & $\begin{array}{l}\text { expected to } \\
\text { increase } 10 \% \text { to } \\
20 \% \text { a year through } \\
\text { the year } 2006\end{array}$ \\
\hline $\begin{array}{c}\text { Grounds } \\
\text { Maintenance } \\
\text { Foreman }\end{array}$ & $\$ 21,506$ a year & $\begin{array}{l}\$ 28,664 \text { a } \\
\text { year with a } \\
\text { four-year } \\
\text { college } \\
\text { degree }\end{array}$ & $\$ 35,121$ a year & $\begin{array}{l}\text { jobs in these fields } \\
\text { outnumber the } \\
\text { people who can fill } \\
\text { them }\end{array}$ \\
\hline $\begin{array}{l}\text { Horseshoers } \\
\text { (Farriers) }\end{array}$ & $\begin{array}{l}\$ 19,218 \text { a year } \\
\text { for full-time } \\
\text { farriers }\end{array}$ & $\begin{array}{l}\$ 23,716 \text { a } \\
\text { year }\end{array}$ & $\begin{array}{l}\$ 27,278 \text { or more } \\
\text { for some } \\
\text { specialists }\end{array}$ & $\begin{array}{l}\text { is in demand } \\
\text { almost everywhere } \\
\text { in the United } \\
\text { States }\end{array}$ \\
\hline Horticulturists & $\begin{array}{l}\$ 28,213 \text { a year } \\
\text { depending on } \\
\text { degree of } \\
\text { education and } \\
\text { employer }\end{array}$ & $\$ 38,079$ & $\$ 46,962$ & $\begin{array}{l}\text { expand at an above } \\
\text { average rate } \\
\text { through the year } \\
2005\end{array}$ \\
\hline Hydrologists & $\begin{array}{l}\$ 34,436 \text { a year } \\
\text { depending on } \\
\text { degree of } \\
\text { education and } \\
\text { employer }\end{array}$ & $\$ 49,133$ & $\$ 59,939$ a year & $\begin{array}{l}\text { expected to } \\
\text { increase } 10 \% \text { to } \\
20 \% \text { through the } \\
\text { year } 2006\end{array}$ \\
\hline $\begin{array}{c}\text { Import-Export } \\
\text { Agent }\end{array}$ & $\begin{array}{l}\text { \$28,674 a year } \\
\text { depending on } \\
\text { firm size; } \\
\text { agents may } \\
\text { work on a } \\
\text { commission }\end{array}$ & $\$ 35,402$ & $\$ 40,716$ & excellent \\
\hline Industrial Designers & $\begin{array}{l}\$ 33,597 \text { a year } \\
\text { with a } \\
\text { bachelor's } \\
\text { degree }\end{array}$ & $\begin{array}{l}\$ 58,265 \mathrm{a} \\
\text { year }\end{array}$ & $\begin{array}{l}\$ 50,180 \text { or more a } \\
\text { year with large } \\
\text { corporations }\end{array}$ & $\begin{array}{l}\text { expected to } \\
\text { increase } 21 \% \text { to } \\
35 \% \text { through the } \\
\text { year } 2006\end{array}$ \\
\hline Industrial Engineers & $\$ 40,732$ & $\begin{array}{l}\$ 58,265 \text { a } \\
\text { year } \\
\text { depending } \\
\text { on degree of } \\
\text { education }\end{array}$ & $\begin{array}{l}\text { \$71,719 a year } \\
\text { depending on } \\
\text { employer and } \\
\text { industry }\end{array}$ & $\begin{array}{l}\text { expected to } \\
\text { increase } 10 \% \text { to } \\
20 \% \text { through the } \\
\text { year } 2006\end{array}$ \\
\hline
\end{tabular}




\begin{tabular}{|c|c|c|c|c|}
\hline $\begin{array}{l}\text { Landscape } \\
\text { Architects }\end{array}$ & $\begin{array}{l}\text { \$22,599 a year } \\
\text { depending on } \\
\text { degree of } \\
\text { education and } \\
\text { employer }\end{array}$ & $\begin{array}{l}\$ 40,355 \text { a } \\
\text { year }\end{array}$ & $\begin{array}{l}\$ 54,471 \text { or more a } \\
\text { year }\end{array}$ & $\begin{array}{l}\text { expected } \\
\text { toincrease } 21 \% \text { to } \\
35 \% \text { through the } \\
\text { year } 2006\end{array}$ \\
\hline Laser Technicians & $\$ 31,101$ a year & $\begin{array}{l}\$ 43,814 \text { a } \\
\text { year } \\
\text { depending } \\
\text { upon } \\
\text { experience }\end{array}$ & $\begin{array}{l}\$ 54,762 \text { a year for } \\
\text { those with } \\
\text { advanced } \\
\text { supervisory } \\
\text { positions, sales and } \\
\text { service }\end{array}$ & $\begin{array}{l}\text { demand for skilled, } \\
\text { experienced } \\
\text { technicians } \\
\text { exceeds the supply }\end{array}$ \\
\hline $\begin{array}{l}\text { Loan Operations } \\
\text { Officers (Financial } \\
\text { Institutions) }\end{array}$ & $\begin{array}{l}\$ 40,700 \text { a year } \\
\text { depending on } \\
\text { degree of } \\
\text { education }\end{array}$ & 40,700 & $\$ 49,288$ a year & $\begin{array}{l}\text { expected to grow } \\
21 \% \text { to } 35 \% \\
\text { through the year } \\
2006\end{array}$ \\
\hline Occupational Titles & $\begin{array}{l}\text { Beginning } \\
\text { Salary* }\end{array}$ & $\begin{array}{l}\text { Average } \\
\text { Salary* }\end{array}$ & Top Salary* & $\begin{array}{c}\text { Occupational } \\
\text { Outlook }\end{array}$ \\
\hline Microbiologists & $\begin{array}{l}\text { \$35,980 a year } \\
\text { depending on } \\
\text { degree of } \\
\text { education }\end{array}$ & $\$ 53,544$ & $\$ 66,446$ & $\begin{array}{l}\text { expected to grow } \\
21 \% \text { to } 35 \% \\
\text { through the year } \\
2006\end{array}$ \\
\hline Nursery Managers & $\begin{array}{l}\$ 19,671 \text { a year } \\
\text { depending on } \\
\text { the size and } \\
\text { success of the } \\
\text { business }\end{array}$ & $\$ 29,356$ & $\begin{array}{l}\$ 36,404 \text { or more } \\
\text { depending on the } \\
\text { skills of the } \\
\text { manager }\end{array}$ & $\begin{array}{l}\text { expected to grow } \\
10 \% \text { to } 20 \% \\
\text { through the year } \\
2006\end{array}$ \\
\hline Packaging Engineers & $\begin{array}{l}\$ 34,366 \text { a year } \\
\text { with a } \\
\text { bachelor's } \\
\text { degree and } \\
\text { depending on } \\
\text { the industry }\end{array}$ & $\begin{array}{l}\$ 52,782 \mathrm{a} \\
\text { year with } \\
\text { experience }\end{array}$ & $\begin{array}{l}\$ 68,721 \text { or more } \\
\text { with a master's } \\
\text { degree and } \\
\text { experience }\end{array}$ & promising \\
\hline $\begin{array}{c}\text { Sales Agents, } \\
\text { Business Services }\end{array}$ & $\$ 30,118$ a year & $\$ 37,176$ & $\begin{array}{l}\$ 42,751 \text { a year for } \\
\text { successful sales } \\
\text { representatives }\end{array}$ & $\begin{array}{l}\text { expected to } \\
\text { increase } 36 \% \text { or } \\
\text { more through the } \\
\text { year } 2006\end{array}$ \\
\hline Sales Managers & $\begin{array}{l}\$ 35,619 \text { a year } \\
\text { with a } \\
\text { bachelor's } \\
\text { degree }\end{array}$ & $\$ 52,381$ & $\$ 65,473$ a year & $\begin{array}{l}\text { expected to } \\
\text { increase } 36 \% \text { or } \\
\text { more through the } \\
\text { year } 2006\end{array}$ \\
\hline Occupational Titles & $\begin{array}{l}\text { Beginning } \\
\text { Salary* }\end{array}$ & $\begin{array}{l}\text { Average } \\
\text { Salary* }\end{array}$ & Top Salary* & $\begin{array}{l}\text { Occupational } \\
\text { Outlook }\end{array}$ \\
\hline Teachers, Secondary & $\mid \$ 23,591$ a year $\mid$ & $\$ 33,216 \mathrm{a}$ & $\$ 41,056$ or more a & expected to \\
\hline
\end{tabular}




\begin{tabular}{|c|c|c|c|c|}
\hline $\begin{array}{c}\text { School } \\
\text { (College/University) }\end{array}$ & & year & year & $\begin{array}{l}\text { increase } 10 \% \text { to } \\
20 \% \text { through the } \\
\text { year } 2006\end{array}$ \\
\hline $\begin{array}{c}\text { Veterinary } \\
\text { Technicians }\end{array}$ & $\begin{array}{l}\$ 19,810- \\
\$ 20,000 \text { a year }\end{array}$ & 24,443 & $\begin{array}{l}\$ 28,113 \text { or more a } \\
\text { year }\end{array}$ & $\begin{array}{l}\text { expected to } \\
\text { increase } 21 \% \text { to } \\
35 \% \text { through the } \\
\text { year } 2006\end{array}$ \\
\hline $\begin{array}{c}\text { Wastewater Plant } \\
\text { Operators }\end{array}$ & $\begin{array}{l}\$ 22,258 \text { a year } \\
\text { for trainees }\end{array}$ & $\begin{array}{l}\$ 33,216 \text { a } \\
\text { year }\end{array}$ & $\begin{array}{l}\$ 42,520 \text { a year for } \\
\text { superintendents of } \\
\text { large plants }\end{array}$ & $\begin{array}{l}\text { expected to } \\
\text { increase } 21 \% \text { to } \\
35 \% \text { through the } \\
\text { year } 2006\end{array}$ \\
\hline
\end{tabular}

* Chronicle Guidance Publications gathers salary information from many sources. Primary sources are the monthly employment and earning figures published by the Bureau of Labor Statistics; salary figures furnished by ERI, the Economic Research Institute in Redmond, Washington; contributions from professionals from all over the United States who have reviewed Chronicle Occupational Briefs; and data supplied by professional organizations.

\section{Chronicle's FFA/Agribusiness home page}

\section{(C) Copyright 2000 by Chronicle Guidance Publications, Inc.}




\section{AGRICULTURE}

Its a time of looking at options, of making choices, and looking ahead. As a student attending Clovis High School you should start thinking about career opportunities and you owe it to yourself to consider a career in agriculture.

There are approximately 22 million people who now work in agriculture and ag related fields. The majority of the jobs are in biotechnology, agribusiness, computers, enginerring, marketing as well as dozens of other occupations related to agriculture.

What ever your aspirations, enrolling in Clovis High Agricultural classes will give yoiu hands on experience as well as skills necessary for college or jobs. Agricultural Science classes help students to master one or more important areas of scientific agriculture and to gain knowledge and understanding of supporting academic areas. Some ag classes maybe used to fulfill all or part of the graduation requirement in science and fine arts. Agriculture courses taken at Clovis High School are an accepted elective for entrance into the Cal State system.

Agribusiness

\section{CAREER OPPORTUNITIES}

Agribusiness involves the supply and sale of products and the provision of services needed for ag production.

Dairy management specialist

Farm appraiser

Fertilizer plant supervisor

Harvest contractor

Biostatician

Field sales representative

Computer programer

Ag Equipment dealer

Ag Specialist

The are dozens of careers available to you. Below is a sample of a just few.

Ag Accountant

Ag Economist

Farm Broadcaster

Land Bank Branch Manager

Farm Appraiser

Photographer

Ag Educator

Foreigh Affairs Official

Publisher

Ag Lawyer

Ag Journalist

Farm Investment Manager

Ag Research/Health Sciences

There is a growing need for new and better ways to feed and clothe our people. Agricultural research and

health sciences can point the way. A sampling of careers in research and science follows.

Agronomist

Plant Geneticist

Marine Biologist

Animal Scientist

Poultry Scientist

Entomologist

Botanist

Organic Chemist

Animal Nutritionist

Veterinarian

Microbiologist

Animal Physiologist

Resource Management

There will always be a need for people who can help us use our renewable natural resources more effectively and efficiently. If you like the outdoors, a career in resource management might be right for you.

Animal Behaviorist

Soil Conservationist

Forest Ranger

Park Ranger

Game Warden

Trapper

Fire Warden

Range Conservationist

Horticulture/Forestry

Careers in these areas include everything from florists to landscape designers to turf managers. Some careers in horticulture and forestry follow.

Floral Designer

Nursery Operator

Turf Manager

Florist

Horticulturist
Landscape Architect

Tree Surgeon

Greenskeeper

Forester

Plant Breeder 


\section{Targeted Dccupations}

The following list and descriptions of targeted occupations have been identified to be viable occupations found within the service a of the Paso Robles High School. An extensive study was jone to determine the program areas of study appropriate to the high school. It should be noted that this list is not absolute, and in fact, presents only a partial list of the jobs available to graduates of the program.

AGRICULTURAL PRODUCTION- USOE COde 01.01

Courses: Plant and Animal Science

The foundation of much of the economy of the united states is the production of food and fiber on our farms, ranches, vineyards, orchards and gardens. Many farms and ranches only grow crops. Other farms and ranches only produce animals or poultry, while many farms and ranches grow crops which are fed to animals and poultry produced on the farm or ranch.

Job Description:

\section{Beef-Cattle Ranch.Foreman}

Supervise and coordinate activities of workers engaged in breeding, feeding, herding, marketing, and segregating beef breeding animals, and in construction and repair of iences, pens, buildings, and other ranch equipment. Inspects cattle, fences, buildings, equipment and feed supplies. Notes tasks to be done and assigns them to crews or individual workers. May perform other duties as assigned such as record keeping and buying and selling cattle. May be responsible for health and sanitation managenent.

\section{Job Description: \\ Beef-Assistant Cattle Ranch Foreman}

Assists with the supervision and coordination of workers engaged in breeding, feeding, herding, marketing, and segregating beef breeding animals, and in construction and repair of fences, pens, buildings and other ranch equipment. Inspects cattle, Fences, buildings, equipment, and Eeed supplies. Notes tasks to be done and assigns them to crews or individual workers. Keeps records and as aists in management.

Beef-Cattle Ranch and Parm Hand

Job Description:

Works on a farm or ranch devoted to the raising of cattle and crops. Attends to cattle, mixes feeds and additives, feeds beef cattle, cleans and sanitizes corrals, sheds, 
maintains buildings, fences and equipment used. Prepares soil for planting. Plants, fertilizes, cultivates, and irrigates crops. Harvests, and stores feed crops raised. operates, repairs and maintains equipment for handiing cattle and growing crops.

Job Description:

Beef-Cowpuncher

Attends to beef cattle on stock ranch. Rides horse to drive cattle in desired direction and to round up stray cattle. Castrates, vaccinates, and brands cattle. Inspects fences from horseback or motor vehicle and repairs them. Rides over the range to search for cattle. Feeds cattle during cold weather. Rounds up horses on open range. Breaks horses and trains them for saddle. Repairs and maintains equipment for feeding and watering
cattie

Job Description:

Beef-Ranch Laborer

Participates in branding, dehorning, and castrating. Herds animals to pens and pastures. Applies prescribed medications. Digs post holes by hand, repairs fences and buildings using hand tools, pulls weeds by hand, removes trash and dirt from watering places. Mixes feed by hand. May ride horseback to assist in herding. Does not operate mobile or stationary power-driven equipment.

Job Description:

\section{Beef-Veterinary Assistant}

Cares for cattle under treatment in hospital pens and shelters for disease and injury. Rides pens and pastures on horseback and detects sick and injured cattle. Drives cattle to and from hospital, operates cattle restraining equipment. Keeps hospital equipment and facilities clean and disinfected and sterilizes equipment. Administers medications and treatment under the direction of the veteriarian. Performs duties such as vaccination, castration, dehorning, and marking, using various methods. Keeps and maintains records of vaccinations, disease, and treatments.

Job Description:

\section{Beef-Cattle Buyer}

Purchases cattle from yards and individual cattle raisers to be fed out for resale to packers. Inspects, grades, and calculates weight of livestock to determine value and yield. Purchases cattle according to authorization or prospect for resale. Contacts carriers to arrange for transportation of cattle to feed lot or other destination. Sells stock to packing houses or other purchasers. Reeps purchasing and selling records. 
Job Description:

Beef-Feed Truck Driver

Drives a special feed hauling truck in transporting

prepared feed to cattle pen feeding bunks as assigned.

unloads truck with the power driven mechanism and

distributes feed in feed bunks. Performs maintenance on

the truck such as lubrication, changing oil, and does minor repair such as replacing light bulbs, fuses, and changing tires. keeps trucks clean. Inspects the truck for proper operation and safety.

Horse Production Employee

Other Titles: Horse Farm Employee, Horse Rancher, Horse Producer, Livestock Farm Employee, Rancher.

Job Description:

Ground trains the horse and trains the horse to drive and/or ride applying handing techniques to handle the horse safely and cares for the horse after work. Compares ethods of breeding, plans and applies a breeding program. Applies sanitation and health practices, recognizes symptoms of common equine diseases and applies a parasite and disease control program by developing a veterinary client relationship. Prepares and exhibits the horse in competition. Grooms the horse caring for the horse's limbs and hooves. Calculates nutritive requirements, evaluates sources of nutrients, prepares feed rations, and feeds horses. Improves and uses pasture. Develops a horse farm record system with budgets and insurance plan to compute taxes and marks the horse for identification. Buys and sells horses comparing and contrastingmarketing methods, selects market, and determines market value and financing method. Identifies major breeds and types of horses, and determines age, weight and height select and judge horses. Installs, maintains, and repairs horse farm buildings, water systems, electrical systems, and training facilities. Evaluates and constructs horse farm builaings and applies environmental control practices. Builds fences and recommends requirements for stalis, hallway or alleyway and tackroom. Practices fir prevention methods and follows proper procedures during fire.

Sheep Rancher

Other Titles: sheep Farmer, Sheep Breeder, sheep

Job Description: Producer, Wool Grower.

Sheep Ranchers are responsible for the success or failure of the sheep production enterprise as they make all the critical management decisions. These decisions include those in marketing, breeding, shearing, feed production, and feeding. They supervise all labor and operations of the sheep ranch. They keep records and develop health and predator control programs. They construct facilities and equipment using hand and power tools. They operate and maintain power machinery and equipment. They perform legislative and service activities. 
Job Description:

Sheep Ranch Foreman

The Sheep Ranch Foremen supervise and coordinate the activities of workers engaged in breeding, lambing, feeding, herding, predator control and in construction and repair of fences, pens, and buildings using hand and power tools. They keep records and make some management decisions. Most of their activities are determined by the sheep rancher. They participate in marketing activiites and have many health related tasks. They perform many technical duties such as castrating and docking and are responsible for some feed production and machinery operation and maintenance.

Sheep Ranch Hand

Other Titles: Farm Hand, Hired Man

Job Description:

Sheep Ranch Hands are involved in a wide variety of farm and ranch chores. They are important, especially during lambing season. They operate and maintain trucks and tractors and perform many routine feeding chores. They build fences and construct small farm and ranch projects such as feed racks and gates, using hand and power tools. They herd sheep and must control predators. They perform many health related chores including such tasks as castrating and docking.

Sheep Herder

Other Titles: Shepheerd, Sheepman, Mutton Puncher,

Job Description: herder and Herdsman.

Sheep Hexders tend flocks of sheep grazing on range or pasture. They are very active with breeding and lambing chores and keep minor records. Providing the flock with feed, performing many technical tasks such as castrating and docking, and some fence construction and repair are all tasks performed by Herders. They operate some machinery when necessary.

Other Title: Camp Jack

Camp Tender

Camp Tenders supervise workers engaged in tending bands of sheep on range or pasturee and keep them supplied with food and other necessities. They perform many technical management tasks, including moving camp. They make some management decisions including evaluating the condition of the sheep and the range. they keep some minor records.

Job Description:

Contract Sheep Shearer-Captain

Contract Sheep Shearers contract with sheep producers to shear their sheep. They and their crew shear the sheep using power driven clippers. Contract sheep shearers 
supervise the crew and perform several management tasks to insure the efficient use of labor and equipment. They keep accurate records and purchase supplies and equipment.

\author{
Forage Producer \\ other Titles: Farmers, Forage crop Grower. \\ Job Description: \\ plants, cultivates, and harvests forages for cash sales \\ and/or feeding to livestock. Selects and buys type and \\ amount of seed to be grown, taking into consideration \\ local growing conditions and market demands. Operates \\ equipment to plow, disk, harrow, and fertilize ground for \\ planting. Plans harvesting, considering ripeness and \\ maturity of plants and weather conditions, and operates \\ forage harvesting equipment. Sells crop or stores it for \\ future sales.
}

\author{
Small Animal Supplier \\ other Titles: Small Animal Farmer, Laboratory Animal \\ Producer, Laboratory Animal Breeder, Laboratory \\ Animal Dealer, Laboratory Animal Vendor, Dealer, \\ Job Description: \\ Commercial Breeder, Laboratory Animal Technician. \\ Supplies small animals, and in some cases large animals, \\ for aboratory use for both private industry and \\ governmental agency testing, teaching, and research \\ purposes, and for pet shops. Must understand and practice \\ specific mating and breeding systems; provide optimum \\ nutrition, feeding, and watering for animals; must also \\ provide proper housing and caging; must keep accurate \\ identification and records of stock, must know the signs \\ of disease and be cognizant of the steps taken to control \\ diseases of small animals. Must have familiarity with the \\ market needs for laboratory animals. Must be familiar \\ with state, federal, and foreign shipping regulations and \\ with the specific requirements for each species for \\ shipping. Must be familiar with loading and transporting \\ procedures. Can be self-employed, employed by a \\ commercial business, research institution, or employed by \\ a governmental agency or university.
}

Job Description:

\title{
Nut Producer
}

Nut producers are persons who establish and/or manage a grove of nut producing trees. they apply the cultural practices necessary to produce quality nuts. They also are responsible for harvesting, storing, and marketing the nuts. 
Sma11 Fruit Farmer

Other Titles: Berry Grower, Fruit Farmer, Fruit-Farm Manager, Commercial Producer of Blueberries, Grapes, Rasberries, and/or strawberries, BIueberry Grower, Rasberry Grower, Strawberry Grower, Vine Grower, Job Descriptions:

Vineyard Manager, Fruit Gardener.

A commercial producer of small fruit is a person competent in managerial and technical skills needed to carry out timely approved practices resulting in maximum profit from the small-fruit enterprises. These skills are needed by owners, managers, and operators. The broad competency areas needed by small fruit producers are as follows: Planning for Production, including acquiring technical knowledge, managing finances, site and variety selection and site prpearation; Growing small fruit crops, including soil preparation, propagation and planting, fertilization, pest control, machinery selection and maintenance;

Harvesting, Processing, storing and Marketing Small-fruit crops, including those operations involved in commercial marketing or operating a pick your own outlet.

Small Grain Producer
Other Titles: Farmer, Grain Farmer, Cash Grain Grower
ption:

Job Description: Plants, cultivates, and harvests one or more small grain crops, such as barley, oats, rice, or wheat for cash sales. Selects and buys type and amount of grain to be grown, taking into consideration local growing conditions and market demands. Operates equipment to plow, disk, harrow, and fertilize ground for planting, and to plant grain. Plans harvesting, considering ripeness and maturity of grain and weather conditions and operates grain-harvesting equipment, sells grain or stores grain
for future sale.

Job Description

\section{Crop Producers- General}

Other Titles: Farmer, Grain Farmer, Cash Grain, Grower.

Plants, cultivates, and harvests one or more grain crops, such as barley, corn, rice, soybeans, or wheat for cash sales. Selects and buys type and amount of grain seed to be grown, taking into consideration local growing conditions and market demands. Operates equipment to plow, disk, harrow, and fertilize ground for planting, and to plant grain. Plans harvesting, considering ripeness and maturity of grain and weather conditions and operates grain harvesting equipment. Sells grain or stores grain
for future sales. 


\begin{abstract}
Other Titles: $\frac{\text { Tree Fruit Grower }}{\text { Tree Fruit Farmer, Tree Fruit Producer. }}$
Job Description: Select and develop sites, prepare site, select varieties, propagat and prune trees, plant trees, maintain soil and trees, and harvest and market fruit. operate and maintain tillage and harvest equipment. Operate roadside markets. Work with management and labor personnel.
\end{abstract}

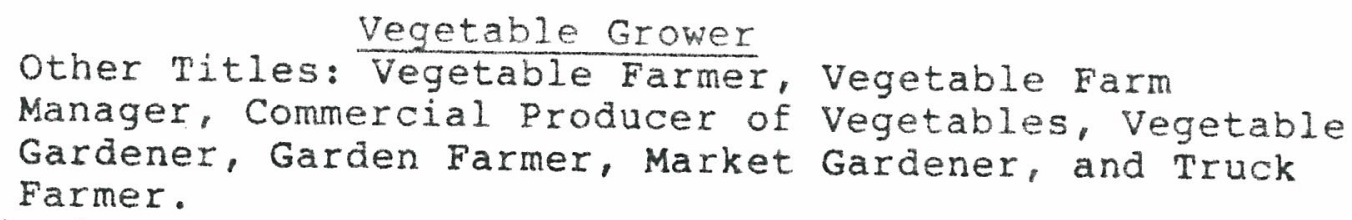


AGRICULTURAL SALES AND SERVICES- USOE COde 10.02

Course: Agribusiness

Thousands of persons in the United states are engagedin agricultural supplies and service occupations which are very essentialto American agriculture. Many of these occupations have only recently emerged on the agricultural scene in this country.

Chemical Applicator

Other Titles: Agricultural Technician, Biological

Technician, Gardener, Groundskeeper, Farm Worker, Job Description:

Equipment Operator, Chemical Equipment Salesperson. Provides information to others for the purpose of selecting chemicals and application equipment; applies chemicals to agricultural and ornamental crops; repairs and maintains equipment for applying agricultural chemicals; uses accepted business procedures for keeping records; practices accepted human relations with work-related personal contacts.

Agri-Chemicals Warehouseman

other Titles: Assistant Plant Manager, Serviceman, Chemical Warehouseman.

Job Description:

The Warehouseman Is responsible for maintaining an accurate inventory at all times; storing compatible materials in proper temperature of facility at all times; possess a working knowleage of chemicals' reactivity, volatility, and stability levels; providing for an appealing display area and arrangement; operating and maintaining storage, handing, and transfer of equipment. Usually under the supervision of the Manager and Assistant Manager.

Agri-Chemicals Trouble shooter

other Titles: Assistant sales Manager, Assistant

Sales Representative, Scout.

Job Description:

The Trouble shooter is responsible for promotion and sale of product and services within assigned area; soil sampling, crop inspection, pesticide performance evaluation, and yield prediction; handing complaints, comments, and suggestions of customers relative to products and services of retail outlets. Usually supervise by the Manager. 
Agri-Chemicals Sales clerk

Other Titles: Office Clerk, Store Clerk, Clerk Typist, Bookkeeper.

Job Description:

The Sales clerk must be able to: prepare and receive orders from customers; prpeare customer invoices; operate wighing equipment, receive money and maintain necessary inventory and financial records; prepare statistical reports for forecast purposes; operate and maintain all office equipment; make recommendations to customers on a limited basis. Sales clerks are responsible to the Manager.

Job Description:

Agri-Chemicals Manager

Other Titles: Plant Manager, Area Supervisor, Office Manager, Sales Manager, Retail Unit manager, plant Superintendent, Lead Operator, store Manager.

The Manager is responsible for implementing and enforcing policies which will maximize profitability and efficiency for the local retail outlet, through effective sales, maintenance, safety, and distribution programs; hires and dismisses employees; orders and maintains adequate amounts of product; and is ultimately responsible for equipinent purchases and repairs. Usually under the supervision of the Regional Sales Representative (or similar title).

\section{Agri-Chemicals Field Technician}

Other Titles: Assistant Manager, Soil and Crop

Job Description:

Specialist, Senior Serviceman.

The Field Technician is required to be competent in all operations of the local retail outlet so that they may temporarily substitute for the Manager in all aspects of tha job except for major financial matters such as purchasing equipment or product and hiring and dismissing employees.

\section{Agri-Chemicals Service Mechanic}

Other Titles: Service Person, Chief Mechanic,

Job Description:

Equipment Manager, Maintenance Supervisor.

The Service Mechanic is responsible for a constant awareness of physical condition of all equipment needed to effectively operate the local retail outlet. This incluaes being able to operate all blending, handing, application transferring, and storing equipment; assemble and maintain all such equipment. 
Job Description:

An Animal Health Technician is a person knowledgeable in the care and handling of animals, and the basic principles of normal and abnormal life processes, and in routine laboratory and animal health care procedures. He or she is primarily an assistant to veterinarians, biological research workers, and other scientists. Examples of the type of work animal technicians perform are:

1. Veterinary Assistant

a. Companion Animal Practice (dogs, cats, pets).

b. Meat Animal Practice (beef, swine, sheep, etc.). c. Mixed Practice ( $a$ and b above)

2. City, State, and Federal Health Agencies.

3. Research and development for government and private industry.

\section{Other Titles: Horseshoer, Shoer, Blacksmith. \\ Job Description:}

A farrier is a skilled craftsman who performs the foliowing major functions: Prepares feet and hooves of horses, mules, and burros, for shoeing; form and shapes

manufactured or handmade shoes; fits and fastens metal shoes to the hooves of horses, mules, and burros; handles animals safely and humanely; secures, utilizes, and maintains hand tools, equipment, and supplies needed for farriery work; diagnoses and corrects foor and gait problems through corrective shoeing; manages scheduling, record keeping, billing, collections, taxes, and other aspects of the farrier business. A farrier secures, applies, and keeps updated in knowledge of: the anatomy of equine feet and legs, techniques in shoeing, use of horseshoeing tools, equipment, and supplies, and customer relations. The Farrier works outdoors or in barns, shops, or other enclosures and is semi-protected from the weather; he or she possesses the strength and endurance to work in a stooping position for long periods while supporting an animal's leg; the person exerts independent judgement in determining work procedures, conforming to quality standards and in the complex shaping/angling functions using hand tools. Persons can become horseshoers by attending public colege or vocational school courses, private schools, or working with a qualified farrier as an apprentice. Farriers are entrepreneurs who may work "on cali," travel a "route, "or work primarily at a ranch, a riding stable, a race track, or other horse-related businesses. 
Manager of Grain, Fertilizex, Feed, and Seed Sales Business Other Titles: Store Manager, Manager Farm Supplies
Store, Elevator Manager.

Job Description:

The Manager of a Grain, Fertilizer, Feed, and seed

Business possesses a good knowledge of the various types of farming and ranching including the application of the basic principles of animal, crop, and soil science, agricultural mechanics, economics, and farm and ranch management; is responsible for the workers required to operate the business, defines and supervises duties of employees; is responsible for purchases of products to be sld at a profit, responsible for the repair and maintenance of a 11 buildings and equipment of the business; promotes good customer relations and keeps superiors informed of company progress and problems; with approval of supervisors makes changes in the business as needed based upon trends and experience. Usually employed by privately owned firms, businesses, and organizations.

Job Description:

Counterman

Must have a wide knowledge of farming and ranching and a good understanding of local livestock and crop production practices and problems. Thoroughly familiar with all products which the business stocks for sale. Knows most customers. Fills customer orders and completes record of sales, Keeps inventory of stock on hand and reorders items in short supply. Is usually employed by provately owned firms, businesses, and organizations.

\section{Other Title: Worker Helper}

Job Description:

A general knowledge of farming and ranching is needed by this employee. He follows instructions of supervisors in filling customer orders, stock supplies, and assists other employees of the business when his services are needed. He helps to keep buildings clean and orderly, helps load trucks, drives trucks and other equipment used in the business. Is usually employed by privately owned firms, businesses, and organizations.

\footnotetext{
Sales and Service Representative

Other Title: Salesman

Job Description:

A thorough knowledge of farming and ranching is a must for this employee. He should quickly recognize the specific needs of prospective customers and provide them with alternative solutions to their needs which the business he represents can provide. He must have a knowledge of the customer's ability to pay for purchases and the amount of credit which may be offered to various customers. He must
} 
have a thorough knowledge of all materials, supplies, and items his company offers for sale, the quantity on hand, their location, and price. He must be considerate with customers and promote good customer relations with the business he represents which is usually privately owned.

\author{
Other ritle: Secretary \\ Job Description: \\ Must have a general knowledge of farming and ranching. \\ Must have good knowledge of keeping financial accounts and \\ records including purchases, sales, charge accounts, \\ monthly billings, and federal and state tax reporting. Is \\ responsible for inventory control. Must have skill in \\ typing. Is usually employed by privately owned firms, \\ businesses, and organizations.
}

Driver

other Titles: Truck Driver, Deliveryman.

Job Description:

Should have a good general knowledge of farming and ranching. Must be familiar with location of customer's fäms ard ranches, including location of nearby towns and centers of transportation, including railroads. Must know how to properly load and unload trucks quickly and safely. Should possess a thorough knowledge of the proper operation and maintenance of trucks, and understand the mechanics of gasoline engines. Must be a skilled and safe driver of trucks and autos; be tactful with customers and promote good customer relations. Must have a good knowledge of all materials and supplies his business sells. Is usually employed by privately owned firms, businesses and organizations

Job Description:

Animal Health Assistant

other Titles: Veterinary-Hospital Attendant,

Veterinary Assistant.

The animal health assistant is responsible for the care of animals under treatment in an animal hospital for disease, injury, or for production of serums; leads, wheels or carries animal from quarters to treatment table and applies restraints or holds it during treatment; sterilizes surgical instruments and other special equipment, such as rubber gloves, syringes, and test tubes, using germicides and autoclave; administers anesthetics, medications and prescribed nursing care under direction of veterinarian; measures, mixes, grinds, and chops specifiea ingredients to prepare food and feeds animals. Bathes and brushes animals and clips thier nails. Sweeps, dusts, mops, and hoses hospital rooms and animal quarters. May receive clients, answer the telephone, make appointments, and accept payment on accounts. 
Pet Shop Worker

Other Titles: Pet Shop Attendant, Aquarist, Aquarium Tankman, Tank Attendant, Tankman.

Job Description:

Cares for birds and animals and fish in a pet shop; feeds and waters birds and animals by replenishing troughs; cleans pens and cages, using scraper and shovel; observes birds and animals for sign of sickness or injury; transfers birds from one cage to another, or removes them according to customer selection, manually or by use of a net. The pet shop worker attends to fish and other marine life; prepares special diets for and feeds fish; cleans bottom and clears away algae on windows of tanks using scrubbing brushes; attends to marine plants and decorations used in live tank displays; observes and reports diseased, injured, or dead fish; gives medical treatments to and provides other necessities for fish as directed; may record number and kinds of fish for inventory purposes. 
ORNAMENTAL HORTICULTURE- USOE COde 01.05

Course: Ornamental Horticulture

Persons who work in occupations that specialize in planting, caring for, and marketing trees, shrubs, plants, flowers, and turf areas are included in this study as being employed in the instructional area of horticulture.

Arboriculture occupations

other Tit les: Trainee, Groundman, Climber, Foreman, Superintendent, Manager.

Description:

Persons in these occupations transplant, prune, repair, protect, and shape trees and shrubs. They remove unwanted trees and shrubs, remove growth obstructing utility lines, and control brush in utility rights-of-way. persons in these occupations must be physically strong, mechanically inclined, and have little fear of heights. They usually are employed by private business services, by municipalities, or by utility companies.

Floriculture- Delivery person

Other Title: Driver

Job Description:

A Floral Delivery person is responsible for prompt, reliable delivery service of floral arrangements and plant materials to the customer. His or her duties include: Taking customer orders, making sale transactions, and guaranteeing careful handling of floral pieces and plants. He or she is responsible for operating and maintaining the delivery vehicle (safety and repair). this occupation is often seasonal or part-time, depending on the size of the business operation.

Floriculture- Salesperson

Other Titles: Designer, Delivery Person, Florist Assistant.

Job Description:

A salesperson deals with people, goods, and services. He or she must have a knowledge and understanding of available plant materials, supplies, and accessories in the shop. His or her duties include advising and recommendng arrangements to the customer, demonstrating various floral products, and explaining price differences. He or she must tactfully handle customer requests and complaints, prepare window displays, maintain a clean and appealing showroom, and should understand basic business principles. In a small flower shop this person may also serve as a designer and/or delivery person. 
Floriculture-Floral Designer

Other Titles: Floral Artist, Floral Arranger,

Job Description: A floral

arranging and the is a person trained in the art of flower work consists of making floral pieces and color. The corsages to wedding bouquets; wreaths ranging from designs; birthdays: holidays; wreaths; sprays; funeral A floral designer is creative and other special occasions. must be capable of interpreting and flexible. He or she pictorial or verbal descripting customer requests (either arrangement) and filling the desired should be able to flowers, tools and supplies and use all the appropriate the kind and amount of customer tastes and of materials needed to satisfy shop this person may also have sales and retail flower as well. $\quad$ also have sales and delivery duties

\section{Other $\frac{\text { Floriculture-Flower shop Manager }}{\text { Title: Retail Florist }}$
iption: \\ Job Description:}

The flower shop manager directs all operations and supervises personnel. He or she must be knowledgeable in business management, and be able to communicate effectively with flower shop personnel. The manager must be able to plan and execute sales strategies, exert strong or she must be able it. The ability to direct a budget and operate within

Job Description:

\section{Grounds Supervisor}

Grounds Supervisors are responsible to the grounds superintendent for maintenance and upkeep of grounds surrounding structures of an industry or business, school, cespital, private estate, municipal park, airport, sizable amount of grounds torise or establishment with a direct the work of "grounds care for. They organize and groundskeepers" and maintain workers" of "assistant concerning maintenance activitiecords and prepare reports of translating blueprints landscape artist into plans prepared by the out landscape plans of their , and may develop and carry requirements of all types of own. They must know cultural operating and maintaining of plant material; be capable of able to design, construct groundskeeping equipment; and be and metal alndscpe struct, and maintain concrete, wood, school technical training into the position. 
Job Description:

Landscape Aide

in estape Aides work under the supervision of crew foreman grading the site and installing. They may assist in soil for planting, and planting drainage systems, prepare otherplant materials, using manual and pow, grass, and equipment. They may help mand power operated pools, and other landscape of Landscape Aide, extensive stuctures. To enter the job experience in a high laboratory of field

ornamental horticulture will be helpful.

Other Title: $\frac{\text { Grounds Worker }}{\text { Jsistant Groundskeeper }}$
Job Description: Grounds workers work under the direction of grounds of an industry caring for the area surrounding structures estate, cemetery, municipal school, hospital, private enterprise or establishment park, airport, or any other grounds to care for. Thent with a sizable amount of groundcover, shrubs they plant and care for turf, perennials. They power operated groundate, maintain, and service hand and employment is provided equipment. Year-round and repair of walks, through construction, maintenance, Completion of a high drives, equipment, and conveniences. ornamental horticulture is hication with training in the job.

Job Description: Crew Foreman-Landscape Planter

Crew Foreman are responsible for supervising crews of be able to translate achitect or planner intoeprints prepared by the landscape They must know and be able workable site layout and plan. grading techniques, sóil to teach their crew members palnting methods grasses. Superviof sidewalks, driveways, layout and construction of plans may also be foreman, completion part of their job. To become a crew or several years of of post-high school technical course required.

Job Description. Randscape and Garden Center Salesperson

The Retail Landscape and Garden Center Salesperson has knowledge of both horticultural and business skills. The 
salesperson possesses desirable personal qualities which enable him/her to effectively represent the business and salesperson uly complete business transactions. The horticultural understands the culture of various gardener. gardener. In addition, the employee cares for and maintains plant materials which are marketed in the garden 


\section{Central Valley Applied Agriculture and Technology Center}

CVAATC

Years 2000-2003

"Transition, Implementation, Acceleration Stages"

\section{YEAR}

2000-01 Transition and Development Stage

* Develop vision statement

* Build trust and enlist help of current CUSD staff

* Assess need/support to refine current ag classes

* $\quad$ Form writing teams to develop new courses

* Develop 7-11 grades recruitment plan

* Develop and clarify relationships with community constituents: CSUF, CART, ROP, Agribusiness et al.

* Pilot current and any refined ag courses, along with new courses

* Establish CSUF UniTrack communication and plans for future CUSD course approval/credit

2001-02 Implementation and Refinement Stage

* Implement, assess, refine AND develop new courses

* Strengthen relationships with community constituents

* Refine recruitment plans for CUSD schools and begin work on regional enrollment strategies/FCOE support

* Student survey developed and administered to assess

* Perceived/real needs being met/going unmet

2002-03 Acceleration Stage

* CUSD and Regional enrollment

* Additional refinement of current courses

* New courses developed 
Central Valley Applied Agriculture and Technology Center

\section{Curriculum Development \\ Progress Summary \\ March 2, 2000}

As of the date of this summary the following characteristics define the direction of the curriculum development efforts:

- New courses will be influenced by four major themes or strands: Sustainable Agriculture Applications, Applied Research, Applied Technology, and Economic and Management Applications

- New courses will be developed with current Regional Occupational Program (ROP) career skills components and/or reflect the philosophy of career skills educational efforts already in place in Clovis Unified and Fresno County

- New courses will integrate and emphasize, whenever reasonable, teacher and student computer skill development and mastery

- New courses will be developed and revised, over time, through input provided by an Agriculture Community network composed of the following: 7-12 teachers, agribusiness leaders/practitioners, higher education, and Fresno County Office of Education and its ROP Network

- Distance learning capabilities of the Applied Ag and Technology Center will help guide the development of new courses and those capabilities will be utilized in the classes where student results/learning can enhanced 


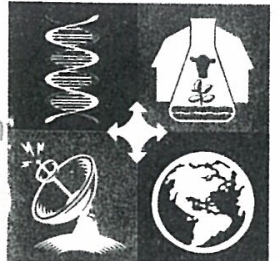

C e n t r a 1 Va 11 e $y$

A p p 1 i e d a n d $\mathbf{T}$ e $\mathbf{c} \mathbf{h}$

8180 E. Donner Clovis, CA 93611 559.327.4320

559.327.4063 Fax
Reagan Educational Center Administration

\section{Linda Hauser}

Asofrtant Superintendent Lyn Snauffer

Assistant Adminfatrator Melissa Macon

Director. Quality Student Seruices

JeffEben

Principal, Clouis Ease High

Stacy Dunnicliff

Principal, Reyburn

Intermedrate

\section{lovis Unified School} fistrict

\section{Administration}

Walter L. Buster, Ed.D. Superintendent

Terry Bradley, Ed.D. Deputy Superintendent

James S. Fugman, Ed.D. Deputy Superfntendent Virginia R. Boris, Ed.D

Associate Superintendent Daniel E. Kaiser, Ed.D. Associate Superintendent William C. McGuire Arsocfate Superintendent

Governing Board

Elizabeth J. Sandoval President

Sandra A. Bengel

Vice President

Jim Van Volkinburg,

D.D.S.

Clenk

Brian D. Heryford

Ginny L. Hovsepian

Richard Lake, C.P.A.

Susan M. Walker,

D.H.Sc.

\section{Memorandum}

All Classes taught within the

Central Valley Applied Agriculture and

Technology Center will contain the following elements in addition to standard approved course

content.

1. FFA

2. Record Keeping with FFA Record Books

3. Leadership Development

4. Supervised Occupational Experience Program

5. Careers In Agriculture

Kevin D. Woodard 裔

Department Chairman

2000- 2001 


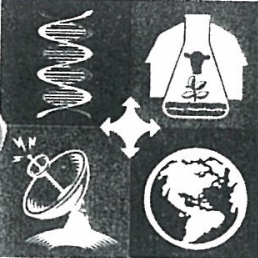

$C$ e n t r a $1 \quad V$ a 11 e y

A p p 1 i e d A griculture

and T e c h n o 1 ogy $\mathrm{d}$ e nt e r

8180 E. Donner Clovis, CA 93611

559.327.4320

559.327.4063 Fax

Reagan Educational Center Admimistration

Linda Hauser

Assistant Superintendent

Lyn Snauffer

Asaistant Adminfotraton

Melissa Macon

Director, Qualley Student

Seruices

Jeff Eben

Principal. Clouis Ease High Schoo

Stacy Dumnicliff

Princlpal, Reyburn

ntermediate

Jlovis Unified School

Jistrict

Administration

Walter L. Buster, Ed.D. Superintendent

Terry Bradley, Ed.D.

Deputy Supenintendent

James S. Fugman, Ed.D.

Deputy Superintendent

Virginia R. Boris, Ed.D

Associate Supenintendent

Daniel E. Kaiser, Ed.D.

Assocfate Superintendene

William C. McGuire

Assocfate Superintendent

\section{Governing Board}

Elizabeth J. Sandoval

President

Sandra A. Bengel

Vice president

Jim Van Volkinburg,

D.D.S.

Clerk

Brian D. Heryford

Ginny L. Hovsepian

Richard Lake, C.P.A.

Susan M. Walker,

D.H.Sc.

\section{Memorandum}

The Agriculture Program within the Clovis Unified School District delivers a broad diverse program that gives students the opportunity meet academic requirements while exploring the agriculture industry.

$\underline{\text { CUSD Graduation Credit }}$

1. Life Science

2. Fine Art

a. Agricultural Science Core I

a. Floral Design

3. Economics

a. Agriculture IV, Ag Economics

UC Acceptance

4. Biological Lab Science

a. Agricultural Science II and IIICombined

b. Agricultural Biology

Articulation Agreements In Place

5. Ag Science I

6. Floral Design

7. Pre-Vet Science

8. Introduction to Ag Mechanics

9. Ag Welding and Fabrication

Kevin D. Woodard

Department Chairman

2000- 2001

"A compelling image of an achievable future." 


\section{COURSE DATA}

AG SCIENCE 1 AB

INSTRUCTOR

KEVIN WOODARD

\section{COURSE DESCRIPTION INFORMATION}

AG SCIENCE $1 \mathrm{AB}$

Prerequisite: none

Duration: Year Course

Ag Science $1 A B$ is designed for the new students interested in the agricultural field. Topics covered include plant science, animal science, ornamental horticulture, agricultural related jobs/careers, public speaking, record keeping, parliamentary procedure, agricultural organizations, judging competitions, supervised occupational experiences and the FFA. Special emphasis is placed upon these areas and their relationship to each other, and to man and his environment.

\section{TEXTBOOK DATA}

TITLE - Agriscience (Fundamentals and Applications)
AUTHOR
PUBLISHER
CPYRT

Elmer L. Cooper Delmar Pub. $\quad 1990$

\section{INSTRUCTIONAL METHODS}

Farm laboratory investigations

Lectures and class discussions

Films

Textbooks

Periodicals

Guest speakers

Enrichment activities (ex: field trips)

\section{EVALUATION METHODS}

Skills proficiency

Written and oral testing

Projects and labs

Overall involvement

\section{FACILITIES}

Classroom

Ag farm facilities

\section{COURSE GOALS}

1. Provide a hands-on approach that will enable students to learn and experience worthwhile concepts of the biological sciences through the agricultural sciences.

Give students a better understanding of themselves and their role in our ecosystem.

To create in the student a continuing, independent and self-involving approach to the biological ag sciences, by providing a firm foundation for later studies in related fields. 


\section{COURSE OBJECTIVES}

- To give basic knowledge and skills for animal science, plant science and agricultural business.

- To understand man's own place in nature.

- To learn about the great diversity of living organisms.

- To understand the nature of scientific inquiry.

\section{COURSE OUTLINE}

1. California Agriculture

a. Economy of California Agriculture

b. State and Local Production

c. California and the National Agricultural Economy

d. California Agriculture and the Global Economy

2. Agriculture and society

a. Agricultural Trends

b. Agencies for Agriculture

3. Agriculture and California resources

a. Agricultural Resources

b. Agriculture and the Environment

c. Energy and Agriculture

4. Domestic animals and production

a. Animal Domestication

b. Animal Protein

5. Animal behavior and biology
a. External Anatomy
b. Animal Behavior
c. Dam/Offspring Interaction
d. Parturition
e. Showmanship

6. Anatomy/physiology
a. Introduction to Anatomy/Physiology
b. Digestive Systems
c. Reproductive Systems
d. Castration

7. Nutrition and feeds
a. Nutrient Needs
b. Classes of Feeds
c. Digestion and Absorption
d. Feed Preparation

8. Animal health
a. Animal Health: Whose Responsibility Is It?
b. Animal Health Evaluation
c. Normal Temperature, Pulse and Respiration
d. Introduction of Parasites
e. Introduction to Vaccinations
f. Development and Types of Immunity

9. Livestock evaluation and selection

a. Livestock Evaluation and Selection

10. Meat grading

a. Meat Grading

b. Wholesale and Retail Cuts

11. Basic Botany 

a. Plant Parts
b. Function of Plant Parts
c. Importance of Plants to Man
d. Classif. Plants by Life Cycle
e. Photosynthesis
f. Plant Growth Requirements
g. Plant propagation lab: from seed

12. Plant nutrition
a. Nutrients essential to plant growth
b. Sources of primary plant nutrients

13. Irrigation
a. Irrigation systems
b. Knowing when to irrigate
c. Irrigation practices

14. Pest management
a. Intro. To plant pests
b. Weed control
c. Damage caused by plant pests
d. Workplace safety

15. Record keeping
a. Introduction to Record Keeping
b. Record Book: Topic Cover, Front Page, \& Planning Calendar
c. Record Book: Calendar
d. Record Book: Bus Agreements/Budget
e. Record Book: Journal
f. Record Book: Loan Payment Summary
g. Record Book: Personal Receipts \& Expenses/Activities

16. FFA LEADERSHIP DEVELOPMENT
a. History of the FFA
b. Chapter Formation \& Aims and Purposes of FFA
c. The Local Chapter and Sectional, Regional and State Organization
d. The National Organization
e. The FFA Emblem and Creed
f. Chapter Officer Duties
g. FFA Jacket, Dress Code, Code of Ethics, Motto and Salute

17. PARLIAMENTARY PROCEDURE
a. Origins of Parli Pro
b. Handling of Motions and Table of Precedence

18. COMMUNICATIONS
a. The Importance of Speaking and Listening
b. Effective Committee Skills
c. Public Speaking Presentations

19. SUPERVISED OCCUPATIONAL EXPERIENCE
a. Introduction to SOEP
b. Getting Started
c. Long Range SOEP. Plans

20. CAREER OPTIONS
a. Importance of work
b. Agriculture Careers
c. Trends in Career Opportunities

21. OCCUPATIONAL GOALS
a. Choosing a Career 


\section{AG.COURSE DATA}

\section{AG SCIENCE 2 AB}

Course Title: Animal Science

\section{COURSE DESCRIPTION INFORMATION}

AG SCIENCE $2 \mathrm{AB}$

Prerequisite: Ag Science $1 \mathrm{AB}$

Duration: Year Course

Ag Science $2 \mathrm{AB}$ is designed for those students interested in the field of animal science. Topics covered include domestic animal production, animal behavior and biology, anatomy and physiology of the farm animal, genetics and breeding, nutrition and feeds, animal health, livestock and meat evaluation, related jobs/careers in the animal industry, public speaking, record keeping, supervised occupational experiences and the FFA.

\section{TEXTBOOK DATA}

1. Animal Science - Ensminger

2. Farm Animal Behavior - Fraser

3. Reproduction in Domestic Animals - Cole

4. Biology - Goodman

5. Feeds and Feeding - Morrison

6. Merck Veterinary Manual

7. Farm Animal Health and Disease Control - Winkler

8. The Stockman's Handbook - Ensminger

9. Principles of Meat Science - Forrest

\section{INSTRUCTIONAL METHODS}

1. Farm laboratory investigations

2. Lectures and class discussions

3. Films/Audiovisual materials

4. Textbooks

5. Periodicals

6. Guest speakers

7. Enrichment activities (ex: field trips)

\section{EVALUATION METHODS}

1. Skills proficiency

2. Written and oral testing

3. Projects and labs/participation

4. Overall involvement 


\section{RESOURCES}

VEP - Cal Poly

IMS - Texas A \& M

NASCO West

Calif. Voc-Ag Curriculums

Hobar Publications

Ohio Curriculum Materials

Carolina Biological Supply Co.

\section{FACILITIES}

1. Classroom

2. Ag farm facilities

\section{COURSE GOALS}

1. Provide a hands-on approach that will enable students to learn and experience worthwhile concepts of the biological sciences through the agricultural sciences.

2. Give students a better understanding of themselves and their role in our ecosystem.

3. To create in the student an advanced, independent and self-involving approach to the science of animal agriculture, by utilizing the foundation developed in previous ag science classes.

\section{COURSE OBJECTIVES}

1. To give advanced knowledge and skills for animal science.

2. To understand the relationship between livestock species and man.

3. To learn about the health and care of domestic livestock.

4. To understand the nature of scientific inquiry.

\section{COURSE OUTLINE}

I. Domestic Animals and Production
A. Animal domestication
B. Growth hormones
C. Animal protein
D. Animal production 
II. Animal Behavior and Biology
A. External anatomy
B. Conception/gestation
C. Cell structure and function
D. Animal cells
E. Nomenclature
F. Species
G. Animal Behavior
H. Dam/offspring interaction
I. Hormones and meat production
J. Natural selection
K. Parturition
L. Showmanship

III. Anatomy/Physiology
A. Intro to anatomy/physiology
B. Digestive system
C. Reproductive system
D. Comparative Anatomy
E. Castration
F. Hormone function
G. Hormone influence
H. Skeletal system

IV. Genetics and Breeding
A. Fertilization
B. Inheritance
C. Geno- and phenotype
D. Dominant and recessive genes
E. Mutation
F. Genetics glossary

V. Nutrition and Feeds
A. Nutrient needs
B. Feed composition
C. Digestion and absorption
D. Feed preparation
E. Protein needs
F. Carbohydrates and fats
G. Vitamins and minerals
H. Feed additives
I. Computing balanced rations 
VI. Animal Health

A. Animal health - "Who's Responsibility is it?"

B. Animal health evaluation

C. Veterinary medicine glossary

D. Normal temperature, pulse and respiration

E. Pathogenic classification and life cycle of common parasites

F. Development and types of immunity

G. Vaccination and administration of biologic agents

VII. Livestock Evaluation and Selection

VIII. Meat Evaluation

A. Meat grading

B. Wholesale and retail cuts

IX. Record Keeping

X. FFA Facts

A. History of the FFA

B. Recognition programs

C. Parliamentary procedure

XI. Careers in agriculture 


\title{
COURSE DATA
}

Title: Agricultural Biology $\mathrm{AB}(\mathrm{P})$

Grade Level: 9-12 Semester/Year Course Year course

Prerequisite(s): Algebra $\mathrm{AB} ; 9^{\text {th }}$ grade level minimum reading level

Please indicate if requesting the following: (High School Only)

- Repeatable Course

$\checkmark$ Meets C.U.S.D. Graduation Requirements

- Meets UC Honors Admittance Requirements

$\checkmark$ Meets UC Regular Admittance Requirements

Course Description:

- Meets CSU Fine Arts Requirements but not UC

Biology is the study of life from the molecular level to the ecosystem level. Interrelationships among living things, their environment, humans, and the environment are emphasized. Special emphasis is placed upon the scientific approach to problem solving in laboratory experiments and the analysis of scientific data. "Hands-on" laboratory experiences incorporating technology are emphasized. When applicable agricultural examples are used to reinforce biological science concepts.

\section{Special Notes:}

Biology is a recommended prerequisite for all the $(\mathrm{P})$ elective courses in Science. A reading level of at east $9^{\text {th }}$ grade and proficiency in Algebra I is strongly recommended for students enrolling in Agricultural siology.

\author{
$\checkmark$ Buchanan High School \\ $\checkmark$ Clovis High School \\ $\checkmark$ Clovis East High School \\ $\checkmark$ Clovis West High School \\ $\checkmark$ Central Valley Applied Agriculture and Technology Center \\ - Enterprise High School \\ - Gateway High School \\ - Alta Sierra Intermediate School \\ 口 Clark Intermediate School \\ - Kastner Intermediate School \\ 口 Reyburn Intermediate School
}


Textbook (Primary textbook if more than one is used):

Title: The Science of Agriculture, A Biological Approach.

Ydition: $1^{\text {st }}$

Author:__Ray V. Harren

Publisher: Delmar Publishers

Copyright Year:_1996

Adoption Year:

\section{COURSE PROFILE}

\section{Course Goals:}

1. Provide student with a scientifically-based awareness of the interrelationships and functional mechanics of all living things.

2. Develop insight and appreciation of the dependence of man on his agriculture and the environment.

3. Challenge the student to approach problems using the scientific method of observation, hypothesis, experimentation, and analysis.

4. Recognize that science is a unique method for accumulating knowledge as well as a body of facts.

- Prepare the student for informed decision-making in social and political situations.

6. Create a desire for life-long learning which will provide a foundation for further studies in Science and Agriculture.

\section{Course Objectives:}

Students will achieve at or above grade level in the following California State Science standards for Biology:

\section{Cell Biology}

Fundamental life process of plants and animals depend on a variety of chemical reactions that are carried out in specialized areas of the organism's cells.

A. All cells are surrounded by a semi-permeable membrane

1. The membrane controls movement of substances into and out-of the cell

2. The membrane controls interaction of cells with their environment.

B. Enzymes are proteins that act as catalysts in living things.

1. Enzymes rely on a specific shape to catalyze chemical reactions.

3. The shape of the enzyme depends upon temperature, $\mathrm{pH}$ (acidity and alkalinity conditions) and concentrations of various ions.

4. Enzymes are unchanged by the chemical reactions they cause.

5. Enzymes do not alter the chemical equilibrium of the reactions they cause. 
6. Most chemical reactions in living things require a specific enzyme

C. All living things (organisms) are made up of one or more cells.

1. There are two basic types of cells; prokaryotic and eukaryotic

2. Prokaryotic cells are much smaller and simpler than eukaryotic cells.

3. Prokaryotic cells do not have a nuclear membrane nor do they have any membrane-bound

4. Eukaryotic cells are much larger than prokaryotic cells. Eukaryotic cells have chromosomes surrounded by a nuclear membrane and have many membrane-bound organelles in their cytoplasm.

5. Bacteria (Kingdom Monera) are one-cell organisms; each bacteria organism is a single prokaryotic cell.

6. Eukaryotic cells make up all organisms except bacteria. Thus, Protists, Fungi, Plants, and Animals are all comprised of one or more eukaryotic cell.

7. Plant cells have cell walls and chloroplasts which are never found in animal cells.

D. Viruses are not made of cells. Viruses require a host cell to reproduce.

1. Viruses consist of a protein coat or "capsule" and a "core" made up of DNA or RNA

2. Viruses are obligate intracellular parasites; they can only multiply within living cells.

E. Biological information is encoded in DNA.

1. All cells contain DNA which makes up the "genetic material".

2. Information in DNA is reproduced in a process called Replication. This occurs each time a cell reproduces. Replication produces identical copies of DNA.

3. DNA stores the information to build proteins.

4. Information in DNA is copied into RNA in a process called Transcription.

5. Information copied from DNA into RNA is used to build proteins in a process called Translation.

6. The flow of information from DNA-to-RNA-to protein is known as the "Central Dogma" of molecular biology.

F. Proteins are macromolecules. To be secreted from an animal cell, a protein must be wrapped in membrane.

1. Proteins that are to be secreted are synthesized by ribosomes located on the rough

2. The protein molecules are then transferred to the Golgi apparatus for processes and packaging into vesicles for secretion.

3. The proteins are secreted by a process called exocytosis.

G. All energy available to living things is light energy trapped by chlorophyll in chloroplasts of photosynthesizing organisms.

1. Photosynthesis stores chemical energy as sugars.

2. Carbon dioxide from the atmosphere is incorporated into the sugar molecules.

H. In all eukaryotic cells, the mitochondria break down glucose to carbon dioxide to free the Chemical bond energy stored in the glucose.

I. Nucleic acids, Proteins, and Polysaccharides are macromolecules. Macromolecules are made up of building blocks called monomers.

1. The monomers of nucleic acids are nucleotides; there are 4 types in DNA

2. The monomers of proteins are amino acids; there are 20 types of amino acids in proteins.

3. Polysaccharides are made up of simple sugars. Starch, cellulose, and glycogen are all made up of many molecules of glucose chemically bonded into giant molecules.

J. Lipids, while not macromolecules, are made of simple subunits.

\section{Genetics}

A. Mutation and sexual reproduction lead to genetic variation in a population.

1. Meiosis is a nuclear division process that occurs in sexual reproduction.

2. Meiosis reduces the chromosome number from diploid $(2 \mathrm{~N})$ to haploid $(\mathrm{N})$ 
3. In meiosis, homologous chromosomes segregate and randomly assort into gametes containing the haploid number of chromosomes. Gametes contain only one of each type of chromosome.

4. Only certain cells in multicellular organisms can undergo meiosis

5. Random segregation of chromosomes during meiosis explains the probability that a particular allele will be in a gamete.

6. Fertilization is the fusion of a sperm and an egg. The sperm and egg are both haploid (N). The resulting zygote (fertilized egg) is thus (2N).

7. Since each individual is the result of fertilization, one-half of each individual's DNA is inherited from each parent.

8. In humans, gender ("sex") is determined by the presence or absence of the "Y" chromosome. If the cells have a "Y" chromosome, the individual is a male. If there is not a "Y" chromosome, the individual is a female. Male cells have "XY" chromosome complement while females have "XX" chromosome complement.

9. Define genotype as the actual genes present and phenotype as the expression of the genotype.

10. Given the genotypes of the parents, determine possible genotypes among the offspring and the probability of each.

B. A multicellular organism develops from a single zygote. Its phenotype is determined by its genotype. The genotype is established at fertilization.

1. Given genotypes of parents and mode of inheritance, predict genotypes and phenotypes of offspring. Include following modes:

a. Dominant and recessive genes

b. X-linked

c. Autosomal genes

2. Explain the genetic basis for Mendel's laws of segregation and independent assortment.

C. Genes are a set of instructions, encoded in the DNA sequence of each organism that specify the sequence of amino acids in proteins characteristic of that organism.

1. Outline the process by which ribosomes synthesize proteins, using tRNAs to translate the genetic information in mRNA.

2. Use the genetic coding rules to predict the sequence of amino acids from a sequence of codons in mRNA.

3. Explain how mutations in the DNA sequence of a gene may or may not affect the expression of the gene or the sequence of amino acids in an encoded protein.

4. Specialization of cells in multicellular organisms is usually due to different patterns of gene expression rather than to differences of the genes themselves.

5. Proteins can differ from one another in the number and the sequence of the amino acids.

D. The Genetic composition of cells can be altered by incorporation of exogenous DNA into the cells.

1. Describe the general structures and functions of DNA, RNA, and protein

2. Use the base-pairing rules to explain the semi-conservative replication of DNA and transcription of information from DNA into RNA.

3. How is biotechnology (recombinant DNA) used to produce novel biomedical and agricultural products.

\section{Ecology}

A. Stability in an ecosystem is a balance between competing effects.

1. Biodiversity is the sum total of different kinds of organisms, and it is affected by alterations of habitats.

2. Analyze changes in an ecosystem resulting from changes in climate, human activity, introduction of non-native species, or changes in population size.

3. The fluctuations in a population's size are determined by relative rates of emigration, immigration, mortality, and natality. 
4. Water, carbon, and nitrogen are cycled between organisms and the abiotic resources in an ecosystem. Oxygen cycles via respiration and photosynthesis.

5. A vital part of an ecosystem is the stability of producers and decomposers.

6. At each link of a food web, some energy is stored in newly made structures but most is dissipated into the environment as heat. This flow of energy is represented in food pyramids.

\section{Evolution}

A. The frequency of an allele in a gene pool of a population depends on many factors, and may be stable or unstable over time.

1. Why natural selection acts on the phenotype rather than the genotype of an organism.

2. Why alleles that are lethal in a homozygous individual may be carried in a heterozygote, and thus maintained in a gene pool.

3. New mutations are constantly being generated in a gene pool.

4. Variation within a species increases the likelihood that at least some members of a species will survive under changed environmental conditions.

B. Evolution is the result of genetic changes that occur in constantly changing environments.

1. Natural selection determines the differential survival of groups of organisms.

2. A great diversity of species increases the chance that at least some organisms survive large changes in the environment.

3. Define genetic drift and tell how genetic drift effects the diversity of organisms in a population.

4. How does reproductive or geographic isolation affect speciation.

5. Analyze fossil evidence with regard to biological diversity, episodic speciation, and mass extinction.

\section{Physiology}

A. As a result of the coordinated structures and functions of organ systems, the internal environment of the human body remains relatively stable (homeostatic), despite changes in the outside environment.

1. Tell how the complementary activity of major body systems provides cells with oxygen and other nutrients and removes toxic waste products such as carbon dioxide.

2. How does the nervous system mediate communication between different parts of the body and interactions with the environment?

3. How do feedback loops in the nervous and endocrine systems regulate conditions within the body.

4. What are the functions of the nervous system and what is the role of neurons in transmitting electrochemical impulses?

5. What are the roles of sensory neurons, interneurons, and motor neurons in sensation, thought, and response?

B. Organisms have a variety of mechanisms to combat disease.

1. What is the role of the skin in providing non-specific defenses against infection?

2. What is the role of antibodies in the body's response to infection?

3. How does vaccination protect an individual from infectious disease?

4. How do bacteria and viruses differ in their requirements for growth and replication?

5. What is the primary defense of the body against bacterial infections? Against viral infections?

6. How do treatments for viral infections and bacterial infections differ?

7. Why is a person with a compromised immune system (for example, a person with AIDS) unable to fight off and survive infections of microorganisms that are usually benign. 


\section{Instructional Methods:}

1. Class discussion

Laboratory investigations

3. Textbook readings and study guide activities

4. Videos and study guides

5. Library/internet research projects

6. Student presentations

7. Field trips

\section{Evaluation Procedures:}

1. Instructor-designed unit examinations and quizzes

2. Laboratory examinations

3. California SAT 9 exam

4. Golden State Exam

\section{COURSE OUTLINE}

I. The Science of Agriculture:
A. The science of life
B. The living condition
C. The chemical basis for life: atoms and molecules

i. Cells; Agriculture's Building Blocks:
A. Membrane structure and function
B. Cell structure: prokaryotic and eukaryotic; plant and animal
C. Cell physiology
D. Photosynthesis and respiration: chloroplasts and mitochondria
E. Nucleic acids: replication, transcription, and translation.

\section{III.Plant and Animal Genetics:}
A. Cell reproduction: mitosis and meiosis
B. Hereditary principles
C. Haploid and diploid number
D. Segregation and independent assortment
E. Genotype and phenotype
F. Animal genetics:

1. Genetic determination of gender

2. Patterns of inheritance

a) Dominant and Recessive Traits

b) X-linkage

\section{Evolution}
A. Mutation and variation in populations
B. Natural selection
C. Evidences for evolution
D. Isolation 
V. Plant and Animal Diseases (recommended optional study)
A. Viruses
B. Bacteria
C. Protozoa

VI. Invertebrate zoology and vertebrates (recommended optional)
A. Sponges and cnidarians
B. Worms
C. Mollusks and echinoderms
D. Arthropoda
E. Insecta
F. Introduction to the vertebrates

VII. Animal Systems

A. Organization of the animal body

B. Digestive system

1. Monogastric

2. Ruminant

3. Nutrition

C. Circulatory and Excretory systems

D. Respiratory system

E. Nervous system

F. Endocrine system

G. Reproductive system

H. Immune system:

VIII. Plants Systems
A. Seed plants
B. Leaf structure and function
C. Root and stem structure and function
D. Plant reproduction

IX. Ecology
A. Communities and ecosystems
B. Biodiversity
C. Food chains and food webs
D. Biomes
E. Population dynamics
F. Cycles in the biosphere: water, carbon, oxygen, nitrogen 


\section{COURSE DATA}

AG SCIENCE $3 \mathrm{AB}$

AGRICULTURE

\section{COURSE DESCRIPTION INFORMATION}

AG SCIENCE $1 \mathrm{AB}$

Prerequisite: Ag Science 1,2

Duration: Year Course

$\mathrm{Ag}$ Science $3 \mathrm{AB}$ is designed for those students interested in the field of plant science and ornamental horticulture. Topics covered include basic botany, plant biology and physiology, soil science, plant pests and diseases, plant propogation, irrigation, record keeping, careers, supervised occupational experiences and FFA.

\section{TEXTBOOK DATA}

Introductory Horticulture - Reiley \& Shry

\section{REFERENCES}

Plant Science: Growth, Development \& Utilization of Cultivated Plants - Hartmann,

Ball RedBook - Greenhouse Growing

Simon \& Schuster's Guide to Plants \& Flowers - Bianchini \& Pantano

Rodale's Illustrated Encyclopedia of Gardening \& Landscape Techniques

Landscape Plants: Thier Identification, Culture \& Use - Bridwell

Applied Science Review: Biology - Pratt

Plant Propagation: Principles \& Practices - Hartmann, Kester, Davies

\section{INSTRUCTIONAL METHODS}

1. Farm laboratory investigations

2. Lectures and class discussions

3. Films

4. Textbooks

5. Periodicals

6. Guest speakers

7. Enrichment activities (ex: field trips)

\section{EVALUATION METHODS}

1. Skills proficiency

2. Written and oral testing 
3. Projects and labs

4. Overall involvement

\section{FACILITIES}

1. Classroom

2. Ag farm facilities

\section{COURSE GOALS}

1. Provide a hands-on approach that will enable students to learn and experience worthwhile concepts of the biological sciences through the plant sciences.

2. Give students a better understanding of the relationship between plants and man

3. To create in the student a continuing, independent and self-involving approach to the plant sciences, by utilizing the foundation formed in previous classes.

\section{COURSE OBJECTIVES/COMPETENCIES}

1. To give advanced knowledge and skills for plant science.

2. To understand the role of plants in nature.

3. To learn about the various facets of the plant science industry.

4. To develop skills in record keeping.

5. To understand the nature of scientific inquiry.

6. To understand proper use of plant fertilizers, herbicides.

7. To effectively demonstrate proper pruning \& grafting techniques.

8. To effectively propagate selected plant species.

9. To correctly identify selected plants by their genus \& specie.

10. To demonstrate correct preparation and use of planting beds.

I. Basic Botany

\section{COURSE OUTLINE}
A. Plant parts
B. Functions of plant parts
C. Importance of plants to man
D. Classification of plants
E. Plant propagation by seed
F. Plant propagation by vegetative methods
G. Plant propagation by mitosis
H. Photosynthesis
I. Respiration 
II. Basic Botany
A. Transpiration
B. Translocation
C. Plant Growth Requirements
D. Propagation lab: seed

III. Soils
A. Soils and plant culture
B. Soil texture and structure
C. Influence of texture on soil
D. Soil erosion and conservation

IV. Plant Nutrition
A. Nutrients essential to plant growth
B. Sources of primary plant nutrients
C. Fertilizer labels and calculations
D. Determining nutrient deficiencies
E. Fertilizer application
F. Organic and inorganic fertilizer

V. Irrigation
A. Irrigation systems
B. Irrigation terminology
C. Knowing when to irrigate
D. Water measurement and soil capacity
E. Irrigation practices

VI. Pest Management
A. Weed control
B. Weed collections
C. Damage caused by plant pests
D. Plant disease identification
E. Disease control
$\mathrm{H}$. Insect control

VII. Pest Management
A. Pest collection
B. Workplace safety
C. Pesticide safety

VIII. Plant Propagation
A. By seed
B. Cuttings
C. Layering 
IX. Plant Identification
A. Classification
B. Trees \& Shrubs
C. Lawns \& Groundcovers
D. Edible Crops
E. House Plants

X. Landscaping

XI. Record Keeping

XII. Careers in Plant Science

XIII. FFA Facts 


\section{COURSE DATA}

EXPLORATORY AGRICULTURE

AGRICULTURE

\section{COURSE DESCRIPTION INFORMATION}

EXPLORATORY AGRICULTURE

Prerequisite: none

Duration: Semester Course

Exploratory Agriculture is designed to give the middle school student an overview of California agriculture, and the ag education program at the high school level.

\section{TEXTBOOK DATA}

TITLE - none

\section{INSTRUCTIONAL METHODS}

1. Farm laboratory investigations

2. Lectures and class discussions

3. Films

4. Textbooks

5. Periodicals

6. Guest speakers

7. Enrichment activities (ex: field trips)

\section{EVALUATION METHODS}

1. Skills proficiency

2. Written and oral testing

3. Projects and labs

4. Overall involvement

\section{$\underline{\text { FACILITIES }}$}

1. Classroom

2. Ag farm facilities

\section{COURSE GOALS}

1. Provide a hands-on approach that will enable students to learn and experience 
worthwhile concepts of the biological sciences through the agricultural sciences.

2. Give students a better understanding of themselves and their role in our ecosystem.

3. To create in the student a continuing, independent and self-involving approach to the biological ag sciences, by providing a firm foundation for later studies in related fields.

\section{COURSE OBJECTIVES}

1. To give basic knowledge and skills for animal science, plant science and agricultural business.

2. To understand man's own place in nature.

3. To learn about the great diversity of living organisms.

4. To understand the nature of scientific inquiry.

\section{COURSE OUTLINE}

I. Foods for the World
A. Foods in History
B. Food Customs
C. The Cost of Food
D. Scarcity and Plenty
E. Food for the Future

II. A Look at US Agriculture
A. History of Ag in the US
B. Ag Areas in US
C. US Ag compared to the World
D. California $\mathrm{Ag}$
1. Ag Products of California
2. How California Ranks in US Production

III Farm Life
A. Country vs. City Life
B. Starting a Farm
C. Part-Time Farming

IV. Livestock Production (Beef, Sheep, Swine, Dairy, Poultry, Horses)
A. Animal Uses
B. History of Domestic Animals
C. Different Species of Livestock
D. Basic Parts of Livestock
E. Basic Terms of Livestock 
V. Plant Science
A. Plant Uses
B. History of Plant Production

VI. Job Opportunity in Agriculture
A. On-Farm Jobs
B. Off-Farm Jobs

VII Agriculture Classes at Clovis High
A. Classes
B. School Farm

VIII. Future Farmers of America
A. What is FFA?
B. SOEP (fairs \& shows)
C. Judging
D. Trips 


\section{COURSE DATA}

\section{INTRODUCTION TO AGRICULTURAL MECHANICS \\ AGRICULTURE}

\section{COURSE DESCRIPTION INFORMATION}

BASIC AG MECHANICS

Prerequisite: None

Duration: Year Course

This course will give students basic skills in shop safety, project design and layout, estimations, carpentry, concrete, masonry, plumbing, electrification, hot and cold metal work, sheet metal fabrication, soldering and welding. Part of the work will include building of projects. Focus will be made on record keeping, FFA and career possibilities in mechanics.

\section{TEXTBOOK DATA}

Text: $\quad$ Elmer L. Cooper, Agricultural Mechanics; Fundamentals \& Applications, 3rd Edition. Delmar Publications 1996.

\section{INSTRUCTIONAL METHODS}

1. Farm laboratory investigations

2. Lectures and class discussions

3. Videos

4. Textbooks

5. Periodicals

6. Guest speakers

7. Enrichment activities (ex: field trips)

\section{EVALUATION METHODS}

1. Skills proficiency

2. Written and oral testing

3. Homework assignments

4. Projects and labs

5. Participation

\section{FACILITIES}

1. Classroom

2. Ag farm facilities

3. Shop and Shop equipment

4. Community and Industry

\section{COURSE GOALS}

1. To be exposed to orientation and safety of Ag Shop Skills.

2. To learn tool identification and use (throughout the year).

3. To know material identification and use (all year).

4. To develop farming skills (development exercises) in Ag shop.

5. To complete required projects

6. To complete individual student projects.

7. To learn shop renovation and reorganization. 


\section{COURSE OBJECTIVES}

1. Student will be able to pass the safety test and review.

2. Student will be able to identify and use agricultural shop tools.

3. Student will be able to identify and use materials properly.

4. Student will be able to work confidently in the following areas:
a. Project Layout
b. Hot Metal
d. Electricity
e. Plumbing
f. Cold Metal
h. Tool Fitting
j. Arc welding
k. Concrete
c. Sheet Metal
g. Rope work
i. Oxy-fuel welding

6. Student will be able to choose, initiate, perform and complete a project.

7. Student will be able to make decisions for improving shop work environment.

\section{COURSE OUTLINE}

1. Careers in agricultural mechanics.

2. Importance of knowing how to use tools and equipment.

3. Safety practices in the shop.

4. Tool identification.

5. Proper use of hand tools.

6. Repair and maintenance of hand tools.

7. Identification and use of hardware and supplies.

8. Making and reading plans.

9. Beginning carpentry.

a. Measuring, marking,

10. Rope Work.

b. Cutting, and assembling woodworking projects.
a. Learn how to tie a knot
d. manger hitch
b. slip knot
g. long splice
j. loop splice
e. half hitch
h. short splice
k. crown splice
c. bowline
f. clove hitch
i. eye splice
1. truckers' hitch

m. Make a rope halter or lead rope.

11. Cold Metal Work

12. Make small hand tools using forging methods:

13. Soldering
a. center punch
d. hinges
b. drift punch
e. metal brackets and latches.
c. cold chisel

a. Prepare metal to solder

d. soldering a patch

b. what metals to solder

e. filling a hole

c. soldering joints

14. Painting
a. different types of paints
d. proper clean-up
b. how paints are used
c. proper methods

15. Plumbing

a. Identification and use of equipment and materials

16. Electrical Work

b. Cut and join galvanized and plastic pipe fittings.

17. Concrete Work

a. Terms, safety and principles of electricity, Wire simple electrical problems
Work

18. Welding

a. Building forms, reinforcing, mixing concrete, pouring and finishing concrete

a. Beginning techniques for arc and oxy-acetylene welding

19. FFA Facts and Record keeping. 


\title{
Agricultural Mechanics. (ROP) Levels I and II
}

\author{
Instructor: Mr. Woodard \\ Class Meets: Periods 6-7 \\ Room: \\ $\mathrm{H} 10$
}

\section{Course Description:}

These courses are designed to develop advanced skills and techniques used in Agricultural Mechanics. All of the concepts introduced will allow the students to perform necessary mechanical work that may arise in production agriculture. Field trips will be used to strengthen understanding of agricultural industries as well as the skills used in agricultural mechanics. Along with written assignments and required projects each student will be required to conceive, design, and produce projects related to agriculture. All students enrolled will develop a personal portfolio containing materials needed for employment. Each level is considered a separate class and a student who completes Level I as a junior may return for Level II the next year. This course transfers to Reedley College.

\section{Prerequisites}

This class is open to all Juniors and Seniors.

\section{Some of the topics to be covered in this class are;}

$\begin{array}{ccc}\text { Shop Safety } & \text { Cold Metal Work } & \text { MIG Welding } \\ \text { Hot Metal Work } & \text { Hand and Power Tool, Care and Use } \\ \text { Arc Welding } & \text { Gas Welding } & \text { TIG Welding } \\ \text { Portfolios } & \text { Job Seeking Skills } & \end{array}$

Ag. Mechanics Competitions

\section{Course Objective}

By the end of this course each student will be able to demonstrate skills and knowledge in the areas of; shop safety, tool use, metal work, wood work, electrification, project design and fabrication, measurement and job seeking skills.

\section{Supplies Needed for Class}

All students are required to bring;

Writing Utensil (daily)

Notebook (daily)

Safety Glasses (daily. no glasses $=$ no work, no work $=$ no credit $)$

Coveralls (students will get credit for dressing out each day)

Shop Card (20 \$ for materials) 


\section{Course Assessment}

The following breakdown will be used to calculate final grades:

\begin{tabular}{|c|c|}
\hline Class Participation: & $10 \%$ \\
\hline Unit Tests: & $20 \%$ \\
\hline Portfolios: & $10 \%$ \\
\hline $\begin{array}{l}\text { Projects: } \\
\text { Final: }\end{array}$ & $50 \%$ \\
\hline Final: & $\frac{10 \%}{100 \%}$ \\
\hline
\end{tabular}

$\begin{array}{ccc}90 \% & -100 \% & \text { A } \\ 80 \%-89 \% & \text { B } \\ 70 \%-79 \% & \text { C } \\ 60 \%-69 \% & \text { D } \\ 59 \text { or below } & \text { F }\end{array}$

\section{Class Participation:}

This is OUR class. Learning can not take place if we are not present, dressed out, and ready to work each day. It is extremely important that I see your smiling face each day. I truly care about your success in this class and your presence may also help others to succeed as well. However, if you must miss class it is your responsibility to get caught up on missed work (notes, handouts, project instructions, etc.)

\section{Portfolios:}

Portfolios are the best opportunity for you to sell yourself. This class is designed to help you focus on a career in the field of agriculture and mechanics. These portfolios contain cover letters, resumes, work samples, writing samples, and letters of recommendation. Regardless of your future plans a strong portfolio will help you succeed.

\section{Unit Tests:}

Unit tests will be given following the completion of each unit. They will be made up primarily of questions covered in assigned reading with the exception of one "Think Piece". The think piece will be a practical test of your knowledge.

\section{Note books:}

This class is full of handouts, notes, quizzes, and stuff. And for all that stuff you will need a place to keep it. A note book is required for this class and will be provided at no cost so long as it is returned in good condition at the end of the year. Please see the attached notebook handout.

\section{Projects:}

In class projects will give you real hands on experience in Agricultural Mechanics. In this class all students will complete all required projects. In addition, each student will be required to plan, design, estimate a bill of materials, and than produce an approved project.

\section{Final:}

The final exam will be comprehensive and will consist of questions previously asked on unit tests. 


\section{Absences}

School Policy - You have as many days to make up missed work as you were
absent.

\section{Tardy Policy}

You will be counted tardy if you are not in the classroom when the bell begins to ring. The Clovis High School Tardy Policy will be strictly enforced.

\section{Student Requirements}

1. Each student must provide or purchase coveralls. It is important that you keep your school clothes neat and clean.

2. Each student, teacher, or guest must wear a pair of safety glasses while in the Ag. Shop. I will provide a supply for the class. These can also be purchased at a welding supply house or hardware store.

3. Each student must purchase a $20 \$$ shop card in the front office.

4. Students will be assigned to lockers. These lockers will be used for shop materials and clothing.

\section{FFA and SOE Projects}

1. FFA - By enrolling in an agriculture class you are a member of the FFA. As a part of your participation grade in this class you must participate in two FFA activities (meeting, contest, fund raiser, project). I will help you accomplish this requirement.

2. SOE - If time permits each student will have the opportunity to enter a project in the Clovis High School Agricultural Mechanics Fair in May. This will be a great opportunity for you to exhibit your skills.

\section{Extra Credit}

At times it will be possible for you to work for extra credit. Do not count on extra credit points. In the event that you desire extra credit you must fill an extra credit request form. This form must be signed by you, your parent or guardian, and
approved by me.

\section{What You Can Expect From Me}

As your teacher you can expect me to;

1. Be prepared to teach each day.

2. Protect and uphold your right to learn each day.

3. Provide you with a safe learning environment.

4. Be there for each of my students to discuss grades, projects, assignments, or just talk about the weather.

\section{Reminders}

This is your class. I promise that you will get out of it twice as much as you put into it. Let's be safe, work hard, and have fun! 


\section{General Class Rules}

1. Always remember SAFETY FIRST.

2. Safety Glasses and Coveralls will be warn by all people in the Ag. Shop at ALL TIMES.

3. All students will be courteous to others and disrespect will not be tolerated.

4. There will be no talking in class unless you are recognized by the teacher first.

5. If you must leave your seat please raise your hand and ask for permission.

6. No hats will be allowed in Classroom. They will be allowed in the Ag. Shop when needed for work.

7. There will be no food or drinks allowed in this Classroom or Ag. Shop.

8. Foul language will not be tolerated for any reason.

9. Any hair long enough to touch the shoulders must be pulled back at all times while in the Ag. Shop..

10 The bell does not dismiss you, I do.

11. If you don't know, ASK ME!!

12. Always remember SAFETY FIRST. 


\section{Mr. Woodard's \\ Classroom Policies and Procedures}

I. Students Bill of Rights

All students in this classroom have the right to a quality education. They have the right to expect top notch performance from their teacher in an environment free of negative criticisms by others. Each individual in this classroom has the right to learn without

interference from those that choose otherwise.

II. Character Counts in this Classroom All students as well as the teacher will be expected to follow the 6 Pillars of Character at all
times.

- Trustworthiness

- Respect

- Responsibility

- Fairness

- $\quad$ Caring

- $\quad$ Citizenship

\section{Student Arrival to and Departure From Class}

All students must be in their assigned seat when the last bell rings. It is your responsibility to respect your classmates as well as the teacher by arriving on time each day. The teacher will advise you as to your unexcused tardy status and follow the CHS tardy policies.

\section{General Classroom and Farm Rules}

A. Be prompt, prepared, and ready to learn each day.

B. No food, drinks, or snacks will be allowed in the

this includes gum and

* Hats and sunglasses are not to be worn in the classroom.

D. *Personal grooming will not be allowed during class time. E. Students Must raise their hand and be recognized by the teacher before getting a
drink or going to the restroom. (their will be no restroom breaks given during the first and last 10 minutes of class)

F. Students are expected to sit properly in their seats at all times. (four on the floor and no feet on furniture)

* Infractions will result in the loss of items until the end of the class period the following day.

V. Daily Routines and Procedures

A. Each day we will use the following format:

B. Students will get their notebook from the cabinet and return to their seat.

C. The prompt will be discussed aloud in class as a review of previously covered

D. Announcements will be kept in class notebooks for a grade)

cements and Questions.

E. New material will be covered. (notes, discussions, reading)

F. Classroom Activities. (labs, exercises, worksheets)

G. Review of days material.

VI. Turning In and Getting Back Student Work

Each class period has been assigned a color that will allow them to stand out in a crowd.

A. Each class has an IN FOLDER as well as an OUT FOLDER.

B. Student work that is to be turned in will be placed into the In Folder for collection.

C. The Out Folder will contain all graded and recorded work that has been turned in Each day a student will distribute the papers during the prompt time. 


\section{Late Work}

It is your right to turn in any missed work at a later date if you have an excused absence it is your responsibility to follow these procedures.
A.
The total time granted for late work will B. In the event that you missed a laboratory exercise you will be required to write a
report on an agricultural or biology related documentary from the television or a
magazine. (see me before you start)
C. Missed (see me before you start)

\section{Extra Credit}

Extra credit is a "privilege" that I allow. However it will only be permitted when the student properly fills out an Extra Credit Request Form and has it signed by a parent or guardian.

\section{Grades}

Grades will be posted in all of my classes. Each student will be assigned a random student number for confidentiality. I will not give anyone your student number. If someone finds your number let me know and I will assign you a new one.

\section{Cheating and Plagiarism}

Cheating and plagiarism will not be tolerated. They go against all 6 pillars of character counts and any students suspected of violating this rule will be referred to their college 


\section{Employability \& Careers}

Students shall appreciate the importance of Supervised Occupational Experience Programs in the total program well as building a portfolio.

Engage in an appropriate S.O.E., taking into consideration individual career goals, and maintain records in the
California Vocational Agriculture Record Book.

2. Understand the importance of positive work ethics and values in the work place.

3. Develop a Portfolio which will include a resume, application, letter of application, letter(s) of recommendation, and work samples.

4. Develop interviewing and job keeping skills.

\section{Safety and First Aid}

Students will demonstrate safe work habits and develop first aid skills.

1. Understand that safety is on going and is practiced daily.

2. Pass appropriate safety tests on equipment students will be using.

3. Demonstrate the appropriate steps to take when handling emergencies.

4. Be familiar with potential hazards and OSHA regulations.

\section{Oxyacetylene/Oxy-Fuel Welding}

Students will be able to perform Oxyacetylene (fuel) welding tasks using appropriate metal preparation
techniques, joints, and filler rods.

1. Use the Oxyacetylene (fuel) equipment to weld mild steel in flat, horizontal, and vertical positions.

2. Use the Oxyacetylene (fuel) equipment to weld pipe in each of the standard joints.

3. Braze weld the butt, fillet, and lap joints.

4. Braze weld pipe in the fillet position.

5. Make appropriate repairs to cast iron using cast iron and brass filler rods.

6. Properly apply other specialty rods including but not limited to hard surfacing.

7. Become familiar with different types of Oxy-Fuel welding systems.

\section{Shielded Metal Arc Welding}

Students will be able to perform arc welding tasks using appropriate joints, electrodes, metal preparation
techniques, and weld current.

1. Arc weld mild steel in flat, horizontal, vertical and overhead positions.

2. Arc weld pipe in each of the standard positions.

3. Make repairs to cast iron using nickel rod.

4. Properly use other specialty rods including those with applications for but not limited to hard surfacing.

5. Properly set the amperage and type of current for the specific application and rod being used.

\section{Metal Cutting}

Students will demonstrate proficiently with various metal cutting techniques.

1. Use the Oxyacetylene (fuel) torch to make cuts in plate and sheet metal using combination, drag, and gouging
tips.

2. Make joint preparations for steel using the Air Arc.

3. Cut non-ferrous metals using the Air Arc. 
4. Cut non-ferrous and nonferrous metals using a plasma arc system.

5. Cut material to length using the hydraulic shear.

6. Make square and angle cuts on different shapes of metal using the band saw, abrasive cut off saw, and /or cold cut saw.

7. Make a pattern for the pantograph - Oxyacetylene duplicator.

8. Use Oxyacetylene pattern cutting machine to cut patterns in mild steel plate.

9. Use Plasma Arc pattern cutting machine to cut patterns in mild steel plate.

\section{Power Tools}

Students will be proficient in the use and application of power tools.

1. Bore and countersink holes in various materials using the portable electric drill, pneumatic drill, and drill press.

2. Grind, buff, and sand metal projects using portable and stationary grinders and sanders.

3. Cut sheet metal using shears, nibblers, saber saw, and circular saw.

4. Demonstrate the use of a manual or hydraulic bending unit (portable or stationary) on angle iron, pipe, flat and round stock.

5. Use a hydraulic iron worker to shear and punch flat stock and angle iron, and to notch pipe.

\section{Project Layout}

Students will become proficient in laying out holes, cuts and angles on metal for specific applications

1. Layout holes and select the correct tools for boring various metals and shapes.

2. Layout square cuts, angles, and compound angle cuts on various metals and shapes

\section{MIG Welding}

Students will demonstrate proficiency using the MIG welding process (GMAW).

1. Using the MIG welder and solid wire, demonstrate welding mild steel in various positions employing the basic joints.

2. Use the MIG welder and inner or outer shield wire to weld mild steel $1 / 4$ " or thicker in the flat position.

3. Demonstrate welding aluminum and/or stainless steel in the flat position using a MIG welder or spool gun.

4. Demonstrate proficiency at MIG machine maintenance and troubleshooting.

\section{TIG Welding}

Students will demonstrate proficiency using the TIG welding process (GTAW).

1. Using the TIG welding process, demonstrate various joints in the flat and horizontal position on aluminum and/or
stainless steel.

2. Have knowledge of other unique or exotic metals that can be welded using the TIG process.

\section{Phase of Metals}

Students will understand the phases of matter and the role temperature and pressure play in the change of phase
of metals.

1. Observe phase change of metal when they are subject to heat and be able to apply the knowledge in practical
applications.

2. Define melting point, thermal conductivity, and grain structure. 
3. Describe the effect of cooling rate on the grain structure of steel.

4. Describe how materials behave under applied pressure and explain how to compensate for pressure problems in various situations where metals are used.

\section{Project Design and Construction}

Students will apply skills learned in welding, metal working, finish work, and project design to plan for and build a complex project.

1. Using actual cost estimates and drawings, complete a plan for a project including: working drawing, bill of materials, and sequence of operations.

2. Employing appropriate tools and skills, manufacture the parts and construct and finish a complex project.

\section{Project Finishing}

Students will demonstrate the skills necessary to finish a project in a professional way.

1. Finish a product to the degree appropriate for the intended use of the item made.

2. Complete the surface preparation of a metal project using proper tools and materials, sealers, paints, etc.

3. Carry a project to completion including all details involved in the production of the finished product.

\section{Alloys}

Students will understand the importance of the synthesis of new alloys which have properties necessary for specific applications and purposes.

1. Explain what alloy steel is and how it is manufactured.

2. List the common additive elements involved in alloy steel production.

3. Identify which qualities are affected by each additive in an alloy.

\section{Basic Machine and Foundry Principals}

Students will demonstrate knowledge and use of machine tools and basic foundry principles.

1. Use a milling machine to square and flatten a surface.

2. Use the mill to make a step, shoulder, and groove in a metal sample.

3. Use the milling machine to machine a key way on a shaft.

\section{Electronics}

Students understand and appreciate the role of electricity in the technological world.

1. Students define electric current and state the difference between conductors and insulators.

2. Students define voltage and amperage and describe their applications in agricultural mechanics

3. Students differentiate between alternating and direct current.

4. Explain the voltage drop and its effect on welding (application) current.

5. Differentiate between open-circuit voltage and are voltage.

6. Students become aware of the prevalence of electromagnetics by investigating the extent to which they are used in arc welders. MIG welders and other electrical shop equipment.

7. Students define electromagnetics and describe their application in the conversion of electrical energy into mechanical energy in electric motors.

8. Students explain the principles of the electric motor. 


\section{Measurement}

Students will understand and are able to read and use a ruler or tape and complete calculations for problems involving
length, area, and volume and weight using American standard and metric measurements.

1. Correctly measure objects with a ruler, tape or framing square.

2. Calculate and solve basic measurement problems including, but not limited to the calculation of board feet, cubic measurement, and standard liquid measurement.

3. Differentiate between American standard and metric measurements units and use in solving problems requiring

4. Use various methods to determine the mass and volume of regular and irregular shaped objects using American standard and metric systems.

\section{Project Design}

Students understand the basic skills required to make simple drawings and be able to interpret a working drawing.

1. Identify the three (3) types of drawings (orthographic, isometric, oblique).

2. Read blueprints including schedules, details, and symbols.

3. Understand the steps necessary to develop plans and apply for building permits and types of other permits related to the construction trade.

4. Interpret a surveyor's field book correctly when reading a map.

\section{Wood Structures}

Students will acquire the knowledge and skills necessary to design

and construct wood structures.

1. Identify the woods, wood substitutes, and materials commonly used in the building of wood structures.

2. Read blueprints including schedules, details and symbols.

3. Make an estimate for a bill of materials using sketches and drawings for a wood structure included on a landscape plan.

4. Identify and use common carpentry tools for the construction and installation of wood structures.

5. Participate in the building of a wood structure.

\section{Concrete Structures}

Students will become aware of the types of concrete structures used in construction and develop an understanding of how
they are installed.

1. Recognize and identify the types of concrete structures used in construction.

2. Develop and estimate the cost for a bill of materials for concrete structures using drawings, plans or blueprints.

3. Identify materials used in the construction of concrete structures.

4. Demonstrate mastery of basic masonry techniques used in the construction of concrete structures.

5. Identify and use basic masonry tools used in the construction of concrete structures.

6. Build a concrete item or structure including the building and eventual removal of the forms for the structure.

7. Determine the structural integrity of a project, by performing a slump test.

\section{Building Components}

Students will familiarize themselves with and be able to identify building components and design features. 
1. Identify foundation components.

2. Differentiate between new and old systems of floor joists.

3. Identify framing components and roof styles

4. Differentiate between types of trusses.

5. Identify by grade the common types of lumber used in construction.

\section{Framing and Forms}

Students will understand the procedures necessary to form or frame a particular structure or foundation.

1. Properly layout a building site with batter boards, lines, and stakes.

2. Be able to properly form, pour, reinforce, and finish a concrete foundation or slab.

3. Install floor joists and sills to specifications.

4. Layout, assemble, erect, and plumb a wall section.

5. Cut out and install common rafters and joists to specifications.

6. Be able to layout, setup, and install a truss.

\section{Fences}

Students will develop the knowledge and skills necessary to design, layout and construct simple fences used for home and
farm applications.

1. Developing a fencing plan, taking in account all pertinent land considerations (e.g., topography property boundaries, etc.).

2. Properly layout a fence line.

3. Have a knowledge of fencing materials and their appropriate uses (i.e., wire, electric, wood, posts, gates, fasteners, pipe, and cables).

4. Repair a wood and/or wire fence.

5. Install a section of fence including a gate.

6. Perform a splice on a wire fence.

7. Properly anchor a fence line.

8. List factors to consider when installing an electric fence.

9. Align posts using a level or a transit.

\section{Surveying}

Students will develop and demonstrate a basic understanding of surveying as it is used in agricultural applications.

1. Perform various measurements for horizontal and vertical applications.

2. Set up and keep a field notebook.

3. Visually identify the builder's, engineer's, and turret levels.

4. Identify and use a direct reading and positive reading rod with an engineer's scale and an architect's scale.

5. Understand and identify applications for profile leveling.

6. Determine distance with the assistance of a transit.

7. Calculate cuts and fills for a given piece of property.

8. Layout a building site for a simple agricultural structure (e.g., pole barn).

9. Understand and be able to read a topographic map.

10. Explain how land is subdivided in California and the United States. 


\section{Irrigation Systems}

Students will develop the knowledge and skills necessary to design, layout and construct simple fences used for home and
farm applications.

1. Differentiate between deep well turbine, low lift, and booster pumps.

2. Properly start and stop a deep well turbine pump.

3. Use and maintain the mechanical device necessary for irrigating crops.

4. Calculate the amount of irrigation water necessary for a field planted with

5. Given the appropriate $\quad$ information

6. $\quad$ Determine the flow, pressure, and appication motor and pump efficiency levels.

Determine the flow, pressure, and application rates of irrigation water given specific information about the equipment used and crop to be irrigated.

7. Understand the principles of injector and vacuum systems used in irrigation.

8. Determine how to channel flow and alter pressure for pressure compensators.

9. Safely handle irrigation equipment and be able to determine when a technician is needed for repairs.

\section{Physical Properties of Materials.}

Students will explain how physical forces effect various types of materials.

1. Define stress and state how it can be measured.

2. Define strain and explain how it can be measured.

3. Differentiate between tensile and compressive strengt and wood substitutes. 4. Describe the concept of elasticity of metals, wood and other materials and explain how this knowledge can be
used in construction.

5.

Differentiate between bending strength, shear strength, fatigue strength, and impact strength; give examples of
each; and demonstrate the effects of one for the class.

\section{Energy}

Students will understand that energy is the ability to do work, that energy comes in many forms and can be converted from
one form to another.

1. Identify the different forms of energy such as heat, light mechanical, and electromagnetic and cite examples of

Observe and describe how heat changes matter and determine whether heating will temporarily or permanently 3. Determine how energy can be channeled to work with the use of energy efficient building materials,
environmental controls.

\section{Electricity}

Students understand and appreciate the role of electricity in the technological world.

Define electric current and state the difference between conductors and insulators.

Define voltage and amperage and describe the application of each in agricultural 
Differentiate between alternating and direct current and identify appropriate applications for each.

4. Describe voltage drop and its effect on welding current, and motor/pump operation.

5. Differentiate between open-circuit voltage and closed circuit voltage.

6. Explain how capacitor start motors function and the principles of single and three phase operation.

7. Develop an appreciation for and general knowledge of new advances in electrical technology including solar energy storage systems and their practical uses.

\section{Electronics}

Students understand the relationship of magnetic forces and electrical currents and investigate the common uses of
magnetism

1. Become aware of the prevalence of electromagnetics by investigating the extent to which they are used in arc welders, MIG welders, and other electrical shop equipment.

2. Define the term electromagnetic and describe applications in the conversion of electrical energy into mechanical energy in electric motor.

3. Explain the principles of operation for the electric motor.

4. Measure the current of motors and determine the efficiency of a specific motors.

5. Understand the principles and value of electrostatic application in agriculture. 


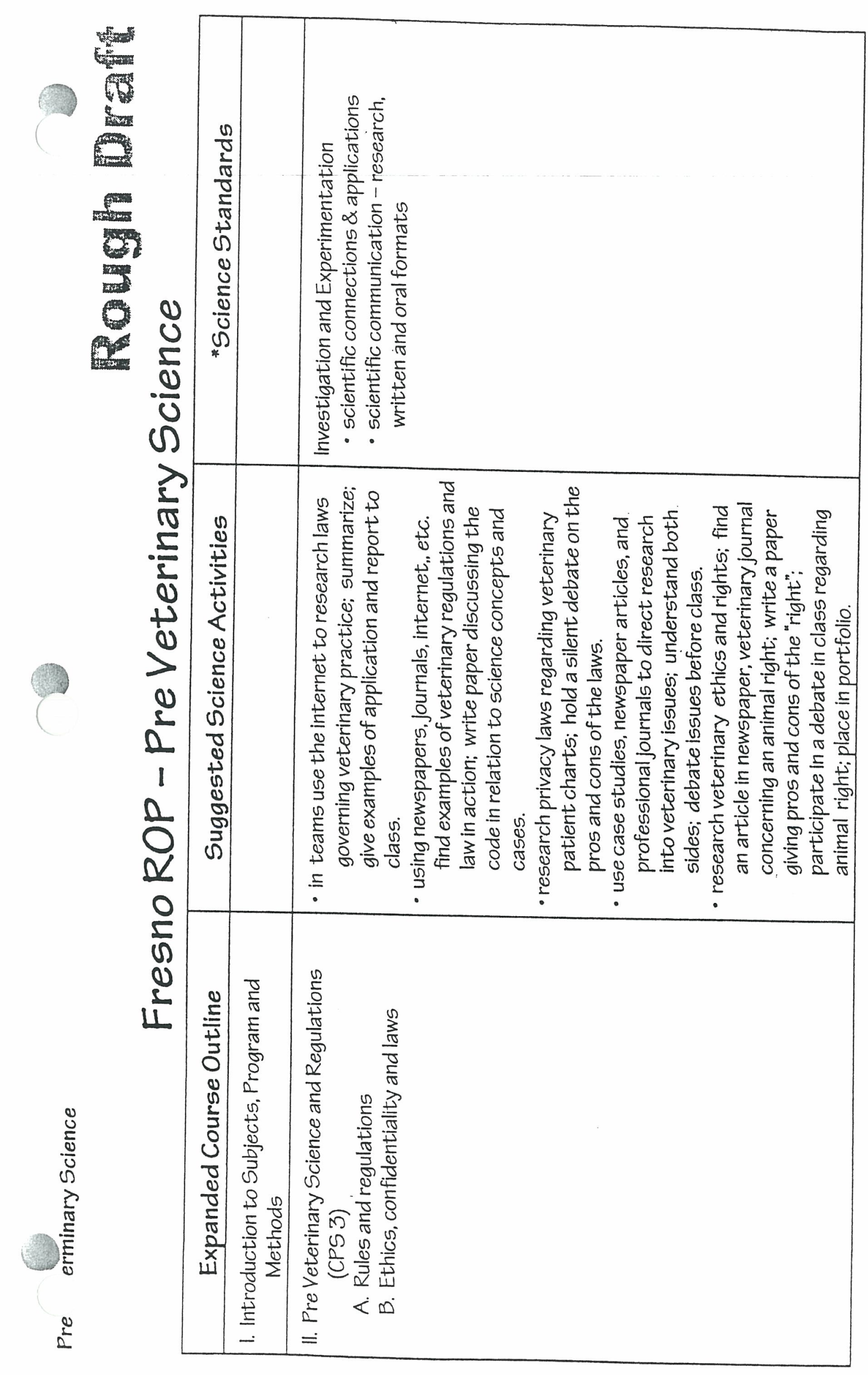




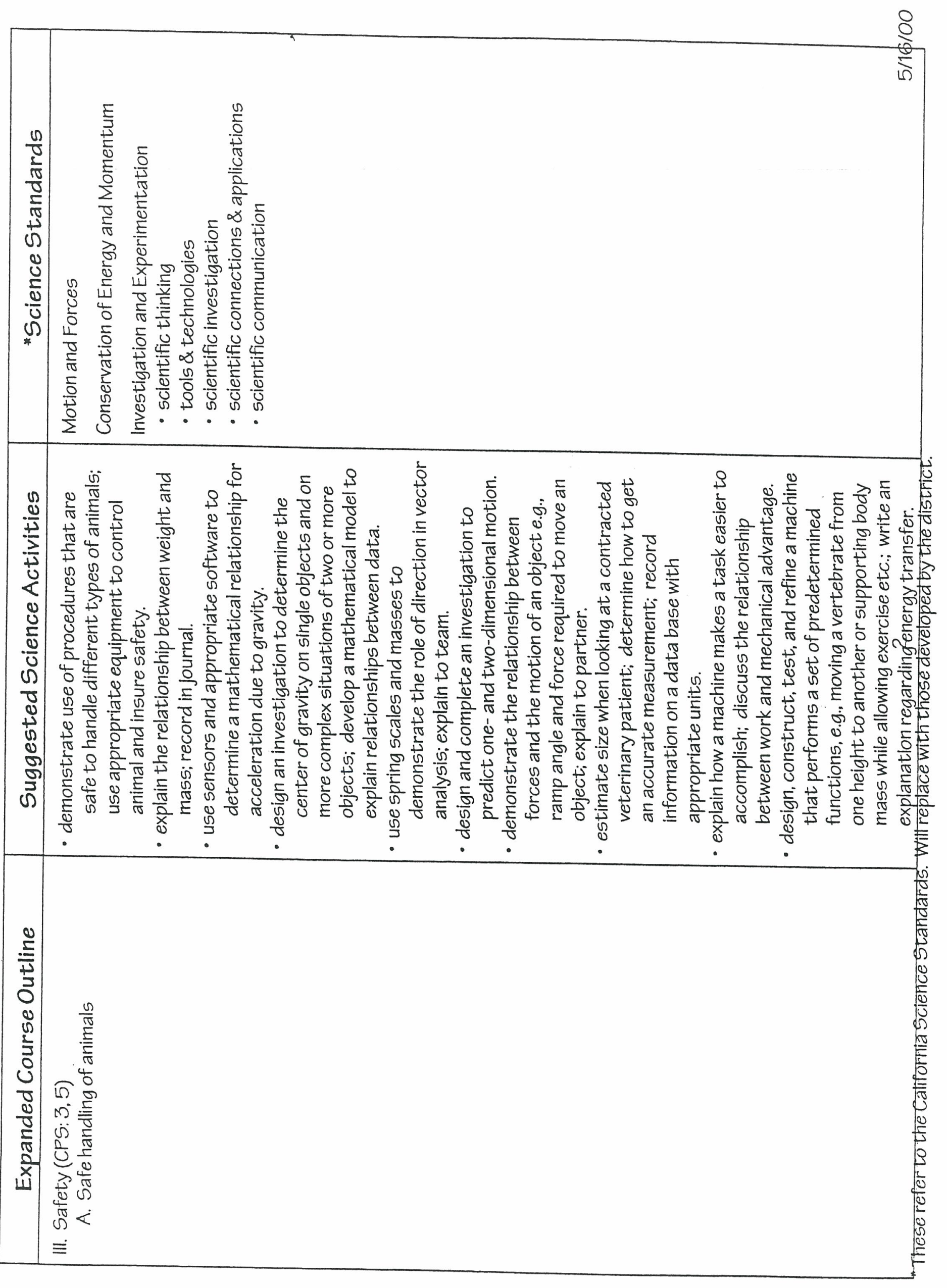




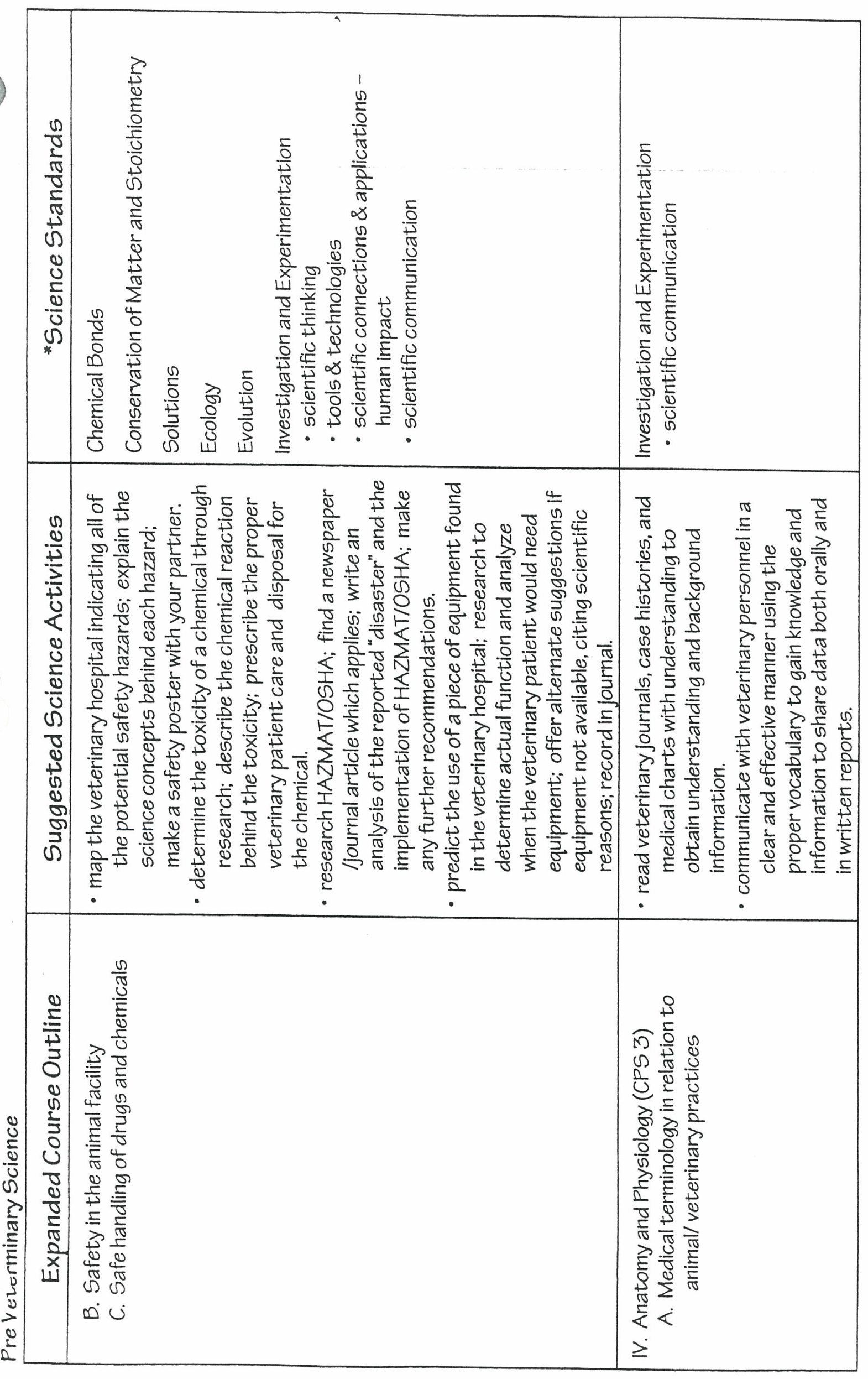




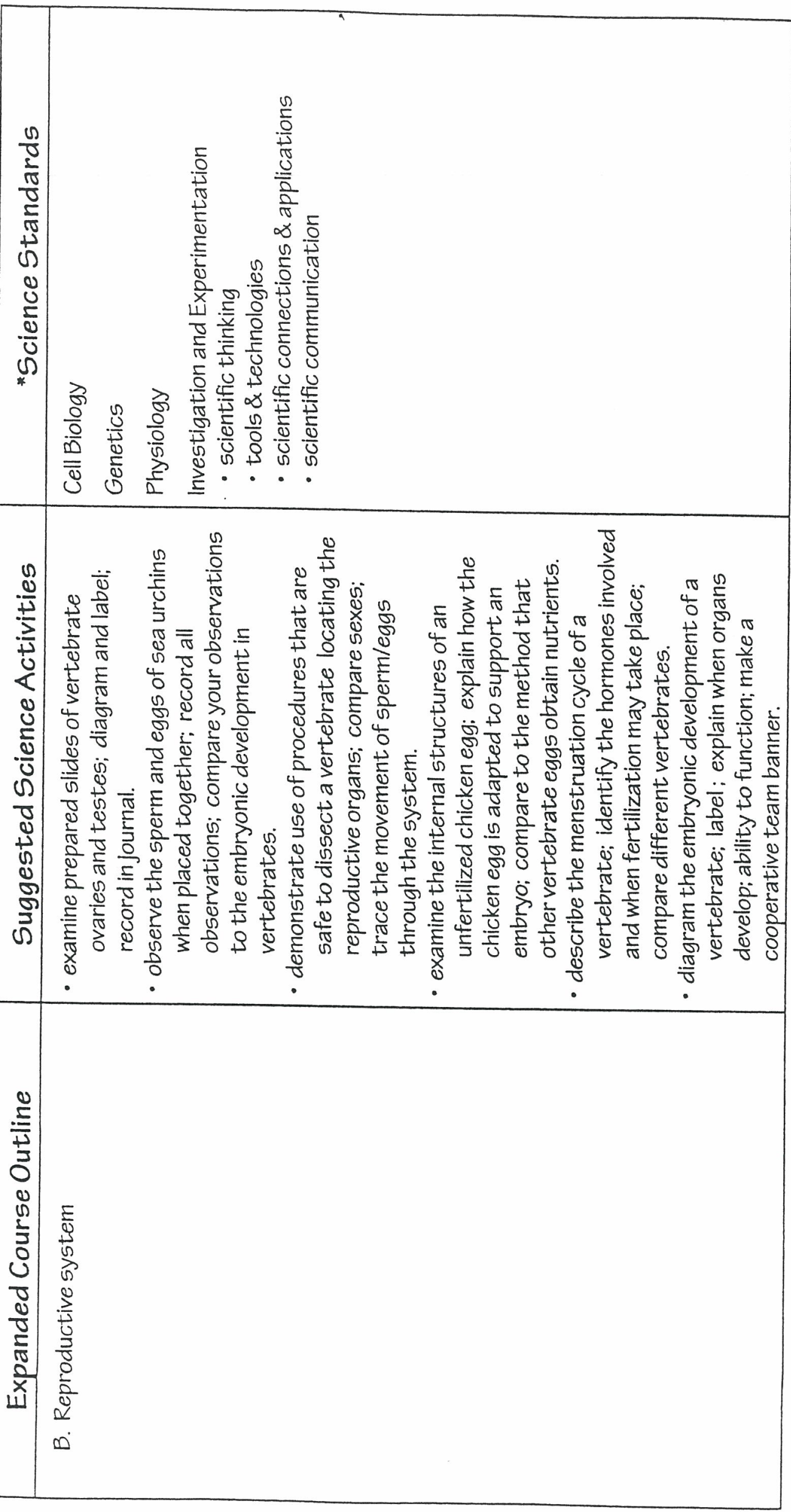




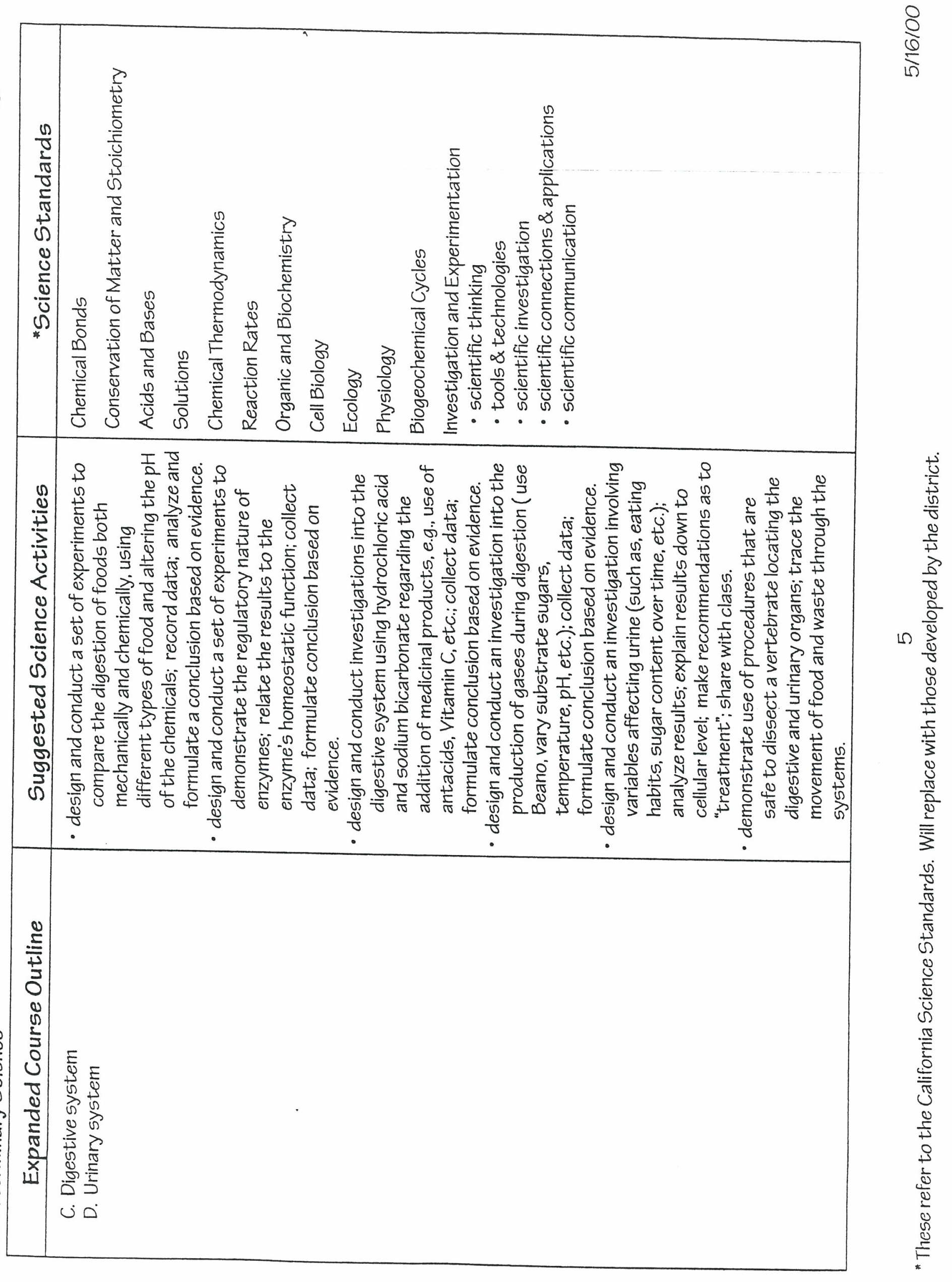




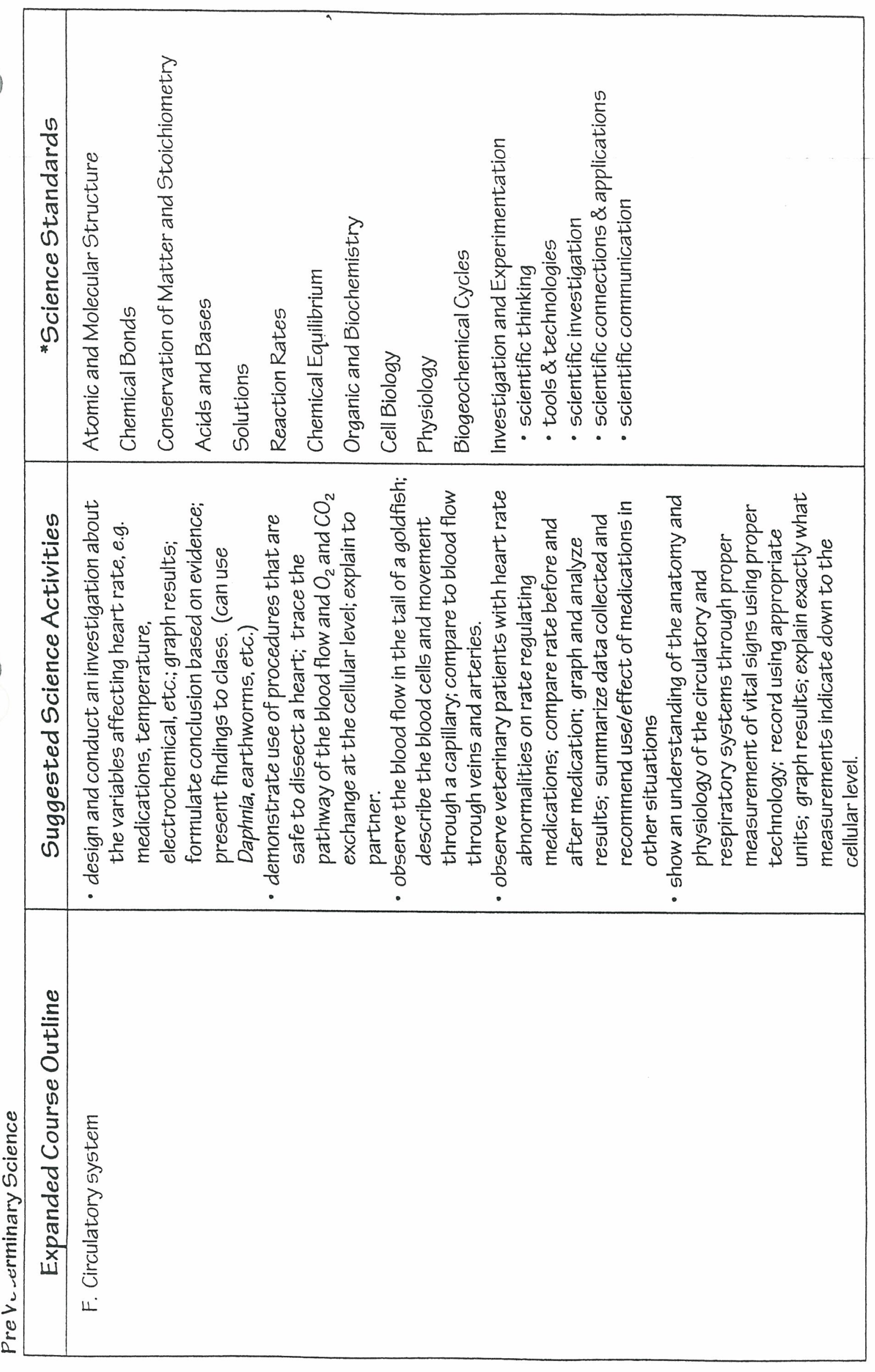




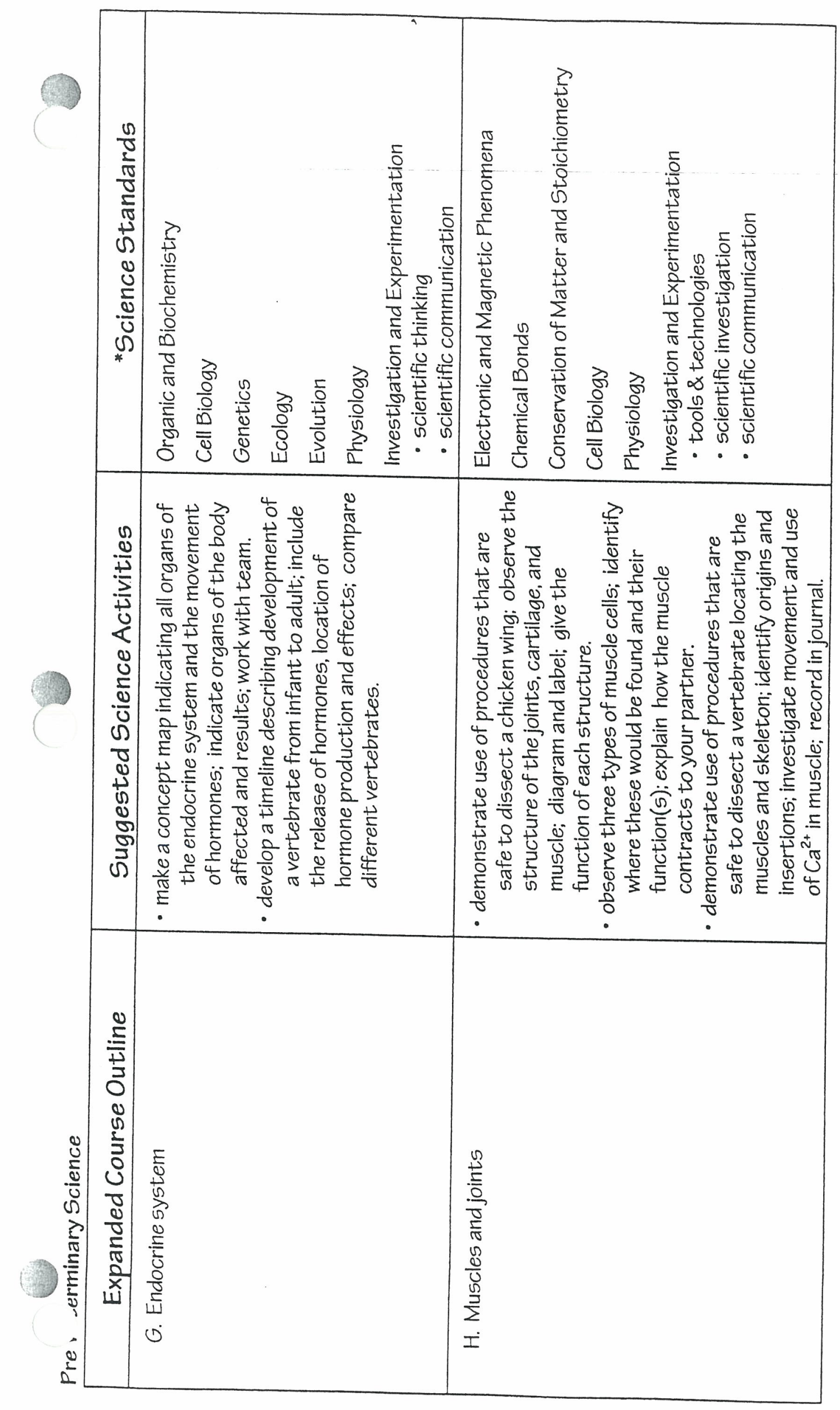




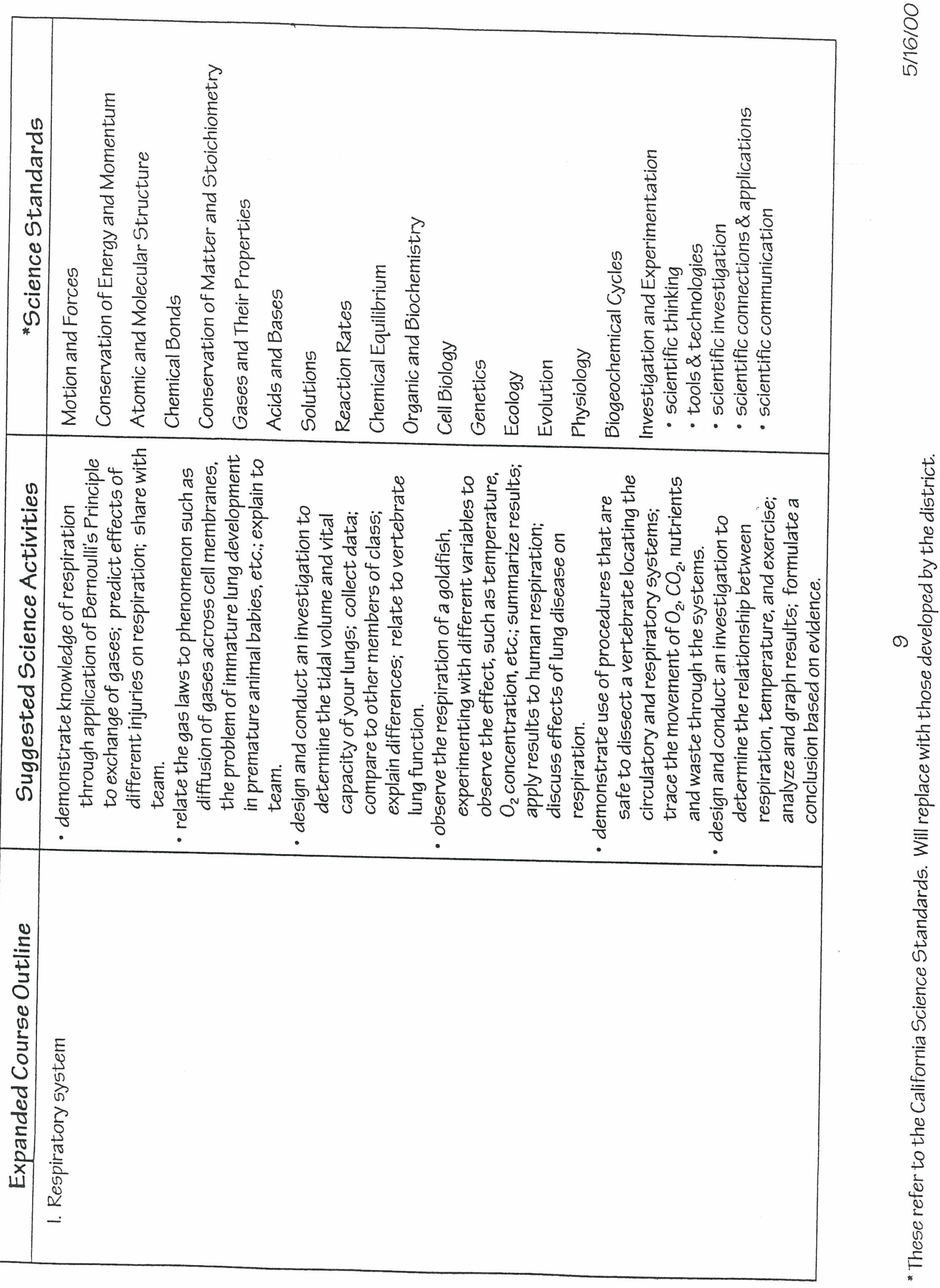




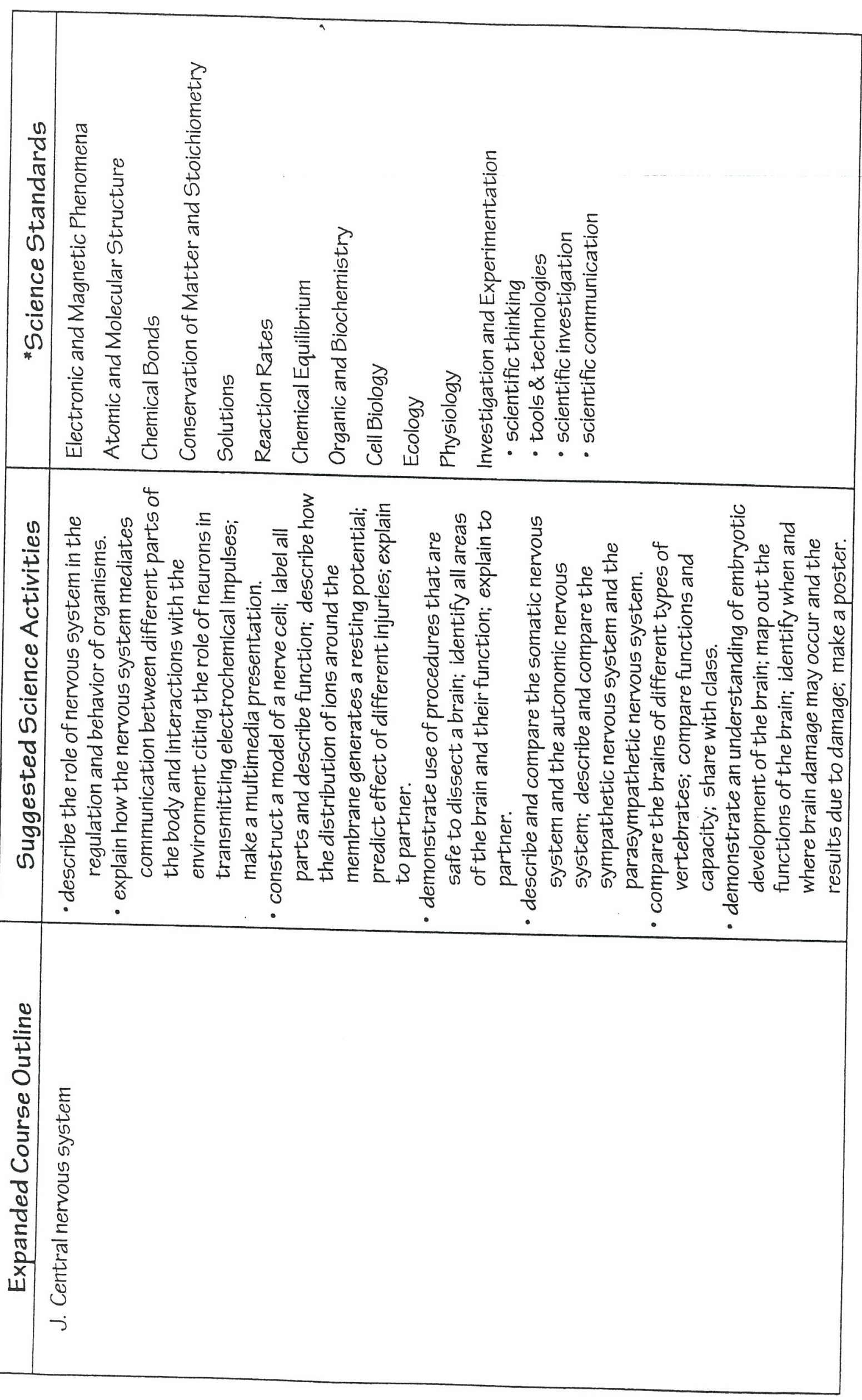




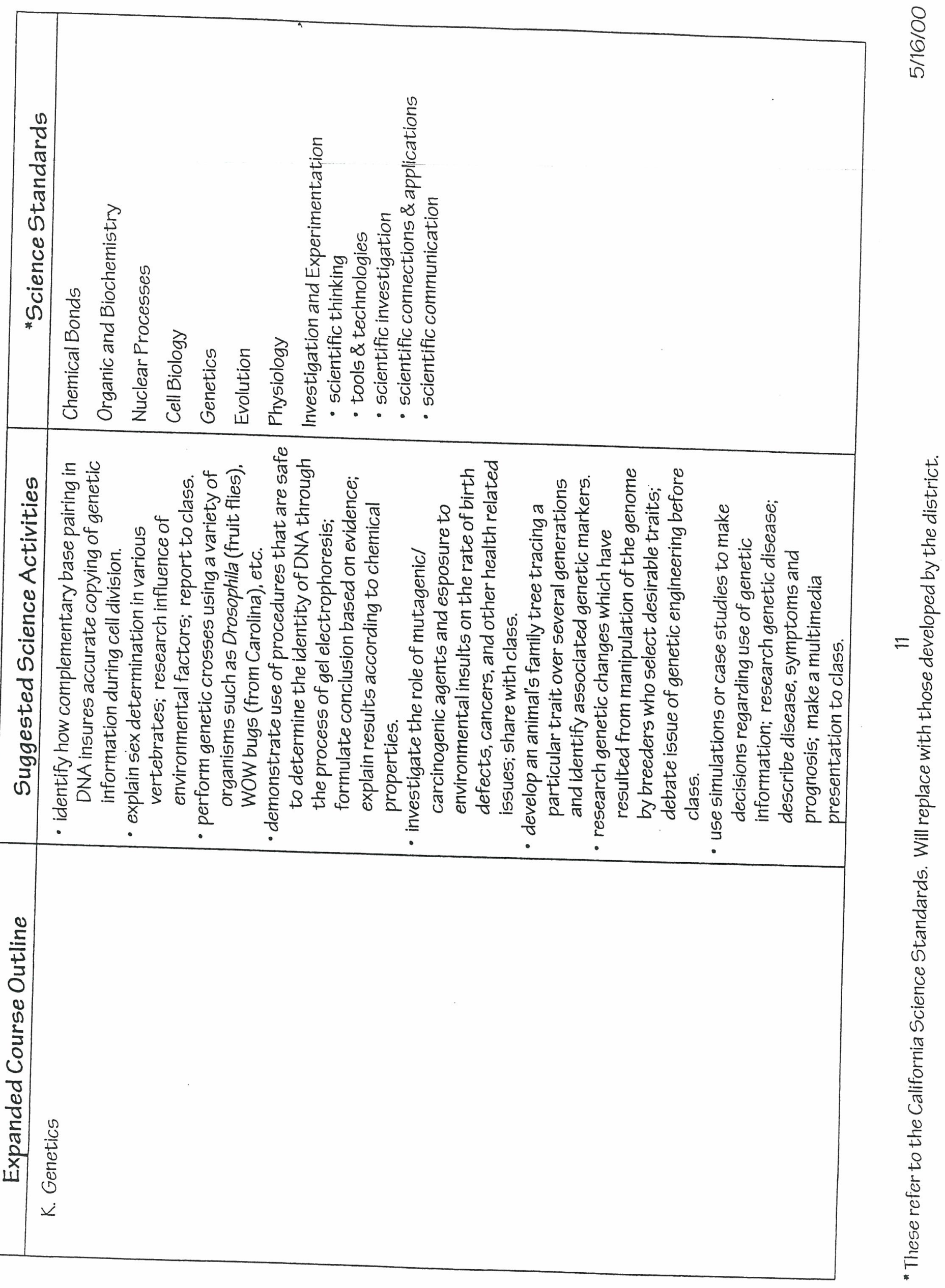




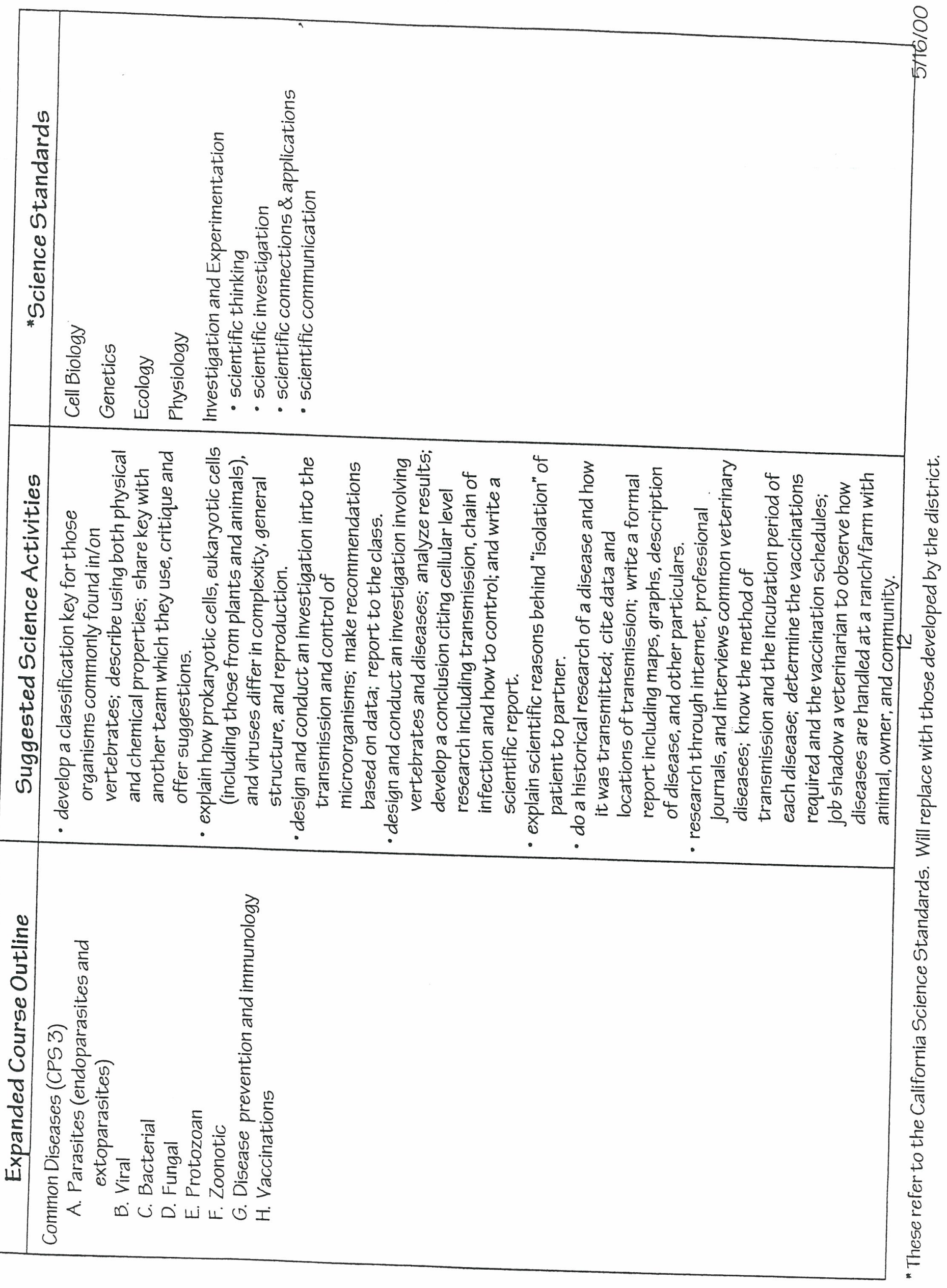




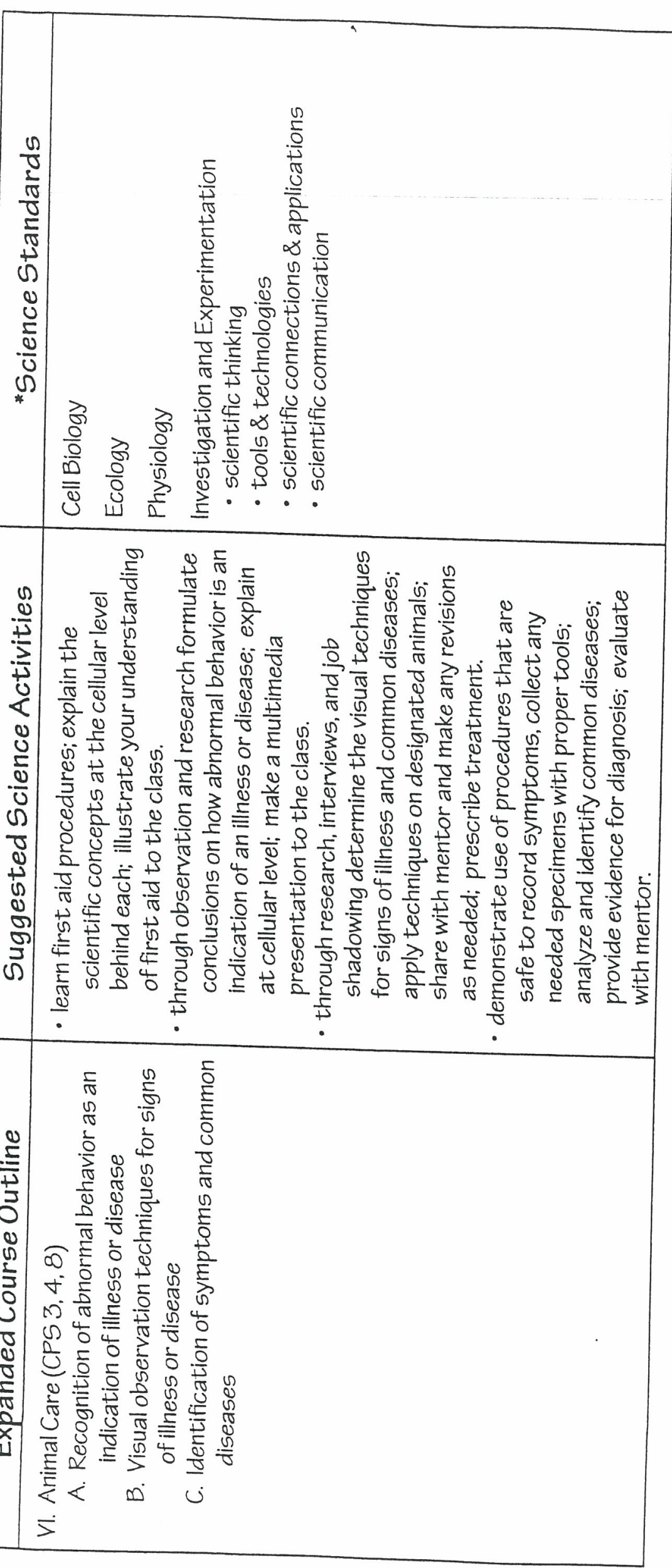




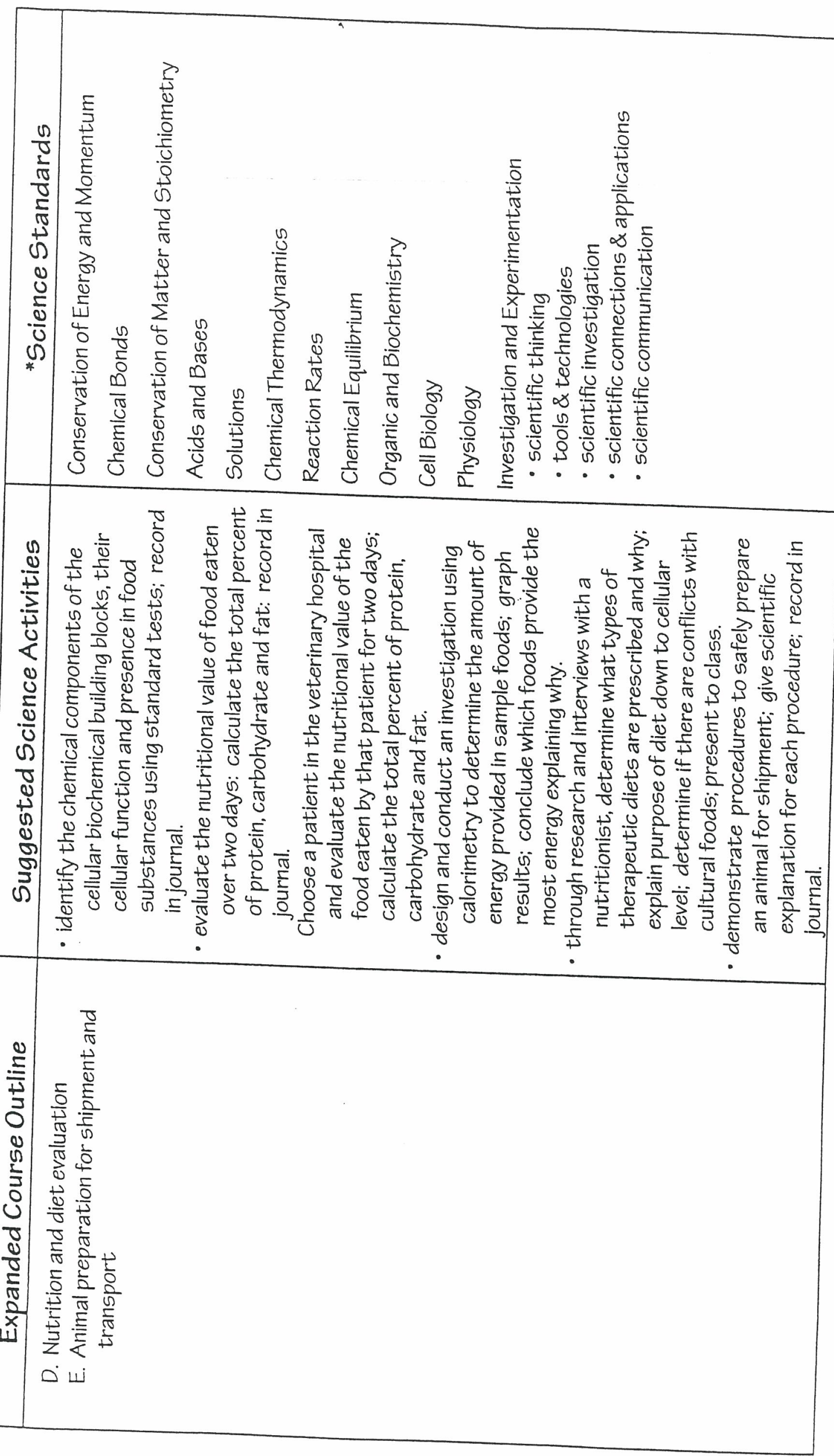




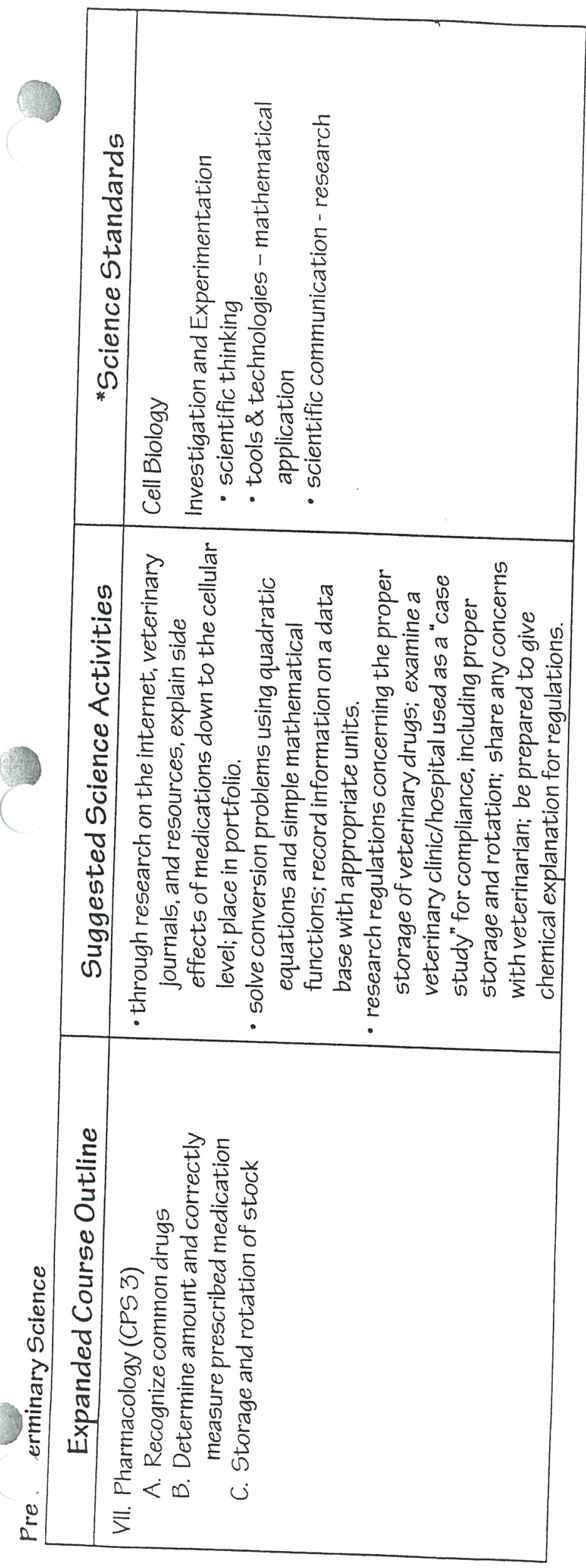

$\frac{8}{0}$

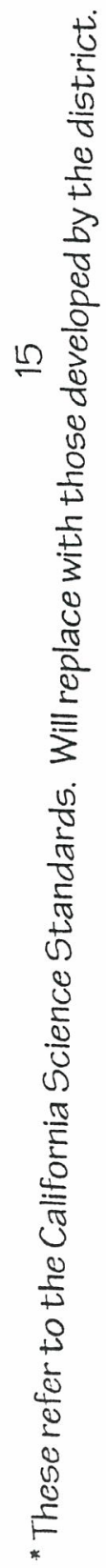



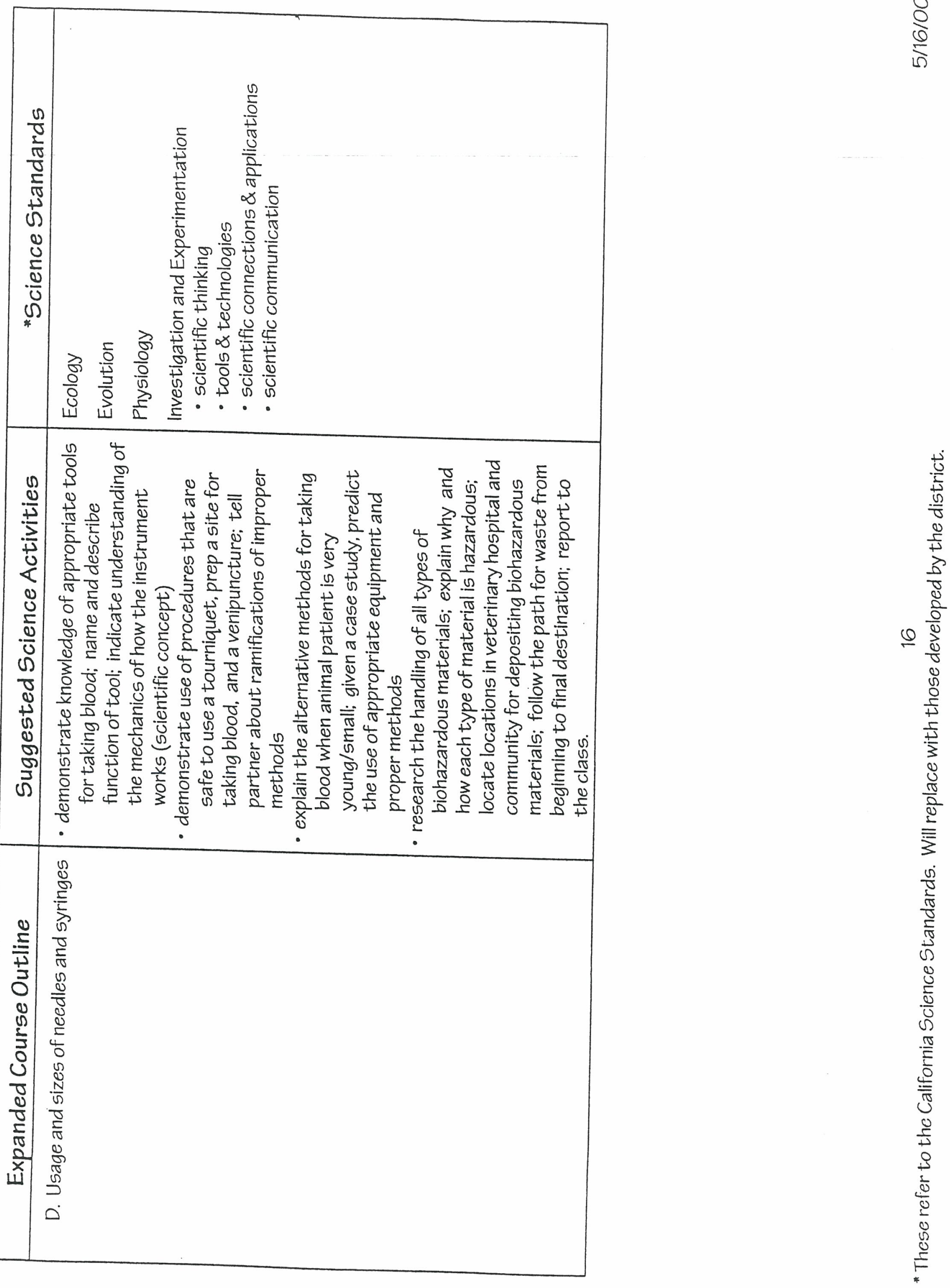


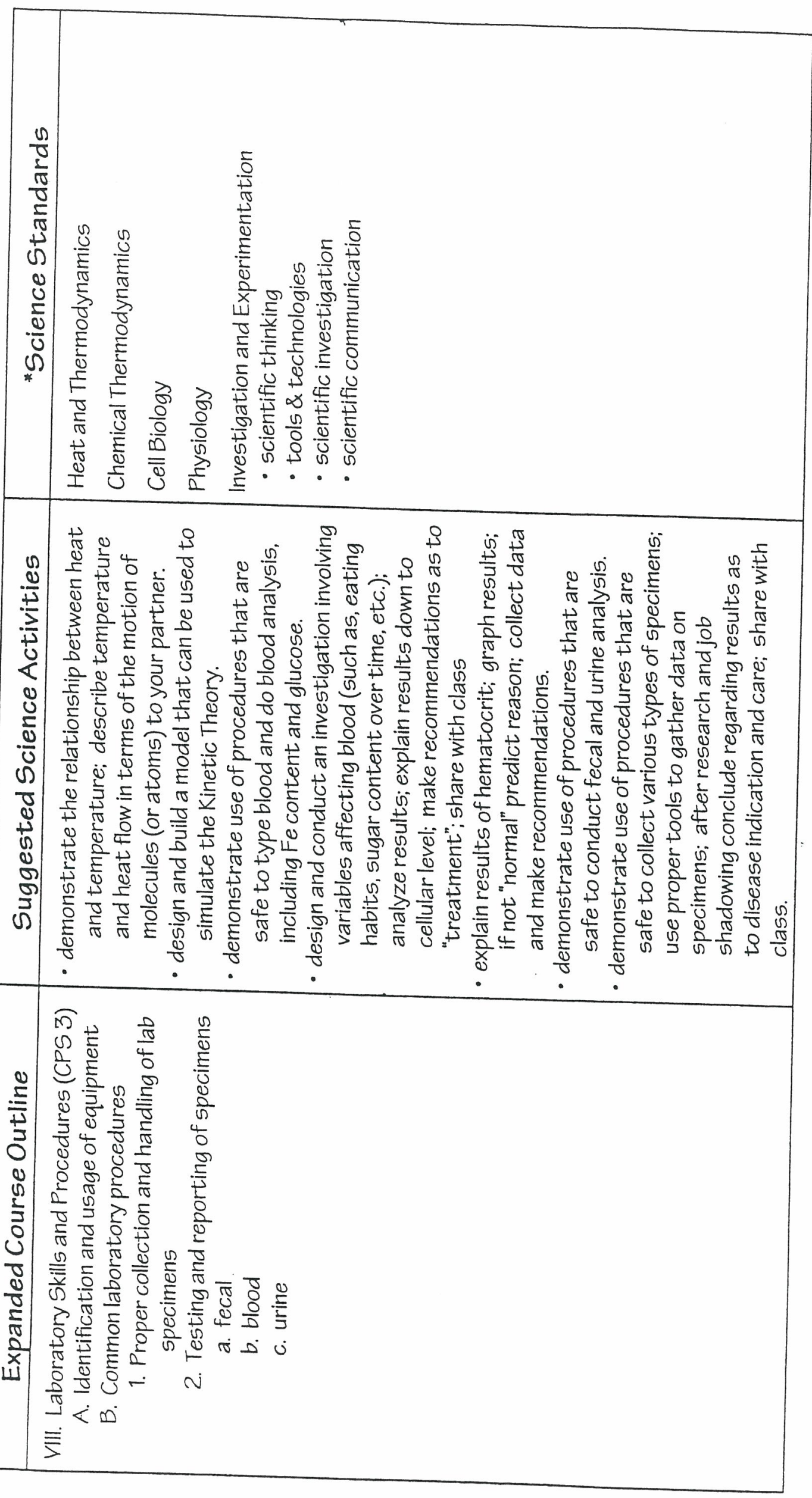




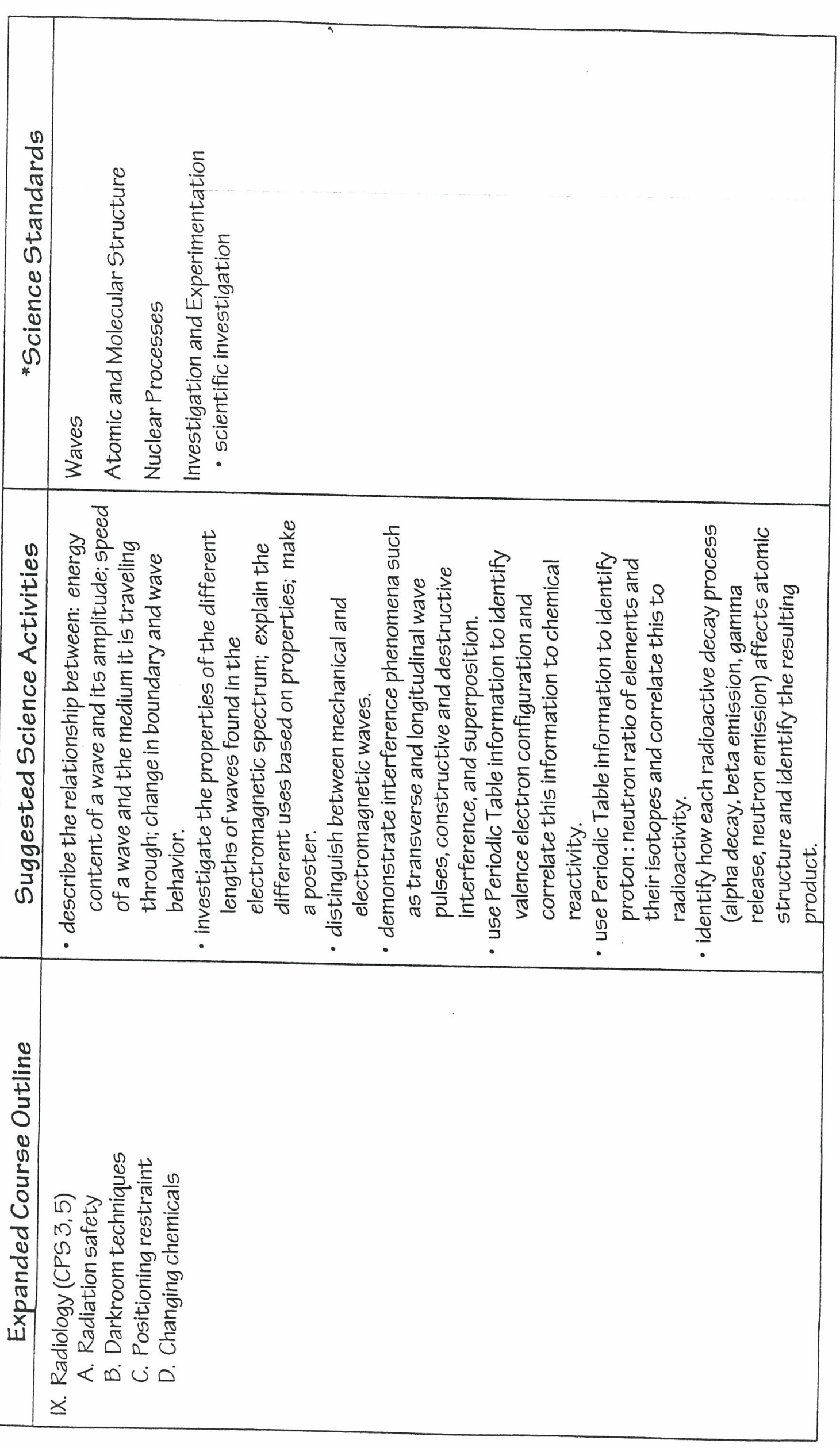




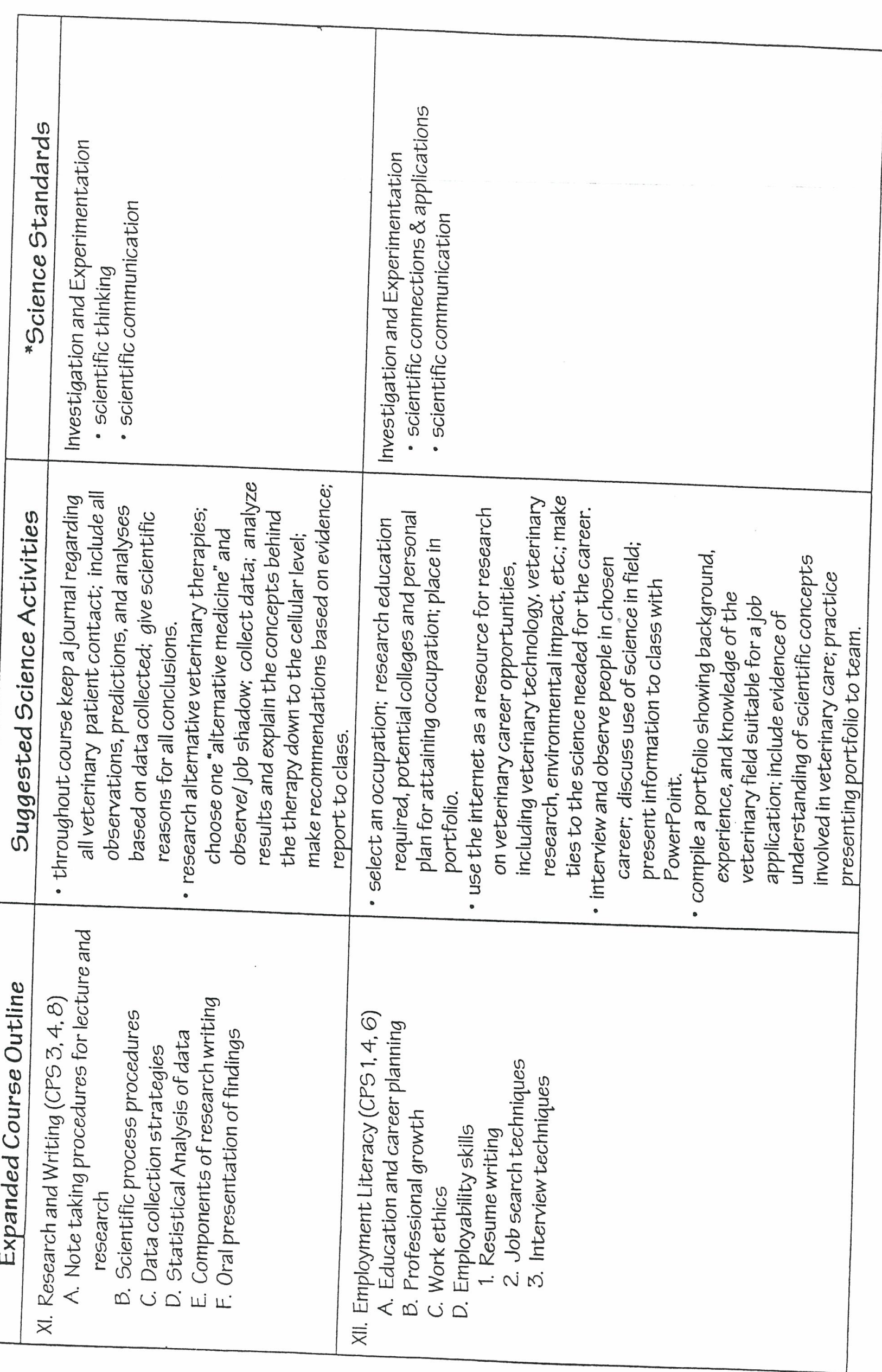

$\frac{\sqrt{2}}{8}$

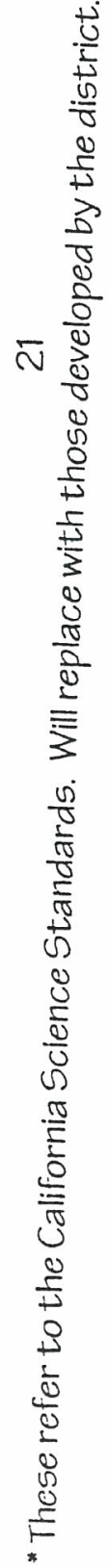




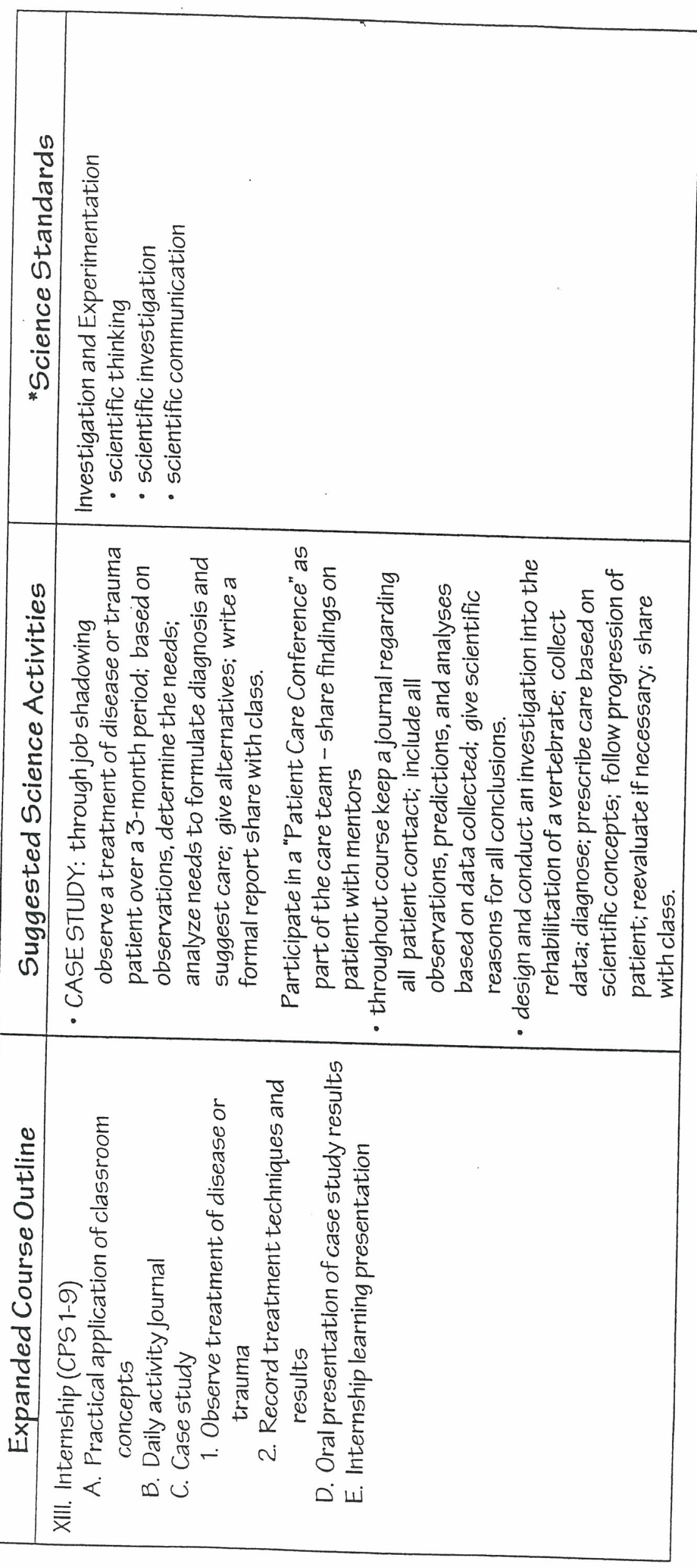




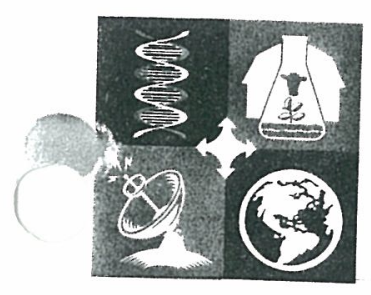

$C$ e n t r a 1

A p p 1 i e d

a $\mathbf{n} \mathbf{d}$

\section{$V$ a 11 e y}

A griculture

8180 E. Donner Clovis, CA 93611

559.327.4320

559.327.4063 Fax

\section{Reagan Educational Center Administration \\ Linda Hauser \\ Asofstane Superintendene Lyn Smauffer \\ Assifant Adminfstrator \\ Melissa Macon \\ Difector. Qualfey Student
Seruices \\ Jefr Principal, Cloufs Ease High
School \\ Stacy Dummicliff \\ Principal, Reyburn \\ ptermediate \\ lovis Uniffed School \\ sistrict}

\section{Administration}

Walter L. Buster, Ed.D.
Superintendent

Terry Bradley, Ed.D.

Depuey Superintendent

James S. Fugman, Ed.D.

Deputy Superintendent

Virginia R. Boris, Ed.D

Assoclate Superfntendent

Daniel E. Kaiser, Ed.D.

Associate Superintendent

William C. McGuire

Assoclate Superintendent

\section{Governing Board}

Elizabeth J. Sandoval

President

Sandra A. Bengel

vice president

Jim Van Volkinburg,

D.D.S.

Clenk

Brian D. Heryford

Ginny L. Hovsepian

Richard Lake, C.P.A.

Susan M. Walker,

D.H.Sc.

\section{Program Completion Standards}

In order for a student within the CUSD Agriculture Program to become a program completer they must received 720 hours of class instruction in four classes of a predetermined sequence of classes. The following list of Program Sequence Options is used to advise students in program planning.

Animal Science Pathway

$\begin{array}{llll}\text { Year } 1 & \text { Ag Sci I } & \text { or } & \text { Ag Biology } \\ \text { Year } 2 & \text { Ag Sci II } & \\ \text { Year 3 } & \text { Ag Sci IV } & \\ \text { Year } 4 & \text { Pre Vet Sci } & \end{array}$

$\frac{\text { Plant Science Pathway }}{\text { Year }}$

$\begin{array}{llll}\text { Year 1 } & \text { Ag Sci I } & \text { or } & \text { Ag Biology } \\ \text { Year 2 } & \text { Ag Sci II } & \text { or } & \text { Ag Environmental Science } \\ \text { Year 3 } & \text { Ag Sci III } & & \\ \text { Year } 4 & \text { Ornamental Horticulture }\end{array}$

Mechanized Agriculture Pathway

$\begin{array}{ll}\text { Year 1 } & \mathrm{Ag} \mathrm{Sci} \mathrm{I} \text { or Ag Biology } \\ \text { Year 2 } & \text { Introduction to Ag Mech } \\ \text { Year 3 } & \mathrm{Ag} \text { Welding and Fabrication } \\ \text { Year } 4 & \text { Precision Agriculture }\end{array}$

Ag Business Pathway

$\begin{array}{ll}\text { Year 1 } & \text { Ag Sci I or Ag Biology } \\ \text { Year 2 } & \text { Ag Sci II } \\ \text { Year 3 } & \text { Concentration Option } \\ \text { Year } 4 & \text { Ag IV , Agri-Business }\end{array}$

General Agriculture Pathway

$\begin{array}{llll}\text { Year 1 } & \mathrm{Ag} \mathrm{Sci} \mathrm{I} & \text { or } & \text { Ag Biology } \\ \text { Year 2 } & \mathrm{Ag} \mathrm{Sci} \mathrm{II} & & \\ \text { Year 3 } & \mathrm{Ag} \mathrm{Sci} \mathrm{III} & & \\ \text { Year 4 } & \mathrm{Ag} \mathrm{IV} & \text { or } & \text { Concentration Option }\end{array}$

"A compelling image of an achievable future." 


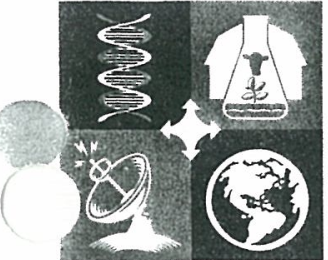

C e 1 tra 1

A p p 1 i e d a n d

8180 E. Donner Clovis, CA 93611 559.327.4320 559.327.4063 Fax

Reagan Educational Center Administration

Linda Hauser

Assistant Superintendent Lyn Snauffer

Assistant Administrator

Melissa Macon

Dinector. Quality Student Seruices

JeffEben

Principal. Clouls East High School

Stacy Dumnicliff

Principal, Reyburn

ntermedice

lovis Unified School istrict

\section{Administration}

Walter L. Buster, Ed.D. Superintendent

Terry Bradley, Ed.D. Deputy Superintendent James S. Fugman, Ed.D. Deputy Superintendent Virginia R. Boris, Ed.D Associate Superintendent Daniel E. Kaiser, Ed.D. Assoclate Superintendent William C. McGuire

Associate Superintendent

\section{Governing Board}

Elizabeth J. Sandoval President

Sandra A. Bengel

vice President

Jim Van Volkinburg,

D.D.S.

Clenk

Brian D. Heryford Ginny L. Hovsepian Richard Lake, C.P.A. Susan $M$. Walker,

D.H.Sc.

\section{Description of Facilities and Major Equipment}

September 2000

One of the goals of the CUSD Agriculture Program is to become a technology model for secondary agricultural education. Currently, the

Ag. Center possesses what may be the most extensive integrated of any program in the state. The following lists contain current

Facilities, and Major Equipment available to students within the CUSD Agriculture Program.

1. Animal Science Facilities

a. Beef and Sheep Facilities

Currently beef projects are housed in conjunction with sheep projects within a Ruminant Unit at the Ag Center. The unit contains 8 large beef stalls and 16 sheep pens, processing facilities, student locker rooms, equipment storage, and feed storage. Full environmental controls consist of 8,30 " box fans, and misters. All environmental controls are operated using programmable sensors. Master site plan makes provisions for a separate sheep unit located to the north of current temporary swine facility.

b. Swine Facilities

Currently the swine projects are housed in a temporary facility that will used at a later time for hay and equipment storage. At this

"A compelling image of an achievable future." 


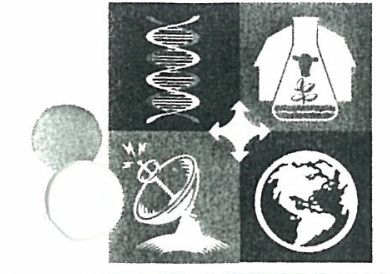

$C$ e 1 t 1 a 1

A p p 1 i e d

a $\mathbf{n} \mathbf{d}$

8180 E. Donner Clovis, CA 93611

559.327.4320

559.327.4063 Fax

Reagan Educational Center Admimistration

Linda Hauser

Assistant Superintendent

Lyn Snauffer

Assistant Adminfstrator

Melissa Macon

Director, Qualley Studene

Seruices

Jeff Eben

Principal, Clovis Ease High

School

Stacy Dumaicliff

Principal. Reyburn

intermedite

lovis Unified School

jistrict

Administration

Walter L. Buster, Ed.D.

Superintendent

Terry Bradley, Ed.D.

Deputy Superintendent

James S. Fugman, Ed.D.

Depuey Superintendent

Virginia R. Boris, Ed.D

Assocfate Superintendent

Daniel E. Kaiser, Ed.D.

Associate Superintendent

William C. McGuire

Assocfate Superintendent

\section{Governing Board}

Elizabeth J. Sandoval

President

Sandra A. Bengel

Vice President

Jim Van Volkinburg,

D.D.S.

Clenk

Brian D. Heryford

Ginny L. Hovsepian

Richard Lake, C.P.A.

Susan M. Walker,

D.H.Sc.
V a 11 e $y$

A griculture

(Swine facilities continued)

time the swine unit consists of a show ring 10 pens and a with a estimated capacity of 100 head of market hogs.

c. Poultry Facilities

The master site plan has made provisions for a Poultry Unit that can be used for three purposed. Facility and equipment may be used to house student projects, house a grower operation of 2,800 birds every 8 weeks, or a layer operation. Currently the Ag Center houses student projects within coops that have been constructed on site by students.

2. Plant Science Facilities

The master site plan makes provisions for the plant science facilities to consist of two Crayvo Duel Zone Greenhouses with full environmental controls. Each growing environment will be monitored by a central computer housed within the Ornamental Horticulture Facility. The $\mathrm{OH}$ facility will consist of a classroom as well as plant tissue lab with laminar flow hoods. The current and future irrigation systems will be contained within this facility with wireless links via PDA. A current 5 -acre pasture although utilized by the animal units will be maintained in conjunction with and under the full authority of the plant science and $\mathrm{OH}$ facility manager. 


\section{$C$ e n t a 1 V a 11 e y \\ A p p 1 i e d A griculture}

\section{Tech o o logy $\mathrm{C}$ enter}

8180 E. Donner Clovis, CA 93611 559.327 .4320

559.327.4063 Fax
Reagan Educational Center Administration

Linda Hauser

Assistant Superintendent Lyn Snauffer

Assistant Administrator Melissa Macon

Director, Quality Serdent Seruices

Jeff Eben Principal, Clouis Ease High
School

Stacy Dunnicliff

Principal, Reyburn

ntermedite

Clovis Unified School District

Administration

Walter L. Buster, Ed.D. Supenintendent

Terry Bradley, Ed.D.

Deputy Superintendent

James S. Fugman, Ed.D.

Deputy Superintendent

Virginia R. Boris, Ed.D

Associate Superfntendent

Daniel E. Kaiser, Ed.D.

Associate Superintendent

William C. McGuire

Associate Superintendent

\section{Governing Board}

Elizabeth J. Sandoval President

Sandra A. Bengel

Vice President

Jim Van Volkinburg,

D.D.S.

Clenk

Brian D. Heryford

Ginny L. Hovsepian

Richard Lake, C.P.A.

Susan M. Walker,

D.H.Sc.
3. Academic Units

Currently the academic portion of the program operates within 4 classrooms on site. Each of the classrooms is wired for network capabilities. The Academic Block consists of the following;

a. Room 901. Triditional Classroom to house 36 students. Room is fully networked for computer use in class.

b. Room 902. Distance Learning Classroom to house 36 students. This is the most advanced video conferencing room at the secondary level in the valley and perhaps the state. The room is equipped to broadcast and receive real time internet communication for Video, Audeo, and Graphics. At this time it is the most logical avenue for regional use of our facility.

c. Rooms 903-904. Biology Laboratory Classrooms to house 36 students. Each shared laboratory Prep Room with fume hood and storage for equipment and supplies. Each Lab contains an Environmental Chamber for class based plant and animal projects.

4. Agricultural Mechanics Facilities

a. Currently our Agricultural Mechanics Facilities consist of a classroom for 36 students, a special projects shop, and main shop with restroom facilities. This 7000 square foot of the facility contains approximately $\$ 100,000$ in equipment and materials. 


\section{$C$ entra $1 \quad V$ a 11 e y}

A p p i e d A griculture and T e c h n o 1 ogy $\mathrm{C}$ e nter

8180 E. Donner Clovis, CA 93611

559.327 .4320

559.327.4063 Fax

Reagan Educational Center Admimistration

\section{Linda Hauser}

Assistant Superintendent Lyn Snauffer

Assistant Adminiserator

Melissa Macon

Director, Quallty Student

Seruices

JeffEben

Principal, Cloufs Ease High School

Stacy Dunnicliff

Principal, Reyburn

intermedice

lovis Unified School

District

Administration

Walter L. Buster, Ed.D. Superintendent

Terry Bradley, Ed.D.

Deputy Superintendent

James S. Fugman, Ed.D.

Deputy Superintendent

Virginia R. Boris, Ed.D

Assocfate Superintendent

Daniel E. Kaiser, Ed.D.

Assocfate Supenintendent

William C. McGuire

Associate Superintendent

\section{Governing Board}

Elizabeth J. Sandoval

\section{President}

Sandra A. Bengel

Vice President

Jim Van Volkinburg,

\section{D.D.S.}

Clenk

Brian D. Heryford Ginny L. Hovsepian Richard Lake, C.P.A. Susan M. Walker, D.H.Sc.

\section{Five Year Facility and Equipment Acquisition Schedule}

September 2000

Phase I of the Central Valley Applied Agriculture and Technology Center was completed in August of 2000. This phase consisted of an Academic Block of 4 classrooms, Agricultural Mechanics Facility, Animal Facilities (beef, sheep, swine), 5-acre pasture, and master site work for the current 11-acre center. The completion of Phase II will be determined by the outcome of a school bond in March of 2001. The following Five-year plan is based on acceptance of this bond measure.

1. 2000-2001

a. Landscaping of Ag Center

b. Purchase Lab Equipment

c. Construct Greenhouses

d. Purchase $2^{\text {nd }}$ Van with Ag Booster Funds

e. Complete Pasture Irrigation

2. 2001-2002

a. Begin Construction

i. Sheep Facilities

ii. Swine Facilities

iii. Poultry Facilities

iv. Ornamental Horticulture Facilities

b. Purchase Walk-In Freezer and Cooler Unit

c. Purchase Textbooks for New Classes Developed

d. Acquire New Tractor and Implements

3. 2002-2003

a. Complete Equipment Acquisition for Shop

b. Complete payment of $2^{\text {nd }}$ Van

c. Purchase 1 Ton Truck

d. Purchase $\mathrm{OH}$ Equipment

e. Purchase Video Surveillance System for Facility

"A compelling image of an achievable future." 


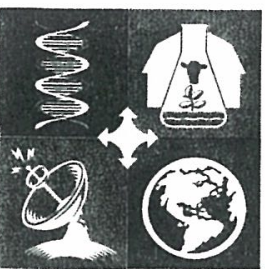

$C$ e in trall 1 V a 11 e $y$

A p p 1 ile d A griculture

and $T$ e c h n o $10 \mathrm{gy} \quad \mathrm{C}$ e nte r

8180 E. Donner

Clovis, CA 93611

$\mathbf{5 5 9 . 3 2 7 . 4 3 2 0}$

559.327.4063 Fax

Reagan Educational

Center Administration

Linda Hauser

Asafstant Superintendent Lyn Snauffer

Assistane Aoministraton

Melissa Macon

Director, Qualley student Seruices

Jeff Eben Principal, Clouis Ease High
School

Stacy Dunnicliff

Principal, Reybuan

ptermedate

Iovis Unified School District

\section{Administration}

Walter L. Buster, Ed.D. Superintendent

Terry Bradley, Ed.D.

Deputy Superintendent

James S. Fugman, Ed.D.

Deputy Superintendent

Virginia R. Boris, Ed.D

Associate Superintendent

Daniel E. Kaiser, Ed.D.

Assocfate Superintendent

William C. McGuire

Assoclate Superintendent

\section{Governing Board}

Elizabeth J. Sandoval

President

Sandra A. Bengel

Vice President

Jim Van Volkinburg,

D.D.S.

Clenk

Brian D. Heryford

Ginny L. Hovsepian

Richard Lake, C.P.A.

Susan M. Walker,

D.H.Sc.
4. 2003-2004

a. Acquire 10 Acres east of current facility

b. Purchase portable Video Conferencing Equipment for use in the farm laboratories.

c. Replace equipment used in transition from old facility.

d. Purchase reference materials for new classes developed 5. 2004-2005

a. Complete payment of 1 Ton Truck

b. Develop newly acquired land to include trees, vines, and experimental crops.

c. Acquire new Orchard Tractor and Implements 


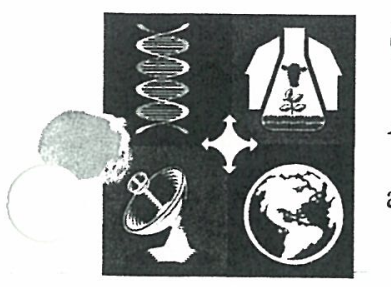

C e n t r a 1

A p p 1 i e d

a $n d$
V a 11 e y

A griculture
Kevin D. Woodard Department Chairman

8180 E. Donner

Clovis, CA 93611

559.327 .4324

559.327.4063 Fax

Reagan Educational Center Administration

Linda Hauser

Area Superintendent

Joe Hernandez

Assistant Administrator

Jeff Eben

Principal, Clovis East High

School

Stacy Dunnicliff

Principal, Reyburn Intermediate

Melissa Macon

Deputy Principal

Clovis Unified School District

Administration

alter L. Buster, Ed.D.

uperintendent

ferry Bradley, Ed.D.

Deputy Superintendent

James S. Fugman, Ed.D

Deputy Superintendent

Virginia R. Boris, Ed.D

Associate Superintendent

Daniel E. Kaiser, Ed.D.

Associate Superintendent

William C. McGuire

Associate Superintendent

Governing Board

Elizabeth J. Sandoval

President

Sandra A. Bengel

Vice President

Jim Van Volkinburg, D.D.S

Clerk

Brian D. Heryford

Ginny L. Hovsepian

Richard Lake, C.P.A.

Susan M. Walker, D.H.Sc.

\section{FIVE YEAR \\ EQUIPMENT ACQUISTION SCHEDULE CLOVIS UNIFIED AGRICULTURE DEPARTMENT}

1. 2001-2002
a. Begin construction on greenhouses.
b. Complete Landscaping
c. Purchase pasture fencing
d. Install irrigation throughout facility
e. Purchase pressure washer
f. Purchase 8 computers for rooms 901 and 904
g. Purchase textbooks

2. 2002-2003
a. Begin construction on Biotech Facility
b. Purchase walk-in cooler
c. Construct callous chamber
d. Pay final payment on $2^{\text {nd }}$ van
e. Purchase 8 computers for rooms 901 and 904
f. Implement acquisition schedule of Federal Technology Grant

3. 2003-2004
a. Complete construction of Biotech Facility
b. Begin Construction of Swine Unit
c. Purchase 2 environmental chambers
d. Purchase 2 incubators
e. Continue acquisition schedule of Federal Technology Grant
f. Purchase New Truck

4. 2004-2005
a. Acquire 10 Acres east of current site
b. Purchase new tractor with implements
c. Continue acquisition schedule of Federal Technology Grant
d. Complete construction of Swine Unit
e. Update computer technology purchased in last 4 years

5. 2005-5006
a. Complete payment schedule for new truck
b. Develop new site to include trees, vines, and demonstration plots
c. Acquire new orchard tractor and implements 
8180 E. Donner Clovis, CA 93611

559.327 .4320

559.327.4063 Fax

Reagan Educational Center Administration

\section{Linda Hauser}

Assistant Superintendent

Lyn Snauffer

Assiscant Adminlstrator

Melissa Macon

Director, Qualley Student Serufces

JeffEben

Paincipal, Clouis Ease High

Stacy Dunnicliff

Principal, Reyburn

intermedrate

\section{Jlovis Unified School} Jistrict

\section{Administration}

Walter L. Buster, Ed.D. Superintendent

Terry Bradley, Ed.D. Deputy Superintendent

James S. Fugman, Ed.D. Deputy Superintendent Virginia R. Boris, Ed.D Assoclate Superintendent Daniel E. Kaiser, Ed.D. Associate Superintendent William C. McGuire

Assoclate Superintendent

\section{Governing Board}

Elizabeth J. Sandoval President

Sandra A. Bengel

Vice President

Jim Van Volkinburg,

D.D.S.

Clenk

Brian D. Heryford

Ginny L. Hovsepian

Richard Lake, C.P.A.

Susan $M$. Walker,

D.H.Sc.

\section{Proficiency Standards for Program Completers}

September 2000

Clovis Unified School District's Agriculture Program is currently reformatting its curriculum to better align with state standards. It is the goal of the faculty to develop a Student Portfolio Program that will reflect Proficiency Standards obtained. Each student will maintain a competency checklist. This checklist when merged with a database program will generate student competency report that will follow them upon exiting the program. The following is a sample program proficiency documentation report. 


\section{ANIMAL HUSBANDRY SKILLS}

\section{$\underline{\text { Physical Skills }}$}

1. Mixing rations for all classes for all purposes

2. Feeding all classes, according to purpose using approved practices

3. Use breeding devices, crates, chutes, etc.

4. Assist at parturition by correcting abnormal presentations

5. Use of veterinary syringe

6. Use balling gun and balling forceps

7. Vaccinate for common diseases

8. Spray, dust, and dip for common external parasites

9. Use the various drenching devices for internal parasites

10. Mixing and/or the use of pre-mixes for the administration of biologics for disease control and prevention

11. Treat common diseases and ailments appropriate to herdsmen

12. Use cannula and trocar for the treatment of bloat in cattle and sheep

13. Use animal thermometer

14. Apply the proper sanitation practices for disease and parasite control and prevention

15. Establish and stock a medicine cabinet

16. Grade fat and feeder animals

17. Operate livestock scales

18. Brand livestock

19. Castrate

20. Set out salt

21. Trim feet

22. Take blood samples

23. Fill out an entry blank for shows

24. Show animals

25. Use a masculator

\section{Managerial Skills}

1. Compute rations for all classes for all purposes

2. Selecting ingredients for rations for all purposes

3. Identify and know characteristics of common feed stuffs

4. Determining feeding schedules

5. Know how to bring animals on full feed and to prevent and/or handle off-feed problems

6. Maintaining feed consumption and cost records

7. Interpreting feed tags

8. Selecting feeding equipment 
9. Determine valve and methods of feeding by-products

10. Compute and compare costs of common feed ingredients according to market price and nutritive value

11. Compute feed conversion ratio

12. Decide when and how to buy feed

13. Be able to judge fat, feeder and breeding animals

14. Selecting all classes of animals on the basis of type

15. Selecting all classes of animals on the basis of pedigree

16. Selecting all classes of animals on the basis of performance

17. Recognize and identify all common breeds of all classes

18. Know characteristics and adaptability of all common breeds of all classes

19. Know proper breeding age and appropriate breeding season for all classes

20. Recognizing heat or estrus in all females

21. Know gestation period for all classes

22. Recognize the signs and symptoms of approaching parturition

23. Supervise and assist at time of parturition

24. Know and evaluate the systems of breeding

25. Know and evaluate the methods of mating

26. Set up and maintain breeding records

27. Know care and management of young animals

28. Know how to fill out registration blanks

29. Complete a transfer of registration blank

30. Interpret a pedigree

31. Know the life cycle of the common internal and external parasites and when to break the cycle

32. Select the appropriate drug or medication to treat for common parasites

33. Complete a breeding certificate

34. Know the management practices for the control and prevention of common diseases and parasites

35. Recognize common diseases

36. Select suitable vaccines for the prevention of common diseases

37. Determine the age, season and exposure conditions that influence the vaccination schedule

38. Be familiar with normal body temperature of animals

39. Select disinfectants appropriate to purpose

40. Recognize when and how to effectively use the services of the veterinarian

41. Calculate daily rate of grain

42. Compute cost of producing 100 pounds of grain

43. Recognize factors involved and calculations of shrinkage

44. Calculate feed cost for 100 pounds of grain

45. Know dressing percentages and how to calculate

46. Compute per cent offspring produced

47. Know and recognize classes and grades of fat and feeder animals

48. Know the primal cuts of livestock carcasses

49. Know the names of the parts of a live animal

50. Set up and maintain a system of production records on an animal unit basis 
51. Establish a complete budget for a livestock operation

52. Establish a calendar of operations for a livestock program

53. Recognize the effects of the longterm cycle and seasonal cycles as an influence of supply

and demand on livestock prices

54. Select time and method to most effectively market livestock and livestock products

55. Recognizing the factors influencing margin and necessary margin

56. Calculate necessary margin

57. Select a ranch to buy, lease or rent

58. Determine the carrying capacity for a pasture, range, and/or a ranch

59. Calculate animal units and animal unit months

60. Be familiar with the space requirements for buildings, corrals, and equipment for all classes

61. Select the appropriate types of buildings and equipment

62. Calculate the storage space required for feeds

63. How to apply for a brand

64. How to use research materials

65. Know laws and regulations pertaining to livestock

66. Organize a layout for efficient livestock operations 


\section{$\underline{\text { Skills for Beef Students }}$}

\section{BEEF}

1. Select cattle on the basis of type

2. Select cattle on the basis of pedigree

3. Select cattle on the basis of performance

4. Identify common feed stuffs

5. Compute balanced rations for cattle of all ages, for all practical purposes

6. Compute average daily grain

7. Compute feed conversion ratio

8. Vaccinate for common diseases

9. Spray, dust and dip for common external parasites

10. Worm cattle, using boluse, drench and feed

11. Trim feet

12. Wash, curl and groom show and sale cattle

13. Mix feed

14. Know life cycle of common internal and external parasites, when to break the cycle and what medication to use

15. Select a cattle ranch to buy, rent or lease

16. Draw plans for complete working corrals

17. Build cattle feeding and working equipment

18. Compute cost of producing 100 pounds of beef

19. Compute calf crop per cent

20. Castrate calves

21. Dehorn cattle

22. Brand cattle

23. Judge beef cattle

24. Show beef cattle

25. Recognize when to call the veterinarian

26. Know how to use the veterinarian

27. Grade Slaughter cattle

28. Know primal cuts of the beef carcass

29. Know how and when to market cattle

30. Be familiar with space requirements for cattle buildings and equipment

31. Calculate a budget for a beef operation

32. Know the names of the parts of a live animal

33. Be familiar with space requirements for cattle buildings and equipment

34. Draw plans for beef buildings and equipment, including corrals, barns, feed bunks, watering devices, etc.

35. Adjust and evaluate weaning weights for a.) age b.) sex and c.) age of dam

36. Compute weight per day of age (WDA) at 365 days and evaluate results

37. Use an index as a selection aid

38. Set up and use a set of financial records for a cow herd

39. Set up and use a set of labor records for a cow herd

40. Set up and use a set of production records for a cow herd 
41. Analyze financial, labor and production records for a cow herd

42. Understand and use the concepts of animal units and animal unit months as related to managerial decisions and analysis

43. Prepare a calendar of operations for a cattle enterprise

\section{$\underline{\text { Physical Skills }}$}

\section{HORSE}

1. Assisting the mare during foaling

2. Saddle training for a specific job

3. Using brushes, curry-comb, and blankets

4. Using a lariat

5. Selecting and using cutting horse

6. Selecting and using trail horse

7. Calculating housing requirements

8. Selecting and using foaling stalls or sites

9. Applying disinfectants to new-born colt's navel

10. Saddle a horse

11. Ride a horse

\section{$\underline{\text { Managerial Skills }}$}

1. Detecting the mare's heat period

2. Weaning a colt

3. Using halter-breaking equipment

4. Using saddle-breaking equipment

5. Selecting a lariat

6. Identifying physical defects and unsoundness in horses 


\section{Physical Skills}

\section{SHEEP}

1. Dock lambs

2. Move ewes and young lambs

3. Tie a fleece and pack a bog of wool

4. Dodge sheep through chute

5. Use a lamb jail

6. Skin a dead lamb

7. Make a gunny sack blanket for sheep

8. Grind clipper blades

9. Using sheep handling devices and equipment

10. Using ear-marking equipment for sheep

11. Identifying burr-bearing weeds in the sheep pasture

12. Training a sheep dog

13. Removing dung tags from ewes prior to breeding

14. Using materials and devices to get a ewe to claim her own or other lambs

15. Shear sheep

16. Using lambing pens

17. Using lamb forceps

18. Using castrating equipment

19. Using docking equipment

20. Using drenching equipment

21. Massaging and treating ewe's inflamed udder

22. Sorting and tying wool

23. Using sheep grooming equipment

24. Determining age of sheep

25. Blocking sheep

26. Holding and moving sheep

27. Using sheep butchering equipment

28. Paint brand sheep

29. Loading sheep

\section{$\underline{\text { Managerial Skills }}$}

1. Grading mutton and lamb

2. Identifying cuts of mutton and lamb

3. Grading wool

4. Maintaining and interpreting wool production records

5. Selection of replacement ewes

6. Preparing flock for breeding season

7. Preparing flock for lambing season

8. Selection of stud Rams 


\section{SWINE}

\section{$\underline{\text { Physical Skills }}$}

1. Stationing self-feeders and watering troughs

2. Using devices to clip needle teeth

3. Using ear-notching equipment for swine

4. Using ear-tagging equipment for swine

5. Holding a pig during castration

6. Castrating a boar with a knife

7. Using hog house cleaning equipment and materials

8. Installation of a greased "rubbing post"

9. Using nose-ringing devices and materials for hogs

10. Using hog-loading devices

11. Applying artificial respiration to new born pigs

12. Using pig washing equipment and materials

13. Using hog grooming equipment

14. Using butchering equipment for swine

15. Know how to ear-notch swine

16. Know numbers involved in ear-notching for swine

17. Using a breeding chute

18. Hand mating of boar

19. Giving injections to swine

20. Blood testing

21. Using hand sprayer for external parasite control

22. Know how to exhibit swine

23. Detecting heat periods

24. Selection of replacement of gilts

25. Identify undesirable traits in swine

26. Know how to measure a swine carcass for certification

27. Getting pigs started on feed

\section{ARTIFICIAL INSEMINATION}

\section{Collecting}

1. Know how to work with bulls (know their habits)

2. Know how to get the bull ready for collecting

3. Know how to get the area ready in which you are going to collect

4. Know how to get the collecting equipment ready

5. Know how to put the equipment together

6. Know how to use the equipment in the proper way

7. Know how to keep the equipment sterile 
8. Know how to keep the semen clean and free from foreign bodies

9. Know what will kill semen

10. Know how to transport semen from collecting area to laboratory

11. Know types of lubricants and how to use them

12. Know correct temperature and amount of water to use in the artificial vagina

13. Know how to clean the sheath of the bull

14. Know the proper technique of collecting the bull

15. Know how to use electrical ejaculator

\section{Processing and Handling}

1. Know how to evaluate semen

a. Visual

b. Microscopic

c. Hemocytometer

d. Photelometer

2. Know how to rate the semen on motility

3. Know how to estimate the number of sperm in semen

4. Know what abnormal sperm looks like

5. Know how to dilute semen

6. Know the common types of diluent used

7. Know how to clean and keep the glassware sterile

8. Know how to use laboratory equipment, freezing, and handling used for semen processing

9. Know how to use antibiotics in semen

\section{Freezing Semen}

1. Know the steps in freezing semen

2. Know how the sperm is protected in freezing process

3. Know the percent of sperm usually killed in freezing

4. Know the characteristics of liquid nitrogen

5. Know the temperature required for storing semen

6. Know what equipment is used in freezing semen

7. Know what rate to dilute semen for freezing

8. Know how long semen will live after freezing

\section{Inseminating}

1. Know the various parts of the reproductive organs

2. Know where the reproductive organs are located in the cow

3. Know where to deposit the semen

4. Know how to remove the semen from the liquid nitrogen

5. Know how to thaw semen

6. Know how to open the vial

7. Know how to read the information on the vials 
8. Know the color code used for the various breeds

9. Know how to lubricate your gloves

10. Know how to stand when breeding the cow

11. Know how to draw the semen from the vial

12. Know how to clean the cow before inserting the tube

13. Know how to insert the tube into the cow

14. Know how to work the tube through the cervix

15. Know how to get all of the semen out of the tube into the cow

16. Know the general cleanup of equipment and area after breeding the cow

\section{Record Keeping}

1. Know how to fill out breeding receipts for both grade cows and registered cows

2. Know how to fill out the breeding records for the dairyman

3. Know how to keep good and accurate records

\section{Salesmanship}

1. Know the steps in selling

2. Know how to talk to your customer

3. Know the advantages of your trade and how to sell them to your customer

4. Know how to use the breeding company's literature

\section{General Skills in Artificial Insemination}

1. Know how long your liquid nitrogen will last in your tank

2. Know what you can and cannot do as a technician, (Veterinary Practice Act)

3. Know the heat cycle of cattle

4. Know the common reproduction diseases of cattle

5. Know the common breeds of cattle and their characteristics

6. Know the various ways bulls can be classified

7. Know the characteristics of an outstanding sire

8. Know how to explain sire records of livestock to dairymen

9. Know how to tell when a cow is in heat

10. Know how to tell if a cow is pregnant

11. Be familiar with the characteristics of dairy and beef cattle

12. Be familiar with the common problems of dairymen

13. Be familiar with the common problems of beef cattle

14. Be able to analyze the weak points of an animal and select a bull which should improve the weak points

15. Be able to saddle and ride a horse

16. Be familiar with fitting and grooming dairy and beef cattle

17. Be familiar with the showing of dairy and beef cattle

18. Be familiar with common types of dairy barns and equipment in the area 
19. Be familiar with common beef breeding operations found in California

20. Be familiar with milk production

21. Be familiar with beef cattle feeding programs

22. Be familiar with dairy cattle feeding programs

23. Be familiar with common methods used to identify animals

24. Be familiar with methods used in testing beef for performance

25. Be familiar with the methods used in proving dairy bulls

26. Be familiar with methods of housing and handling bulls for artificial insemination organizations

27. Be familiar with methods of raising calves

28. Be familiar with proper report writing

29. Be able to speak before farm groups

30. Be able to give demonstrations to various groups

\section{Physical Skills}

\section{DAIRY}

1. Attaching anti-fence jumping devices

2. Identifying colostrum milk

3. Washing, massaging, stimulating cow's udder

4. Using a strip cup

5. Using milking hobbles

6. Hand milking and stripping

7. Attaching the milking machine to the cow

8. Using machine-stripping techniques

9. Using milking utensil washing equipment and materials

10. Reading vacuum pressure gauge on milk machine

11. Regulating milking machine vacuum

12. Using a dipper to take a milk or cream sample

13. Adjusting and reading a milk scale

14. Using a sampling tube to take a milk or cream sample

15. Using home pasteurizing equipment

16. Using dairy cattle clippers

17. Applying antiseptic to navel of new-born calf

18. Using a hypodermic syringe

19. Using milk tubes, teat plugs, dilators

20. Destroying carcasses

21. Culling

22. Scoring dairy barns

23. Identifying breeds

24. Using breeding racks

25. Grading hay

26. Collecting and handling semen

27. Testing milk for solids and acid

28. Testing skim milk and cream for butterfat 
29. Operating refrigerators and coolers

30. Photographing dairy cattle

31. Filling silos

32. Caring for manure

33. Using tape to determine heart girth and weight

34. Clipping hair on flanks and udder

35. Training animal to lead and stand

36. Removing excess teats

37. Interpret feed tag information

38. Using nose tongs

39. Using bull yoking devices

40. Using bull exercise equipment

41. Attaching anti-sucking equipment

42. Using ear-notching equipment

43. Using bull leading equipment

44. Detecting a cow's heat period

45. Using hair brushing and other grooming equipment

46. Selecting and using animal blankets

47. Scoring cream, milk, butter

48. Using a butter trier

49. Filling out a registration blank

50. Filling out a transfer or registration

51. Pulling calves

52. Disinfecting navel of calf

53. Wiping mucus from nostrils and dry

54. Spraying and controlling flies

55. Dehorning calves

a. Caustic method

b. Clippers

c. Electric

56. Dehorning cows and bulls
a. Saw
b. Clippers

57. Stopping bleeding after dehorning

58. Tattooing cattle

59. Branding cattle

60. Neck chaining cattle

61. Trimming hooves on cattle

62. Casting large dairy animals

63. Caring of heifers and cows before and after calving

64. Getting a new-born calf to breathe

65. Teaching calf to drink from bucket or nipple

66. Teaching calf to eat grain and hay

67. Bedding down calves

68. Estimating age of dairy cattle 
69. Castrating cattle

70. Hobbling a bull

71. Building a bull pen

72. Building a calf house

\section{$\underline{\text { Managerial Skills }}$}

1. Selecting and using milking utensil sterilizing materials

2. Selecting and mixing dairy cattle

3. Selecting and using barn cleaning equipment

4. Selecting and using dairy barn and dairy cattle spraying equipment

5. Selecting and using bar disinfecting materials and equipment and sprays

6. Calculating housing requirements

7. Knowing diseases to vaccinate for in this area

8. Selecting and using disease treatment medicines, ointments, salves

9. Recognizing disease and ailment conditions

10. Prevention and treatments of udder ailments

11. Selecting herd sires

12. Selecting replacements

13. Figuring rations
a. calves
b. heifers
c. cows

14. Judging dairy cattle male and female and different age groups

15. Keeping records
a. herd production
b. breeding and calving
c. feed
d. health
e. inventory

16. Computing cost of feed

17. Planning diary barns
a. calf pens
b. bull pens
c. hay barns and shed

18. Planning layout of farm

19. Recognizing and treating common ailments
a. acetonemia
b. mastitis
c. ringworm
d. scorus
e. pinkeye
f. bloat
g. footrot
h. milk fever 
i. lice

j. warts

20. Marketing dairy products

21. Marketing purebred and grade dairy cattle

22. Proving herd sires

23. Selecting and using breeding equipment

24. Selecting, mixing, and feeding ration materials

25. Interpreting a pedigree

26. Interpreting a production record

27. Planning a safe bull pen

28. Planning a calf house

29. Planning a feeding setup to cut down on labor

30. Knowing when to call the veterinarian

31. Know Grade "A" requirements

32. Knowing how to convert the price of milk to the price of butterfat 


\section{POULTRY}

\section{Physical Skills}

1. Wash birds

2. Hold birds

3. Use bird-killing equipment

4. Use scalding equipment

5. Hand-pick foul

6. Catch and properly handle birds

7. Use poultry house cleaning equipment

8. Cool eggs

9. Clean nests

10. Apply hatching egg fumigation

11. Enter houses without scaring poultry

12. Keep litter dry

13. Spread litter

14. Construct simple waters and feeders

15. Assemble automatic feeders and waters

16. Clean and disinfect brooder and layer houses (both dirt and cement floors)

17. Eviscerate poultry

18. Package poultry

19. Pack eggs for market

20. Clean eggs

a. machine

b. hand

1. detergent

2. sandpaper

21. Collect eggs
a. litter floor
b. cages

22. Prepare hover for brooding
a. clean heating element
b. check and adjust thermostat
c. adjust height of hover

23. Treat for mites

24. Treat for lice

25. Chemically caponize cockerels

26. Move
a. feeders
b. shelters
c. waters
d. roost

27. Rotate range for turkeys 
28. Build nests for chickens and turkeys

29. Install automatic feeders and waters

a. range

b. litter floor

30. Treat for worms

31. Artificial inseminate turkeys

a. catch toms

1. strap on table

b. milk toms

c. catch hens

d. break hens

e. inseminate hens

32. Saddle turkeys

33. Debeak poultry

34. Spray for flies

35. Mix feed
a. lift sacks without straining
b. grind feed
c. weigh correct amount of feed
d. run mixer
e. fill sacks

36. Repair automatic lighting equipment

37. Set up wire guards and paper guards for brooding poultry

38. Repair cages

a. doors, etc.

39. Adjust automatic waters

40. Vaccinate

41. Treat diseases with De Hydro-streptomycine

42. Repair brooders

43. Blood test for pollorum

44. Legband birds and wingband chicks

45. Drive electric cart

46. Install thermostat switch

47. Sight level with instrument when leveling cages and water system

48. Hang cages

49. Brace cages with wire from ceiling

50. Install automatic feeders

a. clamp feeders to stacks

b. drive short angle iron stacks

c. lay in dragging chain

d. hook chain to sprocket

e. hook sprocket to motor an pulley

51. Assemble cages

52. Install lights

a. brooder house 

b. layer house

c. turkeys

53. Construct poultry building
a. raising house
b. squaring building
c. roofing

54. Cool birds
a. foggers
b. sprinklers
c. perforated hose

\section{Managerial Skills}

1. Selecting type, breed, and strain of bird

2. Selecting individual birds

3. Select hens for egg production

4. Interpret a feed tag

5. Keep a trap-nest record

6. Calculate numbers or roosters per 100 hens

7. Calculate nest requirements

8. Calculate brooder house requirements

9. Calculate laying house capacity

10. Control diseases and ailments

11. Select and apply disinfectants

12. Grade live fowl

13. Grade dressed fowl

14. Select healthy pullets

15. Cull hens

16. Plan a replacement program

17. Select hatching eggs from proper hens for breeding own strain of chickens and turkeys

18. Select good male birds from good families for strain crossing, etc.

19. Select chicks or hatching eggs from good hatchery or breeder

20. Decide best place to market eggs

21. Figure cost of ration

22. Use antibiotics for medication

23. Balance rations
a. chick starter
b. growing rations for chickens
c. layer ration
d. fryer
e. pullet
f. growing and fattening turkeys

24. Compute proper amount of feed space

25. Judge poultry

26. Select poultry house or ranch to buy 
27. Keep records

28. Select site for houses

a. layer

b. brooder

29. Select birds for show

30. Grade and candle eggs

31. Plan ventilation in old houses

a. brooder and layer houses

32. Compute per cent production and cost

33. Compute feed conversion

34. Compute cost of houses and equipment

35. Plan building

a. brooder house

b. layer houses

1. colony cages

2. single cages

3. double cages

4. litter floor

36. Plan lighting systems

37. Identify diseases and ailments

38. Make managerial decisions from poultry records

39. Determine time and place to market fryers

40. Select manufactured feed

41. Compute daily feed consumption

42. Manage birds that are off feed

43. Perform postmortem and diagnose

44. Use sulfa for coccidiosis

45. Use medication in water for disease control

46. Train chickens and turkeys to roost 


\section{Employability \& Careers}

Students shall appreciate the importance of Supervised Occupational Experience Programs in the total program of agricultural education, and develop those skills necessary to search, locate, and apply for employment, as well as building a portfolio.

1. Engage in an appropriate S.O.E., taking into consideration individual career goals, and maintain records in the California Vocational Agriculture Record Book.

2. Understand the importance of positive work ethics and values in the work place.

3. Develop a Portfolio which will include a resume, application, letter of application, letter(s) of recommendation, and work samples.

4. Develop interviewing and job keeping skills.

\section{Safety and First Aid}

Students will demonstrate safe work habits and develop first aid skills.

1. Understand that safety is on going and is practiced daily.

2. Pass appropriate safety tests on equipment students will be using.

3. Demonstrate the appropriate steps to take when handling emergencies.

4. Be familiar with potential hazards and OSHA regulations.

\section{Oxyacetylene/Oxy-Fuel Welding}

Students will be able to perform Oxyacetylene (fuel) welding tasks using appropriate metal preparation techniques, joints, and filler rods.

1. Use the Oxyacetylene (fuel) equipment to weld mild steel in flat, horizontal, and vertical positions.

2. Use the Oxyacetylene (fuel) equipment to weld pipe in each of the standard joints.

3. Braze weld the butt, fillet, and lap joints.

4. Braze weld pipe in the fillet position.

5. Make appropriate repairs to cast iron using cast iron and brass filler rods.

6. Properly apply other specialty rods including but not limited to hard surfacing.

7. Become familiar with different types of Oxy-Fuel welding systems.

\section{Shielded Metal Arc Welding}

Students will be able to perform arc welding tasks using appropriate joints, electrodes, metal preparation techniques, and weld current.

1. Arc weld mild steel in flat, horizontal, vertical and overhead positions.

2. Arc weld pipe in each of the standard positions.

3. Make repairs to cast iron using nickel rod.

4. Properly use other specialty rods including those with applications for but not limited to hard surfacing.

5. Properly set the amperage and type of current for the specific application and rod being used.

\section{Metal Cutting}

Students will demonstrate proficiently with various metal cutting techniques.

1. Use the Oxyacetylene (fuel) torch to make cuts in plate and sheet metal using combination, drag, and gouging tips.

2. Make joint preparations for steel using the Air Arc.

3. Cut non-ferrous metals using the Air Arc. 
4. Cut non-ferrous and nonferrous metals using a plasma arc system.

5. Cut material to length using the hydraulic shear.

6. Make square and angle cuts on different shapes of metal using the band saw, abrasive cut off saw, and /or cold cut saw.

7. Make a pattern for the pantograph - Oxyacetylene duplicator.

8. Use Oxyacetylene pattern cutting machine to cut patterns in mild steel plate.

9. Use Plasma Arc pattern cutting machine to cut patterns in mild steel plate.

\section{Power Tools}

Students will be proficient in the use and application of power tools.

1. Bore and countersink holes in various materials using the portable electric drill, pneumatic drill, and drill press.

2. Grind, buff, and sand metal projects using portable and stationary grinders and sanders.

3. Cut sheet metal using shears, nibblers, saber saw, and circular saw.

4. Demonstrate the use of a manual or hydraulic bending unit (portable or stationary) on angle iron, pipe, flat and round stock.

5. Use a hydraulic iron worker to shear and punch flat stock and angle iron, and to notch pipe.

\section{Project Layout}

Students will become proficient in laying out holes, cuts and angles on metal for specific applications

1. Layout holes and select the correct tools for boring various metals and shapes.

2. Layout square cuts, angles, and compound angle cuts on various metals and shapes

\section{MIG Welding}

Students will demonstrate proficiency using the MIG welding process (GMAW).

1. Using the MIG welder and solid wire, demonstrate welding mild steel in various positions employing the basic joints.

2. Use the MIG welder and inner or outer shield wire to weld mild steel $1 / 4$ " or thicker in the flat position.

3. Demonstrate welding aluminum and/or stainless steel in the flat position using a MIG welder or spool gun.

4. Demonstrate proficiency at MIG machine maintenance and troubleshooting.

\section{TIG Welding}

Students will demonstrate proficiency using the TIG welding process (GTAW).

1. Using the TIG welding process, demonstrate various joints in the flat and horizontal position on aluminum and/or stainless steel.

2. Have knowledge of other unique or exotic metals that can be welded using the TIG process.

\section{Phase of Metals}

Students will understand the phases of matter and the role temperature and pressure play in the change of phase of metals.

1. Observe phase change of metal when they are subject to heat and be able to apply the knowledge in practical applications.

2. Define melting point, thermal conductivity, and grain structure. 
3. Describe the effect of cooling rate on the grain structure of steel.

4. Describe how materials behave under applied pressure and explain how to compensate for pressure problems in various situations where metals are used.

\section{Project Design and Construction}

Students will apply skills learned in welding, metal working, finish work, and project design to plan for and build a complex project.

1. Using actual cost estimates and drawings, complete a plan for a project including: working drawing, bill of materials, and sequence of operations.

2. Employing appropriate tools and skills, manufacture the parts and construct and finish a complex project.

\section{Project Finishing}

Students will demonstrate the skills necessary to finish a project in a professional way.

1. Finish a product to the degree appropriate for the intended use of the item made.

2. Complete the surface preparation of a metal project using proper tools and materials, sealers, paints, etc.

3. Carry a project to completion including all details involved in the production of the finished product.

\section{Alloys}

Students will understand the importance of the synthesis of new alloys which have properties necessary for specific applications and purposes.

1. Explain what alloy steel is and how it is manufactured.

2. List the common additive elements involved in alloy steel production.

3. Identify which qualities are affected by each additive in an alloy.

\section{Basic Machine and Foundry Principals}

Students will demonstrate knowledge and use of machine tools and basic foundry principles.

1. Use a milling machine to square and flatten a surface.

2. Use the mill to make a step, shoulder, and groove in a metal sample.

3. Use the milling machine to machine a key way on a shaft.

\section{Electronics}

Students understand and appreciate the role of electricity in the technological world.

1. Students define electric current and state the difference between conductors and insulators.

2. Students define voltage and amperage and describe their applications in agricultural mechanics

3. Students differentiate between alternating and direct current.

4. Explain the voltage drop and its effect on welding (application) current.

5. Differentiate between open-circuit voltage and are voltage.

6. Students become aware of the prevalence of electromagnetics by investigating the extent to which they are used in arc welders. MIG welders and other electrical shop equipment.

7. Students define electromagnetics and describe their application in the conversion of electrical energy into mechanical energy in electric motors.

8. Students explain the principles of the electric motor. 


\section{Measurement}

Students will understand and are able to read and use a ruler or tape and complete calculations for problems involving length, area, and volume and weight using American standard and metric measurements.

1. Correctly measure objects with a ruler, tape or framing square.

2. Calculate and solve basic measurement problems including, but not limited to the calculation of board feet, cubic measurement, and standard liquid measurement.

3. Differentiate between American standard and metric measurements units and use in solving problems requiring linear and volumetric calculations.

4. Use various methods to determine the mass and volume of regular and irregular shaped objects using American standard and metric systems.

\section{Project Design}

Students understand the basic skills required to make simple drawings and be able to interpret a working drawing.

1. Identify the three (3) types of drawings (orthographic, isometric, oblique).

2. Read blueprints including schedules, details, and symbols.

3. Understand the steps necessary to develop plans and apply for building permits and types of other permits related to the construction trade.

4. Interpret a surveyor's field book correctly when reading a map.

\section{Wood Structures}

Students will acquire the knowledge and skills necessary to design and construct wood structures.

1. Identify the woods, wood substitutes, and materials commonly used in the building of wood structures.

2. Read blueprints including schedules, details and symbols.

3. Make an estimate for a bill of materials using sketches and drawings for a wood structure included on a landscape plan.

4. Identify and use common carpentry tools for the construction and installation of wood structures.

5. Participate in the building of a wood structure.

\section{Concrete Structures}

Students will become aware of the types of concrete structures used in construction and develop an understanding of how they are installed.

1. Recognize and identify the types of concrete structures used in construction.

2. Develop and estimate the cost for a bill of materials for concrete structures using drawings, plans or blueprints.

3. Identify materials used in the construction of concrete structures.

4. Demonstrate mastery of basic masonry techniques used in the construction of concrete structures.

5. Identify and use basic masonry tools used in the construction of concrete structures.

6. Build a concrete item or structure including the building and eventual removal of the forms for the structure.

7. Determine the structural integrity of a project, by performing a slump test.

\section{Building Components}

Students will familiarize themselves with and be able to identify building components and design features. 
1. Identify foundation components.

2. Differentiate between new and old systems of floor joists.

3. Identify framing components and roof styles

4. Differentiate between types of trusses.

5. Identify by grade the common types of lumber used in construction.

\section{Framing and Forms}

Students will understand the procedures necessary to form or frame a particular structure or foundation.

1. Properly layout a building site with batter boards, lines, and stakes.

2. Be able to properly form, pour, reinforce, and finish a concrete foundation or slab.

3. Install floor joists and sills to specifications.

4. Layout, assemble, erect, and plumb a wall section.

5. Cut out and install common rafters and joists to specifications.

6. Be able to layout, setup, and install a truss.

\section{Fences}

Students will develop the knowledge and skills necessary to design, layout and construct simple fences used for home and farm applications.

1. Developing a fencing plan, taking in account all pertinent land considerations (e.g., topography property boundaries, etc.).

2. Properly layout a fence line.

3. Have a knowledge of fencing materials and their appropriate uses (i.e., wire, electric, wood, posts, gates, fasteners, pipe, and cables).

4. Repair a wood and/or wire fence.

5. Install a section of fence including a gate.

6. Perform a splice on a wire fence.

7. Properly anchor a fence line.

8. List factors to consider when installing an electric fence.

9. Align posts using a level or a transit.

\section{Surveying}

Students will develop and demonstrate a basic understanding of surveying as it is used in agricultural applications.

1. Perform various measurements for horizontal and vertical applications.

2. Set up and keep a field notebook.

3. Visually identify the builder's, engineer's, and turret levels.

4. Identify and use a direct reading and positive reading rod with an engineer's scale and an architect's scale.

5. Understand and identify applications for profile leveling.

6. Determine distance with the assistance of a transit.

7. Calculate cuts and fills for a given piece of property.

8. Layout a building site for a simple agricultural structure (e.g., pole barn).

9. Understand and be able to read a topographic map.

10. Explain how land is subdivided in California and the United States. 


\section{Irrigation Systems}

Students will develop the knowledge and skills necessary to design, layout and construct simple fences used for home and farm applications.

1. Differentiate between deep well turbine, low lift, and booster pumps.

2. Properly start and stop a deep well turbine pump.

3. Use and maintain the mechanical device necessary for irrigating crops.

4. Calculate the amount of irrigation water necessary for a field planted with a specified local crop.

5. Given the appropriate information, determine motor and pump efficiency levels.

6. Determine the flow, pressure, and application rates of irrigation water given specific information about the equipment used and crop to be irrigated.

7. Understand the principles of injector and vacuum systems used in irrigation.

8. Determine how to channel flow and alter pressure for various situations using relief valves, check valves, and pressure compensators.

9. Safely handle irrigation equipment and be able to determine when a technician is needed for repairs.

\section{Physical Properties of Materials.}

Students will explain how physical forces effect various types of materials.

1. Define stress and state how it can be measured.

2. Define strain and explain how it can be measured.

3. Differentiate between tensile and compressive strength and describe how each is used in evaluating metal, wood, and wood substitutes.

4. Describe the concept of elasticity of metals, wood and other materials and explain how this knowledge can be used in construction.

5. Differentiate between bending strength, shear strength, fatigue strength, and impact strength; give examples of each; and demonstrate the effects of one for the class.

\section{Energy}

Students will understand that energy is the ability to do work, that energy comes in many forms and can be converted from one form to another.

1. Identify the different forms of energy such as heat, light mechanical, and electromagnetic and cite examples of each found in their school or home environment.

2. Observe and describe how heat changes matter and determine whether heating will temporarily or permanently change the physical properties of an object.

3. Determine how energy can be channeled to work with the use of energy efficient building materials, environmental controls.

\section{Electricity}

Students understand and appreciate the role of electricity in the technological world.

1. Define electric current and state the difference between conductors and insulators.

2. Define voltage and amperage and describe the application of each in agricultural mechanics. 
3. Differentiate between alternating and direct current and identify appropriate applications for each.

4. Describe voltage drop and its effect on welding current, and motor/pump operation.

5. Differentiate between open-circuit voltage and closed circuit voltage.

6. Explain how capacitor start motors function and the principles of single and three phase operation.

7. Develop an appreciation for and general knowledge of new advances in electrical technology including solar energy storage systems and their practical uses.

\section{Electronics}

Students understand the relationship of magnetic forces and electrical currents and investigate the common uses of magnetism

1. Become aware of the prevalence of electromagnetics by investigating the extent to which they are used in arc welders, MIG welders, and other electrical shop equipment.

2. Define the term electromagnetic and describe applications in the conversion of electrical energy into mechanical energy in electric motor.

3. Explain the principles of operation for the electric motor.

4. Measure the current of motors and determine the efficiency of a specific motors.

5. Understand the principles and value of electrostatic application in agriculture. 


\section{Occupational Competencies}

Animal Care/Veterinarian Science

\section{Course Description:}

A two semester course, designed to prepare students with entry level skills in the animal care field such as: veterinarian aide, kennel, stables or zoo aides. After receiving classroom instruction in areas such as: behavior, handling, grooming, anatomy and physiology, nutrition, reproduction, and genetics. Students will find their own job site the second semester and will work 8 hours a week for three months for a veterinarian, zoo, kennel, ranch or horse facility.

\section{Occupational Competencies:}

\section{Animal Care}

1. A Able to identify breeds, species and sex of animals

B Can properly clean and maintain cages, pens and utensils

C Able to visually observe for illness and abnormal behavior

D Can properly groom various animals

E Can give a medicated bath

F Knows how to trim hooves and nails

$\mathrm{G} \quad$ Prepare and dispense food for animals

I Able to prepare animals for shipping and transport

$\mathrm{J} \quad$ Familiar with common diseases and symptoms in livestock and small animals

$\mathrm{K} \quad$ Know and identify the parts of animals

$\mathrm{L} \quad$ Be familiar with the different digestive tracts for livestock and small animals

$\mathrm{M}$ Identify classes of nutrients and their functions in animal nutrition

$\mathrm{N} \quad$ Know the gestation length, types of reproduction practices in livestock

$\mathrm{O} \quad \mathrm{Be}$ familiar with the endocrine system and the hormones affecting reproduction

\section{Safety}

2. A Can safely handle animals

B Knows proper lifting technique

C Practices good security habits for facilities, locks, latches, and gates

D Follows safe work habits and identifies potential hazards

\section{Examination Assistance}

3. A Demonstrates proper use of assorted restraints

B Demonstrates methods of safe capture

C Able to identify animals dispositions

D Able to correctly take animal vital signs

E Knows how to disinfect an area and equipment 


\section{Medication and Supplies}
4. A Understands label instructions
B Correctly measure prescribed amounts of medication
C Able to store properly and rotate stock
D Can dispose of expired products
E Able to administer medication safely by various methods
F Understands needles and syringes
G Knows procedures for drug related emergencies

I Familiar with common antibiotics, anthelmenics, vitamins

\section{Laboratory Procedures}

5. A Able to identify common parasites

B Can identify the uses of various lab tests

C Understands blood, urine and fecal analysis

D Understands the significance of various lab tests

E Knowledge of $\mathrm{x}$-ray procedures

\section{Treatment and Surgical Skills}

6. A Able to identify instruments

B Practices aseptic technique

C Able to perform surgical prep

D Can clean surgical instruments

E Can repack and autoclave a standard surgical pack

$\mathrm{F} \quad$ Able to clean up after surgery

$\mathrm{G} \quad$ Able to assist in some surgical procedures

\section{Customer/Public Relations}

7. A Can effectively work with clients

B Aware of appearance and manners

C Demonstrates proper telephone etiquette

D Knows how to write notes, memos and receipts

E Able to fill out client information cards

F Able to record animal data and observations

$\mathrm{G}$ Demonstrate basic computer knowledge

$\mathrm{H} \quad$ Able to deal with various personalities, conditions and attitudes

I Maintains a neat and organized work area

$\mathrm{J}$ Abel to communicate effectively with the public

\section{Professional Development}

8. A Demonstrates leadership potential

B Understands career options available and necessary preparation

C Able to fill out an application and prepare a resume

D Demonstrates positive job interview skills

$\begin{array}{ll}\text { E } & \text { Be an active member and participate in FFA activities } \\ \text { F } & \text { Responsible for a SOEP }\end{array}$ 
G Maintains a personal FFA record book related to projects

$\mathrm{H} \quad$ Works toward degree advancement and proficiency awards

I Develop a professional portfolio that reflects knowledge and competencies

$\mathrm{J}$ Participate in community classroom training relevant to career preparation

$\mathrm{K} \quad$ Completed a professional portfolio

\section{Work Attitudes}

9.

10.

A

B

C

D

E

$\mathrm{F}$

G

$\mathrm{H}$

I

$\mathrm{J}$

K

L

A

A

B

C

D Has a professional and business-like attitude

E Exhibits honesty and personal integrity

$\mathrm{G}$ Shows good judgement and common sense in problem solving

$\mathrm{H} \quad$ Is mature and reliable

I Shows positive and cooperative attitude
Accepts constructive criticism in a positive manner

Follows directions well

Exhibits good attendance and punctuality

Works well with minimum of supervision

Is dependable and readily accepts responsibility

Respects authority

Wears clothing appropriate for the job

Utilizes spare time constructively

Works quickly and efficiently

Has a desire to learn and improve

Displays initiative

Is well organized 


\section{R2 Teacher Information Clovis East HS, Clovis Year: 2012}

\begin{tabular}{|c|c|c|c|c|c|c|c|c|c|c|c|}
\hline $\begin{array}{l}\text { Last } \\
\text { Name }\end{array}$ & $\begin{array}{l}\text { First } \\
\text { Name }\end{array}$ & $\mathrm{MI}$ & Gender & Ethnicity & $\begin{array}{c}\text { Total } \\
\text { Years } \\
\text { Teaching } \\
\text { Ag. }\end{array}$ & Credential Type & $\begin{array}{l}\text { 9-Month } \\
\text { Salary }\end{array}$ & $\begin{array}{c}\text { Extended } \\
\text { Contract } \\
\text { Stipend }\end{array}$ & $\begin{array}{c}\text { FFA } \\
\text { Stipend }\end{array}$ & $\begin{array}{l}\text { Department } \\
\text { Head } \\
\text { Stipend }\end{array}$ & $\begin{array}{c}\text { SOE } \\
\text { Period }\end{array}$ \\
\hline Gambril & Steve & B & Male & White & 11 & $\begin{array}{c}\text { Special Secondary, Vocational } \\
\text { Agriculture }\end{array}$ & 59857 & 7450 & 1650 & 0 & $\mathrm{~N}$ \\
\hline Covey & Aireal & & Female & White & 8 & $\begin{array}{c}\text { Special Secondary, Vocational } \\
\text { Agriculture }\end{array}$ & 54941 & 8000 & 1650 & 0 & $\mathrm{~N}$ \\
\hline Valdez & David & $\mathrm{H}$ & Male & Hispanic & 18 & $\begin{array}{c}\text { Special Secondary, Vocational } \\
\text { Agriculture }\end{array}$ & 67086 & 12873 & 1650 & 0 & $\mathrm{~N}$ \\
\hline Woodard & Kevin & D & Male & White & 15 & $\begin{array}{c}\text { Special Secondary, Vocational } \\
\text { Agriculture }\end{array}$ & 68242 & 9382 & 1650 & 2900 & $\mathrm{~N}$ \\
\hline Dias & Ken & $E$ & Male & White & 30 & $\begin{array}{c}\text { Special Secondary, Vocational } \\
\text { Agriculture }\end{array}$ & 71361 & 12400 & 1650 & 0 & $\mathrm{~N}$ \\
\hline
\end{tabular}

\begin{tabular}{|l|l|l|l|l|l|}
\hline Covey, Aireal & \multicolumn{7}{l|}{$\mid$} \\
\hline Schedule & Period & Beginning Time & Course Title & Enrollment & Type \\
\hline 1 & 1 & $7: 50$ & Prep & 0 & Prep \\
\hline 1 & 2 & $8: 50$ & Ag Biology & 32 & Ag Biology \\
\hline 1 & 3 & $9: 58$ & Ag Leadership & 27 & SAE \\
\hline 1 & 4 & $11: 00$ & Ag Biology & 38 & Ag Biology \\
\hline 1 & 6 & $12: 42$ & Ag Biology & 41 & Ag Biology \\
\hline 1 & 7 & $1: 44$ & Ag Biology & 35 & Ag Biology \\
\hline
\end{tabular}

\begin{tabular}{|l|l|l|l|l|l|}
\hline Dias, Ken \\
\hline Schedule & Period & Beginning Time & Course Title & Enrollment & Type \\
\hline 1 & 1 & $7: 50$ & Ag Science 1 & 24 & Agriscience I \\
\hline 1 & 2 & $8: 52$ & Ag Exploration & 29 & Other Ag \\
\hline 1 & 3 & $9: 58$ & Ag Exploration & 34 & Other Ag \\
\hline 1 & 4 & $11: 00$ & Ag Exploration & 18 & Other Ag \\
\hline 1 & 6 & $1: 44$ & Ag Exploration & 28 & Other Ag \\
\hline 1 & 7 & $1: 44$ & Prep & 0 & Prep \\
\hline
\end{tabular}

\begin{tabular}{|l|l|l|l|l|l|}
\hline \multicolumn{6}{|l|}{ Gambril, Steve } \\
\hline Schedule & Period & Beginning Time & Course Title & Enrollment & Type \\
\hline 1 & 1 & $7: 50$ & Ag Mech 2 & 20 & Ag Mechanics \\
\hline 1 & 2 & $8: 52$ & Ag Mech 1 & 26 & Ag Mechanics \\
\hline 1 & 3 & $9: 58$ & Ag Mech 1 & 36 & Ag Mechanics \\
\hline 1 & 4 & $11: 00$ & Ag Mech 1 & 31 & Ag Mechanics \\
\hline 1 & 6 & $12: 42$ & Ag Mech 2 & 23 & Ag Mechanics \\
\hline
\end{tabular}




\begin{tabular}{|l|l|l|l|l|l|}
\hline 1 & 7 & $1: 44$ & Ag Exploration & 32 & Other Ag \\
\hline
\end{tabular}

\begin{tabular}{|l|l|l|l|l|l|}
\hline \multicolumn{2}{|l|}{ Knight, Jennifer } \\
\hline Schedule & Period & Beginning Time & Course Title & Enrollment & Type \\
\hline 1 & 1 & $7: 50$ & Botany & 22 & Plant/Soil Science \\
\hline 1 & 2 & $8: 52$ & Animal Science & 25 & Animal Science \\
\hline 1 & 3 & $9: 58$ & Ag Exploration & 36 & Other Ag \\
\hline 1 & 4 & $11: 00$ & Prep & 0 & Prep \\
\hline 1 & 6 & $12: 42$ & Vet Science & 27 & Animal Science \\
\hline 1 & 7 & $1: 44$ & Vet Science & 27 & Animal Science \\
\hline
\end{tabular}

\begin{tabular}{|l|l|l|l|l|l|}
\hline Valdez, David & \multicolumn{2}{l|}{$\mid$} \\
\hline Schedule & Period & Beginning Time & Course Title & Enrollment & Type \\
\hline 1 & 1 & $7: 50$ & Ag Earth Science & 35 & Forestry/NR \\
\hline 1 & 2 & $8: 52$ & Ag Mech \& Eng 3 & 10 & Ag Mechanics \\
\hline 1 & 3 & $9: 58$ & Prep & 0 & Prep \\
\hline 1 & 4 & $11: 00$ & Ag Earth Science & 26 & Forestry/NR \\
\hline 1 & 6 & $12: 42$ & Ag Welding and Construction & 25 & Ag Mechanics \\
\hline 1 & 7 & $1: 44$ & Ag Welding and Construction & 25 & Ag Mechanics \\
\hline
\end{tabular}

\begin{tabular}{|l|l|l|l|l|l|}
\hline Woodard, Kevin & Course Title & Enrollment & Type \\
\hline Schedule & Period & Beginning Time & Ag. Econ/Government & 21 & Other Ag \\
\hline 1 & 1 & $7: 50$ & AP Environmental Science & 36 & Forestry/NR \\
\hline 1 & 2 & $8: 52$ & Ag Earth Science & 36 & Forestry/NR \\
\hline 1 & 3 & $9: 58$ & AP Environmental Science & 32 & Forestry/NR \\
\hline 1 & 4 & $11: 00$ & AP Environmental Science & 37 & Forestry/NR \\
\hline 1 & 6 & $12: 42$ & Prep & 0 & Prep \\
\hline 1 & 7 & $1: 44$ & & & \\
\hline
\end{tabular}

Printed: 5/24/2013 4:11:52 PM

Site developed and maintained by the California FFA Association. 


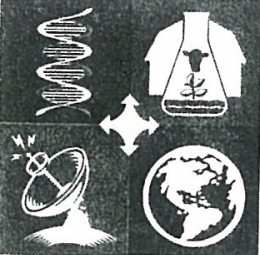

$C$ e $n$ t 1

A p p 1 i e d

a n d $T$ e c h

8180 E. Donner

Clovis, CA 93611

559.327 .4320

559.327.4063 Fax

Reagan Educational

Center Administration

\section{Linda Hauser}

Assistant Superintendent

Lyn Snauffer

Asulstane Adminfatrator

Melissa Macon

Dinector, Qualley Student

Jefferbeu

Paincipal, Cloufs East High

School

Stacy Dumnicliff

Principal, Reyburn

interim

\section{Clovis Unified School \\ Jistrict}

\section{Administration}

Walter L. Buster, Ed.D. Superintendent

Terry Bradley, Ed.D.

Deputy Superintendent

James S. Fugman, Ed.D.

Deputy Superintendent

Virginia R. Boris, Ed.D

Associate Superintendent

Daniel E. Kaiser, Ed.D.

Assocfate Superfintendent

William C. McGuire

Assoclate Superintendene

\section{Governing Board}

Elizabeth J. Sandoval President

Sandra A. Bengel

Vice President

Jim Van Volkinburg,

D.D.S.

Clerk

Brian D. Heryford Ginny L. Hovsepian

Richard Lake, C.P.A.

Susan M. Walker,

D.H.Sc.

\section{Job Site Placements}

\author{
September 2000
}

Clovis Unified School District's Agriculture Program has developed community partnerships that benefit its students. This involvement within the community is key to student application in advanced areas of study. Our Pre-Veterinary Science class enjoys Community Classroom environments throughout the Clovis Area. The Following is an active list of current placement sites within our program. 


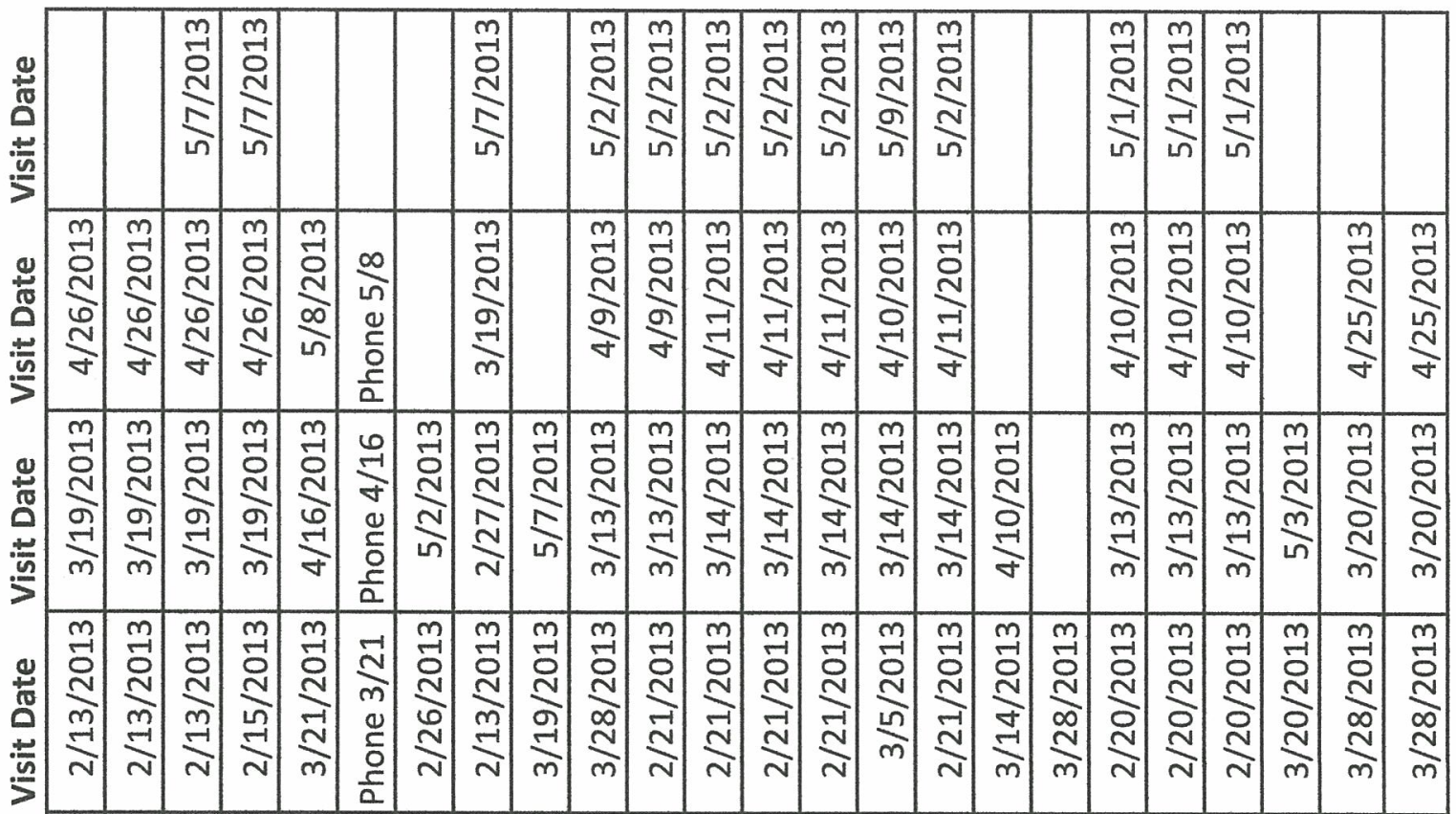
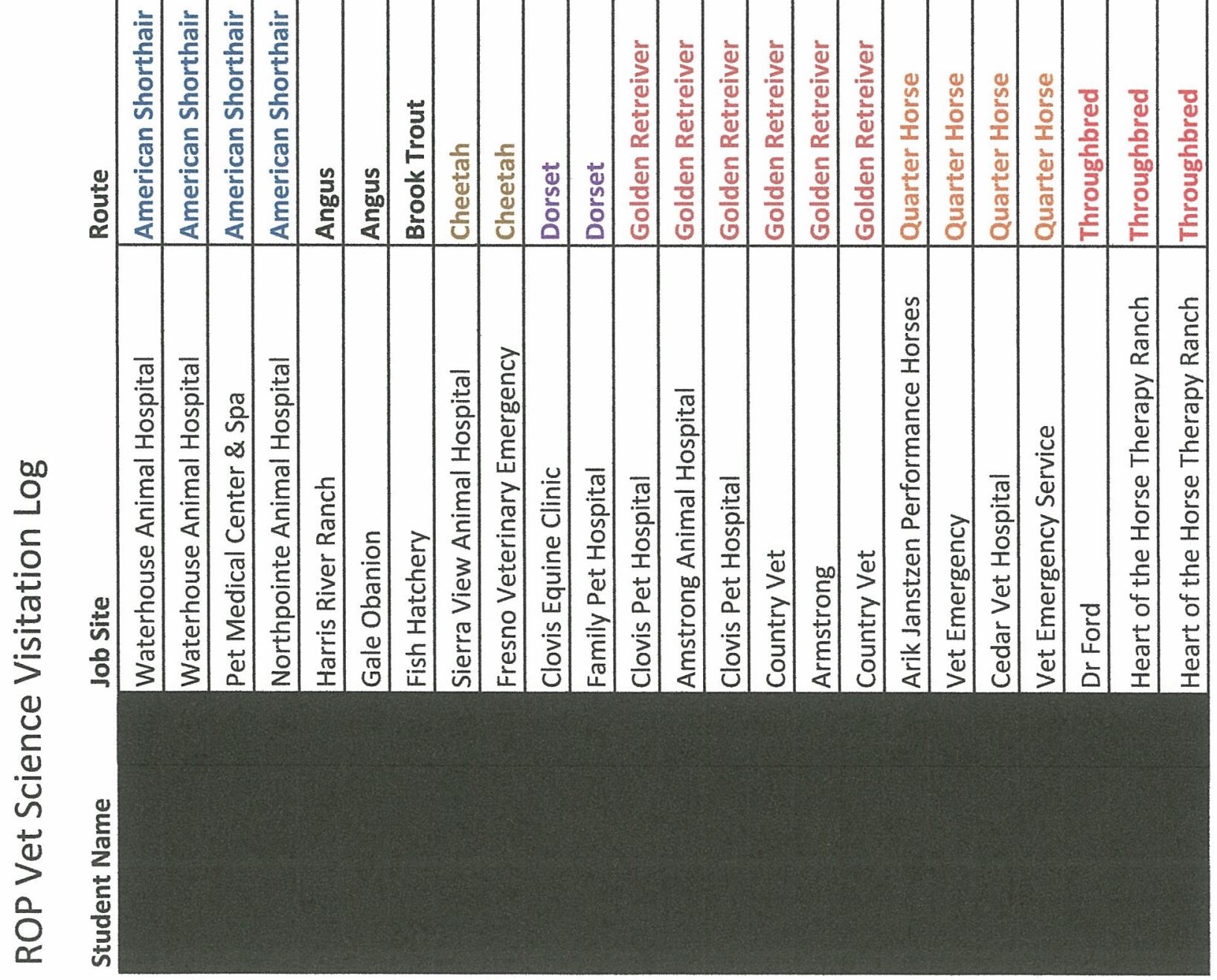


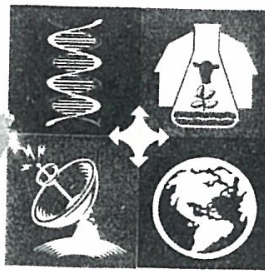

$C$ e n t r a 1

A p p 1 i e d

a n c
8180 E. Donner Clovis, CA 93611

559.327.4320

559.327.4063 Fax
Reagan Educational Center Administration

Linda Hauser

Assfotant Superintendent

Lyn Snauffer

Asalstant Adminfatrator

Melissa Macon Director, Qualley Student
Serulces

JeffEben

Principal, Clouis East High

Stacy Dumnicliff

Principal, Reyburn

intermedite

Jlovis Unified School istrict

\section{Administration}

Walter L. Buster, Ed.D. Superintendent

Terry Bradley, Ed.D.

Deputy Superintendent

James S. Fugman, Ed.D.

Deputy Superintendent

Virginia R. Boris, Ed.D

Associate Superintendent

Daniel E. Kaiser, Ed.D.

Assocfate Supenintendent

William C. McGuire

Assocfate Superintendent

\section{Governing Board}

Elizabeth J. Sandoval President

Sandra A. Bengel

Vice President

Jim Van Volkinburg,

D.D.S.

Clerk

Brian D. Heryford

Ginny L. Hovsepian

Richard Lake, C.P.A.

Susan M. Walker,

D.H.Sc.

\section{Recruitment Activities and Materials}

\section{September 2000}

Clovis Unified School District's Agriculture Program works closely with other schools with the district and surrounding areas. The Program feels Agricultural Literacy is not limited to high school students. For these reason the Agriculture Program is fundamentally Grades 7-12. Students from Clark and Reyburn Intermediate Schools are actively engaged in the Exploratory Agriculture classes. These classes serve a duel purpose for this program. First, they broaden general Agricultural Literacy within the district. In addition, they serve as a feeder program for the department.

Each year the Clovis Unified School District's Agriculture Program hosts a Third Grade Day at the Farm. The program is produced by the students within the program and delivered to third grade students within the district. 
8180 E. Donner Clovis, CA 93611 559.327 .4320

559.327.4063 Fax

Reagan Educational Center Administration

Linda Hauser

Assistant Superintendent Lyn Snauffer

Assistant Adminfatrator Melissa Macon

Director, Qualley Student

Seruices

JeffEben

Pafncipal, Clouis East High
School

Stacy Dumnicliff

Principal, Reyburn

hitermediate

Iovis Unified School Jistrict

\section{Administration}

Walter L. Buster, Ed.D. Superintendent

Terry Bradley, Ed.D.

Deputy Superintendent

James S. Fugman, Ed.D.

Depuey Superintendent

Virginia R. Boris, Ed.D

Assocfate Superintendent

Daniel E. Kaiser, Ed.D.

Associate Supenintendent

William C. McGuire

Assocfate Superintendent

\section{Governing Board}

Elizabeth J. Sandoval

President

Sandra A. Bengel

Vice Presidene

Jim Van Volkinburg,

D.D.S.

Clenk

Brian D. Heryford

Ginny L. Hovsepian

Richard Lake, C.P.A.

Susan M. Walker,

D.H.Sc.

\section{Student Retention Plan}

\author{
2000-2001
}

As with most Agricultural Education Programs at the secondary level Clovis experiences a loss of active students throughout the four-year program. However, unlike most programs the loss is not as prevalent within the year three and year four students. The largest drop by percentage is seen in year two where these students must leave the program to seek science credit in other academic programs. It is the opinion of the Department Chairman that this phenomenon although still observed may not be as prevalent with students within the Reagan Education Center. These Students currently have an eight period day that allows for a larger elective load.

Nevertheless, it is the intent of the program to retain year two students by increasing the number of classes meeting Graduation and UC requirements. The current plan creates a year two-class meeting Physical Science credit. In addition, it is the intent of the program to update current Floral Design course to meet UC Fine Arts Requirements.

The preceding changes within the current program when combined with the inclusion of Senior Government and Economics credit courses should prove to strengthen overall student participation throughout the total program. 


\section{Quality Criteria Ten}

\section{Student to Teacher Ratio}

Even though the quality criteria state that high quality vocational instruction is based upon the maintenance of the appropriate student to teacher ratios, I am proud to say that at Clovis East we do provide quality instruction despite that fact that our District does not and has not supported these criteria. Our agriculture department will continue to fight for the 20:1 ratio especially in the shop classes. In some courses we do meet or come close to meeting the requirement, for example in Botany and Animal Science I have 25 students or less which meets the requirement, but our Ag Biology and Ag Science 1 courses have close to 40:1 ratios. As much as I believe that we should have smaller classes at least we have kids that want to be in our program! Because of the size of our ninth and tenth grade classes we have not had to push as hard on recruitment. If we could know for sure that larger numbers equal the addition of a teacher we could push harder, however we know that is not the case.

Even though our student to teacher ratio may be high in some courses we do have a great system and support for discipline on our campus and for Clovis Unified in general. We have strict policies in place for student behavior and teachers are supported when removing a student from class that may be distracting others or causing a safety situation. Our administration also supports our professional judgment and will remove or relocate students especially in shop classes that may cause safety issues.

\section{Supporting Completion Materials}

Item A- Copies of my class rosters for classes that meet the 25:1 Requirement. 


\section{Class Roster as of $5 / 24 / 2013$}

Ordered by Student

CLOVIS EAST HIGH SCHOOL

\begin{tabular}{|lr}
\hline i: $55725-1$ - Botany P \\
jd: 1
\end{tabular}

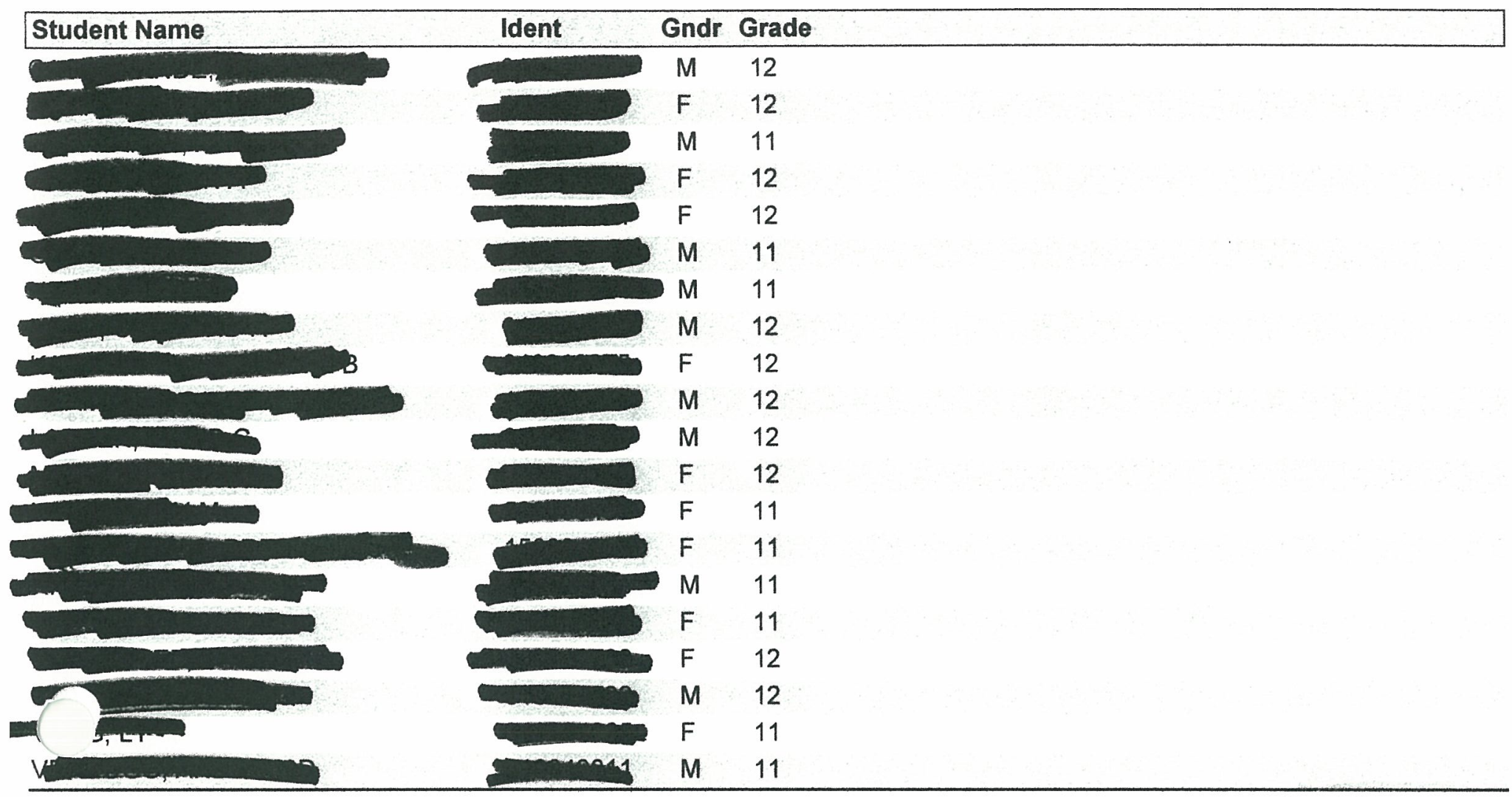


P. od: 4

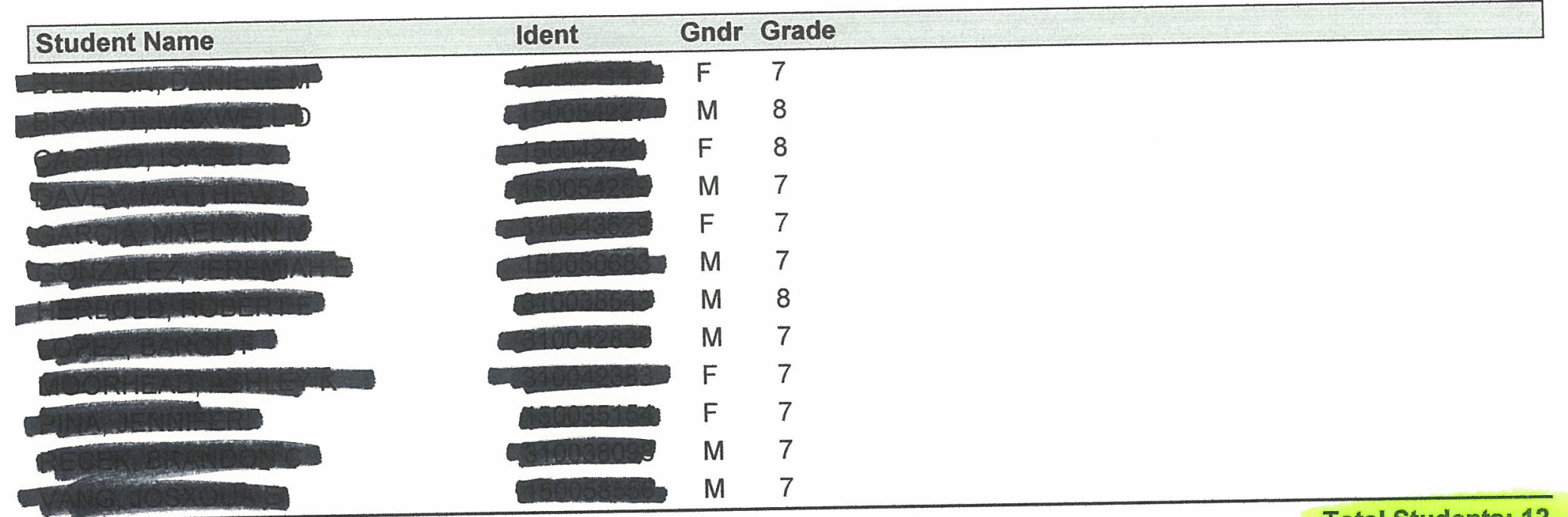




\section{Quality Criteria Eleven}

\section{Full Year Employment}

Prior to 2002 the Clovis Unified Agriculture Department received summer contracts for each full time Agriculture teacher. During this time the Ag Staff was only two or three people and each received forty additional days of pay. However, when the program began to rapidly grow and the new Ag Center opened with five teachers the district decided that it was too expensive to continue funding the Ag teachers at this level. Clovis Unified is a nonUnion district so negotiations began with teachers, Advisory Members and School Site Council Members.

Negations were handled in three phases. Phase one; the school decided that one "Extended Contract" would be granted to cover additional responsibilities after school, on weekends, and during the summer. The staff were asked to present a report that outlined the total number of additional time spent by each teacher outside of class time. The report helped the district realize that additional compensation would be required. Phase two; in the spring of 2002 negotiations continued; this time the focus was the fact that $\mathrm{Ag}$ teachers were now being asked to take a pay reduction while still providing the same level of service. It was at this time that it was determined that current compensation levels would be maintained but frozen at the 2001-2002 levels. Additionally, project supervision periods and FFA Advisor stipends would be revoked to make up for additional expenses. Phase three; unsatisfied with the outcome of phases one and two, the Agriculture Department put together a document entitled Proposal and Justification for Compensation Paid to Agriculture Teachers within Clovis Unified School District. This document was designed to outline the common practice of additional compensation paid to those agriculture teachers performing additional responsibilities beyond the normal scope of the teaching day.

Research showed that agriculture programs State wide routinely compensate teachers at an average rate of 40 duty days above the contracted school year. Additionally compensation in the form of SAE supervision periods and FFA stipends brought the total level of additional compensation value to approximately 60 duty days. For this reason the 
agriculture staff were willing to accept the loss of FFA stipends and SAE periods in exchange for a 60 additional day contract.

Upon receiving the final report and documentation, district administration realized that compensation levels must stay competitive with other school districts. Teachers must be paid for the additional work they perform and that they could not be expected to carry out additional tasks without compensation. It was decided that all teachers would receive extended contracts at a rate of 40 additional duty days and that further negotiations to increase to 60 additional duty days was fully warranted at a later date. Current budget restraints made it difficult to justify additional compensation but FFA stipends were to be reinstated for the 2003-2004 school year.

Currently for the 201-2013 school year each of the six Ag teachers receives a 40 day extended contract frozen at the 2001-2002 levels or in my case frozen at my hire date. This is frustrating for veteran members of our department because movement across the salary scheduled is not matched to the 40 day contract. Because I was hired with 5 years' experience and 50 additional units I started at a higher rate than two teachers who have been here longer than me and both hold Masters Degrees. This year there has also been a change in the way that the extended contracts are paid out. In previous years they were paid in one lump sum at the end of the school year. Now they are paid over a 10 month period included in the regular salary check. Negotiations may resume again shortly regarding this matter as our district announced a review of all salaries due to a large surplus of funds for the upcoming school year. We as an Ag Staff feel this is the opportune time to re-negotiate un freezing the 2001-2002 level pay making the 40 days move along with employees on the salary schedule. Otherwise what incentives do we have for further service years or professional development? Plus I know it could potentially lead to animosity among department members that some people just because their hire date make more than those who have worked here longer and have earned higher levels of education. 


\section{Ag Ed 539 Teacher Internship Report}

\section{Supporting Completion Materials}

Item A- Staff Chart of Responsibilities

Item B- Extended Contract Justification Report

Item C- Information on Extended Contract Freeze sent to head of the Faculty Senate by our Department Chair Kevin Woodard.

Item D- 2012-2013 Summer Work Calendar and Hour Log Sheet 


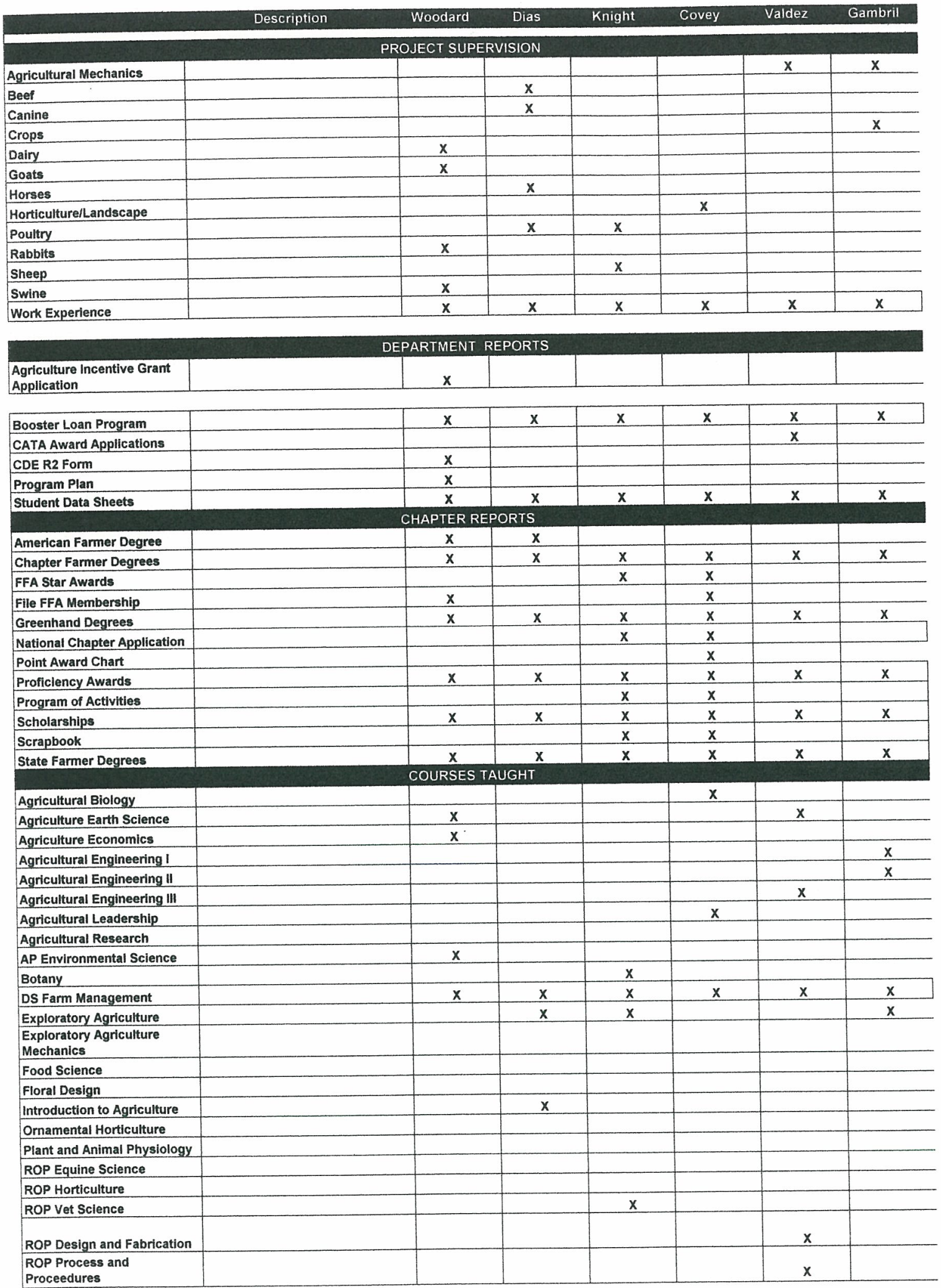




\begin{tabular}{|c|c|c|c|c|c|c|}
\hline & Woodard & Dias & Knight & Covey & Valdez & Gambril \\
\hline \multicolumn{7}{|c|}{ STAFF REPRESENTATION } \\
\hline Academic Supreme Court & $\mathrm{x}$ & & & & & \\
\hline Academic Senate & $x$ & & & & & \\
\hline Accreditation & $\hat{x}$ & $\mathrm{x}$ & $\mathrm{x}$ & $x$ & $x$ & $x$ \\
\hline Advisory Commiltee & $x x$ & $x$ & $x$ & $\bar{x}$ & $x$ & $x$ \\
\hline \begin{tabular}{|l|} 
Articulation Agreements \\
\end{tabular} & $\mathrm{x}$ & $x$ & $\frac{\hat{x}}{x}$ & $\hat{x}$ & $\hat{x}$ & $\frac{n}{x}$ \\
\hline Booster Meetings & $x$ & $x$ & $\bar{x}$ & $x$ & $x$ & $x$ \\
\hline CATA Regional Meetings & $\hat{x}$ & $\hat{x}$ & $\frac{\hat{x}}{x}$ & $\hat{x}$ & $\frac{\hat{x}}{x}$ & $\underline{x}$ \\
\hline CATA Sectional Meetings & $\hat{x}$ & $\frac{a}{x}$ & $\frac{\hat{x}}{x}$ & $\frac{a}{x}$ & $\frac{a}{x}$ & $x$ \\
\hline \begin{tabular}{|l} 
CATA State Meetings \\
\end{tabular} & $\frac{x}{x}$ & $\hat{x}$ & $\hat{x}$ & $x$ & $x$ & $x$ \\
\hline District Communication & $x$ & & & & & \\
\hline \begin{tabular}{|l} 
Faculty Meetings \\
\end{tabular} & $x$ & $\mathrm{x}$ & $\bar{x}$ & $\bar{x}$ & $x$ & $x$ \\
\hline Incentive Grant Review & $x x$ & $\hat{x}$ & $\hat{x}$ & $\hat{x}$ & $x$ & $\mathrm{x}$ \\
\hline
\end{tabular}

SPECIAL ASSIGNMENTS

Department Chairman

Fall Banquet

Fall Classic Showcase

FFA Advisor

FFA Food Drive

FFA Meetings

FFA Officer Retreat

FFA Top 20

Greenhand Advisor

Greenhand Conference

Recruitment

Spring Banquet

Student Teacher Supervision

\begin{tabular}{|c|c|c|c|c|c|c|}
\hline 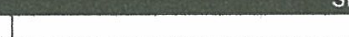 & $x$ & 1 & & & & \\
\hline & $\hat{x}$ & $\mathbf{x}$ & $x$ & $\bar{x}$ & $\mathrm{X}$ & $\mathbf{X}$ \\
\hline & $x$ & $x$ & $\mathrm{x}$ & $x$ & $\bar{x}$ & $x$ \\
\hline & $\bar{x}$ & $x$ & $x x$ & $x x$ & $x$ & $x$ \\
\hline & $x X$ & $x x$ & $x$ & $x$ & $x$ & $\mathrm{X}$ \\
\hline & $\frac{\pi n}{x}$ & $\mathrm{x}$ & $x x$ & $x x$ & $\bar{x}$ & $x$ \\
\hline & $x$ & $x$ & $\overline{x x}$ & $x x$ & $\mathbf{x}$ & $\mathbf{X}$ \\
\hline & $x$ & $x$ & $x x$ & $x x$ & $x$ & $x$ \\
\hline & $x$ & $x$ & $\mathrm{x}$ & $\mathrm{x}$ & $x$ & $x$ \\
\hline & $x$ & $x$ & $x$ & $x$ & $\bar{x}$ & $x$ \\
\hline & $\bar{x}$ & $x$ & $x$ & $x$ & $x$ & $x$ \\
\hline & $x$ & $x$ & $x x$ & $x X$ & $x$ & $x$ \\
\hline & $x x$ & $x x$ & $x$ & $x$ & $x$ & $x$ \\
\hline
\end{tabular}

Beef and Sheep Unit

Swine Unit/ Rabbit Barn

Temporary Swine Barn

Ornamental Horticulture Unit

Pasture

Orchard Vineyard

Shop Complex

Garden Area

Equipment Compound

FACILITY RESPONSIBILITIE

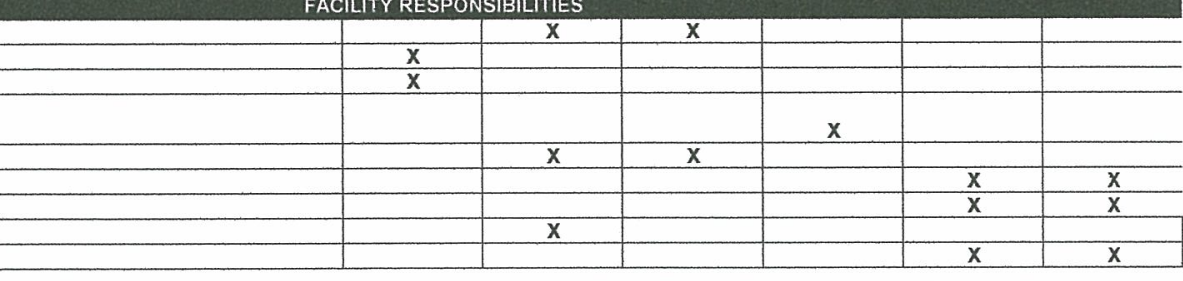

FFA, Advanced Leadership

Conference

FFA, Chapter Officer

Leadership Conference

FFA, Made for Excellence

Conference

FFA, National Convention

American Degrees

FFA, National Finals

FFA, Regional Officer

Leadership Conference

FFA, Sacramento Experience

Conference

FFA, Sectional Bowl-a-thon

FFA, Sectional Officer

Leadership Conference

FFA, State Leadership

Conference

FFA, World Ag Expo

Field Day, Arbuckel

Field Day, CSU Bakersfield

Field Day, Cal Poly Pomona

Field Day, Chico State

Field Day, Exeter

Field Day, Fowler

Field Day, Fresno State

Field Day, Mendota

Field Day, Merced

Field Day, Modesto

Field Day, Monrovia Nursery

Field Day, Reedley College

Field Day, State Finals, Ca

Poly SLO

Field Day, State Finals,

Fresno State

Field Day, State Finals,

Reedley College

Field Day, UC Davis

AQHA World Horse Show

Show, California State Fai

Show, Great Western

Show, Red Wave Classic

Show, The Big Fresno Fair

\begin{tabular}{|l|l|}
\hline & FFA and LEA \\
\hline & \\
\hline & \\
\hline & \\
\hline & \\
\hline & \\
\hline & \\
\hline
\end{tabular}

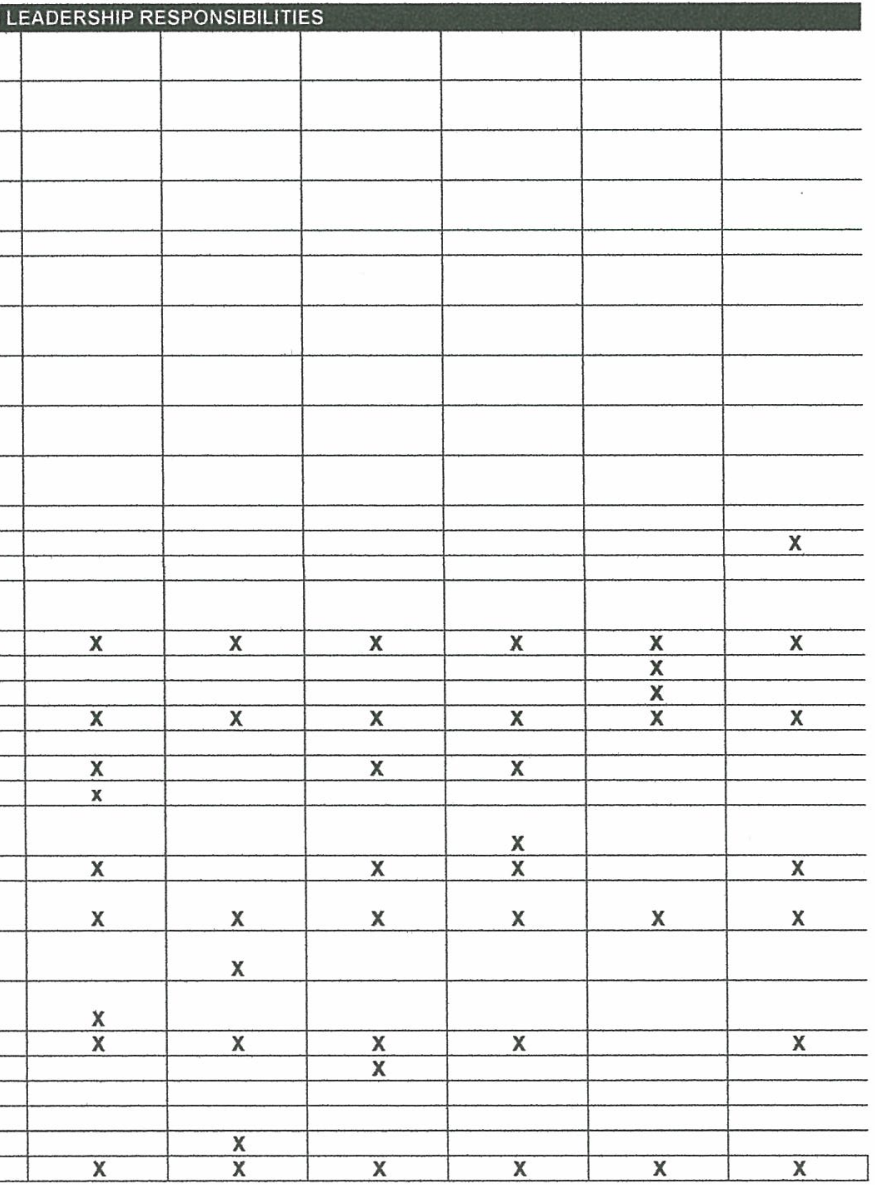




\title{
CLOVIS UNIFIED SCHOOL DISTRICT AGRICULTURE DEPARTMENT
}

\author{
EXTENDED CONTRACT \\ BACKGROUND AND JUSTIFICATION
}

\author{
Revised \\ -Fall 2009-
}




\section{PURPOSE}

The following report has been compiled by the staff of the School of Agriculture Science and Technology (SAST) within the Clovis Unified School District and is intended to be an updated version of an in-depth port originally presented to district administration in the spring of 2002 . The purpose of this report, as with s predecessor, is to demonstrate the continued need for compensation granted to full time Agriculture ieachers within the district. It is the hope of the SAST Staff to give background and create institutional understanding as to why compensation is paid to staff that perform necessary activities and instruction which are integral to the present and future of one of the top agricultural education programs in America.

\section{BACKGROUND AND HISTORY}

In a report generated by the Clovis Unified Agriculture Department in the fall of 2001 the average staff member within the program worked a total of 279 duty days throughout that year. Base salary paid by the district compensates all teachers was based on 186 duty days per year regardless of specific discipline. Historically, additional compensation has been granted to the agriculture staff in the form of "Summer Contracts", Project Supervision Periods, and Stipends for FFA Advisors. This additional compensation was intended to cover the 64 additional duty days required to carry out extended responsibilities.

\begin{tabular}{|c|c|}
\hline Certificated Salaries & 186 Duty Days \\
\hline Project Supervision Period & 31 Duty Days \\
\hline FFA Stipend & 6 Duty Days \\
\hline Summer Contract (Base Salary x .025 x 8 Weeks) & 40 Duty Days \\
\hline Total & 263 Duty Days \\
\hline
\end{tabular}

\begin{tabular}{|c|c|}
\hline Certificated Salaries & 186 Duty Days \\
\hline FFA Stipend & 6 Duty Days \\
\hline Extended Contract (Frozen at 2002-03 rate) & 30 Duty Days \\
\hline Total & 222 Duty Days \\
\hline
\end{tabular}

During the spring semester of the 2001-2002 school-year, the agriculture department staff was notified that the historical compensation schedule would change for the upcoming year. It was proposed that the extended contracts paid to all staff within the agriculture program be "eliminated" due to budget restraints. Likewise, each member of the agriculture staff would no longer receive time compensation during the school day in the form of Project Supervision Periods.

After further negotiation, the staff was granted payment of Extended Contracts with the intent that future funding be frozen at level paid in the June 2002-2003 contracts, regardless of a teacher's position on the salary schedule. Additionally, the members of the department were asked to present a report that outlines how other districts compensate the staff within their agriculture programs. However, within the months between this original assignment and the production of this report the staff was notified that stipends for FFA advisors would also be eliminated.

Gurrently, all members of the Clovis Unified Agriculture Department are performing all necessary duties, espite the extensive uncompensated time required, including classroom instruction, facility management, dvising student projects, working with industry on program development, facility planning, and numerous other tasks without compensation beyond their base salary. 
Despite the fact that this additional funding did not cover the total number of duty days worked, compensation for all agriculture teachers has made it possible to attract and retain a talented staff that is respected rofessionally throughout the nation. This staff has accepted many challenges in and out of the classroom and ot only met these expectations but to exceeded to set higher standards for other programs to follow. The districts Agriculture students have received numerous state and national honors including 13 National Championships, two State FFA Officers and in 2007 Clovis was the home of the National FFA President. Our department has also won State Awards including Top "Small", "Medium", "Large Department" over the past twelve years and is seen as a model for programs throughout the country. Much time is spent giving tours to groups and individuals such as (Iowa State University, Miami Valley, Ohio, Oregon State University, Montana State University, National and State FFA Officers, Ann Veneman, Former Secretary of Agriculture, Jack O'Connell, Former Vice-President Cheney, UC Davis, Cal Poly San Luis Obispo).

\section{CONCLUSION}

Key to the success of the Agriculture Program within the district is its year-round delivery of instruction. As with all other programs in the district the agriculture department delivers a rigorous curriculum and a sequence of classes that give students the skill necessary to perform in our society. However, what makes the Clovis Unified Agriculture Program unique within the district is the additional time dedicated to the advancement of its students. Whether after school, weekends, or summer activities, the Agriculture staff is expected to take learning beyond the classroom. 


\section{Ag Center Enrollment History}

Student enrollment within the Agriculture program has increased from less than 250 students in 1999 to over 50 in 2009 with an additional 250 to $3007^{\text {th }}$ and $8^{\text {th }}$ grade students in our Exploratory Agriculture programs.

nis growth is the result of dedicated teachers and supportive administration that are willing to provide the extra effort to make students successful.

The focus group data supports the idea that just compensation is key to attracting and retaining an agricultural staff that maintains a high level of excellence. Our department at Clovis East is recognized nationally as one of the top schools in the country for Agricultural Education. Yet Clovis agriculture teachers are expected to increase enrollment, develop rigorous academic standards, and maintain the most advanced agricultural facility ever developed while receiving limited resources and compensation.

Therefore, in an effort to resolve the problem of fair compensation, the Clovis Unified Agriculture Department needs to be contracted for an additional Sixty (60) Duty Days per teacher to carry out necessary year round functions within the program. These projects and activities are long-term, continual, and extremely time consuming throughout the year. Early summer is the time that student projects are essential in maintaining and increasing our success level at important competitive events. Students within the program are not capable of accomplishing all these necessary tasks alone. They need the guidance and advice of our staff to insure their success. These responsibilities must be met if we are to maintain the quality agriculture program that we currently have within Clovis Unified. Keeping in mind that the local, state, and federal funds have been used to build what many see to be the most technologically advanced secondary agriculture education facility in the nation, the staff feels that it is our responsibility to provide the highest quality education possible.

\section{Supervised Agricultural Experience Program}

he diverse agriculture program of the School of Agricultural Science and Technology creates an ideal situation rr offering students with comprehensive, year round opportunities. Students are so successful in this award winning and nationally recognized program because of the implementation of the Supervised Agricultural Experience Program (SAEP). The SAE Program requires the time of all agriculture staff members and it is the key component to making this program so successful. The Supervised Agricultural Experience Program is the instruction, guidance, and management of students and their projects outside of the normal school day. Agriculture staff members spend large amounts of time maintaining facilities and equipment, writing grants, purchasing animals, equipment, fertilizers, seed, giving tours, vaccinating animals, picking, pruning, and watering the farm crops.

Although due to budget cuts in 2002, the extended contract stipend was frozen from growing with the annual salary of the individual staff member as well as the Project Supervision Period being cut from all staff members, the School of Agricultural Science and Technology has been fortunate enough to continue to attract and keep innovative teachers that help to maintain the high quality program that Clovis Unified is known for. Although we have worked diligently designing the new facilities, writing innovative course curriculum, maintaining orchards, and hauling kids to fairs and conventions, our biggest contribution is time spent with students. Regardless of state-of-the-art technologies, first class facilities, and rigorous curriculum, the quality time with students always proves to be the most bang for your buck. After all, it's people, not programs! 


\section{Extended Contract Duties}

- Assist, advise, and supervise students concerning on-going projects

- Transport students and animal projects for tagging, inspection, vaccination, and weighing

- Acquire projects for Supervised Agricultural Experience Programs (SAEP)

- Acquire resources for maintaining student projects

- Educate and assist the students with record keeping, award applications, and student loan applications

- Maintain and involve industry contacts

- Host Agricultural Advisory committee meetings

- Facility improvements, management and deferred maintenance

- Program planning and promotion

- Preparation for State Department of Education Incentive Grant Review

- Preparation for Career Development Events for local, regional, state, and national competition

- Transport students to regional, state and national conventions and competitions

- Host Career Development Events

- Agriculture Booster Club supervision

- Professional training/professional development on a quarterly basis

- Attend facility construction meetings and acquire equipment

- Collaborate among other agricultural educators

- Member of Fresno County Office of Educations CTE, ELA, \& Mathematics matrixing project

\section{dditional Components}

- Golden Bell Award winners

- Contribute to the quality of education as noted in the WASC accreditation and National Blue Ribbon award

- California Outstanding Large Agriculture Department

- National and State FFA Presidents

- Program completers have interned at the White House and with Mike Villines

- Students have interned with Chuck and Lynn Poochigian

- Grant writing, facility and equipment development

- Provide consulting and workshop for other school districts

- Participate in the development of nationally published textbooks (Interstate Publishing)

- Provided tours for:

- Dick Cheney

- Jack O'Connell

- Ann Veneman (former U.S. Secretary of Ag)

- Student teachers Cal Poly, San Luis Obispo annually

- Student teachers, CSU Fresno annually

- National FFA Officer team (annually)

- State FFA Office team (annually) 


\section{Extended Contract Job Description for the Clovis Unified Agriculture Staff}

Effective instruction in Agriculture Education extends far beyond the regular school day, school year and school environment. The basic component of instruction occurs as group instruction in the classroom, shop or field throughout the year to all agriculture students to value and understand the vital role of agriculture, food, fiber and natural resources systems in advancing personal and global well being.

Agriculture education prepares students for successful careers and a lifetime of informed choices in the global agriculture systems.

SAE'S - Project visitations. Projects that are housed at home or other facility where teacher visits and advises student. Example of a project visitation would include selection of quality animals (time and travel in the state to select, feeding, health and management of animals, record keeping, sales and marketing, environmental control and reproduction and increasing scope and output of project. Technology will be used through ultrasound, computerized record keeping, embryo transfer, and hydroponics. Fresno Fair - State Fair - On site management of livestock owned by students - Work experience - Exploratory SOE - Improvement .

FFA - Career Development Events: Meat Science, Livestock evaluation, natural resources, Agriculture mechanics, Banking, Farm management and records, Cooperatives, Best Informed Greenhand, Opening and Closing Speaking contest, prepared public speaking, job interview, extemporaneous public speaking, creed speaking, (practice American, State, Chapter and Greenhand Degrees leadership conferences .

Agriculture Boosters- Over seeing the booster organization, attending monthly and bi monthly meetings and attending fundraising events.

Chart of responsibilities 


\section{Agriculture Staff Extended Contracts}

\section{Kevin Woodard}

Sent: Wednesday, May 22, 2013 8:59 AM

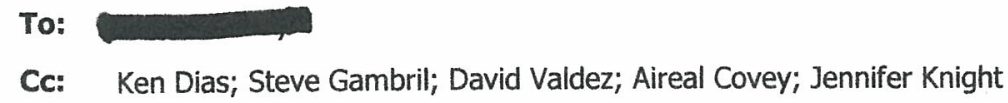

Hello Duane. As you requested on Monday I wanted to send you a little background on our compensation concerns at the $\mathrm{Ag}$ Center.

State-wide, Agriculture Teachers are expected to work year round to cover duties outside of their contracted duty days. Many years ago Clovis Unified developed a formula that determined the additional compensation (Extended Contract) received by the Agriculture Teachers. This contract equated to 40 extra duty days and based on most recent salary schedule. In 2003 the District "froze" our extended contracts and they have stayed flat since that time. I estimated that I personally have lost over $\$ 50,000$ in justified compensation because of the policy (there is no written policy in place). In unionized districts this issue would be negotiated by the union and have probably been resolved after a couple of years. This issue has been brought up many times each year with administration since 2003 and was met with them asking for studies and surveys of state-wide policies. These studies have been done, hours have been tracked. Our case has been stated but still no resolution.

So I bring this to you for guidance. Our "District-Wide" program operates year round and the summer is some of the more demanding times of the year. We work most Saturdays throughout the year not grading papers and prepping for class but working on real world application of life skills. It is time that we are properly compensated for our duties.

Let me know your thoughts.

Kevin Woodard

McFarlane-Coffman

Agriculture Center

kevinwoodard@cusd.com

www.woodardnet.com 
TEACHER

FROM

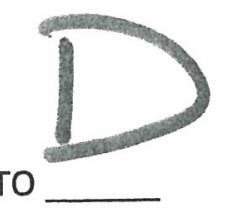

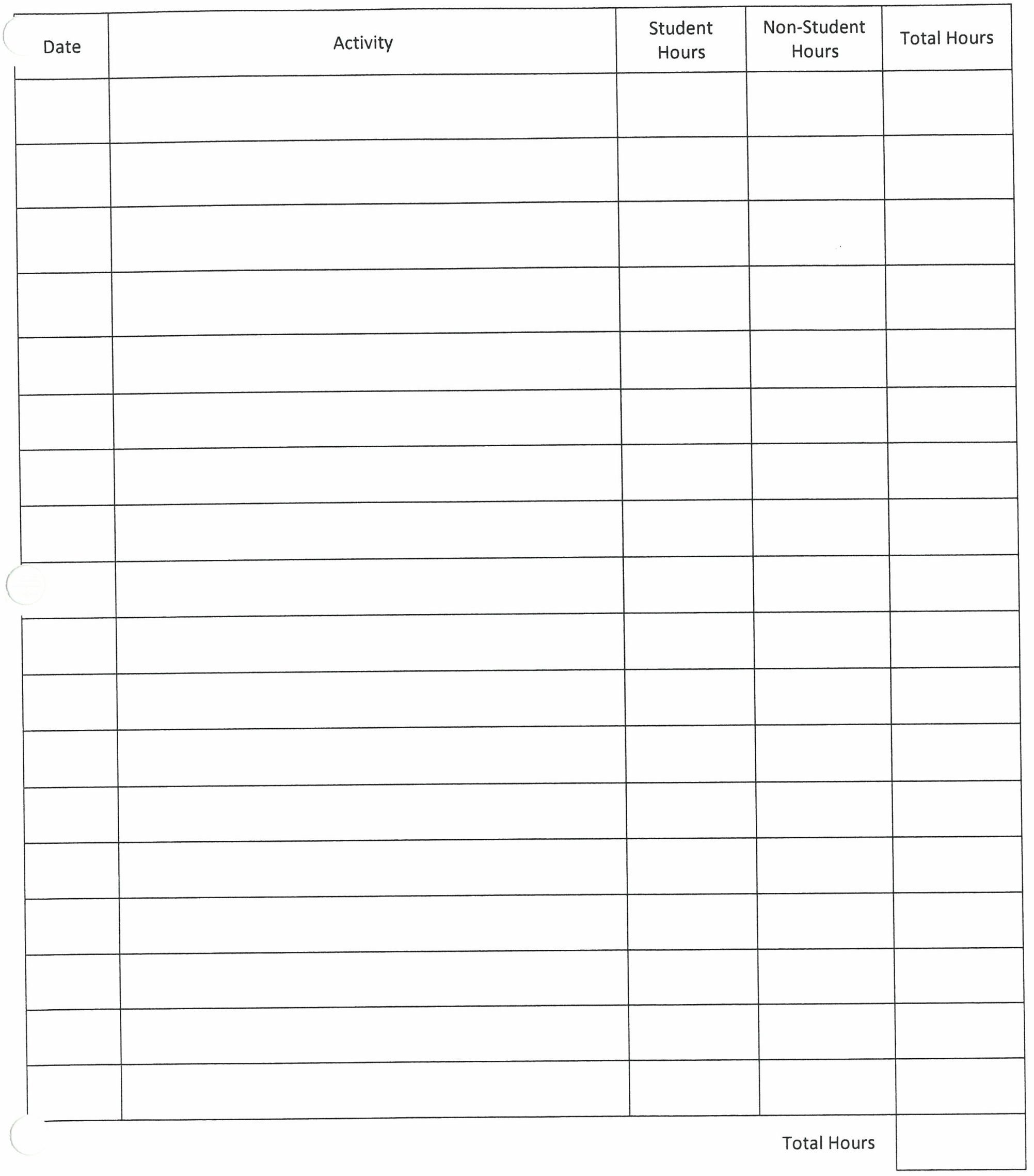




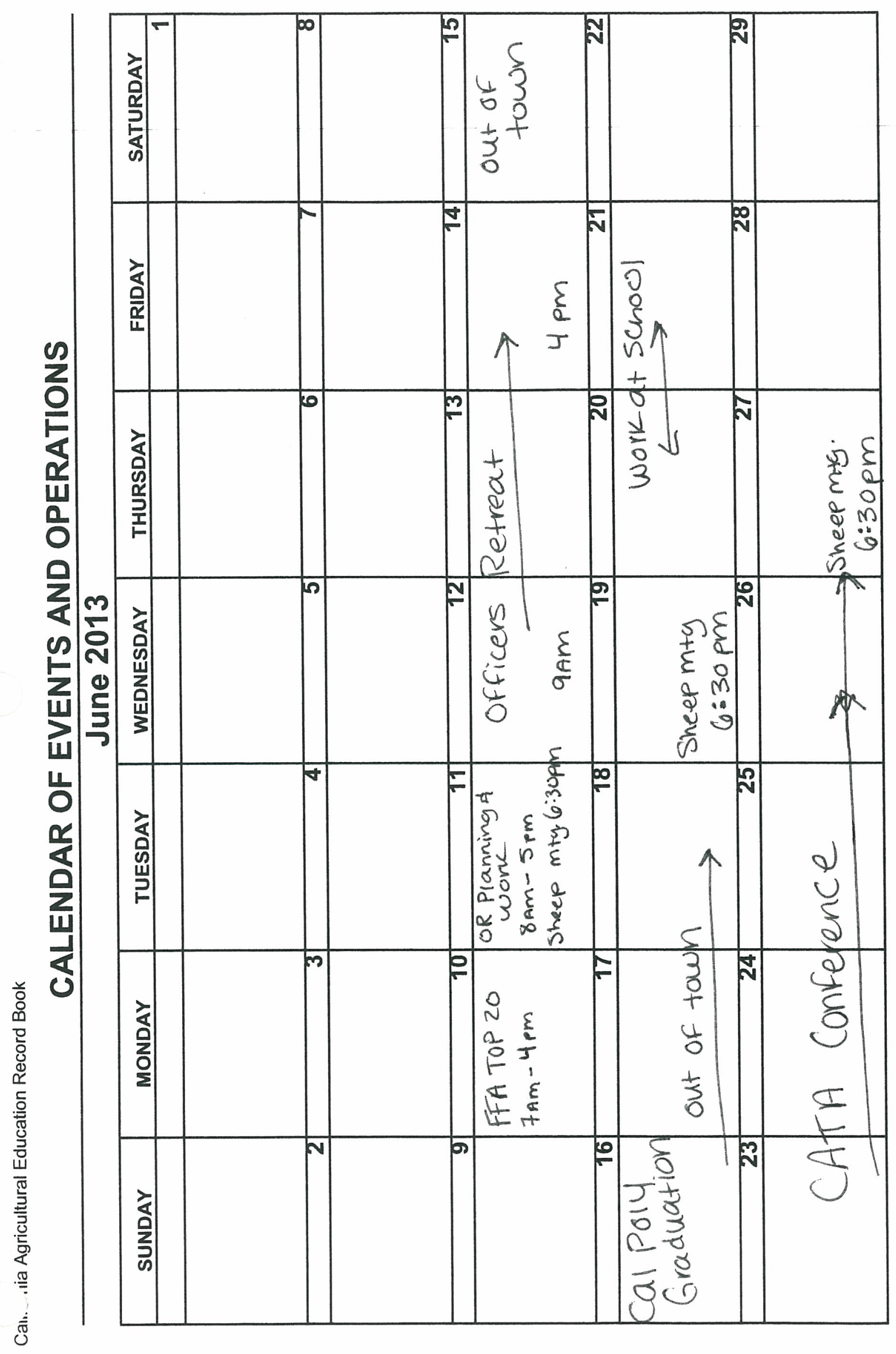

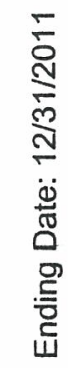




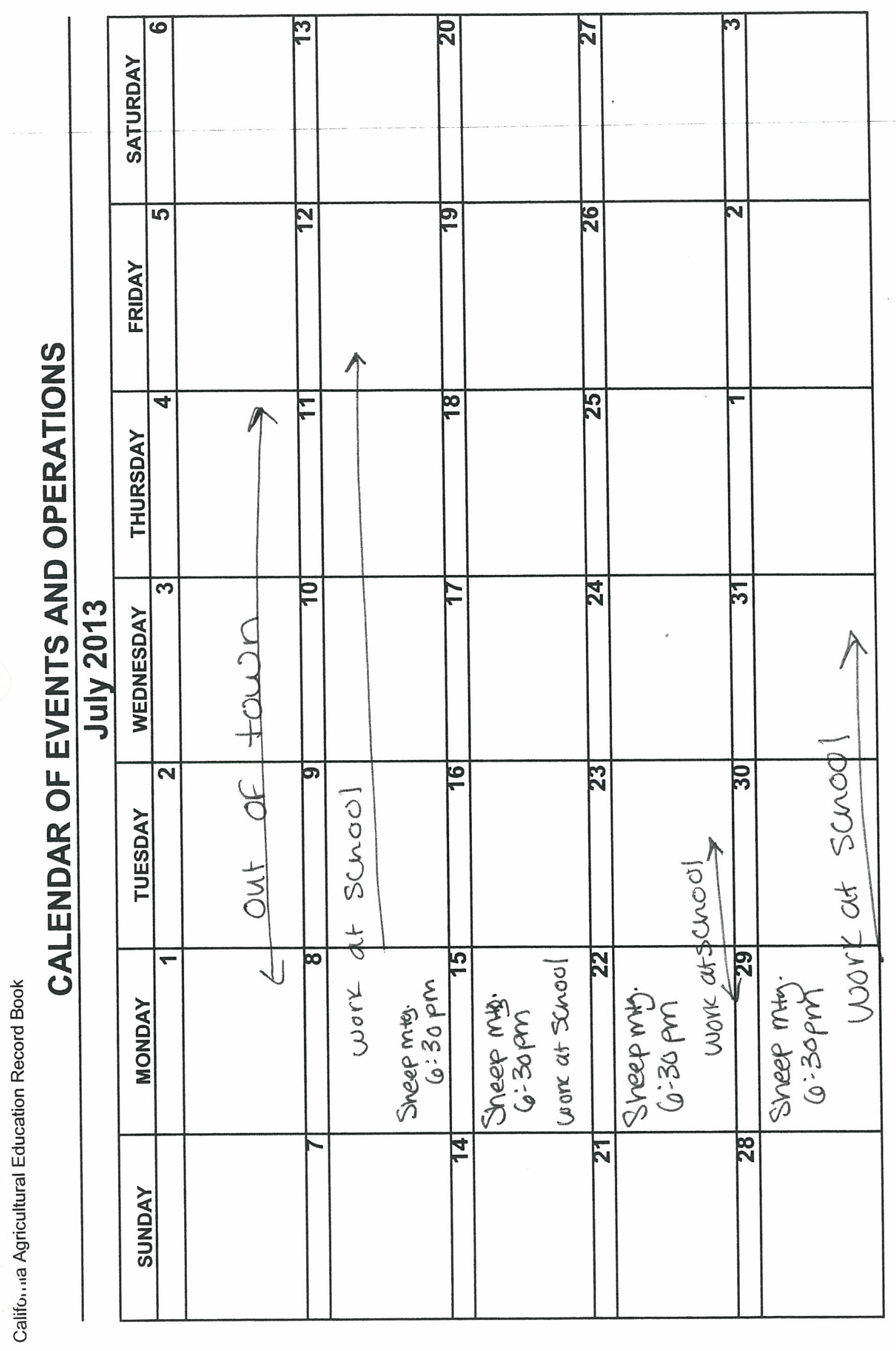

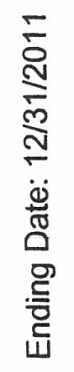




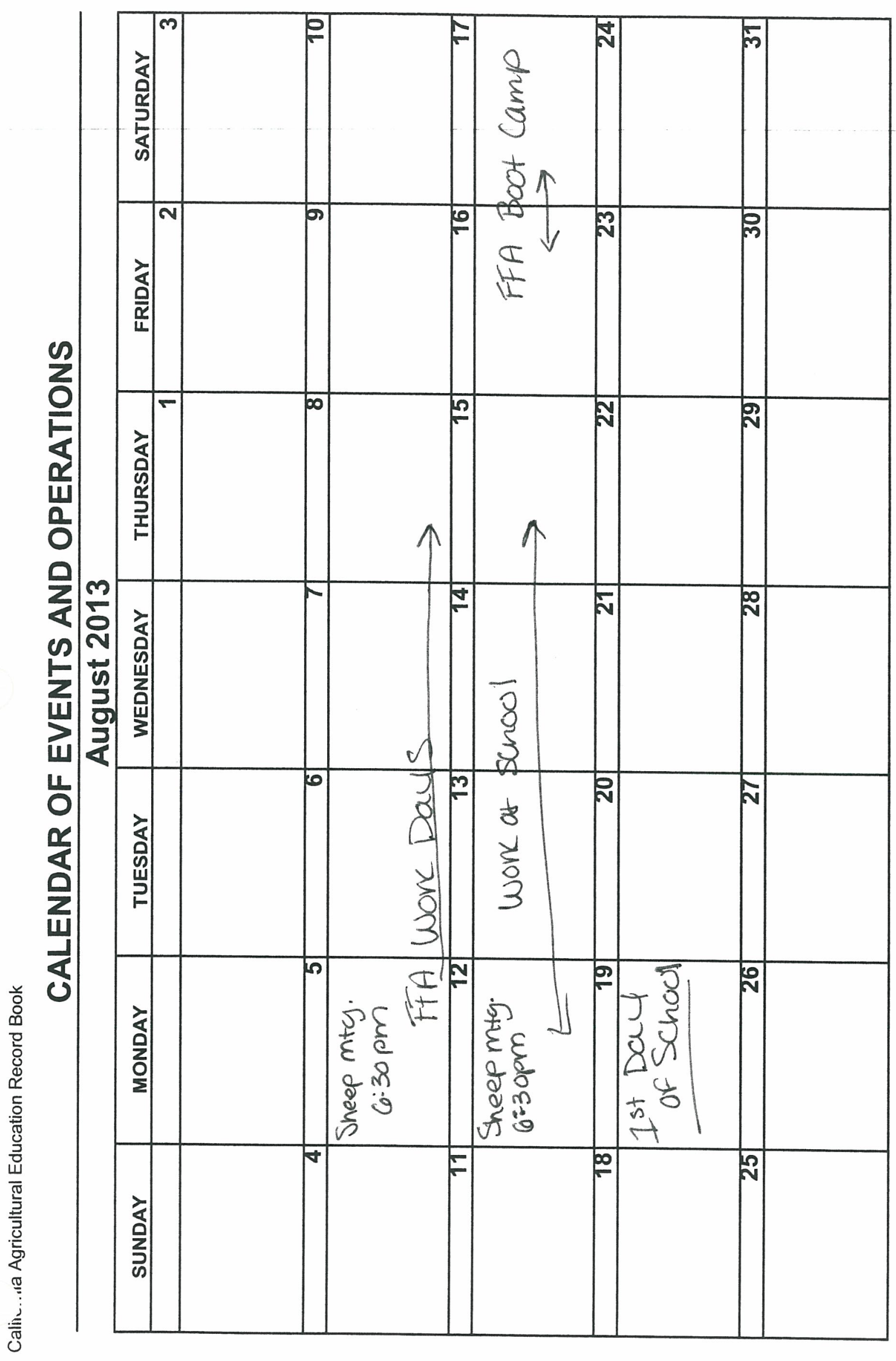

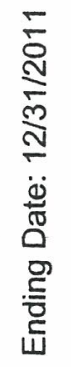




\section{Quality Criteria Two}

\section{Revision of the FFA Chapter Officer Polices and Election Process}

\section{Background:}

When I began this year at Clovis East one of my first encounters with the students was at the Officer Retreat. There was some confusion about when and where it was going to be held as the teacher I was replacing usually handled those matters and it was just a time of shifting duties and change. Mrs. Covey planned for the kids to retreat at Shaver Lake and I met them up there for one day because I still had interviews for the district on the next day. When I met the officer team for the first time they seemed quite young and not as experienced as I would have expected them to be. It was not until later that I found out why the officer team was so young.

After spending some time at Clovis East it became clear that there were parents and community members that were unhappy about how FFA Officer Elections have been ran in the past. Our senior kids were slated against juniors but the juniors had won the elections and people were suspicious because both the President and the Vice-President were children of our Ag Staff. Unfortunately, it seemed like the whole Officer thing was quite political. I even sat in a meeting with Mrs. Covey and the parents of one of the officers as they complained about how we are unfair and favor certain kids in our program. It bothered me that our FFA Officer program, one that I know first-hand can provide excellent leadership training and opportunities was under such scrutiny. There had also been some very nasty political things happening involving an Advisory Member and a certain family 
who has kids in our program. These battles have lead us as an Ag Staff to re think and reevaluate policies in our Department and make sure we as a staff are all on the same page.

After many long lunch periods talking with the other Ag staff members I was determined to present an alternative approach to our Officer Elections. Based on my experience from Central I knew how well a no slate program could work, you got to keep the top end of your kids to serve and no one had to feel like they were up against someone else. I presented the idea to the other Staff and they seemed to think it was a good idea and time for a change. We also agreed we needed to be clearer about the expectations of our Officers and how we would handle students who did not follow them. I began working on new election procedures and cleaning up policies, then collaborated with Mrs. Covey to create the final product. Once we as a staff were happy with the new documents I sent them to our Learning Director for approval.

The policies and procedures were approved and were used this spring to effectively elect our seven new officers. The process worked beautifully and we have not yet had any complaints. The following objectives were successfully met with the adoption of our new policies and Chapter Officer Handbook.

\section{Objectives:}

Objective 1- Adopt a system that eliminates slating of officer candidates, in order to ensure the best and most qualified students are elected. Also help reduce stress on students by not having to run against someone else who may be their friend. 
Met- The new system takes the total number of points into consideration. The student with the highest number of points will be considered for the office that they listed as their first choice on their application.

Objective 2- Adopt a system that is very clear about how officers are elected. There should be no question about what things go on behind closed doors in this process.

Met- The handbook I developed clearly explains the election process, beginning with the minimum qualifications, and then a detailed description of the election process.

Objective 3- Adopt a system that does not place all the weight on member votes. Popularity alone should not elect a student to Chapter Office.

Met- The point total is based on $40 \%$ popular vote, and $60 \%$ from their application, interview, and teacher recommendations. This way a student who does not perform well in these other important areas will not be elected into office. I used a free online grade book to set up the percentage weights so there would not be any room for errors.

Objective 4- Adopt a system that clearly states the expectations for Chapter FFA Officers and how discipline will be handled when elected to office.

Met- The Chapter Officer Policy document was created to outline the expectations for Chapter FFA Officers and establish a three strikes policy for removal of non-compliant officers. 


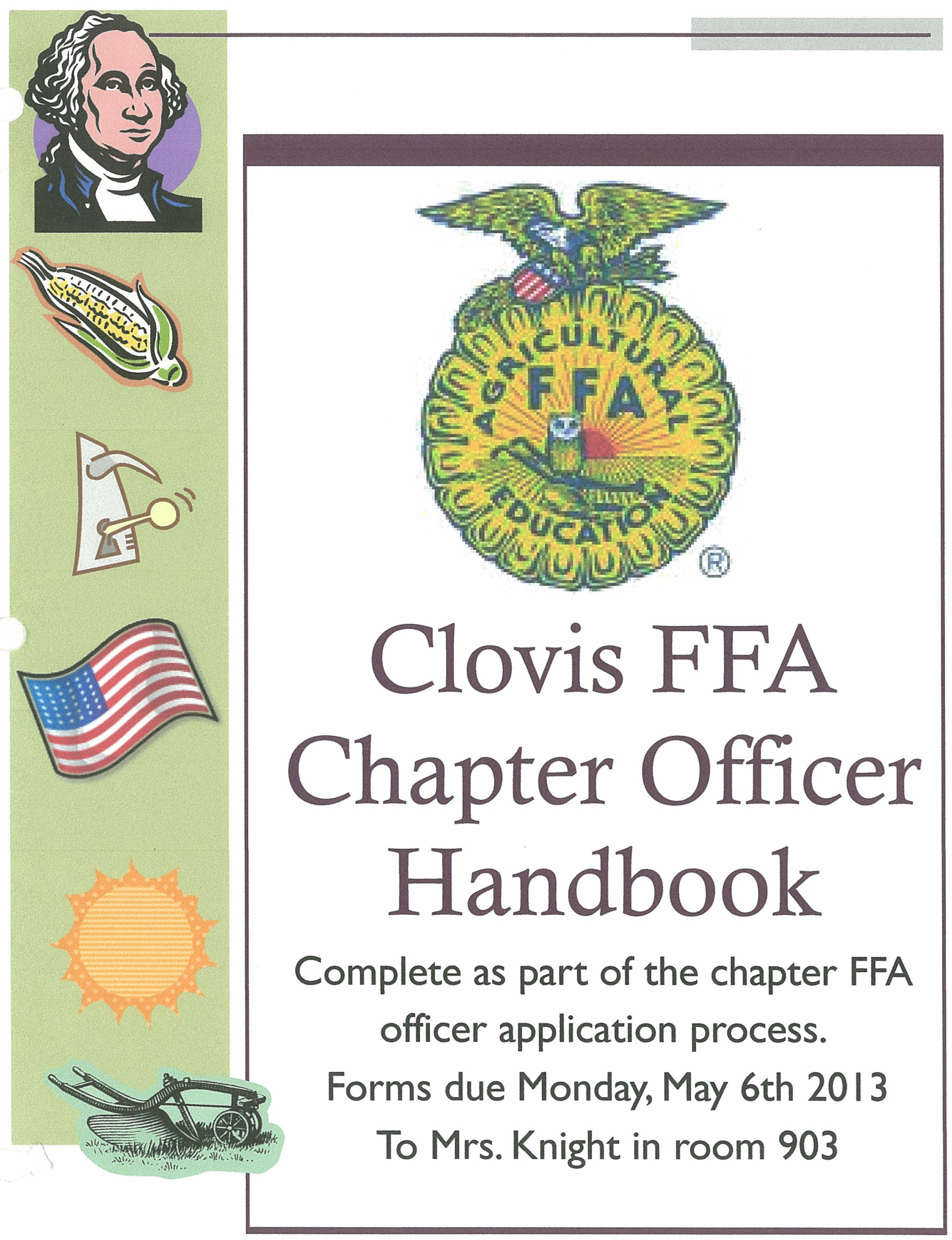




\section{Clovis FFA \\ Chapter Officer Candidate Handbook}

This Handbook contains supplemental Information and Forms that need to be completed as part of the Chapter Officer Application Process.

\section{Introduction:}

Running for Chapter FFA Office is an exciting opportunity that will allow you to develop your leadership skills, gain confidence, and challenge yourself to grow.

Chapter officers serve a vital function in our FFA Chapter by planning, coordinating, and carrying out all our Chapter level activities. Serving as a Chapter Officer is a position of great responsibility and servant leadership. Officer candidates should have a genuine desire to serve our members selflessly and the ability to work with teammates and Ag Advisors in a positive manner.

\section{Organization:}

The Clovis FFA Chapter Officers are overseen by the FFA advisor and the other agriculture teachers, with support by the Administrative Office.

There will be seven elected chapter officers for the school year and the possibility of additional appointed officers, as decided upon by the advisor.

\section{Minimum Qualifications for Candidates:}

To participate in the Clovis FFA Chapter Officer program, officer candidates must meet all of the eligibility requirements:

- Currently be an actively enrolled FFA Member and planning to enroll in Ag Education for the upcoming school year.

- Have and maintain a 2.0 cumulative GPA or higher.

- Have and maintain a clean discipline and attendance record as defined by CUSD policy.

- Complete and submit a signed Chapter Officer Application by the due date.

- Compete and submit signed copies of all required forms that are a part of this handbook on time.

- Go through the chapter interview and voting process.

- If elected, you are required to be enrolled in the Ag Leadership class for the year you serve as an Officer.

- If elected, you are required to attend the mandatory summer Chapter Officer Retreat . 


\section{Election Process:}

All Required Forms must be turned in by the due date in order to schedule an interview. Candidates who do not complete the entire process (paperwork \& Interview) will not be considerd for an office.

\section{The process of election is outlined below}

Candidates will have their application, and teacher reccomendation forms scored. They will also complete an interview and compete for popular votes. The candidates with the highest point total will be elected into office and fill the 7 offices. There will not be slating of candidates for particular offices, as all candidates are competing for office in general and not for any one particular office. Candidates will indicate their office preferences in order (ie $1^{\text {st }}$ choice President, $2^{\text {nd }}$ choice Secretary) on their application. The candidate with the higest score will be considered for the office they indicated as their first choice. The candidate with the second higest score will be considered for their first choice if their first choice was not already filled by the high point winner.

\section{Example:}

If Member A has 92 Points and put President as their first choice for office. Member B has 90 Points and has put President for their first choice and Secretary as their second choice for office, then Member A as having the higher point total would be elected President and Member $B$ would be elected as Secretary.

\section{How Points are Earned}

(15\%) Application

(15\%) Teacher/Adminstrator Reccomendation Forms (30\%) Interview

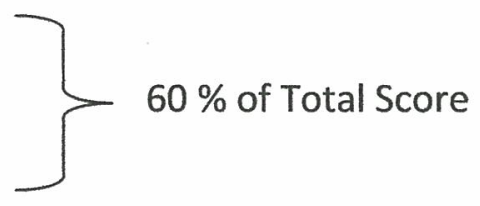

Popular Vote

$$
\} 40 \% \text { of Total Score }
$$

\section{How Applications are Scored}

- The Following Rubric will be used and applications will be blind scored (Name Removed) by two people. Scores are averaged.

- Typed Vs. Handwritten: Typed= 5 Points, Neatly Handwritten = 3-4 Points, Sloppy Handwritten $=2$

- Questions: For Each question 5 Points Possible, 5=Excellent, 4= Good, 3=Average, 2=Poor, 1= very Poor.

- Overall Impression and Sincerity of Thought: 5 Points Possible, 5=Excellent, $4=$ Good, $3=$ Average, $2=$ Poor, $1=$ very Poor. 


\section{How Teacher Recommendations are Scored}

- Forms are scored using a point scale, 5=Excellent Ratings, 4= Good Ratings, 3=Average Ratings, 2=Poor Ratings, $1=$ Very Poor Ratings.

- Scores are raw and not averaged

- Additional comments made on recommendation forms may be used as questions during the interview.

\section{Interview}

- Each candidate will sign up for an interview time when they submit all required forms from the handbook along with their application.

- Candidates are required to wear official FFA dress for their interview

- The interview panel can consist of any combination of the following people:

- FFA Advisor

- Agriculture Teachers

- School Administrators

- Community Members

- All applicants will be asked a set of the same questions, with the exception of a few clarification questions related to responses on their application or teacher recommendation forms.

- Interview Scores will be averaged to make the total score for the candidate.

Things considered in evaluation of interview responses:

$>$ Amount of thought and reflection in answering questions

$>$ Sincerity and Honesty in answers to questions

$>$ Genuine desire to serve our Chapter and its members

$>$ Depth of ideas and explanations given when answering questions

$>$ Professionalism, Poise, and Confidence in front of the interview panel

$>$ Indicators of Leadership and positive core values shared during interview

\section{Popular Vote}

\section{Campaigning for Votes}

Candidates will have the opportunity to film a 1-2 miniute video which will be shown to all FFA members in their Ag classes prior to voting. Video segments will be filmed the day of the candidates interview.

Reccomendations for Video content

- Introduce yourself- Name, grade, year in $\mathrm{Ag}$

- Top 5 FFA/SAE accomplishments or activities

- Personal or Professional traits you possess

- Goals or Ideas you have for the Chapter if elected

One campaign poster will be allowed for each candidate, no larger than 18x24 (Standard Poster Board). Posters must be submitted to the FFA Advisor who will approve them and hang them in a designated poster display area. 


\section{Voting}

Voting will be made available to students in $\mathrm{Ag}$ classes after viewing the video segments from each candidate. Each member may vote for 7 diferent candidates that they would like to see serving as their Chapter FFA Officer Team.

Vote counts will be verified by campus administration and added to the candidates score at a weight of $40 \%$ of their total points.

\section{Discipline:}

All Clovis FFA Chapter Officers will be placed on contracts in accordance with the Clovis FFA Officer Policy Form. The policy form is attached in this handbook.

Each Officer is required to sign the above stated policy form with required signatures and submitt with application package.

Please carefully read all materials and sign accordingly.

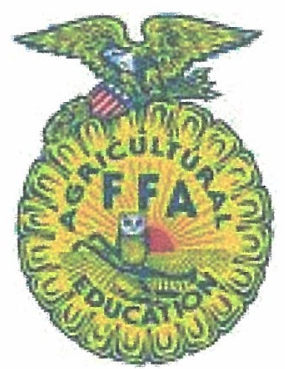

The Clovis Unified School District does not discriminate on the basis of race, color, disability, or national origin in admission or access to and treatment of employment in its programs and activities as required by Title VI, Title IX and Section 504. Complaints: Contact Dr. Cheryl Rogers, Human Resources, (559) 327-9308.

Favor de llamar a la oficina para assistencia en espanol.

Thov tiv tauj peb lub hoob kas yog xav tau kev pab nrog lus Hmoob. 


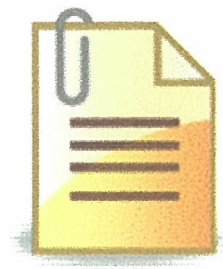

\section{FORMS}

\section{$\checkmark$ Chapter FFA Officer Policy \\ Obtain Signatures- Turn In}

\section{$\checkmark$ Teacher/ Principal Recommendation Forms}

Ask three teachers to complete (1 must be an Ag Teacher, the other 2 non Ag Teachers)

Teachers turn into FFA Advisors directly; you do not collect them

\section{$\checkmark$ GPA and Grade Check Form}

Complete and Turn In

$\checkmark$ Schedule and Important Dates

For Your Reference

\section{$\checkmark$ Officer Duties and Descriptions}

For your Reference

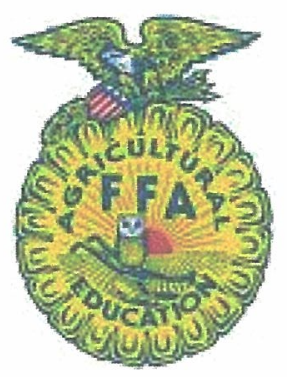




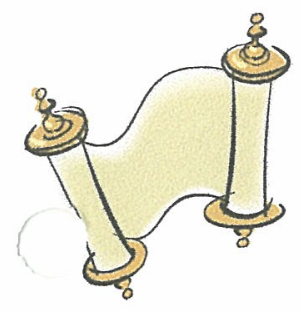

\section{Clovis FFA Chapter Officer Policy Discipline and Evaluation}

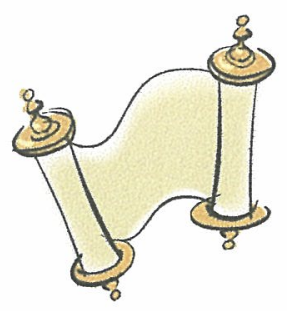

Chapter Officers should have a genuine desire to be part of a team, and should always lead by positive example. They should work with all chapter members and Advisors in a professional manner to complete their duties. The following policy addresses the consequences for not complying with the high standards that come with holding a chapter FFA office.

\section{Expectations}

- Participate in all MANDATORY FFA Activities as listed on the Chapter Officer Calendar.

- Participate actively in other FFA activities

- Complete specific Chapter Officer duties as assigned

- Represent Clovis FFA and the Agriculture Program in a positive and professional manner at all times.

- Communicate with advisors and fellow officers in regards to individual and team business.

- Willingly participate in self and team reflection for the purpose of personal and Chapter improvement.

\section{Discipline}

- A comprehensive discipline program has been established for officer conduct

\section{Excused Actions}

Each officer will receive three "free passes" which can be used to excuse them from an absence or a missed duty that would generally constitute a strike. They DO NOT apply to MANDATORY FFA event attendance or Clovis Unified Student conduct violations. Use of free passes should be cleared by the advisor in advance.

\section{Strikes}

Strikes can be issued for officer absences, not completing assigned duties, and un-professional conduct. Three strikes will be allowed, with the fourth constituting removal from the Officer Team.

\section{Actions that constitute receiving 1 strike:}

- Arriving late to 3 Meetings without an excuse cleared by an advisor.

- Missing a meeting. Meetings include those set by an advisor, monthly meetings, morning meetings and planning meetings. You may only miss a meeting if you talk to an advisor in advance of the meeting date(s).

- Misconduct at a FFA activity or event. Misconduct includes any action that may constitute a school referral.

- Failure to appropriately maintain project conditions or meet the responsibilities of an SAE as determined by the project advisor.

- Talking negatively about members and/or advisors, using profanity, and performing obscene gestures.

- Inappropriate public display of affection on school campus and at FFA events.

- Leaving campus during school hours without being checked out by a parent. 


\section{Clovis FFA}

\section{PRINCIPAL OR TEACHER RECOMMENDATION}

This Student is running for Clovis FFA Chapter Office. This position is one of service and dedication to FFA and all of its members. Please rate this student in each of the following areas. Please be completely honest in your evaluation. This will not be seen by the student or parent. Please complete and remit in a sealed envelope to the Agriculture Department mail bin in the Clovis East front office mailroom, Mrs. Covey in room 910 or Mrs. Knight in room 903. This will help us determine if this student is a good candidate for a leadership position.

Student's Name:

Teacher's Name:

\begin{tabular}{|l|l|l|l|l|}
\hline Category & Outstanding & Good & Fair & Poor \\
\hline Responsibility & & & & \\
\hline Respect for Authority & & & & \\
\hline On Time to Class & & & & \\
\hline Cooperative & & & & \\
\hline Attitude & & & & \\
\hline Gets Along with Others & & & & \\
\hline
\end{tabular}

\begin{tabular}{|l|c|c|}
\hline Recommendation & Yes & No \\
\hline $\begin{array}{l}\text { Would you Recommend this } \\
\text { Student for a Leadership } \\
\text { Position? }\end{array}$ & & \\
\hline
\end{tabular}

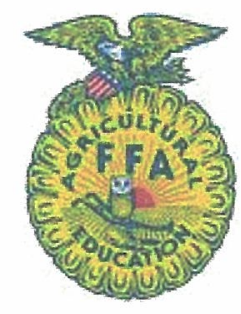

Comments: Please justify the above ratings (especially outstanding and poor ratings). 


\section{Clovis FFA}

\section{PRINCIPAL OR TEACHER RECOMMENDATION}

This Student is running for Clovis FFA Chapter Office. This position is one of service and dedication to FFA and all of its members. Please rate this student in each of the following areas. Please be completely honest in your evaluation. This will not be seen by the student or parent. Please complete and remit in a sealed envelope to the Agriculture Department mail bin in the Clovis East front office mailroom, Mrs. Covey in room 910 or Mrs. Knight in room 903. This will help us determine if this student is a good candidate for a leadership position.

Student's Name:

Teacher's Name:

\begin{tabular}{|l|l|l|l|l|}
\hline Category & Outstanding & Good & Fair & Poor \\
\hline Responsibility & & & & \\
\hline Respect for Authority & & & & \\
\hline On Time to Class & & & & \\
\hline Cooperative & & & & \\
\hline Attitude & & & & \\
\hline Gets Along with Others & & & & \\
\hline
\end{tabular}

\begin{tabular}{|l|c|c|}
\hline Recommendation & Yes & No \\
\hline $\begin{array}{l}\text { Would you Recommend this } \\
\text { Student for a Leadership } \\
\text { Position? }\end{array}$ & & \\
\hline
\end{tabular}

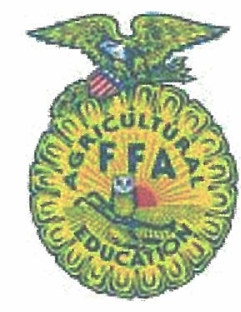

Comments: Please justify the above ratings (especially outstanding and poor ratings).

DO NOT GIVE IT BACK TO THE STUDENT

These are confidential. Thank you for your time! 


\section{Clovis FFA}

\section{PRINCIPAL OR TEACHER RECOMMENDATION}

This Student is running for Clovis FFA Chapter Office. This position is one of service and dedication to FFA and all of its members. Please rate this student in each of the following areas. Please be completely honest in your evaluation. This will not be seen by the student or parent. Please complete and remit in a sealed envelope to the Agriculture Department mail bin in the Clovis East front office mailroom, Mrs. Covey in room 910 or Mrs. Knight in room 903. This will help us determine if this student is a good candidate for a leadership position.

Student's Name:

Teacher's Name:

\begin{tabular}{|l|l|l|l|l|}
\hline Category & Outstanding & Good & Fair & Poor \\
\hline Responsibility & & & & \\
\hline Respect for Authority & & & & \\
\hline On Time to Class & & & & \\
\hline Cooperative & & & & \\
\hline Attitude & & & & \\
\hline Gets Along with Others & & & & \\
\hline
\end{tabular}

\begin{tabular}{|l|c|c|}
\hline Recommendation & Yes & No \\
\hline $\begin{array}{l}\text { Would you Recommend this } \\
\text { Student for a Leadership } \\
\text { Position? }\end{array}$ & & \\
\hline
\end{tabular}

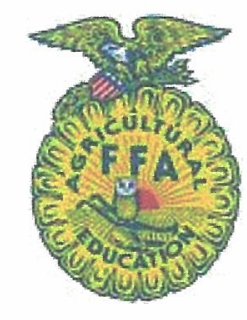

Comments: Please justify the above ratings (especially outstanding and poor ratings). 


\section{Clovis FFA \\ GPA Check Form}

The following student is running for Clovis FFA Chapter Office.

Student Name:

In order to be elligble for chapter office a student must have a 2.0 GPA.

$$
\text { GPA: }
$$

Date:

Signature of School Registrar (Mrs. Dickie):

\begin{tabular}{|c|l|l|l|l|}
\hline \multicolumn{5}{|c|}{ GRA DE CLEARANCE } \\
\hline Period & Subject \& Teacher & Teacher Signature & $\begin{array}{c}\text { Current } \\
\text { Grade }\end{array}$ & Comments \\
\hline 0 & & & & \\
\hline 1 & & & & \\
\hline 2 & & & & \\
\hline 3 & & & & \\
\hline 4 & & & & \\
\hline 5 & & & & \\
\hline 6 & & & & \\
\hline 7 & & & & \\
\hline 8 & & & & \\
\hline
\end{tabular}




\section{Schedule and Important Dates}

Applications Due:

Monday, May $6^{\text {th }} 2013$

To: Mrs. Knight in room 903

Chapter Officer Handbook Forms Due:

Monday, May $6^{\text {th }} 2013$

To: Mrs. Knight 903

Chapter Officer Interviews

May $7^{\text {th }}$ and May $8^{\text {th }} 2013$

$\checkmark$ Be in Official Dress Uniform

$\checkmark$ Campaign Video will be recorded

Voting in Ag Classes

Tuesday, May $14^{\text {th }} 2013$

Spring Banquet- Announcement of The Officer Team

Thursday, May $16^{\text {th }} 2013$

Newly Elected Officers Meeting

Tuesday, May $21^{\text {st }} 2013,7: 00 a m$ room 902 (Blue \& Gold room)

FFA Elected Officers Retreat (MANDATORY!)

Place to be determined

June $12^{\text {th }}, 13^{\text {th }} \& 14^{\text {th }} 2013$

FFA Officers Summer Work Day(s)

Will be scheduled Individually with Officers once elected

August...2013

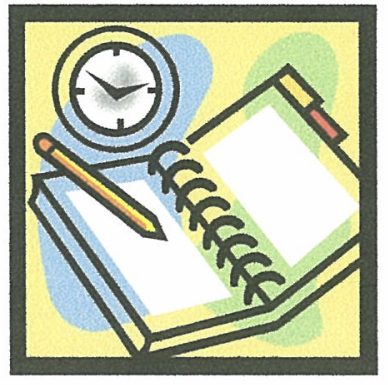




\section{FFA Office Descriptions and Duties}

*President: Shall reside over the executive committee, conduct meetings, appoint standing and special committees, correlate function of the committees, and see to it that they function properly and smoothly, and work closely with the advisor.

Typical Tasks:

- Lead FFA Meetings

- Delegate tasks among officers

- Manage Meeting Schedules and Rotations

- Develop topics for meeting agendas

- Apply for Awards

*Vice-President(s): Shall assist the President in the duties of coordinating FFA activities, act as President in the absence of the President or at such time as the President directs, and attend to coordinating all committees.

Typical Tasks:

- Encourage involvement in SAE's

- Manage Games and Activities at Meetings

- Update and Complete Program of Activities document

- Monitor Recruitment of members

- Promote a Positive Chapter Image

- Manage the FFA Calendar

- Conduct activities that will help improve the community

*Secretary: Shall coordinate agendas, keep an accurate record of all chapter meetings, and handle all corresponding matters.

Typical Tasks:

- Taking Minutes of Meetings

- Typing and posting meeting Agendas

- Communications, phone calls, and E-Mail

- Invitations, Thank You Cards, Programs

- Making Copies

- Keeping activity/meeting attendance records (point award chart)

*Treasurer: Shall handle the funds of the chapter, keep an accurate record of receipts and disbursements, and approve all of the financial spending of the chapter.

Typical Tasks:

- Handle Funds

- Coordinate Fundraising Activities

- Maintain Receipts

- Maintain FFA Account Binder

- Coordinate and monitor raffle at FFA Meetings 
* Reporter: Shall be in charge of informing the membership, community, and fellow chapter of activities and events.

Typical Tasks:

- Writing Articles

- Chair Newsletter Committee

- Provide Public Relations

- Submit Information for morning announcements

- Take Chapter Photographs and organize Photos of events

- Maintain accuracy of Chapter Web Page and help keep up to date

*Sentinel: Shall be in charge of assisting the President in keeping order of the meeting and will be responsible for setting up the meeting room.

Typical Tasks:

- Set up and Clean Up of FFA Activities

- Manage and maintain Official FFA Equipment

- Manage and maintain Music and Video Coordination for FFA Activities

- Coordinate Decorations for events

- Fill out and submit Facility Requests

*Historian: Shall be in charge of keeping an accurate and up to date history of the chapter and its members

Typical Tasks:

- Chairmen of FFA Scrapbook Committee

- Assistant to Chapter Photographer

- Collect and submit photos for the school yearbook

- Record Results from all Contests and Events and keep on file

- Keep track of degrees earned by members and file certificates

- Participate as a member of the Newsletter Committee 


\section{Clovis FFA Chapter Officer Application

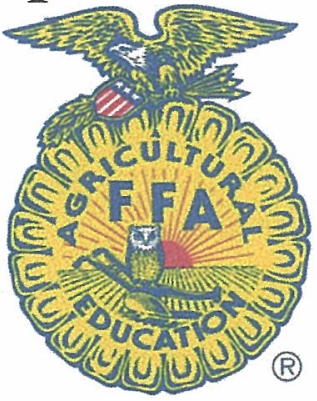

\section{Check-off sheet}

Candidates Name and Grade:

$\square$ Completed Application (in ink or typed) - and all appropriate signatures present.

$\square$ Chapter FFA Officer Policy all signatures obtained.

$\square$ Currently be an actively enrolled FFA Member

$\square$ Enrolled in or planning to enroll in Ag Leadership for the upcoming school year.

$\square$ You have read and understand all contained in the Chapter Officer Handbook.

$\square$ GPA and Grade Check Form is $100 \%$ complete!

$\square$ Available to attend the Summer Officer Retreat and Meetings date TBA

$\square$ Officer Applications Due Monday May $6^{\text {th }} 2013$ to Mrs. Knight, Room 903 


\section{Clovis FFA Chapter Officer Application}

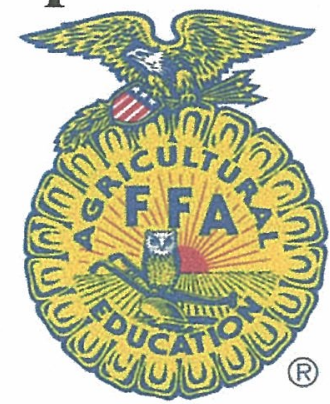

Applicant's Name:

Address:

City:

State:

Zip:

Phone \#:

Cell \#

Grade:

Age:

List the Agriculture classes you have taken each year and plan on taking in the future:

$9^{\text {th }}$ Grade $10^{\text {th }}$ Grade $11^{\text {th }}$ Grade

$12^{\text {th }}$ Grade

Number 1 - 7 by preference, the offices you want to be considered for, if you are elected.

( 1 is your most desired spot and 7 is your least desired spot)

$\begin{array}{llr}\text { _ President } & \text { Vice President } & \text { Secretary } \\ \text { Reporter } & \text { - Sentinel } & \text { _ Historian }\end{array}$

Chapter office you would not accept:

1. What qualities do you possess and how do they qualify you for a chapter office?

2. If elected to a chapter office, what would be your main goal for the chapter? 
3. Being a Chapter Officer is a big time commitment, what other commitments do you have during the year that would compete for your time? Please explain how you plan to manage your time?

4. What do you believe the most important responsibilities of a chapter officer are?

I , hereby approve of my son/daughter,

, running/becoming a Clovis FFA Chapter Officer. I realize that $\mathrm{s}(\mathrm{he})$ is responsible for his/her officer duties and will uphold them to the best of his/her ability. I further understand my student is required to be at all executive committee meetings on time, or will fall subject to the penalties associated with absences and tardiness. I am aware that repeated absences from official officer functions will result in my student's early dismissal from the officer team. I also understand that his/her presence at the officer retreat this summer is mandatory!

Parent/Guardian Signature:

Date:

I understand I am responsible for my duties as a chapter officer and will uphold them to the best of my ability. I further understand I am required to be at all executive committee meetings, on time, or I will fall subject to the consequences associated with absences and tardiness. I am aware that repeated absences from required functions will result in my being dismissed from the officer team early. I further understand that my presence at the officer retreat this summer is mandatory!

Applicant Signature:

Date:

The Clovis Unified School District does not discriminate on the basis of race, color, disability, or national origin in admission or access to and treatment of employment in its programs and activities as required by Title VI, Title IX, and Section 504. Complaints: Contact Dr. Cheryl Rogers, Human Resources, (559) 327-9308.

Favor de llamar a la oficina para asistencia en español.

Thov tiv tauj peb lub hoob kas yog xav tau kev pab nrog lus Hmoob. 


\section{Clovis FFA}

\section{Officer Application Scorecard}

Candidate Name:

Please Rate the Individual on a Scale from 1-5

$1=$ poor, $2=$ needs work, $3=$ =average, $4=$ good, $5=$ excellent

Scored Items
\begin{tabular}{|l|l|}
\hline $\begin{array}{l}\text { Typed= } 5, \text { Neatly Handwriten=3-4, Sloppy } \\
\text { Handwriten=1-2 }\end{array}$ & \\
\hline Question 1 (5 Pts.) & \\
\hline Question 2 (5 Pts.) & \\
\hline Question 3 (5 Pts.) & \\
\hline Question 4 (5 Pts.) & \\
\hline Spelling and Grammar (5 Pts.) & \\
\hline \begin{tabular}{l} 
Overall Impression (5 Pts.) \\
\hline
\end{tabular} & \\
\hline $\begin{array}{l}\text { List of Agriculture Courses shows past commitment } \\
\text { and plans for growth in the Ag Department. (5 Pts.) }\end{array}$ & \\
\hline
\end{tabular}

Total Score for Application ( 40 Points) 


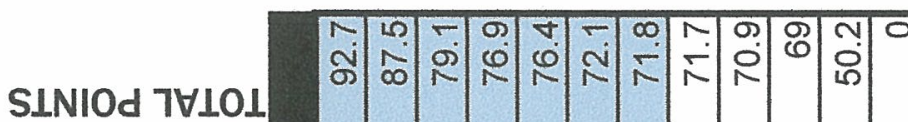

$\varepsilon$ иопрериәшоээәу

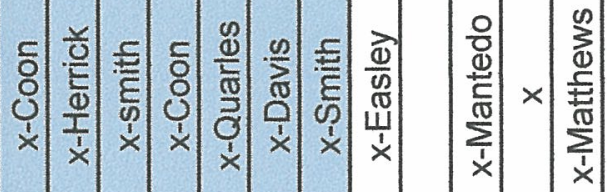

乙 ио!ฺериәшоээәу

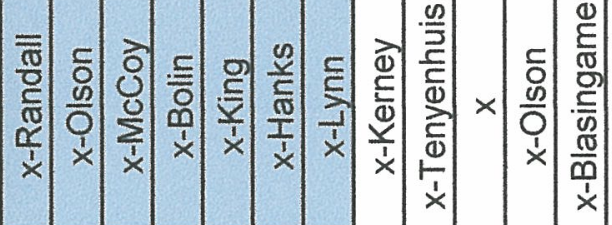

โ иорฺериәшоэวәу

$\times \times \times \times \times \times \times \times \times \times \times$

บวอับว $\forall d \supseteq$

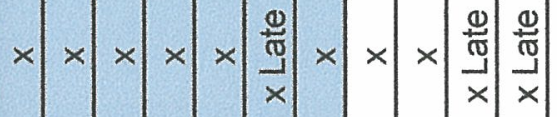

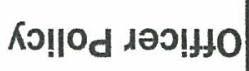

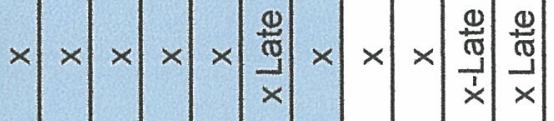

u| uo!̣eग!|dd $\forall$

$$
\times \times \times \times \times \times \times \times \times \times \times
$$

苋

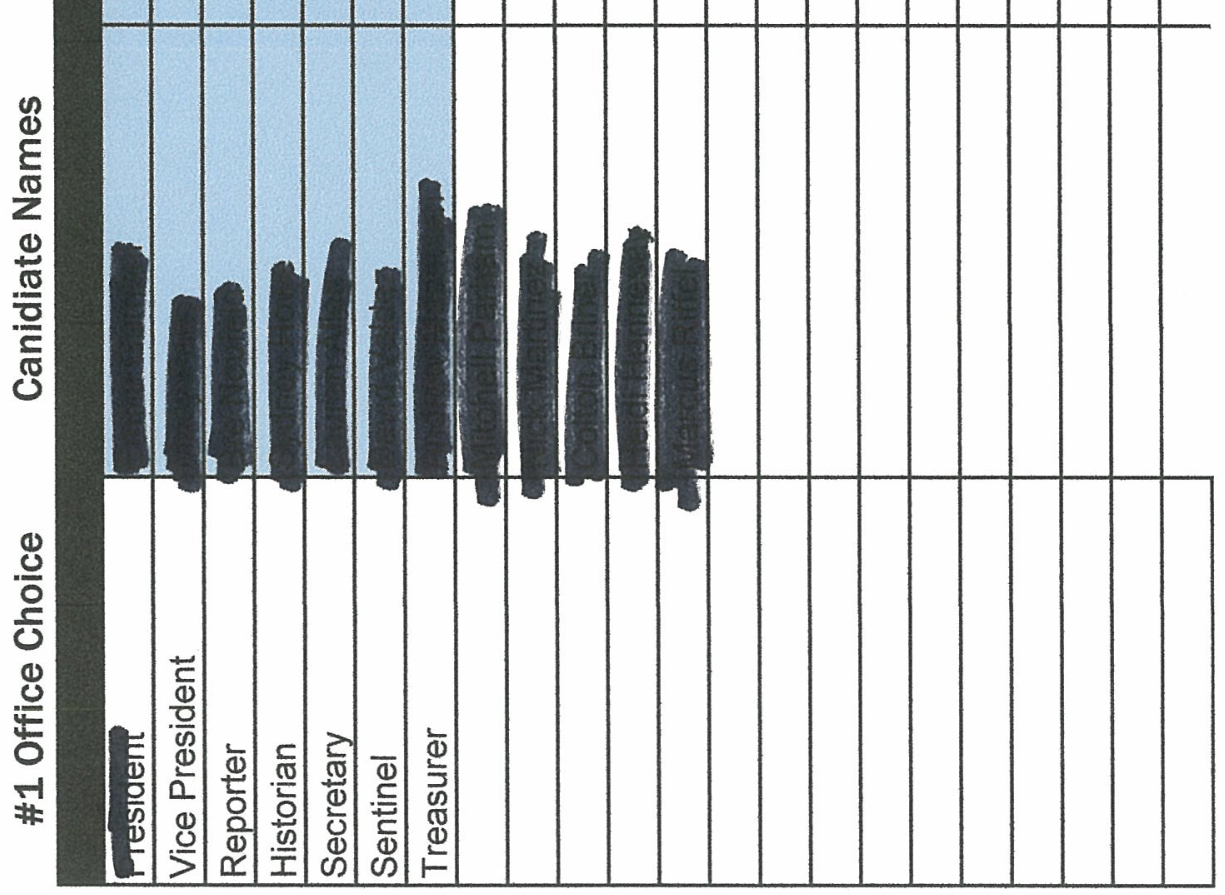


Online Grade Book

Weights for Chapter Officer Elections

Special Marks

Special Mark

\begin{tabular}{|l|l|l|l|}
\hline ex & excused & - & $\%$ \\
\hline ng & not graded yet & - & $\%$ \\
\hline inc & incomplete & - & $\%$ \\
\hline$\checkmark$ & ok & 100 & $\%$ \\
\hline$\checkmark-$ & half & $\%$ \\
\hline & & $\%$ \\
\hline & & $\%$ \\
\hline & & $\%$ \\
\hline
\end{tabular}

Missing Assignments

\begin{tabular}{|l|l|l|l|}
\hline$/$ & missing & $\%$ \\
\hline abs & absent & $\%$ \\
\hline & 0 & $\%$ \\
\end{tabular}

[v] 0 also means Missing

\section{Categories}

Unweighted

(c) Weighted

C. None

\begin{tabular}{l} 
Category \\
\begin{tabular}{|l|l|}
\hline Application & Weight \\
\hline Reccomendations & 15 \\
\hline Interview & 15 \\
\hline Popular Vote & 40 \\
\hline & \\
\hline & \\
\hline & \\
\hline & \\
\hline
\end{tabular} \\
\hline
\end{tabular}

Total

100 
Officer Elections (1) Jennifer Knight 1st Semester, 2013 May 25, 2013

S1

\begin{tabular}{|c|c|c|}
\hline 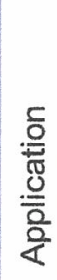 & 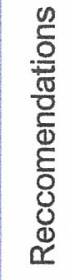 & 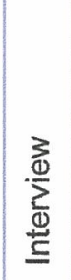 \\
\hline 15 & 15 & 30 \\
\hline
\end{tabular}

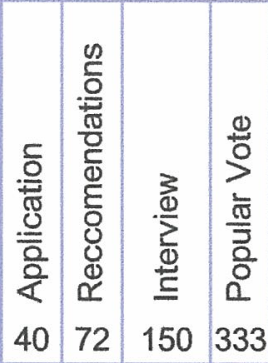

\begin{tabular}{|lll|l|l|l|l|l|l|l|l|}
\hline C- & $71.3 \%$ & 90.0 & 66.7 & 78.0 & 61.0 & 36 & 48 & 117 & 203 \\
\hline
\end{tabular}

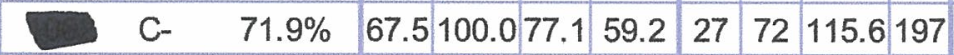

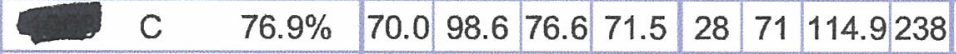

\begin{tabular}{lll|l|l|l|l|l|l|l|l|l|l|l|l|l|}
\hline & A- & $92.7 \%$ & 87.5 & 94.4 & 84.6 & 100.0 & 35 & 68 & 126.9333 \\
\hline
\end{tabular}

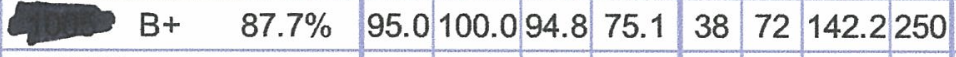

\begin{tabular}{|ll|l|l|l|l|l|l|l|l|}
\hline C+ & $79.5 \%$ & 77.5 & 98.6 & 77.1 & 74.8 & 31 & 71 & 115.7 & 249 \\
\hline
\end{tabular}

\begin{tabular}{|lll|l|l|l|l|l|l|l|}
\hline & C- & $71.4 \%$ & 92.5 & 100.079 .3 & 46.8 & 37 & 72 & 119 & 156 \\
\hline
\end{tabular}

\begin{tabular}{lll|l|l|l|l|l|l|l|l|}
\hline$F$ & $50.2 \%$ & 82.5 & 93.1 & 79.7 & 0.0 & 33 & 67 & 119.5 & 0
\end{tabular}

\begin{tabular}{lll|l|l|l|l|l|l|l|l|}
$\mathrm{S}$ & $\mathrm{C}$ & $73.0 \%$ & 65.0 & 84.7 & 75.3 & 70.0 & 26 & 61 & 113 & 233 \\
\hline
\end{tabular}

\begin{tabular}{llll|l|l|l|l|l|l|}
$\longrightarrow$ & C & $76.5 \%$ & 70.0 & 93.1 & 79.3 & 70.6 & 28 & 67 & 118.9235 \\
\hline
\end{tabular}

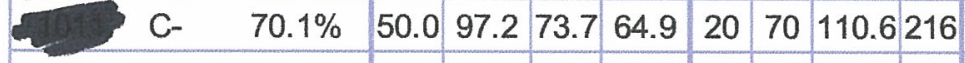




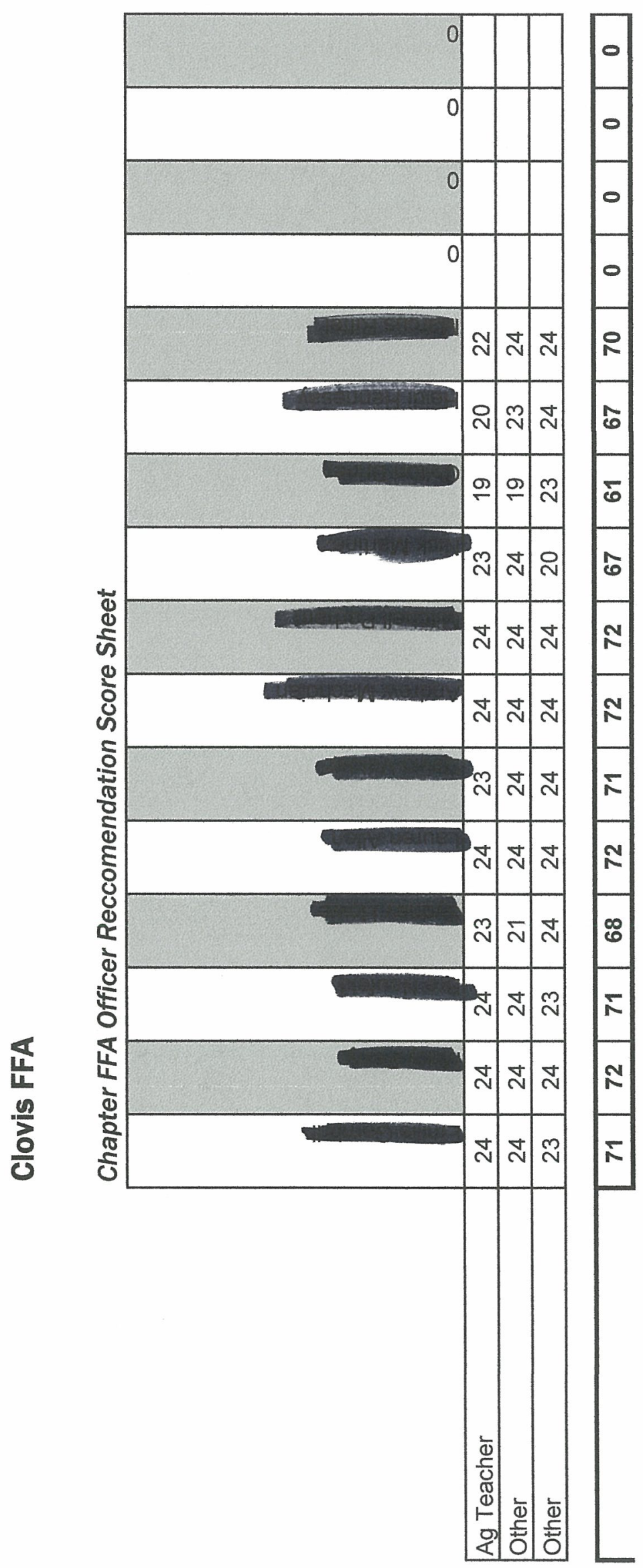




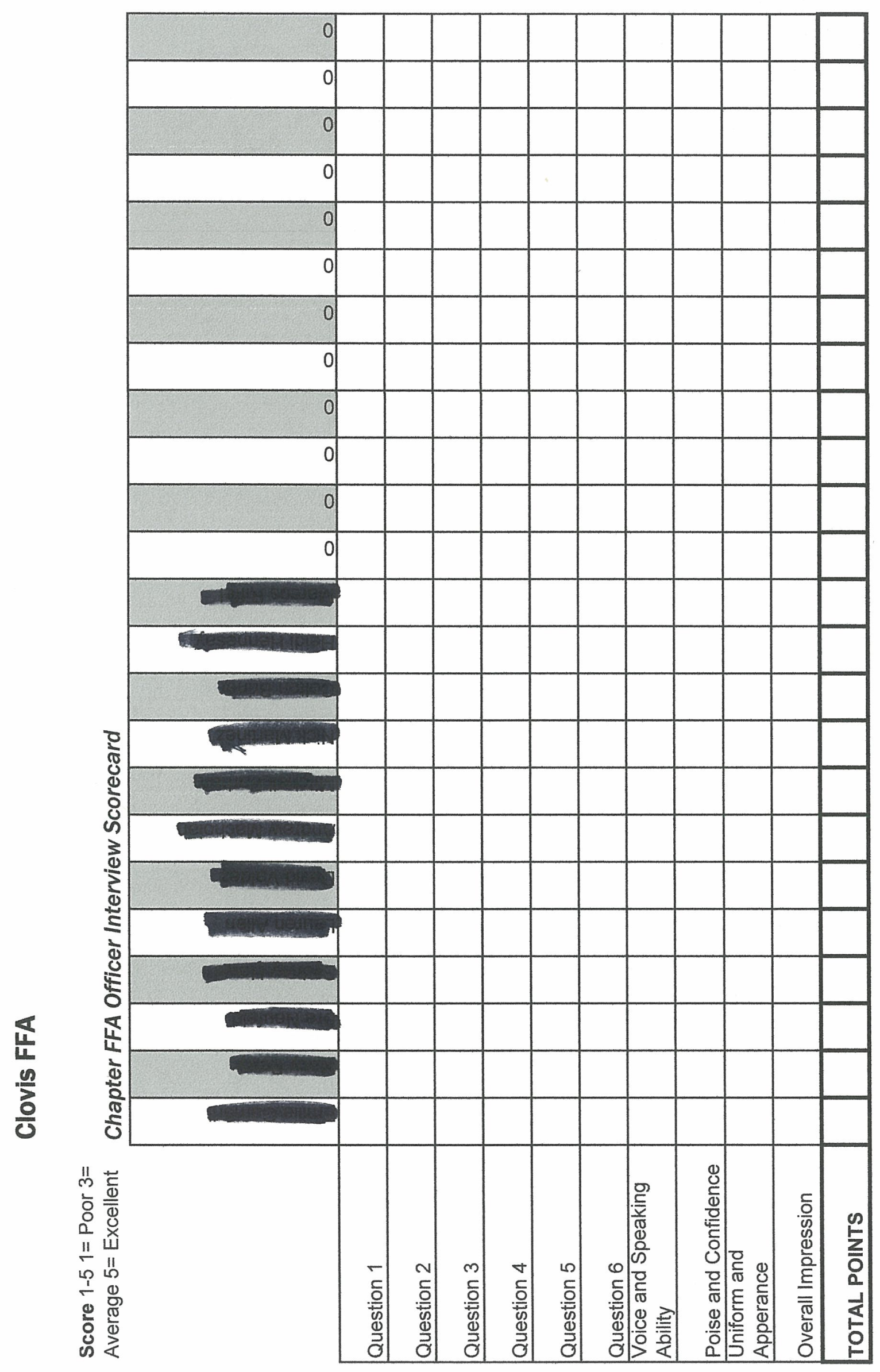




\section{FFA Officer Applicant \\ Informational Meeting}

If you are interested in running for Chapter

FFA Office you should attend the informational meeting on

Wednesday, May Ist at lunch in room 903

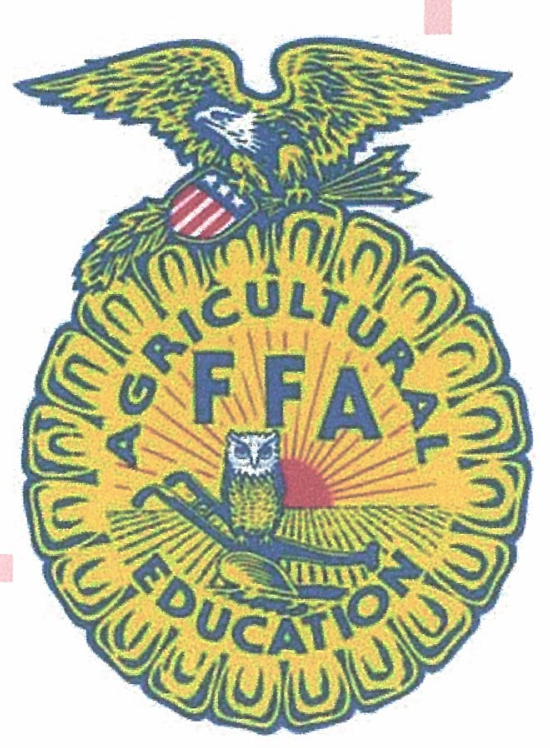




\section{Quality Criteria Three}

Creation of New Farm Use and FFA Exhibitor Contracts for Sheep Projects

\section{Background:}

When I was hired this past August I willingly took over the sheep SAE projects. I was difficult at first trying to figure out who the students were that had projects and who's project belonged to whom, and how they were keeping track of feedings and other things. The previous advisor did not really give me much to go on. I started hanging around in the barn during feeding time to catch kids and their parents and introduce myself. I asked the student when was their normal time for meetings or practices, but they said they had not had a meeting since before school got out. I called a meeting with all the sheep showman who kept their animals at the Ag center and established a once a week meeting schedule with them on Monday evenings. Meeting attendance was not always the best and it was difficult to hold students accountable for attending when I did not know them or could not reach them on the phone. I found some farm contracts in a file folder left by the previous teacher but some students were missing one.

Once school resumed it became much easier to find and meet all the students with projects. I still was met with some opposition from certain students and parents who did not like the way I did things; on the other hand many students were thankful that I did take the time to help them with things like record books and showmanship practices.

I knew that for the next project year I would want to start off right and have clear expectations for students who kept their sheep projects at school and for those students who keep a sheep project at home and wish to show with the Clovis FFA. 
Objective 1- Create a Market lamb Project Info Packet so students and parents can evaluate wither of not this is a good project for them.

Met- I created an informational packet explaining to students and parents the basic costs and time associated with having a market lamb project. If they decide to move forward with the project then they can sign the packet and return to me. I also provide the sheep meeting schedule at this time so they can make sure to schedule their summer plans accordingly.

Objective 2- Collaborate with other Ag Staff to make a new Farm Use contract for Sheep Projects.

Met- I worked with Mr. Gambril, Mr. Woodard, and Mr. Dias to develop a new Sheep SAE Farm Use Contract. See the contract attached in this binder.

Objective3- Create a FFA Exhibitors Contract for those students with sheep SAE's that wish to exhibit at Fairs and Shows under the Clovis FFA name.

Met- This contract was very important because in the past only students who kept their animals at the Ag center signed a contract. Students who keep sheep SAE's at home and plan to show with our Chapter's group at the Fair also need to have expectations. This contract is signed by all students who have a sheep SAE that they wish to show with the Clovis FFA. 


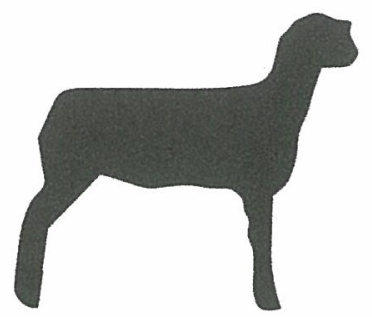

\section{Clovis FFA \\ Market Lamb Project Information 2013}

I am very excited that you have shown an interest in exhibiting a market lamb at this year's Big Fresno Fair. I truly look forward to working with you and your lamb. In order to have a successful year you must be aware of some important information. Hopefully the following information will help you make a final decision about showing a market lamb.

\section{MONEY}

\section{Purchase \& Operation Costs:}

The purchase price of a market lamb will vary, but the average price is $\$ 350$. Mrs. Knight will select a group of lambs and students can select from the group. Some lambs may be different prices $(\$ 250-\$ 375)$ so students may consider the price differences when making their selections. Lambs may be purchased by the individual outside the group Mrs. Knight selects, however you must receive prior approval from Mrs. Knight to do so. Feed costs include grain and hay. Expect to spend $\$ 150$ on grain and $\$ 20$ on hay for the life of the project. Other expenses may include basic medication as recommended by Mrs. Knight or supplies students need for their project. Fair Entry Fees will also be paid by the students. In order to begin this project and select from the group of lambs acquired by Mrs. Knight, a $\$ 250$ non-refundable deposit is required to be submitted and will be credited toward the purchase of your lamb. Please make checks payable to Clovis FFA. Please remember the FFA is not making money on these animals, but we do need to pay the people we buy the animals from. The balance for the purchase price of the animal will be due at the time the student takes ownership of the animal. (May-June)

\section{Cost Breakdown:}

$\begin{array}{ll}\text { Purchase of Animal } & \$ 250-350 \\ \text { Feed (Grain and Hay) } & \$ 170 \\ \text { Fair entries: } & \$ 12 \\ \text { Halter: } & \$ 15 \\ \text { Show supplies: } & \$ 30\end{array}$

TOTAL ESTIMATED COST

Vet bills if your animal becomes sick of injured will be an additional cost

*If an animal dies, the student is still responsible for paying their bills. If students would still like to show an animal, they will have to buy a replacement. Insurance is available and it will be you responsibility to let me know if you would like to insure your animal.

Sale and marketing of project: students will have the opportunity to put up for sale one market animal at the Big Fresno Fair through the Junior Livestock Auction. This auction is like any other livestock auction where people bid on animals to purchase them. However, it is important that you know that simply selling your animal through the auction does not mean you will make money! Successful marketing of your Market Lamb Project is what will ensure that you will break even or make a profit. This marketing of the project is up to the student; The advisor will make suggestions and assist when needed, but the price paid 
for the animal is up to the student promoting their animal and securing a buyer. Here are a few examples that can help illustrate how this could play out for the student.

Student A: Does not have a person lined up to buy their animal at the Big Fresno Fair Livestock Auction, and did not secure any additional bump bids.

Money invested: $\$ 577$

Sale of lamb weighing $145 \mathrm{lbs} @ \$ 2.50 \mathrm{Lb} .=\$ 362.50$

Total LOSS $=\$ 214.50$

- Unfortunately this happens to many students due to the fact they did not market their project, or waited until the last minute to try and do so.

Student B: this student worked hard for several months prior to the Fair to secure a buyer who would purchase their Lamb at a set price at the auction, and then received several bump bids (Monetary Contributions) to their projects.

Money invested: $\$ 577$

Sale of lamb by buyer weighing $145 \mathrm{Lbs} @ 4.50 \mathrm{Lb}=\$ 652.50$

10 bump bids secured for $\$ 30$ each $=\$ 300$

Total INCOME $=\$ 375.50$

- because this student worked hard to market their lamb they were able to earn enough money to reinvest in their project for next year!

\section{TIME \& RESPONSIBILITIES:}

1. This is your market lamb SAE project; therefore it will be your responsibility to feed, water, clean and maintain your animal. As an advisor, I am here to offer suggestions, guidance and directions, not to take care of all the animals. If there is a problem or concern, you are to inform the advisor immediately.

2. In order to show your market lamb at the fair, you will be required to attend weekly practices, feed your lamb, and maintain the facility. Failure to do so will result in a strike. Once you obtain three strikes, your showing privileges for lambs will be revoked.

3. There will be a mandatory parent meeting prior to participation in the Big Fresno Fair. Please be in attendance and inform your parents now.

4. We will go the ranches and sales together if you would like to have input on selecting animals for our group. YOU MAY NOT GO AND PURCHASE A LAMB WITHOUT MY APPROVAL. If you want to do things "on your own" then you can show as an independent and not with our FFA group.

5. Once the lambs are at the school farm, meetings will begin to inform you about the project, for you to work with lamb, showmanship details, etc. You will be required to attend meetings once a week: I will schedule meetings around sports events and other activities.

6. Farm work days- there will be two weekends we have to work on the school farm before the fair. Plan to be there.

7. You can keep your animal at the school farm or at home. There is always risk with keeping your animal at the school farm, and you must remember the school farm is also an educational facility that we share with others. If you keep your animal at the farm then you must read and agree to the farm contract.

8. If you decide to keep your animal at home or off the school farm you must sign and agree to the Clovis FFA Exhibitors contract.

9. You will feed your lamb twice a day! Your lamb does not care if you have a vacation planned; they still need to be fed. Animals must be fed before 9am in the morning and 
between 6:30pm-9:30pm for evening feeding. These times are critical to the animal's growth and health. Feeding late or too early and cause you a strike.

10. You will be missing 2-3 days of schools to participate in the fair. It will be your responsibility to get your work ahead of time and make sure that you are caught up with your work. You will also need to be at the fair all day on Saturday and part of Sunday.

11. In order to receive your fair check from the sale of your animal you must:

a. Complete 2 hours of work on the farm after the fair to help clean our equipment and facilities (checked by teacher).

b. Clean out your pen and locker used that year.

c. Complete your record books (checked by teacher)

d. Complete thank-you letters on nice stationary or thank-you cards. You will show Mrs. Knight the rough and final draft of your thank you letter. If you mail them before you show Mrs. Knight you will re-write them.

e. Pay your bills to the FFA (checked by teacher)

f. Apply for the appropriate FFA degree

\section{I} (student name), have read and understand the information provided in this letter and will be participating in this SAE.

Student Signature:

I ( parent name), have read and understand the information provided in this letter and my child has a permission to participate in the market lamb SAE.

Parent Signature:

Turn in this information packet signed with your deposit to Mrs. Knight in room 903. I am currently looking for prospect show lambs for us. Picking order for Lambs will be based on years shown and who returns all paperwork and deposit first. Please call Mrs. Knight if you have any questions.

(559) 765-9580 Cell

jenniferknight@cusd.com

The Clovis Unified School District does not discriminate on the basis of race, color, disability, or national origin in admission or access to and treatment of employment in its programs and activities as required by Title VI, Title IX and Section 504. Complaints: Contact Dr. Cheryl Rogers, Human Resources, (559) 3279308.

Favor de llamar a la oficina para assistencia en espanol.

Thov tiv tauj peb lub hoob kas yog xav tau kev pab nrog lus Hmoob. 


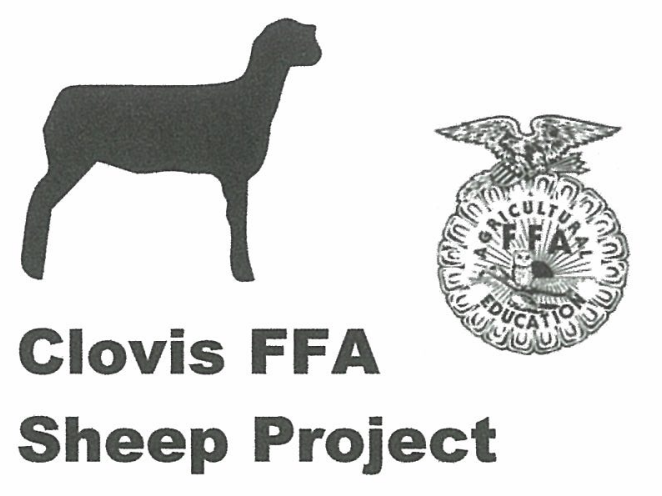

Sheep Exhibitor

Dates and Times

Highlighted are Meetings that are required for Exhibitors keeping their animals at home.

\section{(MUST ATTEND ONE OF THESE TWO Friday MEETINGS)}

Friday, May $10^{\text {th }} 3: 00-4: 30 \mathrm{pm}$ Room 903 , Record Book Start Up Farm and Exhibitors Contract Lamb Care CHOOSE ONE Friday, May $17^{\text {th }} 3: 00-4: 30 \mathrm{pm}$ Room 903

Record Book Start Up Farm and Exhibitors Contract Lamb Care

Monday, May $20^{\text {th }}$ - After School PICKING LAMBS (Take . Ownership) Balance owed DUE

Wednesday, May $29^{\text {th }}-$ Sheep Meeting After School

Monday, June $3^{\text {rd }}$ 3:00pm Meeting and Practice

Tuesday, June $11^{\text {th }} 6: 30 \mathrm{pm}$ Meeting and Practice
Wednesday, June $19^{\text {th }} 6: 30 \mathrm{pm}$ Meeting and Practice

Thursday, June $27^{\text {th }} 6: 30 \mathrm{pm}$ Tagging and Photos for Fair Contracts

Monday, July $8^{\text {th }} 6: 30 \mathrm{pm}$

Fair Entries and Contracts

Tuesday, July $9^{\text {th }} 6: 30 \mathrm{pm}$ MONDATORY Fresno Fair

Exhibitors Meeting

Fresno Fair Grounds 6:30pm

Monday, July $15^{\text {th }} 6: 30 \mathrm{pm}$

Meeting and Practice

Monday, July $22^{\text {nd }} 6: 30 \mathrm{pm}$

Record Book work

Saturday, July $27^{\text {th }} 8: 00 \mathrm{am}-12$ noon

Optional Rate of Gain Weigh in at the Fresno Fair Grounds

Sunday, July $28^{\text {th }}$ (Not a Meeting) ALL FAIR ENTRIES AND CONTRACTS DUE to Fairgrounds

Monday, July $29^{\text {th }} 6: 30 \mathrm{pm}$

Meeting and Practice

Monday, August $5^{\text {th }}$ 6:30pm

Meeting and Practice

Monday, August $12^{\text {th }} 6: 30 \mathrm{pm}$

Meeting and Practice

Monday, August $26^{\text {th }} 3: 00 \mathrm{pm}$

Meeting and Practice

Wednesday, September $4^{\text {th }}$ 3:00pm

Fresno Fair Planning Meeting

Fair Plans and Displays 
Monday, September $9^{\text {th }} 3: 00 \mathrm{pm}$

Meeting and Practice

Shearing for Fall Classic

Tuesday, September $10^{\text {th }}$

Shearing for Fall Classic

Wednesday, September $11^{\text {th }}$

FALL CLASSIC SHOW at CEHS

Monday, September $16^{\text {th }} 3: 00 \mathrm{pm}$

Record Book work

** Fresno Fair Parent Meeting DBD

Possible Dates 9-19 or 9-26

MANTOTORY FOR ALL

EXHIBITORS

Monday, September $23^{\text {rd }}$ 3:00pm

Meeting and Practice

Wednesday, September $25^{\text {th }}$

3:00pm

Work on Fair Displays and Prep

Equipment

Friday, September $27^{\text {th }} 3: 00 \mathrm{pm}$

Work on Fair Displays and Prep

Equipment

Monday, September $30^{\text {th }} 3: 00 \mathrm{pm}$

Meeting and Practice

SHERING DATES, SIGN UP FOR ONE OR THE OTHER

Saturday, October $5^{\text {th }}$ 8:00am

Shearing for Fair

Sunday, October $6^{\text {th }} 8: 00 \mathrm{am}$

Shearing for Fair

Monday, October $7^{\text {th }}$

Group of 3-4 Students

Fresno Fair Haul In

Eveyone else report after school
Tuesday, October $8^{\text {th }}$

At Fair all Day

Weigh In of Lambs

Wednesday, October $9^{\text {th }}$

At Fair all Day

Market Lamb Show

Thursday, October $10^{\text {th }}$

At Fair all Day

Lamb Showmanship

Friday, October $11^{\text {th }}$

Skeleton Crew of 2 Students

Everyone else reports to Fair after

school

Saturday, October $12^{\text {th }}$

At Fair all day

Junior Livestock Auction

Sunday, October $13^{\text {th }}$

Morning Feeding Crew

Evening Feeding Crew

Monday, October $14^{\text {th }}$

Haul Out Crew after school

Monday, October $21^{\text {st }} 3: 00 \mathrm{pm}$

Fresno Fair Clean Up

Record Book work

Fair Check Sign Off Sheets 


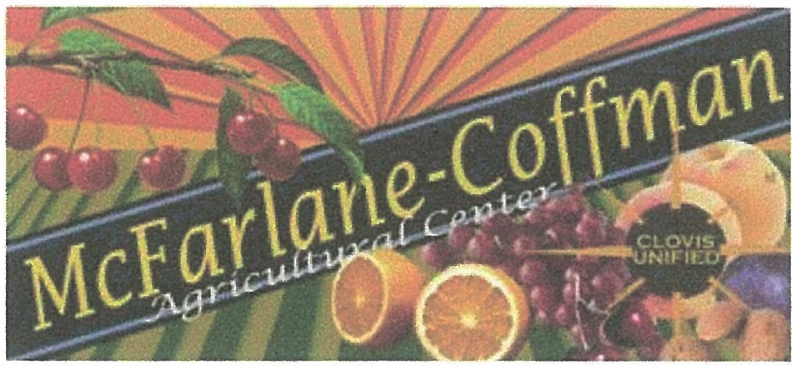

\section{McFarlane Coffman Agriculture Center FARM LABORATORY USE CONTRACT}

The Agriculture Farm Laboratory is an educational facility in Clovis Unified School District that may be utilized by students for keeping Supervised Agriculture Experience (SAE) projects.

\section{GENERAL RULES/CONDITIONS:}

1. This is a CUSD Facility and ALL CUSD rules and policies (including dress code) apply at all times when students are on the Agriculture Center grounds.

- Note that this includes after school hours, evenings, weekends, holidays, and summer vacation.

2. Students who participate in the Clovis East Animal Exhibitor program will agree to and sign the CUSD Code of Conduct for Student Participants in Extra-Curricular/Athletic Activities. (CUSD Board Policy 2505 Section D)

3. Students participating in the Clovis East Animal Exhibitor program will be placed on a "Three Strikes" contract with their advisor. Infractions will be noted and upon the fourth strikeable offense, a code of conduct hearing may be scheduled and the student may lose their opportunity to participate in the Exhibitor program and keep their project at the Agriculture Center.

4. Students who bring guests or family members to the Agriculture Center are responsible for explaining all District Rules and safety precautions to their visitors.

5. Because our Farm is an Educational Facility your project may be used/handled by Ag Teachers or other students for educational or teaching purposes. If you are not ok with this then don't keep your project at the Agriculture Center. All projects are kept here at your own risk.

6. Students, parents, or community members may not just "hang out" at the Agriculture Center.

7. Personal Items brought to the Agriculture Center for use with your project may require the approval of an advisor and should be stored in lockers or other appropriate areas. CUSD and the Agriculture Department are not responsible for loss of personal items. Personal items left out or behind may be discarded. 


\section{ELLIGIBILITY/ PROJECT APPROVAL}

1. In order for a student to participate in a Fair project of any kind the following eligibility requirements apply

- FFA Members grades 9-12 actively enrolled in the Clovis East Agriculture Program or CUSD graduates one year post graduation who have previously been active high school members will be allowed to have a project on the school farm.

- Students must maintain a 2.0 Grade Point Average at every grade reporting period in order to remain eligible to participate in the Exhibitor program. (CUSD Board Policy 2505 Section B)

- If a student falls below a 2.0 for any grading period they will be placed on probation until the next grading period.

- In order to be allowed to participate in the Exhibitor program and keep the project at the Ag Center Farm they must submit a progress report to their SAE advisor every two weeks and attend 3 study table sessions per week.

- If a student falls below a 2.0 GPA for two consecutive grading periods then they will be ineligible to participate in Fairs and Shows with Clovis FFA and will have to remove their project from the Ag Center Farm.

2. All projects brought to the school farm must receive the approval of the Ag Teacher in charge of that particular project area before a project is allowed on the farm.

3. In order for a project to be kept at the Agriculture Center Farm it must be Agricultural in nature.

\section{STUDENT EXPECTATIONS/RESPONSIBILITIES}

1. Students are responsible for the care and maintenance of their project. They will maintain their projects in a manner consistent with or exceeding the standards for that particular agriculture industry. Recommendations for care and management from the project advisor must be followed.

2. Students must keep proper records in their FFA Record Books on all projects kept at the Agriculture Center. The project advisor will check the students Record Book at least twice per year.

3. Students must have a plan for the growth and management of the project.

4. The use of any hazardous chemicals, scalpels, needles, and medications will only be used with the supervision of your project advisor.

5. All bills for feed, materials, or supplies must be paid in a timely manner as outlined by the project supervisor or Ag Teacher.

6. Complete at least 2 hours of farm clean up at the completion of any project to attempt to return the area used to its original clean condition.

7. Follow all specific rules and requirements associated with each individual project type as outlined by the project/species advisor.

8. Follow at all times the Code of Conduct that focuses on the six pillars of Character Counts. 


\section{ANIMAL PROJECTS:}

1. Students with animal projects are expected to uphold all of the responsibilities associated with that animal project as outlined by the Ag Department and Species Advisor.

2. Animals with no agricultural production purpose will not be allowed at the farm. Pets are not projects.

\section{General Animal Project Responsibilities:}

- Purchase your own feed for your animals.

- Proper feeding and watering (Including feeding times, feed quality, and feeding practices)

- Proper cleanliness of animal and immediate area.

- Proper cleaning and management of buildings and equipment.

- Making arrangements for animal feeding and care in case of your absence.

- Maintaining all health and safety standards, medicating sick livestock when necessary.

- Doing your fair share of work on the farm and contributing to the growth and image of our Educational Facility.

- Signing in for feeding, shifts worked, or meetings.

- Attending all scheduled practices and meetings as determined by the species advisor.

- Being actively involved in the growth and development of the animal by spending time working with or training the animal.

- Notify Ag Teachers or species advisor immediately of any problems with your animal or other animals on the farm.

- Breeding projects are only allowed with permission from a species advisor.

- You many not relocate or move animals without prior approval of the advisor.

- You may not euthanize or harvest any animal without permission and supervision from your species advisor, and only the most humane methods will be allowed.

\section{MARKET ANIMALS:}

- Projects classified as market animals must be fed and raised as in a manner consistent with or above the standards for commercially produced market animals.

- Market animals may not remain on the Agriculture Center grounds longer than 30 days following the Big Fresno Fair, unless special arrangements are made with the species advisor.

- Market animals that are sold but return to the school farm will remain under the care of the student who owned it until it is removed from the farm premises.

The Clovis Unified School District does not discriminate on the basis of race, color, disability, or national origin in admission or access to and treatment of employment in its programs and activities as required by Title VI, Title IX and Section 504. Complaints: Contact Dr. Cheryl Rogers, Human Resources, (559) 327-9308.

Favor de llamar a la oficina para assistencia en espanol.

Thov tiv tauj peb lub hoob kas yog xav tau kev pab nrog lus Hmoob. 


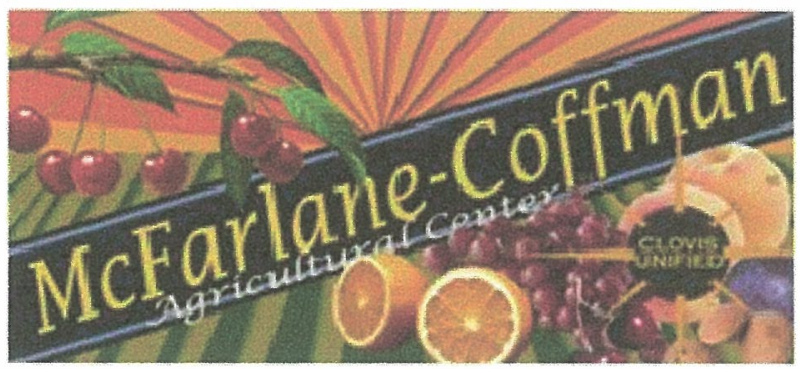

\section{McFarlane Coffman Agriculture Center FARM LABORATORY USE CONTRACT}

Agreement to follow the contract and rules as stated on pages 1-3 of this Farm Use Contract.

\section{AGREEMENT:}

\section{(Please Print Neatly)}

To provide a basis of understanding this agreement is established on and is Effective until This agreement covers the student's SAE in (Ending Date) and is between:

(Project Type) and

(Student Signature)

(Parent Signature)

(Teacher Signature)

\begin{tabular}{c}
\hline (Date) \\
\hline (Date) \\
\hline (Date)
\end{tabular}

CONTACT INFORMATION:

Student Phone Number

Parent Phone Number

Mailing Address

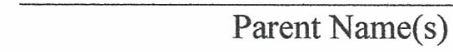

City
Zip

The Clovis Unified School District does not discriminate on the basis of race, color, disability, or national origin in admission or access to and treatment of employment in its programs and activities as required by Title VI, Title IX and Section 504. Complaints: Contact Dr. Cheryl Rogers, Human Resources, (559) 327-9308.

Favor de llamar a la oficina para assistencia en espanol.

Thov tiv tauj peb lub hoob kas yog xav tau kev pab nrog lus Hmoob. 


\section{McFarlane Coffman Agriculture Center FARM LABORATRY USE CONTRACT}

\section{THREE STRIKES FORM \\ DOCCUMENTATION OF VIOLATIONS:}

STUDENT NAME:

STRIKE 1

Date

Project Name

Advisor

Description of Violation and Corrective Action Taken

STRIKE 2

$\overline{\text { Date }} \overline{\text { Project Name }} \overline{\text { Advisor }}$

Description of Violation and Corrective Action Taken

\section{STRIKE 3}

$\overline{\text { Date }} \overline{\text { Project Name }} \overline{\text { Advisor }}$

Description of Violation and Corrective Action Taken

\section{STRIKE 4}

Date

Project Name

Advisor

Code of Conduct Hearing 


\title{
Clovis FFA
}

\section{Sheep Exhibitors Contract and Lamb Care Guide}

\author{
This contract discusses specific rules and guidelines associated with having a sheep SAE project \\ associated with the Clovis FFA Chapter and Clovis East Agriculture Department.
}

\section{Prior to Starting the Project:}

1. All sheep projects must receive the prior approval of Mrs. Knight. DO NOT go purchase a lamb you plan to associate with Clovis FFA without the approval of Mrs. Knight.

2. The budget page and the business agreement page of your record book should be completed prior to the start of the project.

3. Students with market lambs will be expected to attend one weekly meeting/practice with the sheep exhibitors group. A detailed schedule will be provided at the start of the project so students may plan their schedules accordingly.

4. Make sure you have the proper facility ready for your lamb
a. Clean pen with room to move around
b. Shade for the animal to stay cool/place to stay warm and dry when cold
c. No standing water or mud puddles in pen
d. Clean water bucket
e. Feeder (Don't feed animals off the ground)
f. Show Lamb Grain
g. Hay

\section{Feed and Care of your Project:}

\section{Specifics for Animals Kept at the Ag Center}

1. You will purchase your own grain for your market lamb project. We will provide community hay to those who keep their animals at the Ag Center; students will be billed equally for the hay used. The advisor will be responsible for purchasing and stocking hay. I don't recommend sharing grain because it can lead to problems.

2. You will be issued a supply locker and a feed locker. You must store feed and supplies in these lockers. Combination locks will be provided and are the only locks allowed for use.

3. Each exhibitor will have their animal assigned to a specific pen; you will share the back lot of the pen with another exhibitor.

4. You MUST sign in when you feed on your pen time sheet. Each pen has a clipboard with its own timesheet. If you don't sign in Mrs. Knight will assume that you missed a feeding.

5. You will have barn duty once or twice per month. Follow the barn duty schedule posted on the wall near the feed room and sign in when you complete barn duty.

a. BARN DUTY

i. A schedule will be created with all students who keep animals in the barn.

ii. Barn duty must be completed in the morning and in the evening the day you are assigned.

iii. Tasks that must be completed during barn duty:

1. Sweep the entire barn and scoop up and throw away dust and material swept up.

2. Pick up any trash in and around the barn.

3. Empty trash cans in the barn into the dumpster.

4. Roll up all hoses in the barn. 


\section{All Lamb Projects}

\section{Purchase a show quality lamb feed to feed your lamb}

a. Recommendations

i. High Noon by OH Kruse, available at A-1 Feed Store (Academy/Shaw)

ii. Showtime High Energy Lamb by Farmers Feed, available at the Hay Company (McKinley/DelRey)

b. Make sure you have feed on hand, don't wait till you run out to go get more. Lamb performance and health will suffer if you miss a feeding.

c. Inspect feed to make sure it is not dried out, or moldy.

\section{Have plenty of fresh clean water available for your lamb.}

a. Water buckets should be large enough so lambs don't run out too quickly, but not too big to easily dump out and clean.

b. Water must be filled and refreshed daily.

i. Dump old water and fill with fresh water daily!

1. Animal feces can get in water easily, and you would not want to drink water you pooped in!

ii. Clean and scrub water bucket weekly so algae does not grow in bucket.

c. Check water several times per day when it is really hot. Lambs can die quickly if they are without water.

3. Keep your lamb clean and comfortable.

a. Pen area must be cleaned once a week or more.

i. Rake up any manure and spilled hay and grain

b. Wet dirt pens down daily when it is hot or pens get dusty. Dust can cause coughing and lead to other problems like rectal prolapses. Don't get lamb wet though, can increase incidence of club lamb fungus.

c. Don't wash your lamb. The natural oils in their skin are good; washing them too much dries their skin out and can increase problems with fungus.

d. Make sure you lamb has shade and protection from the hot sun!

\section{Feed your lamb properly}

a. This is the most critical part of creating the best and most competitive market lamb.

b. Feed twice a day

i. Morning feedings must be done before 9:00am (lambs don't eat well if it's too hot)

ii. Evening feedings must be done after $5: 00 \mathrm{pm}$ but before $9: 00 \mathrm{pm}$

iii. Feeding times should be at 12 hour intervals; keeping consistent feeding times is what is best for the lamb.

c. Feed in a clean feeder

i. Never feed lambs off the ground, they may contract internal parasite infections!

ii. Clean old or uneaten feed from the feeder at each feeding. Scrub the feeders clean at least once a week.

iii. Remove the feeder from the pen after the lamb has eaten all the feed; this will help keep the feeder clean and you will have less scrubbing to do.

d. Feed show lamb grain and a small amount of roughage.

i. Start by feeding 1 pound $(16 \mathrm{oz}$.) of grain each feeding

1. Mrs. Knight will make recommendations on increasing and decreasing feed based on the weight and progress of your animal.

ii. Feed a good sized handful of roughage (Hay) both feedings

1. At the school farm we will feed Alfalfa Hay

iii. I recommend feeding grain first, then after the lamb eats the grain then provide the hay. 
1. This ensures the lamb will eat all the grain and not just select the hay first. The grain is what provides them with the most complete nutrition to grow and perform. The hay keeps their ruminant digestive tract healthy.

e. Weigh lamb regularly to monitor growth, finish and make needed feed adjustments.

i. Mrs. Knight will advise on needed feeding adjustments.

\section{Monitor your lamb for health problems}

a. Watch your lamb eat! This is when you may really notice health issues.

i. Lambs should get up and be excited to eat; they should go directly to the feed and start to eat when it is provided.

ii. Animals that don't get up or seem eager to eat may be sick.

iii. After the lamb eats then remove the feeder from the pen (cleanliness)

b. Watch for signs of illness and report to Mrs. Knight, I am here to help you!

i. Not getting up to eat

ii. Head down, moving slowly and lethargic behavior.

iii. Yellow, green, or lots of mucous on the nose (a little bit of clear is ok)

iv. Crusty or excessively watery eyes

v. Excessive coughing or hacking

vi. Scours (Diarrhea)

vii. Choking or Vomiting

viii. Limping or not using limbs

ix. Round patches on the wool or skin

x. Crusty scabs on the mouth or lips

xi. Swollen/discolored tongue

c. Learn how to take your lambs temperature; this can tell us a lot about how to treat a sick animal.

i. Taken rectally with a digital thermometer

1. Get a cheap one at the drug store; don't use the one your mom uses for you and your siblings!

ii. Normal Body Temperature $102-103^{0} \mathrm{~F}$

\section{Provide preventative care}

a. De-Worm lamb monthly

i. We will do this as a group at the Ag Center. Exhibitors with projects at home can have Mrs. Knight Help with deworming on a project visit.

b. Trim hooves when necessary.

\section{Practice Showmanship and spend time handling your animal}

a. Use care when catching your animal in the pen as to not injure them.

i. Move slowly and get animal in a corner to grab them by the head.

ii. Don't chase your animal.

b. Practice walking your animal several times a week.

i. Keep their heads help up when walking (establishes good habits for the show)

ii. You can use a rope or nylon halter when walking lambs

c. Practice Showmanship

i. Work on Bracing

ii. Get the animal used to having its legs touched and set up

d. Showmanship practice will be a part of our weekly meetings, students who keep projects at home will be expected to show their progress to Mrs. Knight during project visits. 


\section{Expectations for Clovis FFA Exhibitors}

1. Attend Required Meetings/Schedule Project Visits

a. Exhibitors who keep their animals at the Ag Center must attend the scheduled meetings and practices as provided on the schedule.

b. Exhibitors keeping animals at home must attend the required meetings as indicated on the schedule. They must also schedule a Monthly project visit with Mrs. Knight.

c. Be present at the Fair Contract and Entry Meeting

i. Students may not make entries into the fair under the Clovis FFA group without the signature and approval of Mrs. Knight

\section{Follow all CUSD rules and Policies while participating as a Clovis FFA Exhibitor}

a. Students who participate in the Clovis FFA as a Fair Exhibitor will agree to and sign the CUSD Code of Conduct for Student Participants in Extra-Curricular/Athletic Activities. (CUSD Board Policy 2505 Section D)

\section{Remain Eligible to Participate (Grades)}

a. Students must maintain a 2.0 Grade Point Average at every grade reporting period in order to remain eligible to participate as a Clovis FFA Fair Exhibitor.

(CUSD Board Policy 2505 Section B)

i. If a student falls below a 2.0 for any grading period they will be placed on probation until the next grading period.

ii. In order to be allowed to participate as an exhibitor and keep the project at the Ag Center Farm they must submit a progress report to their SAE advisor every two weeks and attend 3 study table sessions per week.

iii. If a student falls below a 2.0 GPA for two consecutive grading periods then they will be ineligible to participate in Fairs and Shows with Clovis FFA and will have to remove their project from the Ag Center Farm.

4. Care for your project as outlined in this document

a. Students are responsible for the care and maintenance of their project.

b. They will maintain their projects in a manner consistent with or exceeding the standards for that particular agriculture industry.

c. Recommendations for care and management from the project advisor should be followed.

5. Maintain an up to date FFA Record Book

a. Students must keep proper records in their FFA Record Books on all projects associated with the Clovis FFA.

b. The project advisor will check the students Record Book at least twice per year.

\section{Participate with the group for the Fresno Fair}

a. Attend meetings and contribute to the making of the displays and educational material prior to the fair.

b. Coordinate transportation and haul in/out times with Mrs. Knight for exhibitors who keep their lambs at home.

c. Have a positive attitude and maintain a team focus when showing at the fair.

d. Attend the Mandatory Fresno Fair Meeting with a parent.

e. Complete barn duty and follow the assigned Fresno Fair Schedule provided by Mrs. Knight.

7. Pay any fees associated with the Clovis FFA sheep group

a. All exhibitors will pay a $\$ 20$ Fresno Fair Supply Fee, this covers:

i. Shavings for the pens

ii. Show supplies

iii. Use of Clippers and clipper blade sharpening 


\section{Complete ALL Items on the Fair Check sign off sheet prior to Winter Break to receive a Fair Payment}

Check.

a. Students will not receive a Fair Check until they complete the following items prior to winter Break.

i. Completed FFA Record Book for their Lamb Project

ii. All Bills and Fines Paid

iii. Thank You Cards (Show to Advisor BEFORE Mailing)

1. Ag Boosters (They give us money for Fair Supplies each year and make us food)

2. Lamb Buyer and bump or top off bidders

iv. Degree Application or planning check sheet for the next level FFA degree.

v. If animals are kept at the $\mathrm{Ag}$ center then:

1. Two hours of farm clean up

2. Pen and lockers cleaned out

\section{Failure to Meet Expectations or Provide Proper Lamb Care}

Having a live animal project associated with the Clovis FFA is a big responsibility. Showing at the Fair as part of the Clovis FFA group is a privilege. Students who do not meet the expectations as outlined in this document may lose the privilege to show an animal with the Clovis FFA group.

\section{STRIKE CONTRACT}

a. Students participating as a Clovis FFA Sheep Exhibitor will be placed on a "Three Strikes" contract with their advisor. Infractions will be noted and upon the fourth strikeable offense, a code of conduct hearing may be scheduled and the student may lose their opportunity to participate in the SAE program and keep their project at the Agriculture Center, or Show as a member of the Clovis FFA at Fairs and Shows.

i. Receiving a fourth strike will warrant a code of conduct hearing.

b. Actions that constitute a STRIKE

i. Any deviation of the Expectations for Exhibitors or Lamb care as stated in this document.

ii. Missed Meetings

c. Students will be issued warning(s) prior to receiving a strike. When a strike is given both the student and parent will be notified.

\section{FREE PASSES}

a. Students get 3 "Free Passes" from project start to finish. These can be used to excuse an absence to a meeting or practice. There are no EXCUSED absences from practices or meetings.

b. Once the 3 "Free Passes" are used for whatever the reason, the student will be given a strike for continued absences at practices or meetings.

c. Being late or leaving early from meetings/practices can result in a "Free Pass" being used or a strike

The Clovis Unified School District does not discriminate on the basis of race, color, disability, or national origin in admission or access to and treatment of employment in its programs and activities as required by Title VI, Title IX and Section 504.

Complaints: Contact Dr. Cheryl Rogers, Human Resources, (559) 327-9308.

Favor de llamar a la oficina para assistencia en espanol.

Thov tiv tauj peb lub hoob kas yog xav tau kev pab nrog lus Hmoob. 


\section{CLOVIS FFA SHEEP EXHIBITORS CONTRACT AGREEMENT}

Agreement to uphold and follow the

Clovis FFA Sheep Exhibitors contract and lamb care guide as outlined in

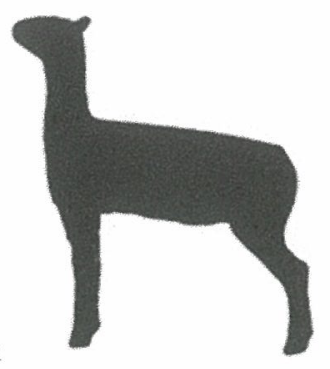

Pages 1-5 of this document.

(Please Print Neatly)

To provide a basis of understanding this agreement is established on and is

Effective until

This agreement covers the student's SAE in and is between:

(Project Type)

and

(Student's name)

(Teacher's name)

(Student Signature)

(Date)

(Parent Signature)

(Date)

(Teacher Signature)

(Date)

\section{CONTACT INFORMATION:}

Student Phone Number

Parent Phone Number

Parent Name(s)

Mailing Address

City

Zip

The Clovis Unified School District does not discriminate on the basis of race, color, disability, or national origin in admission or access to and treatment of employment in its programs and activities as required by Title VI, Title IX and Section 504. Complaints: Contact Dr. Cheryl Rogers, Human Resources, (559) 327-9308.

Favor de llamar a la oficina para assistencia en espanol.

Thov tiv tauj peb lub hoob kas yog xav tau kev pab nrog lus Hmoob. 


\section{Clovis FFA \\ Sheep Exhibitors Contract}

\section{THREE STRIKES FORM \\ DOCUMENTATION OF VIOLATIONS:}

STUDENT NAME:

STRIKE 1

$\overline{\text { Date }} \overline{\text { Project Name }} \overline{\text { Advisor }}$

Description of Violation and Corrective Action Taken

STRIKE 2

$\overline{\text { Date }} \overline{\text { Project Name }} \overline{\text { Advisor }}$

Description of Violation and Corrective Action Taken

\section{STRIKE 3}

$\overline{\text { Date }} \overline{\text { Project Name }} \overline{\text { Advisor }}$

Description of Violation and Corrective Action Taken

STRIKE 4

Date

Project Name

Advisor

Code of Conduct Hearing 